\title{
Inorganic-nucleic acid conjugates for luminescence-sensing and imaging applications
}

by

Elyse Diane Bernard

A thesis submitted to the Faculty of Graduate and Postdoctoral Affairs in partial fulfillment of the requirements for the degree of

Doctor of Philosophy

in

Chemistry

Carleton University

Ottawa, Ontario

(C) 2012, Elyse Diane Bernard 
Library and Archives

Canada

Published Heritage

Branch

395 Wellington Street

Ottawa ON K1A ON4

Canada
Bibliothèque et

Archives Canada

Direction du

Patrimoine de l'édition

395 , rue Wellington

Ottawa ON K1A ON4

Canada
Your file Votre référence

ISBN: 978-0-494-93665-8

Our file Notre référence

ISBN: $978-0-494-93665-8$
NOTICE:

The author has granted a nonexclusive license allowing Library and Archives Canada to reproduce, publish, archive, preserve, conserve, communicate to the public by telecommunication or on the Internet, loan, distrbute and sell theses worldwide, for commercial or noncommercial purposes, in microform, paper, electronic and/or any other formats.

The author retains copyright ownership and moral rights in this thesis. Neither the thesis nor substantial extracts from it may be printed or otherwise reproduced without the author's permission.
AVIS:

L'auteur a accordé une licence non exclusive permettant à la Bibliothèque et Archives Canada de reproduire, publier, archiver, sauvegarder, conserver, transmettre au public par télécommunication ou par l'Internet, prêter, distribuer et vendre des thèses partout dans le monde, à des fins commerciales ou autres, sur support microforme, papier, électronique et/ou autres formats.

L'auteur conserve la propriété du droit d'auteur et des droits moraux qui protege cette thèse. $\mathrm{Ni}$ la thèse ni des extraits substantiels de celle-ci ne doivent être imprimés ou autrement reproduits sans son autorisation.
In compliance with the Canadian Privacy Act some supporting forms may have been removed from this thesis.

While these forms may be included in the document page count, their removal does not represent any loss of content from the thesis.
Conformément à la loi canadienne sur la protection de la vie privée, quelques formulaires secondaires ont été enlevés de cette thèse.

Bien que ces formulaires aient inclus dans la pagination, il n'y aura aucun contenu manquant. 


\section{Abstract}

Aptamers are synthetic, short, single-stranded nucleic acids that are selected through an in vitro process to bind to their targets with high affinity and selectivity. The use of aptamers in many areas, from sensing to medical applications, is being extensively researched. The focus of this work was the development of inorganic-nucleic acid conjugates for use as targeted contrast agents in magnetic resonance imaging (MRI) and in luminescence-based sensors.

More than $30 \%$ of magnetic resonance imaging procedures are performed using contrast agents (CAs) to increase the contrast, and therefore the diagnostic value, of the images. In a proof-of-concept study, the 15 mer thrombin aptamer was conjugated to diethylenetriaminepentaacetic (DTPA) dianhydride to form monoamide and bisamide derivatives of the linear open-chain chelate present in the commonly used gadolinium(III)-based general CA Magnevist $B$. These aptamer-gadolinium(III) singleand double-conjugates, in which one or two aptamer strands, respectively, were covalently bound to DTPA, were tested as potential targeted CAs. Conjugates including a 15 - or 30 -thymine spacer between the aptamer sequence and the chelator were also prepared for the investigation of the possible effects of crowding upon target-binding. The relaxation properties of the conjugates alone and in the presence of either a control or target protein at $9.4 \mathrm{~T}$ were determined and used to select systems for further study at 1.5 T. Double-conjugates were tested in both 1:1 and 1:2 ratios with each respective protein.

The relaxivity values of the conjugate alone and in the presence of either a control or target protein were obtained and compared. The $15 \mathrm{mer}$ aptamer-DTPA-Gd(III) conjugate experienced a $35 \pm 4 \%$ enhancement of relaxivity at $9.4 \mathrm{~T}$ between control and 
target protein cases, and a $20 \pm 1 \%$ enhancement at $1.5 \mathrm{~T}$. The double-conjugate prepared where a 15-thymine spacer was included exhibited enhancements of $47 \pm 15$ and $56 \pm 4 \%$ at 9.4 and $1.5 \mathrm{~T}$, respectively.

A platinum(II) complex that displayed concentration-dependent luminescent properties was prepared for eventual use as a probe to study aptamer folding upon target binding. Preliminary work on the conjugation of this complex to DNA was performed. The possibility of preparing a family of related luminescent platinum(II) complexes was explored. 


\section{Acknowledgements}

I need to begin by thanking my supervisor Dr. Maria DeRosa for being a guiding force throughout my years in her lab group. A cheerleader when my research was not going as expected and honestly excited by what each new development could mean, Maria was very supportive and inspiring.

Thank you to my family. Mom, Dad, and Laura (and the dogs, of course!), you were always a supportive presence despite the distance between us. Always comforting and encouraging when I called to talk, even if you didn't fully understand what I was so upset or happy about. A special thanks to my dad for reading over my thesis.

A big thank you to my flatmate of nearly five years; Becky, we went through a lot while living together and I think that our understanding and encouragement of one another played a huge role in getting me to this point.

To my DeRosa labmates over the years, you enhanced my grad school experience beyond what I could have imagined before starting at Carleton. Dave and Ryan: in the beginning we were the three musketeers and I really enjoyed our sibling-like banter. Daffy, all our shared gym class experiences, talking about characters in books like they are real people, and living with a fellow Leaf fan has been a blast! Mike and Tariq, I loved wandering over to chat with you guys since I could always count on you to make me laugh (with you not at you!). Amanda, my swimming and movie buddy - we can finally get back to doing these things! Maureen, our long lab talks and commiseration when research wasn't going our way helped get me through, along with our shared love of certain musicals. Erin, my original after-hours buddy, and Emily, a new addition to the late-in-the-lab parties, supporting each other through long days (and nights) in the lab 
was a whole lot more fun because of the two of you! Yasir, thanks for always telling me to just get on with it - sometimes that's just what you need to hear. Thanks to Monica, an honourary member of the DeRosa group, for the lovely chats and help with organic chemistry.

To my friend Jessica, thank you for always listening and for your encouragement.

Thank you to Sara for the collaborative work on the modified acetylacetone ligand. Thanks to Alaa and Uyen for help with silanizing capillary tubes.

Thank you to Dr. Jeff Manthorpe for allowing me to use dry THF from your everrunning still. I greatly appreciated your generosity.

Thanks to Keith Bourque for all the help with the NMR experiments and to Karunanithi Rajamanickam and Eve Tsai at The Ottawa Hospital/Ottawa Hospital Research Institute for running samples at low field.

Finally, thanks to Dr. Rocio Aranda-Rodriguez at Health Canada for granting me time off to complete my thesis. Your patience and understanding are much appreciated.

To anyone that $\mathbf{l}$ am forgetting, please excuse this tired thesis-writer. 


\section{Table of Contents}

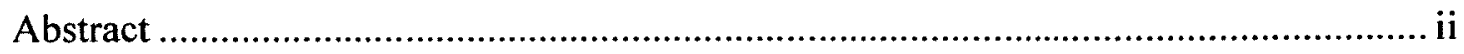

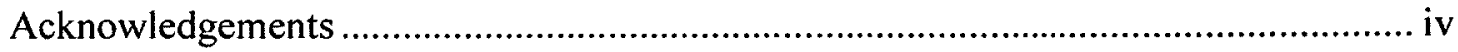

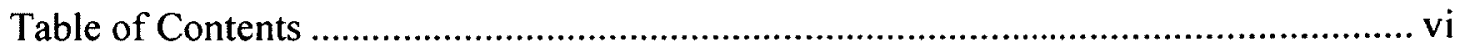

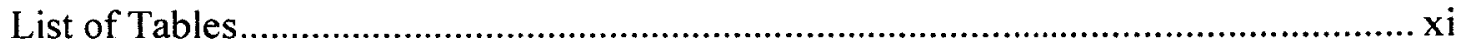

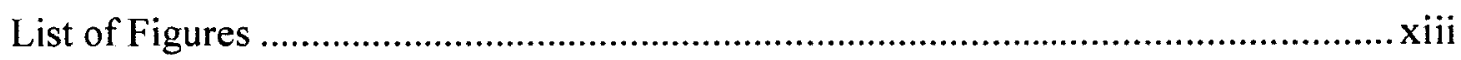

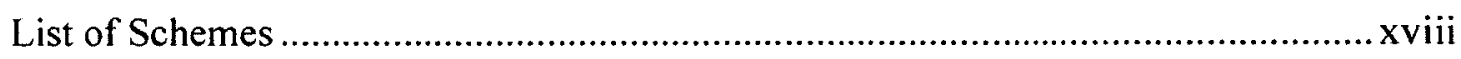

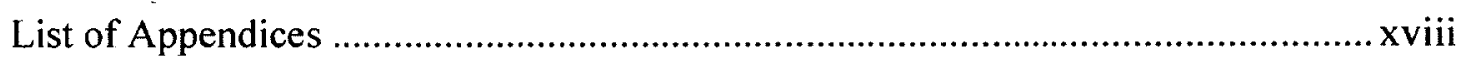

List of Abbreviations.........................................................................................

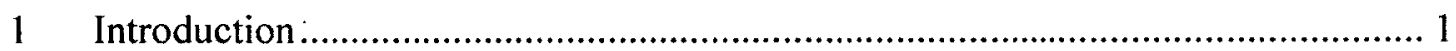

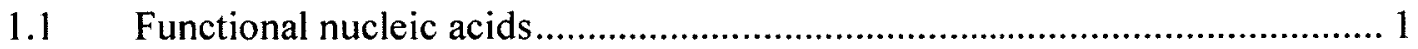

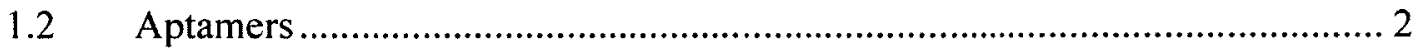

1.2.1 Selection of aptamers using SELEX …............................................... 2

1.2.2 Aptamers versus antibodies............................................................. 4

1.2.3 The 15mer thrombin-binding DNA aptamer....................................... 6

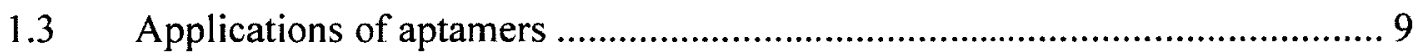

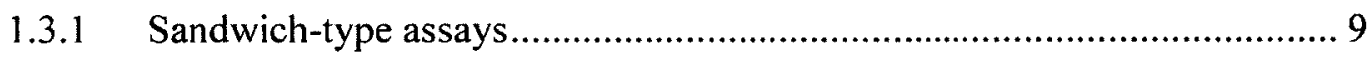

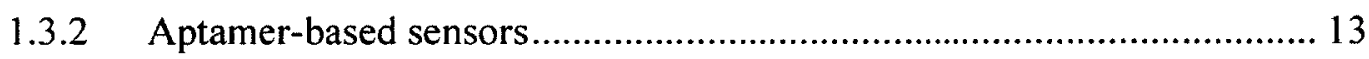

1.3.3 In vivo applications of aptamers ................................................... 17

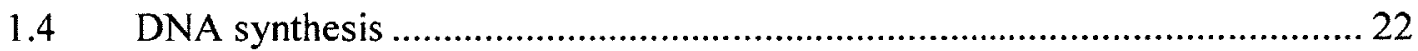

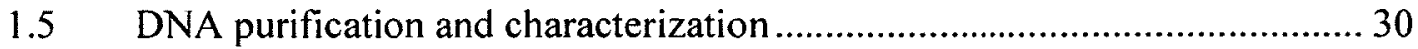

1.5.1 Polyacrylamide gel electrophoresis............................................... 30

1.5.2 Reversed-Phase High-Performance Liquid Chromatography (RPHPLC) .................................................................................... 33

1.5.3 Ultraviolet-Visible (UV-vis) Spectrophotometry................................ 35

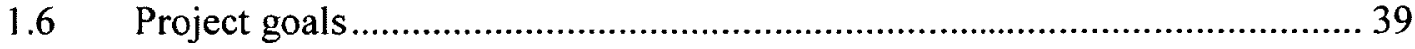

2 Singly-labelled DNA-DTPA conjugates....................................................... 40

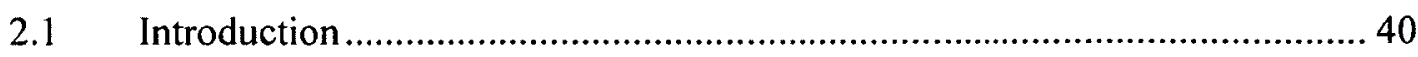

2.1.1 Magnetic resonance imaging (MRI) background theory ..................... 40

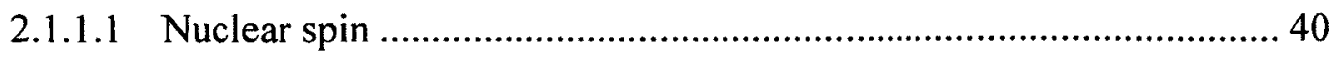

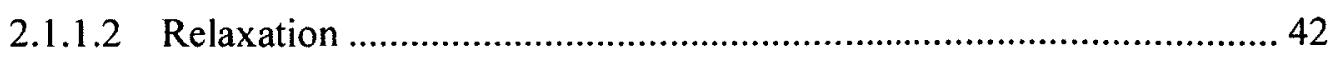


2.1.1.3 Dipole-dipole relaxation mechanisms ................................................ 46

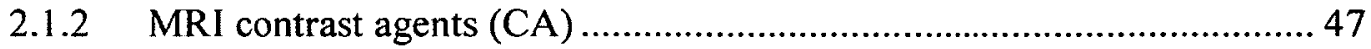

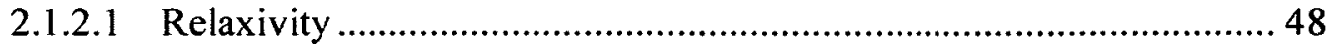

2.1.2.2 Factors that influence the relaxivity of MRI CAs................................ 49

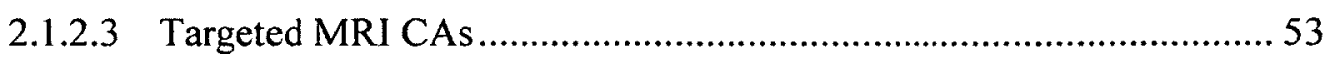

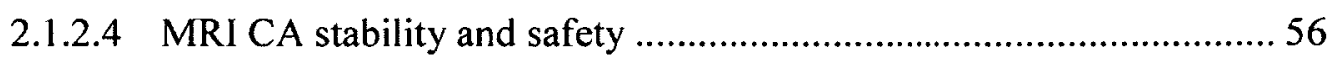

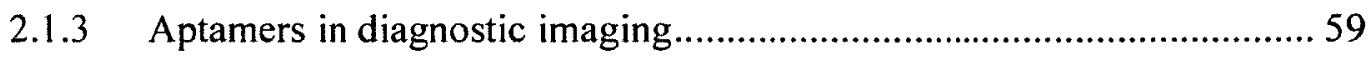

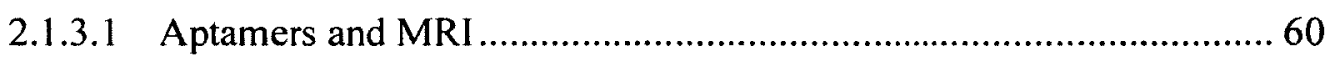

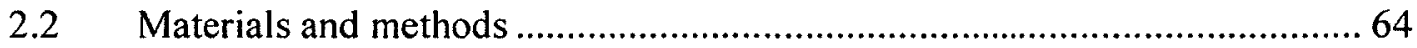

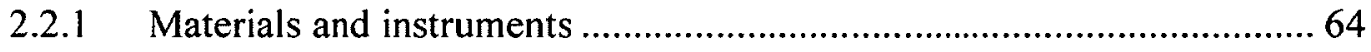

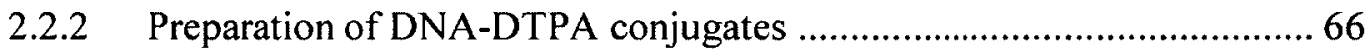

2.2.3 Purification of DNA-DTPA single-conjugates .........................................67

2.2.4 Chelation of Gd(III) with DNA-DTPA single-conjugates...................... 70

2.2.5 Mass spectrometric confirmation of DNA-DTPA single-conjugates ..... 73

2.2.6 Preparation of 15 and 30 polyA oligonucleotides................................... 73

2.2.7 Melting studies on the DNA-DTPA single-conjugates............................ 74

2.2.8 In vitro stability of DNA-DTPA single-conjugates: ................................. 74

2.2.8.1 Stability in serum at $37^{\circ} \mathrm{C}$ by the xylenol orange test ........................ 74

2.2.8.2 Stability in buffer at $37^{\circ} \mathrm{C}$ by the xylenol orange test ....................... 75

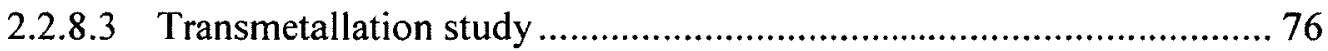

2.2.9 Preparation of samples for $T_{1}$ experiments at high field (9.4 Tesla)....... 77

2.2.10 Preparation of samples for $T_{1}$ experiments at low field (1.5 and 3

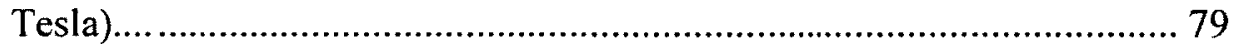

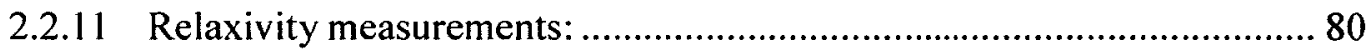

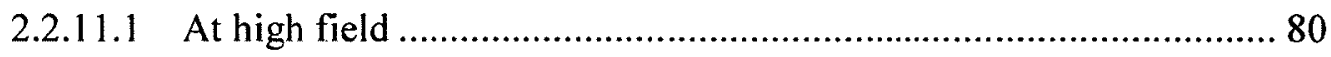

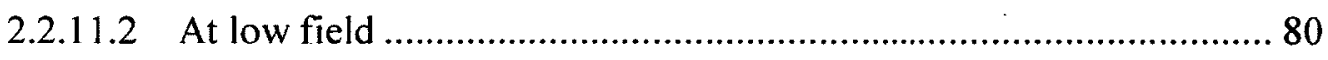

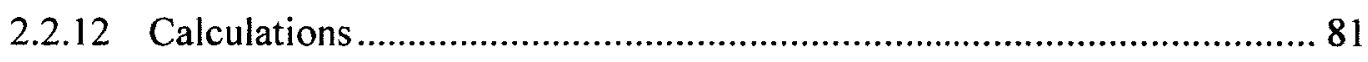

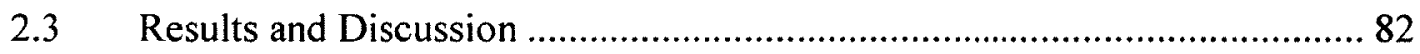

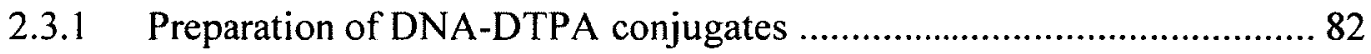

2.3.2 Chelation of Gd(III) with DNA-DTPA conjugates.................................. 85

2.3.3 Preparation of 15 and 30 polyA oligonucleotides................................... 94

2.3.4 Melting Studies ................................................................................ 95

2.3.5 In vitro stability .............................................................................. 97

2.3.5.1 Stability in serum at $37^{\circ} \mathrm{C}$ by the xylenol orange test....................... 97 
2.3.5.2 Stability in buffer at $37^{\circ} \mathrm{C}$ by the xylenol orange test ................. 100

2.3.5.3 Transmetallation study ..................................................... 100

2.3.6 $T_{1}$ experiments and relaxivity data at high field (9.4 Tesla) ................ 105

2.3.7 $\mathrm{T}_{1}$ experiments and relaxivity data at low field ( 1.5 Tesla) ................ 124

2.3.8 Conclusions and Future Work........................................................ 129

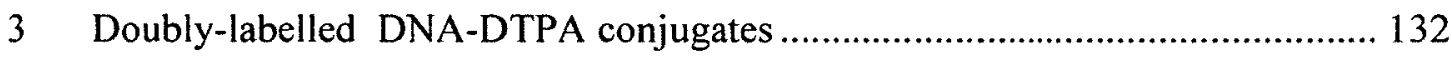

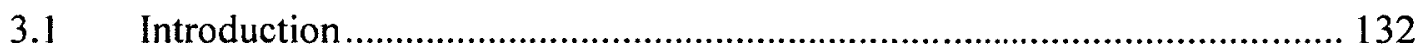

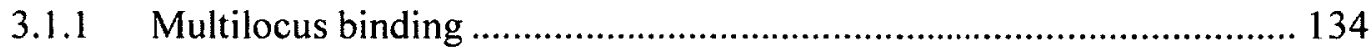

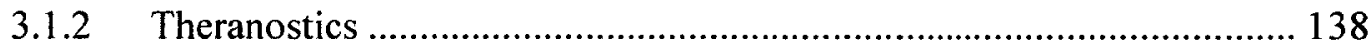

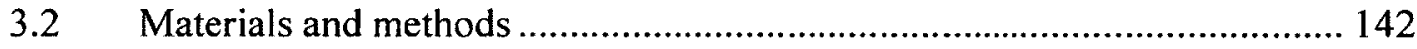

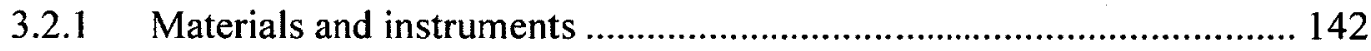

3.2.2 Synthesis of DNA-DTPA conjugates............................................. 142

3.2.3 Purification of DNA-DTPA double-conjugates ............................... 146

3.2.4 Chelation of Gd(III) with DNA-DTPA double-conjugates................. 147

3.2.5 Mass spectrometric confirmation of DNA-DTPA double-conjugates.. 147

3.2.6 Preparation of 15 and 30 polyA oligonucleotides............................. 147

3.2.7 Melting studies on the DNA-DTPA double-conjugates ..................... 148

3.2.8 In vitro stability of DNA-DTPA double-conjugates: Transmetallation

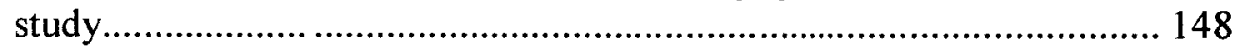

3.2.9 Preparation of samples for $T_{1}$ experiments at high field (9.4 Tesla).... 149

3.2.10 Preparation of samples for $T_{1}$ experiments at low field (1.5 Tesla)..... 150

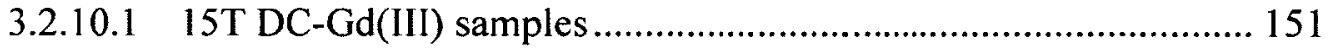

3.2.10.2 DC-Gd(III) samples ......................................................... 151

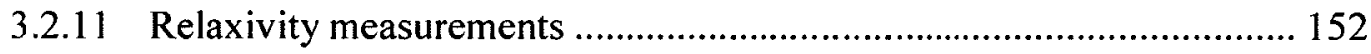

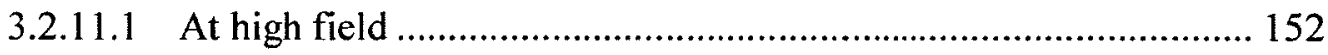

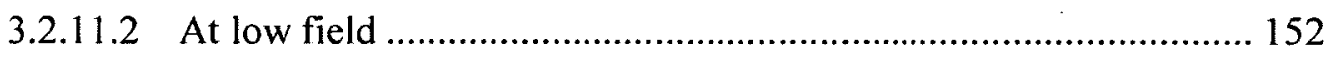

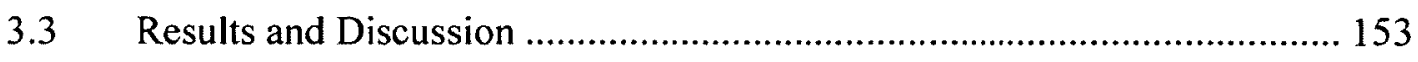

3.3.1 Preparation of DNA-DTPA double-conjugates ................................ 153

3.3.2 Comparison of reaction methods and DNA-DTPA conjugate relative

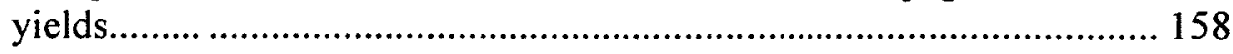

3.3.3 Preparation of DNA-DTPA double-conjugates for further

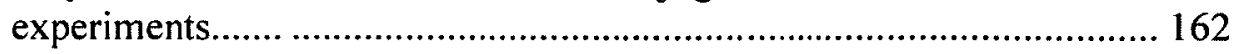

3.3.4 In vitro stability of DNA-DTPA double-conjugates: Transmetallation

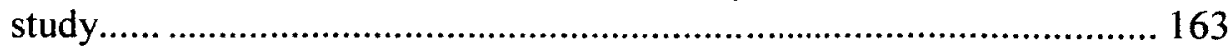


3.3.5 $T_{1}$ experiments and relaxivity data at high field (9.4 Tesla) ................ 166

3.3.6 $T_{1}$ experiments and relaxivity data at low field (1.5 Tesla) ................ 178

3.3.6.1 Experiments on 15T DC-Gd(III) ................................................ 178

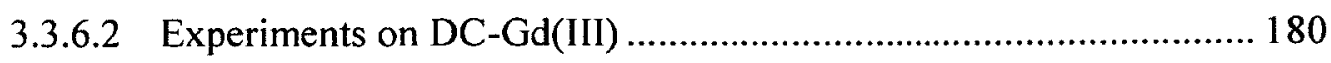

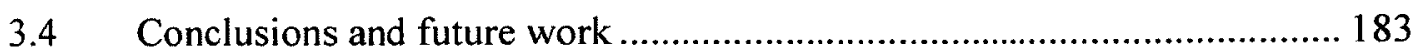

4 Synthesis and characterization of a luminescent platinum(II) complex and its

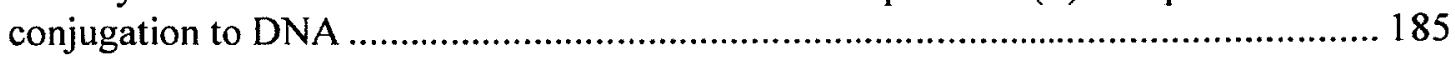

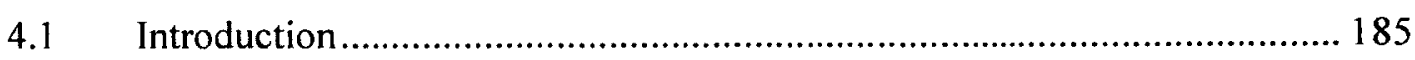

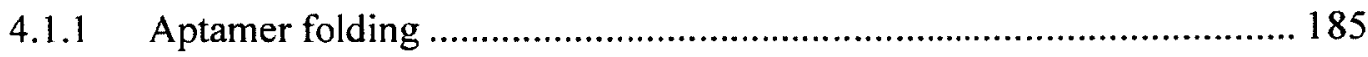

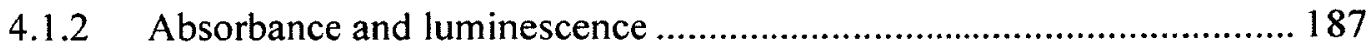

4.1.2.1 Fluorescence resonance energy transfer (FRET) ......................... 189

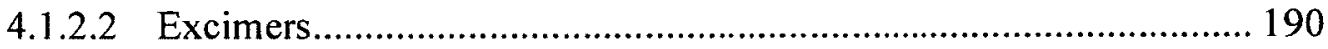

4.1.3 Luminescent probes for the study of aptamer folding upon target

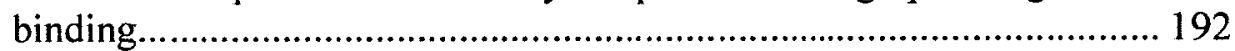

4.1.4 Excimer-forming platinum(II) complexes ...................................... 194

4.1.5 Conjugation through peptide coupling techniques.............................. 196

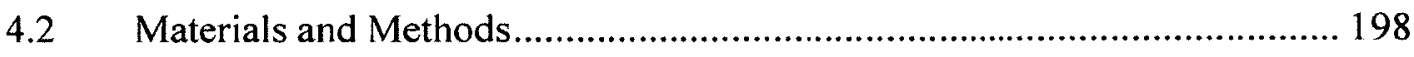

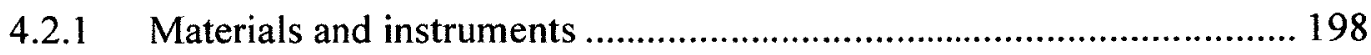

4.2.2 Synthesis of $\mathrm{Pt}(\mathrm{II}) \mu$-dichloro-bridged dimers ................................. 199

4.2.2.1 Synthesis of dimers with 2-phenylpyridine and 2-phenylquinoline ligands..................................................................................... 199

4.2.2.2 Synthesis of a modified phenylpyridine ligand and dimers containing this $2-(2,4$-difluorophenyl)pyridine............................................... 200

4.2.3 Synthesis of the modified acetylacetone ligand (acac-COOH): 8,10 dioxoundecanoic acid ................................................................ 201

4.2.4 Synthesis of luminescent Pt(II) complexes ....................................... 204

4.2.4.1 Synthesis, purification, and characterization of 8,10-dioxoundecanoic acid-2-phenylpyridylplatinum(II); (ppy) Pt(acac-COOH) .............. 204

4.2.4.2 Synthesis and purification attempts for 8,10-dioxoundecanoic acid-2phenylquinolylplatinium(II); (pq) $\mathrm{Pt}(\mathrm{acac}-\mathrm{COOH})$....................... 206

4.2.4.3 Synthesis, purification, and characterization of 8,10-dioxoundecanoic acid-(2-(2,4-difluorophenyl)pyridyl)platinum(II); (2,4-dfppy)Pt(acac-

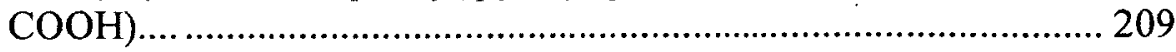

4.2.5 Absorbance and luminescence studies ............................................ 211

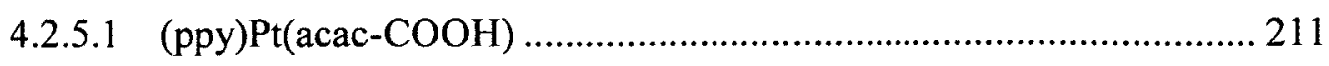

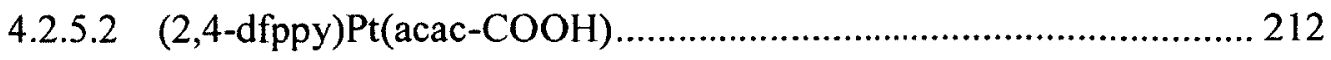


4.2.6 Conjugation of (ppy) Pt(acac-COOH) to the 15 mer thrombin-binding aptamer.

4.2.7 Conjugation of (ppy) Pt(acac-COOH) to complementary strands of DNA

4.2.7.1 Conjugation to a 5 '-amino-modified strand ................................ 215

4.2.7.2 Conjugation to a 3 '-amino-modified strand ................................... 219

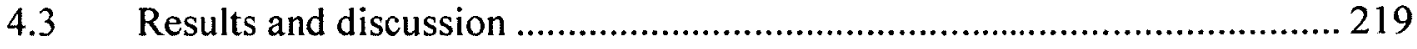

4.3.1 Synthesis of Pt(II) $\mu$-dichloro-bridged dimers ................................... 219

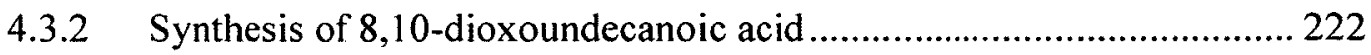

4.3.3 Synthesis of luminescent $\mathrm{Pt}(\mathrm{II})$ complexes ....................................... 225

4.3.3.1 General synthetic route ....................................................... 225

4.3.3.2 Synthesis, purification, and characterization of 8,10-dioxoundecanoic acid-2-phenylpyridylplatinum(II); (ppy)Pt(acac-COOH) .............. 226

4.3.3.3 Synthesis and purification attempts for 8,10-dioxoundecanoic acid-2phenylquinolylplatinum(II); (pq)Pt(acac-COOH) ....................... 230

4.3.3.4 Synthesis, purification, and characterization of 8,10-dioxoundecanoic acid-(2-(2,4-difluorophenyl)pyridyl)platinum(II); (2,4-dfppy)Pt(acac-

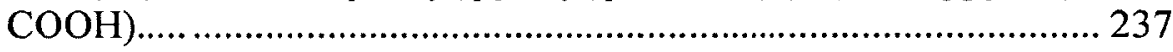

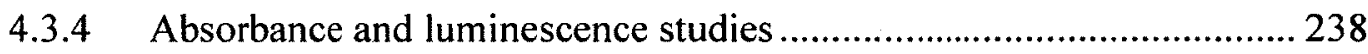

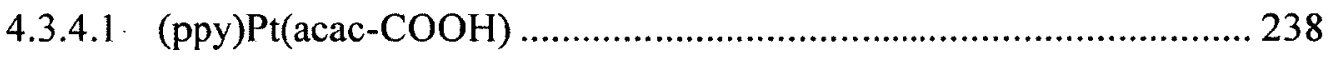

4.3.4.2 (2,4-dfppy)Pt(acac-COOH) ................................................... 243

4.3.5 Conjugation of (ppy) Pt(acac-COOH) to the $15 \mathrm{mer}$ thrombin-binding aptamer..

4.3.6 Conjugation of (ppy)Pt(acac-COOH) to complementary strands of DNA

4.3.6.1 Conjugation to a 5'-amino-modified strand …............................. 251

4.3.6.2 Conjugation to a 3'-amino-modified strand .................................. 262

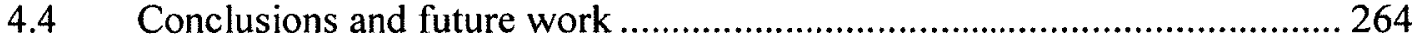

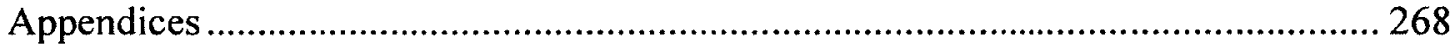

Appendix A Supporting data for singly-labelled DNA-DTPA conjugates ........................268

Appendix B Supporting data for double-labelled DNA-DTPA conjugates .....................2.285

Appendix C Supporting data for work with Pt(II) complexes.........................................294

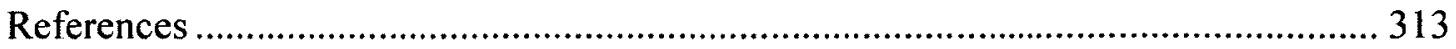




\section{List of Tables}

Table 1.1 Wavelengths of maximum absorbance $\left(\lambda_{\max }\right)$ and molar absorptivities at 260 $\mathrm{nm}\left(\varepsilon_{260}\right)$ for DNA nucleoside-5'-monophosphates.

Table 2.1 Recipes for the preparation of two polyacrylamide gels.

Table 2.2 Values used in calculations of theoretical relaxivities. ${ }^{124,125}$

Table 2.3 Relaxivity Data for Magnevist ${ }^{\circledR}$ and SC-Gd(III) at $9.4 \mathrm{~T}, \mathrm{pH} 7.4$ and $25^{\circ} \mathrm{C}$ in silanized tubes.

Table 2.4 Relaxivity Data for $15 \mathrm{~T} \mathrm{SC-Gd(III)} \mathrm{at} 9.4 \mathrm{~T}, \mathrm{pH} 7.4$ and $25^{\circ} \mathrm{C}$ in silanized tubes.

Table 2.5 Relaxivity Data for $30 \mathrm{~T} \mathrm{SC-Gd(III)} \mathrm{at} 9.4 \mathrm{~T}, \mathrm{pH} 7.4$ and $25^{\circ} \mathrm{C}$ in silanized tubes.

Table 2.6 Relaxivity Data for Magnevist ${ }^{\circledR}$ and SC-Gd(III) at $1.5 \mathrm{~T}, \mathrm{pH} 7.4$ and $25^{\circ} \mathrm{C}$.

Table 3.1 CTAB precipitation guidelines.

Table 3.2 Relaxivity Data for Magnevist ${ }^{\circledR}$ and DC-Gd(III) at $9.4 \mathrm{~T}, \mathrm{pH} 7.4$ and $25^{\circ} \mathrm{C}$. 168

Table 3.3 Relaxivity Data for $15 \mathrm{~T} \mathrm{DC-Gd(III)} \mathrm{at} 9.4 \mathrm{~T}, \mathrm{pH} 7.4$ and $25^{\circ} \mathrm{C}$. 173

Table 3.4 Relaxivity Data for 30T DC-Gd(III) at $9.4 \mathrm{~T}, \mathrm{pH} 7.4$ and $25^{\circ} \mathrm{C}$. 176

Table 3.5 Relaxivity Data for $15 \mathrm{~T} \mathrm{DC-Gd(III)} \mathrm{at} 1.5 \mathrm{~T}, \mathrm{pH} 7.4$ and $25^{\circ} \mathrm{C}$. 179

Table 3.6 Relaxivity Data for DC-Gd(III) at $1.5 \mathrm{~T}, \mathrm{pH} 7.4$ and $25^{\circ} \mathrm{C}$. 181

Table 4.1 Solvent conditions for successful purification of (ppy) Pt(acac-COOH) using silica gel chromatography 206

Table 4.2 Conditions used for breaking $\mu$-dichloro(pq)Pt(II) dimers. 207

Table 4.3 Excitation and emission wavelengths for (ppy)Pt(acac-COOH) solutions of different concentrations.

Table 4.4 Conditions used for RP-HPLC analysis of crude Pt(II) complex-TBA conjugate. 214

Table 4.5 Concentrations for luminescence measurements on DNA isolated using RPHPLC.

Table 4.6 Conditions for RP-HPLC analysis of crude Pt(II) complex-DNA following solid-phase reactions. Note that the TEAA buffer was prepared with $5 \% \mathrm{MeCN}$, so the fraction of $\mathrm{MeCN}$ could be considered to be $5 \%$ higher than the value recorded here. . 218

Table 4.7 Parameterization summary for synthesis and purification of 8,10 dioxoundecanoic acid-2-phenylpyridylplatinum(II); (ppy)Pt(acac-COOH) 227

Table 4.8 Parameterization summary for synthesis of 8,10-dioxoundecanoic acid-2phenylquinolylplatinum(II); (pq)Pt(acac-COOH) 234 
Table 4.9 Purification strategies for (pq)Pt(acac-COOH). The $5.57 \mathrm{ppm}$ peak in $\mathrm{CDCl}_{3}$ was used as the predominant complex peak for comparison......................................... 235

Table 4.10 Summary of MS results for Pt(II) complex-DNA samples. .................... 249

Table 4.11 Comparison of $\mathrm{m} / \mathrm{z}$ values found for $\mathrm{Pt}(\mathrm{II})$ complex-DNA band cut from a polyacrylamide gel with the calculated $\mathrm{m} / \mathrm{z}$ value differences from that of amino-

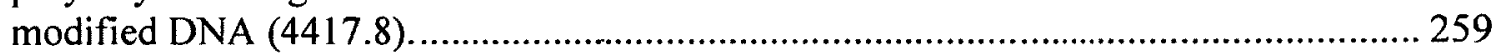

Table 4.12 Comparison of $\mathrm{m} / \mathrm{z}$ values found for DNA-acac-COOH with the calculated

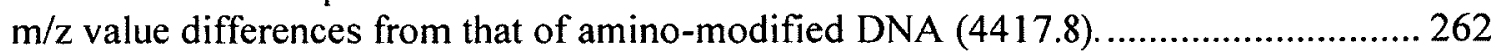

Table 4.13 Summary of MS results for possible 3'-Pt(II) complex-DNA following RP-

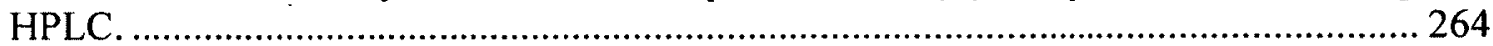




\section{List of Figures}

Figure 1.1 Systematic Evolution of Ligands by EXponential enrichment (SELEX). See text for details. ${ }^{23}$

Figure 1.2 Structure of the $15 \mathrm{mer}$ thrombin-binding DNA aptamer. $\mathrm{M}^{+}$represents a cation that is present to stabilize the G-quadruplex. Modified from references 46 and 47.

Figure 1.3 Structure of a G-tetrad stabilized by a cation. $\mathrm{R}$ represents the sugarphosphate backbone.'

Figure 1.4 Design of aptamer-antibody sandwich ELISA for MUC1-5TR tumour marker detection. Extravidin was used to coat the wells of a 96-well plate for the subsequent immobilization a 5'-biotinylated DNA aptamer selected against MUC1-5TR. MUC1-5TR is a recombinant protein that was prepared containing five tandem repeats of the variable tandem repeat of the $\mathrm{MUCl}$ protein. An anti-MUCl antibody (Ab) was used as a primary antibody and detection was achieved through the use of a secondary $\mathrm{Ab}$ conjugated to an enzyme followed by the addition of the enzyme's substrate. Modified from reference 54 . 10

Figure 1.5 Electrochemical, aptamer-based sensor for the detection of platelet-derived growth factor (PDGF). The star represents the redox active dye methylene blue which is brought closer to the gold electrode upon aptamer-target binding. Modified from reference 56 .

Figure 1.6 DNA sequences and linkages in cocaine-binding aptamer-linked AuNP aggregates. Modified from reference 60 .

Figure 1.7 Modifications for increasing the stability of nucleic acids in vivo. A) The $2^{\prime}$ position can be replaced with a fluoro $(\mathrm{F})$, amino $\left(\mathrm{NH}_{2}\right)$ or $O$-methyl $\left(\mathrm{OCH}_{3}\right)$ group; B) Phosphorothioates can be used in internucleotide linkages; C) End caps that reverse the polarity of the sequence at the 3'-terminus can be added; and D) Heavy tags such as polyethylene glycol (PEG) can be conjugated to the 5'-terminus. Modified from reference 67. 20

Figure $1.8 \quad 5$ '-Dimethoxytrityl- $N$-dimethylformamidine-2'-deoxyguanosine $3 '-[(2-$ cyanoethyl)-( $N, N$-diisopropyl)]-phosphoramidite (dmf-dG-CE phosphoramidite)......... 23

Figure 1.9 5'-DMS(O)MT-Amino-Modifier C6 or 6-(4,4'-dimethoxy-4"methylsulfonyl-tritylamino)hexyl-(2-cyanoethyl)-( $N, N$-diisopropyl)-phosphoramidite. 28

Figure 1.10 3'-Amino-Modifier C7 CPG with fluorenylmethoxycarbonyl (Fmoc) protecting group. Solid circle $=\mathrm{CPG}$ bead; red amino group is protected by Fmoc....... 30

Figure 1.11 Relevant reagents for the preparation of a polyacrylamide gel (A-C) along with the molecular structure of the cross-linked polymer (D) ${ }^{53}$ 31

Figure 2.1 A collection of protons and their precession around an external magnetic field $\left(\mathrm{B}_{0}\right)$ at equilibrium. Slightly more than half of the proton spins (represented by arrows) are aligned with the external field and a little less than half are oriented against it. 
The net magnetization $\left(\mathrm{M}_{\mathrm{z}}\right)$ is therefore in line with the external field. Modified from reference 95

Figure 2.2 Sequence of an inversion-recovery experiment for the measurement of $T_{1}$ relaxation. The value of $t$ remains constant while $\tau$ changes each time through the sequence. See text for further details. Modified from references 96 and 98 .

Figure 2.3 A) Gd(III)-DTPA (Magnevist $(B)$ ); B) Gd(III)-DOTA (Dotarem $(B)$; C)

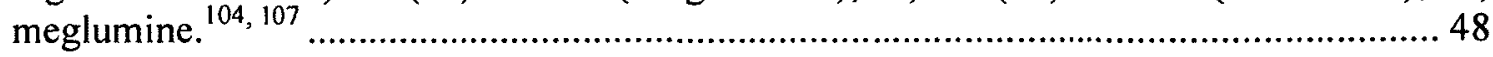

Figure 2.4 The structure of targeted MRI agent MS-325. ${ }^{106}$........................................ 55

Figure 2.5 Set-up of cross-linked dextran-coated superparamagnetic iron oxide (CLIO) nanoparticle system for adenosine-sensing. Two sets of CLIO nanoparticles were prepared with the respective sequences shown in this image in brown and blue. The adenosine aptamer was prepared including a linker portion as shown in green and black, respectively. A) The initial sensor set-up, where the modified aptamer strand was hybridized to the CLIO-supported sequences. A low $T_{2}$ value was achieved with the CLIO nanoparticles held close together. B) The addition of adenosine leads to aptamer folding and the release of one of the CLIO nanoparticles. This results in an increased $\mathrm{T}_{2}$ value compared to that of the system in A. Modified from reference 17.

Figure 2.6 Schematic representation of an adenosine-binding aptamer MRI contrast agent. Binding of the streptavidin-modified aptamer to adenosine leads to the release of the DNA-Gd(III) conjugate. This decrease in the effective mass of the DNA-Gd(III) conjugate results in a measurable drop in relaxivity. Modified from reference 16. 64

Figure 2.7 DNA-DTPA single-conjugates with chelated Gd(III) prepared for this study. A) thrombin aptamer-DTPA, SC-Gd(III); B) thrombin aptamer-15 poly T spacer-DTPA, $15 \mathrm{~T}$ SC-Gd(III); C) thrombin aptamer-30 polyT spacer-DTPA, 30T SC-Gd(III). Reproduced from reference 143 with permission from Springer. 83

Figure 2.8 Xylenol orange

Figure 2.9 Initial xylenol orange calibration solutions (5-25 nmol Gd(III)) and sample (SC filtrate) for determination of $\mathrm{Gd}(\mathrm{III})$ in flow through for SC. Xylenol orange was present in every sample at $15 \mathrm{nmol} / \mathrm{mL}$.

Figure 2.10 Xylenol orange test for determination of Gd(III) in the filtrate of SC (49 nmol) mixed with one molar equivalent of Gd(III). Xylenol orange was present in both samples at $10 \mathrm{nmol} / \mathrm{mL}$ 90

Figure 2.11 Melting curves for 15T SC and 30T SC with equimolar amounts of their respective polyA strands. 96

Figure 2.12 Structures of A) DTPA-Gd(III) (Magnevist(B) and B) DTPA-BMA-Gd(III) (Omniscan ${ }^{\mathrm{TM}}$ ). 102

Figure 2.13 Results of transmetallation experiments where the relaxivity changes of several Gd(III)-containing CAs were monitored over time in the presence of $\mathrm{Zn}$ (II) and phosphate ions at $20 \mathrm{MHz}$ and $37^{\circ} \mathrm{C}$. Modified from reference 140 . 103 
Figure 2.14 Relative paramagnetic longitudinal relaxivity, $R_{1}{ }^{P}(t) / R_{1}{ }^{P}(0)$, of SC-Gd(III) at $37^{\circ} \mathrm{C}$ and $9.4 \mathrm{~T}$ as a function of time after the addition of a molar equivalent of $\mathrm{Zn}$ (II). Reproduced from reference 143 with permission from Springer. 105

Figure 2.15 Schematic-representation of the DNA aptamer-DTPA-Gd(III) conjugates interacting with thrombin. A) SC-Gd(III); B) $15 \mathrm{~T}$ or $30 \mathrm{~T} \mathrm{SC-Gd(III);} \mathrm{C)} 15 \mathrm{~T}$ or 30T SC-Gd(III) when polyT linker is hybridized by a matching 15 or 30 polyA strand. Reproduced from reference 143 with permission from Springer.

Figure 2.16 Chart of the relaxivity values for Magnevist ${ }^{\circledR}$ and SC-Gd(III) at $9.4 \mathrm{~T}, \mathrm{pH}$ 7.4 and $25^{\circ} \mathrm{C}$. Reproduced from reference 143 with permission from Springer. 111

Figure 2.17 Chart of the relaxivity values for single-stranded and hybridized 15T SC$\mathrm{Gd}(\mathrm{III})$ at $9.4 \mathrm{~T}, \mathrm{pH} 7.4$ and $25^{\circ} \mathrm{C}$. Reproduced from reference 143 with permission from Springer. 116

Figure 2.18 Chart of the relaxivity values for single-stranded and hybridized 30T SC$\mathrm{Gd}(\mathrm{III})$ at $9.4 \mathrm{~T}, \mathrm{pH} 7.4$ and $25^{\circ} \mathrm{C}$. Reproduced from reference 143 with permission from Springer.

Figure 2.19 A) Chart of relaxivity data for Magnevist $($ and $\mathrm{SC}-\mathrm{Gd}(\mathrm{III})$ at $1.5 \mathrm{~T}, \mathrm{pH}$ 7.4 and $25^{\circ} \mathrm{C}$. B) Representative $T_{1}$ maps of $0.0125 \mathrm{mM}$ solutions of i) $\mathrm{SC}-\mathrm{Gd}(\mathrm{III})$, ii) SC-Gd(III)+BSA, iii) SC-Gd(III)+pepsin, and iv) SC-Gd(III)+thrombin. An equimolar amount of protein is present, where applicable. Images obtained under the same conditions as in A. Reproduced from reference 143 with permission from Springer. .. 126

Figure 3.1 DNA-DTPA double-conjugates with chelated Gd(III) prepared in this work. A) thrombin aptamer-DTPA, DC-Gd(III); B) thrombin aptamer-15 polyT spacer-DTPA, 15T DC-Gd(III); C) thrombin aptamer-30 polyT spacer-DTPA, 30T SC-Gd(III)........ 133

Figure 3.2 A) Two Gd(III) chelate multimers, one with one targeting group (TG; left) the other with two targeting groups (right). B) Agent with one TG still experiences internal motion. C) Multilocus binding agent with two TGs experiences limited internal motion and therefore a higher relaxivity. Arrows represent the motion of the CAs. When freely rotating bonds are present as in $\mathrm{A}$ and $\mathrm{B}$, the internal motion of these bonds limits the relaxivity achieved by the agent. Modified from reference 155 . 136

Figure 3.3 Schematic diagram of theranostic liposomes. Modified from ref $173 \ldots 139$

Figure 3.4 Schematic representation of the synthetic routes designed to maximize the yield of DNA-DTPA-Gd(III) double-conjugates. 155

Figure 3.5 Representative polyacrylamide gels for the purification of DNA-DTPA conjugates. A) $\mathrm{SC}=$ single-conjugate, $\mathrm{DC}=$ double-conjugate, $19 \%$ gel, absorbance shows $1: 2.5$ ratio of DC to SC prepared with a single addition of DTPA dianhydride; B) $19 \%$ gel, absorbance shows 1:9 ratio of DC to SC prepared using the modular method without the use of $\mathrm{CTAB}$ precipitation; $\mathrm{C}) 15 \mathrm{~T} \mathrm{SC}=15$ polyT $\mathrm{SC}, 15 \mathrm{~T} \mathrm{DC}=15$ polyT $\mathrm{DC}, 12 \%$ gel, absorbance shows $1: 6$ ratio of $15 \mathrm{~T} \mathrm{DC}$ to $15 \mathrm{~T} \mathrm{SC}$ prepared using the modular method; D) $30 \mathrm{~T} \mathrm{SC}=30$ polyT $\mathrm{SC}, 30 \mathrm{~T} \mathrm{DC}=30$ polyT $\mathrm{DC}, 12 \%$ gel, absorbance shows 1:15 ratio of $30 \mathrm{~T} \mathrm{DC}$ to $30 \mathrm{~T}$ SC prepared using the modular method. 
Figure 3.6 Relative paramagnetic longitudinal relaxivity, $R_{1}{ }^{P}(t) / R_{1}{ }^{P}(0)$, of DC-Gd(III) at $37^{\circ} \mathrm{C}$ and $9.4 \mathrm{~T}$ as a function of time after the addition of two molar equivalents of $\mathrm{Zn}(\mathrm{II})$.

Figure 3.7 Schematic of the two possible interactions between the double-conjugates and thrombin. A) 2:1 ratio of protein to conjugate, every aptamer could bind thrombin; B) 1:1 ratio of protein to conjugate, one aptamer in each conjugate could bind to thrombin.

Figure 3.8 Chart of the relaxivity values for Magnevist ${ }^{\circledR}$ and DC-Gd(III) at $9.4 \mathrm{~T}, \mathrm{pH}$ 7.4 and $25^{\circ} \mathrm{C}$. Pepsin is only present where applicable.

Figure 3.9 Chart of the relaxivity values for $15 \mathrm{~T} \mathrm{DC}-\mathrm{Gd}(\mathrm{III})$ at $9.4 \mathrm{~T}, \mathrm{pH} 7.4$ and $25^{\circ} \mathrm{C}$. 174

Figure 3.10 Chart of the relaxivity values for 30T DC-Gd(III) at $9.4 \mathrm{~T}, \mathrm{pH} 7.4$ and $25^{\circ} \mathrm{C}$.

Figure 3.11 Representative $T_{1}$ maps of $0.0134 \mathrm{mM}$ solutions of $A$ ) $15 \mathrm{~T}$ DC-Gd(III), B) 15T DC-Gd(III)+pepsin, and C) $15 \mathrm{~T} \mathrm{DC-Gd(III)+thrombin.} \mathrm{An} \mathrm{equimolar} \mathrm{amount} \mathrm{of}$ protein is present, where applicable. Experiments were performed at $1.5 \mathrm{~T}, \mathrm{pH} 7.4$ and $25^{\circ} \mathrm{C}$.

Figure 3.12 Representative T1 maps of $0.0094 \mathrm{mM}$ solutions of A) Magnevist $($, B) DC-Gd(III), C) DC-Gd(III)+pepsin, and D) DC-Gd(III)+thrombin. An equimolar amount of protein is present, where applicable. Experiments were performed at $1.5 \mathrm{~T}, \mathrm{pH}$ 7.4 and $25^{\circ} \mathrm{C}$. 182

Figure 4.1 Diagram of the physical processes that can occur after a molecule absorbs a photon of UV or visible light. See text for discussion. Modified from reference 53... 188

Figure 4.2 Schematic representation of how an excimer-forming compound could be used to study aptamer folding. See text for discussion. 194

Figure 4.3 Initial emission and excitation spectra obtained for (ppy)Pt(acac-COOH) dissolved in acetone to a concentration of $0.0245 \mathrm{M}$ (Stock 1)....

Figure 4.4 Emission spectra for (ppy) Pt(acac-COOH) in acetone with excitation at 426 $\mathrm{nm}$.

Figure 4.5 Absorbance spectrum for (ppy)Pt(acac-COOH) in acetonitrile at a concentration of $9.4 \times 10^{-6} \mathrm{M}$. 242

Figure 4.6 Emission spectra of (2,4-dfppy) Pt(acac-COOH) in methanol with excitation at $355 \mathrm{~nm} .{ }^{189}$ 244

Figure 4.7 RP-HPLC chromatograms from the analysis of crude Pt(II) complex-TBA. 247

Figure 4.8 Emission spectra for fractions 5 and 6 dissolved in tris buffer to $70 \mu \mathrm{M}$. Excitation at $302 \mathrm{~nm}$.

Figure 4.9 Schematic diagram of a proof-of-concept system using complementary DNA strands... 
Figure 4.10 Results from solution-phase conjugation of (ppy) Pt(acac-COOH) to the 5'terminus of a random DNA sequence. A) PAGE images from absorbance (left) and luminescence (right) channels. The red box indicates how the top band was cut out. B) Emission spectrum of the Pt(II) complex-DNA eluted from the band outlined in A with excitation at $302 \mathrm{~nm}$.

Figure 4.11 Comparative gel for solid-phase Pt(II) complex coupling to ultramild DNA. Lanes: 1 - fluorescently-tagged 18mer; 2 - excess Pt(II) complex after reaction with TSTU; 3 - excess Pt(II) complex after reaction with TSTU plus 10 nmol DNA; 4 - 5 nmol DNA from the first reaction with TSTU upper band; 5 - 5 nmol DNA from the first reaction with TSTU lower band; $6-10 \mathrm{nmol}$ DNA from the fresh reaction using TSTU; 7 $-10 \mathrm{nmol}$ DNA from the fresh reaction using EDC/NHS .........................................256

Figure 4.12 Possible fragment mass values of acac- $\mathrm{COOH}$ when conjugated to DNA. 4.

Figure 4.13 RP-HPLC chromatograms for the purification of $\mathrm{Pt}(\mathrm{II})$ complex-DNA obtained through a solid-phase reaction with 5'-amino-modified DNA. Absorbance at $260 \mathrm{~nm}$ was used to monitor DNA while that at $330 \mathrm{~nm}$ was used to monitor the $\mathrm{Pt}$ (II) complex 


\section{List of Schemes}

Scheme 1.1 Activation of a $3^{\prime}-N, N$-diisopropylphosphoramidite with ETT and subsequent coupling with a second phosphoramidite. $\mathrm{R}^{1}=$ remainder of the nucleoside being activated; $\mathrm{R}^{2}=$ remainder of nucleoside already on the strand. Adapted from reference 1 .

Scheme 1.2 DNA synthesis. 1. Detritylation; 2. Coupling; 3. Capping; 4. Oxidation; 5. Deprotection. Solid circle $=$ controlled pore glass $(\mathrm{CPG})$ bead; $\mathrm{DMT}=$ dimethoxytrityl; Base $=$ adenine, guanine, cytosine or thymine; $\mathrm{Pr}=$ isopropyl; $\mathrm{Ac}=$ acetyl. ${ }^{1}$................. 27

Scheme 2.1 Reaction of DTPA dianhydride with water and amino-modified DNA, where $\mathrm{NH}_{2}$-DNA represents any of the modified sequences present in section $2.2 .2 \ldots . .85$

Scheme 4.1 Peptide coupling reaction using EDC and NHS. 197

Scheme 4.2 Reaction of potassium tetrachloroplatinate(II) with A) 2-phenylpyridine to form $\mu$-dichloro(2-phenylpyridineplatinum(II)) dimers and B) 2-phenylquinoline to form $\mu$-dichloro(2-phenylquinolineplatinum(II)) dimers. ${ }^{196}$

Scheme 4.3 Synthesis of the modified acetylacetone ligand (acac-COOH): $8,10-$ dioxoundecanoic acid. See text for discussion.

Scheme 4.4 General reaction scheme for the synthesis of luminescent, square planar $\mathrm{Pt}$ (II) complexes containing acac-COOH and one of the three cyclometalating $\mathrm{C}^{\wedge} \mathrm{N}$ ligands included at the bottom.

225

Scheme 4.5 Removal of the Fmoc protecting group using piperidine. ${ }^{142}$ 263

\section{List of Appendices}

Appendix A Supporting data for singly-labelled DNA-DTPA conjugates...........268

Appendix B Supporting data for doubly-labelled DNA-DTPA conjugates..........285

Appendix C Supporting data for work with Pt(II) complexes....................294 


\section{List of Abbreviations}

15T DC

$15 \mathrm{~T} \mathrm{SC}$

30T DC

30T SC

acac

acac-COOH

ALISA

AuNPs

6-BrHex

DC

DC

DCA

DCM

DEA

2,4-dfppy

(2,4-dfppy)Pt(acac-COOH)

DIA

$\mathrm{diH}_{2} \mathrm{O}$

DMF
DNA-DTPA double conjugate with 15-thymine spacer between DTPA and TBA aptamer

DNA-DTPA single conjugate with 15-thymine spacer between DTPA and TBA aptamer

DNA-DTPA double conjugate with 30-thymine spacer between DTPA and TBA aptamer

DNA-DTPA single conjugate with 30-thymine spacer between DTPA and TBA aptamer

acetylacetone

modified acetylacetone ligand; 8,10-dioxoundecanoic acid

aptamer-linked immobilized sorbent assay

gold nanoparticles

6-bromohexanoic acid

DNA-DTPA double-conjugate

15 mer thrombin aptamer-containing double-conjugate

dichloroacetic acid

dichloromethane

diethylamine

2-(2,4-difluorophenyl)pyridine

8,10-dioxoundecanoic acid-(2-(2,4-

difluorophenyl)pyridyl)platinum(II)

diisopropylamine

deionized water

dimethylformamide 


\begin{tabular}{|c|c|}
\hline DMSO & dimethyl sulfoxide \\
\hline DMS(O)MT & 4,4'-dimethoxy-4"-thiomethoxytrityl \\
\hline DMT & dimethoxytrityl \\
\hline DTPA & diethylenetriaminepentaacetic acid \\
\hline DTPA-MA & monoamide DTPA \\
\hline DOTA & gadolinium-tetraazacyclododecanetetraacetic acid \\
\hline ELISA & enzyme-linked immunosorbent assay \\
\hline EtOAc & ethyl acetate \\
\hline $\mathrm{EtOH}$ & ethanol \\
\hline HMPA & hexamethylphosphoramide \\
\hline ICP-MS & inductively coupled plasma-mass spectrometry \\
\hline $\mathrm{MeCN}$ & acetonitrile \\
\hline $\mathrm{MeOH}$ & methanol \\
\hline MRI & magnetic resonance imaging \\
\hline n-BuLi & n-butyllithium \\
\hline Ppt & precipitate \\
\hline ppy & 2-phenylpyridine \\
\hline (ppy) $\operatorname{Pt}($ acac-COOH) & 8,10-dioxoundecanoic acid-2-phenylpyridylplatinum(II) \\
\hline $\mathrm{pq}$ & 2-phenylquinoline \\
\hline$(\mathrm{pq}) \mathrm{Pt}(\mathrm{acac}-\mathrm{COOH})$ & 8,10-dioxoundecanoic acid-2-phenylquinolylplatinum(II) \\
\hline QD & quantum dot \\
\hline $\mathrm{SC}$ & single-conjugate \\
\hline SC & $15 \mathrm{mer}$ thrombin aptamer-containing single-conjugate \\
\hline
\end{tabular}


SELEX

TBA

tris

UV-vis
Systematic Evolution of Ligands by EXponential enrichment

thrombin-binding aptamer (might refer to the $15 \mathrm{mer}$ or 29mer)

tris(hydroxymethyl)methylamine

ultraviolet-visible 


\section{Introduction}

\subsection{Functional nucleic acids}

The roles of deoxyribonucleic acid (DNA) and ribonucleic acid (RNA) as genetic material is widely known and taught, but many more functions of nucleic acids have been identified and studied in the past few decades. ${ }^{1,2}$ The first discovery of an enzymatic ribonucleic acid (RNAzyme) was made in the early 1980s. ${ }^{3}$ The knowledge that there were natural nucleic acids with roles other than the storage of hereditary material started a new area of research into these so-called functional nucleic acids. The in vitro discovery of synthetic RNA sequences that could act as enzymes ${ }^{4}$ and/or as molecular receptors (aptamers) ${ }^{5,6}$ were reported by three independent groups of researchers in 1990 , with corresponding reports of functional, single-stranded DNA molecules within a few years. $^{7-9}$

DNA is inherently more stable than RNA, both because the phosphodiester bonds in its backbone are less prone to spontaneous hydrolysis than those in RNA, and because of the extensive presence of ribonucleases in biological samples. ${ }^{2}$ Chemical modifications have been developed to increase the respective biological stabilities of both RNA and DNA as aptamers and nucleic acid enzymes are studied for use as therapeutics. ${ }^{10}$ Functional nucleic acids are also being explored in many other fields, including biosensing, ${ }^{11,}{ }^{12}$ diagnostics, ${ }^{13}, 14$ nanotechnology, ${ }^{15}$ and targeted medical imaging. $^{16-18}$ 


\subsection{Aptamers}

Aptamers are short, synthetic, single-stranded nucleic acids that bind with high affinity and selectivity to their targets. The term 'aptamer' was applied by Ellington and Szostak in 1990 and derives from the Latin word 'aptus', meaning to fit. ${ }^{5}$ The capability of single-stranded nucleic acids to fold into complex tertiary structures is an important property that leads to the specific interactions between each unique aptamer sequence and its target. ${ }^{19}$ The conformation achieved by an aptamer might completely envelop small targets in binding pockets, such as in the cases of theophylline $\mathrm{e}^{19,20}$ and adenosine monophosphate (AMP), ${ }^{19}$ or might interact with a specific region on a larger target, such as the two DNA aptamers for thrombin that bind preferentially to the fibrinogenrecognition $^{8}$ and heparin-binding ${ }^{21}$ sites, respectively. Aptamer folding and aptamertarget interactions will be discussed in more detail in section 4.1.1, while the thrombin aptamers, particularly the 15 mer DNA aptamer that binds to the fibrinogen-recognition site, will be discussed in section 1.2.3.

\subsubsection{Selection of aptamers using SELEX}

Aptamers are selected through an in vitro procedure described as the Systematic Evolution of Ligands by EXponential enrichment (SELEX, see Figure 1.1). ${ }^{6}$ A starting pool composed of $10^{13}$ to $10^{16}$ sequences is prepared where a central random region is flanked by two primer-binding sites (Figure 1.1A). The pool is exposed to the target for which an aptamer is desired (Figure 1.1B) and sequences that do not bind the target are removed (Figure 1.1C). This can be achieved in a variety of ways and is dependent on the particular experimental set-up. For example, the target might be 
immobilized on an agarose column and the nucleic acid pool washed over the modified column material. In this case, the nonbinding sequences would be washed through the column when they failed to bind to the target.

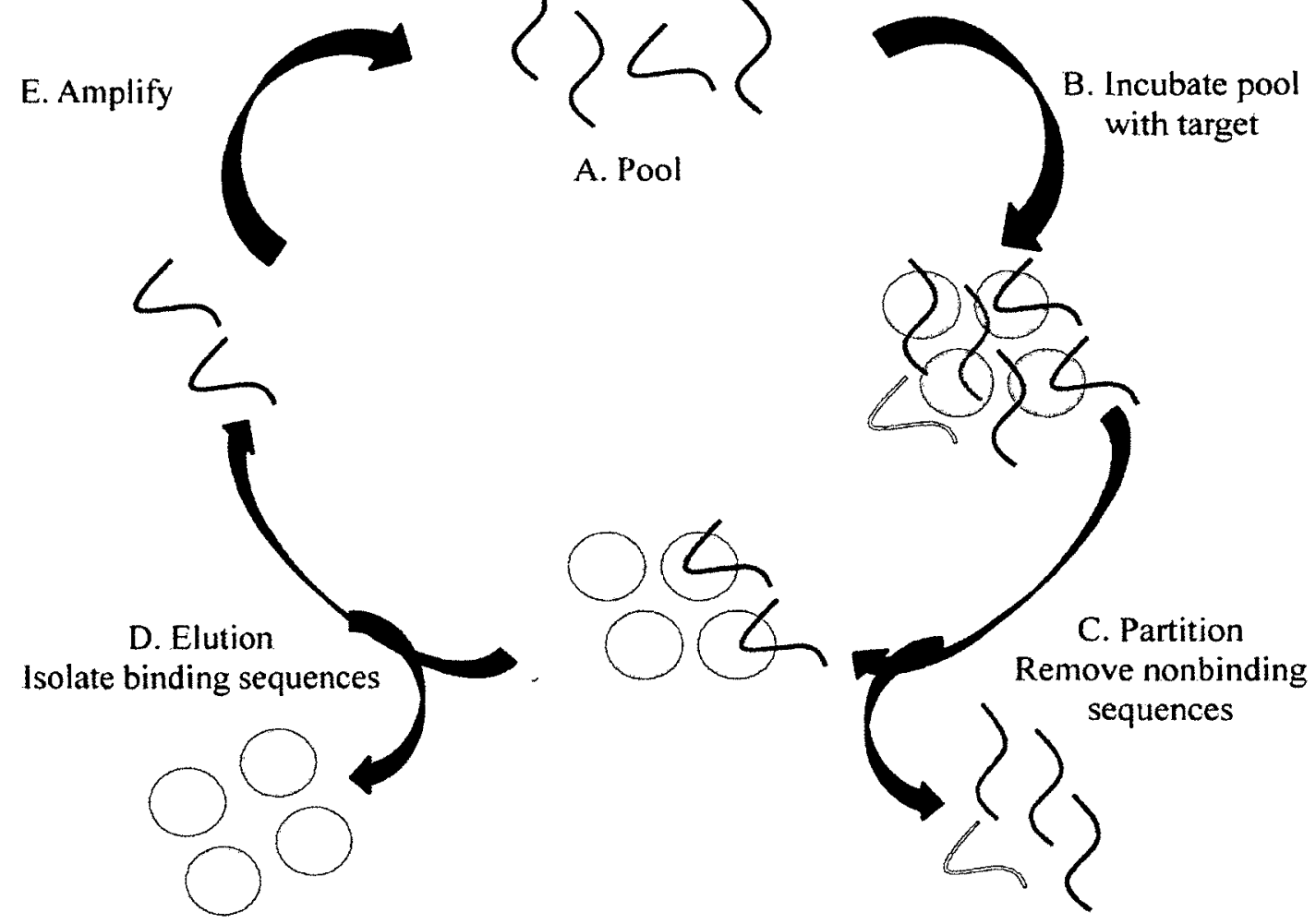

Figure 1.1 Systematic Evolution of Ligands by EXponential enrichment (SELEX). See text for details. $^{23}$

Following partitioning, the elution of binding sequences from the target (Figure 1.1D) is performed to isolate the potential aptamers for amplification using the polymerase chain reaction (PCR, Figure 1.1E). The interactions between the binding sequences and the target might be interrupted through a change in condition, such as increased temperature, a change in $\mathrm{pH}$ or the addition of a chaotropic agent (e.g. urea). 
Amplification of the binding sequences results in an enriched pool with a greater proportion of binders to nonbinders than the original pool. This cycle is repeated until the desired affinity of the pool for the target is achieved or no further enrichment is observed. The final pool is then cloned and sequenced for the identification of specific aptamers for characterization. ${ }^{22}$

Using general as well as modified SELEX procedures, ${ }^{24}$ DNA and RNA aptamers have been developed for a variety of target types. There are reported DNA aptamers for proteins such as human thrombin ${ }^{8,21}$ and immunoglobulin $E(\operatorname{Ig} E)^{25}$ and for small molecules such as cocaine ${ }^{26}$ and ethanolamine. ${ }^{27}$ The development of Cell-SELEX has permitted the use of live cells in selections for the discovery of aptamers that bind to cellsurface proteins in their native conformations. ${ }^{28}$ These aptamers are useful in cell detection and sorting assays. $^{29,30}$

\subsubsection{Aptamers versus antibodies}

Similarities between the properties of aptamers and antibodies have drawn many comparisons between the two with aptamers even being described as chemical antibodies. $^{28}$ The high affinity of both aptamers and antibodies for their respective targets is one common property. It is described using dissociation constants $\left(\mathrm{K}_{\mathrm{d}} \mathrm{s}\right)$, where the lower the $K_{d}$, the higher the affinity. Aptamers have been found with $K_{d}$ values in the nanomolar range down to picomolar levels, while most antibodies have $\mathrm{K}_{d}$ 's between 1 and $10 \mathrm{nM}^{31}$ The recognition of a specific target over other similar species is another mutual characteristic where a classic example is the RNA aptamer for theophylline which binds to its target with an affinity over 10,000 times greater than that for caffeine, which 
differs from theophylline by a single methyl group. ${ }^{32}$ Both of these properties contribute to making aptamers a rival for antibodies in areas ranging from sensing ${ }^{11,33}$ to therapeutics. $^{10,34,35}$

In light of the similarities between them why use aptamers instead of antibodies? Aptamers do offer some advantages. One is the wider variety of targets for which aptamers can be selected including those that do not cause an immune response or are very toxic." It is difficult to obtain high affinity antibodies for metal ions or small molecules, ${ }^{11}$ for example, as well as for toxins such as the mycotoxin fumonisin $\mathrm{B}_{1}$ and the protective antigen toxin of anthrax, both of which have reported DNA aptamers. ${ }^{36,37}$ In addition to an expanded range of targets, aptamers are also cheaper to produce than antibodies and, because they are synthetic, there is little batch-to-batch variation. Chemical modifications and labels can be easily incorporated during the synthesis of nucleic acids, permitting the use of modified nucleotides for increased nucleaseresistance as well as the inclusion of fluorescent or electrochemical tags at specific locations within an aptamer sequence. ${ }^{11,38-40}$ The inclusion of tags or spacers at carefully determined positions (i.e. away from the binding site, away from sites necessary for aptamer-folding) is unlikely to have an impact on aptamer-target binding, while antibodies cannot always be labelled with such precision since native side-chain functional groups are used for their labelling. ${ }^{41}$

Aptamers are mostly less than 100 nucleotides in length and so are smaller than antibodies permitting higher surface coverage when they are immobilized on a surface ${ }^{11}$ as well as the ability to bind to regions on proteins or cells that antibodies cannot access. $^{35}$ The smaller size of aptamers might translate into more of them binding than 
antibodies could when there are multiple targets in a small area, such as in the case of cell-surface receptors, and this could help amplify the signal readout in sensor platforms and so lead to lower detection limits. ${ }^{42}$ Nucleic acid-coated surfaces also generally experience fewer nonspecific interactions than at protein interfaces. ${ }^{43}$

Aptamers can retain their binding properties over a wider range of conditions, such as $\mathrm{pH}$ and temperature, than antibodies, and can even be selected under more "extreme" conditions to ensure they will be useful in a particular environment. Even more advantageous is that aptamers can be thermally denatured and renatured several times without loss of function unlike the majority of antibodies which lose their binding capabilities once denatured. This property permits aptamer-based sensors to be regenerated following the disruption of aptamer-target binding. ${ }^{11}$

\subsubsection{The 15mer thrombin-binding DNA aptamer}

A selection procedure was performed in 1992 by Toole and colleagues ${ }^{8}$ to obtain an aptamer for the enzyme thrombin. Thrombin is of interest because of its two important and opposing functions in the coagulation cascade. It promotes coagulation by converting the protein fibrinogen into an insoluble fibrin clot that then keeps platelets at the site of injury. ${ }^{44}$ Thrombin also acts as an anticoagulant by activating protein $\mathrm{C}$ which leads to the inactivation of coagulation cofactors necessary for thrombin generation. These procoagulant and anticoagulant pathways must be balanced for the coagulation cascade to act efficiently. ${ }^{44}$

Toole et al. isolated a 15mer DNA aptamer that exhibited inhibition of thrombincatalyzed conversion of fibrinogen to fibrin; the consensus 15 mer sequence is 5 '-GGT 
TGG TGT GGT TGG-3' ${ }^{8}$ The authors speculated that the anion-binding exosite (exosite I) at which fibrinogen interacts with thrombin, as well as the overall basic nature of the protein, might have aided in the selection of DNA aptamers, but as they saw a strong sequence dependence in their inhibition studies they suggested that electrostatic interactions alone might not be sufficient for high-affinity aptamer-thrombin binding. ${ }^{8}$ The authors confirmed the binding site of this 15 mer aptamer was exosite I in a separate report $^{45}$ where they found that the aptamer bound to $\alpha$-thrombin but not $\gamma$-thrombin (which has a cleavage site within exosite I) and that the natural thrombin inhibitor hirudin, which is known to bind exosite I, competed with the aptamer for thrombin binding.

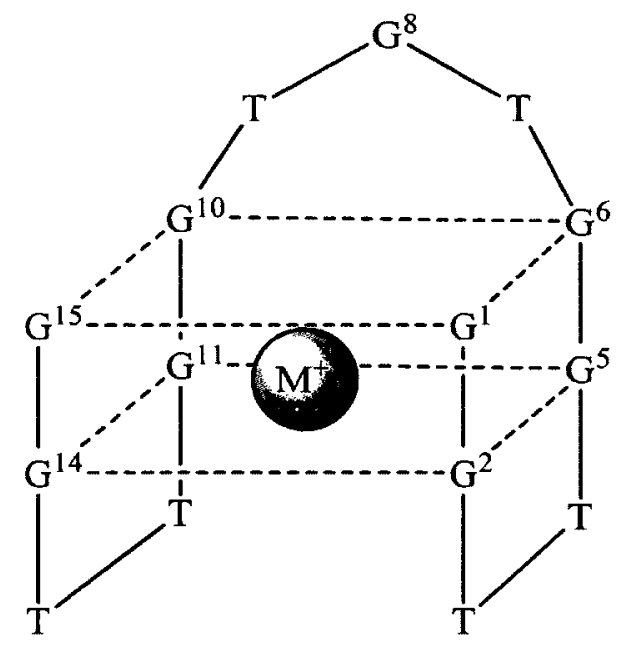

Figure 1.2 Structure of the 15 mer thrombin-binding DNA apta mer. $\mathrm{M}^{+}$represents a cation that is present to stabilize the G-quadruplex. Modified from references 46 and 47.

The tertiary structure of the 15 mer thrombin-binding aptamer (TBA) was studied over the next few years with the reported nuclear magnetic resonance (NMR) spectroscopy and X-ray crystallography results reconciled in a 1996 study by Yeates et 
$a l{ }^{46}$ The chair-like structure of this aptamer is illustrated in Figure 1.2, where the dotted lines connecting guanine residues in the structure represent the hydrogen bonds formed in a G-tetrad. ${ }^{46,47}$ It can be seen that the thrombin-binding aptamer consists of two stacked G-tetrads giving a G-quadruplex that includes a cation located between the tetrads to stabilize the negative charge that results from the carbonyl oxygen atoms pointing into the pore. ${ }^{1}$ This is evident in Figure 1.3, in which the hydrogen-bonding between four guanine residues forming a G-tetrad is illustrated. The potassium cation has been found to be the most effective at stabilizing the chair-like conformation shown in Figure 1.2, so much so that the thrombin-binding aptamer achieves this conformation in solution in the absence of its target. ${ }^{47,48}$ The thrombin-binding aptamer is therefore an example where the addition of the target does not induce a change in conformation. This aptamer also folds in the presence of other cations, with those of ionic radius 1.3-1.5 $\AA$, such as $\mathrm{NH}_{4}{ }^{+}$ and $\mathrm{Sr}^{2+}$, fitting the best within the two G-tetrads. ${ }^{48}$

The 15 mer thrombin-binding aptamer has been extensively researched and is commonly used in proof-of-concept studies that demonstrate the inclusion of aptamers in a variety of applications, some of which are discussed in the following section. Furthermore, while the research discussed in this work deals with the 15 mer thrombin aptamer, it is of interest to note that a separate 29mer DNA aptamer that binds to the heparin-binding exosite (exosite II) on thrombin was isolated by Kubik et al. in $1997 .^{21}$ These two thrombin-binding DNA aptamers have been used in concert in some applications to form sandwich-type assays ${ }^{49,50}$ or to enhance the signal obtained from a sensing system. ${ }^{18,51}$ 


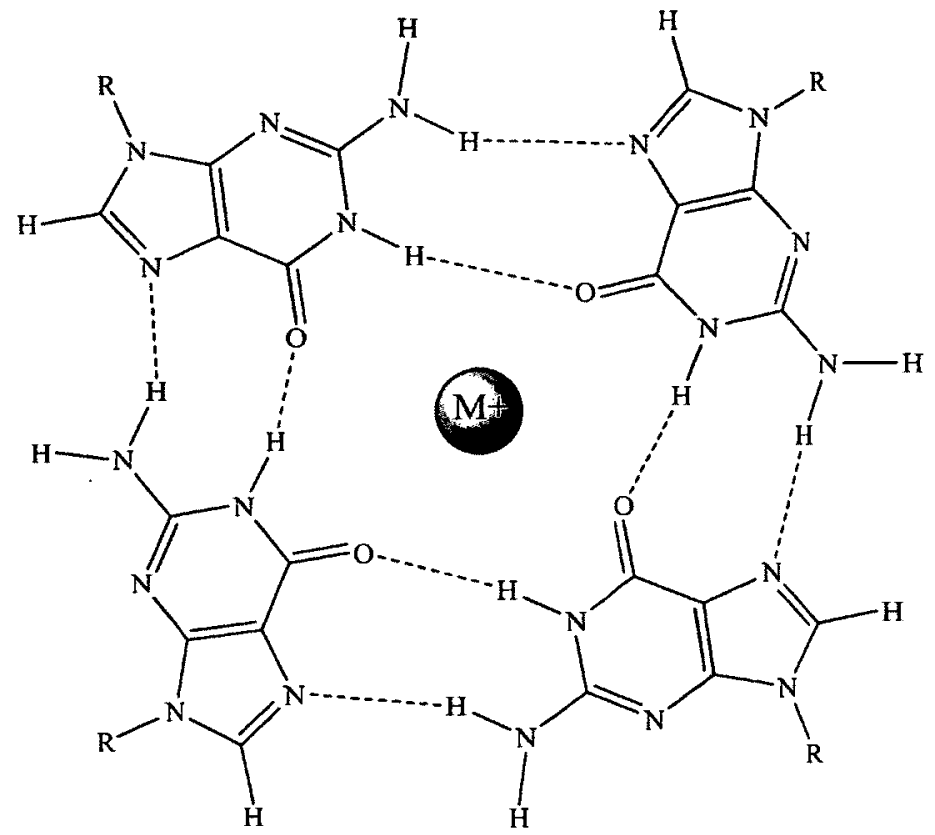

Figure 1.3 Structure of a G-tetrad stabilized by a cation. $R$ represents the sugar-phosphate

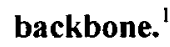

\subsection{Applications of aptamers}

\subsubsection{Sandwich-type assays}

The same properties that cause aptamers to draw many comparisons with antibodies (as discussed above) make them suitable for inclusion in several technologies that already use antibodies. For example, the commonly used sandwich Enzyme-Linked Immunosorbent Assay (ELISA) uses an immobilized antibody (Ab) to capture an antigen of interest and then involves the introduction of a second to simultaneously bind the captured antigen. This second antibody binds to a different portion of the antigen and is previously conjugated to an enzyme. The enzyme's substrate is added and the product of the catalyzed reaction is monitored using absorbance or fluorescence spectroscopy; the amplified signal is used for detection and quantification of the antigen. ${ }^{52,53}$ Aptamers 
have been used in concert with antibodies in assays based on ELISA's set-up as well as in other sandwich-type sensor designs.

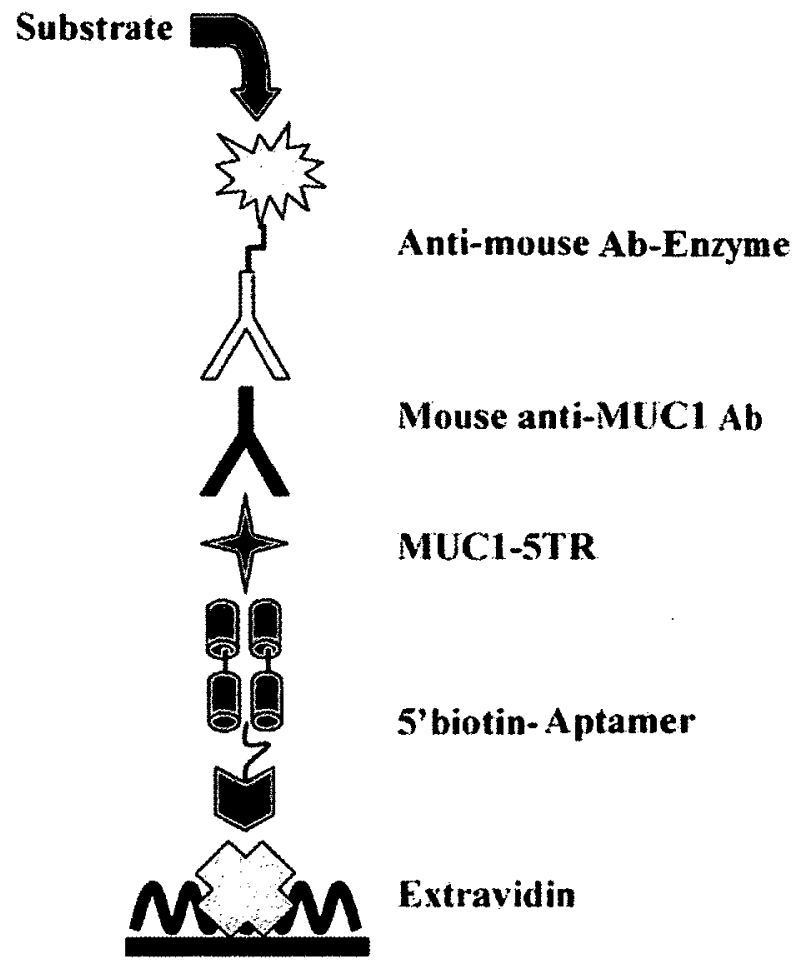

Figure 1.4 Design of aptamer-antibody sandwich ELISA for MUC1-5TR tumour marker detection. Extravidin was used to coat the wells of a 96-well plate for the subsequent immobilization a 5'-biotinylated DNA aptamer selected against MUC1-5TR. MUC1-5TR is a recombinant protein that was prepared containing five tandem repeats of the variable tandem repeat of the MUC1 protein. An anti-MUC1 antibody (Ab) was used as a primary antibody and detection was achieved through the use of a secondary Ab conjugated to an enzyme followed by the addition of the enzyme's substrate. Modified from reference 54.

Ferreira and coworkers selected aptamers against the MUC1 tumour marker and used them in an aptamer-antibody sandwich ELISA as illustrated in Figure $1.4 .^{54}$ This optimized assay design used a 96-well plate coated with extravidin on which biotinylated 
MUC1-binding aptamers were immobilized. Following the capture of the MUC1-5TR protein by the aptamer, an antibody that binds the antigen (the primary Ab) was introduced to complete the sandwich assay. In this case, the primary antibody was not conjugated to an enzyme but rather a secondary antibody that binds the primary antibody was linked to an enzyme and used to produce a signal. ${ }^{54}$ This strategy is used in some assays to avoid the possible reduction (or destruction) of the affinity of the primary antibody for the antigen upon conjugation of the enzyme. ${ }^{41}$ Using this experimental design, the authors reported a detection limit of $1 \mu \mathrm{g} / \mathrm{mL}$ which they hoped to improve upon by further optimization. They suggested that testing spacers of different lengths on the biotinylated end of the aptamer might increase its ability to interact with the target and thus increase the sensitivity of the assay. ${ }^{54}$

The development of an Aptamer-Linked Immobilized Sorbent Assay (ALISA) for the detection of Francisella tularensisis bacteria (a possible bioterrorism agent) by Vivekananda and Kiel was reported in $2006 .{ }^{55}$ They selected aptamers for the bacterial antigen and coated 96-well plates with the resulting aptamer cocktail (i.e. mixture of aptamer sequences). The target was captured by the adsorbed aptamers, and this was followed by the addition of biotin-labelled aptamer cocktail. The biotin was included to permit the use of enzyme-conjugated streptavidin for detection. The streptavidin bound the biotin labels on the aptamers, and the substrate of the enzyme was added to produce a measurable signal. ${ }^{55}$

The authors also designed a sandwich ELISA assay using commercially available antiserum to coat a 96-well plate, an anti-tularemia antibody as primary $\mathrm{Ab}$, and a secondary antibody conjugated to an enzyme for comparison to the ALISA assay. They 
found that the aptamer-based assay gave a lower limit of detection than the ELISA (25 ng versus $100 \mathrm{ng}$, respectively), though the specificities of the two assays for Tularemia antigens versus other bacteria were similar. ${ }^{55}$

These two examples illustrate that aptamers can be used in combination with antibodies to expand the usefulness of existing assay designs. Assays using only aptamers in a sandwich-type set-up have also been reported. For example, the two thrombin-binding aptamers were used together in an assay where detection was achieved with inductively coupled plasma-mass spectrometry (ICP-MS). ${ }^{50}$ Briefly, Le and coworkers conjugated the thiolated 29 mer thrombin-binding aptamer to gold nanoparticles (AuNPs) and biotinylated $15 \mathrm{mer}$ TBA to streptavidin-coated magnetic beads. Upon the introduction of thrombin to a mixture of aptamer-labelled AuNPs and magnetic beads, a sandwich complex was formed as the two aptamers bind to different exosites on thrombin (section 1.2.3). The magnetic beads permitted easy separation of the sandwich complexes from the bulk solution, followed by the dissociation of the complexes with increased temperature. The gold nanoparticles were isolated and analyzed using ICP-MS, with the quantification of ${ }^{197} \mathrm{Au}$ achieved with good reproducibility. The amount of ${ }^{197} \mathrm{Au}$ measured was determined to be directly proportional to the amount of thrombin that had been present in the sandwich complexes, with a detection limit of $0.5 \mathrm{fmol}$ achieved (comparable or better than other aptamerbased assays for thrombin). The authors also reported good specificity of the assay for $\alpha$ thrombin compared to abundant serum proteins and other participants in blood coagulation. 
Assays using aptamers, such as this one, are being developed in an attempt to decrease the time required to obtain results, increase sensitivity, and eventually permit multiplexed analysis (i.e. high-throughput analyses of multiple targets). The increased stability of aptamers compared to antibodies also permits storage of assay components without loss of activity. ${ }^{50}$ This keeps the cost of such assays comparatively low. The use of two recognition elements, antibodies or aptamers, separately or together, to bind the target in sandwich-type assays can lessen false positive results from nonspecific adsorption. Nonspecific interactions at a protein interface are more significant than at a nucleic acid interface, ${ }^{43}$ but in both cases a high specificity binder used for detection can help ensure that only the target of interest is generating a signal.

\subsubsection{Aptamer-based sensors}

Biosensors are devices that produce a signal upon the interaction of a biological component with a target of interest. ${ }^{11,33}$ A common design strategy links a change in the structure of an aptamer to the generated signal; these sensors are called aptamer beacons.

This method can lead to fewer false positives since it depends on a specific interaction. ${ }^{56}$ Many aptamer-based biosensors generate a signal through the inclusion of inorganic complexes, dyes, or nanomaterials through covalent or noncovalent interactions with the aptamer. $^{11,14,33}$ The ease with which aptamers can be modified, often without detriment to their binding abilities when positions for labelling are carefully chosen, can aid in the development and success of direct detection biosensor designs. Direct signal readout from simpler biosensors can be useful where a sandwich-type assay cannot be developed, either because there is not a second aptamer or a readily available antibody available that 
binds to a separate site on a target or because the target is too small to be bound simultaneously by two molecular receptors. ${ }^{43}$ Detection methods used in aptamer biosensing systems include electrochemical, optical, and colourimetric means; an example of each will be discussed here.

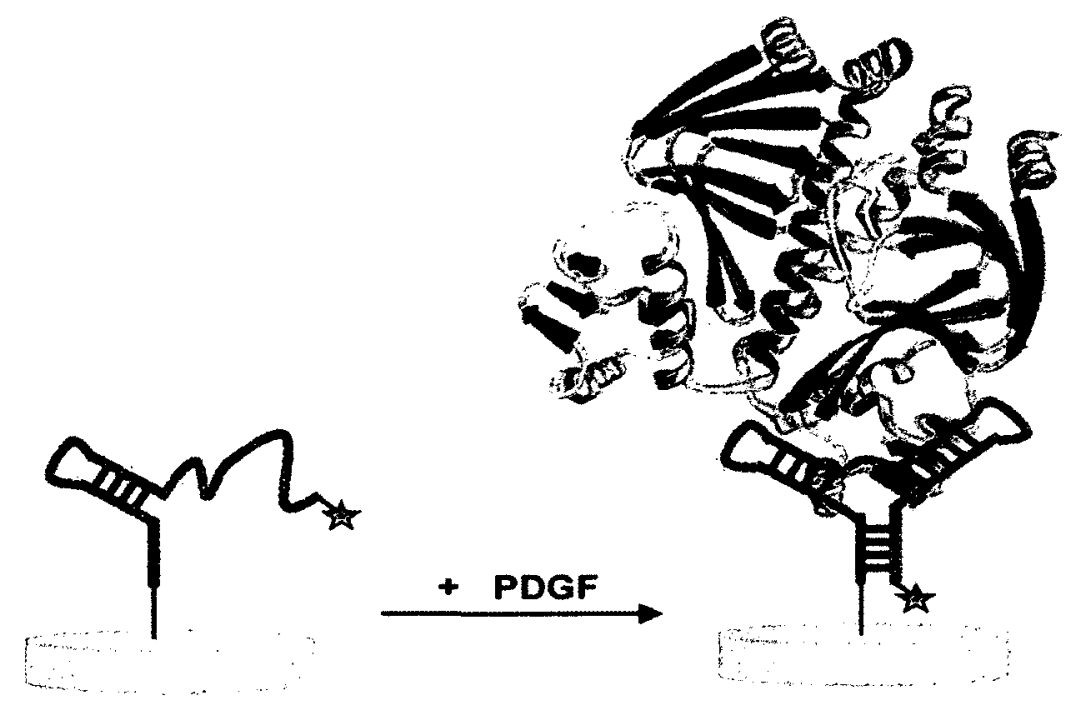

Figure 1.5 Electrochemical, aptamer-based sensor for the detection of platelet-derived growth factor (PDGF). The star represents the redox active dye methylene blue which is brought closer to the gold electrode upon aptamer-target binding. Modified from reference 56.

Biosensors using electrochemical detection as the signal readout have some advantages over optical sensors such as low electroactive background in clinical samples and the rapidly evolving area of microelectronics that has the potential for point-of-care sample testing. ${ }^{56}$ An electrochemical, aptamer-based sensor for the detection of plateletderived growth factor (PDGF) was reported by Plaxco et al. ${ }^{56}$ and is illustrated in Figure 1.5. The authors used the PDGF-binding aptamer tagged with methylene blue, a redox active dye, at the $3^{\prime}$-terminus and a thiol group on the 5 '-terminus for immobilization on a 
gold electrode. The aptamer is believed to be only partially folded when not bound to its target, so the methylene blue tag is kept on average at a greater distance from the electrode prior to target introduction (left side of Figure 1.5). Upon binding PDGF, the aptamer folds into its three-way helix junction motif, ${ }^{57}$ bringing the redox tag closer to the electrode and improving electron-transfer efficiency. The authors reported a significant increase from the background signal obtained with no target present to that obtained following incubation with PDGF in buffer, 50\% serum, and undiluted serum. They were also able to regenerate and reuse the sensor up to 5 times. ${ }^{56}$

As mentioned above, a goal in aptamer sensing is to develop sensors that can be used for point-of-care testing. Therefore, portable devices that incorporate aptamer technology are of great interest. A recent report by Bogomolova and Aldiss $i^{58}$ describes a real-time flow sensor prototype that uses an aptamer for adenosine triphosphate (ATP) ${ }^{59}$ in conjunction with quantum dots (QDs) for fluorescence detection. QDs are atom clusters of a semiconductor material that are prepared on the nanoscale; their size defines the wavelength of light they emit. ${ }^{58}$ In the sensor set-up the biotinylated aptamer is immobilized on a streptavidin-coated glass surface and then hybridized with a complementary DNA sequence conjugated to a QD. Upon the addition of ATP the aptamer changes conformation to bind with it and the QD-conjugated strand is released and washed away by the flow. The removal of QDs from the sensor leads to a decrease in fluorescent signal. Therefore, this is a turn-off sensor in which the measurable signal decreases with increasing target concentration. The aptamer-modified glass coverslips were designed to be disposable so regeneration of the system was not reported. It is 
interesting to note that this design is amenable to multiplexed detection as a variety of QDs with varying fluorescence emission peaks are readily available. ${ }^{58}$

A colourimetric aptamer-based "dipstick" or lateral-flow test reported by Lu and coworkers $^{60}$ was designed in an effort to be user-friendly for those not trained in laboratory techniques. They worked with the ATP aptamer but used it to detect adenosine (to which it is known to bind) ${ }^{59}$ as well as the cocaine aptamer. ${ }^{26}$ The colour change in this sensor was instigated through the use of gold nanoparticles (AuNPs). Because of the size-dependent effects on its localized surface plasmon resonance, dilute AuNPs form a red suspension while aggregated AuNPs appear blue in colour. ${ }^{61,62}$ In the lateral-flow sensor two kinds of DNA-functionalized AuNPs were used, each conjugated to a different DNA sequence (see Figure 1.6). The two DNA sequences on the AuNPs were designed to be complementary to an extended portion of the aptamer being used (AuNP labelled 3 in Figure 1.6) and to partially overlap with the aptamer and the extension (AuNP 4 in Figure 1.6); this second type also included biotinylated DNA. The aptamer-linked AuNP aggregates were spotted near the bottom of the lateral flow devices while a thin line of streptavidin was included farther up the device. Upon dipping the device into a solution that contained the aptamer target, the aptamer folded and disrupted complementary binding. This caused the aggregates to break apart and permitted the AuNPs to move up the device where the AuNPs with biotinylated DNA were captured at the line of streptavidin and formed a red band visible to the naked eye. The authors tested the device using both buffer and human serum to dissolve the targets and found that though the detection limit was higher in serum the device still performed well. ${ }^{60}$ 


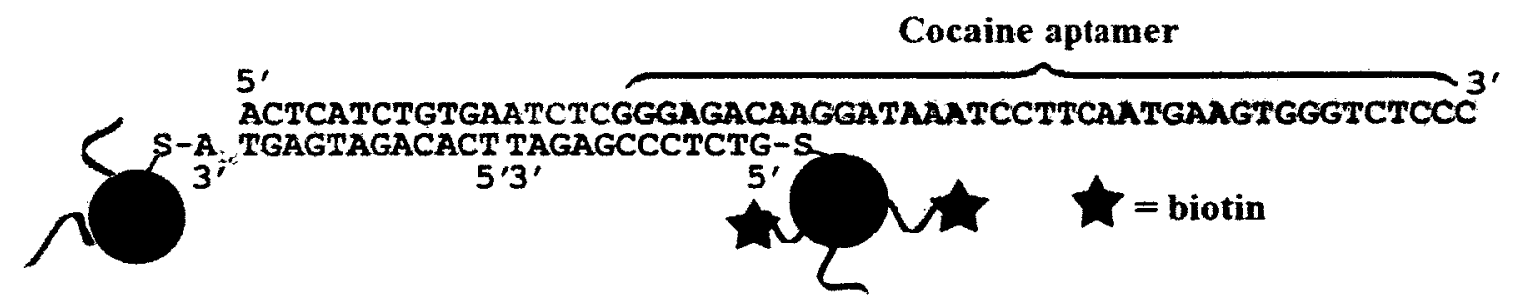

Figure 1.6 DNA sequences and linkages in cocaine-binding aptamer-linked AuNP aggregates. Modified from reference 60 .

The development of biosensors using aptamers is a field in which a great deal of work is being performed and reported. Although aptamer-based sensors are not yet widespread, as the number of reported aptamers for a wider variety of targets increases and as these aptamers are studied and better understood, it is expected that this relatively young field will eventually be comparable to antibody-based systems. ${ }^{11}$

\subsubsection{In vivo applications of aptamers}

The potential to use aptamers as therapeutics or in diagnostic techniques in vivo has not gone unexplored. As mentioned earlier, the $15 \mathrm{mer}$ thrombin-binding aptamer was found to compete with the natural thrombin inhibitor hirudin for binding at exosite $\mathrm{I}^{45}$ and was found to have inhibitory effects on the thrombin-catalyzed conversion of fibrinogen to fibrin in the original selection experiments. ${ }^{8}$ It has since been investigated as a possible anticoagulant along with aptamers for other components of the coagulation pathway. ${ }^{63}$ In addition to being considered as a therapeutic, thrombin-binding aptamers are also being explored for use in thrombus imaging. ${ }^{64,65}$ Thrombosis is the formation of a platelet-fibrin thrombus (i.e. a clot) that occurs with dysregulation of part of the hemostatic system. The resulting thrombus can lead to many serious health 
problems such as heart attacks and strokes. ${ }^{66}$ Radio-labelled thrombin-binding aptamers have been prepared and studied as potential thrombus imaging agents. ${ }^{65}$ As thrombin must bind to fibrin at exosite I to catalyze clot formation, it was demonstrated by Dougan et al. that the 15mer TBA did not bind to model fibrinogen clots in vitro but that the exosite II-binding $29 \mathrm{mer}$ TBA could and did bind to fibrin-bound thrombin. ${ }^{65}$ The authors' in vivo experiments demonstrated some difficulties that must be overcome to lead to a useful TBA imaging agent. For example, slow mass transfer of the aptamers in the clots limits the amount of labelling achieved, and this problem is compounded by the rapid clearance of aptamers from blood. ${ }^{65}$

The problem of rapid clearance of aptamers from the body has been addressed by several researchers through the inclusion of modified sugars in nucleotides, changes in internucleotide linkages, and the conjugation of bulky linkers or molecules to nucleic acids. $^{34,67}$ Sample modifications are illustrated in Figure 1.7. As mentioned previously, RNA is more susceptible than DNA to nucleases because of the abundance of ribonucleases in biological samples, ${ }^{2}$ but DNA is also susceptible to nuclease degradation, and unmodified nucleic acids have half-lives in blood of only minutes. ${ }^{10,67}$ Therefore, the modifications illustrated in Figure $1.7 \mathrm{~A}-\mathrm{C}$ have been developed to increase nucleic acid resistance to nuclease degradation.

Figure 1.7A shows the various substitutions used at the 2'-hydroxyl group (RNA) or 2'-hydrogen (DNA) to impart greater stability; 2'-fluoro pyrimidines, 2'-amino pyrimidines, and 2'-O-methyl ribose purines and pyrimidines. The pyrimidine-based nucleotides are specifically modified because endogenous nucleases have been found to have higher degradation rates when cleaving at pyrimidine residues. ${ }^{67}$ Each of these 
substitutions is particularly useful in RNA as the native 2'-hydroxyl group is directly involved in ribonuclease A (RNase A) hydrolysis, ${ }^{1}$ but they also slow degradation by deoxyribonucleases (DNase). ${ }^{68}$ A SELEX procedure using 2'-deoxy purines and 2'-Omethyl pyrimidines throughout selection resulted in aptamers that demonstrated resistance to DNase I under conditions that lead to degradation in deoxyribonucleic acids. These aptamers also demonstrated high thermal stability as no loss of target-binding ability was found after heating them to $125^{\circ} \mathrm{C}$ in an autoclave. ${ }^{68}$

Another effective modification lies in replacing an oxygen atom with a sulfur atom in the linkages between nucleotides as shown in Figure 1.7B. These phosphorothioate groups provide nuclease resistance, possibly because sulfur is larger than oxygen and its interaction with a nuclease causes the displacement of cations required by the active site of the enzyme. ${ }^{69}$ The inclusion of phosphorothioate groups has also been reported to enhance the affinity of aptamers for their target molecules ${ }^{70,71}$ although the placement of these linkages in aptamers selected using regular phosphate groups must be chosen carefully. The replacement of oxygen atoms with sulfur has also lead to a decreased aptamer-target affinity; the substitution can lead to structural changes in the aptamer which in turn can change key contact points between the aptamer and the target. $^{71}$

The activity of $3^{\prime}$-exonuclease in serum is higher than that of $5^{\prime}$-exonuclease, ${ }^{67}$ and therefore reversing the polarity of a nucleic acid at the 3 -terminus to give the strand two 5'-termini is another strategy used to impart increased nuclease resistance (Figure $1.7 \mathrm{C}) .39,67,72$ 
A)

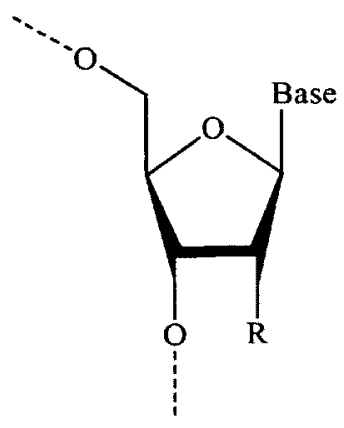

$\mathrm{R}=\mathrm{F}, \mathrm{NH}_{2}, \mathrm{OCH}_{3}$

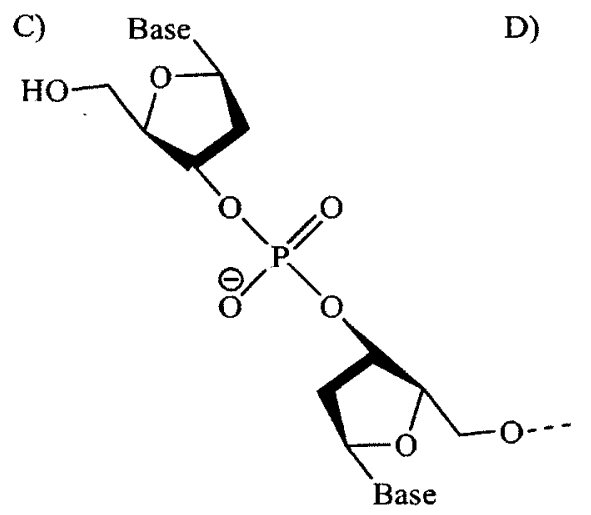

B)

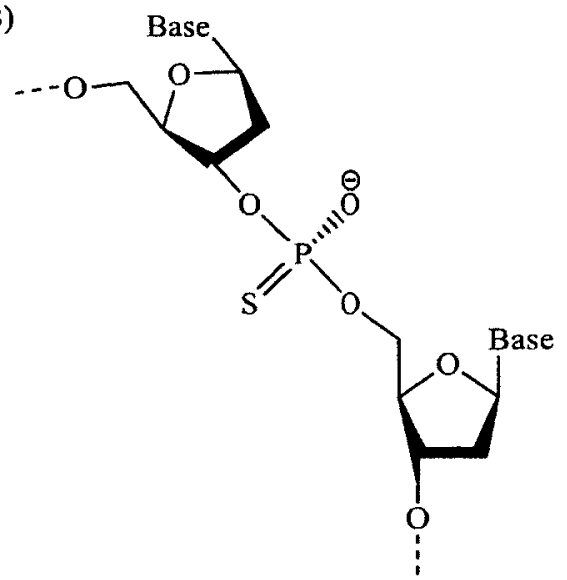

D)

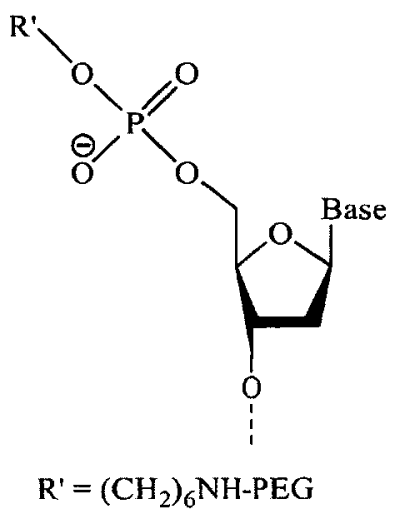

Figure 1.7 Modifications for increasing the stability of nucleic acids in vivo. A) The 2' position can be replaced with a fluoro (F), amino $\left(\mathrm{NH}_{2}\right)$ or $\mathrm{O}$-methyl $\left(\mathrm{OCH}_{3}\right)$ group; B) Phosphorothioates can be used in internucleotide linkages; C) End caps that reverse the polarity of the sequence at the 3'terminus can be added; and D) Heavy tags such as polyethylene glycol (PEG) can be conjugated to the $5^{\prime}$-terminus. Modified from reference 67.

Aside from their susceptibility to degradation by the action of nucleases, aptamers are also generally in the correct molecular mass range $(5-15 \mathrm{kDa}$ or $15-50$ nucleotides long) to be removed from the body via renal filtration. ${ }^{67}$ To improve the pharmacokinetic properties of aptamers they can be conjugated to heavy tags such as polyethylene glycol (PEG; 20 or $40 \mathrm{kDa}$ are typical). The addition of PEG brings the total mass into the 30 - 
$50 \mathrm{kDa}$ range and results in a drop in renal filtration rate that corresponds to a half-life in vivo of days. ${ }^{67}$ Cholesterol has been conjugated to an aptamer for factor IXa to moderately extend its in vivo half-life; the authors reported an increase from 5-10 minutes in pigs following injection to 1-1.5 hours. $^{73}$ The half-lives of possible aptamer therapeutics and diagnostic agents in vivo can therefore be tuned through a combination of modifications for increased nuclease resistance (Figure 1.7A-C, for example) and for increased circulation time (Figure 1.7D).

The aptamer-based drug Pegaptanib (also called Macugen ${ }^{\mathrm{TM}}$ ) has been FDAapproved for the treatment of age-related macular degeneration since December 2004, and there are several other aptamer-based therapeutics currently in clinical trials. ${ }^{10,34,67}$ Pegaptanib is an RNA aptamer that binds to vascular endothelial growth factor (VEGF)165 and was selected from an RNA library that used 2'-fluoro-modified pyrimidines. Following selection and aptamer minimalization it was prepared with $2^{\prime}-O$-methylmodified purines in addition to the 2'-fluoro-pyrimidines and a $3^{\prime}$-' 3 -linked deoxythymidine residue was used to cap the sequence (as in Figure 1.7C). Finally, a 5'conjugated $40 \mathrm{kDa}$ PEG moiety was added to prevent fast renal clearance. ${ }^{74}$ Pegaptanib has a half-life in humans of up to 10 days. ${ }^{67}$

Studies into the safety of aptamers at therapeutically-relevant doses have demonstrated minimal side-effects and a lack of an immune response, thereby suggesting that their incorporation into diagnostic agents could similarly be well-tolerated. ${ }^{34,74}$ The potential use of aptamers in imaging agents will be discussed in Chapter 2 while various therapeutic approaches will be considered in Chapter 3 . 


\subsection{DNA synthesis}

The preparation of labelled aptamers for use in various applications can be automated if the appropriate reagents are available for inclusion on a commercial oligonucleotide synthesizer. When such agents are not available for inclusion in automated synthesis the in-house synthesis of the desired oligonucleotides with appropriate modifiers gives the option of additional reactions being performed while the oligonucleotide is still supported on controlled pore glass (CPG) beads. An advantage of continuing with solid phase reactions is the ease of use of organic reagents at this stage. Once cleaved from the CPG beads on which they are synthesized DNA and RNA are not readily soluble in organic solvents. If the molecule or complex to be used in a labelling reaction is insoluble in aqueous solution, as was the case for both the chelator (Chapters 2 and 3) and the platinum(II) complex (Chapter 4) used in this work, the advantages of reacting CPG-supported DNA prior to cleavage are clear.

The efficient formation of internucleotide bonds in DNA synthesis was developed in the early 1980 s by Caruthers and co-workers. ${ }^{75-77}$ The high efficiency (typically $>98 \%$ per base $)^{1}$ of the phosphite triester, or phosphoramidite, chemistry that they introduced permitted the automation of oligonucleotide synthesis which occurs in a cycle consisting of five stages: 1. detritylation; 2. coupling; 3. capping; 4. oxidation; and 5. deprotection.' These stages are shown in Scheme 1.2.

DNA is synthesized on a solid CPG bead surface, and many of these beads are packed into a column that gives a specific maximum yield. The first deoxyribonucleoside in a synthesized sequence is linked to a CPG bead (the solid circle in Scheme 1.2) through a long chain alkyl amino linker at its deoxyribose 3'-hydroxyl 
prior to the synthetic process. Automated DNA synthesis, therefore, occurs in the $3^{\prime} \rightarrow 5^{\prime}$ direction, as opposed to its $5^{\prime} \rightarrow 3^{\prime}$ synthesis in nature; this method gives high efficiency due to the greater nucleophilic character of the 5'-hydroxyl compared to the 3'-hydroxyl on deoxyribose.'

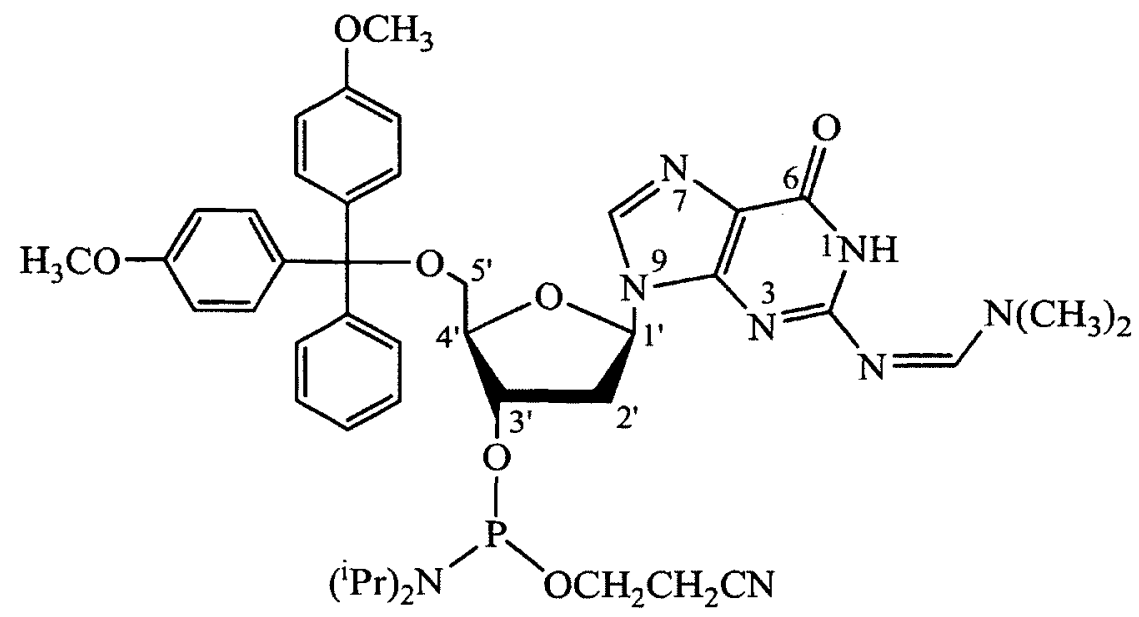

Figure 1.8 5'-Dimethoxytrityl- $N$-dimethylformamidine-2'-deoxyguanosine 3'-[(2-cyanoethyl)-( $N, N$ diisopropyl)]-phosphoramidite (dmf-dG-CE phosphoramidite)

The building blocks used in DNA synthesis are deoxyribonucleoside 3'phosphoramidites, such as that shown for guanosine in Figure 1.8. The structure shown in this figure includes several protecting groups that are present to ensure that only the 5'hydroxyl and 3'-phosphoramidite react, thus resulting in linear DNA with a sugarphosphate backbone comparable to biological DNA. To this end, the 5'-hydroxyl group of each deoxyribonucleoside 3'-phophoramidite synthon must be protected at times during DNA synthesis. For example, upon addition of a new synthon to the CPG beads, the 3 '-phosphoramidite must react with the 5'-hydroxyl group of the previously coupled nucleotide (reaction 2 in Scheme 1.2). To block the 5'-hydroxyl of the newly added 
synthon at this stage, a dimethoxytrityl (DMT) group is included as shown in Figure 1.8. The DMT group is stable to the conditions used during all stages of DNA synthesis except for the step specifically designed to remove it: detritylation (reaction 1 in Scheme 1.2). This reaction proceeds through the addition of dichloroacetic or trichloroacetic acid in dichloromethane and the resultant release of the DMT cation. This cation is bright orange in colour with maximum absorbance at $498 \mathrm{~nm}$, so it can be used to monitor reaction efficiency. ${ }^{1}$

The $3^{\prime}-N, N$-diisopropylphosphoramidites developed by Caruthers et al. $^{78}$ are stable 3'-derivatives of nucleosides that also exhibit efficient reactivity with the 5'hydroxyl of another nucleoside in the presence of a catalyst. ${ }^{1}$ The catalyst is described simply as "Activator" in reaction 2 in Scheme 1.2 ; this represents a solution of 5ethylthio-1H-tetrazole (ETT) in acetonitrile in the syntheses discussed in this work. The reaction between ETT and a $3^{\prime}-N, N$-diisopropylphosphoramidite activates the phosphoramidite making it more reactive towards the 5'-hydroxyl of a nucleoside already incorporated into the sequence on the CPG bead. ' This activation and the resultant coupling of the activated phosphoramidite to another nucleotide are illustrated in Scheme 1.1. It can be seen in this scheme that the 2-cyanoethyl group remains bound to the oxygen atom at the third position on the phosphorus(III) throughout this reaction. It is another protecting group and is included to prevent further reactions from taking place at this position, both during the reactions shown in Scheme 1.1 that result in a phosphite triester, as well as through the remaining capping and oxidation stages (reactions 3 and 4, respectively, in Scheme 1.2). 2-cyanoethyl is used as a protecting group both because of 
its stability through these reactions and the ease of its removal at the end of DNA synthesis.

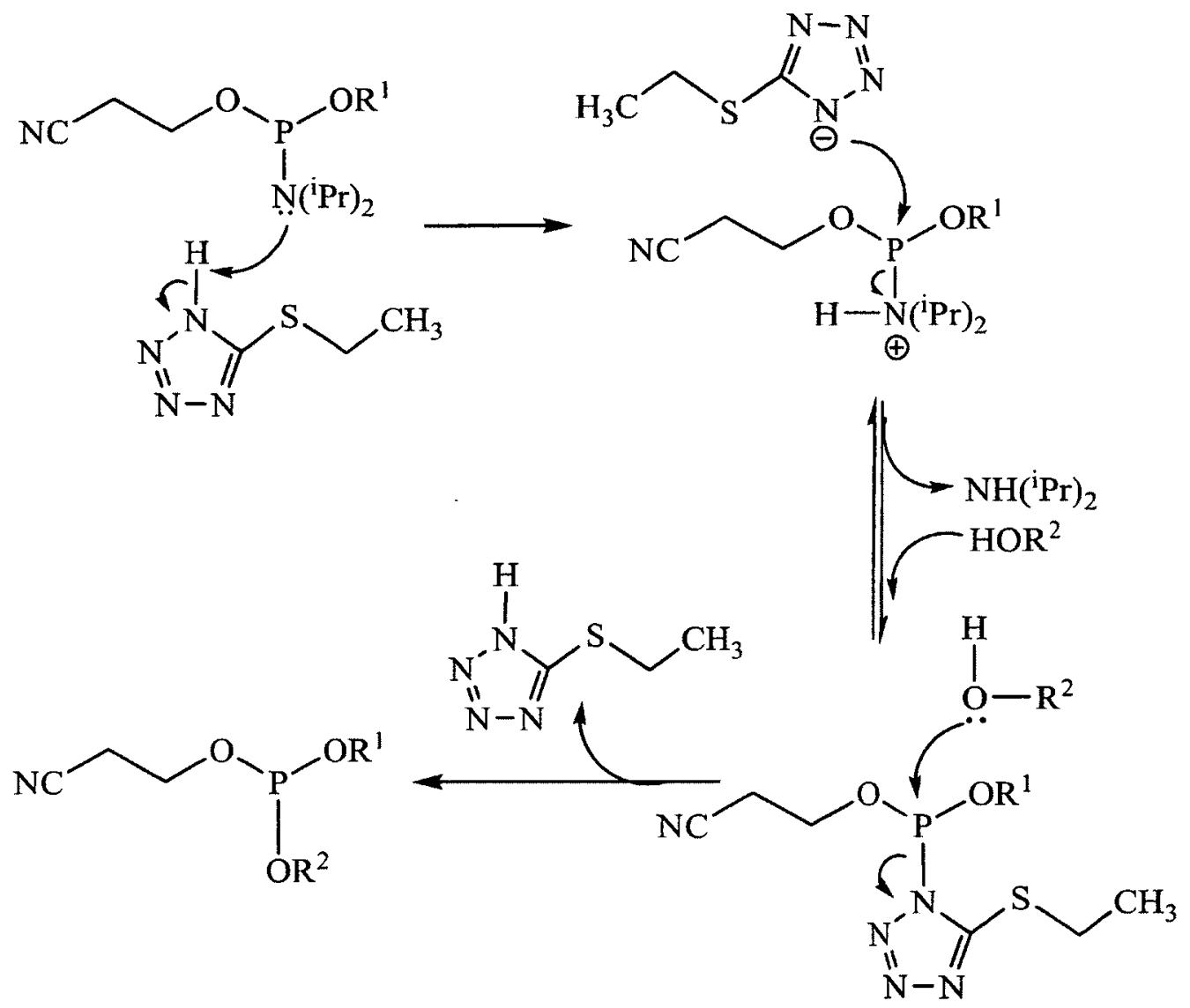

Scheme 1.1 Activation of a 3'- $N, N$-diisopropylphosphoramidite with ETT and subsequent coupling with a second phosphoramidite. $R^{1}=$ remainder of the nucleoside being activated; $\mathbf{R}^{2}=$ remainder of nucleoside already on the strand. Modified from reference 1.

The exocyclic amino groups of adenine, cytosine, and guanine must also be protected throughout DNA synthesis (thymine does not have an exocyclic amino group). The structure for 5'-dimethoxytrityl- $N$-dimethylformamidine-2'-deoxyguanosine $3^{\prime}-[(2-$ cyanoethyl)-( $N, N$-diisopropyl)]-phosphoramidite (dmf-dG-CE phosphoramidite) in 
Figure 1.8 includes a dimethylformamidine protecting group on guanine's exocyclic amino group. In adenine and cytosine, benzoyl and acetyl groups, respectively are typically used for protection of their exocyclic amino groups. ${ }^{\prime}$ Like the 2-cyanoethyl group on the 3 ' phosphorus, the protecting groups used for the exocyclic amino groups of the bases withstand the conditions used throughout DNA synthesis and are only removed once the synthesis is complete.

The coupling of a new phosphoramidite to the growing DNA sequence (reaction 2 in Scheme 1.2) is about $98 \%$ efficient.' Therefore, at each position there are some sequences supported on the CPG beads that did not experience proper coupling to the next phosphoramidite and would then result in sequences with deleted nucleotides if they were extended further. The capping step (reaction 3 in Scheme 1.2) is performed following coupling to ensure that the unreacted 5'-hydroxyl is blocked from further coupling reactions. This is achieved through the addition of 2,6-lutidine/acetic anhydride and $N$-methylimidazole in separate tetrahydrofuran (THF) solutions. ${ }^{1}$ These reagents result in stable 5 -acetylated nucleobases that will not be further extended in coupling reactions.

Once capping is complete, the next stage of DNA synthesis is oxidation (reaction 4 in Scheme 1.2). Iodine is used in THF with pyridine present at this stage. The pyridine neutralizes the hydrogen iodide released as the iodine oxidizes the phosphite triester to a phosphate triester. ${ }^{1,77}$ Once oxidation is complete, the cycle begins again with detritylation of the protected 5'-hydroxyl and continues until the entire desired sequence is synthesized. 


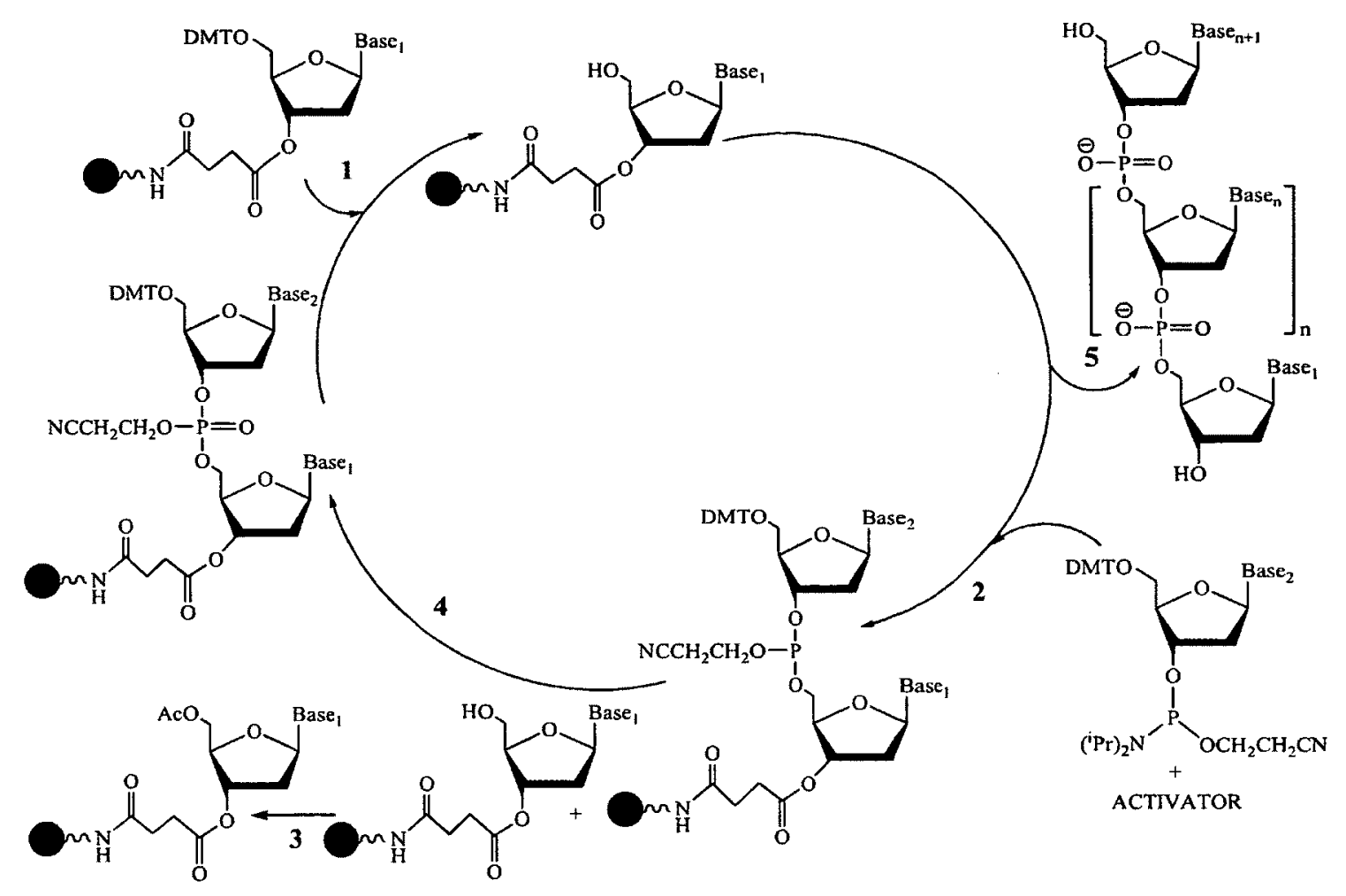

Scheme 1.2 DNA synthesis. 1. Detritylation; 2. Coupling; 3. Capping; 4. Oxidation; 5. Deprotection. Solid circle $=$ controlled pore glass $(\mathrm{CPG})$ bead; DMT $=$ dimethoxytrityl; Base $=$ adenine, guanine, cytosine or thymine; $\mathrm{PPr}=$ isopropyl; $\mathrm{Ac}=$ acetyl. ${ }^{1}$

Following synthesis of the full sequence, a final detritylation step is performed to remove the 5'-DMT group on the last nucleotide prior to deprotection and cleavage of the DNA from the CPG beads using concentrated ammonium hydroxide (reaction 5 in Scheme 1.2$){ }^{77}$ In this reaction, the ester bonds through which the DNA is bound to the CPG beads are hydrolyzed by the strong base. Heating the CPG beads in ammonium hydroxide at $50^{\circ} \mathrm{C}$ also removes the 2-cyanoethyl protecting groups from the phosphorus $(\mathrm{V})$ centre and the protecting groups on the exocyclic amino groups of the 
bases. ${ }^{1}$ Following deprotection, the DNA is isolated from the ammonium hydroxide solution by removing the solution from the CPG beads and drying it down under vacuum.

In cases where the presence of the DMT protecting group will aid with purification, as when using reversed-phase high performance liquid chromatography (RPHPLC), the final detritylation step is not performed during automated synthesis and the DMT group is removed manually following purification. ${ }^{1}$ In cases where a modifier is included on the 5 ' end of a DNA sequence for reaction with a label it is also convenient to leave the trityl protecting group on the $5^{\prime}$ end immediately following DNA synthesis. The relevant example for this work is the inclusion of an amino modifier at the $5^{\prime}$ terminus of synthesized aptamers or random deoxyribonucleotides.

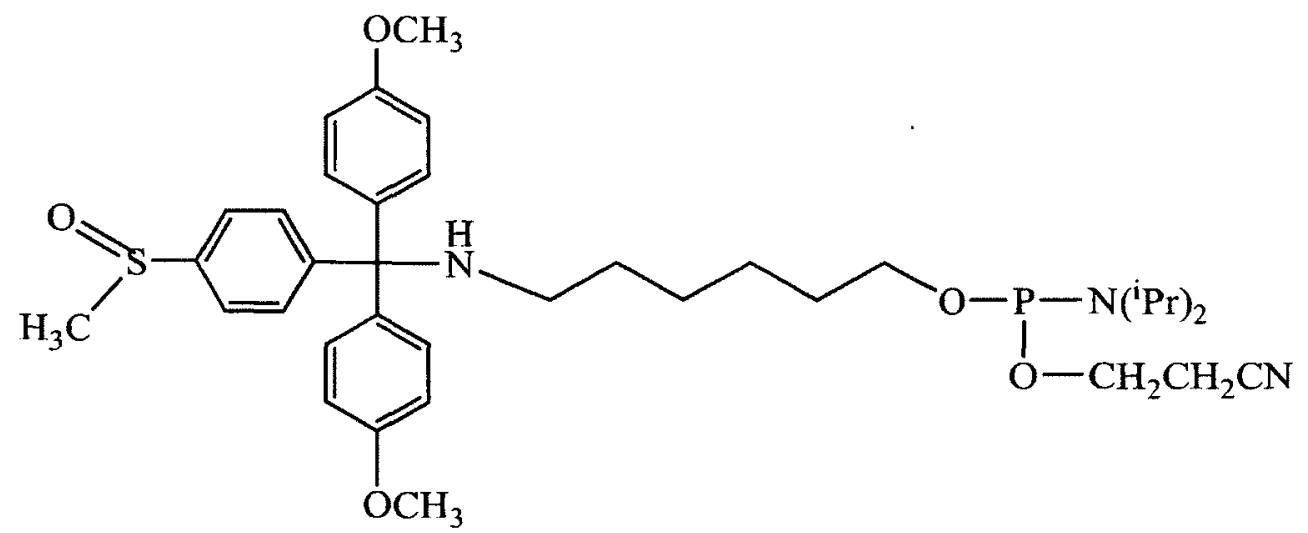

Figure $1.9 \quad 5^{\prime}$-DMS(O)MT-Amino-Modifier C6 or 6-(4,4'-dimethoxy-4''-methylsulfonyltritylamino)hexyl-(2-cyanoethyl)-( $N, N$-diisopropyl)-phosphoramidite.

The 5'-DMS(O)MT-amino modifier C6, or 6-(4,4'-dimethoxy-4"-methylsulfonyltritylamino)hexyl-(2-cyanoethyl)-( $N, N$-diisopropyl)-phosphoramidite, is shown in Figure 1.9. It consists of a phosphite triester identical to those in the four regular phosphoramidites, a hexyl chain linker, and finally an amino group that is protected by 
the DMS(O)MT group. This protecting group was developed by the company Glen Research as the equivalent to DMT protection of hydroxyl groups for the amino group in a terminus modifier. ${ }^{79}$ DMT is unsuitable as a protecting group for amino groups as it is too unstable for regular use, demonstrating a significant loss of DMT from the amine during deprotection. The DMS $(\mathrm{O}) \mathrm{MT}$ protecting group withstands both DNA synthesis conditions as well as regular deprotection protocols and provides the same advantages for purification as the DMT group, if required. ${ }^{80}$ The DMS(O)MT cation that is released upon detritylation of the modifier is more stable than previous protecting groups used for amino modifiers and is more efficiently removed using standard deblocking protocols (see previous detritylation discussion). ${ }^{79}$ The cation has a distinct red colour in solution, with its wavelength of maximum absorbance being $523 \mathrm{~nm}$, differing from the orange colour of the DMT cation. ${ }^{79}$ Removal of the DMS(O)MT group is possible while the DNA is still supported on the CPG beads, permitting solid phase reactions with desired compounds to be performed, or while the DNA is in solution following deprotection for further use in solution-phase reactions.

The 5'-DMS(O)MT-amino-modifier $\mathrm{C} 6$ was used when labelling at the $5^{\prime}$ terminus of synthesized DNA was required, and 3'-amino-modifier CPG beads with the fluorenylmethoxycarbonyl (Fmoc) protecting group bound to the reactive amine were used for $3^{\prime}$ modification $^{81}$ (Figure 1.10). These modified CPG beads were used in DNA synthesis and then in a solid phase reaction prior to deprotection of the DNA. The deblocking protocol for the removal of the Fmoc group differs from that of the trityl protecting groups: instead of using an acid, a base (piperidine) is used to deblock the reactive amine. This will be discussed further in Chapter 4 . 


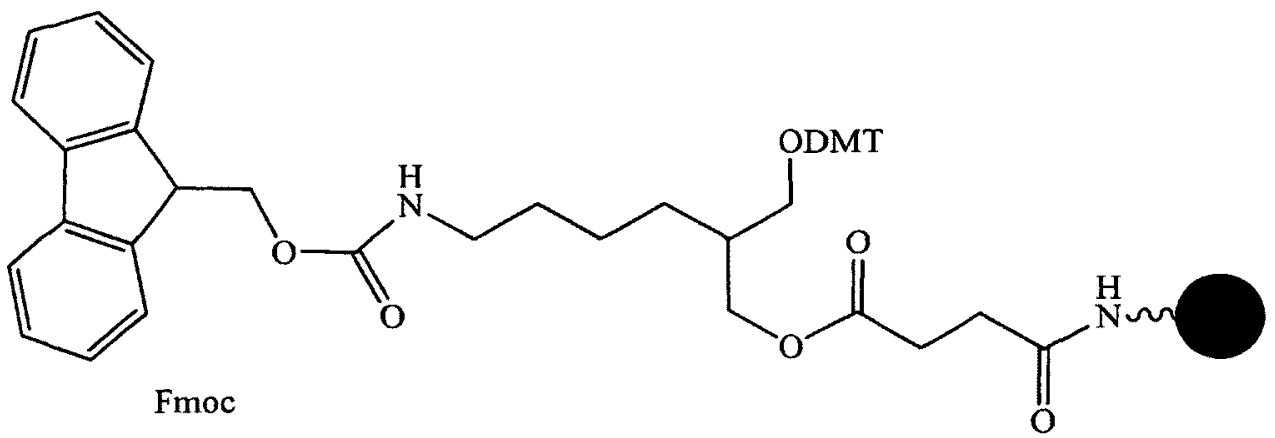

Figure 1.10 3'-Amino-Modifier C7 CPG with fluorenylmethoxycarbonyl (Fmoc) protecting group. Solid circle $=$ CPG bead; red amino group is protected by Fmoc.

\subsection{DNA purification and characterization}

Following DNA synthesis, purification using polyacrylamide gel electrophoresis (PAGE) or reversed-phase high-performance liquid chromatography (RP-HPLC) can be performed to separate failed strands from complete sequences. If crude DNA was used in a labelling reaction without purification, these techniques can be used to isolate the labelled product from both the short, failed strands of DNA and any full strands that remain unlabelled following the reaction. The characteristic absorbance of DNA at about $260 \mathrm{~nm}^{1}$ permits its detection in both of these purification methods, as well as the quantification and further characterization of the purified DNA (i.e. melting temperature of hybridized strands).

\subsubsection{Polyacrylamide gel electrophoresis}

Electrophoresis works on the principle that charged molecules or particles move in an electric field. The use of a polymer gel matrix to which the electric field is applied permits good resolution of macromolecules based on their size or shape, as well as charge, since the polymers typically used can be prepared with a variety of pore sizes. ${ }^{82}$ 
The negative charges along the sugar-phosphate backbone cause nucleic acids to move towards the anode, ${ }^{1,82}$ but since the charge per unit length of a nucleic acid is approximately the same in all cases ${ }^{\prime}$ it will be the size of the strands that lead to differing mobilities. The pores in the gel matrix therefore allow smaller strands to move through the gel faster than longer ones. ${ }^{82}$ When urea is included in the gel matrix, as in this work, the DNA applied to the gel is denatured ${ }^{1}$ and so is separated based on size but not shape (i.e. the DNA strands are linearized). ${ }^{83}$ The addition of formamide to the DNA samples prior to gel loading also helps ensure the DNA is denatured. ${ }^{82,84}$

A)<smiles>C=CC(N)=O</smiles>

Acrylamide
B)<smiles>C=CC(=O)NCNC(=O)C=C</smiles>

$N, N$-Methylenebisacrylamide
C)<smiles>CN(C)CCN(C)C</smiles>

$N, N, N^{\prime}, N^{\prime}$-Tetramethylethylenediamine (TEMED)

D)

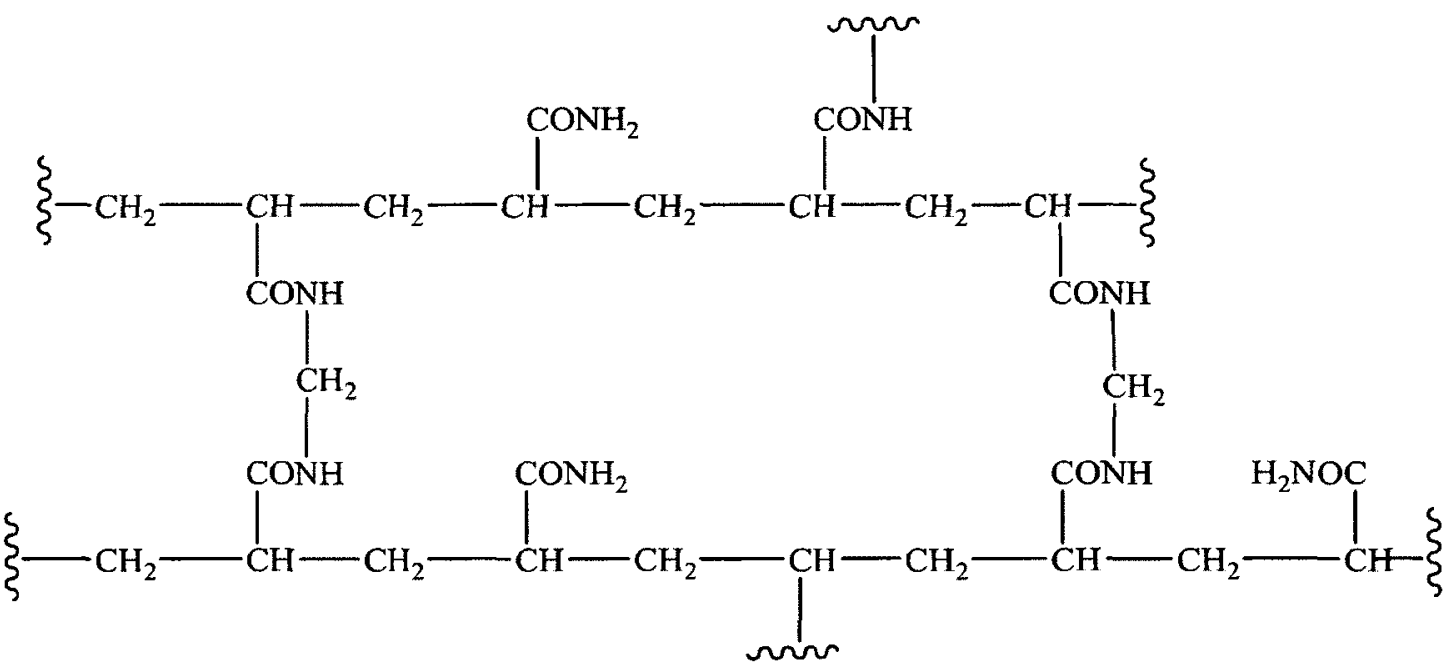

Figure 1.11 Relevant reagents for the preparation of a polyacrylamide gel (A-C) along with the molecular structure of the cross-linked polymer (D). ${ }^{53}$ 
Polyacrylamide is the material used in gel electrophoresis that gives the widest range of pore sizes and the higher the percentage of acrylamide used, the smaller the pores. ${ }^{82}$ Figure 1.11 illustrates the molecular structures of the acrylamide monomer (A), the cross-linker $N, N^{\prime}$-methylenebisacrylamide (B), and one of the polymerization initiators used, $N, N, N^{\prime}, N^{\prime}$-tetramethylethylenediamine (TEMED, C). The other initiator used is ammonium persulfate which decomposes to give persulfate radicals $\left(\mathrm{SO}_{4}{ }^{-}\right)^{82}$ TEMED accelerates the decomposition of persulfate to produce these radicals; the radicals then initiate vinyl addition polymerization of acrylamide and $N, N^{\prime}$ methylenebisacrylamide. ${ }^{85}$ Figure $1.11 \mathrm{D}$ shows two polyacrylamide chains linked together by $N, N^{\prime}$-methylenebisacrylamide, forming a pore. ${ }^{53,82}$

As polyacrylamide adheres well to glass, a polyacrylamide gel prepared between two glass plates can be vertically oriented with sample-loading wells located at the top of the gel. ${ }^{82}$ Upon application of an electric field DNA moves down the gel according to size, as discussed previously. The progress of the sample bands can be monitored through DNA absorbance or the fluorescence of a tag on the DNA. ${ }^{1}$ Once separation is achieved the desired bands are cut out of the gel and the DNA is recovered. One method used to remove the purified DNA from polyacrylamide gel involves crushing the gel and then adding aqueous buffer into which the DNA can diffuse. Freezing the gel/buffer slurry allows ice crystals to form and further disrupt the acrylamide matrix. Following this step with a rapid thaw of the frozen slurry helps decrease the elution time as well as increasing the yield of DNA recovered; up to $80 \%$ recovery has been reported for a 20 mer oligonucleotide, although recovery generally decreases as the length of the DNA increases. $^{83}$ It should also be noted that with an increased percentage of acrylamide 
comes a decrease in the percentage of recovered DNA with an average yield of $50 \%$ expected. ${ }^{83}$

\subsubsection{Reversed-Phase High-Performance Liquid Chromatography (RP-HPLC)}

Chromatography works on the principle that a solute can transfer between phases depending on its affinity and interactions with each respective phase. In highperformance liquid chromatography (HPLC) beads 3-5 $\mu \mathrm{m}$ in diameter packed into a column serve as the stationary phase and a solution containing the solutes of interest is forced through the beads at high pressure. ${ }^{53}$ Reversed-phase high-performance liquid chromatography (RP-HPLC) uses a nonpolar stationary phase and more polar solvent to achieve separation of solutes that have at least one hydrophobic region to interact with the column material. ${ }^{53}$

Recall that at the end of DNA synthesis, a final detritylation reaction is performed to remove the DMT group protecting the 5'-hydroxyl of the last nucleotide added to the sequence (section 1.4, reaction 1 in Scheme 1.2). This step, however, need not be performed during automated DNA synthesis; the DNA can be deprotected and still retain this final DMT protecting group for its use as a hydrophobic tag in RP-HPLC (Figure 1.8). ${ }^{1}$ Failed sequences do not have a DMT group on their $5^{\prime}$-terminus as they are capped with an acetyl group (reaction 3 in Scheme 1.2) so they elute from the reversed-phase column very quickly. Full sequences with the 5'-DMT group intact will be retained in the HPLC column as the DMT group is attracted to the nonpolar beads. ${ }^{1}$ The DNA transfers between the stationary and mobile phases as the solvent is forced through the column and 
solvent molecules compete with it for sites on the stationary phase. ${ }^{53}$ DNA is eluted from the column using a continuous gradient of acetonitrile (MeCN) in aqueous buffer. ${ }^{86}$

Chromatograms are graphs that illustrate the response of a detector with elution time. $^{53}$ Spectrophotometric detectors are commonly used in HPLC systems for solute detection. An ultraviolet detector is useful for solutes that absorb ultraviolet light ${ }^{53}$ and so can be used for the detection of DNA (absorbs at about $260 \mathrm{~nm}$ ). ${ }^{1}$ Fluorescence detectors can also be useful if the DNA is labelled with a fluorescent molecule.

As discussed, RP-HPLC can be useful in purifying crude DNA following synthesis, but it can also be used to purify labelled DNA following a reaction with a molecule or complex of interest as long as the label has hydrophobic properties to act in place of the 5'-DMT group. For example, in Chapter 4 the conjugation of a platinum(II) complex to DNA will be discussed. Attempts to purify these conjugates using RP-HPLC were made as the complex contained organic ligands that would be expected to interact with the nonpolar reversed-phase column. Crude DNA could be used in the reaction and the failed sequences would be easily separated from the complete labelled and unlabelled DNA strands as discussed above. The labelled DNA would be slightly larger than the unlabelled strands and would contain a hydrophobic entity and so would elute later than the unlabelled DNA.

DNA that contains several guanine residues, such as the 15 mer thrombin aptamer, $^{8}$ tend to form intra- or intermolecular G-quadruplexes. ${ }^{84}$ As in PAGE, separation of DNA based on size rather than shape is desirable in RP-HPLC for efficient peak resolution and, therefore, purification of labelled and unlabelled DNA. Denaturing conditions that were applicable in PAGE (i.e. urea, formamide) are too harsh for use on a 
RP-HPLC column, ${ }^{84}$ but an organic cation that contains a positively charged portion for interaction with the anionic sugar-phosphate backbone of the DNA as well as a hydrophobic portion for interaction with the stationary phase can be effectively used to linearize DNA. A commonly used example is triethylammonium acetate (TEAA). ${ }^{86}$ The triethylammonium cation, $\left(\mathrm{CH}_{3} \mathrm{CH}_{2}\right)_{3} \mathrm{~N}^{+}$, associates with the negatively charged phosphate groups on the DNA backbone leading to a hydrophobic coating. The longer a strand of DNA, the more TEAA cations will associate with it, and the stronger its resultant interaction with the nonpolar column. Therefore larger DNA strands will be more strongly retained on the column than short strands and will elute later. ${ }^{86}$

\subsubsection{Ultraviolet-Visible (UV-vis) Spectrophotometry}

Light can be described as having properties of both particles and waves. Light waves consist of electric and magnetic fields that oscillate in planes perpendicular to each other. The distance from the crest of one peak to that of the next is defined as the wavelength $(\lambda)$, and the number of complete oscillations that the wave makes every second is defined as the frequency (v). Each photon (i.e. particle) of light has a particular energy that is inversely related to wavelength, as described by equation 1.1 where $h$ is Planck's constant and $\mathrm{c}$ is the speed of light. ${ }^{53}$

$$
E=\frac{h c}{\lambda} \quad[1.1]
$$

When a molecule absorbs ultraviolet (UV, $\lambda \approx 10-380 \mathrm{~nm}$ ) or visible (vis, $\lambda=$ $380-780 \mathrm{~nm}$ ) light the molecule enters an excited state in which electrons are promoted to higher-energy orbitals. ${ }^{53}$ When these electrons relax back to lower energy orbitals the molecule will emit light; ${ }^{.53}$ this is luminescence and will be discussed in more detail in 
Chapter 4. For the purpose of understanding how DNA is quantified using ultravioletvisible (UV-vis) spectrophotometry, its absorption of light at about $260 \mathrm{~nm}^{\prime}$ and the measurement of that absorption must be discussed.

The absorbance of a sample is the amount of the irradiating light that did not pass through the sample or was not transmitted. Transmission $(\mathrm{T})$ is expressed as the fraction of irradiating light that passed through the sample (P) compared to its initial irradiance $\left(\mathrm{P}_{0}\right)$, as in equation 1.2:

$$
T=\frac{P}{P_{0}}
$$

Absorbance $(\mathrm{A})$ is related to transmission as in equation 1.3:

$$
A=\log \left(\frac{P_{0}}{P}\right)=-\log T
$$

The measurement of a sample's absorbance is useful because of the linear relationship between absorbance and the concentration of the absorbing species. This is described by Beer's law

$$
A=\varepsilon b c
$$

where $c$ is the concentration of the light-absorbing species in mol/L (M), b is the pathlength the light travelled in $\mathrm{cm}$ (typically $1 \mathrm{~cm}$ ), and $\varepsilon$ is the molar absorptivity (or molar extinction coefficient) in $\mathrm{M}^{-1} \mathrm{~cm}^{-1}$. Absorbance is unitless. The molar absorptivity value depends on the wavelength of light, as does the absorbance, ${ }^{53}$ and for quantification of a chemical species the wavelength of maximum absorbance (or molar absorptivity) is generally considered.

To determine the concentration of DNA in a sample by measuring its absorbance it is necessary to know the molar absorptivity of the DNA sequence present. The pentose 
and phosphate moieties in the sugar-phosphate backbone of nucleic acids do not exhibit UV absorption above $230 \mathrm{~nm}$, so the absorbance of DNA at about $260 \mathrm{~nm}$ arises from the electronic transitions within the purine and pyrimidine moieties of the bases.' Each base, when considered alone, has a unique wavelength of maximum absorbance, ${ }^{1,87}$ but molar absorptivities of each base at the average wavelength of $260 \mathrm{~nm}$ are used when quantifying DNA (see Table 1.1). The molar absorptivity of a single-stranded DNA (ssDNA) molecule is calculated by summing the molar absorptivities of the individual nucleotides multiplied by the number of each nucleotide present in the sequence. ${ }^{88}$ In the example of the $15 \mathrm{mer}$ thrombin aptamer (see section 1.2 .3 ), ${ }^{8}$ there are 9 guanosine and 6 thymine residues present, so the overall molar absorptivity of this aptamer is $155700 \mathrm{M}^{-1}$ $\mathrm{cm}^{-1}[(9 \times 11500)+(6 \times 8700)]$. Once the absorption spectrum of a solution of ssDNA has been obtained, the absorbance at $260 \mathrm{~nm}$ and the molar absorptivity of the sequence can be used to calculate the concentration of DNA present using Beer's Law (equation 1.4).

Table 1.1 Wavelengths of maximum absorbance $\left(\lambda_{\max }\right)$ and molar absorptivities at $260 \mathrm{~nm}\left(\varepsilon_{260}\right)$ for DNA nucleoside-5'-monophosphates.

\begin{tabular}{|c|c|c|}
\hline DNA nucleoside-5'-monophosphate & $\lambda_{\max }(\mathrm{nm})^{[\mathrm{a}]}$ & $\varepsilon_{260}\left(\mathrm{M}^{-1} \mathrm{~cm}^{-1}\right)^{[\mathrm{b}]}$ \\
\hline adenosine & 259 & 15400 \\
cytosine & 271 & 7400 \\
guanosine & 252 & 11500 \\
thymine & 267 & 8700 \\
\hline [a] From reference 87. & & \\
[b] From reference 88. & \\
\hline
\end{tabular}


The molar absorptivity values for the individual nucleotides in Table 1.1 are applicable to ssDNA, but the interactions between the bases in double-stranded DNA (dsDNA) lead to a reduction in absorption (and $\varepsilon_{260}$ ) of up to $30 \%$. This effect is called hypochromicity. ${ }^{1}$ In hybridized DNA the bases can share $\pi-\pi$ electron interactions when the purine and pyrimidine rings are stacked face-to-face. This affects the transition dipoles of the bases that arise upon interaction of light with the DNA and leads to the drop in absorbance demonstrated by dsDNA., 89

The change in absorbance between dsDNA and ssDNA proves useful in determining the melting temperature $\left(T_{m}\right)$ of two hybridized DNA strands. The melting temperature is the temperature at which there is a transition from a double-stranded helical pair of DNA to two unstacked single DNA strands. ${ }^{1}$ A melting curve is obtained by monitoring the absorbance of a DNA sample at $260 \mathrm{~nm}$ over a range of temperatures. The absorbance of the DNA will increase as the temperature increases and provides the energy to break the hydrogen bonds between base pairs in the hybridized DNA, eventually resulting in two completely separate strands. The midpoint of the curve is taken as the melting temperature. ${ }^{1}$ The $T_{m}$ of a specific pair of DNA strands depends on base composition, monovalent cation concentration, and the length of the duplex. The base composition plays a role since G-C base pairs have three hydrogen bonds compared to the two in A-T pairs; it will therefore take more energy and result in a higher $T_{m}$ when there is a higher G-C content in a DNA duplex. Since monovalent cations help stabilize dsDNA, a lower salt concentration will lead to a lower $T_{m}$. Finally, for dsDNA that is shorter than a few hundred base pairs, the $T_{m}$ will be affected by the length of the duplex; longer sequences will have higher melting temperatures and steeper melting curves.' 
Performing a melting study on a duplex can help ensure that the proper conditions are used to promote hybridization (i.e. annealing) of complementary DNA strands, as described in sections 2.2 .7 and 2.3.4. It can also provide information about changes in the stability of a duplex upon the inclusion of modified bases or labels.'

\subsection{Project goals}

The following two chapters will discuss the development of thrombin aptamergadolinium(III) conjugates as a proof-of-concept system for the use of aptamers in targeted magnetic resonance imaging (MRI). Chapter 2 will deal with aptamer conjugates in which one aptamer strand is conjugated to the metal chelator diethylenetriaminepentaacetic acid (DTPA). The development of the methods needed to prepare, purify, and characterize the DNA-DTPA conjugates will be discussed along with their relaxation properties under different conditions and at different magnetic field strengths. Chapter 3 will focus on the various reaction conditions used while attempting to optimize the conjugation of two thrombin aptamer strands to one DTPA moiety. The relaxation properties of these double-conjugates will also be explored.

Chapter 4 encompasses a separate project in which a luminescent platinum(II) complex was synthesized, purified, and characterized for eventual use in the study of aptamer folding upon target binding. Work on the conjugation of the isolated platinum(II) complex to a DNA aptamer and random sequence DNA strands will be discussed. 


\section{Singly-labelled DNA-DTPA conjugates}

\subsection{Introduction}

Due to their molecular recognition abilities and minimal side-effects in vivo, aptamers have been investigated for use in a variety of targeted imaging applications. For example, studies have been reported on the use of functionalized superparamagnetic iron oxide nanoparticles, ${ }^{17,}{ }^{18}$ quantum dot-aptamer conjugates, ${ }^{90}$ fluorescently-labelled aptamers, ${ }^{9 !}$ radio-labelled aptamers ${ }^{92}$, and a smart aptamer-based magnetic resonance imaging (MRI) contrast agent. ${ }^{16}$ These systems will be discussed further following a general introduction to MRI.

\subsubsection{Magnetic resonance imaging (MRI) background theory}

\subsubsection{Nuclear spin}

Magnetic resonance imaging (MRI) is a common medical diagnostic technique used to obtain three-dimensional images of soft tissue with high resolution. ${ }^{93}$ It works on the same principles as nuclear magnetic resonance (NMR) spectroscopy but is used to obtain images as opposed to spectra. These techniques are made possible by the intrinsic property of nuclear spin. Nuclei with unpaired proton and neutron spins exhibit this property therefore it occurs in isotopes with an odd atomic number and/or an odd atomic mass. ${ }^{94}$ Protons, with an atomic number of 1 and no neutrons $\left({ }^{1} \mathrm{H}\right)$, have a nuclear spin (I) of $1 / 2$ and since they are abundant in biological systems they are used to create the images obtained using MRI. ${ }^{95,96}$

The intrinsic spin of a proton can be visualized as a spherical particle spinning about its own axis with a resulting angular momentum. Since it is a charged particle, a 
spinning proton generates a magnetic field and so is influenced by external magnetic fields to which it is exposed. ${ }^{96}$ Consider a collection of protons placed into an external magnetic field designated $\mathrm{B}_{0}$, as in Figure 2.1. Each proton may exist in one of two alignment states with respect to the external field; they might line up with or against the applied field. This is evident when using equation 2.1 (recall that I is nuclear spin): $:^{95}$

$$
(2 \times I)+1
$$

A small majority of proton spins in a population will align with the external magnetic field since this is the lower energy state and so will result in a net magnetization of the sample in the direction of $\mathrm{B}_{0}$ (Figure 2.1).

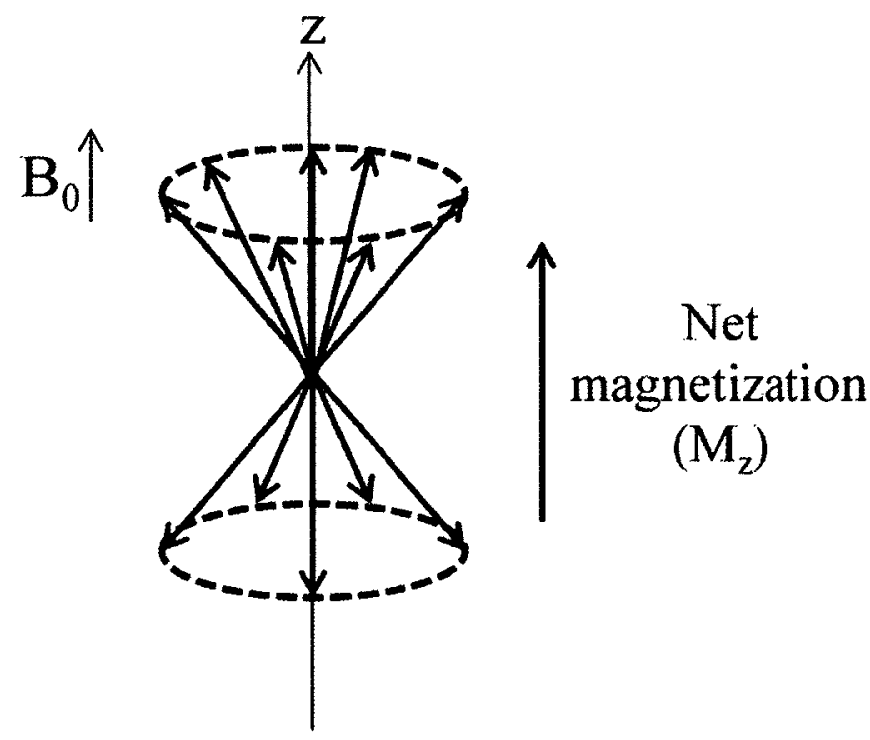

Figure 2.1 A collection of protons and their precession around an external magnetic field $\left(B_{0}\right)$ at equilibrium. Slightly more than half of the proton spins (represented by arrows) are aligned with the external field and a little less than half are oriented against it. The net magnetization $\left(\mathbf{M}_{2}\right)$ is therefore in line with the external field. Modified from reference 95. 
Figure 2.1 also illustrates that though only two states are allowed when using quantum mechanics to describe the behaviour of protons in an external magnetic field, protons actually precess around the axis of the external field. ${ }^{95}$ The frequency of this precession in an external magnetic field is characteristic of each nucleus with the intrinsic property of spin and is referred to as the Larmor frequency $\left(\omega_{0}\right)$. It is calculated using equation 2.2 where $\omega_{0}$ is the Larmor frequency in megahertz $(\mathrm{MHz}), \gamma$ is the gyromagnetic ratio (a proportionality constant unique to each element), and $B_{0}$ is the magnetic field strength of the external field in tesla (T). The gyromagnetic ratio of protons is $42.58 \mathrm{MHz} / \mathrm{T}^{96}$

$$
\omega_{0}=\gamma \times B_{0}
$$

At equilibrium, the precessional motion of a population of protons around the axis of an external field is randomly distributed. As a result, the net magnetization in the plane perpendicular to $\mathrm{B}_{0}$ (i.e. the $x y$ plane as the $z$-axis is always aligned with $\mathrm{B}_{0}$ ) is equal to zero. $^{95}$ When there is no net magnetization in the xy-plane, no signal is emitted by the system. ${ }^{96}$

\subsubsection{Relaxation}

Signal contrast in MR images is dependent on the relaxation times of in vivo water protons. The relaxation time is the time required to reach the equilibrium arrangement of proton spins (as in Figure 2.1) once a population of protons is placed in an external magnetic field. The relaxation time depends on the physical environment in which the protons are located. For example, different tissues have different relaxation 
times when exposed to the same external field. ${ }^{95}$ There are two types of relaxation: longitudinal (or spin-lattice) relaxation and transverse (or spin-spin) relaxation. ${ }^{97}$

Longitudinal relaxation is also referred to as $T_{1}$ relaxation, and is achieved upon full recovery of the net magnetization aligned with $B_{0}$ (i.e. in the positive $z$-direction) at equilibrium. It is also called spin-lattice relaxation because the protons at higher energy (or those aligned against $\mathrm{B}_{0}$ ) in a non-equilibrated system transfer energy to the surroundings, or the lattice, in order to achieve relaxation. ${ }^{95,97}$ In $\mathrm{T}_{1}$-weighted $\mathrm{MR}$ images, tissues with shorter $T_{1}$ values will appear brighter than those with longer $T_{1}$ 's because they relax more quickly and emit more of a signal. ${ }^{96}$

Transverse relaxation, or $T_{2}$ relaxation, is the time required for complete loss of net magnetization in the transverse or $x y$-plane (i.e. perpendicular to $\mathrm{B}_{0}$ ); recall that when proton spins are equilibrated, there is no net transverse magnetization and so this process is also a return to equilibrium. ${ }^{95}$ The term spin-spin relaxation also applies because, in this case, energy is transferred between nuclei that are aligned with $\mathrm{B}_{0}$ (i.e. in the lower energy state) and not with the surroundings. ${ }^{97} \mathrm{~T}_{2}$-weighted $\mathrm{MR}$ images have the opposite trends of $T_{1}$-weighted images: tissues with shorter $T_{2}$ values will be darker than those with longer $T_{2}$ 's since the signal only persists while there is some net transverse magnetization. $^{95,96}$

The pulse sequence for an inversion-recovery experiment along with representative images of the net magnetization of the system are illustrated in Figure 2.2. This type of experiment is generally used to determine $T_{1}$ relaxation (or obtain $T_{1}$ weighted MR images), but it can be used to measure $T_{2}$ relaxation as well. ${ }^{96}$ For the purposes of this work, the determination of $T_{1}$ relaxation is illustrated and will be 
discussed in reference to a chemical sample as opposed to a biological specimen (i.e. a human subject).

A)
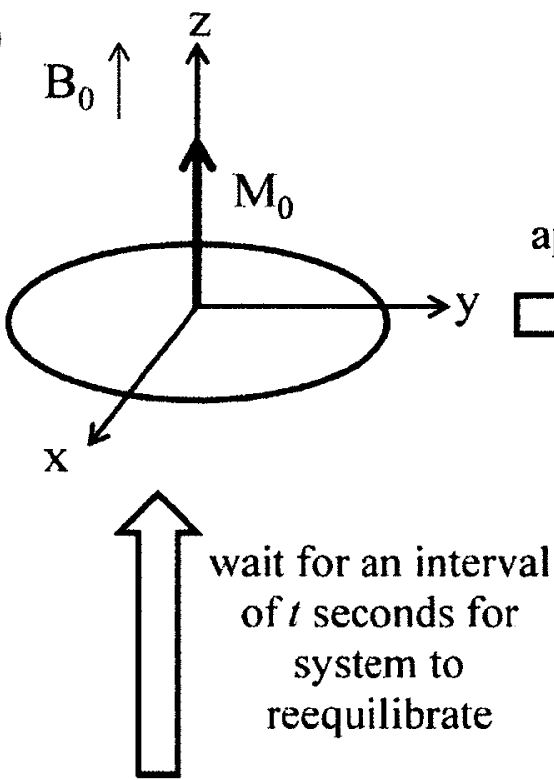

D)

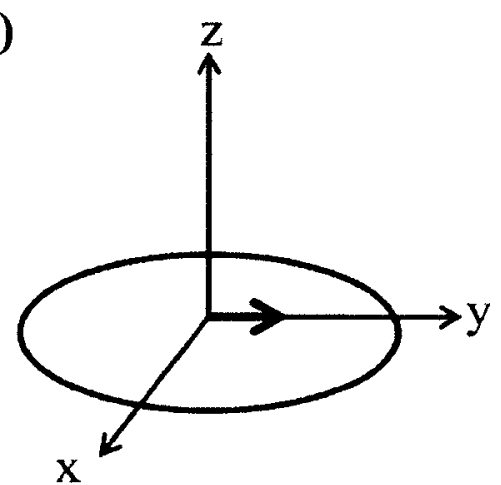

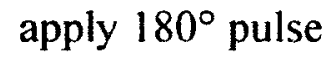

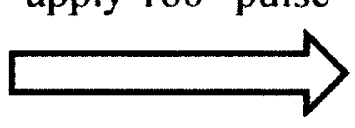

B)

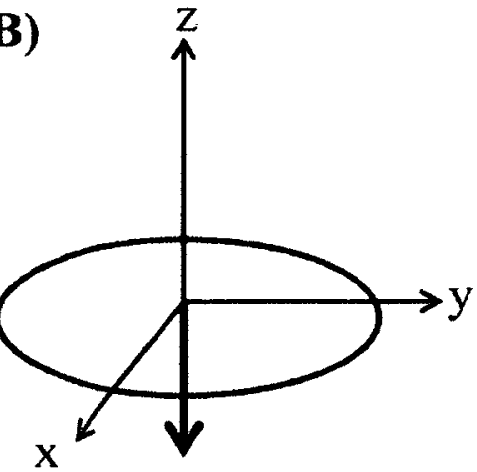

wait for an
interval of
$\tau$ seconds

C) apply $90^{\circ}$ pulse then acquire data

Figure 2.2 Sequence of an inversion-recovery experiment for the measurement of $T_{1}$ relaxation. The value of $t$ remains constant while $\tau$ changes each time through the sequence. See text for further details. Modified from references 96 and 98.

When a sample is placed in an external magnetic field, $\mathrm{B}_{0}$, the net longitudinal magnetization $\left(\mathrm{M}_{0}\right)$ of the sample will be aligned with $\mathrm{B}_{0}$, as discussed above. This is 
illustrated in Figure $2.2 \mathrm{~A}$ by the bold arrow on the positive $\mathrm{z}$-axis. To perturb the proton spins from their equilibrium positions, an electromagnetic wave with a frequency matching the Larmor frequency of a proton can be applied; this transfers energy into the system. With a radio frequency pulse of the correct power and duration, precise deflection of the longitudinal magnetization by a certain angle can be achieved. ${ }^{96}$ For example, the application of a $180^{\circ}$ pulse will result in $\mathrm{M}_{0}$ being deflected to the negative $\mathrm{z}$-axis as in Figure 2.2B. At this point of the experiment, there is a wait period of tau $(\tau)$ seconds to allow the partial or full recovery of equilibrium/longitudinal magnetization (partial recovery is illustrated in Figure 2.2C). No signal is emitted at this point as there is no net magnetization in the xy-plane. After the wait period of length tau seconds, a $90^{\circ}$ pulse is applied to the sample which results in the deflection of $M_{0}$ into the $x y$-plane. $A$ signal is emitted due to the resulting precession of magnetization in the xy-plane (Figure 2.2D) and thus data is acquired at this point. The system is allowed to reequilibrate for time $\mathrm{t}$ seconds and the process illustrated in Figure 2.2 is repeated with different values of tau each time..$^{96,97}$

The intensity of the signal at time tau $\left(I_{\tau}\right)$ and the tau values themselves are used to solve for $T_{1}$ using equation $2.3:^{.97}$

$$
I_{\tau}=I_{e q}\left[1-a \exp \left(-\frac{\tau}{T_{1}}\right)\right]
$$

The software used to run NMR spectrometers include programs that can solve this equation simultaneously for $I_{e q}$ (intensity at equilibrium), $a$ (a constant), and $\mathrm{T}_{1}$ using the data obtained from a minimum of ten points (i.e. ten tau values). ${ }^{97}$ 


\subsubsection{Dipole-dipole relaxation mechanisms}

As mentioned above, the intrinsic spin of a proton generates a local magnetic field or a dipole. Consider a sample in which many protons are located in close proximity, such as in water. If the local field generated by one dipole resonates at the Larmor frequency this local field can cause transitions in a separate, nearby dipole analogous to the effects of a radio frequency pulse as discussed above. ${ }^{95,99}$ This is referred to as the dipole-dipole mechanism of relaxation, and it is similar to one of the relaxation mechanisms induced in the presence of a paramagnetic species. ${ }^{99}$

A paramagnetic species, such as gadolinium(III), is an atom or molecule that has one or more unpaired electrons in its valence orbitals and so displays a net magnetic moment in line with an applied external field. ${ }^{95,96}$ Superparamagnetic species have particularly pronounced paramagnetic characteristics, that is, a greatly increased magnetic moment. This results from the increased mobility of electrons when paramagnetic ions are arranged in a fixed crystal lattice. ${ }^{96}$ The net magnetic moment of a paramagnetic species leads to the generation of a local magnetic field as the atom or molecule tumbles in solution. This local field can interact with proton dipoles in the same way that the spin of one proton can affect another (as described above). When a paramagnetic species' local magnetic field resonates at or close to the Larmor frequency of a proton, it will affect the orientation of nearby proton spins and therefore lead to relaxation. ${ }^{99}$ The dipole-dipole (DD) relaxation ("through-space") mechanism between a paramagnetic species and nearby protons has a substantial effect on the longitudinal $\left(\mathrm{T}_{1}\right)$ relaxation rate $\left(R_{1}=1 / T_{1}\right)$ of a sample, while scalar, or contact ("through-bonds"), relaxation has a negligible effect. ${ }^{95,} 100$ The relaxation-enhancing properties resulting 
from such effects have led to the development and use of paramagnetic agents in MRI to improve contrast in and increase the diagnostic value of the images obtained.

\subsubsection{MRI contrast agents (CA)}

Since the approval in 1988 of the first gadolinium(III) chelate for use as an MRI contrast agent (CA), research in the area of MRI CAs has greatly expanded. ${ }^{101}$ More than $30 \%$ of all clinical MRI scans are performed using a Gd(III)-based $\mathrm{CA}^{102}$ and the development of new CAs is a highly active area of research. Gadolinium(III) is useful as a proton relaxation agent because it is the only ion with seven unpaired electrons, ${ }^{101}$ but it cannot be administered as a free ion because of its high toxicity $\left(\mathrm{LD}_{50}=0.1 \mathrm{mmol} \mathrm{kg}\right.$ $\left.{ }^{1}\right)^{101,103}$ As a result, it must be administered as part of a stable chelate with a multidentate ligand such as diethylenetriaminepentaacetic acid (DTPA, Figure 2.3A) or tetraazacyclododecanetetraacetic acid (DOTA, Figure 2.3B). ${ }^{93,101}$ These chelators are examples of the two structural types of Gd(III)-based CAs currently approved for use: open-chain or linear (DTPA) and macrocyclic (DOTA). It can be seen in Figure 2.3A and $\mathrm{B}$ that when chelated with $\mathrm{Gd}(\mathrm{III})$ these ligands form ionic species, and Figure $2.3 \mathrm{C}$ illustrates the counter-ion used in the formulations of these two CAs: Magnevist ${ }^{\circledR}$ consists of a dimeglumine salt and Dotarem $\left(\right.$ is a meglumine salt, respectively. ${ }^{104}$ Despite there often being a need to image only a specific organ or tissue, many currently

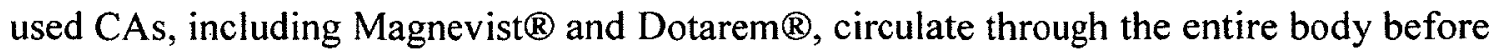
being eliminated. ${ }^{93}$ This requires large standard doses of $0.1 \mathrm{mmol} \mathrm{kg}^{-1}$ to cause a detectable signal change in an MR image; ${ }^{105}$ this translates to micromolar concentrations needed at imaging sites. ${ }^{106}$ 


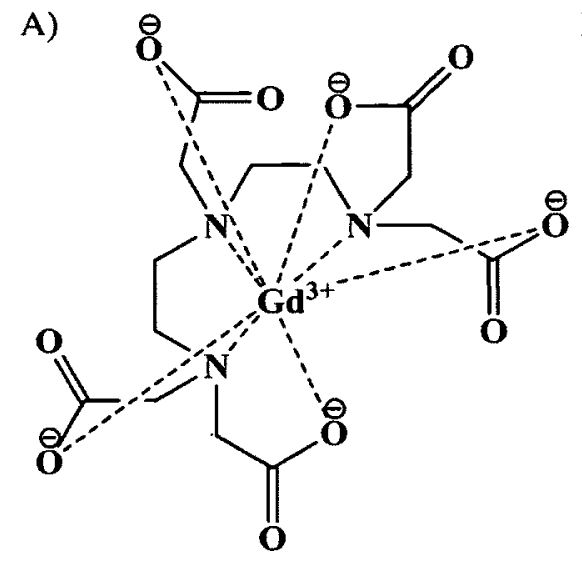

Figure 2.3 A) Gd(III)-DTPA (Magnevist®); B) Gd(III)-DOTA (Dotarem®); C) meglumine. ${ }^{104,107}$
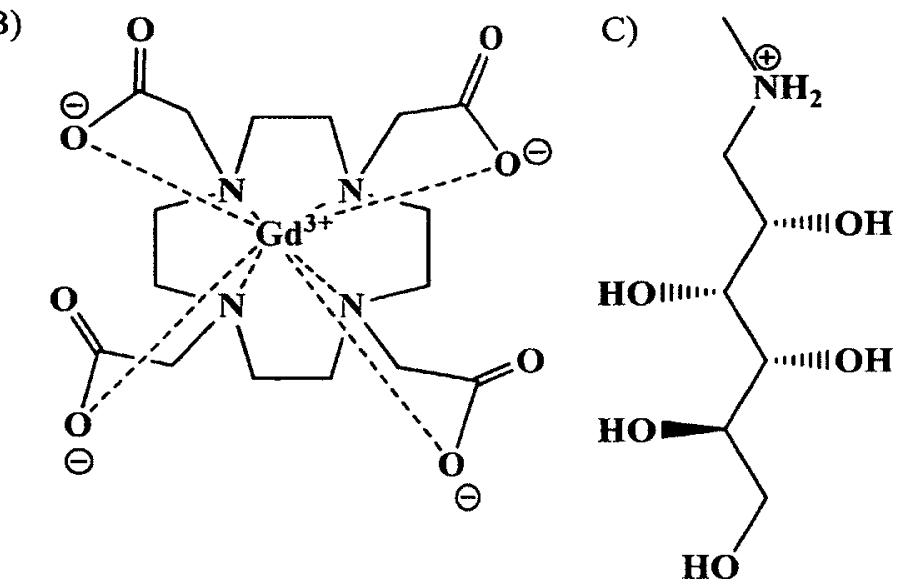

Effective targeted MRI CAs that would collect at specific areas of interest are greatly desirable as an alternative to the nonspecific agents. Targeted CAs would require the administration of relatively lower doses than general CAs, as well as providing sharper contrast in MR images. ${ }^{108}$ Some targeted MRI CAs are already in clinical use, such as MS-325 (Ablavar $\left.{ }^{\circledR}\right)$ to image blood vessels ${ }^{109}$ and superparamagnetic iron oxide nanoparticles (Feridex I.V.(B) to image liver lesions. ${ }^{110}$ Much research is being done to develop or improve targeted MRI CAs using paramagnetic liposomes, ${ }^{111,112}$ polymers, ${ }^{113-}$ 116 iron oxide nanoparticles, ${ }^{17,117,118}$ proteins, $^{19-121}$ and nucleic acids. ${ }^{16,122,123}$ Some examples will be presented in sections 2.1.2.3 and 2.1.3.1. First a discussion of what makes a good CA, aside from paramagnetism, will be presented.

\subsubsection{Relaxivity}

In order to compare the effectiveness of existing and newly developed CAs, or variations on CAs already approved for use, their relaxivities are determined and reported, first under in vitro conditions and then in in vivo experiments. Relaxivity is the 
concentration-dependent effect of a $\mathrm{CA}$ on nearby water proton relaxation in a magnetic field. $^{101}$ Since contrast agents containing the paramagnetic Gd(III) ion affect the relaxation of water protons, it is through the measurement of $T_{1}$ or $T_{2}$ relaxation at varying concentrations of a CA that its respective relaxivity values, $r_{1}$ and $r_{2}$, can be determined. The relationship used to determine relaxivity is shown in equation 2.4:

$$
\frac{1}{T_{i}^{o b s}}=\frac{1}{T_{i}^{d i a}}+r_{i}\left[G d^{3+}\right]
$$

where $\mathrm{i}$ is 1 or 2 (for longitudinal and transverse relaxation, respectively), $T_{i}^{o b s}$ represents the $T_{i}$ value obtained experimentally, $T_{i}^{d i a}$ is the diamagnetic contribution to $T_{i}$ (in other words, the $\mathrm{T}_{\mathrm{i}}$ of the sample without the paramagnetic species present), $r_{i}$ is the relaxivity in $\mathrm{mM}^{-1} \mathrm{~s}^{-1}$, and $\left[G d^{3+}\right]$ is the concentration of the $\mathrm{Gd}(\mathrm{III})$-based contrast agent in $\mathrm{mM}$. $T_{i}$ values are expressed in seconds. Equation 2.4 illustrates that the relaxivity is the slope of a $\frac{1}{T_{i}^{o b s}}$ versus $\left[G d^{3+}\right]$ curve. $^{101}$ Gadolinium(III)-based CAs induce better contrast enhancement in $T_{1}$-weighted $M R$ images than in those that are $T_{2}$-weighted, ${ }^{101}$ so the remaining discussion will focus on longitudinal $\left(T_{1}\right)$ relaxation. CAs with higher $r_{1}$ values allow faster restoration of longitudinal magnetization, which in turn enables the high frequency pulses needed for rapid, high resolution $T_{1}$-weighted imaging. ${ }^{101}$

\subsubsection{Factors that influence the relaxivity of MRI CAs}

The gadolinium(III) ion in a CA can effectively decrease the $T_{1}$ relaxation of nearby water protons through directly coordinating one or more water molecules (innersphere contribution, IS) and by influencing those in molecules that hydrogen-bond to the complex or its counter-ions (second-sphere contribution, SS) and that diffuse freely next 
to the complex (outer-sphere contribution, OS). Therefore, the overall relaxivity is made up of contributions from each of these solvation spheres as in equation $2.5:^{124}$

$$
r_{1}^{o b s}=r_{1}^{I S}+r_{1}^{S S}+r_{1}^{o S}
$$

The inner-sphere contribution to relaxivity $\left(r_{1}^{I S}\right)$ is summarized by the SolomonBloembergen-Morgan theory as in equation $2.6:^{102,125}$

$$
r_{1}^{I S}=\frac{\left[G d^{3+}\right] q}{55.5} \frac{1}{T_{1 M}+\tau_{m}} \quad[2.6]
$$

where the concentration of the Gd(III)-based agent is in moles per litre, $q$ is the number of inner-sphere water molecules coordinated directly to the $\mathrm{Gd}(\mathrm{III})$ ion, $T_{1 M}$ is the longitudinal relaxation time of the bound water protons, and $\tau_{m}$ is the mean residence time of the inner-sphere water molecules in seconds. The first term represents the molar fraction of the bound water molecules. ${ }^{102}$

As discussed in section 2.1.1.3, longitudinal relaxation is significantly affected through the interactions of the dipoles of the paramagnetic species and of water protons. The relaxation rate of the bound water protons $\left(\frac{1}{T_{1 M}}\right)$ is therefore equal to the relaxation rate resulting from the dipole-dipole (DD) mechanism $\left(\frac{1}{T_{1}^{D D}}\right)$, the equation for which is shown below: $:^{125}$

$$
\frac{1}{\tau_{1}^{D D}}=\frac{2}{15} \frac{\gamma_{I}^{2} g^{2} \mu_{B}^{2}}{r_{G d H}^{6}} S(S+1)\left(\frac{\mu_{0}}{4 \pi}\right)^{2}\left[\frac{7 \tau_{c 2}}{1+\omega_{S}^{2} \tau_{c 2}^{2}}+\frac{3 \tau_{c 1}}{1+\omega_{I}^{2} \tau_{c 1}^{2}}\right]
$$

The constants used in equation 2.7 are summarized in section 2.2.12, Table 2.2. The dipolar relaxation rate $\left(\frac{1}{T_{1}^{D D}}\right)$ is related to the strength of the magnetic field, which is demonstrated through the inclusion of the electron $\left(\omega_{S}\right)$ and nuclear $\left(\omega_{l}\right)$ Larmor 
frequencies in equation 2.7. ${ }^{101}$ Recall that the nuclear Larmor frequency is calculated using equation 2.2, and is field dependent. The Larmor frequency of an electron is 658 times that of a proton at a specific magnetic field. ${ }^{125}$

Continued consideration of equation 2.7 also shows that dipolar relaxation is affected by the longitudinal and transverse correlation times, $\tau_{c i}$ with $\mathrm{i}=1$ and 2 , respectively. The correlation time is expressed in seconds, and the values of $\tau_{c i}$ must be calculated using equation 2.8 since the correlation time has contributions from the rotational correlation time $\left(\tau_{R}\right)$, the mean residence time of the inner-sphere water molecules $\left(\tau_{m}\right)$, and the relaxation time of the unpaired electrons $\left(T_{i e}\right)$.

$$
\frac{1}{\tau_{c i}}=\frac{1}{\tau_{R}}+\frac{1}{\tau_{m}}+\frac{1}{T_{i e}}
$$

All of these values are expressed in seconds and they represent the different dynamic processes occurring on a molecular level. ${ }^{101}$

The rotational correlation time is the tumbling rate of a $\mathrm{CA}$ and is therefore related to its size. Small molecule CAs such as Magnevist巴 and Dotarem $\circledast$ have $\tau_{R}$ values in the picosecond range ${ }^{93,126}$ and exhibit relatively field independent $r_{1}$ relaxivity due to this fast tumbling. ${ }^{124}$ The mean residence time of water molecules $\left(\tau_{m}\right)$ directly coordinated to $\mathrm{Gd}(\mathrm{III})$ is inversely related to the exchange rate $\left(k_{\text {ex }}\right)$ of the water molecules at the ion centre. With a faster exchange rate (i.e. lower $\tau_{m}$ ), more water molecules directly associate with the Gd(III) centre, experience enhanced relaxation, and are exchanged with molecules in the bulk solution (i.e. the second- and outer-spheres). However, $\tau_{m}$ must also be long enough to permit efficient relaxation of the coordinated water protons for optimal relaxation enhancement; there is therefore an optimal range for 
$\tau_{m}{ }^{124}$ Furthermore, when $\tau_{m} \ll T_{1 M}$, the relaxation of the bulk solution can be described as the relaxation rate enhancement of the coordinated water molecule $\left(\frac{1}{T_{1 M}}\right)$, as clear from equation $2.6 .^{101}$

The longitudinal and transverse electronic relaxation times (i.e. relaxation of the unpaired electrons), $T_{1 e}$ and $T_{2 e}$, represent the thermal transitions of Gd(III)'s unpaired electrons between low and high energy states. ${ }^{107}$ Their respective relaxation rates are field-dependent and can be calculated using equations 2.9 and 2.10 . In these equations, $\mathrm{C}$ represents the expression shown in equation $2.11 .^{125} \Delta^{2}$ is the mean-square zero-field splitting (ZFS) energy. ${ }^{127}$ Zero-field splitting is the loss of degeneracy of electron spin states when there is no external field applied; ${ }^{128}$ this can be caused by the collision of solvent molecules or by molecular vibrations. ${ }^{101} \tau_{\mathrm{v}}$ is the correlation time for the modulation of the ZFS interaction. ${ }^{127}$

$$
\begin{gathered}
\frac{1}{T_{1 e}}=2 C\left(\frac{1}{1+\omega_{S}^{2} \tau_{v}^{2}}+\frac{4}{1+4 \omega_{S}^{2} \tau_{v}^{2}}\right) \\
\frac{1}{T_{2 e}}=C\left(\frac{5}{1+\omega_{S}^{2} \tau_{v}^{2}}+\frac{2}{1+4 \omega_{S}^{2} \tau_{v}^{2}}+3\right) \\
C=\frac{1}{50} \Delta^{2} \tau_{\mathrm{v}}\{4 S(S+1)-3\}
\end{gathered}
$$

Working backwards from equation 2.11 to equation 2.6 allows for the theoretical calculation of a CA's relaxivity at a particular concentration (since $\left[G d^{3+}\right]$ is part of equation 2.6) as described in the supplementary material of a report by Yang et al. ${ }^{125}$ Values for parameters such as $\Delta^{2}$ and $\tau_{v}$ have been reported for many existing CAs from experiments using nuclear magnetic resonance dispersion (NMRD), among other methods. ${ }^{126,127,129}$ In NMRD, the longitudinal proton relaxation in the presence of a CA 
is measured as a function of magnetic field and a number of parameters can be calculated based on the results. ${ }^{127}$ The values of such parameters can be used as a starting point in theoretical calculations. This will be described in more detail in sections 2.3.6 and 2.3.7.

In addition to the effects molecular processes such as $\tau_{R}$ and $\tau_{m}$ have on relaxivity, the solution in which a $\mathrm{CA}$ is located can influence its relaxivity. This is exemplified by the nonlinear effects of varying viscosities on CA relaxivity, with longer relaxation times seen at low and high viscosity and shorter relaxation times at intermediate viscosities. $^{130}$ This can be explained by considering the fluctuations in a CA's local magnetic field as its correlation time changes under various viscosity conditions. As discussed above, the closer the frequency of the agent's local magnetic field to the Larmor frequency of a proton, the greater its effect on relaxation times. Considering all CA complex molecules in a solution, fewer achieve tumbling rates that closely match the Larmor frequency of a proton under both low and high viscosity conditions. $^{130}$

\subsubsection{Targeted MRI CAs}

Much of the research on CA improvement has focused on ligand optimization for enhanced relaxivity. Optimization of parameters such as hydration number and mean residence time of the water molecules can lead to improved efficacy, ${ }^{131}$ so families of Gd(III) chelates in which two water molecules can coordinate to the metal ion (i.e. $q=2$ in hydroxypyridinone-based agents) are under study for future use as MRI CAs. ${ }^{93}$

Another approach to the improvement of CAs is to use targeted agents that experience an increase in size upon target-binding that can translate into a measurable 
increase in the relaxivity achieved compared to the CA alone. ${ }^{106,106}$ The rapid tumbling of small molecular contrast agents such as DTPA-Gd(III) and DOTA-Gd(III) causes their local magnetic field to fluctuate on the gigahertz level; recall that proton nuclear spins resonate on the order of megahertz. The interaction of a small molecule with a larger moiety, such as a protein, slows the tumbling, and therefore the magnetic field fluctuation, and brings it closer to that of protons and makes the CA a more effective relaxation agent. This effect is called the receptor-induced magnetization enhancement (RIME). ${ }^{106}$ Thus, the use of molecular recognition agents, such as antibodies and aptamers, in targeted CAs could be an effective strategy, not only due to the active targeting capability afforded by the recognition moiety but also due to the relaxivity improvement from the increase in size upon binding due to the RIME effect. From the molecular tumbling standpoint, it is plausible that initially smaller CAs based on nucleic acid aptamers would provide a greater change in relaxivity upon target binding, as opposed to initially larger CAs based on antibodies. ${ }^{122}$ Molecular tumbling is, of course, only one contributing factor among several that must be correctly balanced ${ }^{93}$ to achieve an enhancement of relaxivity when an aptamer-based CA binds to its target. Such targeted agents could also have the previously mentioned advantages of lower required doses and potentially sharper contrast in MR images due to high localized concentrations. $^{108}$

The MRI contrast agent MS-325 (Ablavar $(\mathbb{B})$ is approved for use in imaging blood vessels. MS-325 consists of a modified DTPA backbone chelated with Gd(III); ${ }^{109}$ its structure is illustrated in Figure 2.4. The biphenylcyclohexyl moiety connected to the DTPA backbone through a phosphodiester linkage interacts noncovalently with serum 
albumin, the most abundant protein in blood plasma. ${ }^{106}$ The relaxivity of the proteinbound agent (in vitro in human plasma) is over 8 times or $700 \%$ higher than its relaxivity unbound in buffer. ${ }^{109}$ The half-life of MS-325 in vivo is extended as a result of its interaction with albumin, and this increases the effectiveness of a single dose. The interaction merely delays filtration through the kidneys since a small fraction of the agent will remain unbound in the blood due to the reversible nature of its interaction with albumin. ${ }^{106}$ The reversible binding of this agent to albumin is comparable to the binding of an aptamer, such as the thrombin-binding aptamer, to its target. In both cases, an equilibrium is established between the free binder $(A)$ and free target $(T)$ and the complex they form upon interaction $(\mathrm{C})$, as shown in equation $2.12 .^{132}$

$$
\mathrm{A}+\mathrm{T} \rightleftharpoons \mathrm{C}
$$

The existence of some unbound MS-325 ensures that the CA is gradually removed from the body, ${ }^{106}$ which is important with all Gd(III) agents due to the toxicity of free Gd(III).

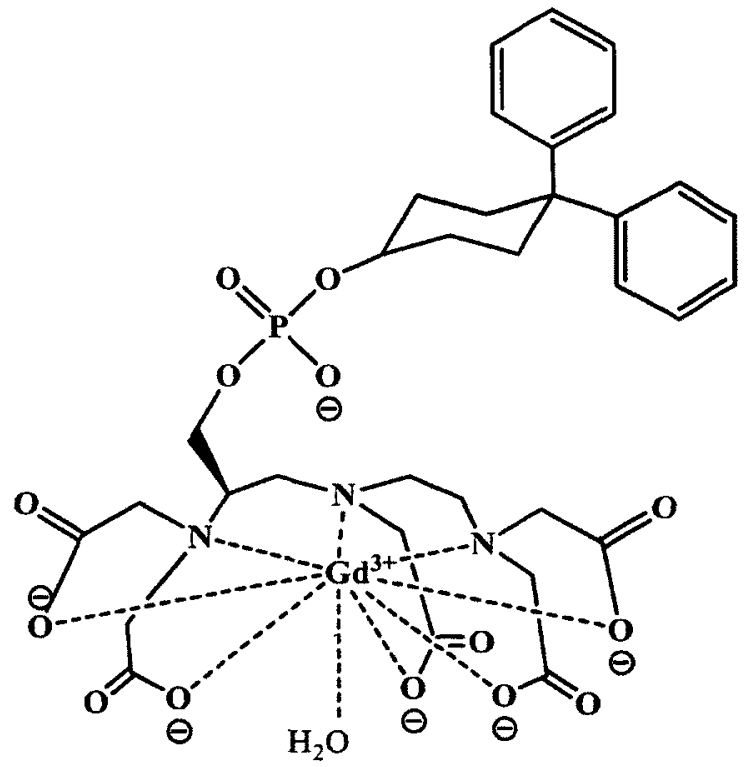

Figure 2.4 The structure of targeted MRI agent MS-325. ${ }^{106}$ 
The use of an antisense oligonucleotide-based probe as an MRI contrast agent was explored by Hines et al. where they developed a paramagnetic DNA 6mer that was complementary to a macromolecular target, $5 \mathrm{~S}$ rRNA. ${ }^{122}$ The complementary 6 mer was conjugated to DTPA. Following the addition of $\mathrm{Gd}(\mathrm{III})$ for chelation, the relaxivity of the paramagnetic oligonucleotide was measured alone and in the presence of the target at two magnetic fields. The authors found enhancements of the complementary 6 mer's relaxivity when in the presence of $5 \mathrm{~S}$ rRNA of 16 and $15 \%$ at 6.3 and $1.5 \mathrm{~T}$, respectively. They also determined the relaxivity of the paramagnetic $6 \mathrm{mer}$ in the presence of a protein target to which it did not bind and was about the same mass as their target, 5S rRNA, to determine how much of this enhancement resulted from viscosity effects upon introduction of the protein target. In the presence of this control protein, relaxivity enhancements of 3 and $7.7 \%$ at 6.3 and $1.5 \mathrm{~T}$, respectively, were found when compared to the conjugate's relaxivity alone. Therefore this system demonstrated that a net relaxivity enhancement was caused through interaction of an antisense paramagnetic oligonucleotide with a macromolecular target, with net relaxivity increases at 6.3 and 1.5 T of 13 and $7.3 \%$, respectively.

\subsubsection{MRI CA stability and safety}

In 2006, the first report linking the use of Gd(III)-based CAs with the occurrence of a condition called nephrogenic systemic fibrosis (NSF) in patients with impaired renal function was published. ${ }^{133,134}$ NSF causes thickening and hardening of the skin and so is often characterized by a skin biopsy, but it can also involve muscle, tendons, the lungs 
and the heart, among other organs. ${ }^{133,135}$ It can result in significant disability or even death. $^{134}$

Once the link between the administration of Gd(III)-based CAs to patients with kidney disease was identified, further exploration of the subject led to the identification of the marketed contrast agents most commonly associated with occurrences of NSF. As of 2008 , an overwhelming majority of high-risk patients with NSF had been injected with DTPA-BMA-Gd(III) (gadodiamide or Omniscan ${ }^{\mathrm{TM}}$ ), ${ }^{134}$ an agent based on the DTPA frame but with two amide groups replacing two of the carboxylate groups (see Figure 2.12B in section 2.3.5.3 for its structure). It has been proposed that $\mathrm{Gd}(\mathrm{III})$-based CAs pose a risk based on four properties of the chelates used. A relationship between dosage and risk of NSF has been identified, and CAs with high relaxivities can be administered in lower doses while still providing good contrast. Therefore, agents with higher relaxivities are thought to be safer. There is also the method by which the $\mathrm{CA}$ is removed from the body. Most Gd(III)-based CAs are eliminated in urine, and therefore they have increased half-lives in patients with impaired kidney function. There are some approved CAs that are partially eliminated by hepatic excretion since they bind to proteins in vivo (such as MS-325). ${ }^{104,136}$ Finally, the kinetic and thermodynamic stabilities of the chelates have been studied in more detail ${ }^{104,137,138}$ as these properties are thought to explain the differential risks of approved Gd(III)-based CAs. ${ }^{136}$

The stability of $\mathrm{Gd}(\mathrm{III})$-based CAs to dechelation of $\mathrm{Gd}(\mathrm{III})$ is specifically suspected of importance in the occurrence of NSF, though the pathogenesis of NSF is still being studied and Gd(III) complexes, not just free $\mathrm{Gd}(\mathrm{III})$, are also suspected of being bioactive. ${ }^{135}$ Recall that free $\mathrm{Gd}(\mathrm{III})$ is toxic. This is due to its ionic radius being 
close to that of calcium(II) (107.8 versus $114 \mathrm{pm}$, respectively). ${ }^{104}$ Gadolinium(III) can therefore replace calcium(II) in vivo and disrupt normal biological processes such as those that depend on an influx of calcium(II) (i.e. muscle contraction) or enzymes activated by calcium(II). ${ }^{104}$ Therefore, it is not only due to the positive correlation between Gd(III)-based CA administration and NSF that the stability of the chelates is important.

The thermodynamic stability of Gd(III)-based CAs is influenced by three structural factors of the chelating ligands used. The basicity of the ligand, which affects whether the chelate is ionic or nonionic, is important since anionic groups on the ligand will bind more strongly to the Gd(III) ion than nonionic groups. Secondly, the greater the number of five-membered rings formed between the $\mathrm{Gd}(\mathrm{III})$ ion and the donor atoms of the ligand, the greater the stability. There are six such rings in linear agents, such as DTPA-Gd(III) and DTPA-BMA-Gd(III), but eight in macrocyclic agents such as DOTAGd(III). Finally, there is the macrocyclic effect, which refers to the cavity size of the chelates with ring structures that "wrap" themselves around the Gd(III) ion. DOTA$\mathrm{Gd}(\mathrm{III})$, as an ionic macrocylic agent, has the highest thermodynamic stability of currently marketed Gd(III)-based CAs. ${ }^{104}$

The kinetic stability of Gd(III)-based CAs is also highly dependent on the chelate's ionic state. ${ }^{104,136}$ As discussed earlier, both DTPA-Gd(III) and DOTA-Gd(III) are ionic chelates that require the presence of a counter-ion (meglumine) in their formulations (Figure 2.3) while DTPA-BMA-Gd(III) is a nonionic agent. The kinetic stability of ionic CAs has been found to be higher than that of nonionic agents such as DTPA-BMA-Gd(III). ${ }^{104,137}$ The process of transmetallation, or the replacement of 
Gd(III) in a chelate by a different metal ion, is a kinetic process ${ }^{104}$ that will be discussed in greater detail in section 2.3.5.3. Generally, macrocyclic chelates are more stable to transmetallation than linear chelates. This is thought to be due to the greater conformational mobility of linear agents. Linear CAs are able to unravel from around the Gd(III) ion, particularly those with fewer ionic donor groups, such as DTPA-BMAGd(III), that are likely more flexible than ionic agents such as DTPA-Gd(III). In fact, agents such as MS-325 that have an aromatic substituent included on the DTPA backbone show better resistance to transmetallation. This might be due to steric effects. $^{104}$

Overall, new occurrences of NSF are decreasing as a result of changes in screening of patients and the reduction in dosages of Gd(III)-based CA administered. The risk of NSF is still restricted to those with impaired renal function, and Gd(III)-based CAs are considered safe for patients without this risk factor. ${ }^{136}$

\subsubsection{Aptamers in diagnostic imaging}

The use of aptamers in various diagnostic imaging agents is being explored by several research groups. A recent example of a radiolabelled RNA aptamer for potential use as a targeted agent in positron emission tomography (PET) explored the conjugation of four different metal chelators to an aptamer for prostate specific membrane antigen (PSMA). The chelation of copper-64 by two of the chelator-aptamer conjugates was achieved with high labelling efficiency, and these conjugates retained the unmodified aptamer's binding affinity and specificity for PSMA in vitro. ${ }^{92}$ This could lead to a 
highly efficient PET contrast agent if one of these agents is as effective and is safe to use in vivo.

The research discussed in this chapter involves a proof-of-concept study on the development of $15 \mathrm{mer}$ thrombin-binding aptamer-gadolinium(III) conjugates for targeted MR imaging. There have been a handful of studies on the incorporation of aptamers into MRI CA systems; these are described below.

\subsubsection{Aptamers and MRI}

The use of cross-linked dextran-coated superparamagnetic iron oxide (CLIO) nanoparticles functionalized with adenosine ${ }^{17}$ and thrombin-binding aptamers ${ }^{18}$ in two separate studies have been reported for potential use in $T_{2}$-weighted $M R$ imaging. Superparamagnetic iron oxide nanoparticles have been approved for use in the targeted imaging of liver lesions in a formulation called Feridex I.V.®, ${ }^{110}$ as mentioned previously. Used in $\mathrm{T}_{2}$-weighted imaging, the presence of these nanoparticles shortens the $T_{2}$ relaxation rates of nearby water protons. ${ }^{110}$ This translates to darker areas in MR images and improved contrast. ${ }^{17}$ CLIO nanoparticles exhibit a phenomenon called magnetic relaxation switching (MRS) which refers to their increased effectiveness as $T_{2}$ relaxation agents when assembled than when dispersed in solution. ${ }^{18}$

In the example of CLIO nanoparticles modified with adenosine-binding aptamers, the authors designed a "turn-on" sensor which resulted in brighter spots in $\mathrm{T}_{2}$-weighted MR images upon addition of the target, adenosine. They used two sets of CLIO nanoparticles that had been functionalized with different oligonucleotide sequences that were complementary to a linker added to the 5'-terminus of the adenosine-binding 
aptamer as well as part of the aptamer sequence. This is clearly illustrated in Figure $2.5 \mathrm{~A}$ where the two CLIO nanoparticles are held together through complementary binding to the extended aptamer sequence. Near such an assembly, $T_{2}$ relaxation of water protons was shortened and the $T_{2}$-weighted images obtained without adenosine present or with it present at low concentrations (i.e. $\leq 1.0 \mathrm{mM}$ ) had corresponding dark spots.

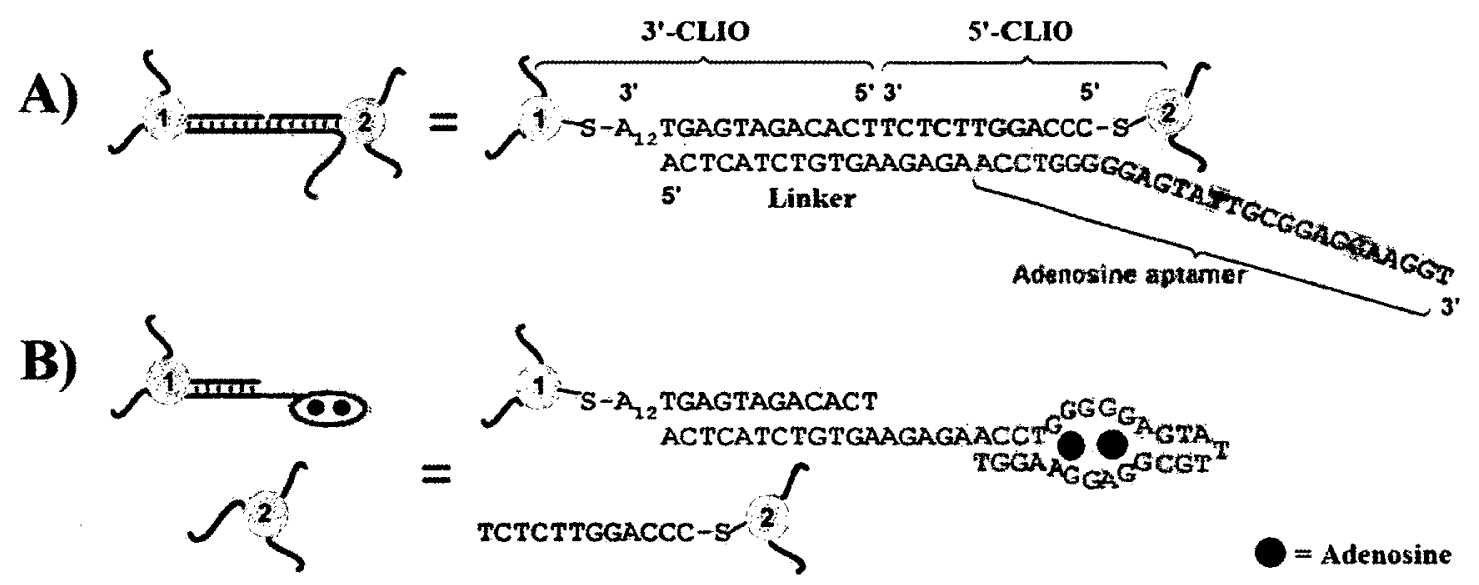

Figure 2.5 Set-up of cross-linked dextran-coated superparamagnetic iron oxide (CLIO) nanoparticle system for adenosine-sensing. Two sets of CLIO nanoparticles were prepared with the respective sequences shown in this image in brown and blue. The adenosine aptamer was prepared including a linker portion as shown in green and black, respectively. A) The initial sensor set-up, where the modified aptamer strand was hybridized to the CLIO-supported sequences. A low $T_{2}$ value was achieved with the CLIO nanoparticles held close together. B) The addition of adenosine leads to aptamer folding and the release of one of the CLIO nanoparticles. This results in an increased $T_{2}$ value compared to that of the system in $A$. Modified from reference 17.

The addition of adenosine (brown circles in Figure 2.5B) at various concentrations caused folding of the aptamer upon interaction with the target, and greater dispersion of the CLIO nanoparticles was achieved with higher concentrations of adenosine. With the 
addition of $2.5 \mathrm{mM}$ adenosine, the MR images obtained were completely bright. Therefore, this system successfully demonstrated the use of aptamer-functionalized CLIO nanoparticles in a "turn-on" MRI sensing system; that is, the brightness of the images increased with increasing target concentration.

In $T_{2}$-weighted $M R I$, it is desirable for water proton $T_{2}$ relaxation times to decrease in the presence of an agent. The same group that reported the adenosinebinding aptamer CLIO nanoparticle sensor also designed a "turn-off" system ${ }^{18}$ using the two thrombin-binding DNA aptamers introduced in section 1.2.3. In this system, one set of CLIO nanoparticles was functionalized with the $15 \mathrm{mer}$ TBA and a second set was functionalized with the $29 \mathrm{mer}$ TBA. Recall that these two aptamers bind to the fibrinogen- and heparin-binding exosites of thrombin, respectively. ${ }^{8,21}$ In the absence of thrombin, a mixture of the two sets of functionalized CLIO nanoparticles has a high $\mathrm{T}_{2}$ since the nanoparticles remain dispersed in solution; the images of such solutions remain bright. The addition of thrombin to mixtures of the two sets of CLIO nanoparticles caused the nanoparticles to assemble as the aptamers simultaneously bound to their respective bonding sites on the protein. A concentration as low as $10 \mathrm{nM}$ thrombin began causing this assembly, as indicated by darker $T_{2}$-weighted images. When thrombin reached a concentration of $50 \mathrm{nM}$, the solution was completely dark. ${ }^{18}$ Tests of both CLIO nanoparticle-based systems in diluted human serum demonstrated higher limits of detection for each target but confirmed their utility in biological fluids. ${ }^{17,18}$

A recent study by Tan and coworkers also used aptamer-conjugated magnetic (iron oxide) nanoparticles (ACMNPs) in a magnetic relaxation switch sensor with the goal of developing a method by which to analyze and identify different cancer cell lines 
through pattern recognition. ${ }^{118}$ To this end, the aptamers used in this work targeted six different cell surface receptors. The receptors are expressed in different amounts by different cancer cell lines, and some are specific to a particular type of cancer. Six cancer cell lines and one normal cell line were individually tested with each of the six types of aptamer-conjugated magnetic nanoparticles and the $T_{2}$ relaxation times measured. Where the aptamers bound to many of their target receptors on the cell surfaces, a greater change in $T_{2}$ was found compared to samples with ACMNPs but no target cells. ${ }^{118}$ The expression of the varying cell surface receptors in each cell line was determined using this method, with a pattern determined for each cell type. The capability of this system to quantify small amounts of target cells in buffer and biological media was confirmed and it therefore shows promise as a useful tool in cancer cell detection and eventually cancer screening. ${ }^{118}$

One aptamer-based $T_{1}$ relaxation agent has heretofore been reported. Lu and coworkers incorporated the adenosine-binding aptamer into a targeted MRI CA system where DOTA-Gd(III) was conjugated to a short DNA strand complementary to part of the extended aptamer; this is illustrated in Figure 2.6. ${ }^{16}$ The aptamer strand included a biotin modifier at its $5^{\prime}$-terminus so that the size and mass of the CA could be increased through interaction with streptavidin. The mass of the hybridized form (left-hand side of Figure 2.6) was about $70 \mathrm{kDa}$. The addition of adenosine caused the aptamer to fold so that it could interact with its target, and this caused displacement of the complementary strand conjugated to DOTA-Gd(III) (right-hand side of Figure 2.6). The mass of the Gd(III)-strand was about $4 \mathrm{kDa}$, and this 17.5 times drop in mass, and therefore size, of the CA would be expected to change its $\tau_{R}$ and therefore its relaxivity. The authors 
reported a drop of about $34 \%$ in relaxivity in the presence of $5 \mathrm{mM}$ adenosine in this turn-off system. ${ }^{16}$

\section{Aptamer strand 5'Biotin-CACTGACCTGGGGGAGTATTGCGGAGGAAGGT} Gd-strand $3^{\prime}-\mathrm{NH}_{2}-$ GTGACTGGACC

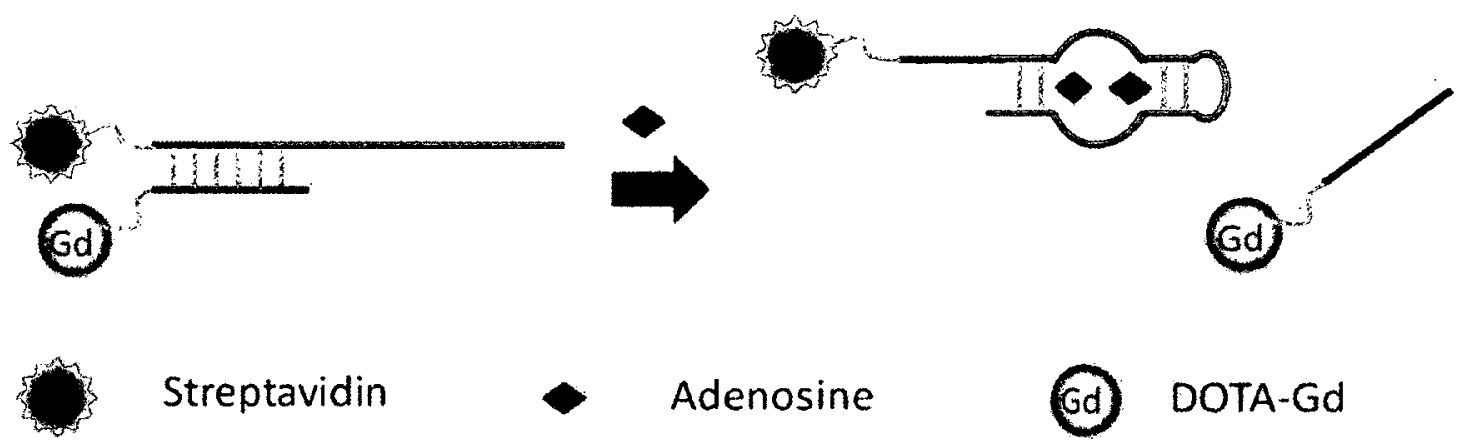

Figure 2.6 Schematic representation of an adenosine-binding aptamer MRI contrast agent. Binding of the streptavidin-modified aptamer to adenosine leads to the release of the DNA-Gd(III) conjugate. This decrease in the effective mass of the DNA-Gd(III) conjugate results in a measurable drop in relaxivity. Modified from reference 16.

In $\mathrm{T}_{1}$-weighted $\mathrm{MRI}$ it is more desirable to have a targeted $\mathrm{CA}$ that results in a turn-on signal with target-binding, and that was the goal of the work presented in this and the following chapter. Research on the conjugation of the thrombin aptamer to DTPA, both directly and with the inclusion of polynucleotide spacers, will be presented.

\subsection{Materials and methods}

\subsubsection{Materials and instruments}

DTPA (diethylenetriaminepentaacetic acid) dianhydride (98\%), gadolinium(III) chloride hexahydrate $(99.999 \%)$, and xylenol orange disodium salt were obtained from Sigma-Aldrich. Zinc(II) chloride was purchased from Fluka. Pepsin and bovine serum 
albumin were obtained as lyophilized powders from Sigma-Aldrich, thrombin was obtained either in lyophilized form (as a gift from The Ottawa Hospital/Ottawa Hospital Research Institute) or in solution (Haematologic Technologies Inc.). Magnevist ${ }^{\circledR}$ was donated by The Ottawa Hospital/Ottawa Hospital Research Institute. Chemicals for the preparation of polyacrylamide gels were obtained from BioShop®.

DNA was prepared on a Bioautomation MerMade 6 Oligonucleotide synthesizer using standard phosphoramidite chemistry. Phosphoramidites and DNA synthesis reagents, including the 5'-DMS $(\mathrm{O}) \mathrm{MT}$-amino modifier $\mathrm{C} 6$, were purchased from Glen Research. Deionized water was prepared using a Millipore Milli-Q water system. DTPA-DNA conjugates were purified by denaturing polyacrylamide gel electrophoresis (PAGE; Hoefer SE 600 Chroma Unit). Desalting was performed using Amicon Bioseparations Stirred Ultrafiltration Cells containing Ultrafiltration Membranes (1000 Da cut-off, Millipore). 15 and 30 polyA oligonucleotides were purified using Clarity QSP cartridges (Phenomenex). Separation of DTPA-DNA conjugates from free Gd(III) after chelation was achieved using Amicon Ultra Centrifugal Filter Units $(0.5 \mathrm{~mL}, 3 \mathrm{kDa}$ cut-off, Millipore). DTPA-DNA conjugates were quantified by monitoring absorbance at $260 \mathrm{~nm}$ with a Cary 300 Bio UV-Visible spectrophotometer. Purified DTPA-DNA conjugates were sent to Novatia, LLC (New Jersey) for FIA/MS (flow injection analysis mass spectrometry) analysis for molecular weight confirmation. A $400 \mathrm{MHz}(9.4 \mathrm{~T})$ Bruker NMR spectrometer (Carleton University) and a 1.5 T Siemens Symphony clinical MR scanner (The Ottawa Hospital, Civic Campus) were used for determination of $\mathrm{T}_{1}$ relaxation. 


\subsubsection{Preparation of DNA-DTPA conjugates}

To prepare the DNA-DTPA conjugates SC, 15T SC, and 30T SC, the following sequences of DNA were prepared, respectively:

Thrombin aptamer: ${ }^{8}$ 5'-NH2-( $\left.\mathrm{CH}_{2}\right)_{6}$-GGT TGG TGT GGT TGG-3'

Thrombin aptamer with 15 polyT spacer: 5 '- $\mathrm{NH}_{2}-\left(\mathrm{CH}_{2}\right)_{6}-(\mathrm{T})_{15}$ GGT TGG TGT GGT TGG-3'

Thrombin aptamer with 30 polyT spacer: 5'- $\mathrm{NH}_{2}-\left(\mathrm{CH}_{2}\right)_{6}-(\mathrm{T})_{30}$ GGT TGG TGT GGT TGG-3'

Directly following synthesis, the DNA columns containing $500 \AA$ controlled pore glass (CPG) beads were placed on the cleaving chuck and rinsed sequentially with 300 $\mu \mathrm{L}$ of anhydrous $\mathrm{MeCN}, 3 \times 300 \mu \mathrm{L}$ of $10 \% \mathrm{DEA}$ in anhydrous $\mathrm{MeCN}$, and $2 \times 200 \mu \mathrm{L}$ of anhydrous MeCN. For immediate conjugation to DTPA dianhydride, removal of the DMS(O)MT protecting group from the synthesized DNA was performed by washing the beads with $3 \times 300 \mu \mathrm{L}$ of deblock solution ( $2 \%$ dichloroacetic acid in dichloromethane). The characteristic absorbance of $\mathrm{DMS}(\mathrm{O}) \mathrm{MT}^{+}$in the deblock solution (collected separately from the MeCN washes; $\lambda_{\max }=523 \mathrm{~nm}$ ) confirmed DNA modification. The column material was once again rinsed with $\mathrm{MeCN}(2 \times 300 \mu \mathrm{L})$ to ensure the removal of all free DMS(O)MT ${ }^{+}$, and the $\mathrm{CPG}$ beads were transferred to $1.5 \mathrm{~mL}$ Eppendorf tubes. A 42-fold excess of DTPA dianhydride in anhydrous DMSO was added to each column of DNA (each of which had a maximum yield of $1 \mu \mathrm{mol}$ ) and the reaction was left to proceed overnight on a shaker. The CPG beads were then collected at the bottom of the tubes through centrifugation (5 minutes at $10,000 \mathrm{~g}$ ) and the DTPA dianhydride solution was removed via pipette. To remove any remaining DTPA dianhydride, $500 \mu \mathrm{L}$ more 
anhydrous DMSO were added to the beads. The mixture was vortexed, centrifuged, and the solution removed as above. The DNA-DTPA conjugates were then deprotected and removed from the $\mathrm{CPG}$ beads though a treatment with $\mathrm{NH}_{4} \mathrm{OH}(28 \%)$ for 24 hours at room temperature or $37^{\circ} \mathrm{C}$. The deprotected $\mathrm{DNA}$ in $\mathrm{NH}_{4} \mathrm{OH}$ was isolated from the $\mathrm{CPG}$ beads using filter tubes (RAININ Microfilterfuge ${ }^{\text {TM }}$ Tubes $0.45 \mu \mathrm{m}$ uncharged nylon-66 filters) or by pipette. After the samples were dried under vacuum, the crude DNA-DTPA was quantified using the characteristic absorbance of DNA at $260 \mathrm{~nm}$.

The single-conjugates were also isolated during the purification of the reaction mixtures resulting from the methods used to maximize the yield of the respective doubleconjugates (section 2.3.1).

\subsubsection{Purification of DNA-DTPA single-conjugates}

Denaturing polyacrylamide gel electrophoresis (PAGE) was used to purify the DNA-DTPA conjugates. Approximately $300-400 \mathrm{nmol}$ of crude DNA-DTPA were run per gel, where the acrylamide percentages used were $19 \%$ for SC, 12 or $15 \%$ for 15 T SC and $12 \%$ for $30 \mathrm{~T} \mathrm{SC}$, respectively. Gels were prepared by stirring the requisite amounts of the reactants (see Table 2.1) while heating the reaction mixture to $37^{\circ} \mathrm{C}$, then gravity filtering the hot solution into a clean beaker. Once the reaction solution had cooled to room temperature, $450 \mu \mathrm{L}$ of a freshly prepared $10 \%$ ammonium persulfate solution and $35 \mu \mathrm{L}$ of $N, N, N^{\prime}, N^{\prime}$-tetramethylethylenediamine (TEMED) were added and mixed with gentle swirling. The solution was then poured between prepared glass plates and a 15 lane comb or preparative scale comb with two reference lanes was inserted. After 30 
minutes, the comb was removed and the lanes rinsed with $\mathrm{diH}_{2} \mathrm{O}$ to remove any traces of the reaction solution. The gel unit was assembled with $1 \times$ TBE used as running buffer.

The crude DNA-DTPA was quantified using the characteristic absorbance of DNA at $260 \mathrm{~nm}$ so as to avoid overloading the gels. The extinction coefficients of the expected single- and double-conjugates were used to determine the respective theoretical yields of each if all of the DNA present was incorporated into only one type of conjugate. The average of the two values was determined and taken to be representative of the reaction resulting in a 1:1 ratio of single- to double-conjugate. This value was used to determine the amount of crude DNA-DTPA to load into a single lane on a polyacrylamide gel.

The amount of DNA that could be loaded in one lane of a polyacrylamide gel without overloading had been found by a colleague to be $\sim 20 \mathrm{nmol}$. Twenty microlitres was the maximum volume of a DNA solution run per lane, therefore the concentration of DNA-DTPA required in order that $20 \mu \mathrm{L}$ contained $20-30 \mathrm{nmol}$ was determined using the previously computed average. For gels in which a preparative scale comb was used, it was estimated that about an extra third of the amount of DNA loaded in a gel with 15 lanes could be purified.

Crude DNA-DTPA was dissolved in a 1:1 solution of $\mathrm{diH}_{2} \mathrm{O}$ and formamide and then heated at $70^{\circ} \mathrm{C}$ for 5 minutes. Samples were loaded onto the cast gels, with a maximum volume of $40 \mu \mathrm{L}$ per lane when applicable. A fluorescein-labelled 18 mer was used on several gels as a means to track the progress of the DNA-DTPA conjugates. A constant current of $30 \mathrm{~mA}(19 \%)$ or $45 \mathrm{~mA} \mathrm{(12} \mathrm{and} \mathrm{15 \% )} \mathrm{was} \mathrm{used} \mathrm{for} \mathrm{each} \mathrm{gel} \mathrm{unit} \mathrm{(2}$ gels). Gels were imaged using the UV-Vis channel on an AlphaImager ${ }^{\circledR}$ (Alpha 
Innotech). Representative gel images for each of the DNA sequences used in reactions with DTPA dianhydride are shown in Figure 3.5 and are discussed in more detail in section 3.3.2.

Bands were cut out and the gel crushed prior to the addition of 6 volume equivalents of Tris buffer $(10 \mathrm{mM}, \mathrm{pH} 7.4)$. The mixture was frozen at $-80^{\circ} \mathrm{C}$ and left shaking at $37^{\circ} \mathrm{C}$ overnight. The solution was isolated from the crushed gel using syringe filters (CELLTREAT $0.45 \mu \mathrm{m}$ PES membrane) or filter tubes (RAININ Microfilterfuge ${ }^{\mathrm{TM}}$ Tubes $0.45 \mu \mathrm{m}$ uncharged nylon-66 filters).

Table 2.1 Recipes for the preparation of two polyacrylamide gels.

\begin{tabular}{|c|c|c|c|}
\hline Chemical & $12 \%$ acrylamide & $15 \%$ acrylamide & $19 \%$ acrylamide \\
\hline Urea $(\mathrm{g})$ & 31.5 & 31.5 & 31.5 \\
Acrylamide stock $(\mathrm{mL})$ & 23.5 & 29.5 & 37.5 \\
$5 \times \mathrm{TBE}$ buffer $(\mathrm{mL})$ & 15 & 15 & 15 \\
$\operatorname{diH}_{2} \mathrm{O}(\mathrm{mL})$ & 14 & 8 & 0 \\
\hline
\end{tabular}

The purified DNA-DTPA conjugates were desalted using the Amicon Bioseparations Stirred Ultrafiltration Cell. Briefly, the solution isolated from the gel was filtered through a 1000 Da cut-off Ultrafiltration Membrane (Millipore) under high pressure. The DNA-DTPA conjugates were retained on the filter and were washed with $\mathrm{diH}_{2} \mathrm{O}$ to remove any salts lingering from the buffer. The DNA conjugates were removed from the filter via the repeated addition of water to the filter to dissolve the DNA, and collection of these washings in Eppendorf or Falcon tubes by pipette. The washings were 
dried under vacuum and the DNA-DTPA conjugates were then quantified using UV-Vis spectroscopy.

\subsubsection{Chelation of $G d(I I)$ with DNA-DTPA single-conjugates}

The initial attempt to chelate Gd(III) with SC was performed with $\sim 59 \mathrm{nmol}$ of the conjugate. Ammonium acetate buffer $(0.5 \mathrm{M}, \mathrm{pH} 6)$ was prepared containing $\sim 0.9$ $\mathrm{mM} \mathrm{Gd(III).} \mathrm{Five} \mathrm{molar} \mathrm{equivalents} \mathrm{of} \mathrm{Gd}(\mathrm{III})$ were added to the $\mathbf{S C}$ sample. The conjugate and Gd(III) were shaken overnight and then desalted. Ethanol precipitation was attempted, where the conjugate-Gd(III) solution was split into three $\sim 110 \mu \mathrm{L}$ portions which were each placed into separate $1 \mathrm{~mL}$ aliquots of chilled ethanol. The three mixtures were placed on dry ice for about an hour then centrifuged at $-9^{\circ} \mathrm{C}$ for 30 minutes. Pellets were not visible in the tubes and because the success of the precipitation was not clear, the solutions were dried under vacuum in preparation for another procedure.

The next desalting attempt was made using an Amicon Bioseparations Stirred Ultrafiltration Cell. The conjugate and Gd(III) solution was diluted to $1 \mathrm{~mL}$ total, run through an Ultrafiltration Membrane (1000 Da cut-off), and rinsed with $4 \times 1 \mathrm{~mL}$ portions of $\mathrm{diH}_{2} \mathrm{O}$. All washings were collected in clean Eppendorf tubes and dried under vacuum. The conjugate was removed from the filter as previously described (section 2.2.3) and the washings were tested for the presence of $\mathrm{Gd}(\mathrm{III})$ using the xylenol orange test.

For the xylenol orange test, ${ }^{139}$ a series of calibration solutions was prepared in which the amount of xylenol orange was kept constant while the ratio of Gd(III) to dye 
was varied. Concentrations between 0 and $50 \mathrm{nmol} / \mathrm{mL}$ of $\mathrm{Gd}(\mathrm{III})$ were used and the amount of xylenol orange was chosen to be in the middle of the range used for a particular calibration series (typically 10,15 or $20 \mathrm{nmol} / \mathrm{mL}$ ). An equivalent amount of dye to that used in the calibration solutions was also added to the excess salt fraction isolated from the DNA-DTPA conjugate: in this first case, the washings from the stirred cell. Ammonium acetate buffer $(0.5 \mathrm{M}, \mathrm{pH}$ 6) was used as diluent to make every sample up to $1 \mathrm{~mL}$ total volume and the solutions were mixed for 30 minutes to ensure equilibration. The absorbance of every calibration and sample solution was measured in triplicate and the calibration curve was then used to quantify the amount of free $\mathrm{Gd}(\mathrm{III})$ in the excess salt fraction.

Following the determination that excess Gd(III) could not readily be removed from a DNA-DTPA conjugate sample, only one molar equivalent of Gd(III) was mixed with a fresh $49 \mathrm{nmol}$ sample of SC. The Gd(III) was added following dissolution of the conjugate in Tris buffer (50 mM, pH 7.4) containing $140 \mathrm{mM} \mathrm{NaCl}$. Following overnight incubation, the SC-Gd(III) was isolated using a stirred cell and the washings were analyzed using the xylenol orange test as described above. The success of this second procedure led to the optimized experimental protocol. Conjugates were shaken overnight and desalted using an ultrafiltration cell (as described above) or Amicon Ultra Centrifugal Filter Units (desalting tubes).

Desalting using the filter units was performed as follows. The chelation solution was added to the desalting tube filter in $450 \mu \mathrm{L}$ portions and centrifuged at $13,000 \mathrm{~g}$ for 30 minutes after each addition. The filtrate was removed from the desalting tube after each 30 minute period and combined in $2 \mathrm{~mL}$ Eppendorf tubes. Following filtration of all 
of the chelation solution, the retentate was washed with three portions $(300-400 \mu \mathrm{L})$ of $\mathrm{diH}_{2} \mathrm{O}$ to ensure removal of buffer salts and free $\mathrm{Gd}(\mathrm{III})$. The retentate was removed from the filter through the addition of $50 \mu \mathrm{L} \mathrm{diH} \mathrm{d}_{2} \mathrm{O}$ followed by inversion of the filter and collection in a new tube by centrifugation $(1,000 \mathrm{~g}$ for 3 minutes). Prior to performance of the xylenol orange test, the efficiency of desalting was confirmed through quantification of the conjugate in the retentate by UV-visible spectroscopy. If more than $10 \mathrm{nmol}$ of the conjugate had been lost to the filtrate, it was desalted for a second time.

All of the filtered buffer and $\mathrm{diH}_{2} \mathrm{O}$ washings were dried down under vacuum and the excess salts combined to undergo a xylenol orange test ${ }^{139}$ for the quantification of free Gd(III). If required following this test more Gd(III) was added to the conjugate and the process was repeated until the calculated percentage of excess conjugate in the retentate reached a minimum.

A small amount of conjugate was commonly lost to the filtrate during desalting. Attempts were made to recover lost SC-Gd(III) from the filtrate and xylenol orange sample from an early test. Ethanol precipitation was tried first. Ethanol $(1 \mathrm{~mL})$ was mixed with $50 \mu \mathrm{L} \mathrm{NaCl}(3 \mathrm{M})$ and chilled in dry ice. The SC-Gd(III) and xylenol orange solution had been dried to $\sim 50 \mu \mathrm{L}$. This solution was added to the chilled ethanol solution and placed on dry ice for about an hour. Following centrifugation at $-9^{\circ} \mathrm{C}$ for 30 minutes, the liquid phase was removed from the pelleted DNA. The separation of the conjugate from xylenol orange was also attempted using an ultrafiltration cell. The procedure used was identical to that described above for the first chelation experiment. 


\subsubsection{Mass spectrometric confirmation of DNA-DTPA single-conjugates}

FIA/MS analysis confirmed the molecular weight of the DNA-DTPA conjugates: SC m/z calc'd: 5276; found: 5279. 15T SC-Gd(III) m/z calc'd: 9997; found: $9997.30 T$ SC m/z calc'd 14402; found: 14406.

\subsubsection{Preparation of 15 and 30 polyA oligonucleotides}

The polyA strands corresponding to the polyT spacer portions of the modified thrombin aptamers were also synthesized on the MerMade 6 Oligonucleotide synthesizer (without the final deblock performed when Clarity QSP cartridges were used for purification). Following overnight deprotection in $\mathrm{NH}_{4} \mathrm{OH}$ at $55^{\circ} \mathrm{C}$, the polyA DNA was purified by PAGE (19\%, see section 2.2.3) or through the use of one Clarity QSP $3 \mathrm{~mL}$ cartridge for each column of DNA.

The cartridges were conditioned using $2 \times 1.5 \mathrm{~mL} \mathrm{MeOH}$ under vacuum at $2-3$ " $\mathrm{Hg}$ and then equilibrated with $2 \times 1.5 \mathrm{~mL} \mathrm{diH}_{2} \mathrm{O}$ at $3-4 " \mathrm{Hg}$. DNA loading buffer was added to each DNA sample in a 1:1 ratio with the $\mathrm{NH}_{4} \mathrm{OH}(1 \mathrm{~mL}$ of each). The DNA solutions were then loaded onto their respective cartridges and run through under vacuum at 3-4" Hg. Diluted DNA loading buffer $\left(1.5 \mathrm{~mL}\right.$ total, $1: 1$ with $\mathrm{diH}_{2} \mathrm{O}$, vacuum at $3 " \mathrm{Hg}$ ) was used to wash each cartridge, and then the DNA was detritylated with $1.5 \mathrm{~mL}$ aqueous $2 \%$ DCA (4-5" Hg). An orange band was observed on the sorbent within the cartridge at this stage, indicating that the freed trityl was retained. The cartridges were each rinsed with $2 \times 1.0 \mathrm{~mL} \mathrm{diH} 2 \mathrm{O}(4-5 " \mathrm{Hg})$ and then the sorbent was dried by increasing the vacuum to 10 " $\mathrm{Hg}$ for 2 minutes. Finally, the DNA was eluted using $2 \mathrm{~mL}$ per cartridge of a $20 \%$ $\mathrm{MeCN}$ in Tris $(10 \mathrm{mM}, \mathrm{pH} \sim 8)$ solution under vacuum at $3-4 " \mathrm{Hg}$. The DNA was then 
desalted, dried down and quantified prior to use. The molecular weights of the polyA strands were confirmed using FIA/MS analysis: 15 polyA m/z calc'd: 4636.2 ; found: 4634.9. 30 polyA m/z calc'd: 9334.3 ; found: 9333.2 .

\subsubsection{Melting studies on the DNA-DTPA single-conjugates}

Melting studies were performed on a Cary 300 Bio UV-Visible spectrophotometer with a Varian temperature controller to confirm hybridization under the conditions used. For these experiments, a $1.7 \mu \mathrm{M}$ solution of $15 \mathrm{~T} \mathrm{SC}$ and $1.2 \mu \mathrm{M}$ solution of $30 \mathrm{~T} \mathrm{SC}$ were prepared, respectively, using thrombin aptamer buffer $(50 \mathrm{mM}$ Tris, pH 7.4140 $\mathrm{mM} \mathrm{NaCl}, 1 \mathrm{mM} \mathrm{MgCl}, 5 \mathrm{mM} \mathrm{KCl}$ ) as the diluent. In each case, the conjugate and its corresponding polyA sequence were mixed in a $1: 1$ ratio and made up to $3 \mathrm{~mL}$ total. The mixture was heated at $70^{\circ} \mathrm{C}$ for 5 minutes then allowed to cool to room temperature prior to being transferred to a cuvette. A micro stir bar was used to ensure even heating of the solution. Absorbance of the solution was monitored at $260 \mathrm{~nm}$ and recorded at $1{ }^{\circ} \mathrm{C}$ intervals as the temperature was increased from $17-90^{\circ} \mathrm{C}$, held at $90^{\circ} \mathrm{C}$ for 5 minutes, and then decreased from $90-20^{\circ} \mathrm{C}$. The temperature was set to change $1^{\circ} \mathrm{C}$ per minute.

\subsubsection{In vitro stability of DNA-DTPA single-conjugates:}

\subsubsection{Stability in serum at $37^{\circ} \mathrm{C}$ by the xylenol orange test}

A portion of SC-Gd(III) was dissolved in bovine serum to a concentration of about $0.1 \mathrm{mM}$. Half of the solution was removed to a new Eppendorf tube, and both tubes were heated to $37^{\circ} \mathrm{C}$ using a heat block. One tube was removed after a period of 1 hour while the other was left at $37^{\circ} \mathrm{C}$ for 24 hours. Following removal, the solutions were cooled to room temperature and desalted using the centrifugal filter units as 
described in section 2.2.4. UV-visible spectra were obtained for the retentates and filtrates in an attempt to confirm high efficiency of the desalting technique. However, the DNA peak at $260 \mathrm{~nm}$ was obscured by the protein peak at $280 \mathrm{~nm}$ (proteins that were present in the bovine serum). The filtrates were desalted a second time each and the xylenol orange test was performed with $10 \mathrm{nmol}$ of dye in each sample.

A solution of $\mathrm{Gd}(\mathrm{III})$ in serum was prepared to a concentration of about $0.075 \mathrm{mM}$ to test the possibility that any free Gd(III) would by masked during the xylenol orange test through binding to proteins in the serum. The solution was heated at $37^{\circ} \mathrm{C}$ for 1 hour then desalted twice, as in the case of SC-Gd(III) in serum above. The xylenol orange test was performed on the filtrate with $10 \mathrm{nmol}$ of dye in each solution.

\subsubsection{Stability in buffer at $37^{\circ} \mathrm{C}$ by the xylenol orange test}

A portion of SC-Gd(III) was dissolved to a concentration of $0.17 \mathrm{mM}$ in thrombin aptamer buffer $\left(\mathrm{pH} \mathrm{7.4).} \mathrm{This} \mathrm{was} \mathrm{then} \mathrm{heated} \mathrm{at} 37^{\circ} \mathrm{C}\right.$ for 3 hours in order to test the stability of $\mathrm{Gd}(\mathrm{III})$ chelation in the conjugate at a biologically relevant temperature. The sample was desalted using centrifugal filter units as described in section 2.2 .4 after heating, with the first 30 minutes of filtration performed at $37^{\circ} \mathrm{C}$.

The amount of $\mathrm{Gd}(\mathrm{III})$ in the excess salt fraction from this sample was measured using a xylenol orange test as described in section 2.2 .4 with $15 \mathrm{nmol}$ of dye per solution. This value was compared to the amount of conjugate, and therefore Gd(III), originally present to determine the percentage loss of Gd(III). 


\subsubsection{Transmetallation study}

A $5 \mathrm{~mm}$ NMR tube was silanized using the reagent Sigmacote $($ as instructed.

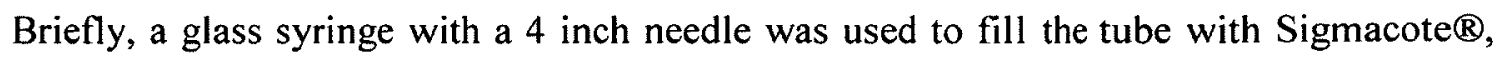
and then to remove most of the solution a few minutes later. The tube was emptied by pouring the remaining Sigmacote ${ }^{\circledR}$ into a vial. The tube was left in a fume hood overnight prior to being placed in an oven at $100^{\circ} \mathrm{C}$ for 30 minutes to stabilize the coating. The NMR tube was rinsed with deionized water, re-dried at $100^{\circ} \mathrm{C}$, and placed in a desiccator to await use.

Phosphate buffer was prepared (pH 7, $26 \mathrm{mM} \mathrm{KH}_{2} \mathrm{PO}_{4}, 41 \mathrm{mM} \mathrm{Na} \mathrm{HPO}_{4}$ ) in $\mathrm{diH}_{2} \mathrm{O}$ and precise aliquots were dried under vacuum to isolate the salts. The buffer was reconstituted in $\mathrm{D}_{2} \mathrm{O}$ and used to dissolve a portion of SC-Gd(III) to a concentration of $0.05 \mathrm{mM}$. This sample was placed in the silanized $5 \mathrm{~mm}$ NMR tube.

Following the estimation of the $T_{1}$ value of this solution $(t=0)$ at $37^{\circ} \mathrm{C}$ on a $9.4 \mathrm{~T}$ NMR spectrometer, an equimolar amount of $\mathrm{Zn}$ (II) was added from a $\mathrm{ZnCl}_{2}$ stock solution. The reaction solution was kept at $37^{\circ} \mathrm{C}$ for approximately 4 days and $\mathrm{T}_{1}$ values were obtained periodically to demonstrate the extent of transmetallation.

The observed relaxivity was calculated using $R_{1}=\left(1 / T_{1}\right),{ }^{137,140}$ where the $T_{1}$ values were estimated using the null spectrum of a $180-\tau-90$ pulse program, where $T_{1}=\tau$ $/ \ln 2 .^{141}$ The Bruker $t 1$ irld pulse program was used with the recycle delay (d1) set to 30 seconds and the value of $\tau(\mathrm{d} 7)$ adjusted until the peak of interest (water, $\sim 4.7 \mathrm{ppm}$ ) was nulled. The null point was identified as the case when the integrations of positive and negative peaks in each spectrum (determined using the software) were found to be approximately equal. 
The paramagnetic longitudinal relaxation rates $\left(R_{1}{ }^{P}\right)$ were calculated from the observed relaxivity values through the subtraction of the diamagnetic contribution of water proton relaxation rate. This was determined to be $\sim 0.077 \mathrm{~s}^{-1}$, which is the observed relaxivity for the phosphate buffer reconstituted in $\mathrm{D}_{2} \mathrm{O}$.

\subsubsection{Preparation of samples for $T_{1}$ experiments at high field (9.4 Tesla)}

Thrombin aptamer (TBA) buffer (50 mM Tris, pH $7.4140 \mathrm{mM} \mathrm{NaCl}, 1 \mathrm{mM}$ $\mathrm{MgCl}_{2}, 5 \mathrm{mM} \mathrm{KCl}$ ) was lyophilized and reconstituted with $\mathrm{D}_{2} \mathrm{O}$ to obtain the relaxivity measurement buffer. DTPA-Gd(III) was prepared in-lab as follows: DTPA dianhydride was dissolved in $\mathrm{D}_{2} \mathrm{O}(99.9 \%)$ and mixed in a $1: 1$ ratio with $\mathrm{Gd}(\mathrm{III})$ in deuterated TBA buffer at a concentration of $0.1 \mathrm{mM}$. Lyophilized thrombin was dissolved to a known concentration in TBA buffer and requisite volumes were added to conjugate samples where applicable. Pepsin was obtained as a powder (Sigma-Aldrich) and was also dissolved in TBA buffer and added to samples as required.

For $\mathrm{T}_{1}$ experiments, samples were injected using a 4 inch needle into capillary tubes (Kimble Kontes LLC, borosilicate glass, O.D. $\times$ length $1.5-1.8 \times 90 \mathrm{~mm}$ ) which were silanized using the reagent Sigmacote $(\mathbb{R}$ as described in section 2.2.8.3. The capillary tubes were placed into regular $5 \mathrm{~mm}$ NMR tubes and were held upright with a piece of narrow plastic tubing. It was determined that a minimum volume of $50 \mu \mathrm{L}$ could be used in one of these tubes for $T_{1}$ measurements. Following determination of its $T_{1}$, a sample was removed from the capillary tube using a 4 inch needle. All concentrations but the lowest in a particular series were then diluted using deuterated TBA buffer to achieve the next desired concentration. 
The experiments on each of the three conjugates SC-Gd(III), 15T SC-Gd(III), and 30T SC-Gd(III) were performed on three different series. Each conjugate was run alone in solution in the first series, in a 1:1 ratio with thrombin in the second series, and in a 1:1 ratio with pepsin (used as a control) in the third series. 15T SC-Gd(III) and 30T SC-Gd(III) required an additional three series each for the inclusion of their corresponding polyA strands for hybridization (this was not performed for 15T SCGd(III)+BSA). Magnevist $(\mathbb{B}$, the formulation of DTPA-Gd(III) used as an approved CA, was used as a control for these experiments after testing a sample of DTPA-Gd(III) prepared as previously described.

For these experiments, the conjugates were dissolved in buffer and refolded prior to the addition of proteins. To fold the aptamer, the conjugate samples were dissolved in relaxivity buffer, denatured by heating at $70^{\circ} \mathrm{C}$ for 5 minutes, and then cooled to room temperature. The most concentrated solution of each conjugate mixed with a protein were prepared by dissolving the conjugate in relaxivity buffer and adding the required amount of protein to achieve a 1:1 molar equivalency. The solution was then shaken for half an hour to allow it to equilibrate prior to $T_{1}$ measurement.

The solutions were prepared from isolated portions between 6 and $15 \mathrm{nmol}$ of the respective conjugates, with the highest concentration in each series adjusted to a value between 0.025 and $0.1 \mathrm{mM}$ so that the initial total volume was $80 \mu \mathrm{L}$ or greater. PolyA strands were added in a 1:1 ratio to the conjugates prior to refolding to permit hybridization where applicable. 


\subsubsection{Preparation of samples for $T_{1}$ experiments at low field (1.5 and 3 Tesla)}

Plastic cuvettes (4.5 mL, Brandtech Brand) were taped together to hold the sample solutions in each sample set. All solutions were made up to a volume of $1 \mathrm{~mL}$. Lyophilized pepsin was dissolved in TBA buffer and used in these experiments as in the 9.4 $\mathrm{T}$ samples. Thrombin was used as purchased from Haematologic Technologies Inc. (i.e. in $50 \% \mathrm{v} / \mathrm{v}$ glycerol/water solution).

A set of SC-Gd(III) at concentrations of $0.05,0.025,0.0125,0.005,0.0025$, and $0.0005 \mathrm{mM}$ was prepared for $\mathrm{T}_{1}$ experiments on a $3 \mathrm{~T} \mathrm{MRI}$ spectrometer to determine the detection limit of the instrument. A cuvette containing buffer was included as well. Three scans were performed on this set of samples over the course of two days. The lowest concentration of SC-Gd(III) tested was found to be near the detection limit of the instrument (i.e. its $T_{1}$ was about the same as that for buffer alone).

Five sample sets were prepared for simultaneous $T_{1}$ determination at low field (1.5 T). Sets including SC-Gd(III) consisted of solutions at $0.0125,0.0094,0.0070$, 0.0053 , and $0.0040 \mathrm{mM}$ and, where appropriate, an equimolar amount of protein to match the conditions used at 9.4 T. Magnevist ${ }^{\circledR}$ was diluted to concentrations of $0.025,0.0125$, 0.0062 , and $0.0031 \mathrm{mM}$. All solutions were prepared using TBA buffer and were made

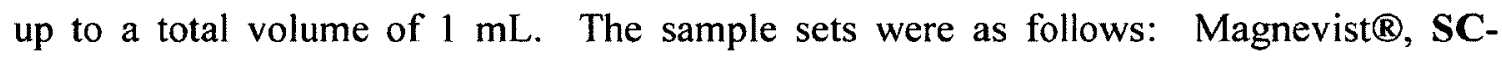
Gd(III), SC-Gd(III) and thrombin, SC-Gd(III) and pepsin, and SC-Gd(III) and BSA. Two scans were performed on these samples. 


\subsubsection{Relaxivity measurements:}

\subsubsection{At high field}

A $400 \mathrm{MHz}(9.4 \mathrm{~T})$ Bruker NMR spectrometer was used for the collection of $\mathrm{T}_{1}$ data. A minimum of three concentrations of a conjugate in the range of 0.003 to $0.1 \mathrm{mM}$ were used in each series. Magnevist ${ }^{\circledR}$ and DTPA-Gd(III) prepared in the lab (as described in section 2.2.9) were run at concentrations of $0.1,0.05,0.025$, and 0.0125 $\mathrm{mM}$, and deuterated buffer was run as a control with $0 \mathrm{mM} \mathrm{Gd(III).} T_{1}$ values were obtained for each concentration in triplicate at room temperature $\left(25^{\circ} \mathrm{C}\right)$. An inversion recovery program (Avance Version) was used from the standard Bruker pulse program library to measure $T_{1}$ relaxation. $T_{1}$ values were then automatically calculated using the supplied Bruker NMR Relaxation Guide. Molar relaxivity $\left(r_{i}, \mathrm{mM}^{-1} \mathrm{~s}^{-1}\right)$ was obtained from plots of relaxation time $\left(\frac{1}{T_{1}}, \mathrm{~s}^{-1}\right)$ versus gadolinium(III) concentration $(\mathrm{mM})$ as discussed in section 2.1.2 and illustrated by equation 2.4. Linear regression calculations were performed to determine the slope of the curve in each series and the associated error.

\subsubsection{At low field}

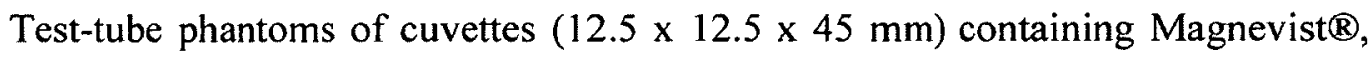
DTPA-Gd(III) or SC-Gd(III) at various concentrations were obtained by colleagues in the C2 Neurosciences Unit at The Ottawa Hospital/Ottawa Hospital Research Institute (OHRI) using a 1.5 or $3 \mathrm{~T}$ (as indicated in section 2.2.10) clinical MR scanner (Siemens Symphony). The temperature within the gantry was maintained at approximately $25^{\circ} \mathrm{C}$ using air conditioners. The pulse sequence for $T_{1}$ and $R_{1}$ estimation followed Spin Echo 
(Saturation Recovery) techniques; repetition time (TR) 100, 300, 800, 1500, 3000, 6000 and $8000 \mathrm{~ms}$; echo time (TE) $12 \mathrm{~ms}$; field of view (FOV) $150 \mathrm{~mm}$; matrix size $128 \times 128$; slice thickness $8 \mathrm{~mm}$; and non-averaged.

The signal intensity of the phantoms was measured 2-3 times (as indicated in section 2.2.10) using a square region of interest, and the values thus obtained were averaged (where applicable). The $T_{1}$ values of each phantom were calculated from signalto-noise ratio in successive images using different TR by non-linear fit. Next, $r_{1}$ (relaxivity $\mathrm{mM}^{-1} \mathrm{~s}^{-1}$ ) values were estimated from the $T_{1}$ values of serial phantoms with four or five different sample concentrations. Relaxivity is the slope of the linear fit plotted of the relaxation rate $\left(1 / \mathrm{T}_{1}\right)$ versus the concentration $(\mathrm{mM})$ using an in-house Matlab program.

\subsubsection{Calculations}

Values of $\tau_{R}$ and $\tau_{m}$ were adjusted and used in equations 2.6 to 2.11 as described by Yang et al. ${ }^{125}$ to achieve theoretical $r_{1}^{I S}$ values similar to those found experimentally. The constants used in the calculations are summarized in Table 2.2. Values for $\Delta^{2}, \tau_{v}$, $\tau_{R}$, and $\tau_{m}$ reported for monoamide derivatives of DTPA-Gd(III) by Tóth et al. ${ }^{126}$ were used as starting points in the calculations (as described in sections 2.3.6 and 2.3.7) due to the monoamide framework in the DNA-DTPA-Gd(III) conjugates. 
Table 2.2 Values used in calculations of theoretical relaxivities. ${ }^{124,125}$

\begin{tabular}{|c|c|c|}
\hline Symbol & Description & Value \\
\hline$\gamma_{I}$ & gyromagnetic constant for the proton & $2.675 \times 10^{8} \mathrm{~T}^{-1} \mathrm{~s}^{-1}$ \\
$g$ & electronic $g$-factor of Gd(III) & 2.0 \\
$\mu_{B}$ & Bohr magneton & $9.274 \times 10^{-24} \mathrm{~J} \mathrm{~T}^{-1}$ \\
$\mathrm{~S}$ & spin of Gd(III) & $7 / 2$ \\
$r_{G d H}$ & Gd-H bond distance & $3.0 \times 10^{-10} \mathrm{~m}$ \\
$\mu_{0}$ & permeability of a vacuum & $1.257 \times 10^{-6} \mathrm{~N} \mathrm{~A}^{-2}$ \\
$\omega_{I}$ & frequency of a proton & $\gamma_{I} \times$ magnetic field (T) \\
$\omega_{S}$ & frequency of an electron & $658 \times \omega_{I}$ \\
\hline
\end{tabular}

\subsection{Results and Discussion}

\subsubsection{Preparation of DNA-DTPA conjugates}

Three thrombin aptamer-DTPA single-conjugates, SC, 15T SC and 30T SC were prepared through the reaction of amino-modified DNA with DTPA dianhydride (Figure 2.7). All three DNA sequences used included the 15 mer thrombin aptamer, but the $15 \mathrm{~T}$ and 30T DNA strands included 15 and 30 thymine residues, respectively, on the 5 end of the aptamer (see section 2.2.2 for sequences). The $5^{\prime}$ end of each respective sequence included an amino-modifier protected with a 4,4'-dimethoxy-4"-thiomethoxytrityl (DMS(O)MT) group (see Figure 1.9).

For the reaction with DTPA dianhydride, the DNA was left bound to the CPG beads on which it was synthesized and was rinsed with $\mathrm{MeCN}$ and a $10 \%$ diethylamine (DEA) solution to remove and inactivate any reactants or side-products leftover from DNA synthesis. The DEA rinse was used because of the production of acrylonitrile 
during deprotection: it is produced through the $\beta$-elimination of the 2-cyanoethyl protecting group on the phosphate triester.' Primary amines, such as that in the aminomodifier, can react with acrylonitrile and be inactivated ${ }^{142}$ as shown in equation 2.13:

$$
\mathrm{R}-\mathrm{NH}_{2}+\mathrm{CH}_{2}=\mathrm{CH}-\mathrm{CN} \rightarrow \mathrm{R}-\mathrm{NH}-\mathrm{CH}_{2}-\mathrm{CH}_{2}-\mathrm{CN} \quad \text { [2.13] }
$$

Therefore, washing with a $10 \%$ DEA solution ensures that any acrylonitrile present in the column is inactivated by the reaction with DEA, and this wash is followed by another with $\mathrm{MeCN}$ to remove the aminonitrile product from the CPG beads.

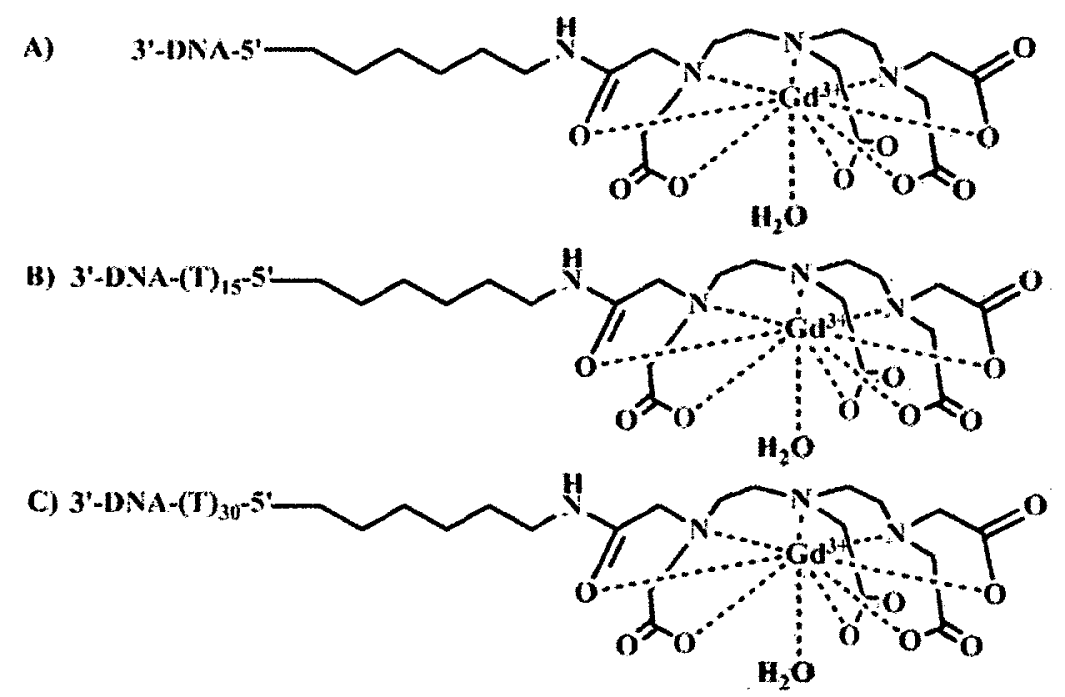

Figure 2.7 DNA-DTPA single-conjugates with chelated Gd(III) prepared for this study. A) thrombin aptamer-DTPA, SC-Gd(III); B) thrombin aptamer-15 polyT spacer-DTPA, 15T SCGd(III); C) thrombin aptamer-30 polyT spacer-DTPA, 30T SC-Gd(III). Reproduced from reference 143 with permission from Springer.

Once the presence of acrylonitrile was no longer a concern, the DMS(O)MT group blocking the reactive amine on the modifier was removed by rinsing the CPG 
beads with $3 \times 300 \mu \mathrm{L}$ of deblock solution. These washings were collected to allow for the qualitative assessment of modifier and DNA synthesis efficiency. The DMS(O)MT cation is a distinctive red colour in deblock solution, which is visibly different from the orange observed when the DMT cation is removed from phosphoramidites during synthesis. If the amino-modifier failed to be incorporated into the majority of sequences produced during a DNA synthesis, the isolated deblock solution from rinsing the CPG beads at this stage was bright orange. The DNA would not be used for reaction with DTPA dianhydride in such cases. When the DNA synthesis produced a high yield of amino-modified sequences, the deblock solution was red and the reaction was performed following a final rinse of the $\mathrm{CPG}$ beads with $\mathrm{MeCN}$ to remove any lingering DMS(O)MT cations or deblock solution.

The CPG beads were reacted with a 42 -fold excess of DTPA dianhydride in anhydrous DMSO. The high excess of DTPA dianhydride was used both to ensure that the amino-modified DNA was the limiting reagent and also due to the moisture sensitivity of the anhydride groups. They readily undergo hydrolysis in the presence of water as shown for the anhydride on the left in Scheme 2.1, and despite precautions such as storage under argon and the use of anhydrous DMSO, it is likely that some of the DTPA no longer contained the reactive anhydride groups.

The reaction of the anhydride groups with the reactive amines on the modified DNA strands proceeds as demonstrated for the anhydride on the right in Scheme 2.1. When a DNA strand was conjugated to DTPA through its reaction with one anhydride group and the other anhydride was hydrolyzed, as shown in Scheme 2.1, DNA-DTPA single-conjugates were formed. When both anhydride moieties reacted with amino- 
modified DNA, double-conjugates were produced (these will be discussed in detail in chapter 3). While hydrolysis of one of the cyclic anhydrides produces two carboxylic acid groups, the reaction of an amino-modified strand of DNA with the second cyclic anhydride results in the formation of an amide bond and one carboxylic acid, as is evident in both Figure 2.7 (showing the three single-conjugates synthesized) and Scheme 2.1. The chelating backbone in the single-conjugates are therefore monoamide derivatives of the parent DTPA ligand (see Figure 2.3A). The importance of this amide in the chelating backbone of the DNA-DTPA conjugates will be considered later with regards to its chelation stability towards Gd(III) (sections 2.3 .2 and 2.3.5.3) and the relaxation properties of the conjugates (section 2.3.6).

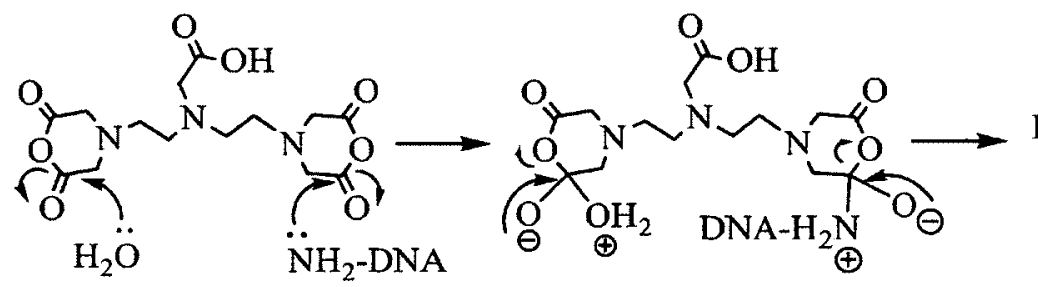<smiles>O=C(O)CN(CCN(CC(=O)O)CC(=O)N[Na])CC(=O)O</smiles>

Scheme 2.1 Reaction of DTPA dianhydride with water and amino-modified DNA, where $\mathrm{NH}_{2}$-DNA represents any of the modified sequences present in section $\mathbf{2 . 2 . 2}$.

\subsubsection{Chelation of $G d(I I I)$ with DNA-DTPA conjugates}

The first attempt to chelate Gd(III) with SC and demonstrate the success and extent of chelation using a xylenol orange test demonstrated that an excess of Gd(III) should not be added to the conjugates.

Xylenol orange is a dye that chelates to several metal ions in acidic solution ${ }^{139}$ and is commonly used to demonstrate Gd(III) chelation in CAs. ${ }^{144,} 145$ The stability 
constant $(\operatorname{logK})$ of xylenol orange and $\mathrm{Gd}(\mathrm{III})$ has been reported to be about 4.5 (at $\mathrm{pH}$ $5.5,298 \mathrm{~K}$, mixed in a $1: 1$ ratio). ${ }^{139}$ Its structure is shown in Figure 2.8. An acetate buffer was chosen as the diluent for the dye and all solutions in the xylenol orange test based on a literature example. ${ }^{145,144}$ The buffer was prepared to a $\mathrm{pH}$ of 6 to remain in the range where the Gd(III)-xylenol orange complex would be stable ${ }^{139}$ and to minimize the acidity in case the DNA-DTPA conjugates needed to be recovered from a solution containing dye. At this $\mathrm{pH}$, xylenol orange has two distinct absorption peaks, at 450 and $570 \mathrm{~nm}$, respectively. ${ }^{139}$ When complexed with a metal ion, the peak at $570 \mathrm{~nm}$ increases in absorbance ${ }^{139}$ as illustrated in Figure 2.9.

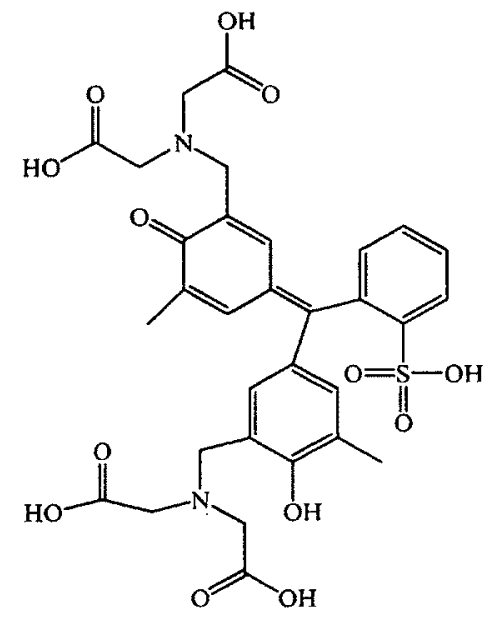

Figure 2.8 Xylenol orange

The spectra in Figure 2.9 are from the first attempt made to chelate SC with Gd(III) and then demonstrate the extent and effectiveness of chelation using xylenol orange. Five molar equivalents of $\mathrm{Gd}($ III) were added to a $\sim 59 \mathrm{nmol}$ portion of $\mathbf{S C}$ and the mixture was left on a shaker overnight to promote chelation. Initially, ethanol precipitation was attempted as a method by which to isolate SC-Gd(III) from the excess $\mathrm{Gd}(\mathrm{III})$ in solution. The lack of visible pellets resulting from the precipitation was not 
unusual given the small amount of DNA present, but a desire to ensure maximum recovery of SC lead to the decision to dry the solutions and attempt the separation by a different method.

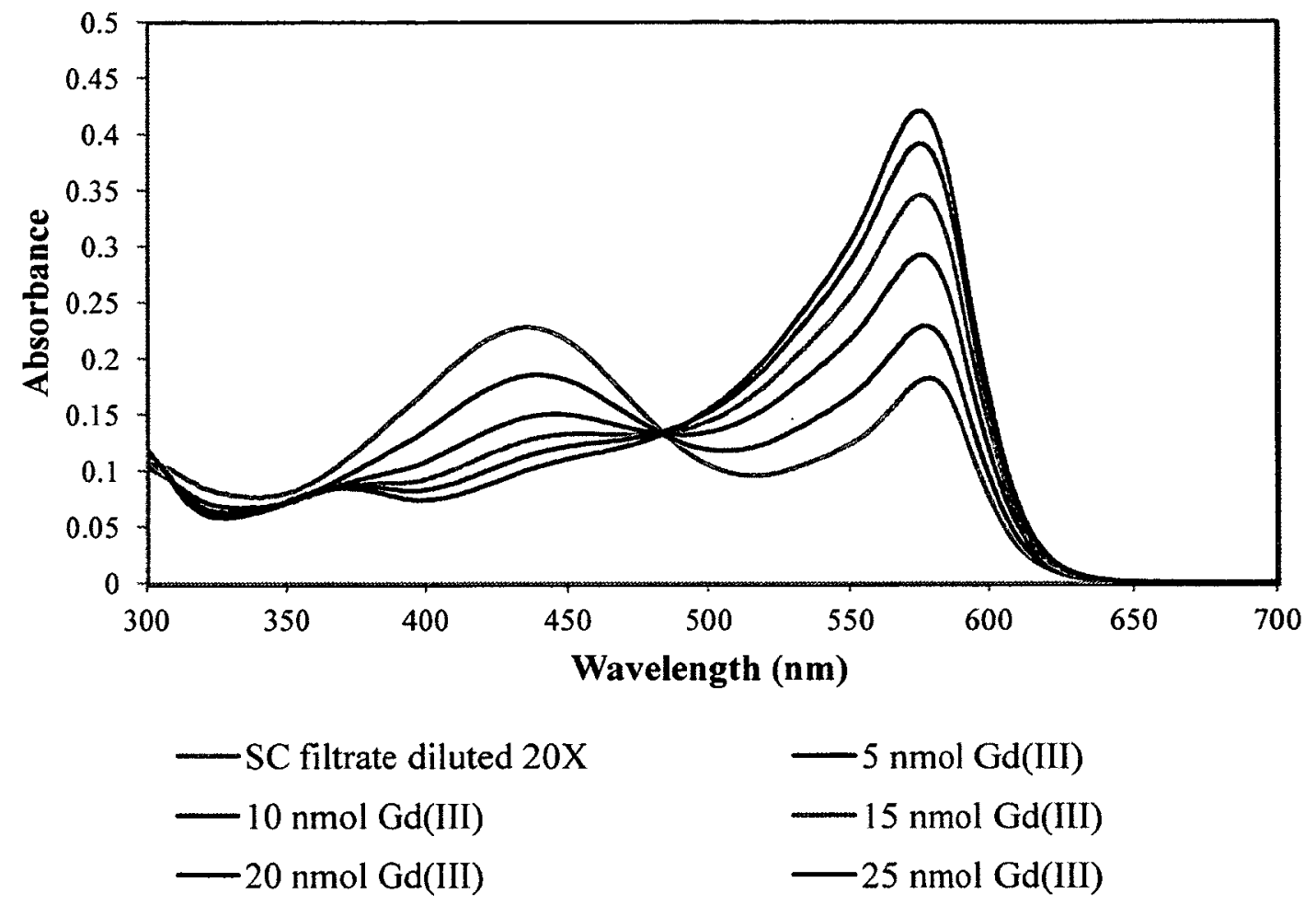

Figure 2.9 Initial xylenol orange calibration solutions (5-25 nmol Gd(III)) and sample (SC filtrate) for determination of $\mathrm{Gd}(\mathrm{III})$ in flow through for SC. Xylenol orange was present in every sample at $15 \mathrm{nmol} / \mathrm{mL}$.

An ultrafiltration cell was then used for the isolation of SC-Gd(III) from the solution containing excess Gd(III). It was expected that SC-Gd(III) would be retained on the filter and that the excess Gd(III) would be washed through in the filtrate alongside salts from the buffer. The filtrate was therefore collected, dried under vacuum, and all the salts were combined when reconstituted in $\mathrm{diH}_{2} \mathrm{O}$. The amount of $\mathrm{Gd}(\mathrm{III})$ expected to 
be in the filtrate was determined based on the excess Gd(III) added to SC, and the solution was diluted 20 times to ensure that the concentration fell within the range of the calibration solutions. Each sample in the series tested included $15 \mathrm{nmol}$ of xylenol orange and was made up to $1 \mathrm{~mL}$ total volume. The calibration solutions contained varying amounts of Gd(III) from 5 to $25 \mathrm{nmol}$. Prior to the acquirement of absorbance spectra for each sample in triplicate, the samples were mixed for 30 minutes to ensure equilibration between the dye and $\mathrm{Gd}(\mathrm{III})$ ions. As evident from the spectra in Figure 2.9, the amount of $\mathrm{Gd}(\mathrm{III})$ in the diluted filtrate (orange line) was below $5 \mathrm{nmol}$, and it was determined that with such a high dilution an accurate measurement of Gd(III) present using the resultant calibration curve was unobtainable. Figure 2.9 also illustrates that the peak at lower wavelength decreases in absorbance as the concentration of Gd(III) increases, demonstrating the opposite trend of the peak at $577 \mathrm{~nm}$.

A new batch of calibration solutions were prepared along with a filtrate sample that was merely diluted two times. The calibration curve obtained in this case is shown in Figure A-1 (Appendix A), where each point represents the average absorbance at 577 $\mathrm{nm}$ of the three spectra obtained for one solution. The absorbance at $577 \mathrm{~nm}$ was used instead of the expected $570 \mathrm{~nm}^{139}$ since it was observed to be the wavelength with maximum absorbance under the conditions used. From this data, it was determined that the filtrate contained about $26 \mathrm{nmol}$ of Gd(III), which was much lower than the expected $236 \mathrm{nmol}$ of Gd(III) that were added in excess of SC. It was hypothesized that the positively-charged $\mathrm{Gd}(\mathrm{III})$ ion could become associated with the negatively-charged backbone of the DNA in addition to being chelated by DTPA, and that the desalting conditions were not strong enough to break these electrostatic interactions. 
Once it was evident that excess Gd(III) could not be easily removed from a DNADTPA conjugate, the following procedure was developed for the chelation of Gd(III) and the follow-up xylenol orange test using a fresh portion of SC (49 nmol). The DNADTPA conjugate was dissolved in Tris buffer $(50 \mathrm{mM}, \mathrm{pH} 7.4)$ containing $140 \mathrm{mM} \mathrm{NaCl}$. Saturation of the negatively-charged DNA backbone with sodium ions prevented the association of $\mathrm{Gd}(\mathrm{III})$ with the backbone and therefore promoted its chelation with the DTPA moiety. Additionally, only one molar equivalent of Gd(III) was added to the conjugates to further ensure that Gd(III) was chelated by the DTPA and not associated with the DNA backbone. After the sample was left overnight to allow adequate time for chelation, the excess salts, including any free $\mathrm{Gd}(\mathrm{II})$, were isolated from the conjugate using an ultrafiltration cell in early experiments, and then centrifugal filter units in later experiments.

The amount of Gd(III) present in the filtrate in these experiments was expected to be low as no excess Gd(III) was added to the conjugates, so the calibration series always included a solution with the same concentration of xylenol orange as in the rest of the calibration samples but no $\mathrm{Gd}(\mathrm{III})$. It was often observed that the absorbance of the filtrate salts with the dye would be about the same as this $0 \mathrm{nmol}$ Gd(III) calibration solution. In such cases, this was taken as evidence that few or no Gd(III) ions were present in the filtrate and, therefore, it had all chelated to the DTPA in the conjugate. An example of this is shown in Figure 2.10 for the first sample of SC that had been mixed in a $1: 1$ ratio with $\mathrm{Gd}(\mathrm{III})$, as described above, and where an equivalent amount of xylenol orange $(10 \mathrm{nmol} / \mathrm{mL})$ is present in both samples. When the spectra of a conjugate's filtrate and the $0 \mathrm{nmol} \mathrm{Gd}(\mathrm{III})$ calibration solution were identical, or nearly so, as in 
Figure 2.10, the portion of conjugate under consideration was deemed ready for $T_{1}$ experiments.

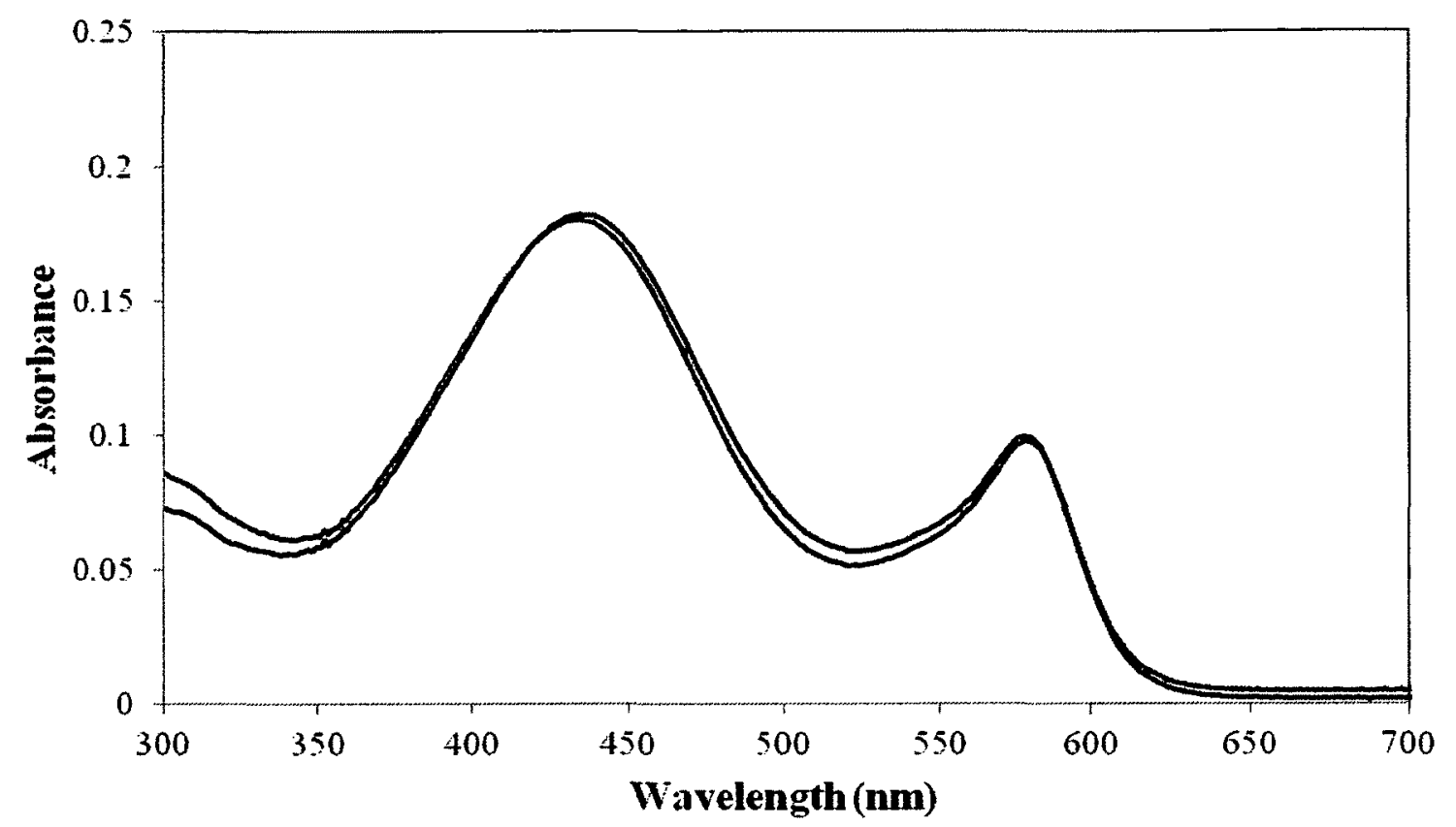

- SC filtrate $-0 \mathrm{nmol}$ Gd(III)

Figure 2.10 Xylenol orange test for determination of Gd(III) in the filtrate of SC (49 $\mathrm{nmol})$ mixed with one molar equivalent of Gd(III). Xylenol orange was present in both samples at $10 \mathrm{nmol} / \mathrm{mL}$.

Of the $49 \mathrm{nmol}$ of SC used in the optimization experiment, only $32 \mathrm{nmol}$ of SCGd(III) was recovered from the stirred cell filter. It was typical to see a loss of some DNA-DTPA conjugate during the desalting step, using either the stirred cells or desalting tubes. Attempts were made in an early experiment to separate SC-Gd(III) from the dye following the performance of a xylenol orange test. Ethanol precipitation was tried first, but the pellet of DNA recovered remained orange, indicating that the dye was still present and had not remained in the ethanol as desired. A stirred cell with a filter of 1000 Da cut- 
off was tried next, with the sample dissolved in $1 \mathrm{~mL} \operatorname{diH}_{2} \mathrm{O}$ and washed with three subsequent $1 \mathrm{~mL}$ portions of the same. Even chelated to Gd(III), xylenol orange would be expected to cross through the filter ( $874 \mathrm{Da}$ ) while SC-Gd(III) (5434 Da) should be retained. The spectra in Figure A-2 illustrate that while a higher ratio of xylenol orange (see peaks in inset) to SC-Gd(III) (peaks at $260 \mathrm{~nm}$ ) was present in the flow through for the first couple of millilitres pushed through the filter (blue and red lines, respectively), the filter allowed more of the DNA-DTPA conjugate through in later washes, resulting in an inefficient separation of conjugate and dye.

The low recovery of DNA-DTPA conjugates from the stirred cells was repeatedly observed, so they were relegated to use only when it was necessary to isolate the conjugates from high volume $(>3 \mathrm{~mL})$ solutions, such as following PAGE purification. Following chelation with Gd(III), the conjugates and excess salts were separated using desalting tubes instead. As the separation of xylenol orange from SC-Gd(III) was not achieved in the experiments discussed above, the amount of conjugate recovered from the retentate following desalting was determined prior to performing the xylenol orange test on the flow through. Generally, if more than $10 \mathrm{nmol}$ of the conjugate was missing from the retentate and found in the flow through using UV-visible spectroscopy, the flow through was desalted for a second time. Some loss of the conjugate through its sticking to the filter was also inevitable, which is why the amount of conjugate in the flow through would be determined prior to the second desalt.

When the amount of Gd(III) ions measured in the filtrate salts was nonzero, the quantity was compared to the total amount of $\mathrm{Gd}(\mathrm{III})$ initially added to the conjugate and a percentage of conjugate without chelated $\mathrm{Gd}(\mathrm{III})$ was calculated. From these 
calculations, the percentage of conjugate without chelated Gd(III) was generally found to be in the range of 0 to $9 \%$. In cases where the percentage of the conjugate without chelated $\mathrm{Gd}(\mathrm{III})$ exceeded $10 \%$, an appropriate portion of $\mathrm{Gd}(\mathrm{III})$ was added to the conjugate for the achievement of molar equivalency between the conjugate and the ion. The sample was desalted and the xylenol orange test performed once again, and the process was repeated as required until the percentage of conjugate without chelated Gd(III) was below $10 \%$.

In one unusual case, following the addition of a molar equivalency of Gd(III) to a portion of 30T SC $(\sim 160 \mathrm{nmol})$, about $24 \%$ of the conjugate was found to remain unchelated by the xylenol orange test. It was necessary to prepare more calibration solutions than originally expected for this test once the absorbance spectra were obtained and the peak at $577 \mathrm{~nm}$ for the filtrate sample was outside the range of calibration

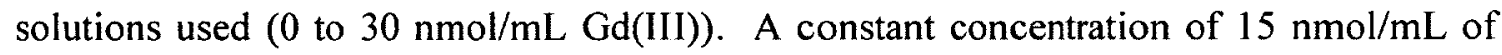
xylenol orange had been used for this series of samples, and as illustrated in Figure A-3, two distinct linear relationships were found for the calibration solutions.

When the amount of xylenol orange in the solutions was in the middle of the range of $\mathrm{Gd}(\mathrm{III})$ concentrations, as in the series at low concentrations in Figure A-3 (solid markers), a greater change in absorbance at $577 \mathrm{~nm}$ was observed with added Gd(III), as reflected by the slope. For the high Gd(III) concentration range (Figure A-3, open markers), the amount of xylenol orange present was much lower than that of Gd(III) and so the absorbance change with increasing concentration of the metal ion can be seen to be an order of magnitude lower. It is expected that as the concentration of $\mathrm{Gd}(\mathrm{III})$ increases, even when in excess of xylenol orange, the equilibrium between free $\mathrm{Gd}(\mathrm{III})$ and that 
chelated with the dye would positively shift, resulting in the increase in absorbance seen over this higher concentration range. The calibration curve resulting from the high concentrations was therefore used to determine the amount of Gd(III) in the filtrate and from that, the percentage of conjugate without chelated Gd(III).

Following the determination of the amount of $30 \mathrm{~T} \mathrm{SC}$ that still required chelation by $\mathrm{Gd}(\mathrm{III})$, enough $\mathrm{Gd}(\mathrm{III})$ was added to make up the difference and the entire process was repeated. In the second xylenol orange test performed for this sample, approximately $9 \%$ of the conjugate was found to remain without chelated Gd(III). It is possible that the 45mer DNA strand present in 30T SC interferes with the chelation of Gd(III) by the DTPA moiety through steric effects, leading to the difficulty in obtaining complete chelation. Furthermore, though the conditional stability constant $\left(\log \mathrm{K}^{\prime}\right)$ of DTPA with Gd(III) has been reported as 17.7 at $\mathrm{pH} 7.4,{ }^{138}$ the conjugates prepared here are in fact monoamide derivatives of DTPA, where one of the carboxylate groups in the backbone of DTPA is replaced with an amide group as a result of the conjugation reaction (see Figure 2.7). The electrostatic attraction of the monoamide DTPA (DTPA$\mathrm{MA}$ ) to Gd(III) is lower than that of DTPA, and as a result the kinetics of chelation might be affected. ${ }^{104}$ DTPA and other open-chain chelators used as MRI CAs have been reported to be less kinetically stable overall than macrocyclic chelators such as DOTA. ${ }^{137}$, 138 This includes gadodiamide, the bisamide derivative of DTPA. At a pH of 7.4, the rates of dissociation of Gd(III) from commonly studied gadolinium(III)-containing CAs are low, ${ }^{138}$ but the effect of the DNA strands in the DNA-DTPA conjugates on the kinetics of chelation is as yet unknown. Therefore, upon achievement of a percentage of 
conjugate without chelated Gd(III) below $10 \%$, the 30T SC-Gd(III) sample discussed above was considered ready for use in $T_{1}$ experiments.

Finally, gadolinium(III) chelation of the single-conjugates when stored at $-20^{\circ} \mathrm{C}$ for a period of about four months was tested using a representative sample of 15 T SCGd(III). It was dissolved in $\operatorname{diH}_{2} \mathrm{O}$ and desalted using a centrifugal filter unit as described above. The filtrate was tested for the presence of Gd(III) using xylenol orange, and the spectra from this experiment are shown in Figure A-4. It can be seen that very little Gd(III) was detected in the filtrate, and it was calculated to be a loss of about $1.3 \%$ of the total Gd(III) initially present in the sample. Therefore the stability of the chelate in the single-conjugates was found to be stable by the xylenol orange test after storage at $-20^{\circ} \mathrm{C}$ for a period of approximately four months.

\subsubsection{Preparation of 15 and 30 polyA oligonucleotides}

Conjugates with 15 and 30 polythymine spacers included between the DNA aptamer and DTPA moieties (15T SC and 30T SC) were prepared to address the possibility of a slowed water exchange rate for the gadolinium(III) complex due to steric hindrance from the folded thrombin aptamer located directly next to DTPA, particularly when the aptamer interacts with its target (see Figure 2.15). The inclusion of spacers consisting of nucleotides rather than one made of polyethylene glycol (PEG), for example, allowed for control over linker flexibility to see what effect that might have on the relaxivities; the single-stranded polyT linker would be expected to be more flexible than one that is hybridized with a matching polyA strand. PolyA strands of 15 and 30 nucleotides were therefore prepared for use in melting studies to confirm hybridization, 
as well as for relaxivity studies. Following their synthesis using standard phosphoramidite chemistry, the polyA strands were purified either using PAGE or Clarity QSP 3 mL cartridges.

The advantage of using the cartridges for purification was the rapidity of the procedure. Following the overnight deprotection and cleavage of the DNA from the CPG beads, the $\mathrm{NH}_{4} \mathrm{OH}$ solution could be applied to the cartridge columns after dilution with DNA loading buffer. The Clarity QSP cartridges were used as directed by the company (section 2.2.6) to purify the completed DNA sequences from the failed sequences and then to detritylate the DNA prior to its removal from the column. The DNA could then be desalted directly from the elution solution using centrifugal filter units, dried under vacuum and quantified.

\subsubsection{Melting Studies}

Melting studies were performed to determine the conditions necessary for hybridization between the polyT spacers in $15 \mathrm{~T} \mathrm{SC}$ and 30T SC and their respective matching polyA strands. In each case, the conjugate was mixed with an equimolar amount of the appropriate polyA strand and diluted using thrombin aptamer buffer. The solution was then heated at $70^{\circ} \mathrm{C}$ for 5 minutes before being allowed to cool to room temperature. This step was performed to denature the DNA portion of the conjugate and promote hybridization between the polyT spacers and matching polyA strands. The melting studies were used to confirm whether or not this protocol was appropriate for use in the preparation of hybridized samples for the $T_{1}$ experiments. Once cool, the solution was transferred to a cuvette and a micro stir bar was used during the studies to ensure 
even heating throughout the solution. The absorbance of the solution was recorded at $260 \mathrm{~nm}$ at $1^{\circ} \mathrm{C}$ intervals between the temperatures of 17 and $90^{\circ} \mathrm{C}$.

Recall that dsDNA experiences hypochromicity (section 1.5.3). As the sample of hybridized DNA was heated and the two strands separated, the absorbance increased ${ }^{1}$ as clearly illustrated in the melting curves obtained for the two conjugates as shown in Figure 2.11. The dotted curve illustrates that $\mathbf{1 5 T} \mathbf{S C}+$ polyA has a melting temperature between 35 and $45^{\circ} \mathrm{C}$, as this is where the inflection point of the curve is located. For $30 \mathrm{~T}$ SC+polyA, the melting temperature is between 55 and $60^{\circ} \mathrm{C}$. Therefore, in both cases heating the conjugate to $70^{\circ} \mathrm{C}$ will denature the DNA, and cooling in the presence of their respective polyA strands will promote hybridization.

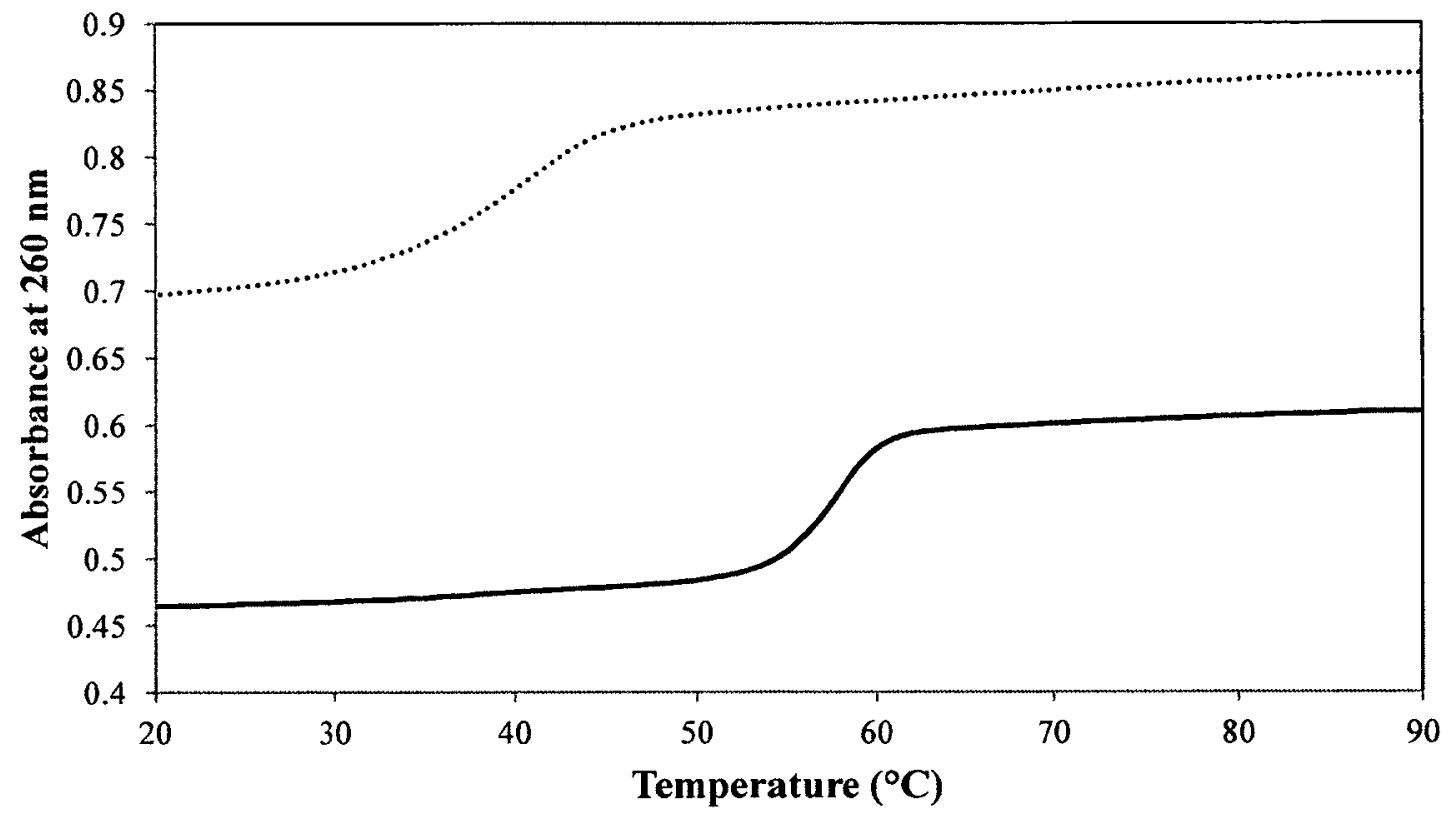

$15 \mathrm{~T} \mathrm{SC}+15$ polyA $-30 \mathrm{~T} \mathrm{SC}+30$ polyA

Figure 2.11 Melting curves for 15T SC and 30T SC with equimolar amounts of their respective polyA strands. 


\subsubsection{In vitro stability}

The stability of $\mathrm{Gd}(\mathrm{III})$ chelation within CAs is an important concern, as free $\mathrm{Gd}(\mathrm{III})$ ions are toxic $\left(\mathrm{LD}_{50}=0.1 \mathrm{mmol} \mathrm{kg}^{-1}\right)^{103}$ and have been implicated as a potential culprit in the development of nephrogenic systemic fibrosis (NSF) in patients with impaired renal function ${ }^{133,146,147}$ (section 2.1.2.4). Dechelated gadolinium(III) has been investigated as a possible culprit in the development of NSF, though recent studies have indicated that gadolinium(III) complexes might also be bioactive. ${ }^{135}$ Thus, the stability of SC-Gd(III) was examined using two approaches: a xylenol orange test and a transmetallation study.

\subsubsection{Stability in serum at $37^{\circ} \mathrm{C}$ by the xylenol orange test}

The stability of the DNA-DTPA conjugates with regards to Gd(III) loss in serum at the biologically relevant temperature of $37^{\circ} \mathrm{C}$ was sought as an indication of the potential safety of these conjugates as targeted MRI CAs. To this end, a portion of SCGd(III) was used as a representative system and dissolved in bovine serum to a concentration of about $0.1 \mathrm{mM}$. The sample was split between two Eppendorf tubes and both were heated at $37^{\circ} \mathrm{C}$. The clearance half-life of DTPA-Gd(III) is reported to be 1.6 hours (assuming normal renal function), ${ }^{105}$ while unmodified DNA aptamers have a lifetime of only minutes in blood. ${ }^{10,64,67}$ Upon consideration of these clearance times, one tube was removed from the heat block after 1 hour. As a test of long term stability under these biologically relevant conditions, the other half of the sample was left at $37^{\circ} \mathrm{C}$ for a total of 24 hours. After being cooled to room temperature following the respective heating times, each solution was desalted using a centrifugal filter unit. It was impossible 
to determine the efficiency of retention of SC-Gd(III) in the filter units for these samples as protein from the serum was present in both the retentate and filtrate, and the absorbance of the proteins at $280 \mathrm{~nm}$ obscured the $260 \mathrm{~nm}$ absorbance of the DNA in the conjugate. The filtrates from both 1 and 24 hours of heating were desalted for a second time as in cases where greater than $10 \mathrm{nmol}$ of the conjugate had been lost to the filtrate (section 2.2.4), but it was still impossible to quantify the conjugate in retentate or filtrate. The filtrates were tested using xylenol orange at this point.

The presence of SC-Gd(III) in the filtrate could have caused bias in the results if the Gd(III) ions bound to xylenol orange more strongly than to the DTPA moiety in the conjugate. The stability constant of xylenol orange with $\mathrm{Gd}(\mathrm{III})$, as mentioned earlier, is about $4.5\left(\mathrm{pH} 5.5,298 \mathrm{~K}\right.$, mixed in a $1: 1$ ratio). ${ }^{139}$ The conditional stability constants (logK') of DTPA and DTPA-BMA (DTPA-bisamide) are reported as 17.7 and $14.9(\mathrm{pH}$ 7.4), respectively, ${ }^{138}$ so the monoamide derivative in SC-Gd(III) would be expected to have a stability constant in this range, and therefore higher than that of Gd(III) with xylenol orange. It was therefore concluded that the possible presence of SC-Gd(III) in the filtrate should not affect the results obtained using the xylenol orange test.

The spectra obtained from the test on the filtrates from 1 and 24 hours of heating, along with the calibration solutions containing 0 and $5 \mathrm{nmol}$ of $\mathrm{Gd}(\mathrm{III})$, respectively, are shown in Figure A-5. These results illustrated that out of a possible $27 \mathrm{nmol}$ of Gd(III) that might have been lost from each of the portions of SC-Gd(III) heated in serum, less than $1 \mathrm{nmol}$ was found in the filtrate by the xylenol orange test for both cases. The amount of Gd(III) in the filtrate for the sample heated for 1 hour was about $2 \%$ of the original amount, while for the sample heated for 24 hours was about $1 \%$ of the Gd(III) 
initially present chelated to SC. These results were initially encouraging as they demonstrated little loss of chelated Gd(III) from SC-Gd(III) under conditions representing those encountered in vivo.

As the amounts of Gd(III) measured in the filtrates were about the same, and was slightly lower after 24 hours of heating compared to only 1 hour, it was determined that a solution of $\mathrm{Gd}(\mathrm{III})$ in bovine serum should be tested in an identical experiment to test whether the contents of the serum could interfere with the quantification of Gd(III) by the xylenol orange test. If proteins present in the serum bound to $\mathrm{Gd}(\mathrm{III})$ with higher affinity than xylenol orange, particularly since the protein-Gd(III) interactions would have formed prior to addition of the dye, it is likely that few, if any, free Gd(III) ions would be detected in this test. For this test, $15 \mathrm{nmol}$ of Gd(III) were added to bovine serum for the preparation of a $0.075 \mathrm{mM}$ solution. Following heating at $37^{\circ} \mathrm{C}$ for 1 hour and two desalting steps, the xylenol orange test was performed on the filtrate. The resulting spectra are illustrated in Figure A-6. They demonstrate that little Gd(III) was detected in the filtrate; $0.8 \mathrm{nmol}$ of $\mathrm{Gd}(\mathrm{III})$ was detected, which was only $5 \%$ of the Gd(III) that should have been measured. Bovine serum albumin (BSA) present in the serum likely played a role in removing free Gd(III) from solution as it has been reported as having a $\operatorname{logK}$ value of $6.16 \pm 0.08$ for this ion. ${ }^{148}$ It was therefore concluded that the results of the xylenol orange test used to measure freed Gd(III) from SC-Gd(III) in bovine serum were unreliable. 


\subsubsection{Stability in buffer at $37^{\circ} \mathrm{C}$ by the xylenol orange test}

Once the in vitro test of $\mathrm{Gd}(\mathrm{III})$ chelation stability in bovine serum was discounted and the Gd(III)-binding properties of BSA were considered, a simple test for the determination of the chelation stability of SC-Gd(III) at $37^{\circ} \mathrm{C}$ was developed. Considering the in vivo clearance times of DTPA-Gd(III) and unmodified DNA aptamers mentioned above (1.6 hours and minutes, respectively, section 2.3.5.1), a portion of the SC-Gd(III) was dissolved to $0.17 \mathrm{mM}$ in thrombin aptamer buffer and subsequently heated at $37^{\circ} \mathrm{C}$ for 3 hours. The conjugate was then desalted, with the conjugate solution filtered at $37^{\circ} \mathrm{C}$ to ensure any Gd(III) freed from SC-Gd(III) as a result of the temperature passed into the filtrate. The washes of the retentate were performed at room temperature so as not to cause any additional Gd(III) to become unchelated at this stage. The amount of $\mathrm{Gd}(\mathrm{III})$ in the filtrate was quantified using xylenol orange as in the optimized protocol described above (sections 2.2 .4 and 2.3.2). The spectra from this test are shown in Figure A-7 and the calibration curve in Figure A-8, respectively. About 0.9 nmol of Gd(III) were detected in the filtrate, representing about $2 \%$ of the $43 \mathrm{nmol}$ of SC-Gd(III) initially present. This relatively low level of loss, in combination with the expectation that the required dose of a targeted system should be lower than that of a general agent $\left(0.1 \mathrm{mmol} \mathrm{kg}^{-1}\right.$ for DTPA-Gd(III)),${ }^{105}$ indicated good chelation stability of SC-Gd(III) under the conditions used.

\subsubsection{Transmetallation study}

The dechelation of gadolinium-based contrast agents in vivo has become the focus of much research and can be a function of the thermodynamic stability of the complex or 
of transmetallation. $^{104}$ There are several endogenous metal ions present in the human body, but the only endogenous ion that has high enough association constants with common chelators and is present at a sufficient concentration in vivo to potentially displace chelated gadolinium(III) is zinc(II). ${ }^{137,140}$ As such, Laurent et al. reported a method by which to measure the transmetallation of a $\mathrm{Gd}(\mathrm{III})$ complex through monitoring its change in relaxivity over time in the presence of $\mathrm{Zn}(\mathrm{II}) .^{137,140}$

In the reported study, ${ }^{137}$ the Gd(III)-containing chelate under consideration was dissolved in phosphate buffer to a concentration of $2.5 \mathrm{mM}$ and an equimolar amount of $\mathrm{Zn}(\mathrm{II})$ was added. The initial observed relaxivity value $(\mathrm{t}=0)$ at $37^{\circ} \mathrm{C}$ and $20 \mathrm{MHz}$ was then determined, where $R_{1}=1 / T_{1}$. Throughout the experiment, the sample solution was maintained at $37^{\circ} \mathrm{C}$ and the relaxivity was monitored through the periodic determination of $T_{1}$. Phosphate buffer was used as the diluent to take advantage of the very low solubility of $\mathrm{Gd}\left(\mathrm{PO}_{4}\right)\left(\mathrm{K}_{\mathrm{sp}}=10^{-22.26} \mathrm{M}^{2}\right) .{ }^{140} \mathrm{As} \mathrm{Gd}(\mathrm{III})$ ions were replaced in the chelate by diamagnetic $\mathrm{Zn}$ (II) over time, they were removed from solution in precipitated $\mathrm{Gd}\left(\mathrm{PO}_{4}\right)$ and their effect on the relaxivity of water protons became negligible. ${ }^{140}$ Equations 2.14 to 2.16 (adapted from reference 140) illustrate the transmetallation process and equation 2.16 represents the resultant precipitation of Gd(III) with phosphate:

$$
\begin{gathered}
\text { Chelator-Gd }{ }^{(\mathrm{n})-} \rightleftharpoons \text { Chelator }^{(\mathrm{n}+3)-}+\mathrm{Gd}^{3+} \\
\text { Chelator }{ }^{(\mathrm{n}+3)-}+\mathrm{Zn}^{2+} \rightleftharpoons \text { Chelator- } \mathrm{Zn}^{(\mathrm{n}+1)-} \\
\mathrm{Gd}^{3+}+\mathrm{PO}_{4}{ }^{3-} \rightarrow \mathrm{Gd}\left(\mathrm{PO}_{4}\right)_{\text {insoluble }}
\end{gathered}
$$

The changes in observed relaxivity over time for several $\mathrm{Gd}(\mathrm{III})$-containing chelates were determined by Laurent et al. using this experimental procedure. Representative results are illustrated in Figure 2.13. 
The reported transmetallation studies on DTPA-Gd(III) and DTPA-BMA-Gd(III) (gadodiamide or Omniscan ${ }^{\mathrm{TM}}$ ) are of particular interest in the case of SC-Gd(III). The conjugation reaction reported herein results in DNA-DTPA conjugates that are monoamide derivatives of DTPA (see Figure 2.7); that is, one of the five carboxylate groups present in the DTPA backbone is replaced with an amide group upon reaction of amino-modified DNA with DTPA dianhydride. This leads to the chelating moiety in SCGd(III) and the other single-conjugates falling between that of DTPA and DTPA-BMA, the latter of which is a bisamide variant of DTPA (see Figure 2.12). Figure 2.13 includes the results of the transmetallation studies reported by Laurent et al. for both of these CAs. It can be seen that the relaxivity of DTPA-Gd(III) decreased by approximately $15 \%$ about 3 hours after $\mathrm{Zn}(\mathrm{II})$ was added to it and by about $50 \%$ after 50 hours. ${ }^{140}$ DTPA-BMAGd(III) demonstrated much lower stability than that of DTPA-Gd(III). In only 70 minutes, the relaxivity of gadodiamide had dropped by $20 \%$, and after about 50 hours it had dropped to slightly more than $10 \%$ of its initial value. ${ }^{137,140}$ It could be hypothesized that, based strictly on the chelator, SC-Gd(III) would demonstrate a stability to transmetallation between those reported for these two commonly used CAs.

A)

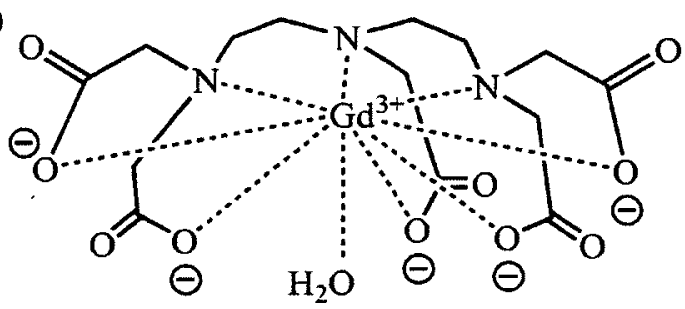

B)

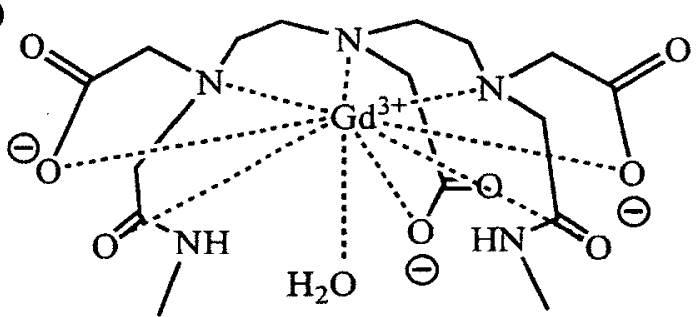

Figure 2.12 (Omniscan ${ }^{\mathrm{TM}}$ ). 


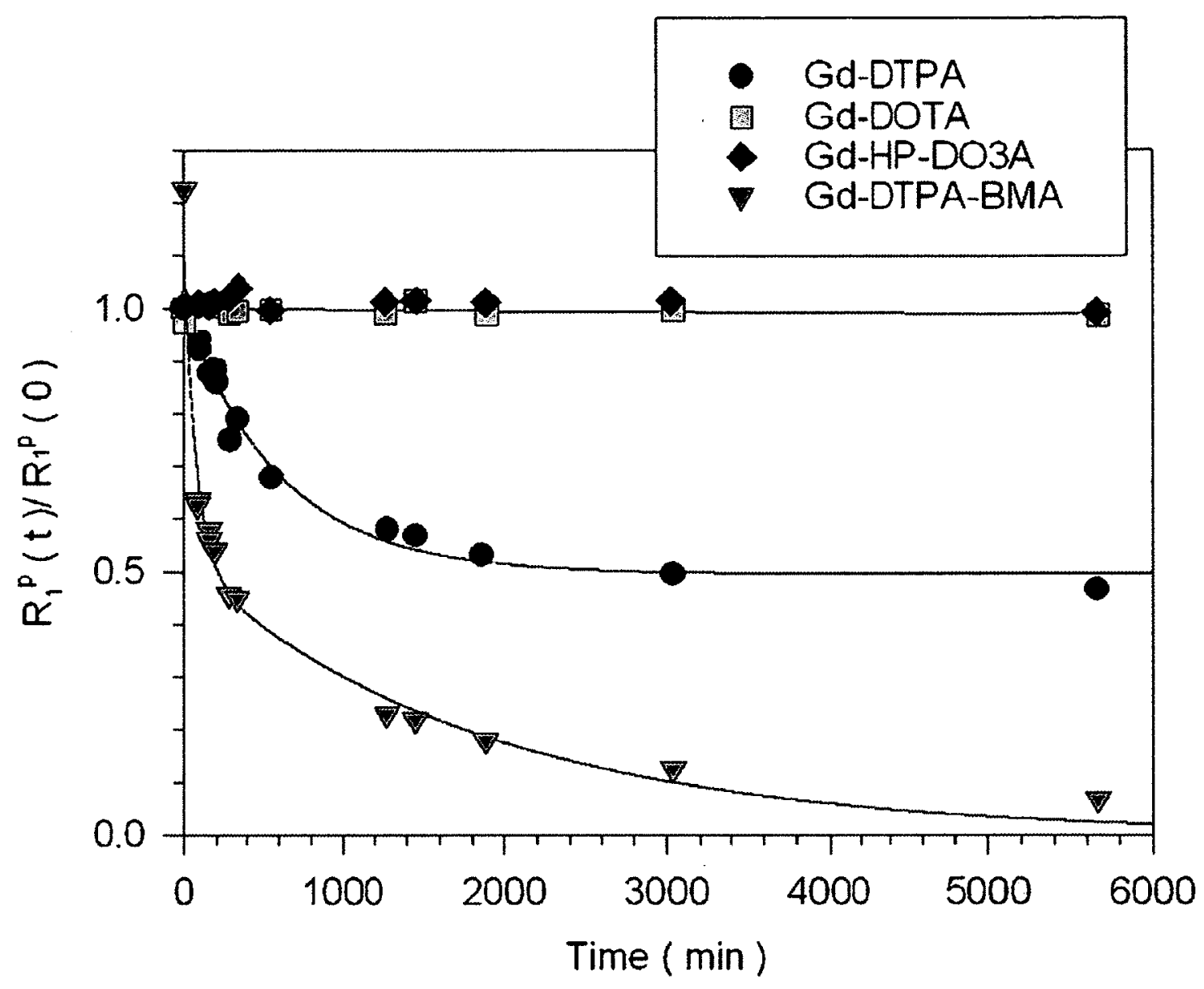

Figure 2.13 Results of transmetallation experiments where the relaxivity changes of several Gd(III)-containing CAs were monitored over time in the presence of $\mathrm{Zn}$ (II) and phosphate ions at 20 MHz and $37^{\circ} \mathrm{C}$. Modified from reference 140 .

Laurent et al. also reported the kinetics of the transmetallation process for a variety of DTPA-bisamides. ${ }^{137}$ Upon comparison it was generally found that chelates with more bulky amide substituents displayed kinetics closer to the parent DTPA-Gd(III) chelate while the primary bisamide complex had the lowest stability. The tertiary bisamide derivative studied actually showed better stability than that of DTPA-Gd(III), and it merely consisted of two methyl groups substituted on the amide nitrogen. ${ }^{137}$ The 
DNA aptamer bonded through an amide, therefore, could be sufficiently large to help offset the loss of stability expected from the replacement of a carboxylate group by an amide.

In order to determine how the conjugation of the thrombin aptamer to DTPA$\mathrm{Gd}(\mathrm{III})$ affected the extent of transmetallation of $\mathrm{Gd}(\mathrm{III})$ by $\mathrm{Zn}(\mathrm{II})$, the relaxivity of SCGd(III) at $37^{\circ} \mathrm{C}$ was monitored over time. The conjugate was dissolved in deuterated phosphate buffer to a concentration of $0.05 \mathrm{mM}$ and its initial observed relaxivity value $(t=0)$ was determined, where $R_{1}=1 / T_{1}$. Due to the time sensitive nature of this study, the $T_{1}$ values were estimated on a 9.4 T NMR spectrometer using the null spectrum of a 180 $\tau-90$ pulse program, where $T_{1}=\tau / \ln 2 .{ }^{141}$ The reaction solution was kept at $37^{\circ} \mathrm{C}$ for approximately 4 days, with $T_{1}$ values estimated periodically to monitor transmetallation. The paramagnetic longitudinal relaxivities $\left(\mathrm{R}_{1}{ }^{\mathrm{P}}\right)$ were then calculated from the observed relaxivity values through subtraction of the diamagnetic contribution of water proton relaxation $\left(0.077 \mathrm{~s}^{-1}\right)$. The diamagnetic contribution (see equation 2.4 ) was found through the determination of the relaxivity of the deuterated phosphate buffer at $37^{\circ} \mathrm{C}$ using the same method as described for the sample solution.

The relative paramagnetic relaxivity values, $\mathrm{R}_{1}{ }^{\mathrm{P}}(\mathrm{t}) / \mathrm{R}_{1}{ }^{\mathrm{P}}(0)$, of $\mathrm{SC}-\mathrm{Gd}(\mathrm{III})$ were determined and plotted as a function of time after the addition of $\mathrm{Zn}$ (II), as illustrated in Figure 2.14. It can be seen that after about 3 hours that $95 \%$ of the relaxivity was retained compared to the approximately $85 \%$ reported by Laurent et al. for DTPA-Gd(III) and only about $50 \%$ for DTPA-BMA-Gd(III). ${ }^{137,140}$ After about 50 hours, though, the relaxivity of SC-Gd(III) had dropped to $37 \%$ of its initial value, which is lower than the approximately $50 \%$ change reported for DTPA-Gd(III) but better than the $10 \%$ retention 
of relaxivity for DTPA-BMA-Gd(III). ${ }^{140}$ The transmetallation study on SC-Gd(III), therefore, indicates that the stability of the conjugate remains closer to that of DTPAGd(III) than DTPA-BMA-Gd(III). This likely results from a combination of its being a monoamide rather than a bisamide and from the steric hindrance to transmetallation lent by the presence of the DNA aptamer. Overall, the transmetallation results for SCGd(III) are promising when considering the possible future use of such conjugates as targeted MRI contrast agents, since both DTPA-Gd(III) and gadodiamide are already commonly used general contrast agents.

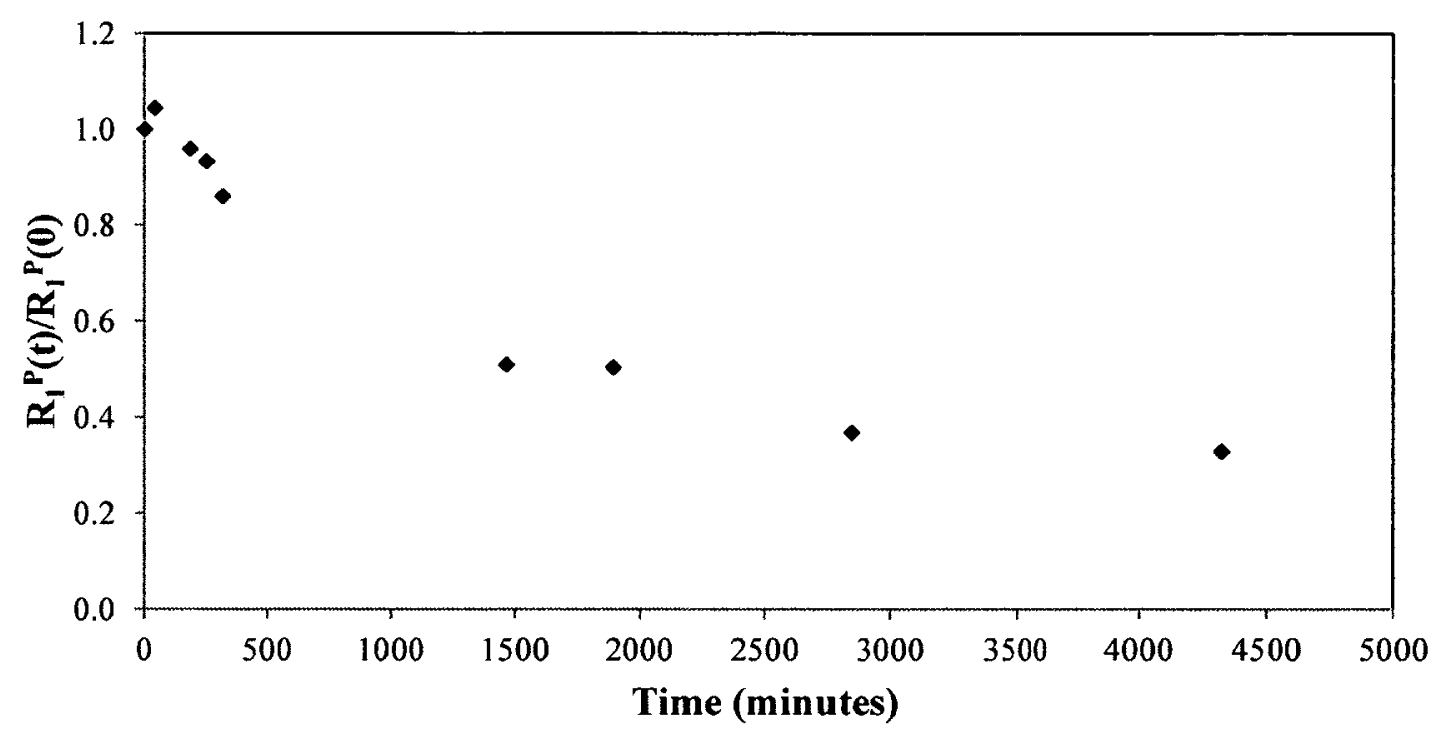

Figure 2.14 Relative paramagnetic longitudinal relaxivity, $\mathbf{R}_{1}{ }^{\mathrm{P}}(t) / \mathbf{R}_{1}{ }^{\mathrm{P}}(0)$, of $\mathrm{SC}-\mathrm{Gd}(\mathrm{III})$ at $37^{\circ} \mathrm{C}$ and 9.4 $\mathrm{T}$ as a function of time after the addition of a molar equivalent of $\mathrm{Zn}$ (II). Reproduced from reference 143 with permission from Springer.

\subsection{6 $T_{1}$ experiments and relaxivity data at high field (9.4 Tesla)}

Following the optimized chelation of Gd(III) to SC detailed in section 2.3.2 and confirmed in Figure 2.10, the appropriate conditions for the determination of $T_{1}$ for these 
samples at high field had to be determined. Due to the limited amount of SC-Gd(III) available for the initial set of $T_{1}$ experiments, it was necessary to devise an experimental set-up that would permit volumes of less than $100 \mu \mathrm{L}$ to be run on the $400 \mathrm{MHz}(9.4 \mathrm{~T})$ NMR spectrometer. For these low volumes to extend high enough in a $5 \mathrm{~mm}$ NMR tube to ensure accurate detection, glass capillary tubes of 1.5-1.8 $\mathrm{mm}$ outer diameter were used to hold the samples inside NMR tubes. The capillary tubes were held upright using a piece of narrow plastic tubing that was immobilized by passing it through a hole in the centre of the NMR tube's lid. The hydroxyl-terminated surface of the glass tubes ${ }^{149}$ can result in adsorption of charged species, thus changing their concentrations in the bulk solution. ${ }^{150}$ The capillary tubes in which the samples were placed for $T_{1}$ experiments at 9.4 $\mathrm{T}$ were therefore silanized using Sigmacote ${ }^{\circledR}$ as instructed (see section 2.2.8.3 for details) to convert the surface silanol groups on the glass to a neutral, hydrophobic thin film that would prevent the adsorption of proteins and other charged species. ${ }^{151}$ The advantage of using capillary tubes over thick-walled $5 \mathrm{~mm}$ NMR tubes or NMR insert tubes was the ease with which the sample solutions could be recovered following $T_{1}$ measurement. A high recovery was necessary to ensure that each successive concentration could be achieved through dilution due to the limited amount of sample available. The use of 4 inch needles permitted reasonably efficient injection and removal of sample solutions from the capillary tubes.

The three conjugates, SC-Gd(III), 15T SC-Gd(III), and 30T SC-Gd(III), were split into portions of 6 to $15 \mathrm{nmol}$, depending on the total amount isolated following successful chelation of Gd(III). There was enough SC-Gd(III) and 15T SC-Gd(III) for the highest concentration used to be $0.1 \mathrm{mM}$ in most cases. Less 30T SC-Gd(III) was 
isolated, and so the highest concentrations of its series were 0.05 or $0.025 \mathrm{mM}$. A minimum of three concentrations were used in $T_{1}$ experiments in all datasets and deuterated thrombin aptamer (TBA) buffer (relaxivity measurement buffer) was used as diluent. The number of points and the concentration ranges used are evident upon consideration of the plots of relaxation time $\left(\frac{1}{T_{1}}, \mathrm{~s}^{-1}\right)$ versus gadolinium(III) concentration (mM) for each conjugate. The conjugates were thermally denatured to permit refolding of the thrombin-binding aptamer prior to the performance of $T_{1}$ experiments since inconsistent results were obtained when this step was not performed (data not shown). This might be especially important in the conjugates containing thymine spacers as the additional nucleotides in the DNA portions could interact with the aptamer region and cause it to misfold.

The relaxivity of a contrast agent is influenced by a number of factors, one of which is the rotational correlation time $\left(\tau_{\mathrm{R}}\right)$, as mentioned in section 2.1.2.2. The rotational correlation time is related to the tumbling rate of the $\mathrm{CA}$, as it is the time it takes for reorientation of the $\mathrm{Gd}(\mathrm{III})-\mathrm{H}_{2} \mathrm{O}$ bond ${ }^{100}$ (see Figure 2.7). The short $\tau_{R}$ values of small CAs such as DTPA-Gd(III) and DOTA-Gd(III) limit their relaxivities, and so conjugation of these agents to macromolecules in an effort to increase $\tau_{R}$ has been a common approach in the design of high relaxivity agents. ${ }^{101}$

If the binding between aptamer and target interfered with the exchange of water molecules at the $\mathrm{Gd}(\mathrm{III})$ centre, thus increasing the value of $\tau_{m}$, increasing the space between the aptamer-protein and chelator moieties of the conjugate might diminish this effect. Since the 15 mer thrombin-binding aptamer folds into a chair-like structure 
(Figure 1.2$)^{46}$ in the presence of the potassium cation, ${ }^{47,48}$ it can be expected to mostly be folded in the relaxivity measurement buffer (in which $5 \mathrm{mM} \mathrm{KCl}$ is included), and even this folded DNA strand located immediately next to the DTPA-Gd(III) might be large enough to interfere with water exchange at the ion. Nucleotide spacers of 15 and 30 thymine residues ( 15 and 30 polyT) were therefore included between the $15 \mathrm{mer}$ thrombin-binding aptamer and DTPA in the conjugates 15T SC-Gd(III) and 30T SCGd(III), respectively. The spacers are represented in Figure $2.15 \mathrm{~B}$ and $\mathrm{C}$ as purple lines. As discussed earlier (section 2.3.3), the use of spacers composed of nucleotides allowed for control over linker flexibility to see what effect that might have on the relaxivities. The pink line in Figure $2.15 \mathrm{C}$ represents the matching polyA strand when hybridized to the polyT spacer.

$\mathrm{T}_{1}$ experiments for the three conjugates, SC-Gd(III), 15T SC-Gd(III), and 30T SC-Gd(III), were run in triplicate on a 9.4 T NMR spectrometer for each concentration within three series: 1) conjugate alone, 2) conjugate in a 1:1 ratio with thrombin and 3) conjugate in a 1:1 ratio with pepsin (used as a control). The choice of pepsin as a control was guided by the need to account for the viscosity changes caused by the addition of thrombin. It was determined that a protein of comparable size to thrombin $(36.7 \mathrm{kDa})$ that would have no specific affinity for the thrombin aptamer should be used as a control to help eliminate viscosity effects from the factors affecting our relaxivity results. Pepsin $(35 \mathrm{kDa})$, an enzyme that is inactivated in solution at $\mathrm{pH}>7$, was chosen as our control based on its size and its lack of interaction with the thrombin aptamer. ${ }^{12}$ 
A)
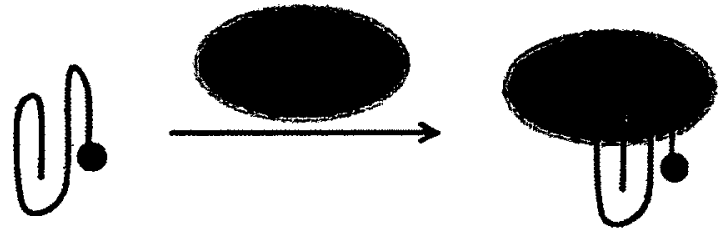

B)
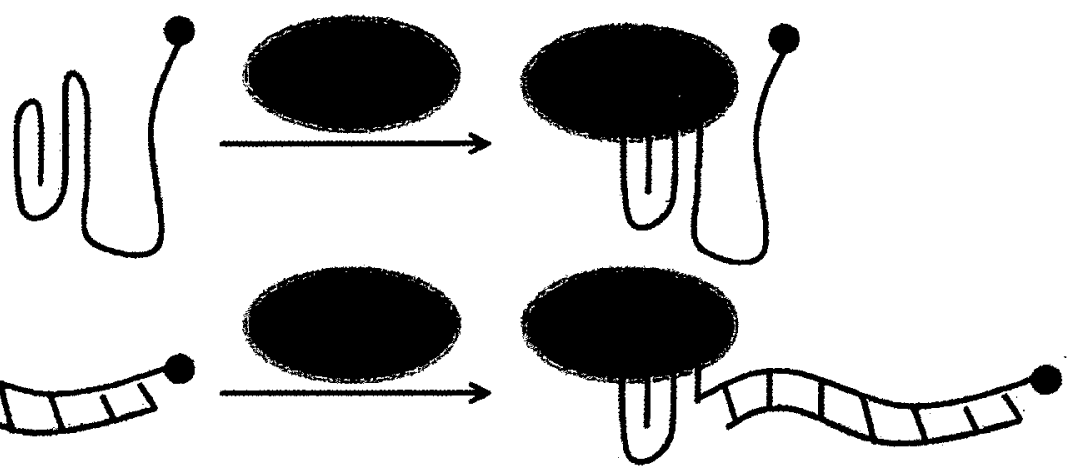

Thrombin aptamer $3^{\prime}$-GGT TGG TGT GGT TGG-5'

PolyT spacer ( $\mathrm{T}_{15}$ or $\mathrm{T}_{30}$ for $15 \mathrm{~T}$ SC-Gd(II) and 30T SC-Gd(III), respectively)

PolyA hybridizing strand ( $A_{15}$ or $A_{30}$ for $15 T$ SC and $30 T$ SC, respectively)
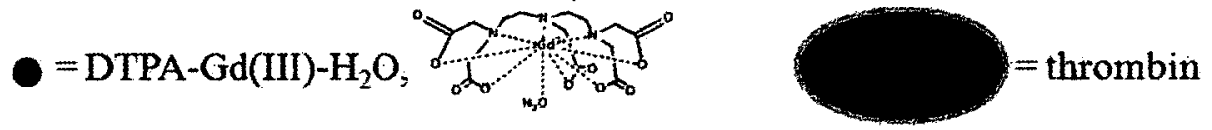

Figure 2.15 Schematic representation of the DNA aptamer-DTPA-Gd(III) conjugates interacting with thrombin. A) SC-Gd(III); B) $15 T$ or 30T SC-Gd(III); C) $15 \mathrm{~T}$ or 30T SC-Gd(III) when polyT linker is hybridized by a matching 15 or 30 polyA strand. Reproduced from reference 143 with permission from Springer.

The relaxivities of lab-prepared DTPA-Gd(III) and Magnevist® (formulation of DTPA-Gd(III) used as a CA) were determined under the conditions used for the conjugates. The relaxivities were calculated to be $5.75 \pm 0.05$ and $5.01 \pm 0.08 \mathrm{mM}^{-1} \mathrm{~s}^{-1}$, respectively (see Figure A-10). These values are comparable to those reported in literature for DTPA-Gd(III) when $\mathrm{D}_{2} \mathrm{O}$ was used as the solvent at the same magnetic field strength used in our experiments $\left(9.4 \mathrm{~T}, 5.25\right.$ and $\left.5.5 \mathrm{mM}^{-1} \mathrm{~s}^{-1}\right) .^{152,153}$ DTPA-Gd(III) typically has a relaxivity of $4.3 \mathrm{mM}^{-1} \mathrm{~s}^{-1}$ under low field conditions $\left(0.47 \mathrm{~T}, 25^{\circ} \mathrm{C}\right.$, 
aqueous solution), ${ }^{101,154}$ and it has been found to display decreasing relaxivity with increasing field strength, ${ }^{127,155}$ as mentioned earlier. The higher relaxivities observed at 9.4 $\mathrm{T}$ in the aforementioned cases are likely attributable to the higher viscosity of $\mathrm{D}_{2} \mathrm{O}$ compared to $\mathrm{H}_{2} \mathrm{O}\left(\sim 1.09{ }^{156}\right.$ versus $0.890 \mathrm{mPa} \cdot \mathrm{s}^{157}$ respectively, at $\left.25^{\circ} \mathrm{C}\right)$. The higher relaxivity found for the DTPA-Gd(III) prepared in-lab compared to Magnevist $®$ could be a result of incomplete chelation of Gd(III) by DTPA when mixed in a $1: 1$ ratio or a result of viscosity. Magnevist $®$ was therefore considered a better control and will be the only one of these results compared to the conjugate relaxivities in the following discussion.

Results from the SC-Gd(II) experiments are summarized in Figure A-11, Figure 2.16, and Table 2.3. The increase in relaxivity of $26 \pm 2 \%$ from Magnevist ${ }^{\circledR}$ to $\mathrm{SC}$ Gd(III) is likely an effect of the increase in size of the CA with the addition of the thrombin aptamer. Alone, the small DTPA-Gd(III) complex tumbles at a fast rate causing fluctuations in its local magnetic field. The addition of the thrombin aptamer to the Gd(III) complex seems to slow the overall rotational correlation time, $\tau_{R}$, bringing this local magnetic field's fluctuation frequency closer to the Larmor frequency of a proton and increasing its effectiveness as a relaxation agent. ${ }^{106}$ This can be compared to receptor-induced magnetization enhancement (RIME) where an enhancement of relaxivity is experienced by a small complex when it binds to a larger target. ${ }^{106}$ In the case of SC-Gd(III), simply increasing the size and mass of the CA by conjugating DTPA-Gd(III) to the $15 \mathrm{mer}$ thrombin-binding aptamer gives an enhancement of relaxivity, even without aptamer-target binding. 


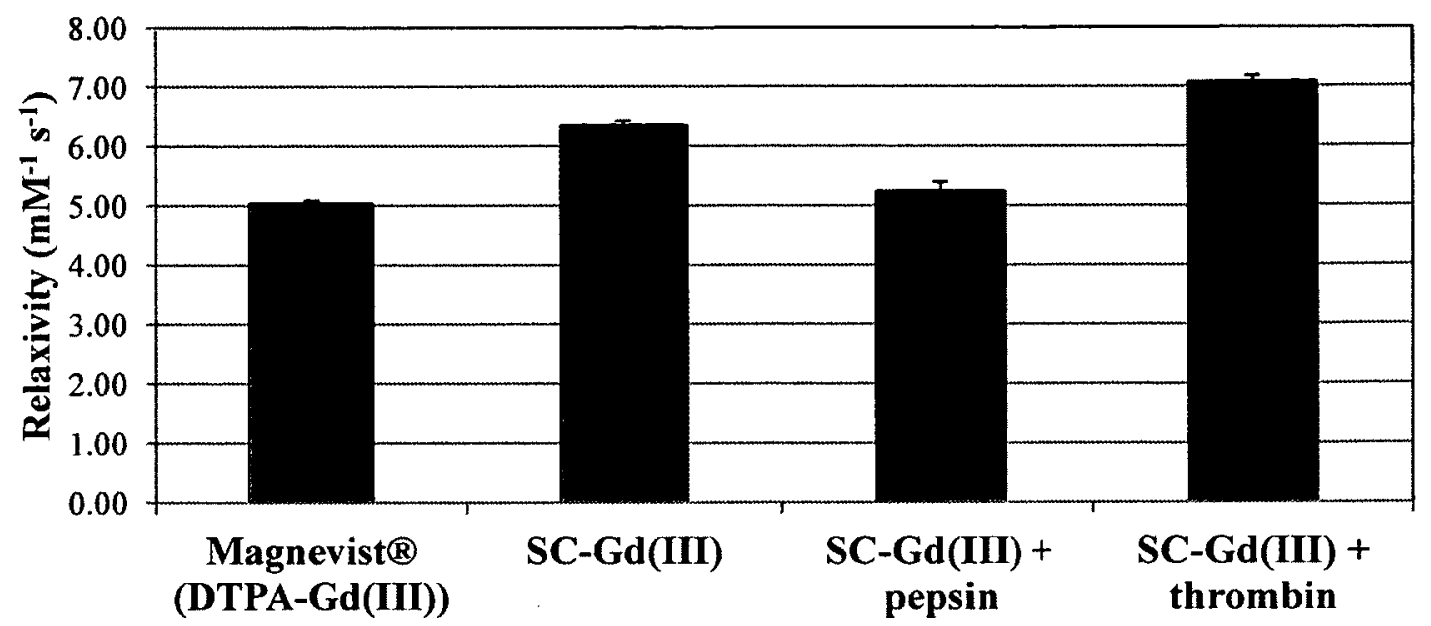

Figure 2.16 Chart of the relaxivity values for Magnevist ${ }^{8}$ and SC-Gd(III) at 9.4 T, pH 7.4 and $25^{\circ} \mathrm{C}$. Reproduced from reference 143 with permission from Springer.

Table 2.3 Relaxivity Data for Magnevist ${ }^{\circ}$ and SC-Gd(III) at $9.4 \mathrm{~T}, \mathrm{pH} 7.4$ and $25^{\circ} \mathrm{C}$ in silanized tubes.

\begin{tabular}{|c|c|c|c|}
\hline Sample & Relaxivity $\left(\mathrm{mM}^{-1} \mathrm{~s}^{-1}\right)$ & Protein & $\begin{array}{c}\text { Enhancement of } \\
\text { relaxivity (\%) }\end{array}$ \\
\hline Magnevist@ & $5.03 \pm 0.06$ & - & - \\
SC-Gd(III) & $6.36 \pm 0.07$ & - & $26 \pm 2^{[\mathrm{a}]}$ \\
SC-Gd(III) & $5.2 \pm 0.2$ & pepsin (control) & - \\
SC-Gd(III) & $7.09 \pm 0.10$ & thrombin (target) & $35 \pm 4^{[\mathrm{b}]}$ \\
\hline
\end{tabular}

[a] Comparing the relaxivity of SC-Gd(III) to that of Magnevist $(B)$

[b] Comparing the relaxivity of SC-Gd(III)+thrombin to that of SC-Gd(III)+pepsin

As mentioned in previous sections, an amide is present in the DTPA backbone of our conjugates that is not present in the parent molecule (Figure 2.7). In addition to its possible effects on the stability of the chelate, it is necessary to consider how this amide could influence the relaxivities of our conjugates. It has been reported that the replacement of a carboxylate group with an amide in the inner-sphere of a Gd(III) 
chelator causes the water-exchange rate to decrease by about a factor of four. ${ }^{126}$ This is attributed to two factors. First, the water-exchange process within nine-coordinate linear Gd(III) complexes is dissociative. The one inner-sphere water molecule, that is, the water molecule directly bound to the $\mathrm{Gd}(\mathrm{III})$ ion in the ninth coordination position, must dissociate from the ion for it to be replaced by another water molecule. The gadoliniumamide oxygen distance is longer than that for a carboxylate oxygen, leading to less crowding in the inner sphere of the complex and therefore less steric pressure for the water to leave. Secondly, the inner-sphere water molecule will be held more tightly by the Gd(III) due to a stronger electrostatic attraction in a monoamide DTPA frame where the positive charge is less shielded. ${ }^{126}$ This slower water-exchange, and therefore the increased lifetime of inner-sphere water molecules, $\tau_{m}$, can limit the relaxivity of a CA as fewer relaxed water molecules are exchanged with the bulk. ${ }^{124}$ However, the replacement of a carboxylate with an amide in the backbone also increases the overall size of the chelate and, therefore, its rotational correlation time $\tau_{R} \cdot{ }^{126}$ The inclusion of two amide groups in DTPA-BMA-Gd(III) does not greatly affect its relaxivity in comparison to that of DTPA-Gd(III); at various field strengths their relaxivities are reportedly very similar. ${ }^{158}$ With a measurable relaxivity enhancement observed after the overall size increase from DTPA-Gd(III) to SC-Gd(III), it would seem that the expected drop in water-exchange rate in our monoamide derivative is not a limiting factor. We therefore hypothesized that aptamer-target binding would produce further relaxivity gains, as per the RIME effect.

The study of an antisense paramagnetic oligonucleotide reported in 1999 by Hines et al., discussed in section 2.1 .2 .3 , found a relaxivity enhancement of $16 \%$ at $6.3 \mathrm{~T}$ when 
their complementary 6mer-DTPA-Gd(III) bound to its macromolecular target (5S rRNA, $\cong 40 \mathrm{kDa}$ ), as expected considering the RIME effect. They compared this to the $3 \%$ enhancement caused by the change in viscosity upon the addition of a control molecule with about the same mass as the 5S rRNA to their paramagnetic oligonucleotide at 6.3 $\mathrm{T}^{122}$ One might anticipate an increase in relaxivity with an increase in viscosity as molecular tumbling can be expected to be slower in a more viscous solution. ${ }^{101}$ However, it has been found that the dependence of relaxation time on viscosity is not linear, ${ }^{130}$ as discussed in section 2.1 .2 .2 . In the case of SC-Gd(III), a drop in relaxivity upon addition of our control (pepsin) to SC-Gd(III) was observed rather than an enhancement (see Figure 2.16 and Table 2.3). This is unrelated to any change to SCGd(III) itself as pepsin does not have specific affinity for the thrombin aptamer. ${ }^{12}$ The solution, however, does become more viscous and more crowded with the pepsin present, and this appears to negatively impact the tumbling rate of SC-Gd(II).

The addition of thrombin to SC-Gd(III) will also cause crowding and an increase in viscosity of the solution, thus making a comparison of this relaxivity value to that obtained with pepsin present more relevant than a comparison with SC-Gd(III) or DTPA-Gd(III) alone. The difference between the relaxivities of SC-Gd(III) with pepsin and with thrombin can then be isolated to thrombin's interaction with the aptamer portion of SC-Gd(III) and the resulting overall increase in the conjugate's size. An increase in relaxivity of $35 \pm 4 \%$ (Table 2.3 ) over the sample containing pepsin supports a decrease in the rate of molecular tumbling being the dominant factor. The enhancement demonstrated in the SC-Gd(III) system illustrates that aptamer-gadolinium(III) conjugates shows comparable, and perhaps better, promise as targeted MRI contrast 
agents compared to the antisense paramagnetic oligonucleotide discussed above. ${ }^{122}$ With a similar sized target $(36.7 \mathrm{kDa}$ versus $\cong 40 \mathrm{kDa})$, our system achieved a greater enhancement in relaxivity upon target binding ( $35 \pm 4 \%$ versus a net change of $13 \%)$.

To determine how changes to $\tau_{m}$ and $\tau_{R}$ theoretically affect the relaxivities obtained for SC-Gd(III) at $9.4 \mathrm{~T}$, modified equations from theory developed by Bloembergen and Solomon ${ }^{100}$ were used as described by Yang et al. ${ }^{125}$ These are equations 2.6 to 2.11 in section 2.1.2.2; as mentioned in that section, working backwards through these equations allows for the theoretical calculation of a CA's relaxivity at a particular concentration. The value of $\frac{1}{T_{1 m}}$, or the longitudinal inner sphere relaxation rate, was calculated for when $0.1 \mathrm{mM}$ of the contrast agent was present and was compared to the experimental values obtained for SC-Gd(III) alone and with thrombin at this concentration $\left(\sim 0.72\right.$ and $0.77 \mathrm{~s}^{-1}$, respectively). The $\tau_{R}$ value reported by Tóth $e t$ al. $(143 \mathrm{ps})^{126}$ for a DTPA-monoamide (DTPA-MA) matching the modification in our conjugates was used as a starting point in the calculations. The terms related to electronic relaxation (mean-square zero-field splitting (ZFS) energy, $\Delta^{2}$, and the correlation time for the modulation of the ZFS interaction, $\tau_{v}$ ) were held constant at the reported values for DTPA-MA $\left(2.6 \times 10^{20} \mathrm{~s}\right.$ and $3 \mathrm{ps,}$, respectively) ${ }^{126}$ since electronic relaxation has a less pronounced effect on the observed relaxivity at high fields than at low fields. ${ }^{100,101}$ The $\tau_{m}$ for DTPA-MA is reportedly on the order of $10^{-7} \mathrm{~s}$, so it was desirable to get values of $\frac{1}{T_{1 m}}$ at this residence time that were close to the experimental results and the value of $\tau_{R}$ was adjusted to this end. If $\tau_{R}$ and the size of the CA are assumed to be linearly related, multiplying the value for DTPA-MA by 11 times would approximate the change in mass 
from DTPA-Gd(III) to SC-Gd(III). A $\tau_{m}$ on the order of $10^{-9} \mathrm{~s}$ would be required to produce a match in experimental and theoretical values in this case, which seems unlikely for a monoamide derivative of DTPA. The $\tau_{R}$ was therefore adjusted until a maximum $\frac{1}{T_{1 m}}$ of $0.73 \mathrm{~s}^{-1}$ was found for $\tau_{m}$ of $10^{-7} \mathrm{~s}$. This resulted when the $\tau_{R}$ of DTPA-MA was increased 3-fold, which indicates that the freely rotating $\mathrm{C}_{6}$ linker between the chelate and the DNA does not rotate entirely independent of the aptamer and aptamer-target (i.e. is not fully decoupled). The implications of this will be discussed in further detail below.

The thrombin aptamer folds into a G-quadruplex conformation that interacts with the fibrinogen-binding exosite of thrombin. In SC-Gd(III) this would hold the comparatively bulky thrombin right next to the DTPA-Gd(III) moiety and could interfere with the exchange of water molecules at the Gd(III) centre (Figure 2.15). As discussed above, the presence of an amide in the DTPA backbone of our conjugates is already detrimental to the water-exchange process, and a spacer between the aptamer and chelate portions of our conjugate was included in an effort to avoid further limiting $\tau_{m}$. Two different spacer lengths were incorporated: 15 polyT and 30 polyT to yield 15 T SCGd(III) and 30T SC-Gd(III), respectively (see Figure 2.7). It was possible to explore the effect of spacer flexibility on relaxivity through the hybridization of matching polyA strands to these conjugates.

The 15T SC-Gd(III) relaxivity data summarized in Table 2.4 and Figure 2.17 (and illustrated in Figures A-12 and A-13) shows a drop in the relaxivity of the conjugate alone compared to the values found for SC-Gd(II) and DTPA-Gd(III) (Table 2.3). 


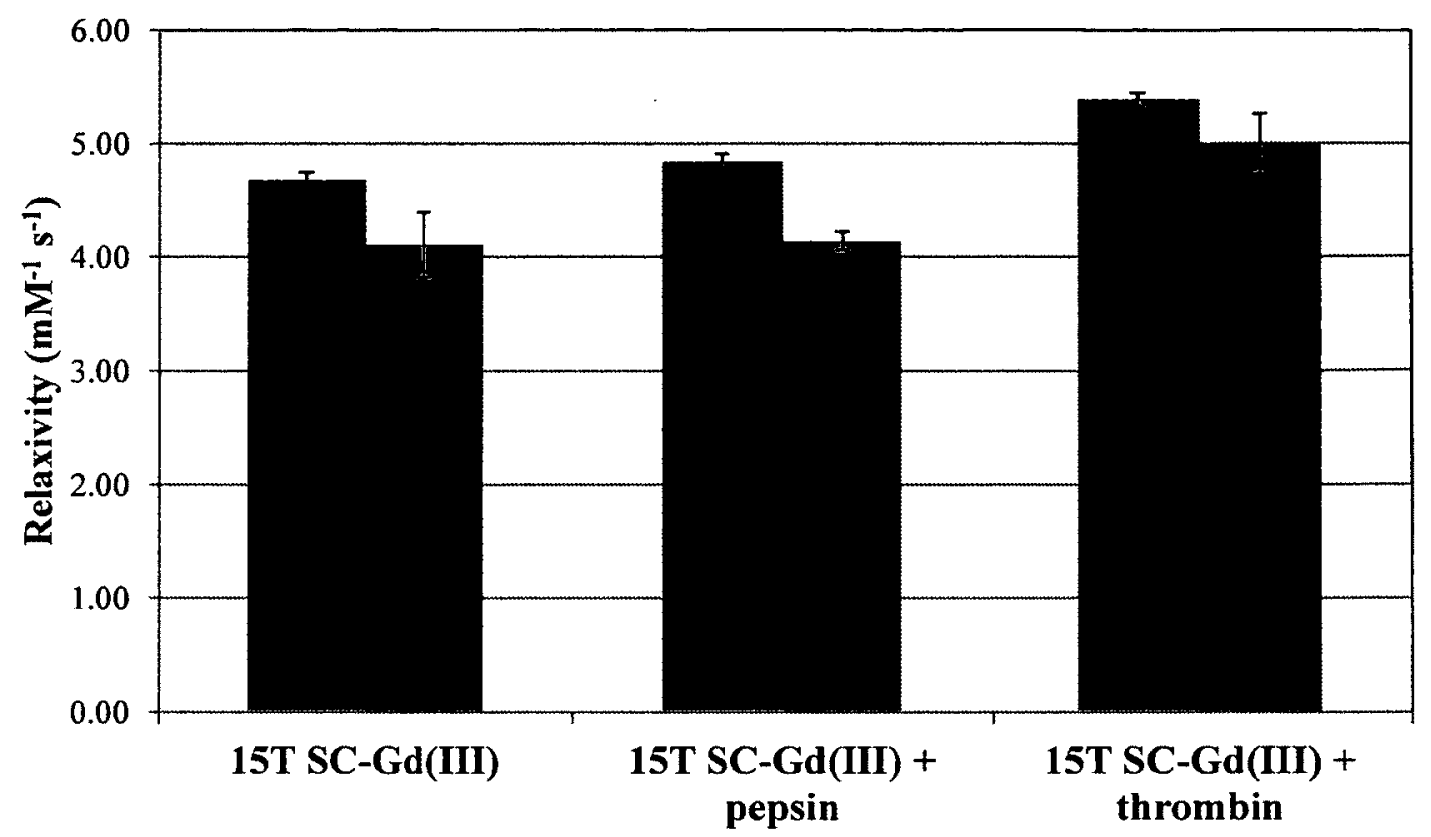

- Single-stranded - Hybridized with polyA

Figure 2.17 Chart of the relaxivity values for single-stranded and hybridized 15T SC-Gd(III) at 9.4

T, pH 7.4 and $25^{\circ} \mathrm{C}$. Reproduced from reference 143 with permission from Springer.

Table 2.4 Relaxivity Data for 15T SC-Gd(III) at 9.4 T, pH 7.4 and $25^{\circ} \mathrm{C}$ in silanized tubes.

\begin{tabular}{|c|c|c|c|}
\hline Sample & Relaxivity $\left(\mathrm{mM}^{-1} \mathrm{~s}^{-1}\right)$ & Protein & $\begin{array}{l}\text { Enhancement of } \\
\text { relaxivity }(\%)\end{array}$ \\
\hline \multirow{3}{*}{ 15T SC-Gd(III) } & $4.68 \pm 0.07$ & - & $-7 \pm 2^{[\mathbf{a}]}$ \\
\hline & $4.84 \pm 0.06$ & pepsin (control) & - \\
\hline & $5.39 \pm 0.06$ & thrombin (target) & $11 \pm 2^{[b]}$ \\
\hline \multirow{3}{*}{$\begin{array}{l}\text { 15T SC-Gd(III) } \\
+ \text { polyA }\end{array}$} & $4.1 \pm 0.3$ & - & $-18 \pm 6^{[\mathrm{c}]}$ \\
\hline & $4.14 \pm 0.08$ & pepsin (control) & \\
\hline & & & $21 \pm 7^{[\mathrm{d}]}$ \\
\hline \multicolumn{4}{|c|}{ [a] Comparing the relaxivity of $\mathbf{1 5 T}$ SC-Gd(III) to that of DTPA-Gd(III) } \\
\hline \multirow{2}{*}{\multicolumn{4}{|c|}{$\begin{array}{l}\text { [b] Comparing the relaxivity of } 15 \mathrm{~T} \mathrm{SC-Gd(II)+thrombin} \mathrm{to} \mathrm{that} \mathrm{of} 15 \mathrm{~T} \mathrm{SC-Gd(III)+pepsin} \\
\text { [c] Comparing the relaxivity of } 15 \mathrm{~T} \mathrm{SC-Gd(III)+polyA} \mathrm{to} \mathrm{that} \mathrm{of} \mathrm{DTPA-Gd(III)}\end{array}$}} \\
\hline & & & \\
\hline \multicolumn{4}{|c|}{$\begin{array}{l}\text { [d] Comparing the relaxivity of } \mathbf{1 5 T} \text { SC-Gd(IID)+polyA+thrombin to that of } 15 \text { T SC- } \\
\text { Gd(IIN+polyA +pepsin }\end{array}$} \\
\hline
\end{tabular}


Doubling the length of the DNA portion of the aptamer-DTPA-Gd(III) conjugate by adding the 15 polyT spacer changes the viscosity and crowding of the solution, and it seems that the change in molecular tumbling due to the larger size of the conjugate is not great enough to balance these effects. Furthermore, the length of the spacer could be leading to more crowding at the Gd(III) centre, contrary to its desired effect, as the flexible polyT portion could be permitting the folded thrombin aptamer to more directly block water molecule exchange at the DTPA-Gd(III) moiety. As the $\tau_{R}$ of a CA increases, the water-exchange rate becomes a more limiting factor in achieving high relaxivity. ${ }^{159} \mathbf{1 5 T}$ SC-Gd(III) would be expected to have a higher $\tau_{R}$ than SC-Gd(III) due to its higher mass, so the drop in relaxivity of $7 \pm 2 \%$ for $15 \mathrm{~T} \mathbf{S C}-\mathbf{G d}(\mathbf{I I I})$ compared to DTPA-Gd(III) suggests that slow water-exchange imposed by the monoamide moiety or resulting from steric constraints could be a dominant factor affecting relaxivity in this system.

The decrease in relaxivity between DTPA-Gd(III) and 15T SC-Gd(III) could also be an effect of internal motion. In bifunctional CAs such as our conjugates, a flexible linker joining the chelate and the targeting moiety allows the chelate to rotate freely. As a result, the rotational motion that the inner-sphere water molecule experiences is a combination of this fast internal motion and the slower global motion of the entire CA. At high fields, it has been suggested that the internal motion of the metal chelate in a targeted $\mathrm{CA}$ should be decoupled from the slower rotational tumbling to maximize relaxivity and that this can be achieved through the use of a flexible linker. ${ }^{124}$ This is because intermediate values of $\tau_{R}$ provide higher relaxivity in contrast agents at high field, whereas at low field the RIME effect proves so effective because of the higher $\tau_{R}$ 
values achieved upon target binding. ${ }^{124,160}$ The amino modifier used for conjugation of the DNA to DTPA dianhydride in this work provides a flexible $\mathrm{C}_{6}$ linker between the metal chelate and the DNA (Figure 2.7). The $26 \pm 2 \%$ increase in relaxivity observed between SC-Gd(III) and Magnevist ${ }^{\circledR}$ implies that the overall increase in size due to the presence of the aptamer lead to a beneficial change in $\tau_{R}$, as discussed above. Had the internal motion been fully decoupled from the global rotation, the relaxivity would not be expected to differ from that of DTPA-Gd(III) as it would reflect the relaxivity of the chelate alone. The calculations performed and described above indicate that the $\tau_{R}$ of SC-Gd(III) could be about 3-fold higher than that of DTPA-MA, supporting a slight increase in $\tau_{R}$ with addition of the aptamer. The increase was not, however, as large as could be reasonably expected with the 11 times higher mass of the conjugate compared to DTPA-Gd(III). In the case of 15 T SC-Gd(III), the small drop in relaxivity of $7 \pm 2 \%$ compared to DTPA-Gd(III) could indicate that the net $\tau_{R}$ experienced by the chelate in this conjugate was closer to that of free DTPA-Gd(III); that is, the rotation around the linker was more effectively decoupled from the global rotation in this case.

The inclusion of pepsin does not change the relaxivity value much in the case of 15T SC-Gd(III) (Figure 2.17). Thrombin, however, causes an increase in relaxivity as observed in the case of SC-Gd(III). The change in the global molecular tumbling of 15T SC-Gd(II) upon target binding could be positively influencing the net tumbling of the chelate and reducing the effects of internal motion. Thrombin binding to the aptamer portion of the DNA could also be limiting any interference it was having in the waterexchange process. For example, the comparatively bulky thrombin might not permit the DNA to move as near to the chelate as it could while target-free (Figure 2.15B). The 
relaxivity enhancement, however, is not as large as that seen for SC-Gd(III) when comparing the values obtained in the presence of thrombin and pepsin, here only $11 \pm 2 \%$ compared to $35 \pm 4 \%$ for SC-Gd(III). Overall, the inclusion of a single-stranded 15 polyT spacer did not improve the ability of the thrombin aptamer-DTPA-Gd(III) conjugate to act as a targeted MRI contrast agent.

If the flexibility of the polyT spacer was leading to the observed decrease in relaxivity of $\mathbf{1 5 T}$ SC-Gd(III) compared to SC-Gd(III), hybridization of the 15 polyT portion with 15 polyA DNA could diminish these effects and help improve the enhancement (Figure 2.15C). This was in fact observed with a slight improvement in relaxivity enhancement found in the case of hybridized $15 \mathrm{~T}$ SC-Gd(III) compared to unhybridized conjugate $(21 \pm 7$ and $11 \pm 2 \%$ respectively, see Table 2.4$)$, despite the overall drop in relaxivities found for $\mathbf{1 5 T}$ SC-Gd(III)+ polyA compared to those for $\mathbf{1 5 T}$ SCGd(III) on its own (Figure 2.17). The changes in viscosity and crowding in the solution upon addition of the polyA strands in a 1:1 ratio with $\mathbf{1 5 T}$.SC-Gd(III) could be the reason for the decreased relaxivities measured, with the decrease in flexibility of the polyT spacer upon hybridization resulting in a change in $\tau_{R}$ that partially compensates for these effects and results in the slightly better relaxivity enhancement. If slow waterexchange caused the decrease in relaxivity, it is unlikely to be due to steric hindrance from the folded thrombin aptamer as it would be held farther from the chelate on average in the hybridized case (Figure 2.15C) than when single-stranded (Figure 2.15B). It is, however, possible that the hybridizing polyA strand could be misaligned with the polyT spacer, and any overhang at the $5^{\prime}$ end of the conjugate could be blocking the Gd(III) centre and slowing water exchange. Whether that is the case or it is the effect of the 
monoamide DTPA backbone, slow water-exchange and greater influence of internal motion are likely the underlying factors resulting in decreased relaxivity in the case of 15T SC- Gd(III)+polyA.

A DNA-DTPA conjugate including a polyT spacer containing thirty thymine nucleotides was also prepared to test whether moving the folded thrombin aptamer even farther away from the chelate, while also increasing the overall size of the conjugate, would lead to greater relaxivity gains. The relaxivity data for this conjugate is summarized in Figure 2.18 and Table 2.5, and illustrated in Figure A-14 (single-stranded) and Figure A-15 (hybridized). The relaxivity of 30T SC-Gd(III) on its own in solution was found to be higher than that for SC-Gd(III) $\left(8.5 \pm 0.2 \mathrm{mM}^{-1} \mathrm{~s}^{-1}\right.$ compared to $6.34 \pm 0.07 \mathrm{mM}^{-1} \mathrm{~s}^{-1}$ ), suggesting that the increase in size is large enough to compensate for any increase in the solution's viscosity and crowding. This also implies that the net motion of the chelate is not completely decoupled from that of the conjugate's global tumbling rate, as in the case of SC-Gd(III) and unlike that of 15T SC-Gd(III). The 30 polyT spacer could simply be keeping the folded aptamer farther from the chelate on average than the 15 polyT spacer does, both avoiding steric hindrance at the Gd(III) centre and increasing the global $\tau_{R}$ of the conjugate. Overall, the result is that $30 \mathrm{~T}$ SCGd(III) is a more effective relaxation agent than both SC-Gd(III) and 15T SC-Gd(III) when compared to the parent DTPA-Gd(III), as 30T SC-Gd(III) demonstrates a relaxivity enhancement over the chelate alone of $70 \pm 5 \%$ (Table 2.5 ). The presence of the spacer also seems to be beneficial in this case whereas the 15 poly $\mathrm{T}$ spacer proved detrimental overall. 
Table 2.5 Relaxivity Data for 30T SC-Gd(III) at 9.4 T, pH 7.4 and $25^{\circ} \mathrm{C}$ in silanized tubes.

\begin{tabular}{|c|c|c|c|}
\hline Sample & Relaxivity $\left(\mathrm{mM}^{-1} \mathrm{~s}^{-1}\right)$ & Protein & $\begin{array}{c}\text { Enhancement of } \\
\text { relaxivity (\%) }\end{array}$ \\
\hline \multirow{3}{*}{ 30T SC-Gd(II) } & $8.5 \pm 0.2$ & - & $70 \pm 5^{[\mathrm{a}]}$ \\
& $4.14 \pm 0.08$ & pepsin (control) & - \\
& $5.0 \pm 0.3$ & thrombin (target) & $28 \pm 2^{\text {[b] }}$ \\
\hline 30T SC-Gd(III) & $8.86 \pm 0.07$ & - & $76 \pm 2^{[\mathrm{c}]}$ \\
+ polyA & $6.5 \pm 0.4$ & pepsin (control) & - \\
& $7.9 \pm 0.1$ & thrombin (target) & $23 \pm 6^{[\mathrm{d}]}$ \\
\hline
\end{tabular}

[a] Comparing the relaxivity of 30T SC-Gd(III) to that of DTPA-Gd(III)

[b] Comparing the relaxivity of 30T SC-Gd(III)+thrombin to that of 30T SC-Gd(III)+pepsin

[c] Comparing the relaxivity of 30T SC-Gd(III)+polyA to that of DTPA-Gd(III)

[d] Comparing the relaxivity of 30T SC-Gd(III)+polyA+thrombin to that of 30T SCGd(III)+polyA+pepsin

When the protein control and target were added and the respective relaxivity values for the two cases obtained, 30T SC-Gd(III) did not improve on the results obtained for SC-Gd(III). Overall, higher relaxivities were found for 30T SC-Gd(III), but the enhancement of relaxivity due to thrombin binding determined for 30T SCGd(III) (either single stranded or hybridized with 30 polyA) was similar or worse than that of SC-Gd(III) (compare Table 2.3 and Table 2.5). There is a lower change in mass between the target-bound and free 30T SC-Gd(III) (about 3.5 times) compared to SCGd(III) (about 8 times), and this might be limiting the relaxivity enhancements achieved for the larger conjugate. These results illustrate the complexity of the factors affecting relaxivity in these systems. 


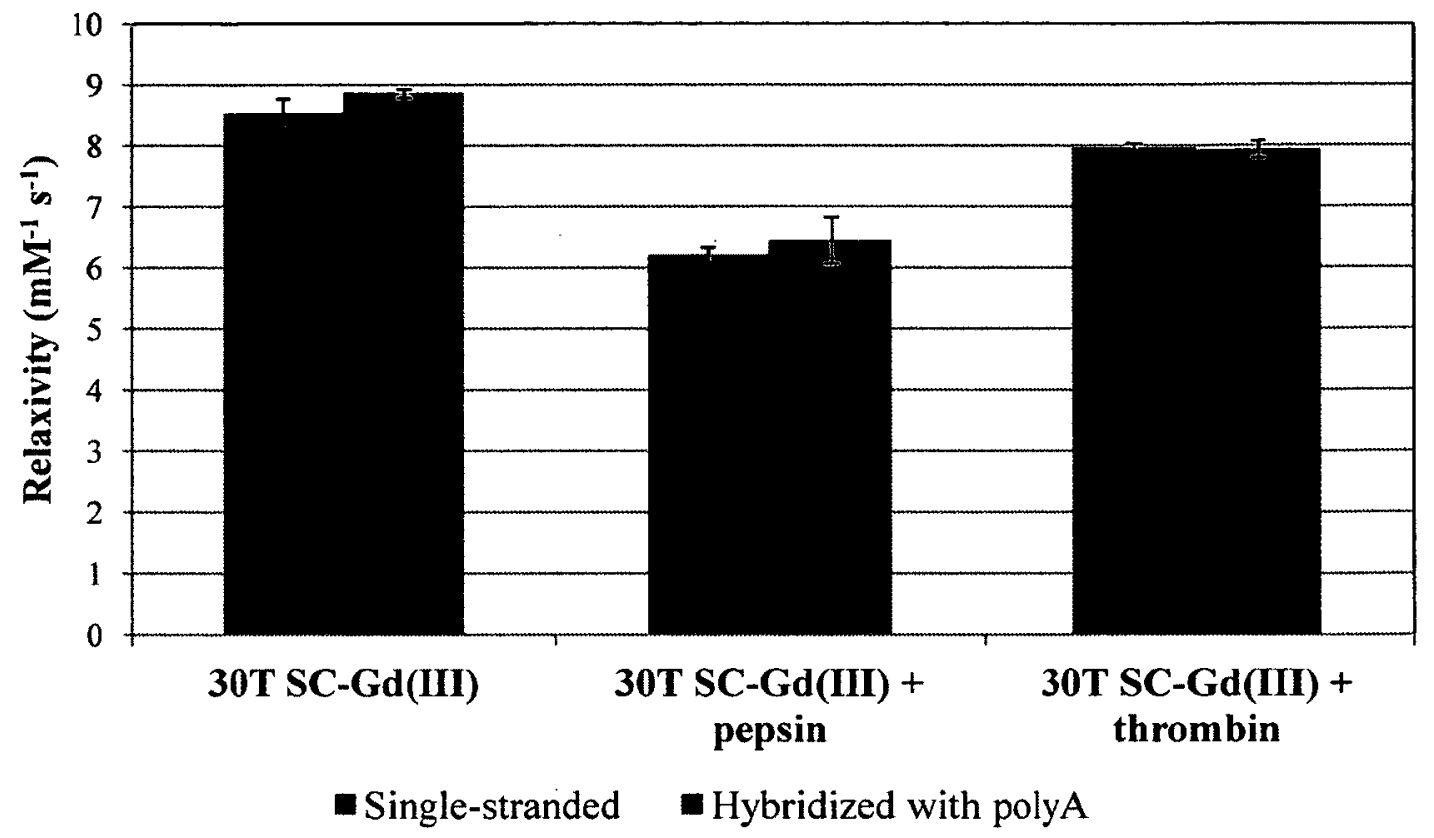

Figure 2.18 Chart of the relaxivity values for single-stranded and hybridized 30T SC-Gd(III) at 9.4 T, pH 7.4 and $25^{\circ} \mathrm{C}$. Reproduced from reference 143 with permission from Springer.

The crowding introduced by the addition of a protein and the increase in viscosity seem to be greatest for 30T SC-Gd(III), not unexpected given that this is the conjugate containing the longest DNA portion of the three tested. A solution containing 30T SCGd(III) compared to one containing the same concentration of $15 T$ SC-Gd(III) or SCGd(III) would be the most crowded to begin with, and the addition of protein, and later the hybridizing 30 polyA strands, would cause further impedance to water-exchange at the Gd(III) centre. This effect can be seen when considering that the relaxivity values of 30T SC-Gd(III) in the presence of pepsin and of thrombin are both lower than that for the conjugate on its own (Figure 2.18). In comparison, SC-Gd(III) shows a drop from the relaxivity of the conjugate alone once pepsin is added and the viscosity changes, but 
then an increase in relaxivity over the original value when thrombin is included (Figure 2.16). Even for $\mathbf{1 5 T}$ SC-Gd(III) the observed relaxivity in the presence of thrombin is higher than that of the conjugate alone, in both the hybridized and single-stranded cases (Figure 2.17). The partial recovery of relaxivity exhibited by $30 \mathrm{~T}$ SC-Gd(III) with the specific interaction of thrombin and its aptamer when compared to the control suggests that the overall size increase helps the chelate achieve a more favourable net $\tau_{R}$, even under high viscosity conditions. However, the relaxivity of 30T SC-Gd(III) with thrombin is only slightly higher than that of SC-Gd(III) with thrombin $\left(7.96 \pm 0.07 \mathrm{mM}^{-1}\right.$ $\mathrm{s}^{-1}$ compared to $7.09 \pm 0.10 \mathrm{mM}^{-1} \mathrm{~s}^{-1}$ ), perhaps in part due to the smaller size difference between free and bound conjugate, as mentioned above. The hybridization of 30T SCGd(III) with 30 mer polyA strands does not significantly change the relaxivity values obtained, although it results in a slight reduction in the observed enhancement of relaxivity from the control to the target sample when compared to the single-stranded values (Table 2.5). This suggests that any improvement due to increasing the spacing and size of the conjugate upon hybridization may be negated by increased viscosity and crowding, along with an even smaller change in mass upon the binding of thrombin.

Considering all the aforementioned results, it was concluded that the original conjugate, SC-Gd(III), would be best suited for continuing experiments under clinical, low field conditions. It demonstrated the best relaxivity enhancement upon target binding while experiencing minimal viscosity-related effects, and was the simplest system with the largest mass difference between free and target-bound cases. 


\subsection{7 $T_{1}$ experiments and relaxivity data at low field (1.5 Tesla)}

The capability of measuring $T_{1}$ values for multiple samples simultaneously on a 1.5 or $3 \mathrm{~T}$ clinical MR scanner reduced the time required to obtain data. Plastic cuvettes were taped together in a line or in a grid as necessary to hold the samples. All samples were made up to $1 \mathrm{~mL}$ total volume; in the $1 \mathrm{~cm}$ wide cuvettes, this meant the samples were all $1 \mathrm{~cm}$ in height. This was necessary because when placed in a 1.5 or $3 \mathrm{~T}$ MRI spectrometer data is obtained in slices perpendicular to the horizontal applied magnetic field $\left(\mathrm{B}_{0}\right) .{ }^{96}$ Trying to obtain data from samples much less than $1 \mathrm{~cm}$ in height leads to low signal-to-noise ratios, and therefore more error associated with the data.

The lowest concentrations of Magnevist $\circledast$ and SC-Gd(III) at which a measurable change in water proton $\mathrm{T}_{1}$ relaxation could be detected on 1.5 and $3 \mathrm{~T}$ MRI spectrometers were unknown by our collaborators at The Ottawa Hospital/Ottawa Hospital Research Institute. Several concentrations of SC-Gd(III) were therefore prepared to test the range of concentrations that could be detected on an MRI spectrometer. The $3 \mathrm{~T}$ MRI spectrometer was available for use for these samples. The concentrations tested in this trial were $0.05,0.025,0.0125,0.005,0.0025$, and $0.0005 \mathrm{mM}$. Three scans were performed on the samples over two consecutive days. The relaxivity calculated for SCGd(III) differed in the three scans (data not shown). This indicated that the changes in relaxivity were likely related to error associated with removing the samples from the MRI between scans; the placement of the samples within the spectrometer could not be precisely the same in each scan and the instrument had to be set up anew each time the sample was removed and placed back into the MRI. This demonstrated the necessity of performing scans multiple times, which would also provide information on the error 
between scans under the conditions used. Furthermore, the lowest concentration tested $(0.0005 \mathrm{mM})$ was found to have a similar $T_{1}$ to that of TBA buffer and thus be near the detection limit of the instrument. This concentration was therefore omitted in future experiments.

Five sample sets were considered in the final experiments performed on SCGd(III) at 1.5 T: 1) Magnevist( ${ }^{\circ}$, 2) SC-Gd(III), 3) SC-Gd(III) and thrombin in a 1:1 ratio, 4) SC-Gd(III) and pepsin in a 1:1 ratio (control 1) and 5) SC-Gd(III) and BSA in a 1:1 ratio (control 2). All sample solutions were prepared using TBA buffer and were made up to a total volume of $1 \mathrm{~mL}$ as in the trial experiments. They were run in plastic cuvettes that were taped together in a $5 \times 5$ grid, with one extra cuvette containing TBA buffer alone added to the Magnevist ${ }^{\circledR}$ set.

BSA was found to be a reasonable secondary control under the conditions used at low field despite its larger size and therefore different effect on viscosity compared to thrombin and pepsin. The results of the low field experiments are summarized in Figure 2.19, Figure A-16, and Table 2.6.

The relaxivity of SC-Gd(III) was found to be $103 \pm 2 \%$ greater than that of Magnevist ${ }^{\circledR}$ under the conditions used at $1.5 \mathrm{~T}$. The relaxivity values upon addition of thrombin and the non-target proteins are all higher than that of SC-Gd(III) alone at low field. This supports the hypothesis that the change in viscosity affected by protein addition is different under the less constrained conditions used here compared to at $9.4 \mathrm{~T}$. The relaxivity of SC-Gd(III) in a 1:1 ratio with $\mathrm{BSA}$ is higher than that with pepsin present, suggesting that the larger BSA changes the viscosity in a way that leads to a more optimal net $\tau_{R}$ experienced by the conjugate. 


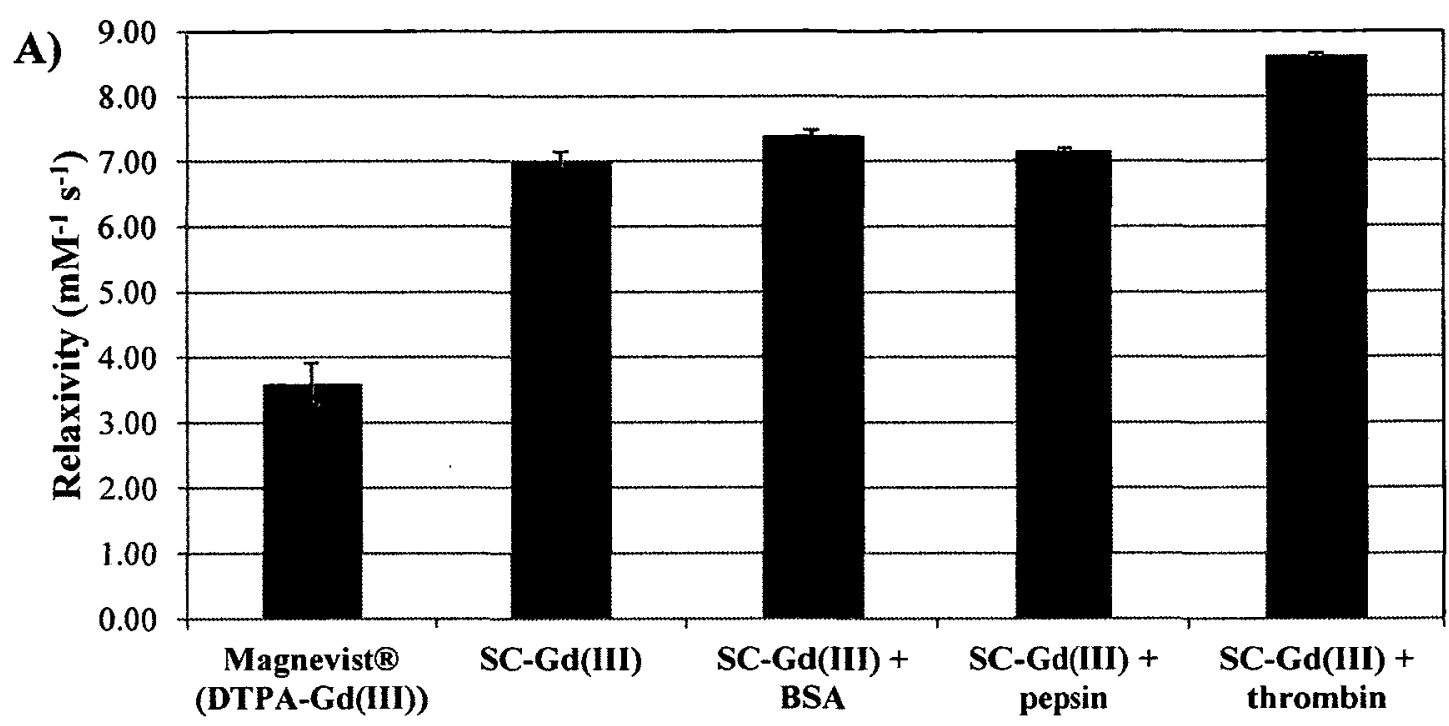

B) i)
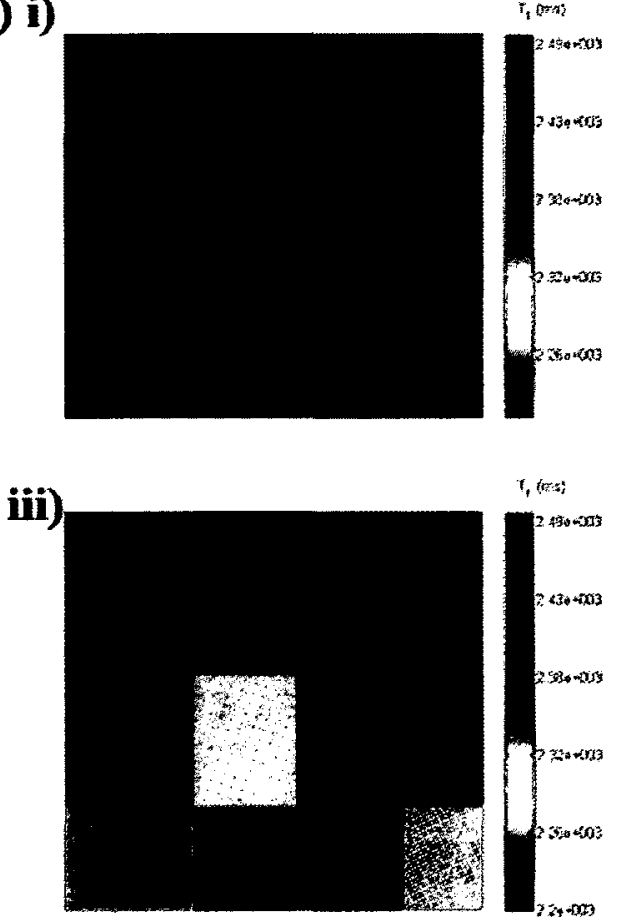

ii)

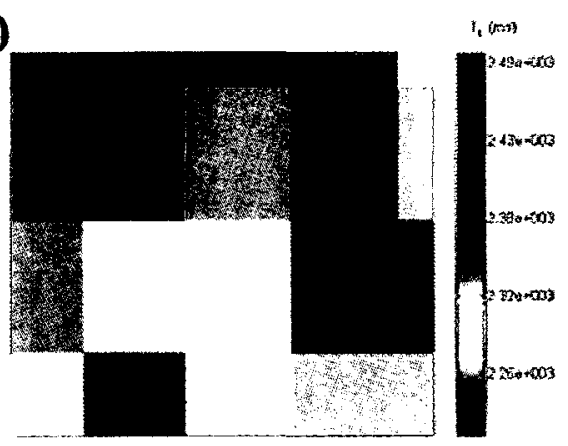

iv)

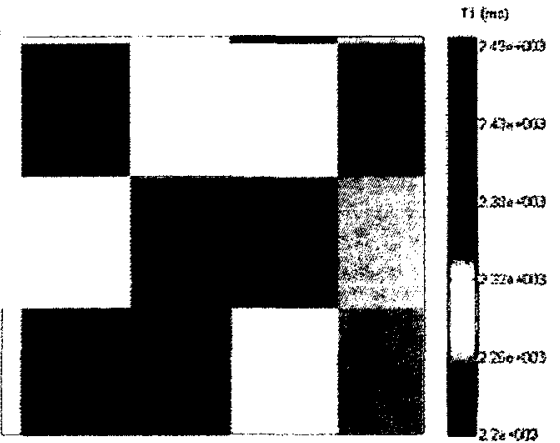

Figure 2.19 A) Chart of relaxivity data for Magnevist ${ }^{\circledR}$ and SC-Gd(III) at $1.5 \mathrm{~T}, \mathrm{pH} 7.4$ and $25^{\circ} \mathrm{C}$.

B) Representative $T_{1}$ maps of $0.0125 \mathrm{mM}$ solutions of i) SC-Gd(III), ii) SC-Gd(III)+BSA, iii) SCGd(III)+pepsin, and iv) SC-Gd(III)+thrombin. An equimolar amount of protein is present, where applicable. Images obtained under the same conditions as in $A$. Reproduced from reference 143 with permission from Springer. 
Table 2.6 Relaxivity Data for Magnevist ${ }^{\circ}$ and SC-Gd(III) at $1.5 \mathrm{~T}$, pH 7.4 and $25^{\circ} \mathrm{C}$.

\begin{tabular}{|l|c|c|c|}
\hline Sample & Relaxivity $\left(\mathrm{mM}^{-1} \mathrm{~s}^{-1}\right)$ & Protein & $\begin{array}{c}\text { Enhancement of } \\
\text { relaxivity (\%) }\end{array}$ \\
\hline Magnevist $®$ & $3.60 \pm 0.32$ & - & - \\
SC-Gd(III) & $7.009 \pm 0.141$ & - & $95 \pm 13^{\text {[a] }}$ \\
SC-Gd(III) & $7.398 \pm 0.0863$ & BSA (control) & - \\
SC-Gd(III) & $7.167 \pm 0.0337$ & pepsin (control) & - \\
SC-Gd(III) & $8.624 \pm 0.0374$ & thrombin (target) & $17 \pm 1^{[\mathrm{b}]}, 20 \pm 1^{[\mathrm{c}]}$ \\
\hline [a] Comparing the relaxivity of SC-Gd(III) to that of DTPA-Gd(III) \\
[b] Comparing the relaxivity of SC-Gd(III)+thrombin to that of SC-Gd(III)+BSA \\
[c] Comparing the relaxivity of SC-Gd(III)+thrombin to that of SC-Gd(III)+pepsin \\
\hline
\end{tabular}

Despite the slightly enhanced relaxivity afforded to SC-Gd(III) through the viscosity changes upon non-target protein introduction, the addition of thrombin to the conjugate provided relaxivity enhancements of $20 \pm 1 \%$ and $17 \pm 1 \%$ in comparison to pepsin and BSA, respectively (Table 2.6). As illustrated in Figure 2.19B, these enhancements translate into visible differences in the MR images obtained for SCGd(III) alone and in the presence of each protein, with the brightest image that of SCGd(III) in the presence of thrombin. The observable differences in the MR images indicate that the relaxivity enhancements found for SC-Gd(III) between control and target cases are in an appropriate range for this system to be further considered as a targeted MRI contrast agent. However, changes that would increase the enhancement further would undoubtedly prove beneficial for practical applications.

The observed relaxivity enhancements can likely be attributed to the eight times increase in mass and the corresponding change in $\tau_{R}$ experienced by the chelate moiety upon aptamer-target binding. The modified Solomon-Bloembergen equations were again used as described above with the most important changes being the use of $0.0125 \mathrm{mM}$ as 
the concentration of $\mathrm{CA}$ to match the highest concentration tested at $1.5 \mathrm{~T}$ and the testing of $\tau_{v}$ values of both $3 \mathrm{ps}^{126}$ and 40 ps. Reported $\tau_{v}$ values for polymeric DTPA-MA derivatives fall in the range $38-41 \mathrm{ps}^{161}$ and these agents are closer in size to DNADTPA-MA derivatives than DTPA-MA alone. Furthermore, as mentioned above, electronic relaxation makes a greater contribution to the observed relaxivity at low fields. It was determined that a $\tau_{v}$ of $40 \mathrm{~s}$ was necessary to obtain values of $\frac{1}{T_{1 m}}$ about 0.42 and $0.44^{\mathrm{s}-1}$ to match those found experimentally for SC-Gd(III) alone and with thrombin, respectively. Once again considering the case of $\tau_{\mathrm{m}}$ being $10^{-7} \mathrm{~s}, \mathrm{a} \frac{1}{T_{1 m}}$ of $0.44 \mathrm{~s}^{-1}$ was achieved when the $\tau_{R}$ was 50 times that of DTPA-MA. The mass difference between DTPA-Gd(III) and SC-Gd(III) with thrombin is 82-fold, therefore this result again suggests that the rotation of the chelate is not fully decoupled from the global motion of the whole agent. Under the same conditions, a $\frac{1}{T_{1 m}}$ of $0.42 \mathrm{~s}^{-1}$ was achieved with a $\tau_{R} 30$ fold that of DTPA-MA. This is greater than the 11 -fold mass difference of DTPAGd(III) and SC-Gd(III), but as the mass differences are merely useful for the estimation of $\tau_{R}$ values to test, this discrepancy is unsurprising. This result agrees with the previous calculations and the experimental results in that the chelate portion of SC-Gd(III) is only partially decoupled from the global motion of the whole agent.

The relaxivity enhancements found at $1.5 \mathrm{~T}$ are on the same order of magnitude as that found for SC-Gd(III) at $9.4 \mathrm{~T}(35 \pm 4 \%$, Table 2.3). However, the RIME effect has been reported to be more effective at low fields than at high due to the greater relaxivitylimiting effects of short $\tau_{\mathrm{R}}$ values at low magnetic fields. ${ }^{106,124,162}$ Unlike at high fields, however, at low fields it has generally been found to be more effective to have a rigid 
linker connecting the imaging and targeting moieties in targeted CAs or the chelates and scaffold in multiplexed CAs (those that contain many Gd(III) ions per molecule) as this decreases the unfavourable effects of internal motion on relaxivity. This phenomenon has been observed and studied in a variety of systems, including protein-targeting CAs, ${ }^{106,160,163}$ dendrimer and other scaffolds for multiplexed CAs, ${ }^{159,} 162$ and lipid nanoparticle CAs. ${ }^{11}$ Overall, these studies strove to simultaneously optimize the waterexchange rates and rotational correlation times of the chelates tested, and less flexible linkers permitted the achievement of higher relaxivity values. Greater relaxivity gains and relaxivity enhancement might have been observed for SC-Gd(III) if a more rigid linker were connecting the chelate to the aptamer. However, the expected slow waterexchange due to the monoamide backbone of the chelate in SC-Gd(III) could also be playing a more significant role at low field as it has been reported that there is a more narrow ideal range of $\tau_{m}$ values for a $\mathrm{CA}$ with optimized $\tau_{\mathrm{R}}$ at low field than at high field. $^{124}$ It is also of interest to note that the relaxivity enhancement of SC-Gd(III) at high field could have been affected by the use of a deuterated buffer as solvent. As mentioned previously, the viscosity of $\mathrm{D}_{2} \mathrm{O}$ is higher than that of $\mathrm{H}_{2} \mathrm{O}$, and the relaxivity of Magnevist $\left(\right.$ at $9.4 T$ seems to be increased when $T_{1}$ experiments are performed in $\mathrm{D}_{2} \mathrm{O}$.

\subsubsection{Conclusions and Future Work}

The research performed on the preparation and testing of aptamer-gadolinium(III) conjugates has demonstrated that aptamer-target binding leads to improved relaxivity, with the original aptamer-DTPA single-conjugate, SC-Gd(II), showing an enhancement 
of $35 \pm 4 \%$ at $9.4 \mathrm{~T}$ and $20 \pm 1 \%$ at $1.5 \mathrm{~T}$ due to target binding. The inclusion of a polyT spacer composed of 15 or 30 nucleotides to add spatial separation between the folded thrombin aptamer and the DTPA-Gd(III) portion of the conjugate did not significantly improve relaxivity at $9.4 \mathrm{~T}$. The 15 polyT spacer proved more detrimental than the 30 polyT spacer when considering both the relaxivities and the relaxivity enhancements achieved upon thrombin-binding in the case where the spacer was left single-stranded. The flexibility of the spacer regions did not appear to have a great effect on the relaxivity values measured as their hybridization with matching polyA strands only slightly affected the relaxivity enhancements determined, positively in the case of $15 T$ SC-Gd(III) and negatively for 30T SC-Gd(III). From these observations, we conclude that spacer portions may not be required to improve relaxivity in all systems and were unnecessary for the system studied.

SC-Gd(III) showed a higher relaxivity enhancement than a complementary oligonucleotide-based $\mathrm{Gd}(\mathrm{III})$ system reported to have a $16 \%$ increase in proton relaxation in a comparable study. ${ }^{122}$ Lu et al. recently reported a turn-off aptamergadolinium conjugate using the adenosine aptamer (Figure 2.6 in section 2.1.3.1) that gave a drop in relaxivity of about $34 \%$ upon target binding (measured at $1.41 \mathrm{~T}$ and $\left.37^{\circ} \mathrm{C}\right)$. With the conjugation of streptavidin to the aptamer to increase the overall starting mass, their system resulted in a 17.5 times decrease in mass upon introduction of the target. ${ }^{16}$ This change in mass is more than twice that of the mass change for SC-Gd(III) upon target binding, but the changes in relaxivity measured at low field are similar $(-34 \%$ versus $20 \pm 1 \%$ ) despite the different system designs and chelates used (DOTA-Gd(III) versus DTPA-Gd(III)). As illustrated by Figure 2.19B and the MR images published by 
Lu et al., the changes in relaxivity upon aptamer-target binding in these systems demonstrate observable changes in vitro when imaged at low field. As turn-on $T_{1}$ weighted imaging is more desirable for medical diagnoses, ${ }^{16}$ further optimization of aptamer-gadolinium(III) conjugate systems like that reported herein will be explored in the future with the achievement of higher relaxivity enhancements of particular interest.

The incorporation of aptamers for membrane-bound targets, such as proteins or lipids, or for targets that are larger than thrombin into aptamer-gadolinium(III) conjugates could result in even greater relaxivity enhancements as the change in rotational correlation time would be expected to be greater than in our study, as demonstrated by the relaxivity change reported by Lu et al. ${ }^{16}$ Current efforts in whole-cell SELEX to obtain aptamers for membrane-bound targets and at the same time identify biomarkers for diseases such as cancer could lead to the development of valuable targeted MRI contrast agents. $^{164-166}$ At present, the targeting of $\alpha$-thrombin could still prove useful for precise MR imaging of internal bleeding and blood clotting processes. ${ }^{167,168}$ For instance, there is interest in MR imaging of coronary thrombosis and pulmonary embolism, circumventing the conventional, much more invasive, angiography and angioscopy procedures. $^{169}$ As discussed in section 1.3.3, the 29mer TBA would prove more useful in this area of research than the 15 mer TBA and future work could include the incorporation of the 29mer TBA into DNA-DTPA-Gd(III) conjugates like those studied herein.

Studies on chelate-aptamer spacing may be valuable in other aptamer-target systems or within a matrix more closely mirroring in vivo conditions, such as human serum, as the CA would have negligible influence on physiological viscosity. For example, the conformations that other aptamers achieve upon target binding might 
directly interfere with the effectiveness of the chelated Gd(III) as a $T_{1}$ relaxation agent. The addition of a spacer between the aptamer and the chelator could prevent this interference in such a situation. In some systems the conjugation of the chelate might detrimentally alter the aptamer-target interaction. In such cases, the inclusion of a spacer might prove necessary to retain aptamer-target binding and therefore the targeting capability of the CA. Therefore, the optimization of a particular aptamer-gadolinium(III) conjugate for use as a targeted CA might necessarily involve testing spacers of various compositions and lengths for their effects on CA performance.

The investigation of various linkers of differing rigidity between the DNA and chelate moieties might also prove useful in future studies. Increasing the rigidity of the linker could aid in the achievement of higher relaxivity enhancements upon aptamertarget binding, particularly at low field as discussed above. Furthermore, chelators other than the monoamide derivative of DTPA used herein might provide more advantageous properties, such as more optimal water-exchange rates, and therefore increase the value of aptamer-gadolinium(III) conjugates for use as targeted MRI contrast agents.

\section{3 . Doubly-labelled DNA-DTPA conjugates}

\subsection{Introduction}

The incorporation of aptamers into a variety of applications, including sensing, therapeutics, and diagnostics, has been introduced and discussed in the previous chapters. While DNA-DTPA single-conjugates were prepared and their function as potential 
targeted MRI contrast agents was explored, the corresponding DNA-DTPA doubleconjugates represented in Figure 3.1 were also identified and isolated.

A)

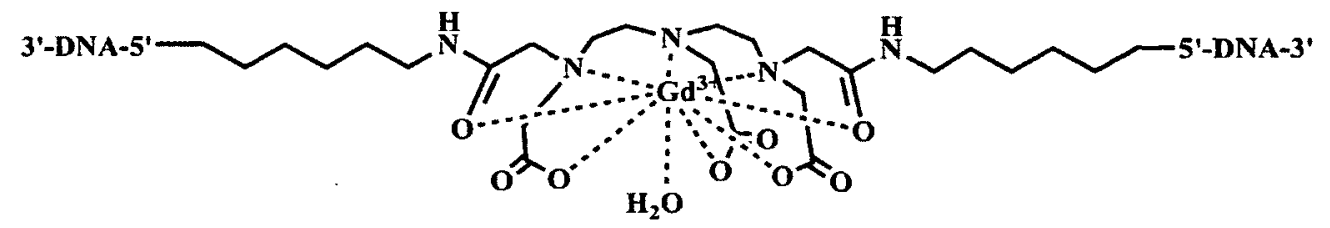

B)

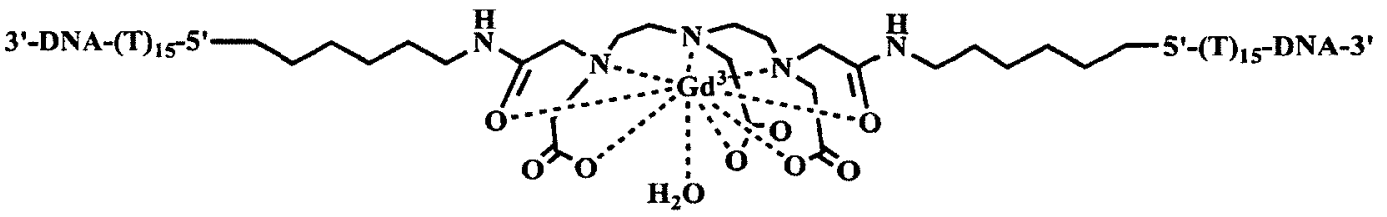

C) $3^{\prime}-\mathrm{DNA}-(\mathrm{T})_{30} 0^{-5^{\prime}}$

Figure 3.1 DNA-DTPA double-conjugates with chelated Gd(III) prepared in this work. A) thrombin aptamer-DTPA, DC-Gd(III); B) thrombin aptamer-15 polyT spacer-DTPA, 15T DCGd(III); C) thrombin aptamer-30 polyT spacer-DTPA, 30T SC-Gd(III).

The yield of these double-conjugates (DCs) was always a great deal lower than that of the related single-conjugates, so different synthetic strategies were explored in an effort to maximize the conjugation of two DNA aptamer strands to a single DTPA dianhydride molecule. While the work performed thus far on DNA-DTPA DCs has been restricted to symmetric conjugates, the future development of asymmetric DCs for potential use as theranostic or multimodal agents is envisioned. The inclusion of two targeting moieties in a single agent, whether they bind the same target or not, could also prove useful in achieving a greater relaxation enhancement upon target binding. 


\subsubsection{Multilocus binding}

Two strategies have been used to improve the relaxivity values, and so the sensitivities, of MRI contrast agents. One includes increasing the number of Gd(III) ions in one molecule of an agent. The other involves optimizing the parameters, such as the rotational correlation time, $\tau_{R}$, and water exchange rate, that influence the overall relaxivity of a CA. ${ }^{160}$ Receptor-induced magnetization enhancement (RIME) is an example using the second strategy listed above. Upon binding of an agent, specifically or nonspecifically, to a target protein, for example, the $\tau_{R}$ experienced by the CA changes from that of a small molecule to that of the protein. This is particularly effective at the low fields typically used to obtain diagnostic MR images. ${ }^{124}$

The effectiveness of either RIME or the inclusion of multiple Gd(III) ions in a CA has been found to be limited by the effects of internal motion. This concept was introduced in Chapter 2 (section 2.3.6) in relation to the $\mathrm{C}_{6}$ linker used to connect the DNA aptamer strands in the single-conjugates to the DTPA molecule (the same linker is present in the double-conjugates; see Figure 3.1). Flexible linkers, such as the one used in this work, joining the chelate and targeting moieties in bifunctional CAs can rotate freely. This results in the inner-sphere water molecule experiencing a net rotational motion that is a combination of the fast internal motion caused by the freely rotating linker and the slower global motion of the entire CA molecule. ${ }^{124,160}$ Recall that at high field it has been suggested that the internal motion experienced by the chelate should be fully decoupled from the overall slower tumbling rate of the CA since intermediate values of $\tau_{R}$ result in higher relaxivities at high fields. ${ }^{124}$ Greater rigidity in the linker 
between the chelate and targeting moieties has been found to be effective at increasing relaxivities of CAs at low field. ${ }^{111,160}$

One method that has been used to achieve greater rigidity is the design of a CA that contains two targeting vectors for multilocus binding. Consider the schematic representation of two Gd(II) chelate multimer agents in Figure 3.2A. Both agents contain four Gd(III) chelates connected by flexible linkers. The one shown on the left, however, only has one targeting group (TG) while the agent on the right has two targeting groups. ${ }^{155}$ In a study reported in 2005 by Caravan et al., ${ }^{160}$ a tetrameric DTPA-Gd(III) agent was prepared to study how the two agent designs represented in Figure 3.2 would compare. The targeting group used was a biphenyl albumin-binding group. They found that the agent with two TGs both bound with higher affinity for human serum albumin than the agent with one $\mathrm{TG}$, and it had a greater per Gd(III) relaxivity (39.1 versus 26.2 $\mathrm{mM}^{-1} \mathrm{~s}^{-1}$, respectively, at $37^{\circ} \mathrm{C}$ and $20 \mathrm{MHz}$ ). These relaxivity values were both higher than that for an identical agent prepared without the inclusion of a targeting group (15 $\left.\mathrm{mM}^{-1} \mathrm{~s}^{-1}\right){ }^{160}$ When bound to albumin the agent prepared with one TG (Figure 3.2B) experiences an overall slower global rotation and thus an increased in relaxivity. However, the linkers within the Gd(III) chelate multimer chain are flexible and the overall effect on relaxivity is inhibited by the internal motion within the chain (represented by the arrows in Figure 3.2). The agent with two targeting groups was found to have a higher relaxivity due to the limited internal motion experienced within the $\mathrm{Gd}(\mathrm{III})$ multimer chain as a result of its binding to albumin through two points (i.e. multilocus binding). ${ }^{155,160}$ 


\section{B)}
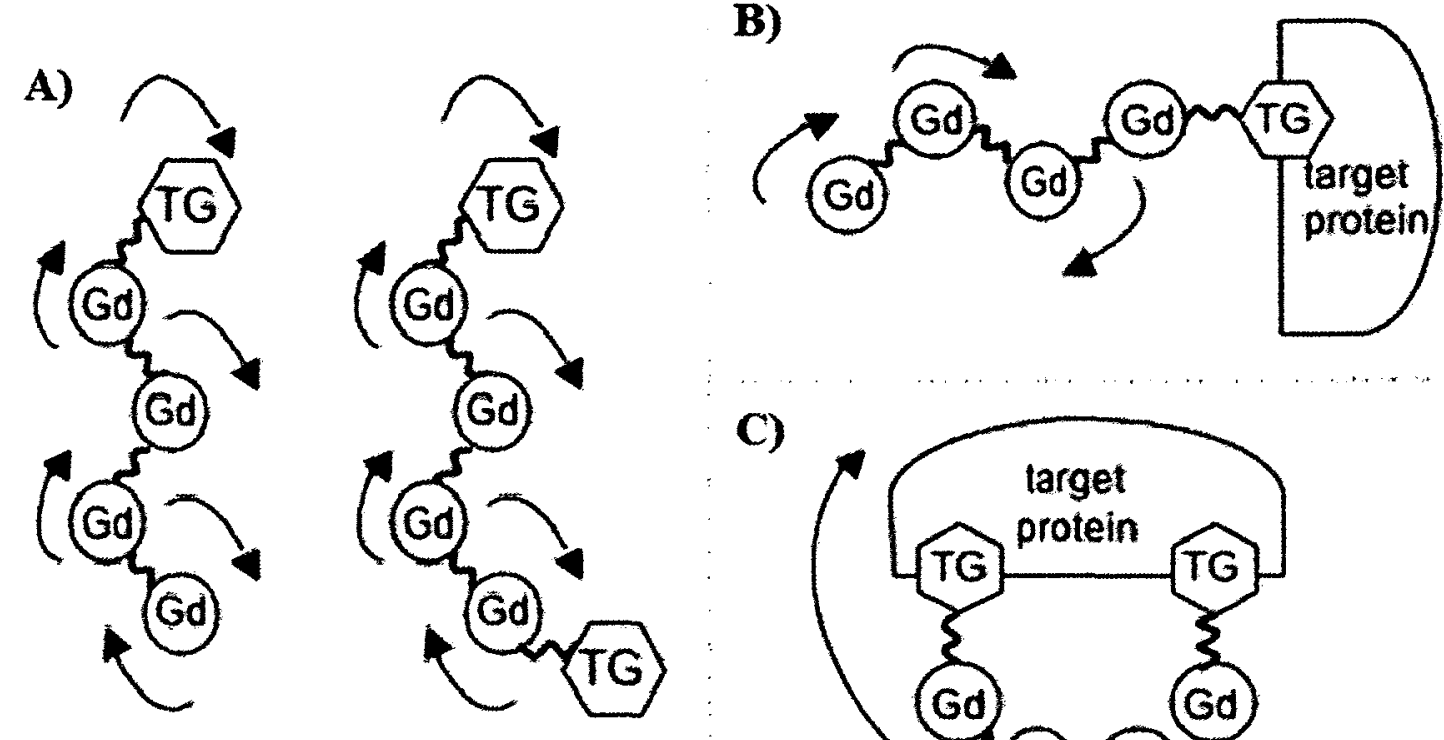

C)

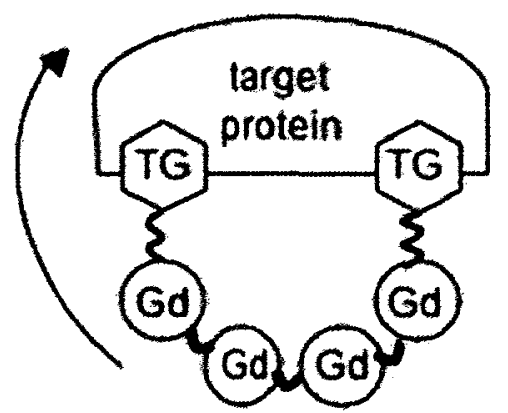

Figure 3.2 A) Two Gd(III) chelate multimers, one with one targeting group (TG; left) the other with two targeting groups (right). B) Agent with one TG still experiences internal motion. C) Multilocus binding agent with two TGs experiences limited internal motion and therefore a higher relaxivity. Arrows represent the motion of the CAs. When freely rotating bonds are present as in $A$ and $B$, the internal motion of these bonds limits the relaxivity achieved by the agent. Modified from reference 155 .

The per Gd(III) relaxivities of these agents was lower than that of MS-325 $\left(46 \mathrm{mM}^{-1} \mathrm{~s}^{-1}\right)$, an albumin-binding agent described in section 2.1.2.3 and shown in Figure 2.4, which binds to albumin through a different targeting group. However, consider that each of the Gd(III) chelate multimers contains four ions and so their per molecule relaxivities are considerably higher than that of MS-325..$^{160}$

The potential to use DNA-DTPA double-conjugates as targeted CAs with multilocus binding ability could prove useful even without the presence of several 
chelates within one molecule. If the aptamers used targeted groups on cell surfaces, for example, a high concentration of the agent might gather at the desired location and an increase in sensitivity might be observed as a result. The two aptamers used could bind two different targets that are located near to each other such as the two binding sites on thrombin, for example. ${ }^{8,21}$

Another method that could prove useful in future work with aptamer-based targeted CAs could be the design of an agent where the $\mathrm{Gd}(\mathrm{III})$ chelate was at the barycenter of the molecule. Port et al. prepared a molecule called gadomelitol which is basically DOTA-Gd(III) with large hydrophilic groups attached through the $\alpha$-carbons of the four acetate arms (the structure of this chelate is shown in Figure 2.3B). ${ }^{170}$ Though there is flexibility within the hydrophilic groups, the $\mathrm{Gd}(\mathrm{III})-\mathrm{H}_{2} \mathrm{O}$ vector cannot rotate independently from the motion of the entire molecule. ${ }^{155}$ This lead to the achievement of an enhanced relaxivity of $39.0 \mathrm{mM}^{-1} \mathrm{~s}^{-1}$ (at $37^{\circ} \mathrm{C}$ and $20 \mathrm{MHz}$ ) compared to that of DOTA-Gd(III) $\left(3.5 \mathrm{mM}^{-1} \mathrm{~s}^{-1}\right.$ at $\left.20 \mathrm{MHz}\right){ }^{170}$ Enhanced relaxivity has also been observed where DOTA-Gd(III) was bound to two hydrophobic lipid chains $\left(34.8 \mathrm{mM}^{-1} \mathrm{~s}^{-1}\right.$ at $25^{\circ} \mathrm{C}$ and $20 \mathrm{MHz}$ ) compared to one lipid chain $\left(15.4 \mathrm{mM}^{-1} \mathrm{~s}^{-1}\right)$ in a study by Botta et al. ${ }^{111}$ Therefore, it seems that conjugating at least two large groups on adjacent acetate arms of DOTA-Gd(III) limits the internal motion within the agent and leads to a greater enhancement of relaxivity than where only one group is present. The preparation of DNA-DOTA-Gd(III) conjugates where two to four DNA strands are included could lead to high relaxivity targeted CAs when at least one of the DNA strands is an aptamer. Furthermore, a mixture of DNA and other hydrophilic or hydrophobic groups could be 
used in concert to prepare agents with high relaxivity and, potentially, the ability to form structures such as micelles. ${ }^{111,171}$

\subsubsection{Theranostics}

Theranostics is a term used to describe materials that are designed for simultaneous therapeutic and diagnostic imaging modalities. ${ }^{172}$ Therefore, theranostic agents contain both therapeutic drugs and diagnostic imaging agents that are administered within the same dose. The inclusion of imaging agents alongside therapeutic drugs is useful for monitoring the diseased tissue, the effects of the drug on the tissue, and the kinetics of the agent's delivery. ${ }^{172}$ As a result, the development of personalized medical treatments is a significant driving force in theranostics research. ${ }^{172}$

In addition to the advantageous characteristics of theranostic agents described above, some of the designs used permit the inclusion of imaging agents for multiple imaging methods. For example, a recent report by Bao et al. ${ }^{173}$ describes a theranostic liposome-based drug delivery system that includes agents for MR, near-infrared (NIR) fluorescent, and nuclear imaging. Including multiple diagnostic imaging agents can increase the value of the information collected, with the imaging modalities complementing each other and compensating for the shortcomings of each method. For example, while MR imaging provides higher spatial resolution and better image contrast, nuclear imaging has a higher sensitivity. ${ }^{172,173}$ The inclusion of MRI contrast agents in the liposomes reported by Bao et al. allowed for 3D high-resolution information to be obtained detailing the microdistribution of the liposomes in tumors in animal studies. This was deemed important for the prediction of cancer therapy response. Quantitative 
information on liposomal distribution inside the tumors was obtained using nuclear images, while NIR fluorescent images provided information on the systemic distribution and locoregional retention of the agent.

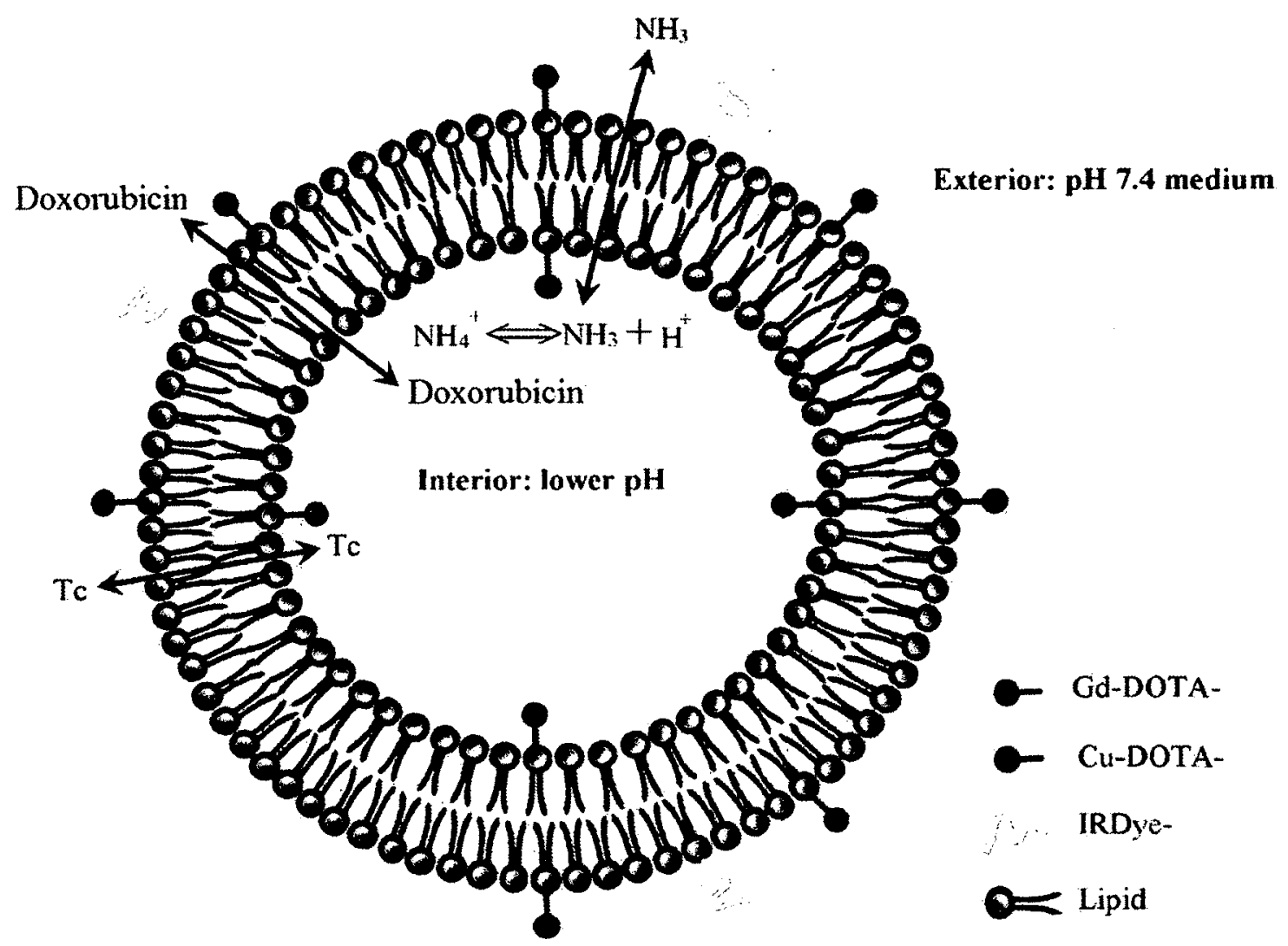

Figure 3.3 Schematic diagram of theranostic liposomes. Modified from ref 173 .

The incorporation of the various imaging agents used in this study into multimodal liposomes is illustrated in the schematic in Figure 3.3. DOTA-Gd(III) and unchelated DOTA were conjugated to the lipids used to form the liposomes and are shown on the hydrophilic outer and inner surfaces of the lipid bilayer. The unchelated DOTA was included for radiolabelling of previously prepared Gd(III)-liposomes with ${ }^{64} \mathrm{Cu}$ for use in PET imaging. A dye for use in NIR fluorescence imaging was also 
conjugated to the lipids used for liposome formation, and these are represented on the outer surface of the liposome in Figure 3.3 as a yellow line. Finally, the gamma imaging radionuclide technetium-99m $\left({ }^{99 \mathrm{~m}} \mathrm{Tc}\right)$ and the chemotherapeutic drug doxorubicin were loaded into prepared Gd(III)-liposomes using an ammonium/pH gradient. ${ }^{173}$

The liposomes prepared were found to be stable over 6 months of storage, which demonstrates their potential as commercial agents. They were tested in vivo through intratumoral infusion in rats and images were obtained using each imaging modality possible considering the agents included. The distribution and retention of the liposomes in the tumors in this preliminary study were therefore monitored using MRI, PET, gamma camera imaging, and NIR fluorescent imaging. These imaging methods do not interfere with one another, and the initial results obtained in this study suggest that they will prove complementary as these multifunctional liposomes are studied further as theranostic agents. $^{173}$

Aptamers have also been incorporated into theranostic systems. ${ }^{90,174}$ One example is that of quantum dot-aptamer conjugates prepared using the RNA aptamer for prostate specific membrane antigen (PSMA). ${ }^{90}$ Recall that quantum dots (QD) can be used for fluorescence detection, thus these conjugates provide targeted imaging. They were also prepared to deliver the drug doxorubicin through its intercalation within the one double-stranded cytosine-guanine site in the RNA aptamer. Doxorubicin is known to fluoresce, and the authors predicted and confirmed that upon its intercalation within aptamers conjugated to the QDs the drug and the QD fluorescence were both quenched. The fluorescence of the QDs was quenched by doxorubicin, while intercalation caused the fluorescence of doxorubicin to be quenched. Therefore, when fully prepared, the 
conjugates were in the "off" state. Upon binding of the aptamer portion of the conjugate to PSMA, the agents were endocytosed into the target prostate cancer cells and doxorubicin was gradually released, either through dissociation or biodegradation of the aptamers. The authors found that prostate cancer cells were specifically targeted by the conjugates using the imaging capabilities of the system; once the drug was no longer intercalated with the aptamer, the QD fluorescence could be used to monitor the conjugates. Furthermore, the target cells experienced significantly greater cytotoxicity following exposure to the conjugate than non-target cells. ${ }^{90}$

In the future, nucleic acid-DTPA double-conjugates could potentially be designed for use as theranostic agents. While one of the nucleic acid strands used could still be an aptamer to provide targeting capabilities to the agent, the second strand could be used to deliver a therapeutic agent to the targeted area. For example, it could be an aptamer for a drug. Since aptamer-target interactions occur as equilibria (see section 2.1.2.3), the drug would eventually be released in vivo, and with the targeting capability of the agent it would predominantly be released near the desired target; a tumor, for example. The second nucleic acid included in the double-conjugate could also be used to carry small interfering RNA (siRNA) to target areas. Aptamers have already been studied for their potential use in the targeted delivery of both drugs and siRNA. ${ }^{38}$ The use of nucleic acidDTPA double-conjugates would provide another system by which this could be accomplished with the advantage of simultaneous MR imaging capabilities when Gd(III) is chelated to the DTPA moiety.

This work encompasses initial syntheses strategies for maximal symmetric DNADTPA double-conjugate yields that could be extended to the preparation of asymmetric 
conjugates. The possible use of the prepared conjugates as targeted MRI contrast agents was then studied at both high $(9.4 \mathrm{~T})$ and low $(1.5 \mathrm{~T})$ fields, as for the single-conjugates discussed in the previous chapter.

\subsection{Materials and methods}

\subsubsection{Materials and instruments}

All materials and instruments used for this work were the same as those used in Chapter 2. Please see section 2.2.1.

\subsubsection{Synthesis of DNA-DTPA conjugates}

The DNA-DTPA double-conjugates DC, 15T DC, and 30T DC were prepared using the following sequences of DNA, respectively:

Thrombin aptamer: ${ }^{8}$ 5'-NH2-( $\left.\mathrm{CH}_{2}\right)_{6}$-GGT TGG TGT GGT TGG-3'

Thrombin aptamer with 15 polyT spacer: $5^{\prime}-\mathrm{NH}_{2}-\left(\mathrm{CH}_{2}\right)_{6}-(\mathrm{T})_{15}$ GGT TGG TGT GGT TGG-3'

Thrombin aptamer with 30 polyT spacer: $5^{\prime}-\mathrm{NH}_{2}-\left(\mathrm{CH}_{2}\right)_{6}-(\mathrm{T})_{30}$ GGT TGG TGT GGT TGG-3'

DNA was synthesized without the final deblock of the 5'-DMS(O)MT-amino modifier C6 performed.

Directly following synthesis, the DNA columns containing $500 \AA$ controlled pore glass (CPG) beads were placed on the cleaving chuck and rinsed sequentially with 300 $\mu \mathrm{L}$ of anhydrous $\mathrm{MeCN}, 3 \times 300 \mu \mathrm{L}$ of $10 \% \mathrm{DEA}$ in anhydrous $\mathrm{MeCN}$, and $2 \times 200 \mu \mathrm{L}$ of anhydrous MeCN. For the one-pot synthesis route, all prepared DNA columns were stored at $-4^{\circ} \mathrm{C}$ until required. For the modular synthetic route and modified one-pot 
synthetic route developed, half of the columns prepared were deprotected at room temperature or $37^{\circ} \mathrm{C}$ for 24 hours in $\mathrm{NH}_{4} \mathrm{OH}(28 \%)$ ( $1 \mathrm{~mL}$ per column) while the other half were stored at $-4^{\circ} \mathrm{C}$ until required. The deprotected DNA in $\mathrm{NH}_{4} \mathrm{OH}$ was isolated from the CPG beads using filter tubes (RAININ Microfilterfuge ${ }^{\mathrm{TM}}$ Tubes $0.45 \mu \mathrm{m}$ uncharged nylon-66 filters) or by pipette. At this point, DNA was generally quantified using the characteristic absorption peak at $260 \mathrm{~nm}$ in order to ensure a high yield of DNA was obtained prior to reaction with DTPA dianhydride.

For DNA conjugation to DTPA dianhydride in all synthetic routes, the stored DNA columns were brought to room temperature, placed on the cleaving chuck, and rinsed with $300 \mu \mathrm{L}$ of anhydrous $\mathrm{MeCN}$. Removal of the DMS(O)MT protecting group from the CPG-supported DNA was performed by washing the beads with $3 \times 300 \mu \mathrm{L}$ of deblock solution ( $2 \%$ dichloroacetic acid in dichloromethane). The characteristic absorbance of $\mathrm{DMS}(\mathrm{O}) \mathrm{MT}^{+}$in the deblock solution (collected separately from the $\mathrm{MeCN}$ washes) confirmed DNA modification. The column material was once again rinsed with MeCN $(2 \times 300 \mu \mathrm{L})$ to ensure the removal of all free DMS(O) $\mathrm{MT}^{+}$, and the CPG supports were transferred to $1.5 \mathrm{~mL}$ Eppendorf tubes. From this point, different synthetic routes were developed in an effort to maximize the amount of double-conjugate (two DNA strands conjugated to one DTPA molecule) obtained.

In the modular synthetic route, a 42 -fold excess of DTPA dianhydride in anhydrous DMSO was added to the CPG-supported DNA immediately following the deblocking procedure described above. After shaking the reaction mixture for 4 hours, the CPG beads were collected at the bottom of the tubes through centrifugation (5 minutes at $10,000 \mathrm{~g}$ ) and the DTPA dianhydride solution was removed via pipette. To 
remove any remaining DTPA dianhydride, $500 \mu \mathrm{L}$ more anhydrous DMSO were added to the beads. The mixture was vortexed, centrifuged, and the solution removed as above. About $300 \mu \mathrm{L}$ anhydrous DMSO was then added to just cover the CPG beads.

The modified and original one-pot synthetic methods were initiated in an identical fashion. DTPA dianhydride in anhydrous DMSO $(0.04 \mathrm{M})$ was titrated into each CPG bead-anhydrous DMSO $(100-200 \mu \mathrm{L})$ slurry immediately following the manual deblocking procedure detailed above. To each column of CPG-supported DNA, the DTPA dianhydride solution was added in six increments of $25 \mathrm{nmol}$ with 10 minutes of shaking between additions, followed by six increments of $50 \mathrm{nmol}$ with 5 minutes of shaking between additions. Another $1 \mu \mathrm{mol}$ of DTPA dianhydride was then added in six increments with 5 minutes of mixing between additions, followed by another micromole in three equal portions. Finally, a single addition of $0.8-1 \mu \mathrm{mol}$ of DTPA dianhydride was added to ensure that its final concentration was about a 3- to 4-times that of the DNA. For the one-pot synthetic route, the reaction mixture was left on a shaker overnight at room temperature.

Concurrently to the conjugation of CPG-supported DNA to DTPA dianhydride, the DNA that had already been cleaved from the CPG beads for the modular and modified one-pot synthetic routes was manually deblocked in solution. The $\mathrm{DMS} \mathrm{O}) \mathrm{MT}^{+}$group was removed by a 90 minute treatment with $80 \%$ glacial acetic acid in $\mathrm{diH}_{2} \mathrm{O}(200 \mu \mathrm{L}$ per column of DNA) or a 5 minute treatment with $2 \%$ dichloroacetic acid (DCA) in $\operatorname{diH}_{2} \mathrm{O}(100 \mu \mathrm{L}$ per column of DNA), followed by extraction of the DNA via ethanol precipitation. Each portion of DNA was added to a solution of $1 \mathrm{~mL}$ EtOH and $50 \mu \mathrm{L}$ of $3 \mathrm{M} \mathrm{NaCl}$ and placed on dry ice for about 1 hour. The precipitated DNA 
was isolated through centrifugation at $-9^{\circ} \mathrm{C}$ and $10,000 \mathrm{~g}$ for $15-30$ minutes. As much of the solution as possible was removed via pipette, with care not to disturb the pellet. Any remaining liquid was removed under vacuum, then the DNA was re-dissolved in a minimum volume of $\mathrm{diH}_{2} \mathrm{O}(50-200 \mu \mathrm{L})$ and either added to the CPG-supported DNADTPA or precipitated out of aqueous solution using hexadecyltrimethylammonium bromide $(\mathrm{CTAB})$ as described in literature. ${ }^{175}$

Table 3.1 CTAB precipitation guidelines.

\begin{tabular}{|c|c|}
\hline $\begin{array}{c}\text { Volume used to dissolve DNA } \\
(\mu \mathrm{L})\end{array}$ & $\begin{array}{c}\text { First CTAB addition } \\
(\mu \mathrm{L})\end{array}$ \\
\hline 50 & 2 \\
100 & 4 \\
150 & 6 \\
200 & 8 \\
\hline
\end{tabular}

Briefly, an $8 \%(w / v)$ solution of CTAB in $\mathrm{diH}_{2} \mathrm{O}$ was prepared. Depending on the volume used to dissolve the DNA, an initial volume of the CTAB solution was added to the resuspended DNA (see Table 3.1) and the sample was mixed on a vortex. The solution was then centrifuged for 2 minutes at $15,000 \mathrm{~g}$ to collect $\mathrm{CTA}^{+}$-DNA as a pellet. This process was repeated with the following $\mathrm{CTAB}$ additions half the volume of the first one. When it appeared that DNA was no longer precipitating out of solution the procedure was halted since excess $\mathrm{CTAB}$ in solution would permit the $\mathrm{CTA}^{+}$-DNA to redissolve. At this point, most of the solution was removed from the CTA ${ }^{+}$-DNA pellet by pipette and the pellet was completely dried under vacuum. The CTA ${ }^{+}-$DNA was then suspended in anhydrous DMSO $(\sim 200 \mu \mathrm{L})$ and combined with the CPG-supported DNA- 
DTPA representing the other half of the synthesized DNA. The mixture was left on a shaker overnight.

The CPG beads were isolated through centrifugation (10 minutes at $10,000 \mathrm{~g}$ ), the solution was pipetted off, and about $300-500 \mu \mathrm{L}$ of anhydrous DMSO was added to help remove unreacted $\mathrm{CTA}^{+}$-DNA or DTPA dianhydride, depending on the synthetic route used. This process was repeated to remove the rinse DMSO, and then the CPG beads were treated with $\mathrm{NH}_{4} \mathrm{OH}$ for 24 hours at room temperature or $37^{\circ} \mathrm{C}$ to cleave and deprotect the support-bound DNA-DTPA conjugates. The deprotected DNA was isolated from the CPG beads as described above and quantified using its characteristic absorbance at $260 \mathrm{~nm}$.

\subsubsection{Purification of DNA-DTPA double-conjugates}

Denaturing polyacrylamide gel electrophoresis (PAGE) was used to purify the DNA-DTPA conjugates. Approximately $300-400 \mathrm{nmol}$ of crude DNA-DTPA were run per gel, where the acrylamide percentages used were $19 \%$ for DC and 12 or $15 \%$ for $\mathbf{1 5 T}$ DC and 30T DC, respectively. Gels were prepared and run and the double-conjugates isolated from the gel as described in section 2.2.3 for the single-conjugates. Recall that the single-conjugates were often isolated as products of the reaction methods used to maximize the yield of the respective double-conjugates described in the previous section. Many of the methods and protocols used for the single-conjugates were therefore used in parallel for isolation and preparation of the double-conjugates for further study.

The purified DNA-DTPA conjugates were desalted using a $1000 \mathrm{Da}$ cut-off Ultrafiltration Membrane in the Amicon Bioseparations Stirred Ultrafiltration Cell. 
Following recovery of the conjugates from the filter, they were quantified using UV-Vis spectroscopy.

\subsubsection{Chelation of Gd(III) with DNA-DTPA double-conjugates}

Due to the lower quantities of the double-conjugates isolated compared to the single-conjugates, only the optimized method for the chelation of Gd(III) by the DTPA moiety developed with tests on SC-Gd(III) (sections 2.2.4 and 2.3.2) was used for the double-conjugates. Briefly, following resuspension in tris buffer $(50 \mathrm{mM}, 140 \mathrm{mM} \mathrm{NaCl}$, $\mathrm{pH} 7.4$ ), the quantified DNA-DTPA double-conjugates were combined with $\mathrm{Gd}(\mathrm{III})$ in ammonium acetate $(0.5 \mathrm{M}, \mathrm{pH} 6)$ in $1: 1$ molar equivalents. Conjugates were shaken overnight, desalted (using Amicon Ultra Centrifugal Filter Units), and tested for free Gd(III) with a xylenol orange test ${ }^{139}$ as previously described. If required, more Gd(III) was added to the conjugate and the process was repeated until the percentage of unchelated Gd(III) had reached a minimum.

\subsubsection{Mass spectrometric confirmation of DNA-DTPA double-conjugates}

FIA/MS analysis confirmed the molecular weight of the DNA-DTPA doubleconjugates: DC m/z calc'd: 10165; found: 10167. 15T DC-Gd(III) m/z calc'd: 19447; found: 19448. 30T DC m/z calc'd 28417; found 28418 .

\subsubsection{Preparation of 15 and 30 poly $A$ oligonucleotides}

The polyA strands corresponding to the polyT spacer portions of the modified thrombin aptamers were also synthesized on the MerMade 6 Oligonucleotide synthesizer and purified using Clarity QSP cartridges as described in section 2.2.6. 


\subsubsection{Melting studies on the DNA-DTPA double-conjugates}

Melting studies on the DNA-DTPA double-conjugates were performed on 0.87 and $0.60 \mu \mathrm{M}$ solutions of $15 \mathrm{~T} \mathrm{DC}$ and $30 \mathrm{~T} \mathrm{DC}$, respectively. The conjugates were

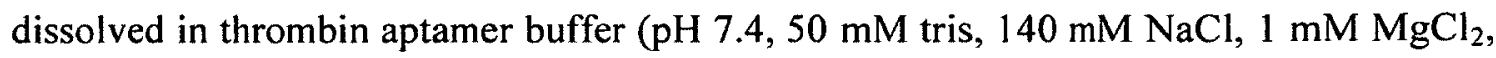
$5 \mathrm{mM} \mathrm{KCl}$ ) in a 1:2 ratio with the corresponding polyA strand. The solutions were heated at $70^{\circ} \mathrm{C}$ for 5 minutes then allowed to cool to room temperature. Each solution was placed into a cuvette along with a micro stir bar to ensure even heating of the sample. Absorbance was monitored at $260 \mathrm{~nm}$ and recorded at $1^{\circ} \mathrm{C}$ intervals as the temperature increased from $17-90^{\circ} \mathrm{C}$ at a rate of $1^{\circ} \mathrm{C}$ per minute. The temperature was held at $90^{\circ} \mathrm{C}$ for 5 minutes before decreasing from $90-20^{\circ} \mathrm{C}$.

\subsubsection{In vitro stability of DNA-DTPA double-conjugates: Transmetallation study}

A portion of DC-Gd(III) was dissolved in deuterated phosphate buffer ( $\mathrm{pH} \mathrm{7,26}$ $\mathrm{mM} \mathrm{KH}_{2} \mathrm{PO}_{4}, 41 \mathrm{mM} \mathrm{Na}_{2} \mathrm{HPO}_{4}$ ) to a concentration of $0.05 \mathrm{mM}$ and placed in a silanized $5 \mathrm{~mm}$ NMR tube. The buffer and silanized tube were both prepared as for the transmetallation experiment on SC-Gd(III) (section 2.2.8.3). The $T_{1}(t=0)$ of the DCGd(III) solution was estimated at $37^{\circ} \mathrm{C}$ on a $9.4 \mathrm{~T}$ NMR spectrometer and then a $2: 1$ molar equivalent of $\mathrm{Zn}(\mathrm{II})$ was added from a $\mathrm{ZnCl}_{2}$ stock solution. The reaction mixture was maintained at $37^{\circ} \mathrm{C}$ for a period of approximately 4 days and $T_{1}$ values were estimated periodically as a measure of the extent of transmetallation.

The observed relaxivity was calculated using $R_{1}=\left(1 / T_{1}\right),{ }^{137,140}$ where the $T_{1}$

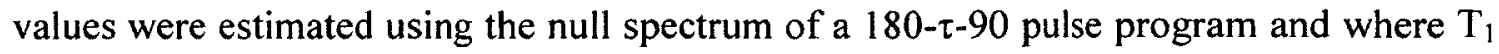
$=\tau / \ln 2 .^{141}$ The Bruker $t$ lirld pulse program was used with the recycle delay, $\mathrm{d} 1$, set to 
30 seconds and the value of $\tau, \mathrm{d} 7$, adjusted until the peak of interest (water, $\sim 4.7 \mathrm{ppm}$ ) was nulled. The null point was identified as the case when the integrations of positive and negative peaks in each spectrum (determined using the software) were found to be approximately equal.

The same value of the diamagnetic contribution determined for the experiment on SC-Gd(III) applied for the DC-Gd(III) experiment since the same buffer was used. The paramagnetic longitudinal relaxation rates $\left(\mathrm{R}_{1}{ }^{\mathrm{P}}\right)$ of $\mathbf{D C}-\mathbf{G d}(\mathrm{II})$ were therefore also calculated by subtracting the diamagnetic contribution $\left(\sim 0.077 \mathrm{~s}^{-1}\right)$ from the observed relaxivity values.

\subsubsection{Preparation of samples for $T_{1}$ experiments at high field (9.4 Tesla)}

As the double-conjugates each contain two thrombin aptamer strands, there were two possible cases to consider when adding target or control protein: $2: 1$ and 1:1 protein to conjugate ratios. In the $2: 1$ case, enough protein was present for every aptamer in a solution of a double-conjugate to be interacting with a protein. In the 1:1 case, there was one protein per double-conjugate, so on average one aptamer on each double-conjugate would have a protein present in solution with which to interact (see Figure 3.1). With both cases to consider each of the three conjugates DC-Gd(III), 15T DC-Gd(III), and 30T DC-Gd(III) were run in five sets of $T_{1}$ experiments. These were 1) conjugate alone in solution, 2) conjugate with thrombin in a $2: 1$ ratio, 3) conjugate with pepsin (control) in a 2:1 ratio, 4) conjugate with thrombin in a 1:1 ratio, and 5) conjugate with pepsin in a 1:1 ratio. In the cases of $15 T$ DC-Gd(III) and 30 T DC-Gd(III), an additional five sets 
of experiments were run using the same protein content as listed above, but with their matching polyA strands hybridized to their polyT spacers.

The $T_{1}$ measurement buffer was thrombin aptamer buffer $(\mathrm{pH} 7.4,50 \mathrm{mM}$ tris, $140 \mathrm{mM} \mathrm{NaCl}, 1 \mathrm{mM} \mathrm{MgCl}, 5 \mathrm{mM} \mathrm{KCl}$ ) that had been lyophilized and reconstituted using $\mathrm{D}_{2} \mathrm{O}$.

Portions of 7, 3, and $2 \mathrm{nmol}$ for DC-Gd(III), 15T DC-Gd(III), and 30T DCGd(III), respectively, were dissolved in $\mathrm{T}_{1}$ measurement buffer, heated at $70^{\circ} \mathrm{C}$ for 5 minutes, and then allowed to cool to room temperature in order to ensure the aptamer was folded into its most stable conformation. For the sample sets requiring the addition of protein, it was added to the re-folded DNA-DTPA and the sample solution was agitated for half an hour to promote equilibration.

In cases requiring hybridization of the polyA strands, they were added in a $2: 1$ ratio to the conjugates prior to the samples being heated as described above.

Capillary tubes were silanized using the reagent Sigmacote ${ }^{\circledR}$ as previously described (section 2.2.8.3). For $T_{1}$ experiments, DNA-DTPA-Gd(III) or Magnevist ${ }^{\circledR}$ samples were injected into silanized capillary tubes which were then placed into $5 \mathrm{~mm}$ NMR tubes. A narrow plastic tube was used to hold the capillary tubes upright. A minimum volume of $50 \mu \mathrm{L}$ was used in these tubes for the determination of $T_{1}$ values.

\subsubsection{Preparation of samples for $T_{1}$ experiments at low field (1.5 Tesla)}

Lyophilized pepsin was dissolved in TBA buffer for use in these experiments while thrombin was used as purchased from Haematologic Technologies Inc. (i.e. in 50\% $\mathrm{v} / \mathrm{v}$ glycerol/water solution). 


\subsubsection{15T DC-Gd(III) samples}

Little 15T DC-Gd(III) was available for $T_{1}$ experiments at low field $(\sim 35 \mathrm{nmol})$. Three sets of 15T DC-Gd(III) at concentrations of $0.0134,0.0100,0.0075,0.0057$, and $0.0042 \mathrm{mM}$ were prepared. The sample sets were as follows, where the conjugate and protein ratio was 1:1 where applicable: 15T DC-Gd(III), 15T DC-Gd(III) and thrombin, and 15T DC-Gd(III) and pepsin. A set of DTPA-Gd(III) solutions was also prepared (using chelate prepared in the lab) with concentrations of $0.0125,0.0094$, $0.0070,0.0053$, and $0.0040 \mathrm{mM}$. All sample solutions were made up to a total volume of $280 \mu \mathrm{L}$ so as to completely fill $0.2 \mathrm{~mL} \cdot \mathrm{PCR}$ tubes. The tubes were taped together with each row holding one sample set. An extra tube containing TBA buffer was included in the same row as the DTPA-Gd(III) samples.

\subsubsection{DC-Gd(III) samples}

Three sample sets of DC-Gd(III) were prepared for simultaneous $T_{1}$ measurement at $1.5 \mathrm{~T}$ along with Magnevist ${ }^{8}$. All solutions were prepared to a total volume of $1 \mathrm{~mL}$ and were placed in $4.5 \mathrm{~mL}$ plastic cuvettes. The sample sets were DCGd(III), DC-Gd(III) with thrombin, DC-Gd(III) with pepsin, and Magnevist ${ }^{\circledR}$. The conjugate to protein ratio was 1:1 where applicable, and pepsin was dissolved in $50 \% \mathrm{v} / \mathrm{v}$ glycerol and $\mathrm{diH}_{2} \mathrm{O}$ to match the conditions of the thrombin solution used. All series were prepared at concentrations of $0.0125,0.0094,0.0070$, and $0.0053 \mathrm{mM}$. 


\subsubsection{Relaxivity measurements}

\subsubsection{At high field}

$\mathrm{T}_{1}$ determination at high field was performed on a $400 \mathrm{MHz}(9.4 \mathrm{~T})$ Bruker NMR spectrometer. Four or five concentrations of a conjugate in the range of 0.003 to 0.05 $\mathrm{mM}$ were used for each series of samples. Magnevist ${ }^{\circledR}$ was run at concentrations of 0.1 , $0.05,0.025$, and $0.0125 \mathrm{mM}$. The highest concentration for each case was prepared and each successive concentration was obtained through dilution of the previous solution following measurement of its $T_{1}$ relaxation time.

$T_{1}$ values were obtained for each concentration in triplicate at room temperature $\left(25^{\circ} \mathrm{C}\right)$. An inversion recovery program (Avance Version) was used from the standard Bruker pulse program library to measure $T_{1}$ relaxation. $T_{1}$ values were then automatically calculated using the supplied Bruker NMR Relaxation Guide. Molar relaxivity $\left(\mathrm{r}_{\mathrm{i}}, \mathrm{mM}^{-1} \mathrm{~s}^{-1}\right)$ was obtained from plots of relaxation time $\left(\frac{1}{T_{1}}, \mathrm{~s}^{-1}\right)$ versus gadolinium(III) concentration (mM) as discussed in section 2.1.2 and illustrated by equation 2.4. Linear regression calculations were performed to determine the slope of the curve in each series and the associated error.

\subsubsection{At low field}

Test-tube phantoms of cuvettes $(4.5 \mathrm{~mL})$ or $200 \mu \mathrm{L}$ PCR tubes containing Magnevist $®$, 15T DC-Gd(III) or DC-Gd(III) were obtained by colleagues in the C2 Neurosciences Unit at the Ottawa Hospital Research Institute (OHRI) using a $1.5 \mathrm{~T}$ clinical MR scanner (Siemens Symphony). The temperature within the gantry was maintained at approximately $25^{\circ} \mathrm{C}$ using air conditioners. The pulse sequence for $T_{1}$ and 
$R_{1}$ estimation followed Spin Echo (Saturation Recovery) techniques; repetition time (TR) $100,300,800,1500,3000,6000$ and $9000 \mathrm{~ms}$; echo time (TE) $12 \mathrm{~ms}$; field of view (FOV) $150 \mathrm{~mm}$; matrix size $128 \times 128$; slice thickness $8 \mathrm{~mm}$ for 15 T DC-Gd(III) and 6 mm for DC-Gd(III); and non-averaged.

\subsection{Results and Discussion}

\subsubsection{Preparation of DNA-DTPA double-conjugates}

The use of DTPA dianhydride in the synthesis of DNA-DTPA conjugates led to the formation of two unique products: a single-conjugate where one DNA aptamer was covalently bound to a DTPA molecule, and a double-conjugate where two DNA aptamers were conjugated to one DTPA molecule. A low yield of the double-conjugate, DC, was obtained when an excess of DTPA dianhydride was added to the 15 mer thrombin-binding aptamer (see section 1.2.3). Attempts to maximize the yield of the double-conjugates led to the development of a modular synthetic route and a one-pot synthetic route. The 15 mer thrombin aptamer was used in each case, along with two variations in which poly $\mathrm{T}$ spacers of 15 or 30 thymine residues, respectively, were included on the $5^{\prime}$ end of the aptamer (see section 3.2.2 and Figure 3.1). The 5 ' ends of the respective DNA sequences were modified with amino groups through which the reaction with DTPA dianhydride took place.

The first synthetic variation developed was modular in nature. The experiments described herein focused on double-conjugates in which the two DNA strands were identical (symmetric DC), but this first method could be adapted to permit the conjugation of two different sequences of DNA to the same DTPA molecule (asymmetric 
DC), the implications of which were discussed in the introduction. In this method, half of the DNA was left bound to the CPG-beads after synthesis and was reacted with a 42 -fold excess of DTPA dianhydride in anhydrous DMSO following manual deblocking of the amino-modifier (Figure 3.4A). Figure 3.4B represents the possible CPG-supported products of this reaction: one DTPA dianhydride molecule could react with two strands of DNA near each other on a bead, or one anhydride moiety could react with the aminomodifier on a DNA strand leaving the other free for further reaction. The ratio of products in Figure 3.4 does not reflect the experimental yield obtained, which is discussed in more detail in section 3.3 .2 , but rather the possible synthetic routes to give the single- and double-conjugate products.

In an effort to maximize the yield of double-conjugate, the addition of aminomodified DNA in solution following a four hour reaction time between CPG-supported DNA and DTPA dianhydride was performed. Following DNA synthesis, half of the columns prepared were immediately deprotected and removed from the CPG beads. The blocking group was left on the 5' amino-modifier and therefore had to be removed in solution prior to the addition of this DNA to the CPG-supported DNA-DTPA. This was originally accomplished with a 90 minute treatment with $80 \%$ glacial acetic acid. In later reactions this was performed by a 5 minute treatment with $2 \%$ DCA, which decreased the time required for this step without observable detriment. In fact, a red oil generally appeared in the solution upon addition of the $2 \%$ DCA solution, providing a qualitative indication that the $\mathrm{DMS}(\mathrm{O}) \mathrm{MT}^{+}$group was being removed which was not observed when using the $80 \%$ glacial acetic acid treatment. 
A)

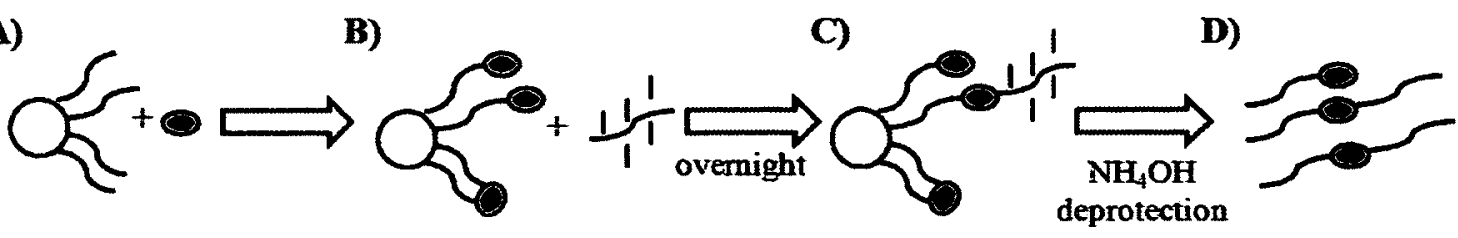

Amino-modified thrombin aptamer 3'-GGT TGG TGT GGT TGG- $\left(\mathrm{CH}_{2}\right)_{6}-\mathrm{NH}_{2}-5$ ',

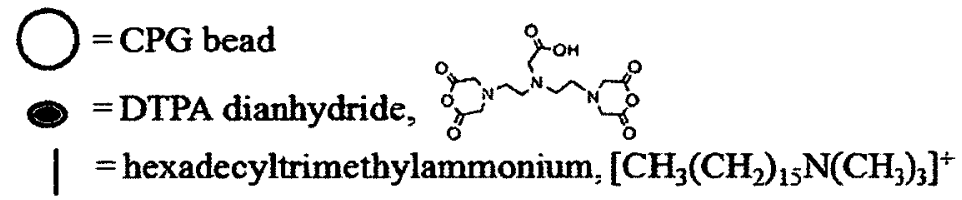

Figure 3.4 Schematic representation of the synthetic routes designed to maximize the yield of DNA-DTPA-Gd(III) double-conjugates.

In both cases, the manual deblocking reaction was quenched upon addition of the reaction solution to a cooled $\mathrm{EtOH}$ and $\mathrm{NaCl}$ solution for ethanol precipitation of the amino-modified DNA. The pelleted DNA was thoroughly dried following precipitation to remove any traces of EtOH and was then resuspended in a minimum volume of $\mathrm{diH}_{2} \mathrm{O}$.

In earlier reactions using the $15 \mathrm{mer}$ thrombin aptamer, the deblocked, and therefore reactive, amino-modified DNA was added at this point to the CPG-supported DNA-DTPA. Due to the hydrolysis of anhydride groups in water (equation 3.1 below), the addition of an aqueous aptamer solution was not ideal.

$$
\mathrm{R}-(\mathrm{C}=\mathrm{O})-\mathrm{O}-(\mathrm{C}=\mathrm{O})-\mathrm{R}+\mathrm{H}_{2} \mathrm{O} \rightarrow 2 \mathrm{RCOOH}
$$

However, DNA does not readily dissolve in organic solvents ${ }^{175}$ and so could not have been added to the CPG-supported DNA-DTPA in anhydrous DMSO as in the case of DTPA dianhydride. The precipitation of DNA with hexadecyltrimethylammonium bromide (CTAB) has been reported as a method by which to achieve solubilization of DNA in aprotic organic solvents such as DMSO and DMF. ${ }^{175}$ The CTA ${ }^{+}$-DNA aptamer 
pellet that resulted from the precipitation was readily soluble in anhydrous DMSO and therefore permitted the addition of amino-modified aptamer in solution to the CPGsupported DNA-DTPA, as illustrated in Figure 3.4B and C. The reaction was left to proceed overnight, and then the resulting DNA-DTPA conjugates were deprotected and cleaved from the CPG beads using $\mathrm{NH}_{4} \mathrm{OH}$ (Figure 3.4D).

Though some of the DTPA dianhydride will react through both anhydride moieties with two amino-modified aptamer strands located close together on the CPG beads (Figure 3.4B), the addition of a high excess of DTPA dianhydride as described above is likely to result in a higher percentage of single-conjugates than if the DTPA dianhydride was added in small amounts to the DNA. Therefore, the modular synthetic route discussed above could be used to prepare asymmetric double-conjugates with two different sequences of DNA per conjugate. One sequence would be left on the CPG beads while the other would be added in solution. Provided that the two nucleic acid sequences were of different lengths, any double-conjugate resulting from the bridging of DTPA between two CPG-supported strands could be removed during purification using denaturing polyacrylamide gel electrophoresis (PAGE). The yield of symmetric DCs might be improved, however, if DTPA dianhydride was gradually added to CPGsupported amino-modified DNA in small amounts. This approach was taken in the onepot synthetic route.

A report by Sleiman et al. ${ }^{176}$ discussed the synthesis of transition metal linked, branched oligonucleotides. The authors connected two CPG-supported DNA strands through a ruthenium(II) complex that included two $n$-hexyl spacers. Combined with the previous observation that a low yield of double-conjugate was obtained upon reaction of 
an excess of DTPA dianhydride with amino-modified DNA, this paper served as inspiration for the one-pot synthetic route. This method was developed in an attempt to maximize the amount of symmetric double-conjugate obtained, and was further modified in an effort to achieve this goal.

As described in section 3.2.2, the CPG beads supporting amino-modified aptamers were mixed with anhydrous DMSO, and a stock solution of DTPA dianhydride was titrated into this mixture in small increments with time for the reaction to occur between additions. An excess of DTPA dianhydride was achieved in the end, but time was allowed for the small amounts added to react with the modified CPG-supported DNA to encourage the formation of double-conjugates on the beads. A modified version of this synthetic route included the addition of amino-modified aptamer in solution following the titration of DTPA dianhydride (Figure 3.4B and C). This was intended to combine the two strategies developed for maximizing the yield of the double-conjugates, though the design of the one-pot method is such that symmetric conjugates should be the desired product. Even if the aptamer added in solution was different from the CPGsupported sequence, the titration of DTPA dianhydride into the bead mixture would likely skew the ratio of DC products to the symmetric conjugate. Though this experiment was not attempted, it might provide a method by which to determine the amount of symmetric DC which forms strictly during the titration portion and the amount that forms during the overnight reaction period in the presence of excess DTPA dianhydride. 


\subsubsection{Comparison of reaction methods and DNA-DTPA conjugate relative yields}

The crude reaction mixtures from the syntheses described above were subjected to denaturing polyacrylamide gel electrophoresis (PAGE) for purification of the products. The double-conjugates were readily separated from the single-conjugates and unreacted DNA due to their greater mass. Figure 3.5 contains representative images of gels from the purification of conjugates prepared using the 15 mer thrombin aptamer (Figure $3.5 \mathrm{~A}$ and $\mathrm{B}$ ), the aptamer with a 15 polyT spacer (Figure $3.5 \mathrm{C}$ ), and the aptamer with a 30 polyT spacer (Figure 3.5D). The bands for the respective double-conjugates were cut out and DNA-DTPA extracted and desalted as described above (section 2.2.3). In many cases, as mentioned earlier, the single-conjugates were isolated in parallel to the doubleconjugates.

Upon consideration of the relative absorbances and widths of the respective single- and double-conjugate bands in the images in Figure 3.5, it is clear that the singleconjugates were always obtained in higher yield than the double-conjugates. This is especially evident when one considers that each double-conjugate has a molar absorptivity that is twice that of its corresponding single-conjugate. This is reflected in the double- to single-conjugate ratios that were determined based on the absorbances of the bands in these representative gel images: $1: 2.5$ and 1:9 for the TBA conjugates, 1:6 for the TBA-15 polyT spacer conjugates, and 1:12 for the TBA-30 polyT spacer conjugates. The TBA conjugates, SC and DC, were prepared using nearly every experimental method outlined above. The gel image in Figure 3.5B was obtained following a modular reaction in which $\mathrm{CTAB}$ was not used to solubilize the DNA added in solution; it was therefore dissolved in water for addition to the CPG-supported DNA- 
DTPA. Despite the chance of hydrolysis of the anhydride moieties on the DTPA molecule (as in equation 3.1), the ratio of double- to single-conjugate resulting from the reaction was low at 1:6. It is possible that a good deal of the $\mathbf{D C}$ formed during the initial reactions where water was present occurred through the reaction of DTPA dianhydride with two CPG-supported DNA strands (Figure 3.4B). This is supported through the comparison of the ratios of double- to single-conjugate calculated based on the yield after isolation from the gel and desalting. Following recovery of the DNA from the gel represented in Figure 3.5B, a ratio of 1:8 was found. For a reaction in which a 42 -fold excess of DTPA dianhydride was added all at once to CPG-supported amino-modified TBA (Figure 3.5A), the ratio of DC to SC was $1: 2.5$ considering the absorbances of the bands on the gel and 1:5 upon recovery of the conjugates. The formation of DC strictly between CPG-supported DNA strands therefore seems to have been a significant contribution to the total amount of this conjugate prepared.

The preparation of DC and SC was also performed using the modular method, where the entire procedure was performed in organic solution following CTAB precipitation of TBA and using the one-pot method where DTPA dianhydride was introduced via titration. The results of the reactions using both of these methods were similar or identical to that found where water was used; that is, the ratio of DC to SC recovered following PAGE was 1:8 at most. The use of the varying methods to maximize the yield of DC therefore seems to have been unnecessary. Furthermore, for future modular reactions in which an asymmetric double-conjugate is desired, shortening the reaction time between the CPG-supported DNA and DTPA dianhydride might prove necessary to decrease the amount of symmetric DC formed between CPG-supported 
strands. The simultaneous addition of DTPA dianhydride and solubilized CTA ${ }^{+}$-DNA to the CPG-supported DNA could be attempted and reaction conditions optimized from there.

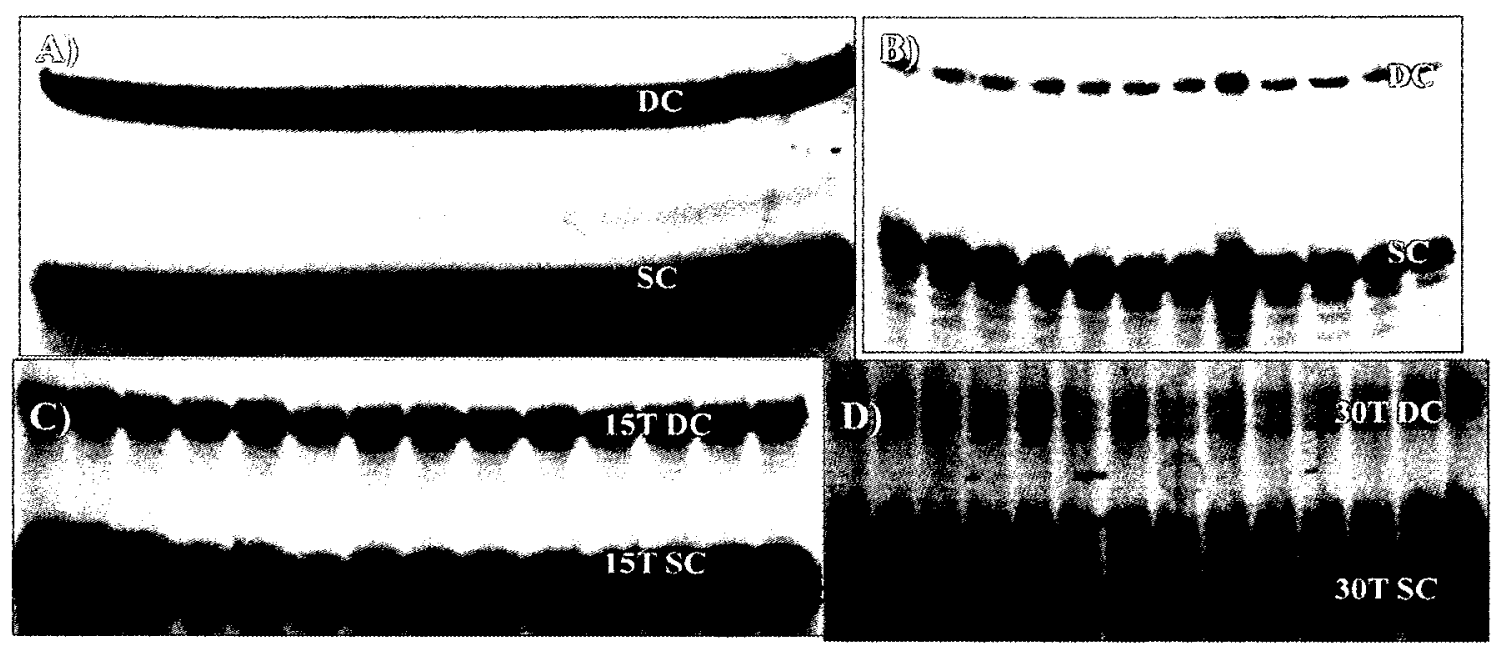

Figure 3.5 Representative polyacrylamide gels for the purification of DNA-DTPA conjugates. A) $\mathrm{SC}=$ single-conjugate, $\mathrm{DC}=$ double-conjugate, $19 \%$ gel, absorbance shows 1:2.5 ratio of $\mathrm{DC}$ to $\mathrm{SC}$ prepared with a single addition of DTPA dianhydride; B) 19\% gel, absorbance shows 1:9 ratio of DC to SC prepared using the modular method without the use of CTAB precipitation; C) $15 T$ SC $=15$ polyT SC, $15 \mathrm{~T}$ DC $=15$ polyT $\mathrm{DC}, 12 \%$ gel, absorbance shows $1: 6$ ratio of $15 \mathrm{~T}$ DC to $15 \mathrm{~T}$ SC prepared using the modular method; D) $30 \mathrm{~T} \mathrm{SC}=30$ polyT $\mathrm{SC}, 30 \mathrm{~T} \mathrm{DC}=30$ polyT $\mathrm{DC}, 12 \%$ gel, absorbance shows 1:15 ratio of 30T DC to 30T SC prepared using the modular method.

The conjugates prepared using the thrombin-binding aptamer with 15 and 30 polyT spacers were only obtained using the modular and modified one-pot reaction methods. The 1:6 ratio of $15 \mathrm{~T}$ DC to $15 \mathrm{~T}$ SC found for the gel in Figure $3.5 \mathrm{C}$ is representative of the best product ratio seen for these conjugates, as well as the only example where the ratio of products on the gel closely matched that of the conjugates isolated from the gel (1:5). The gel image was obtained during purification of a reaction 
performed using the modular method. Due to the low amount of 15T DC typically obtained following purification and desalting ( $40 \mathrm{nmol}$ or less), several reactions to obtain this double-conjugate were performed where the corresponding single-conjugate was not isolated following PAGE. This prevents a relative comparison of the two conjugates yields from being determined. Since there were only a small number of cases where both conjugates were isolated, a method that consistently resulted in more $\mathbf{1 5 T}$ DC relative to $15 \mathrm{~T}$ SC cannot be established. This will be addressed further below.

The 1:15 ratio of 30T DC to 30 T SC determined through the comparison of the absorbances on the gel in Figure 3.5D is lower than any of the ratios found upon isolation of these conjugates following PAGE. The best recovery of these conjugates resulted in a 1:35 ratio of $\mathbf{3 0 T}$ DC to $30 \mathrm{~T}$ SC following a separate modular reaction. Since the amount of 30T DC isolated from each reaction used to prepare it was typically about 12 nmol or less no matter what method was used to prepare it, several reactions were performed without the isolation of the corresponding single-conjugate following PAGE. It is therefore impossible to say whether either the modular or modified one-pot method resulted in a better relative yield of this double-conjugate.

Considering the faint absorbance of 30T DC on the gel in Figure 3.5D compared to the shorter double-conjugates (Figure 3.5A, B, and C), it is possible that the estimated absorbance from the gel is too high and therefore that the ratio of products based on the gel is too low. A decreased recovery of DNA from polyacrylamide gels with increasing length of the nucleic acid strands has been reported, as previously mentioned (section 1.5.1). ${ }^{83}$ This could also explain why so little 15T and 30T DC were recovered following 
PAGE purification and why the estimated ratios based on the gels were so different from those realized upon isolation of the conjugates.

Based on the collective data obtained and observations made throughout the synthesis of all of the DNA-DTPA conjugates, the overall success of the DNA synthesis, including the extent of amino-modification, the dryness of the DMSO, and the reactivity of the DTPA dianhydride were all more important factors than the method used for conjugate preparation. When newly acquired anhydrous DMSO and DTPA dianhydride were used in the reaction where an excess of DTPA dianhydride was added to the CPGsupported 15 mer thrombin-binding aptamer (as described above for the gel in Figure 3.5A), the lowest ratio of $\mathbf{D C}$ to $\mathbf{S C}$ out of all the reactions was obtained. Therefore, a more direct comparison between the methods would be more useful to determine the best one for the preparation of each double-conjugate. For example, both the modular and one-pot method could be performed simultaneously on DNA prepared during one synthesis.

\subsubsection{Preparation of DNA-DTPA double-conjugates for further experiments}

Following isolation and quantification of the double-conjugates eluted from polyacrylamide gels, they were chelated with Gd(III) using the method developed working with the single-conjugates. Once a test using xylenol orange demonstrated that a minimum percentage (less than $10 \%$ ) of a particular conjugate sample was not chelated to Gd(III), it was set aside for use in $T_{1}$ experiments or, in the case of DC-Gd(III), for a transmetallation study. 
Melting studies on 15T and 30T DC were also performed using the same conditions as those used for the corresponding single-conjugates, with the exception of the ratio of DC to polyA strands. As there are two DNA strands present in each doubleconjugate, the appropriate polyA strand was added in a 2:1 ratio with the conjugate. The results of the melting studies performed for $15 \mathrm{~T}$ and 30T DC are shown in Figure B-1 (Appendix B). They confirmed that hybridization occurred between the conjugates and their matching polyA strands under the conditions used, with melting temperatures between 30 and $40^{\circ} \mathrm{C}$ for $15 \mathrm{~T} \mathrm{DC}$ and between 55 and $60^{\circ} \mathrm{C}$ for $30 \mathrm{~T} \mathrm{DC}$.

\subsubsection{In vitro stability of DNA-DTPA double-conjugates: Transmetallation study}

It was mentioned earlier that the toxicity of the gadolinium(III) ion is not the only concern when considering the stabilities of gadolinium-based CAs to dechelation. There is also the connection between the administration of such CAs to patients with impaired renal function and the occurrence of nephrogenic systemic fibrosis (NSF) with which to be concerned. ${ }^{133,146,147}$ As discussed in section 2.1.2.4, it was determined that a majority of the high-risk patients who had developed NSF following injection with a gadoliniumbased CA had been given DTPA-BMA-Gd(III), the bisamide version of DTPA. ${ }^{134}$ The DNA-DTPA double-conjugates prepared in this work have the bisamide DTPA frame bound to two aptamers as a result of the conjugation reaction (see Figure 3.1). Therefore, when considering the stability of DC-Gd(III) to transmetallation by $\mathrm{Zn}(\mathrm{II})$, it is most relevant to compare the results to those reported for DTPA-BMA-Gd(III).

The transmetallation experiment was performed as described for SC-Gd(III) (section 2.3.5.3) except that two molar equivalents of $\mathrm{Zn}(\mathrm{II})$ were mixed with the DC- 
Gd(III). Since a 1:1 molar ratio of $\mathrm{Zn}(\mathrm{II})$ to a $\mathrm{Gd}(\mathrm{III})$-based CA is higher than what would be experienced in vivo, ${ }^{140}$ the use of the $1: 1$ and $2: 1$ ratios reported herein for SCGd(III) and DC-Gd(III), respectively, both result in an overestimation of the rate of transmetallation expected under biological conditions.

The results of the transmetallation study on DC-Gd(III) are shown in Figure 3.6. After being heated for about 3 hours at $37^{\circ} \mathrm{C}$ in the presence of $\mathrm{Zn}(\mathrm{II})$, about $15 \%$ of the initial relaxivity of the sample was lost as $\mathrm{Zn}(\mathrm{II})$ replaced $\mathrm{Gd}(\mathrm{III})$ in DC-Gd(III). Compared to the reported transmetallation of DTPA-BMA-Gd(III), which as illustrated in Figure 2.13 experienced about a $50 \%$ drop in relaxivity after the same amount of time, ${ }^{140}$ it seems that DC-Gd(III) initially displays higher stability. This could be due to steric hindrance resulting from the presence of the DNA strands since it has been reported that chelates with bulky amide substituents display kinetic stabilities more similar to the parent DTPA-Gd(III) chelate than to the bisamide derivative. ${ }^{137}$ In fact, following 3 hours of heating in the presence of $\mathrm{Zn}(\mathrm{II}), \mathrm{DTPA}-\mathrm{Gd}(\mathrm{III})$ was reported to lose about $15 \%$ of its relaxivity just as DC-Gd(III) did. ${ }^{140}$ Therefore, the initial stability of DC-Gd(III) to transmetallation by $\mathrm{Zn}$ (II) was approximately the same as for DTPA-Gd(III) and so was slightly worse than that found for SC-Gd(III) ( $~ 5 \%$ lost in 3 hours, Figure 2.14$)$.

Though the initial stability of DC-Gd(III) was determined to be better than DTPA-BMA-Gd(III), after about 25 hours (1500 minutes) the loss of relaxivity, and so of Gd(III), for the two chelates was approximately equal (between $75-80 \%$ loss). Furthermore, as the experiment was monitored for about another 50 hours, the results for DC-Gd(III) remained about the same as those for DTPA-BMA-Gd(III) since they both retained less than $10 \%$ of their initial relaxivities at the end of this period. This 
demonstrates considerably less stability to transmetallation than DTPA-Gd(III) as well as SC-Gd(III) after the same amount of time, with about 50 and $30 \%$ of their initial relaxivities retained, respectively.

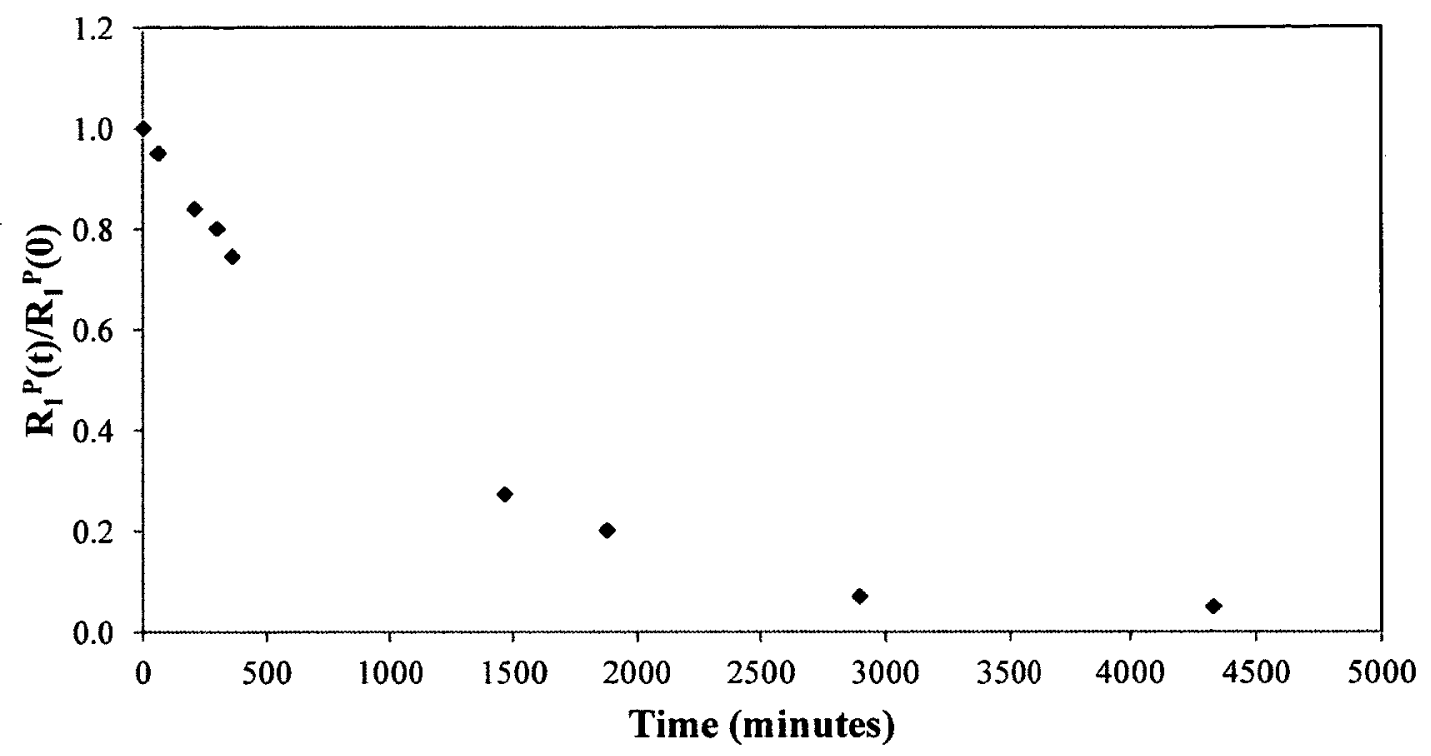

Figure 3.6 Relative paramagnetic longitudinal relaxivity, $R_{1}{ }^{\mathrm{P}}(t) / \mathbf{R}_{1}{ }^{\mathrm{P}}(0)$, of $\mathrm{DC}-\mathrm{Gd}(\mathrm{III})$ at $37^{\circ} \mathrm{C}$ and 9.4 $\mathrm{T}$ as a function of time after the addition of two molar equivalents of $\mathrm{Zn}(\mathrm{II})$.

It therefore seems that the favourable effect of steric hindrance conferred by the DNA strands for the first few hours of the transmetallation study could not compensate for the presence of the two nonionic amide groups on the DTPA framework as more time passed. It is suspected that the weaker binding of amide groups compared to carboxylate groups in open-chain chelates might permit greater flexibility and conformational mobility. ${ }^{104}$ The DNA aptamer strands might have helped prevent the normally rapid unraveling $^{104}$ of the DTPA-BMA backbone in DC-Gd(III) for the first few hours following the introduction of $\mathrm{Zn}(\mathrm{II})$, but steric hindrance alone does not seem able to 
entirely prevent the ligand from unwrapping itself around the Gd(III) ion. ${ }^{104}$ Eventually, the structure likely changed enough for the $\mathrm{Zn}(\mathrm{II})$ ions to have easier access to the pocket where Gd(III) was chelated, increasing the likelihood of transmetallation.

The clearance half-life of DTPA-BMA-Gd(III) is 1.3 hours where there is normal renal function, similar to the 1.6 hours for DTPA-Gd(III). ${ }^{105}$ Therefore, the higher stability of DC-Gd(III) to transmetallation in the first three hours of exposure to $\mathrm{Zn}$ (II) compared to DTPA-BMA-Gd(III) coupled with the lower dosage requirements for targeted CAs demonstrate the promise of using such a conjugate as a targeted MRI contrast agent in the future.

\subsection{5 $T_{1}$ experiments and relaxivity data at high field (9.4 Tesla)}

The conditions used in experiments performed on the DNA-DTPA singleconjugates at high field (section 2.3.6) were also used during the $T_{1}$ experiments performed on the double-conjugates.

$\mathrm{T}_{1}$ experiments for the three double-conjugates prepared, DC-Gd(III), 15T DCGd(III), and 30T DC-Gd(III), were performed in triplicate on a $9.4 T$ NMR spectrometer for four or five concentrations of each sample series. Each DNA-DTPA double-conjugate was run alone in solution in one of these series. It was then necessary to run four series in which protein were included for each double-conjugate, two including thrombin and two including pepsin (the control). This was due to the presence of two aptamer strands in each individual conjugate molecule. As illustrated in Figure $3.7 \mathrm{~A}$, if a 2 to 1 ratio of protein to DC was used in preparation of a sample, there would theoretically be one protein for each aptamer strand in the sample. Therefore each 
double-conjugate was run in a control and a target series where the respective protein was present at twice the concentration of the conjugate. Considering the possible future use of DNA-DTPA asymmetrical double-conjugates where only one of the DNA strands might be an aptamer for a target relevant to imaging, the inclusion of the control or target protein in an equimolar concentration with each conjugate was also tested. This is represented in Figure 3.7B and these two series were the last two obtained for each double-conjugate.

A)
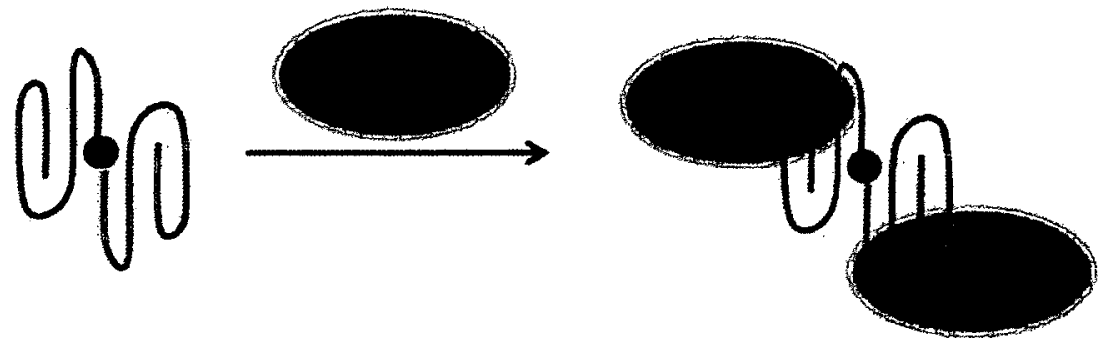

B)
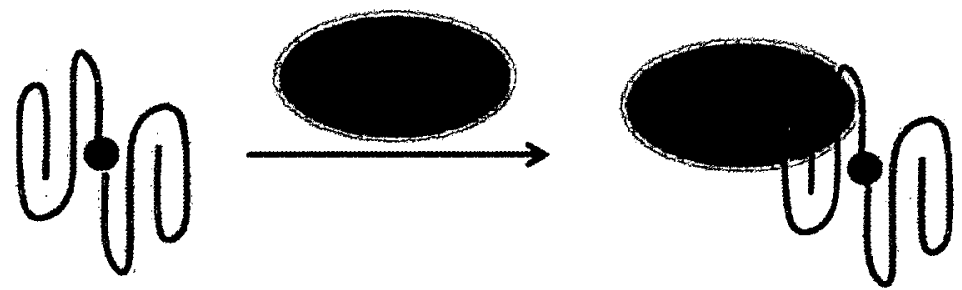

Thrombin aptamer 3'-GGT TGG TGT GGT TGG-5'

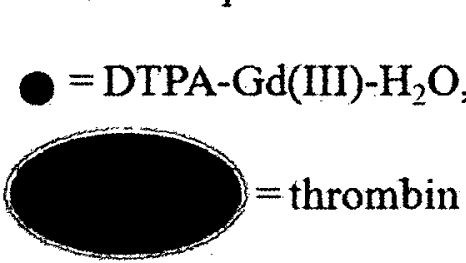

Figure 3.7 Schematic of the two possible interactions between the double-conjugates and thrombin. A) 2:1 ratio of protein to conjugate, every aptamer could bind thrombin; B) 1:1 ratio of protein to conjugate, one aptamer in each conjugate could bind to thrombin. 
Results from the DC-Gd(III) experiments are summarized in Table 3.2 and Figure 3.8, where the relaxivity of Magnevist ${ }^{\circledR}$ obtained during the single-conjugate experiments is included for comparison. The relaxivity plots used to obtain this data are shown in Figure B-2.

Table 3.2 Relaxivity Data for Magnevist 8 and DC-Gd(III) at 9.4 T, pH 7.4 and $25^{\circ} \mathrm{C}$.

\begin{tabular}{|c|c|c|c|}
\hline Sample & Relaxivity $\left(\mathrm{mM}^{-1} \mathrm{~s}^{-1}\right)$ & Protein & Enhancement of relaxivity $(\%)$ \\
\hline Magnevist $\circledast$ & $5.03 \pm 0.06$ & - & - \\
\hline \multirow{5}{*}{ DC-Gd(III) } & $6.0 \pm 0.1$ & - & $20 \pm 3^{[\mathrm{a}]}$ \\
\hline & $6.9 \pm 0.1$ & Pepsin (2:1) & - \\
\hline & $6.3 \pm 0.2$ & Thrombin $(2: 1)$ & $-8 \pm 3^{[b]}$ \\
\hline & $5.6 \pm 0.1$ & Pepsin $(1: 1)$ & - \\
\hline & $6.4 \pm 0.2$ & Thrombin (1:1) & $13 \pm 3^{[c]}$ \\
\hline \multicolumn{4}{|c|}{$\begin{array}{l}\text { [b] Comparing relaxivity of DC-Gd(III) with thrombin (2:1) to that of } \mathbf{D C}-\mathbf{G d}(\mathbf{I I I}) \text { with pepsin } \\
(2: 1)\end{array}$} \\
\hline \multicolumn{4}{|c|}{$\begin{array}{l}\text { [c] Comparing relaxivity of } \mathbf{D C}-\mathbf{G d}(\mathbf{I I I}) \text { with thrombin (1:1) to that of } \mathbf{D C}-\mathbf{G d}(\mathbf{I I I}) \text { with pepsin } \\
(1: 1)\end{array}$} \\
\hline
\end{tabular}

The rotational correlation time, $\tau_{R}$, of DC-Gd(III) would be expected to be larger than that of SC-Gd(III) with the possibility of an increase in relaxivity as a result. The relaxivity of DC-Gd(III), however, is slightly lower than that of SC-Gd(III), at $6.0 \pm 0.1$ versus $6.36 \pm 0.07 \mathrm{mM}^{-1} \mathrm{~s}^{-1}$. An increase in relaxivity similar to that for SC-Gd(III) was therefore found for DC-Gd(III) when compared to Magnevist ${ }^{\circledR}(26 \pm 2 \%$ versus $20 \pm 3 \%$, respectively) even though the double-conjugate is almost twice the mass, and so about twice the size, of the single-conjugate. An increased $\tau_{R}$ seemingly could not make up for the decreased water exchange rate that would be expected for a bisamide derivative of 
DTPA. Recall that a decrease by about a factor of four has been reported for the replacement of each carboxylate group in a DTPA frame by an amide. ${ }^{126}$ Therefore, the water exchange rates experienced by the double-conjugates would be expected to be lower than those of the single-conjugates which were monoamide derivatives of DTPA.

In section 2.3.6, equations 2.6 to 2.11 (section 2.1.2.2) ${ }^{125}$ were used to theoretically predict the rotational correlation time $\tau_{R}$ for SC-Gd(III), resulting in a value of the longitudinal inner sphere relaxation rate, $\frac{1}{T_{1 m}}$, that was nearly equal to the experimental values found for the conjugate alone and in the presence of thrombin. The reported lifetime of the inner-sphere water molecules, $\tau_{m}$, for DTPA-MA is on the order of $10^{-7}$ seconds, ${ }^{126}$ so the value of $\tau_{R}$ was adjusted from that reported for DTPA-MA until the value of $\frac{1}{T_{1 m}}$ at this residence time was similar to that obtained experimentally. The same approach was taken for calculations performed in an attempt to theoretically predict $\tau_{R}$ for DC-Gd(III) but using values corresponding to DTPA-BMA-Gd(III) instead of the monoamide derivative. The reported $\tau_{m}$ for DTPA-BMA-Gd(III) is on the order of $10^{-6}$ seconds while the $\tau_{R}$ is on the order of $10^{-12}$ seconds $(167 \mathrm{ps}) .{ }^{126}$ The terms related to electronic relaxation $\left(\Delta^{2}\right.$ at $0.38 \times 10^{20} \mathrm{~s}, \tau_{\mathrm{v}}$ at $\left.34 \mathrm{ps}\right)$ were held steady during the calculations since, as mentioned earlier, electronic relaxation has less of a pronounced effect on the observed relaxivity at high fields than at low fields. ${ }^{100,101}$

The highest concentration of DC-Gd(III) used for the determination of $T_{1}$ was $0.05 \mathrm{mM}$; the theoretical calculation of $\frac{1}{T_{1 m}}$ was therefore performed using this concentration. The conjugate alone experimentally gave a value of $0.38 \mathrm{~s}^{-1}$ at this 
concentration, while with thrombin (in both ratios tested) the value of $\frac{1}{T_{1 m}}$ was about $0.40 \mathrm{~s}^{-1}$. The mass of DC-Gd(III) is approximately 20 times higher than that of DTPABMA-Gd(III), so if the motion experienced by the chelate moiety was coupled to the overall global motion of the conjugate the effective $\tau_{R}$ would be expected to be about 20 times larger than that of DTPA-BMA-Gd(III). Recall, however, that the flexible $\mathrm{C}_{6}$ linkers used to connect the DNA strands to the DTPA moiety can freely rotate and so result in partial or complete decoupling of the local motion experienced by the Gd(III) chelate from the global motion of the entire conjugate (section 2.3.6). ${ }^{124}$ In the case of DC-Gd(III), the use of the modified Bloembergen and Solomon equations ${ }^{100,125}$ did not allow for theoretical values of $\frac{1}{T_{1 m}}$ that matched those found experimentally to be determined except where $\tau_{m}$ was on the order of $10^{-9}$ seconds. For $\tau_{m}$ values on the order of $10^{-6} \mathrm{~s}$, like that reported for the bisamide derivative of DTPA, the highest value of $\frac{1}{r_{1 m}}$ achievable using equations 2.6 to 2.11 was about $0.26 \mathrm{~s}^{-1}$ when the reported $\tau_{R}$ of DTPA-BMA-Gd(III) was multiplied by two. The failure of these equations to model the experimental results could be due to the greater influence of internal motion in DCGd(III) on the relaxivity of the agent when compared to SC-Gd(III). Modified equations that incorporate the Lipari-Szabo model-free approach ${ }^{129,160,177}$ might be more useful in modelling the DC-Gd(III) results, however experimental data obtained using nuclear magnetic relaxation dispersion (NMRD) would provide more substantial information about the values of $\tau_{m}$ and $\tau_{R}$ for the system ${ }^{127,129}$ and would therefore prove more helpful in understanding its relaxation properties. 
The relaxation enhancements observed for DC-Gd(III) in the presence of one and two molar equivalents of thrombin when compared to the corresponding pepsin case were $13 \pm 3 \%$ and $-8 \pm 3 \%$, respectively. Therefore, under the conditions used an enhancement is only found in the case where, on average, one of the aptamers is interacting with thrombin (see Figure 3.7). It seems that the four times increase in mass with the interaction of DC-Gd(III) with one thrombin molecule is able to overcome the negative effect the addition of the protein has on the viscosity of the solution, as demonstrated by the drop in relaxivity with the addition of pepsin in a $1: 1$ ratio with the conjugate $(5.6 \pm 0.1$ versus $6.0 \pm 0.1 \mathrm{mM}^{-1} \mathrm{~s}^{-1}$ for DC-Gd(III), see Table 3.2 and Figure 3.8). The addition of two molar equivalents of pepsin, however, shows the opposite effect on viscosity with an increased relaxivity compared to that of the conjugate alone. Recall that viscosity does not affect relaxivity in a linear manner and so its effects are not readily predicted (section 2.3.6). A drop in relaxivity between the pepsin and thrombin cases where two molar equivalents of protein are present compared to DC-Gd(III) demonstrates that the two times greater mass, and therefore approximately two times greater size, when two thrombin molecules are bound is detrimental to the overall relaxivity. It is possible that the two proteins held in such close proximity to the DTPA-Gd(III) chelate negatively affected water exchange due to steric effects. The decreased relaxivities of DC-Gd(III) alone and with thrombin compared to those for SC-Gd(III) (Table 2.3) could be the combined result of a lower water exchange rate, steric effects, and greater internal motion.

The inclusion of 15 and 30 polyT spacers in the $15 \mathrm{~T}$ and 30T DNA-DTPAGd(III) conjugates was used to test whether holding the folded thrombin-binding aptamer 
farther away from the Gd(III) chelate on average might help improve the rate of water exchange by reducing any possible steric hindrance (see Figure 2.15 ). The hybridization of the polyT spacers with matching polyA DNA strands was also tested as a means of rigidifying the spacer portion and so further ensuring that the folded aptamer was held away from the chelate.

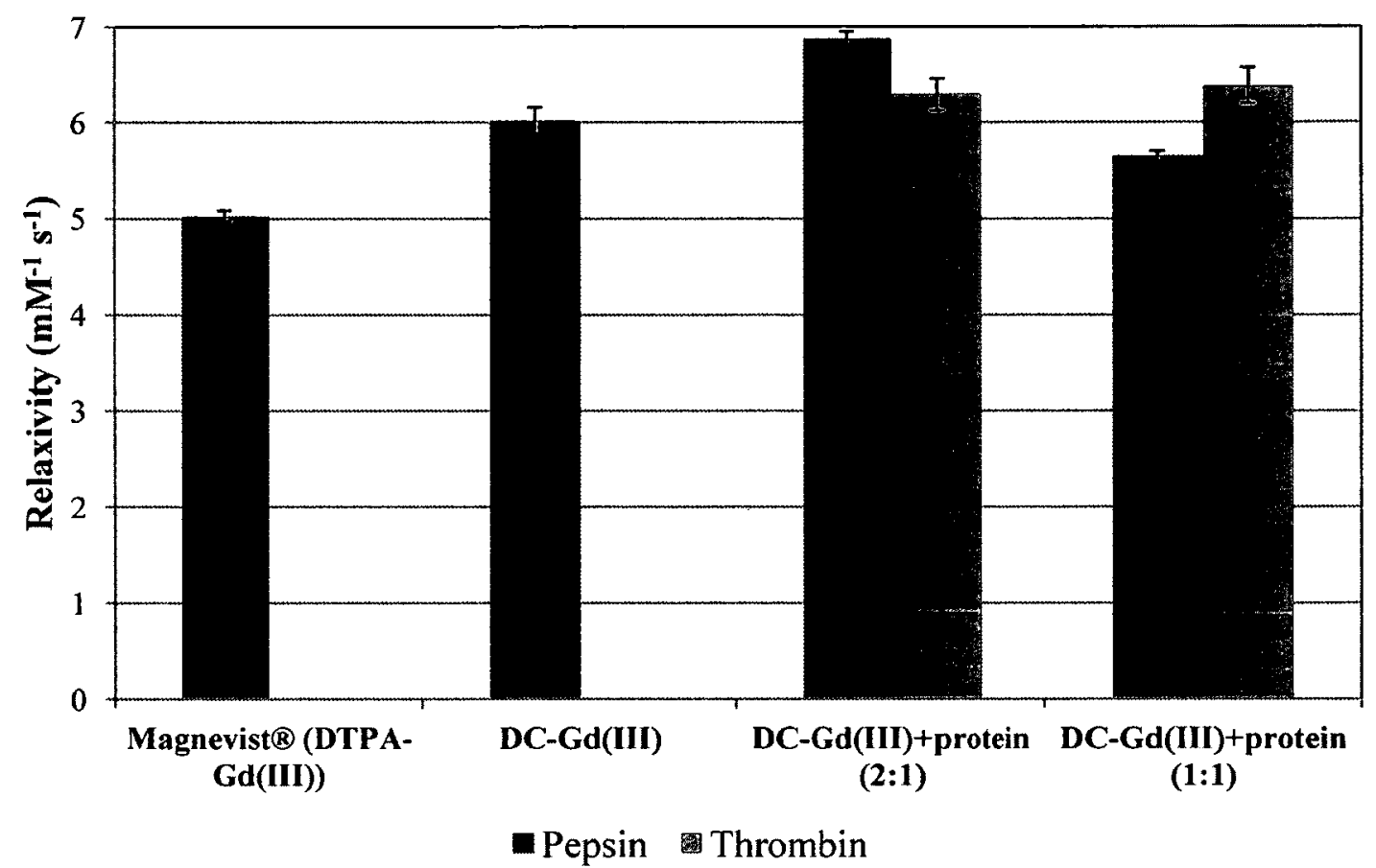

Figure 3.8 Chart of the relaxivity values for Magnevist ${ }^{\circledR}$ and DC-Gd(III) at $9.4 \mathrm{~T}, \mathrm{pH} 7.4$ and $25^{\circ} \mathrm{C}$. Pepsin is only present where applicable.

The relaxivity data for $\mathbf{1 5 T}$ DC-Gd(III) is summarized in Table 3.3 and Figure 3.9 (the relaxivity plots are shown in Figures B-3 and B-4). It can be seen that overall the relaxivities for this conjugate are higher than those of DC-Gd(III). Furthermore, the only single-conjugate that had some relaxivity values at about $8 \mathrm{mM}^{-1} \mathrm{~s}^{-1}$ was $30 \mathrm{~T}$ SC- 
Gd(III). These are the highest relaxivity values found for any of the DNA-DTPA conjugates prepared, including 30T DC-Gd(III) which will be discussed shortly. It can be seen that 15T DC-Gd(III) alone and hybridized with matching polyA strands showed relaxivity enhancements over that of Magnevist ${ }^{\circledR}$ of $62 \pm 6$ and $74 \pm 5 \%$, comparable only to the enhancements found for 30T SC-Gd(III) (see Table 2.5).

Table 3.3 Relaxivity Data for 15T DC-Gd(III) at $9.4 \mathrm{~T}, \mathrm{pH} 7.4$ and $25^{\circ} \mathrm{C}$.

\begin{tabular}{|c|c|c|c|}
\hline Sample & Relaxivity $\left(\mathrm{mM}^{-1} \mathrm{~s}^{-1}\right)$ & Protein & Enhancement of relaxivity (\%) \\
\hline \multirow{5}{*}{ 15T DC-Gd(III) } & $8.1 \pm 0.3$ & - & $62 \pm 6^{[a]}$ \\
\hline & $8.7 \pm 0.4$ & Pepsin (2:1) & - \\
\hline & $10.1 \pm 0.4$ & Thrombin $(2: 1)$ & $17 \pm 7^{[b]}$ \\
\hline & $9.0 \pm 0.4$ & Pepsin $(1: 1)$ & - \\
\hline & $13 \pm 1$ & Thrombin (1:1) & $47 \pm 15^{[\mathrm{c}]}$ \\
\hline \multirow{5}{*}{$\begin{array}{c}\text { 15T DC-Gd(III) } \\
+ \text { polyA }\end{array}$} & $8.7 \pm 0.3$ & - & $74 \pm 5^{[d]}$ \\
\hline & $8.5 \pm 0.2$ & Pepsin (2:1) & - \\
\hline & $9.33 \pm 0.07$ & Thrombin $(2: 1)$ & $10 \pm 3^{[\mathrm{e}]}$ \\
\hline & $9.6 \pm 0.2$ & Pepsin (1:1) & - \\
\hline & $8.9 \pm 0.1$ & Thrombin $(1: 1)$ & $-7 \pm 2^{[f]}$ \\
\hline \multicolumn{4}{|c|}{$\begin{array}{l}\text { [b] Comparing relaxivity of } \mathbf{1 5 T} \text { DC-Gd(III)with thrombin }(2: 1) \text { to that of } \mathbf{1 5 T} \text { DC-Gd(III) } \\
\text { with pepsin }(2: 1)\end{array}$} \\
\hline \multicolumn{4}{|c|}{$\begin{array}{l}\text { [c] Comparing relaxivity of } \mathbf{1 5 T} \text { DC-Gd(III) with thrombin }(1: 1) \text { to that of } \mathbf{1 5 T} \mathbf{D C}-\mathbf{G d}(\mathbf{I I I}) \\
\text { with pepsin }(1: 1)\end{array}$} \\
\hline \multicolumn{4}{|c|}{ [d] Comparing relaxivity of $\mathbf{1 5 T} \mathbf{D C}-\mathbf{G d}(\mathbf{I I I})+$ polyA to that of DTPA-Gd(III) } \\
\hline \multicolumn{4}{|c|}{$\begin{array}{l}\text { [e] Comparing relaxivity of } \mathbf{1 5 T} \text { DC-Gd(III) + polyA with thrombin }(2: 1) \text { to that of } \mathbf{1 5 T} \text { DC- } \\
\text { Gd(III) + polyA with pepsin }(2: 1)\end{array}$} \\
\hline \multicolumn{4}{|c|}{$\begin{array}{l}\text { [f] Comparing relaxivity of } \mathbf{1 5 T} \text { DC-Gd(III) }+ \text { polyA with thrombin }(1: 1) \text { to that of } \mathbf{1 5 T} \text { DC- } \\
\text { Gd(III) }+ \text { polyA with pepsin }(1: 1)\end{array}$} \\
\hline
\end{tabular}

The size of 15T DC-Gd(III) seems to be uniquely suitable for balancing all of the parameters that contribute to the relaxivity of the DNA-DTPA conjugate systems studied 
herein. Though the solutions of this conjugate with two molar equivalents of thrombin would be expected to be the most crowded considered thus far, both from the size of the conjugate itself and the presence of the protein, it still experienced a net enhancement of

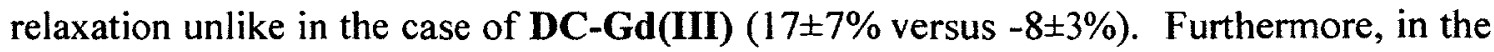
presence of one molar equivalent of protein, the greatest enhancement of relaxivity for any of the conjugates studied was achieved: $47 \pm 15 \%$.

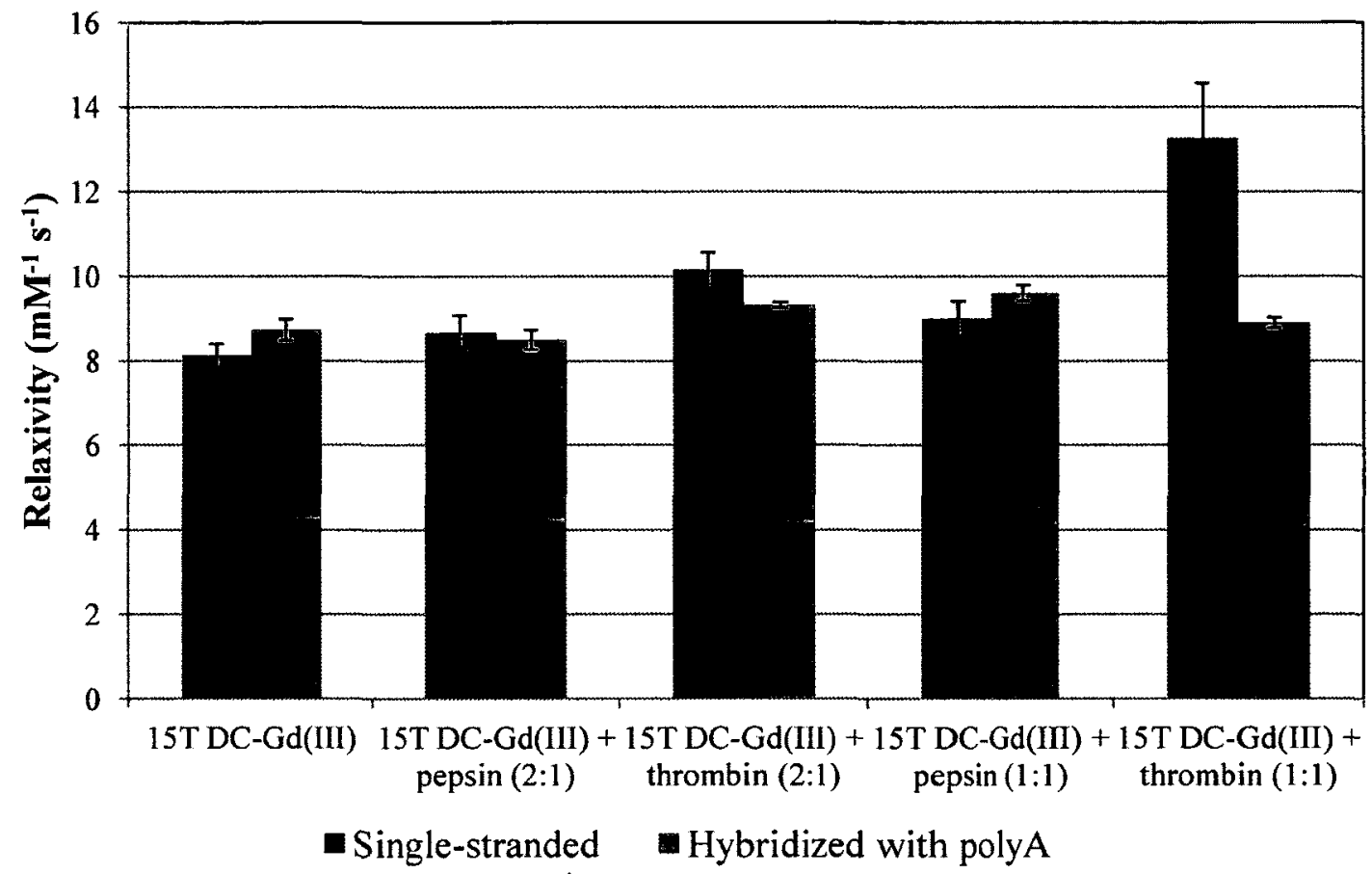

Figure 3.9 Chart of the relaxivity values for $15 \mathrm{~T}$ DC-Gd(III) at $9.4 \mathrm{~T}, \mathrm{pH} 7.4$ and $25^{\circ} \mathrm{C}$.

The flexibility of the 15 polyT spacers does not greatly affect this conjugate. Figure 3.9 demonstrates that it was only in the case where 15T DC-Gd(III) was mixed in a 1:1 ratio with thrombin that its hybridization with matching polyA strands proved significantly detrimental to the relaxivity achieved. This is the opposite of what was 
found for the corresponding single-conjugate; in that case, the more rigid hybridized form showed a slightly better relaxation enhancement than the single-stranded case.

Based on the observation that hybridization only greatly affects the one sample series, the average size of 15 T DC-Gd(III) when interacting with one thrombin molecule, and the resulting effective $\tau_{R}$ experienced by the $\mathrm{Gd}(\mathrm{III})$ chelate, seems to be the most significant parameter for this conjugate. When bound to one thrombin, $\mathbf{1 5 T}$ DC-Gd(III) only increases in size three-fold while when bound to two thrombin molecules it would increase in size about five-fold. Particularly at high field, however, a greater $\tau_{R}$ is not necessarily beneficial; intermediate values have been found to be most effective for achieving high relaxivities. ${ }^{124}$ This could be why the hybridization of $15 T$ DC-Gd(III) with the polyA strands proves so detrimental when the proteins are present in one molar equivalent. The addition of the two 15 polyA strands would be expected to rigidify the poly $\mathrm{T}$ spacer portions of the conjugate, as previously discussed. This would result in the agent being larger overall and its experiencing a different global tumbling rate than the single-stranded version. If the tumbling rate of the single-stranded conjugate combined with the internal motion of the $C_{6}$ linkers joining the DNA and the DTPA moieties to give the most optimal $\tau_{R}$ of any of the possible CAs studied here, then any significant changes, such as the hybridization of two polyA strands, could be detrimental to the relaxivity observed.

The 15T DC-Gd(III) conjugate therefore proved to show the greatest relaxation enhancement of all the agents tested, with its superior performance when only binding one target molecule suggesting that the dosage of such agents might need to be carefully optimized to obtain the best possible contrast in MR images. 
Table 3.4 Relaxivity Data for 30T DC-Gd(III) at 9.4 T, pH 7.4 and $25^{\circ} \mathrm{C}$.

\begin{tabular}{|c|c|c|c|}
\hline Sample & Relaxivity $\left(\mathrm{mM}^{-1} \mathrm{~s}^{-1}\right)$ & Protein & Enhancement of relaxivity (\%) \\
\hline \multirow{5}{*}{ 30T DC-Gd(III) } & $5.2 \pm 0.3$ & - & $3 \pm 6^{[\mathrm{a}]}$ \\
\hline & $7.11 \pm 0.10$ & Pepsin $(2: 1)$ & - \\
\hline & $4.9 \pm 0.4$ & Thrombin (2:1) & $-31 \pm 6^{[\mathrm{b}]}$ \\
\hline & $5.4 \pm 0.4$ & Pepsin (1:1) & - \\
\hline & $5.6 \pm 0.5$ & Thrombin $(1: 1)$ & $4 \pm 13^{[c]}$ \\
\hline \multirow{5}{*}{$\begin{array}{l}\text { 30T DC-Gd(III) } \\
+ \text { polyA }\end{array}$} & $5.1 \pm 0.8$ & - & $2 \pm 15^{[\mathrm{d}]}$ \\
\hline & $5.7 \pm 0.4$ & Pepsin (2:1) & - \\
\hline & $5.3 \pm 0.6$ & Thrombin $(2: 1)$ & $-7 \pm 14^{[\mathrm{e}]}$ \\
\hline & $4.7 \pm 0.4$ & Pepsin (1:1) & - \\
\hline & $5.4 \pm 0.1$ & Thrombin $(1: 1)$ & $15 \pm 10^{[f]}$ \\
\hline \multicolumn{4}{|c|}{ [a] Comparing relaxivity of $30^{\prime} \mathbf{T} \mathbf{D C}-\mathbf{G d}(\mathbf{I I I})$ to that of DTPA-Gd(III) } \\
\hline \multicolumn{4}{|c|}{$\begin{array}{l}\text { [b] Comparing relaxivity of } \mathbf{3 0 T} \text { DC-Gd(III)with thrombin }(2: 1) \text { to that of } 30 \text { TC-Gd(III) } \\
\text { with pepsin }(2: 1) \\
\text { [c] Comparing relaxivity of } \mathbf{3 0 T} \mathbf{D C}-\mathbf{G d}(\mathbf{I I I}) \text { with thrombin }(1: 1) \text { to that of } 30 \text { T } \mathbf{D C}-\mathbf{G d}(\mathbf{I I I}) \\
\text { with pepsin }(1: 1)\end{array}$} \\
\hline \multicolumn{4}{|c|}{ [d] Comparing relaxivity of $\mathbf{3 0 T} \mathbf{D C}-\mathbf{G d}(\mathrm{III})+$ polyA to that of DTPA-Gd(III) } \\
\hline \multicolumn{4}{|c|}{$\begin{array}{l}\text { [e] Comparing relaxivity of } 30 \text { T DC-Gd(III) + polyA with thrombin }(2: 1) \text { to that of } 30 \text { T DC- } \\
\text { Gd(III) + polyA with pepsin }(2: 1)\end{array}$} \\
\hline \multicolumn{4}{|c|}{$\begin{array}{l}\text { [f] Comparing relaxivity of } \mathbf{3 0 T} \text { DC-Gd(III) + polyA with thrombin }(1: 1) \text { to that of } \mathbf{3 0 T} \text { DC- } \\
\text { Gd(III) + polyA with pepsin }(1: 1)\end{array}$} \\
\hline
\end{tabular}

The results of the relaxivity studies performed on 30T DC-Gd(III) further demonstrate that, at least under the conditions used, the optimal tumbling rate and net $\tau_{R}$ for the DNA-DTPA conjugates seems to have been achieved in the 1:1 mixtures of 15T DC-Gd(III) and thrombin and increasing the size further is unnecessary or detrimental. This is obvious when considering the relaxation enhancements reported in Table 3.4. Not only does 30T DC-Gd(III) not improve on the relaxivity of Magnevist ${ }^{\circledR}$ despite its 55fold larger mass, none of the enhancements of relaxivity determined are significant. The 
errors found for all of the relaxivities are high $\left(0.1 \mathrm{mM}^{-1} \mathrm{~s}^{-1}\right.$ or greater $)$ and the relaxivities are almost all nearly the same as that of Magnevist $\left(5.03 \pm 0.06 \mathrm{mM}^{-1} \mathrm{~s}^{-1}\right)$. This suggests that the global and internal motions for this conjugate could be fully decoupled and that the net $\tau_{R}$ experienced by the DTPA-Gd(III) chelate is that of the chelate alone. Since the $\tau_{R}$ of small molecule agents such as DTPA-Gd(III) and DTPABMA-Gd(III) are very low (i.e. fast tumbling) and intermediate $\tau_{R}$ values are more desirable at high fields, ${ }^{124}$ the relaxivities of the conjugate under fully decoupled conditions remain low.

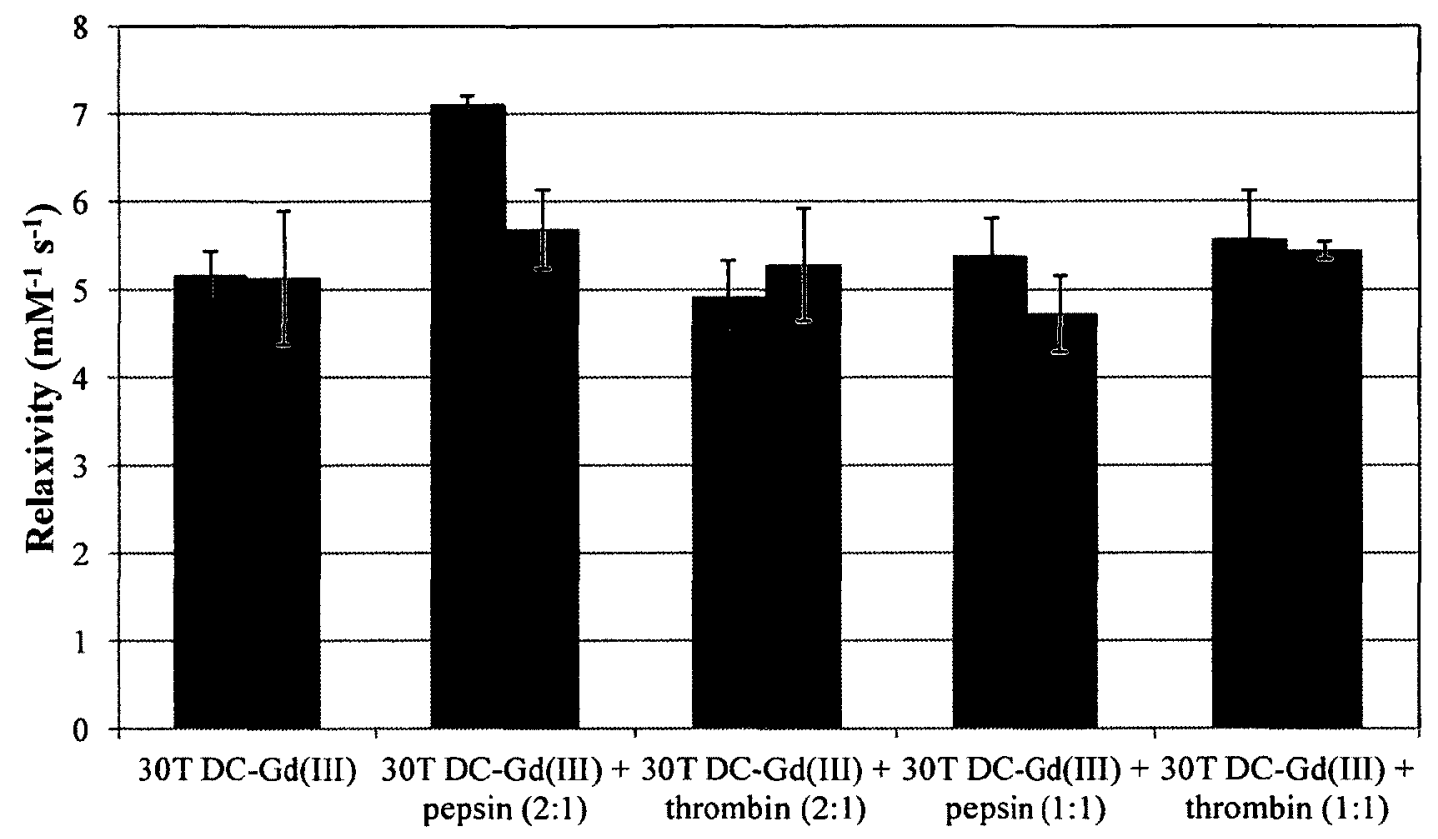

- Single-stranded Hybridized with polyA

Figure 3.10 Chart of the relaxivity values for $30 \mathrm{~T}$ DC-Gd(III) at $9.4 \mathrm{~T}, \mathrm{pH} 7.4$ and $25^{\circ} \mathrm{C}$.

The hybridization of the conjugate with matching polyA strands might be slightly beneficial, but due to the high error values it is impossible to say for sure. The relaxivity 
plots are shown in Figures B-5 and B-6 and the data is illustrated in Figure 3.10, further demonstrating that little change was observed among the relaxivities determined. The viscosity change upon the addition of two molar equivalents of pepsin seems to slightly enhance the relaxivity of 30T DC-Gd(III) in what is the only example of a change in the conditions to significantly affect its relaxivity but in an example that does not actually lead to a beneficial change that might be used in targeted imaging.

\subsection{6 $T_{1}$ experiments and relaxivity data at low field (1.5 Tesla)}

\subsubsection{Experiments on 15T DC-Gd(III)}

15T DC-Gd(III) was the first double-conjugate to be prepared for $T_{1}$ experiments at low field. The typically low yields of the double-conjugates restricted the amount of 15 TC-Gd(III) available for these experiments to about $35 \mathrm{nmol}$. As a result, lower total sample volumes needed to be used in order to obtain sample concentrations similar to those in the experiments on SC-Gd(III) at low field (section 2.3.7). Therefore, instead of using cuvettes to hold the sample solutions, PCR tubes that were completely filled by $280 \mu \mathrm{L}$ samples were used and taped together with each row holding a different sample set.

The data obtained from the $T_{1}$ experiments on 15T DC-Gd(III) alone and in a $1: 1$ ratio with thrombin and pepsin is summarized in Table 3.5 and Figure A-7. Though the concentration range used for the sample series tested here was about the same as that used in the $1.5 \mathrm{~T}$ experiments on SC-Gd(III), the reduced sample volume resulted in lower sensitivity. This is particularly evident upon consideration of the relaxivity curves in Figure A-7. All of the samples being run on the 1.5 T MRI spectrometer are very 
small when compared to the bore of the MRI magnet and it seems that the use of $280 \mu \mathrm{L}$ samples decreases the signal-to-noise ratio compared to the use of $1 \mathrm{~mL}$ samples. The results obtained for $\mathbf{1 5 T} \mathbf{D C}$-Gd(III) at $1.5 \mathrm{~T}$ are promising, with an enhancement of relaxivity of $56 \pm 4 \%$ calculated where thrombin was present compared to the conjugate with pepsin. This is a similar result to that obtained at $9.4 \mathrm{~T}(47 \pm 15 \%)$ and it suggests that the net $\tau_{R}$ of this conjugate is also highly effective at low field.

Table 3.5 Relaxivity Data for 15T DC-Gd(III) at $1.5 \mathrm{~T}$, pH 7.4 and $25^{\circ} \mathrm{C}$.

\begin{tabular}{|c|c|c|c|}
\hline Sample & Relaxivity $\left(\mathrm{mM}^{-1} \mathrm{~s}^{-1}\right)$ & Protein & Enhancement of relaxivity (\%) \\
\hline \multirow{3}{*}{$\begin{array}{c}\text { 15T DC- } \\
\text { Gd(III) }\end{array}$} & $5.6 \pm 0.1$ & - & - \\
\cline { 2 - 4 } & $5.5 \pm 0.2$ & Pepsin (1:1) & - \\
\cline { 2 - 4 } & $8.5 \pm 0.1$ & Thrombin $(1: 1)$ & $56 \pm 4^{[\mathrm{a}]}$ \\
\hline
\end{tabular}

[a] Comparing relaxivity of $\mathbf{1 5 T}$ DC-Gd(III) with thrombin (1:1) to that of $\mathbf{1 5 T}$ DC-Gd(III) with pepsin $(1: 1)$

A)

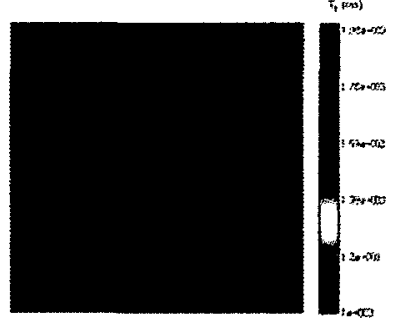

B)

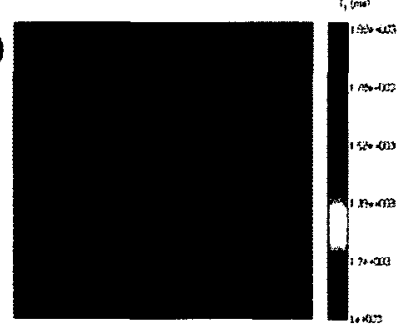

C)

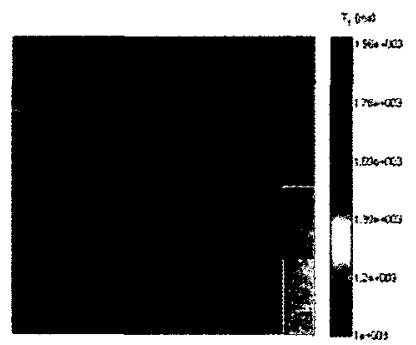

Figure 3.11 Representative $T_{1}$ maps of $0.0134 \mathrm{mM}$ solutions of A) $15 \mathrm{~T}$ DC-Gd(III), B) $15 T$ DCGd(III)+pepsin, and C) 15T DC-Gd(III)+thrombin. An equimolar amount of protein is present, where applicable. Experiments were performed at $1.5 \mathrm{~T}, \mathrm{pH} 7.4$ and $25^{\circ} \mathrm{C}$. 
The $T_{1}$ maps shown in Figure 3.11 illustrate that there is a detectable change as $0.0134 \mathrm{mM}$ solutions of 15T DC-Gd(III) alone (A), with pepsin (B), and with thrombin (C) are considered. The visual change, however, does not seem as great as would be expected using an agent that proved to have the highest relaxation enhancement at $9.4 \mathrm{~T}$ and an enhancement of $56 \pm 4 \%$ at $1.5 \mathrm{~T}$. The sensitivity of the instrument when using the low volume samples in PCR tubes is likely why a greater difference is not observed in the $T_{1}$ maps.

\subsubsection{Experiments on $D C-G d(I I I)$}

Samples of DC-Gd(III) were prepared for study at $1.5 \mathrm{~T}$ with a total volume of 1 $\mathrm{mL}$ since the use of low volume samples of 15 T DC-Gd(III) in PCR tubes seemed to result in decreased sensitivity. The conditions therefore matched those used for SCGd(III) in low field experiments. DC-Gd(III) was used since it also demonstrated relaxation enhancement when mixed in a 1:1 ratio with thrombin compared to the control at $9.4 \mathrm{~T}(13 \pm 3 \%$, Table 3.2$)$, and it could be isolated in larger amounts than $15 \mathrm{~T}$ DCGd(III). The relaxivity results are summarized in Table 3.6 and the relaxivity plots are shown in Figure B-8.

The relaxation enhancement found for DC-Gd(III) over Magnevist $\circledast$ at $1.5 \mathrm{~T}$ is $27 \pm 2 \%$, which is similar to the value found at $9.4 \mathrm{~T}(20 \pm 3 \%)$. The enhancements seen between the relaxivities obtained in the presence of the control (pepsin) and thrombin at high and low fields are also the same within error ( $13 \pm 3 \%$ versus $11 \pm 1 \%$, respectively). These results further support the discussion above with regards to the failure to theoretically match the experimental results using the modified Bloembergen and 
Solomon equations. ${ }^{125}$ If the chelate is experiencing a great deal of internal motion, calculations that take this into account are likely necessary to match the experimental values. Recall that small CAs such as DTPA-Gd(III) and DTPA-BMA-Gd(III) have similar relaxivities over a wide range of magnetic field strengths. ${ }^{124,158}$ If the chelate in DC-Gd(III) is greatly decoupled from the global motion of the agent, it would help explain why the relaxivities found for this agent at $1.5 \mathrm{~T}$ are about the same as those found at 9.4 T when the RIME effect tends to have a greater influence at low fields. ${ }^{124}$

Table 3.6 Relaxivity Data for DC-Gd(III) at $1.5 \mathrm{~T}$, pH 7.4 and $25^{\circ} \mathrm{C}$.

\begin{tabular}{|c|c|c|c|}
\hline Sample & Relaxivity $\left(\mathrm{mM}^{-1} \mathrm{~s}^{-1}\right)$ & Protein & Enhancement of relaxivity (\%) \\
\hline Magnevist@ & $2.75 \pm 0.05$ & - & $27 \pm 2^{[\mathrm{a}]}$ \\
\hline \multirow{3}{*}{ DC-Gd(III) } & $3.49 \pm 0.04$ & - & - \\
\cline { 2 - 4 } & $6.09 \pm 0.04$ & Pepsin $(1: 1)$ & - \\
\cline { 2 - 4 } & $6.77 \pm 0.04$ & Thrombin $(1: 1)$ & $11 \pm 1^{[\mathrm{b}]}$ \\
\hline
\end{tabular}

[a] Comparing relaxivity of DC-Gd(III) to that of Magnevist $\otimes$

[b] Comparing relaxivity of DC-Gd(III) with thrombin (1:1) to that of DC-Gd(III) with pepsin $(1: 1)$

A brief test using equations 2.6 to 2.11 was performed to determine if the low field results could be successfully modelled. A longitudinal inner sphere relaxation rate, $\frac{1}{T_{1 m}}$, of about $0.4 \mathrm{~s}^{-1}$ at a concentration of $0.0125 \mathrm{mM}$ would need to be calculated to match the experimental results obtained for the conjugate alone and in the presence of thrombin. As in the high field case, this could not be achieved using the same reported parameters of DTPA-BMA-Gd(III) as described in section 3.3 .5 with a $\tau_{m}$ on the order of $10^{-6}$ seconds, as expected for the bisamide derivative of DTPA. This lends further 
support to the theory that the chelate in this agent experiences a great deal of internal motion.
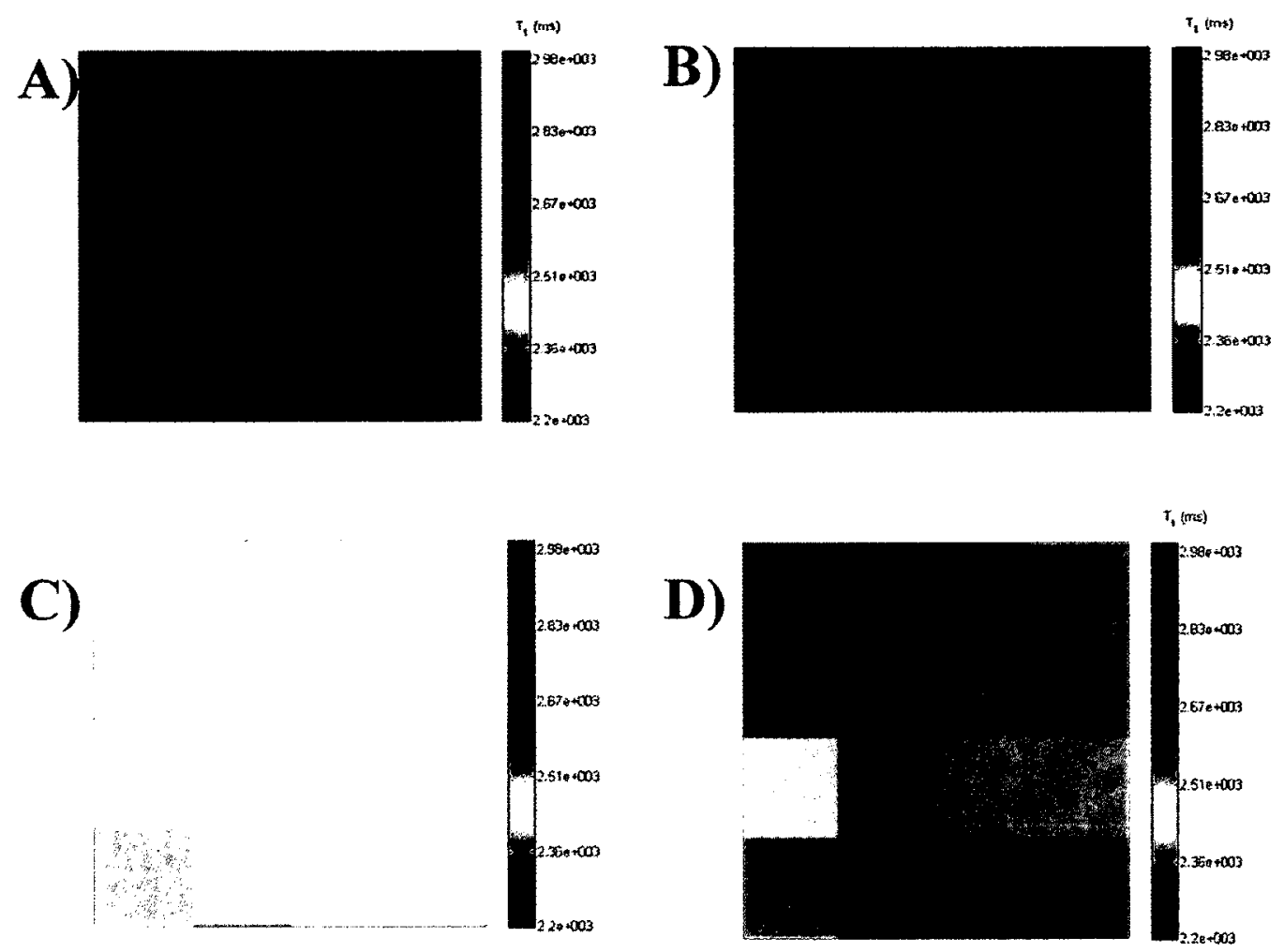

Figure 3.12 Representative T1 maps of $0.0094 \mathrm{mM}$ solutions of A) Magnevist ${ }^{\circledR}$, B) DC-Gd(III), C) DC-Gd(III)+pepsin, and D) DC-Gd(III)+thrombin. An equimolar amount of protein is present, where applicable. Experiments were performed at $1.5 \mathrm{~T}, \mathrm{pH} 7.4$ and $25^{\circ} \mathrm{C}$.

The $T_{1}$ maps shown in Figure 3.12 for $0.0094 \mathrm{mM}$ solutions of Magnevist $(\mathrm{B})$ (A), DC-Gd(III) alone (B), then DC-Gd(III) with pepsin (C) or thrombin (D) illustrate that the binding of the conjugate to its target causes a visible difference when compared to all other cases. The difference in the image obtained with pepsin present $(C)$ compared to 
those for Magnevist ${ }^{\circledR}$ and the conjugate alone can be attributed to the change in viscosity upon introduction of the protein. It appears as though agents similar to DC-Gd(III) could therefore prove to be a useful targeted MRI CA under optimized conditions. These conditions would need to take into account that DC-Gd(III) shows an enhancement in relaxation upon binding one molar equivalent of its target. This binding scenario would be built-in to the design of an asymmetric double-conjugate where the second DNA strand was used to carry a therapeutic drug, for example.

\subsection{Conclusions and future work}

The prepared DNA-DTPA double-conjugates were synthesized using a variety of methods in an effort to maximize their relative yield to their respective single-conjugates, but a more direct comparison between the methods would be necessary to determine if any gave significantly better results than the others. For example, the methods could be performed simultaneously on DNA synthesized at the same time to help eliminate differences related to the success of the DNA synthesis and modification. Furthermore, the solvent and DTPA dianhydride should be kept as dry as possible, and new portions of both should be ordered regularly to ensure maximum reactivity. The design of new symmetric or asymmetric double-conjugates for use as contrast agents or theranostic agents, respectively, was mentioned in the introduction to this chapter. A more detailed look at the synthetic methods and their further optimization would prove useful for future work preparing such agents.

The relaxivity studies performed at $9.4 \mathrm{~T}$ demonstrated that 15 T DC-Gd(III) experienced the highest relaxation enhancement upon target-binding of all the DNA- 
DTPA conjugates prepared (47 $15 \%$, Table 3.3$)$ when mixed with an equimolar amount of protein. Hybridization with matching polyA strands destroyed this enhancement, suggesting that the single-stranded agent might have the optimal $\tau_{R}$ among all of the conjugates studied. This suggests that although the addition of spacers between the aptamer and chelate moieties might prove unnecessary in some systems, the added mass, and therefore size, contributed by the spacers and even their flexibility might improve the relaxation properties in some conjugates. Therefore, the inclusion of oligonucleotide spacers in future conjugates should be considered and possibly tested.

When tested at low field, a visible difference in the obtained $T_{1}$ maps (Figure 3.11) for 15T DC-Gd(III) was seen, but due to the small amount of the conjugate available for testing relaxivity data could not be obtained. DC-Gd(III) did, however, demonstrate similar relaxation properties at $1.5 \mathrm{~T}$ to those at $9.4 \mathrm{~T}$ when mixed $1: 1$ with thrombin compared to the control. Therefore, the potential for the use of DNA-DTPA double-conjugates as targeted MRI contrast agents was demonstrated in this work. Expanding to the use of other Gd(III) chelators, such as DOTA, in the future could both enhance the stability of the agents and provide methods by which to achieve high relaxivity values. For example, as described in section 3.1.1 the conjugation of two DNA strands on adjacent acetate arms of DOTA can restrict the internal motion experienced by the chelate and so cause an enhancement in its relaxivity through the greater global motion of the agent. 


\section{Synthesis and characterization of a luminescent platinum(II) complex and its conjugation to DNA}

\subsection{Introduction}

\subsubsection{Aptamer folding}

The concept of single-stranded nucleic acid folding and its importance in influencing the interactions between aptamers and their targets was introduced in section 1.2. As previously mentioned, some aptamers envelop their small molecule targets in binding pockets while others interact with a specific portion of a larger target such as a protein. ${ }^{19}$ Aptamer-target binding results from a number of molecular level interactions between the nucleic acid and the target molecule. Hydrogen-bonding, stacking, and electrostatic intermolecular interactions can all contribute to the specificity of an aptamer's binding to its respective target. ${ }^{19}$ Due to these interactions, the structures that aptamers adopt upon target recognition often require the target's presence; that is, aptamers are often unfolded and unstructured prior to target binding and the molecular interactions with their targets stabilize the three dimensional structures formed. In fact, the in vitro selection of aptamers ensures that the resultant sequences adopt conformations that are optimized for target recognition rather than for any other purpose. $^{19}$

The 15 mer thrombin-binding aptamer is an unusual example in that it achieves its G-quadruplex conformation in the absence of its target, as discussed in section 1.2.3..$^{19,47,48}$ It was also previously mentioned that its structure was solved using both NMR spectroscopy and X-ray crystallography. ${ }^{46}$ These are the two experimental 
methods generally used to solve nucleic acid structures, with NMR spectroscopy performed on solution-phase samples and X-ray crystallography performed on solid samples.

NMR spectroscopy is useful in determining the conformations of short nucleic acids in solution. Detailed information might be obtained from NMR data on the bonding within a nucleic acid strand and the three-dimensional arrangement of the bonds. ${ }^{1,} 178$ However, the low natural abundancies of NMR active nitrogen and carbon isotopes result in low sensitivities when using ${ }^{15} \mathrm{~N}$ or ${ }^{13} \mathrm{C}$ NMR. Enriching nucleic acids with these isotopes helps increase the sensitivity of the experiments ${ }^{179}$ but is expensive. Furthermore, the interpretation of the data obtained using one- or two-dimensional NMR techniques is complicated due to the high number of nuclei under consideration (i.e. hundreds of proton signals). ${ }^{179}$

X-ray crystallography permits atomic-level resolution of three-dimensional structures and is an important method used to determine the structures of biological macromolecules. It is possible to crystallize nucleic acids, however it is difficult to obtain crystals with the good diffraction qualities required for study using $x$-ray crystallography. Another complication when using this technique arises from the contacts between neighbouring oligonucleotide strands in crystals and the unknown effect of these interactions on the structure of the individual strands. ${ }^{1}$

The determination of an aptamer's conformation upon interaction with its target is required for the design of various applications into which the aptamer is being incorporated as well as to gain a fundamental understanding of the interaction. Some applications require that tags of some sort be covalently bound to the aptamer strand, 
such as in the electrochemical, aptamer-based sensor designed for the detection of platelet-derived growth factor (PDGF) discussed in section 1.3.2. The introduction of such tags does not often interfere with aptamer folding or the interaction of an aptamer with its target unless they are placed within an important binding region. Therefore, some previous knowledge of the tertiary structure of an aptamer and its binding domains is useful when designing sensor systems or including modifications for other purposes. It is proposed herein that luminescence-based probes could be used for the study of aptamer folding upon target binding. The preparation of luminescent platinum(II) complexes and preliminary experiments on the conjugation of one of the complexes to DNA will be described.

\subsubsection{Absorbance and luminescence}

A brief introduction to absorbance was given in section 1.5 .3 in the discussion of UV-visible spectrophotometry. It was mentioned that when a molecule absorbs light it is promoted from the ground electronic state, $S_{0}$, to an excited electronic state, $S_{1}$, in which electrons are promoted to higher energy orbitals. This is represented in Figure 4.1 by the green arrow. It can be seen in the diagram that absorption of a photon is a fast process: on the order of $10^{-15} \mathrm{s.}^{53}$

Once in the excited state, the molecule has greater vibrational and rotational energy than it did in the ground state. The excited molecule will generally lose some of the absorbed energy through a radiationless transition. This is represented by the purple dashed arrows in Figure 4.1 and it occurs due to loss of energy of the molecule as it collides with other molecules (for example, solvent molecules). Once the excited 
molecule has relaxed to the lowest vibrational level of $S_{1}$ in this manner, it can experience one of three processes. It can undergo internal conversion (labelled in Figure 4.1 and shown as a grey arrow) and enter an excited vibrational level of $\mathrm{S}_{0}$. The excited molecule can then relax back to the ground state through a radiationless transition process as described above. When all of the energy absorbed from a photon is lost in such a manner it is entirely converted into heat. ${ }^{53}$

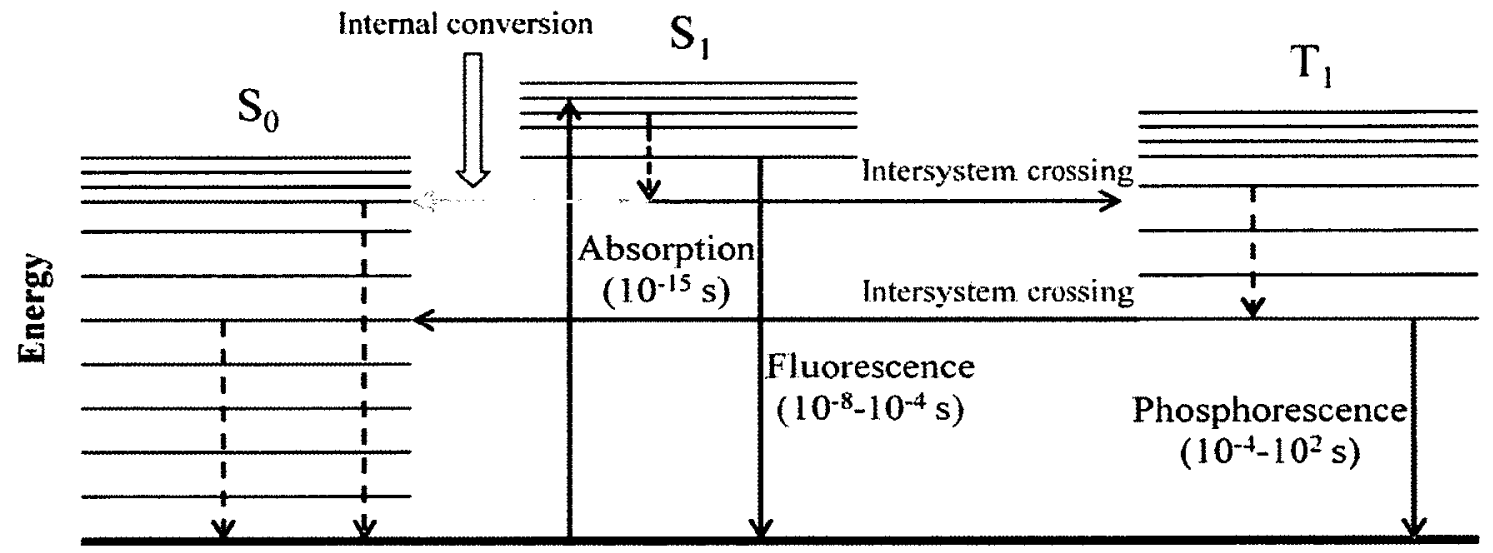

Figure 4.1 Diagram of the physical processes that can occur after a molecule absorbs a photon of UV or visible light. See text for discussion. Modified from reference 53.

The other two processes that an excited molecule can experience once in the lowest vibrational state of $S_{1}$ result in luminescence. Luminescence is the emission of light from an excited molecule. ${ }^{53}$ When the excited molecule relaxes directly from $S_{1}$ back to the ground electronic state $S_{0}$, the radiational transition is called fluorescence (blue arrow). This process occurs on the order of $10^{-8}$ to $10^{-4}$ seconds. The excited molecule can also cross from $S_{1}$ into an excited vibrational level of $T_{1}$ instead of relaxing to the ground state, which is called intersystem crossing (orange arrows), and it 
subsequently experiences radiationless relaxation to the lowest vibrational level of $T_{1}$ (purple dashed arrow). When the molecule then relaxes back to the ground electronic state $\mathrm{S}_{0}$ through the emission of a photon, the process is called phosphorescence. This process has a longer lifetime than fluorescence at $10^{-4}$ to $10^{2}$ seconds and is thus the rarer of the two types of luminescence..$^{53}$

Finally, intersystem crossing from $T_{1}$ to $S_{0}$ can also occur (brown arrow) and thus result in a radiationless transition in which no light is emitted when the molecule relaxes back to its ground electronic state. ${ }^{53}$

The energy at which a molecule emits light is always lower than the energy of the photon absorbed. This means that the wavelength of light emitted is always longer (i.e. red-shifted) than that of the incoming photon. The radiationless relaxation of an excited molecule within an excited state, $S_{1}$ or $T_{1}$, to the lowest vibrational level of the state is the reason behind this phenomenon. Therefore, there is always some loss of energy as the result of a radiationless transition before the emission of light through a luminescent process. $^{53}$

\subsubsection{Fluorescence resonance energy transfer (FRET)}

Fluorescence resonance energy transfer (FRET) is a phenomenon in which the emission of an excited-state fluorescent donor molecule excites a fluorescent acceptor molecule. ${ }^{53}$ This occurs when the emission spectrum of the donor molecule overlaps with the excitation spectrum of the acceptor molecule. The rate of energy transfer is dependent on the distance between the donor and acceptor species and is typically measured over distances of 20 to $100 \AA .^{180}$ As the fluorescent signal measured from a 
FRET pair is distance-dependent, this effect has been used to study interactions between biomolecules ${ }^{1,180}$ or in the design of biosensors. ${ }^{11,181}$ As an example, the two thrombinbinding aptamers were incorporated into a FRET-based sensor. ${ }^{181}$ The 15 - and $29 \mathrm{mer}$ thrombin-binding aptamers were each extended using a non-nucleotide-based linker, and a short DNA sequence was added at the end of each linker. The 7 mer regions used at the end of the linkers were complementary to one another and, therefore, when the two aptamers were in close proximity (i.e. binding thrombin) they would end up being linked through hybridization of the complementary regions. Three different donor-acceptor dye pairs were tested for use in this sensor by their inclusion at the end of the complementary oligonucleotide regions with all three resulting in functional molecular beacons for thrombin-sensing. ${ }^{181}$

\subsubsection{Excimers}

The formation of excimers is another unique phenomenon that occurs among certain luminescent chemical species. The term "excimer" refers to an excited dimer that is dissociated when in the ground state. That is, a stable dimer that is then excited does not qualify as an excimer. ${ }^{182}$ A brief discussion of how an excimer is formed will help clarify this point.

Consider a luminescent molecule ${ }^{1} \mathrm{M}$ that is excited upon absorption of a photon $\left({ }^{1} M^{*}\right)$ and then relaxes back to the ground state emitting light at wavelength $\lambda_{M}$ as in equation $4.1 .^{182}$

$$
{ }^{\mathrm{l}} \mathrm{M}^{*} \rightarrow{ }^{\mathrm{l}} \mathrm{M}+\lambda_{\mathrm{M}}
$$


The excited molecule might quench its own luminescence by colliding with an unexcited molecule; when these molecules become associated with one another, they form an excited dimer or excimer as in equation $4.2 .^{182}$

$$
{ }^{1} \mathrm{M}^{*}+{ }^{1} \mathrm{M} \rightleftharpoons{ }^{1} \mathrm{D}^{*}
$$

The excimer does not exist in the ground state; it dissociates back into two unexcited molecules and emits light at a longer wavelength than the single molecule (equation $4.3)^{182}$

$$
{ }^{1} \mathrm{D}^{*} \rightarrow{ }^{1} \mathrm{M}+{ }^{1} \mathrm{M}+\lambda_{\mathrm{D}}
$$

It is therefore possible to distinguish between monomer (single molecule) and excimer emission; excimer emission is red-shifted from that of the monomer. Furthermore, excimer emission is structureless. ${ }^{182}$

The differences in the emission spectra of excimers compared to monomers permit the use of excimer-forming chemical species as structural probes and in sensors in the same way as FRET donor-acceptor pairs are used. A significant difference between these two phenomena is the distance over which the two luminescent moieties can interact. As mentioned above, FRET can typically be measured when the acceptor and donor are 20 to $100 \AA$ apart. ${ }^{180}$ Excimers form when two appropriate molecules are within a few angstroms of each other. For example, pyrene is a commonly used excimerforming molecule that requires about $3.4 \AA$ between co-planar, stacked molecules for efficient excimer formation. ${ }^{183}$ The planarity of aromatic compounds such as pyrene allows for efficient stacking and $\pi$-orbital interaction of two molecules and, thus, the formation of an excimer. ${ }^{183}$ 
A potassium ion-sensing system using the 15 mer thrombin-binding aptamer was reported by $\mathrm{Ma}$ et al. in $2010 .^{184}$ Recall that this aptamer folds into its chair-like conformation in the presence of certain cations, with particular stability of the structure achieved in the presence of the potassium cation (section 1.2.3). ${ }^{47,48}$ Hence, a sensor in which the aptamer is prevented from folding through partial complementarity with a second oligonucleotide strand was designed where the addition of $\mathrm{K}^{+}$would cause the aptamer to fold and, therefore, disrupt the interstrand hydrogen-bonding. The nonaptamer oligonucleotide was labelled on both the 5'- and $3^{\prime}$-termini with pyrene, and it was designed to fold into a hairpin when single-stranded. The formation of the hairpin brought the pyrene-labelled termini together resulting in excimer emission that could be measured for the determination of potassium ion concentration. ${ }^{184}$

\subsubsection{Luminescent probes for the study of aptamer folding upon target binding}

As previously discussed, NMR and X-ray crystallography are generally used to determine the structure of nucleic acids, including those of aptamers. Both processes have their advantages and disadvantages (see section 4.1.1). A new method by which to determine aptamer conformation using luminescence-based probes could complement the traditionally used methods and provide an alternative when needed. It is therefore proposed that excimer-forming luminescent molecules could be used to this end.

The choice of monomer-excimer based emission changes rather than a FRETbased system for the study of aptamer folding is based on the difference in proximity required in each case. The height of a base pair in duplex DNA is approximately $3.4 \AA .{ }^{\prime}$ Recall that this is the same distance required for efficient excimer formation between two 
pyrene molecules ${ }^{183}$ and that other excimer-forming compounds also need to approach within a few angstroms to form an excimer. FRET occurs over a greater distance and therefore could not be used to detect changes in conformation over as little space as a monomer-excimer system could be used.

The proposed luminescence-based system is represented in Figure 4.2. Two molecules of an excimer-forming compound would be conjugated to two distinct points on an aptamer strand. In Figure $4.2 \mathrm{~A}$ the two molecules are conjugated to the termini of the aptamer, while in Figure $4.2 \mathrm{~B}$ they are conjugated to neighbouring nucleotides. When the two luminescent molecules are conjugated at distant locations of the aptamer, as in Figure 4.2A, the emission found in the absence of the target would be that of the monomer. Upon addition of the target and aptamer-folding, if the two nucleotides at which the compound was conjugated were brought into close proximity, a shift to excimer emission would be seen. The opposite case is illustrated in Figure 4.2B where the two molecules that initially could form an excimer might be pulled away from each other upon target binding. In this second case, a shift from monomer to excimer emission would be observed. In a comprehensive study of a particular aptamer, the two molecules would be conjugated to varying positions on the aptamer in separate experiments to study how different portions of the strand changed conformation upon target binding. A ratio of monomer to excimer emission would be expected to be observed when the two luminescent molecules were held at certain distances from each other, so a ratiometric method, as in FRET-based studies, ${ }^{185}$ could be used in the interpretation of the results. This experimental design would not work for an aptamer that does not change conformation upon target binding, but since a number of aptamers selected and reported 
do experience a conformational change it would be expected to be a valuable tool in the study of aptamers.

A)

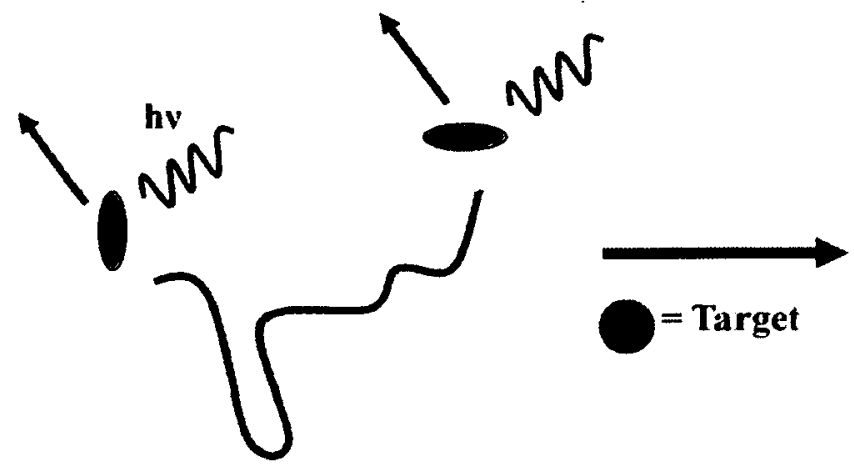

Monomer Emission

B)

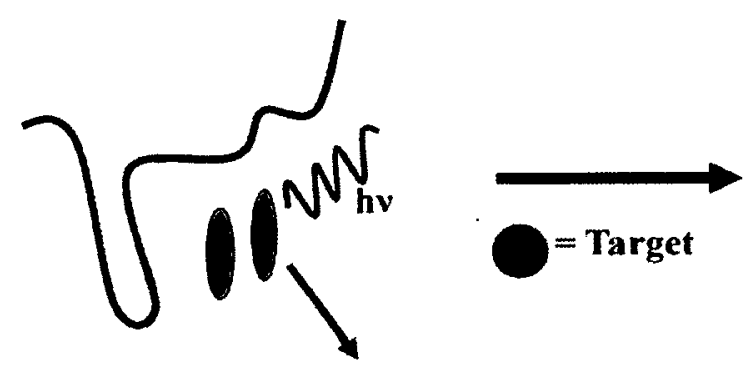

Excimer Emission

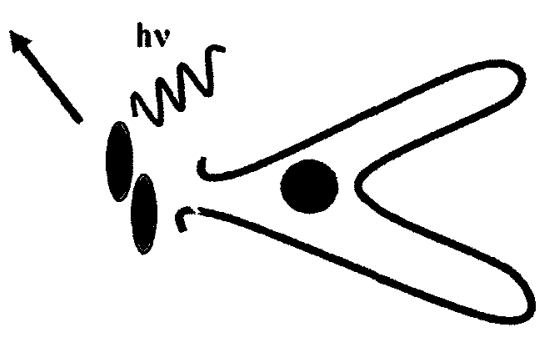

Excimer Emission

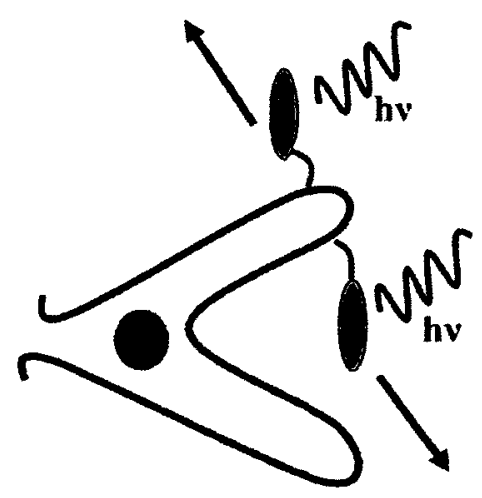

Monomer Emission

Figure 4.2 Schematic representation of how an excimer-forming compound could be used to study aptamer folding. See text for discussion.

\subsubsection{Excimer-forming platinum(II) complexes}

Several square planar platinum(II) complexes containing aromatic ligands emit at room temperature in solution ${ }^{186}$ and have been found to form excimers. ${ }^{187}$ Such complexes benefit from having metal centred (MC) excited states that are higher in energy than the metal-to-ligand charge transfer (MLCT) and intraligand states. This property prevents quenching of the emission from the MLCT or intraligand states and 
leads to enhanced luminescence efficiency of such complexes. ${ }^{186}$ Platinum(II) complexes including $\mathrm{C}^{\wedge} \mathrm{N}$ bidentate aromatic chelates are very stable and are known to be highly emissive. The preparation of heteroleptic $\left(\mathrm{C}^{\wedge} \mathrm{N}\right) \mathrm{Pt}(\mathrm{LX})$ complexes as opposed to those with two $\mathrm{C}^{\wedge} \mathrm{N}$ ligands provides the opportunity to adjust properties such as solubility and charge as the LX ligand is varied. ${ }^{186}$

The nature of the LX ligand also affects the electronic properties of $\left(\mathrm{C}^{\wedge} \mathrm{N}\right) \operatorname{Pt}(\mathrm{LX})$ complexes. In a study by Thompson et al., ${ }^{186}$ several Pt(II) square planar complexes were prepared using a $\mathrm{C}^{\wedge} \mathrm{N}$ ligand and a $\beta$-diketonate ligand (chelating through $\mathrm{O}^{\wedge} \mathrm{O}$ ). The electron-withdrawing ability of the $\mathrm{C}^{\wedge} \mathrm{N}$ cyclometalating ligand was found to have a strong effect on the emission wavelength and so, using one of two $\mathrm{O}^{\wedge} \mathrm{O}$ ligands, the authors were able to prepare complexes that emitted throughout the visible spectrum by varying the nature of the $\mathrm{C}^{\wedge} \mathrm{N}$ ligand. ${ }^{186}$

Based on the Thompson study it was hypothesized that excimer-forming $\mathrm{Pt}(\mathrm{II})$ complex luminescence-based probes for the study of aptamer folding could be prepared. Furthermore, the preparation of $\mathrm{Pt}(\mathrm{II})$ complexes with different $\mathrm{C}^{\wedge} \mathrm{N}$ cyclometalating ligands would result in a family of luminescent probes with a range of emissive properties. Unlike organic excimer-forming species, this could allow for the use of multiple Pt(II) complexes in aptamer folding studies or in aptamer-based sensors. The Thompson study used acetylacetone as one of the $\mathrm{O}^{\wedge} \mathrm{O}$ chelates incorporated into the Pt(II) complexes. ${ }^{186}$ For conjugation of a $\mathrm{Pt}(\mathrm{II})$ complex to a nucleic acid strand, a reactive functional group on one of the chelates would be required. In 2002, Weizman and Tor reported the synthesis of a modified acetylacetone ligand in which a carboxylic acid group was included to allow conjugation to nucleotides. ${ }^{188}$ This ligand was therefore 
prepared for inclusion in the $\mathrm{Pt}(\mathrm{II})$ complexes synthesized for use as luminescent probes. One Pt(II) complex including this modified acetylacetone ligand has already been prepared and reported in the B.Sc. thesis of a colleague. ${ }^{189}$ Research reported herein describes the efforts made to expand the number of Pt(II) complexes available for use as luminescent-probes.

\subsubsection{Conjugation through peptide coupling techniques}

The formation of peptide bonds and the development of a variety of peptide coupling reagents is an area of organic synthesis that has been studied for many decades. As such, a number of different reagent families have been developed for use in peptide coupling reactions. ${ }^{190,191}$ Though developed for the coupling of amino acids, the chemistry behind these reactions can be extended for use in the formation of peptide bonds between a chemical species with an amino group and one with a carboxylic acid group and thus can be used in conjugation reactions for the labelling of DNA. The carboxylic acid group is activated through a reaction with a coupling agent that introduces an electron-withdrawing group. ${ }^{191}$

Carbodiimide-mediated reactions are traditionally used to form peptide bonds as part of a combination method where an appropriate activator is also used. ${ }^{190}$ For example, the reagents used in this work were $N$-ethyl- $N^{\prime}-(3-$ dimethylaminopropyl)carbodiimide (EDC) along with $N$-hydroxysuccinimide (NHS) for the formation of an active ester at the carboxylic acid group of a $\mathrm{Pt}(\mathrm{II})$ complex which was being conjugated to amino-modified DNA. The reaction is illustrated in Scheme 4.1. The reaction of EDC with a carboxylic acid leads to the formation of an ester with a very 
reactive leaving group. This is then reacted with NHS to give a more stable yet active ester in which the carbonyl group remains susceptible to nucleophilic attack by an amine at room temperature leading to the formation of a peptide bond. ${ }^{191}$ Though commonly used, the use of EDC in coupling reactions is not without its disadvantages. The reactive intermediate formed by the reaction of EDC and a carboxylic acid can undergo an undesirable rearrangement to give a stable, inert compound. This reaction is irreversible; it uses up the acidic species without formation of the desired peptide bonds, and is reportedly very fast in $N, N$-dimethylformamide (DMF). ${ }^{191}$ The formation of an active ester with an agent such as NHS can help suppress the formation of this side product, thus increasing the efficiency of carbodiimide-mediated reactions. ${ }^{191}$

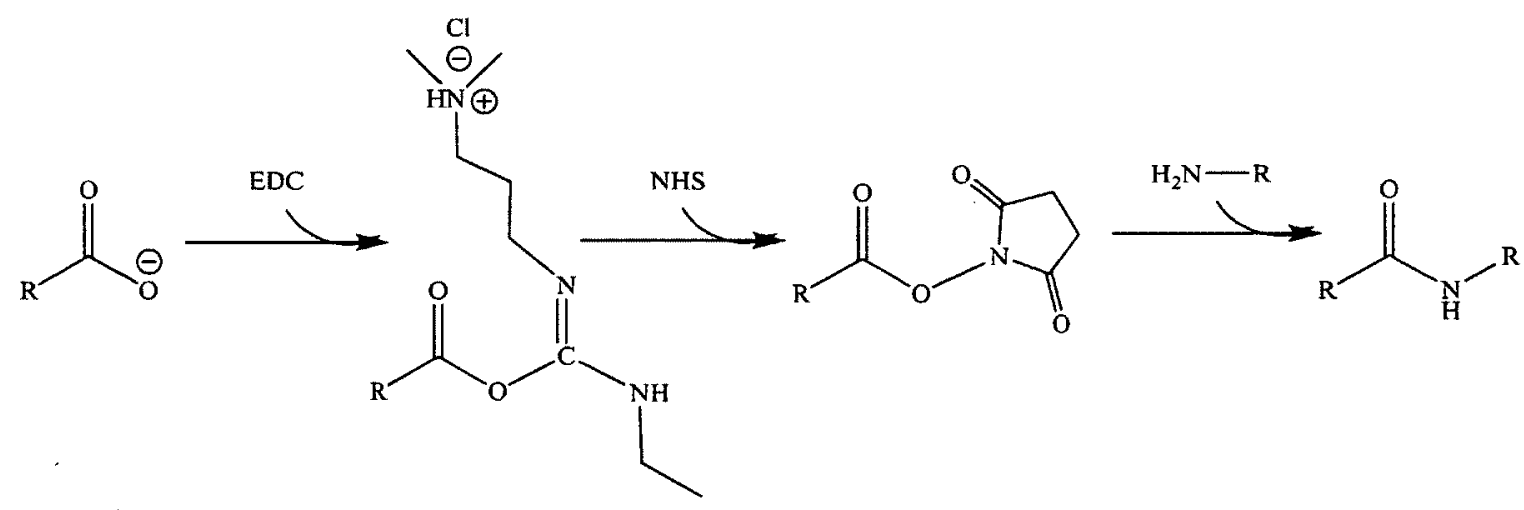

Scheme 4.1 Peptide coupling reaction using EDC and NHS.

Aminium/uronium salts are another class of peptide coupling reagent. They contain a positive carbon atom that reacts with a carboxylic acid group in the presence of a tertiary base, such as diisopropylethylamine (DIPEA), to generate an active ester. The aminium salt used herein was a derivative of hydroxysuccinimide, 2-succinimido-1,1,3,3- 
tetramethyluronium tetrafluoroborate (TSTU). It is thought that the use of agents such as TSTU leads to the formation of both an $\mathrm{O}$-acyl active ester and a less reactive $\mathrm{N}$-acyl active ester that exist in equilibrium with each other. This is one stage at which the use of TSTU can lead to poor coupling. TSTU can also form an unwanted guanidine sideproduct through direct reaction with the amine group, which tends to occur when an excess of the agent is used or when the preactivation of the carboxylic acid is slow. ${ }^{191}$

Both the EDC/NHS and TSTU/DIPEA coupling reagents were used in conjugation reactions between amino-modified DNA and a $\mathrm{Pt}(\mathrm{II})$ complex.

\subsection{Materials and Methods}

\subsubsection{Materials and instruments}

Potassium tetrachloroplatinate(II) (99.9\% metals basis, Pt $46.61 \%)$ was purchased from Alfa Aesar. Unless otherwise specified, solvents were obtained from Caledon. Acetylacetone ( $\geq 99 \%$, acac), 2-phenylpyridine (98\%, ppy), 2-pheynylquinoline (99\%, pq), 6-bromohexanoic acid (98\%, 6-BrHex), n-butyllithium (2.5 M in hexanes, $n$-BuLi), diisopropylamine (99\%, DIA), hexamethylphosphoramide (99\%, HMPA), $N$-ethyl- $N^{\prime}-(3-$ dimethylaminopropyl)carbodiimide (EDC), diisopropylethylamine (DIPEA), 2succinimido-1,1,3,3-tetramethyluronium tetrafluoroborate (TSTU), and all other chemicals were obtained from Sigma-Aldrich unless otherwise noted. 2-Ethoxyethanol and dimethylformamide were obtained from Fluka.

A 1200 Series Agilent Technologies High-Pressure Liquid Chromatograph (HPLC) was used with a Zorbax 300Ext - C18 reverse phase column for DNA 
purification. A Fluorolog (Horiba Jobin Yvon) was used for luminescence measurements.

\subsubsection{Synthesis of Pt(II) $\mu$-dichloro-bridged dimers}

\subsubsection{Synthesis of dimers with 2-phenylpyridine and 2-phenylquinoline ligands}

Platinum(II) $\mu$-dichloro-bridged dimers were prepared by reacting potassium tetrachloroplatinate(II) with 2.5 equivalents of 2-phenylpyridine (ppy, MW $155.2 \mathrm{~g} / \mathrm{mol}$ )

or 2-phenylquinoline (pq, MW $205.25 \mathrm{~g} / \mathrm{mol}$ ) as previously described. ${ }^{186}$ About $0.4 \mathrm{~g}$ $(0.96 \mathrm{mmol})$ of $\mathrm{K}_{2} \mathrm{PtCl}_{4}(\mathrm{MW} 415.088 \mathrm{~g} / \mathrm{mol})$ was placed in a 2-neck round-bottom flask. A 3:1 mixture of 2-ethoxyethanol and $\mathrm{diH}_{2} \mathrm{O}$ was used as solvent and the reaction mixture was left stirring under argon at $80^{\circ} \mathrm{C}$. The reaction was maintained at this temperature and was shielded from light. Argon was flowed through the round-bottom flask and condenser while the temperature stabilized, after which the system was isolated and left under a blanket of argon. After about 16 hours, the reaction mixture was cooled to room temperature and enough $\mathrm{diH}_{2} \mathrm{O}$ was added to double the volume of the solution. This caused $\mu$-dichlorobis(2-phenylpyridylplatinum(II)) (MW $771.466 \mathrm{~g} / \mathrm{mol}$ ) or $\mu$ dichlorobis(2-phenylquniolineplatinum(II)) (MW $871.56 \mathrm{~g} / \mathrm{mol}$ ) to precipitate out of solution.

The precipitate was collected on a sintered glass funnel using vacuum filtration. The funnel was covered with parafilm with some holes poked in it and was stored in a desiccator until the collected solid was dry. It was then removed from the funnel using a spatula, placed in a preweighed glass vial, and stored in a desiccator. The product yield was calculated and the dimers were characterized using ${ }^{1} \mathrm{H}$ NMR. The NMR samples 
were prepared in deuterated dimethyl sulfoxide (DMSO-d6) or deuterated chloroform $\left(\mathrm{CDCl}_{3}\right)$.

4.2.2.2 Synthesis of a modified phenylpyridine ligand and dimers containing this 2-(2,4difluorophenyl)pyridine

The syntheses of the modified phenylpyridine ligand, 2-(2,4difluorophenyl)pyridine, and the platinum(II) $\mu$-dichloro-bridged dimers containing this ligand were reported in the B.Sc. thesis of Boshra Hosseini ${ }^{189}$ and will be only briefly described herein.

2-(2,4-Difluorophenyl)pyridine was prepared using a procedure modified from that reported by Lohse et al. ${ }^{192} 1.42 \mathrm{~g}$ of 2-bromopyridine $(9 \mathrm{mmol}), 1.66 \mathrm{~g}$ of phenylboronic acid (10.5 mmol), and $0.085 \mathrm{~g}$ of tetrakis(triphenylphosphine)palladium catalyst $(0.2 \mathrm{mmol})$ were added to $12 \mathrm{~mL}$ of $2 \mathrm{M} \mathrm{K}_{2} \mathrm{CO}_{3}$ and $10 \mathrm{~mL}$ of dimethoxyethane. The reaction was performed under argon for 18 hours at $90^{\circ} \mathrm{C}$. Following separation of the organic and aqueous phases, the aqueous phase was extracted using ethyl acetate (EtOAc). The organic layers were washed with water, dried over $\mathrm{MgSO}_{4}$, and concentrated. Silica gel column chromatography was used to purify the desired product using $20 \%$ EtOAc in hexanes as the mobile phase. The product was a clear yellow liquid. $\mu$-Dichlorobis(2-(2,4-Difluorophenyl)pyridylplatinum(II)) was prepared using the same method as that described in the previous section, but using $0.19 \mathrm{~g}$ of $2-(2,4-$ difluorophenyl)pyridine $(1 \mathrm{mmol})$ and $0.22 \mathrm{~g}$ of $\mathrm{K}_{2} \mathrm{PtCl}_{4}(0.52 \mathrm{mmol})$ and $50 \mathrm{~mL}$ of a $3: 1$ 2-ethoxyethanol/water solution. ${ }^{189}$ 


\subsubsection{Synthesis of the modified acetylacetone ligand (acac-COOH): 8,10-}

dioxoundecanoic acid

Tetrahydrofuran (THF) was dried by refluxing it in the presence of lithium aluminum hydride. About $10 \mathrm{~mL}$ and $40 \mathrm{~mL}$ portions of dry THF were removed from a still and injected in previously flame-dried and argon-purged 3-neck round-bottom flasks. This reaction was modified from that reported by Weizman and Tor. ${ }^{188}$

Part 1 of the reaction was performed in the $10 \mathrm{~mL}$ portion of THF. The THF was cooled to $0^{\circ} \mathrm{C}$ in an ice-water bath and about $80 \mathrm{mg}(\sim 10 \mathrm{mmol})$ of lithium hydride ( $\left.\mathrm{LiH}\right)$ and about $2 \mathrm{~g}(\sim 10 \mathrm{mmol})$ of 6-bromohexanoic acid (BrHex, MW $195.06 \mathrm{~g} / \mathrm{mol})$ were added to the flask. The reaction mixture was left stirring under argon gas for a minimum of one hour or until part 2 of the reaction was complete. It was then cooled to $-78^{\circ} \mathrm{C}$ using a dry ice-acetone bath.

Part 2 of the reaction was performed in the $40 \mathrm{~mL}$ portion of THF which was cooled to $-78^{\circ} \mathrm{C}$. Lithium diisopropylamide (LDA) was prepared in situ through the reaction of $4 \mathrm{~mL}(28 \mathrm{mmol})$ diisopropylamine (DIA) and $10.4 \mathrm{~mL}(112 \mathrm{mmol})$ of $\mathrm{n}$ butyllithium (n-BuLi) for 30 minutes. About $1.8 \mathrm{~mL}$ of dry hexamethylphosphoramide (HMPA) and $1.02 \mathrm{~mL}(10 \mathrm{mmol})$ of acetylacetone (acac, MW $100.116 \mathrm{~g} / \mathrm{mol})$ were added to the LDA solution and were left to react for 30 minutes at $-78^{\circ} \mathrm{C}$.

For the third and final part of this reaction, the reaction mixture from part 2 was transferred to the lithium 6-bromohexanoate suspension from part 1 using a syringe while keeping both mixtures at $-78^{\circ} \mathrm{C}$. This final reaction mixture was stirred while in a dry ice-acetone bath for 1 hour, in an ice-water bath for 1 hour, and then at room temperature 
for 1 hour. Deionized water was then added to quench the reaction (typically about 25 $\mathrm{mL}$ was used).

The water layer was extracted with $3 \times 100 \mathrm{~mL}$ of DCM. The organic phase was dried over magnesium sulfate $\left(\mathrm{MgSO}_{4}\right)$, concentrated, and characterized using ${ }^{1} \mathrm{H} \mathrm{NMR}$ to ensure no product was lost at this stage. The water layer was acidified to $\mathrm{pH} \sim 3$ using $4 \mathrm{M}$ hydrochloric acid $(\mathrm{HCl})$ and using $\mathrm{pH}$ indicator strips to monitor the $\mathrm{pH}$. The resultant water/oil mixture was extracted with $3 \times 100 \mathrm{~mL}$ portions of diethyl ether. The organic phase was dried over $\mathrm{MgSO}_{4}$, concentrated, and the crude product was characterized using ${ }^{1} \mathrm{H}$ NMR. The samples for NMR were prepared using deuterated chloroform $\left(\mathrm{CDCl}_{3}\right)$.

Purification of the modified acetylacetone ligand (acac-COOH) from the reaction mixture was performed using silica gel chromatography. The reaction mixture was dissolved in dichloromethane (DCM) and dried onto a small amount of silica which was then added to the top of a silica gel column. Two different running solvent systems were used in the course of this work. In one, the column was prepared using silica and DCM and the running solvent used was $30 \%$ ethyl acetate (EtOAc) in DCM. In the second, the column was prepared using silica and DCM mixed with $1 \%$ acetic acid $\left(\mathrm{CH}_{3} \mathrm{COOH}\right)$ by volume. The inclusion of $1 \% \mathrm{CH}_{3} \mathrm{COOH}$ in DCM when preparing the column and in the initial running solvent helped keep the carboxylic acid groups in acac- $\mathrm{COOH}$ and $\mathrm{BrHex}$ protonated and prevented these compounds from sticking to the silica gel. ${ }^{193} \mathrm{DCM}+1 \%$ $\mathrm{CH}_{3} \mathrm{COOH}$ was also used as the running solvent to start this column with the addition of $30 \% \mathrm{EtOAc}$ in DCM with $1 \% \mathrm{CH}_{3} \mathrm{COOH}$ when required to further move coloured bands down the column. Fractions were monitored by thin layer chromatography (TLC) using 
$30 \%$ EtOAc in DCM as the running solvent and bromocresol green dye as an indicator for carboxylic acids. Bromocresol green was dissolved in a 4:1 mixture of ethanol (EtOH) and deionized $\mathrm{H}_{2} \mathrm{O}$ and the solution was neutralized with sodium hydroxide $(\mathrm{NaOH})$. For development of TLCs, a few drops of this solution was diluted in $\sim 40 \mathrm{~mL}$ EtOH and the TLCs were individually placed in the diluted bromocresol green solution for a few seconds. Fractions with similar TLC profiles were concentrated and characterized using ${ }^{1} \mathrm{H} \mathrm{NMR} . \mathrm{CDCl}_{3}$ was used as solvent for the NMR samples.

The wet sample silica portion was typically orange in colour and an orange band was the first seen moving down the column both when the column was prepared with $1 \%$ acetic acid $\left(\mathrm{CH}_{3} \mathrm{COOH}\right)$ in DCM and when it was prepared with DCM alone. The orange band was found to contain the unreacted starting materials, acac and BrHex, both by TLC and ${ }^{1} \mathrm{H}$ NMR performed on the concentrated fractions. Acetylacetone was distinguished in TLC through its strong absorbance of UV light and its high mobility on the TLC plates. 6-Bromohexanoic acid was only clearly visualized once the TLC plates were developed in a solution of bromocresol green dye, which stains carboxylic acids yellow. 6-Bromohexanoic acid also had a high mobility on the TLC plates using $30 \%$ EtOAc in DCM as the mobile phase.

The orange band containing the starting materials was followed by a pale yellow or yellow-orange band of acac-COOH. The modified acac ligand was discernible on the TLC plates through its high UV absorbance and its decreased mobility when compared to the starting materials; the difference between the mobilities of acac and acac-COOH was particularly useful since they were both so highly absorbent. 
If the acac-COOH (MW $213 \mathrm{~g} / \mathrm{mol}$ ) isolated from a column did not form pale, almost colourless crystals, but the ${ }^{1} \mathrm{H}$ NMR showed few impurities, the sample was recrystallized. Dichloromethane was added to the product just until it fully dissolved and then hexanes were added until crystals of acac-COOH crashed out of solution. The crystals were too fine to remove from solution by vacuum filtration using an ultrafine sintered glass funnel, so it was necessary to remove the solvents via pipette with as little perturbation of the crystals as possible.

\subsubsection{Synthesis of luminescent Pt(II) complexes}

The first step in the preparation of luminescent $\mathrm{Pt}(\mathrm{II})$ complexes containing 8,10 dioxoundecanoic acid and either 2-phenylpyridine or 2-phenylquinoline was to break the Pt(II) $\mu$-dichloro-bridged dimers by removal of the chloride ions. Once this was achieved, acac-COOH was added for its complexation with the $\mathrm{Pt}(\mathrm{II})$ ion.

4.2.4.1 Synthesis, purification, and characterization of 8,10-dioxoundecanoic acid-2phenylpyridylplatinum(II); (ppy)Pt(acac-COOH)

About $0.1 \mathrm{~g}(0.136 \mathrm{mmol})$ of $\mu$-dichlorobis(2-phenylpyridylplatinum(II)) was mixed with $0.07 \mathrm{~g}(0.280 \mathrm{mmol})$ of silver trifluoromethanesulfonate $\left(\mathrm{CF}_{3} \mathrm{SO}_{3} \mathrm{Ag}, \mathrm{MW}\right.$ $256.94 \mathrm{~g} / \mathrm{mol}$ ) in $80 \mathrm{~mL}$ of acetone. The reaction mixture was left stirring at $50^{\circ} \mathrm{C}$ under argon while sheltered from light. This reaction was typically left to proceed for 18-24 hours at which point it was cooled to room temperature. The solid silver chloride $(\mathrm{AgCl})$ formed during the reaction was removed by gravity filtration of the reaction mixture into a clean round-bottom flask. 
About $0.07 \mathrm{~g}(0.345 \mathrm{mmol})$ of acac-COOH and $0.5 \mathrm{~mL}(3.6 \mathrm{mmol})$ of triethylamine (TEA, MW $101.19 \mathrm{~g} / \mathrm{mol}$ ) were added to the solution of broken $\mu$ dichloro(ppy)Pt(II) dimers. This reaction mixture was stirred at $40^{\circ} \mathrm{C}$ and room temperature intermittedly over the course of 2-3 days, with heat applied for 10-15 hours total during this time, and the reaction was left at room temperature overnight. The progression of the reaction was monitored by TLC and ${ }^{1} \mathrm{H}$ NMR. Using TLC, where $30 \%$ EtOAc in DCM was the running solvent, the reaction mixture was compared to the starting materials (i.e. $\mathrm{Pt}(\mathrm{II})$ dimers and acac-COOH) and the characteristic luminescence and TLC staining upon exposure to UV light of the desired complex. In ${ }^{1} \mathrm{H} N M R$, the integrations of the acac- $\mathrm{COOH}$ and complex peaks $\left(\delta 5.66\right.$ and $5.59 \mathrm{ppm}$ in $\mathrm{CDCl}_{3}$, respectively) were compared. The reaction was ended when the presence of the complex was apparent using ${ }^{1} \mathrm{H}$ NMR. The reaction mixture was gravity filtered into a clean round-bottom flask to remove a solid brown side-product prior to its concentration under vacuum.

Purification of (ppy)Pt(acac-COOH) was achieved using silica gel chromatography. The crude product was dissolved in acetone or methanol and dried onto a small amount of silica. This sample silica was added to the top of a prepared column. Several solvent systems were tested and used to purify the complex from remaining starting materials. The successful solvent gradients used all began with a column prepared with silica and toluene. Due to greater silica gel swelling in toluene compared to DCM and EtOAc, which were the other solvents used, it was necessary to change to high DCM content with a slow gradient. The purification conditions used to successfully purify (ppy)Pt(acac-COOH) from the crude reaction mixture are summarized in Table 
4.1. Where $1 \%$ acid was included in all solvents used, $\mathrm{CH}_{3} \mathrm{COOH}$ was added as $1 \%$ of the total solvent volume. Fractions with similar TLC profiles were concentrated together and further characterized using ' $\mathrm{H}$ NMR with $\mathrm{CDCl}_{3}$ or acetone-d6 used as solvent.

The first band to move through and off the column contained unreacted platinum dimers. They moved as a yellow band that emitted green light and were identifiable through their characteristic movement on TLC plates (mobile phase $30 \%$ EtOAc in DCM). The complex moved through the column in a second yellow band that also emitted green light. Following the band of complex was an orange band that did not fluoresce. This was determined to be acac-COOH by ${ }^{1} \mathrm{H}$ NMR in a case where it was nearly pure when recovered.

ESI mass spectrometry (MS) was used to further characterize the complex. Calc'd m/z 563.8 , found $\mathrm{m} / \mathrm{z} 563$.

Table 4.1 Solvent conditions for successful- purification of (ppy)Pt(acac-COOH) using silica gel chromatography

\begin{tabular}{|c|c|c|}
\hline Acid included? & Column prepared using: & Gradient slowly changed to: \\
\hline no & toluene & DCM, 30-100\% EtOAc in DCM \\
yes & toluene & DCM \\
yes & toluene & DCM, 30\% EtOAc in DCM \\
yes & toluene & $30 \%$ EtOAc in DCM \\
\hline
\end{tabular}

\subsubsection{Synthesis and purification attempts for 8,10-dioxoundecanoic acid-2-} phenylquinolylplatinium(II); (pq)Pt(acac-COOH)

About $0.12 \mathrm{~g}(0.138 \mathrm{mmol})$ of $\mu$-dichlorobis(2-phenylquinolylplatinum(II)) and $0.07 \mathrm{~g}(0.280 \mathrm{mmol})$ of silver trifluoromethanesulfonate $\left(\mathrm{CF}_{3} \mathrm{SO}_{3} \mathrm{Ag}\right)$ were dissolved in 
one of the solvents or solvent mixtures listed in Table 4.2 and heated for 20-24 hours at the corresponding temperature. The reaction was performed under argon and while shielded from light. Following the reaction period, the reaction mixture was cooled to room temperature and gravity filtration was used to remove the precipitated $\mathrm{AgCl} .{ }^{1} \mathrm{H}$ NMR was used to qualitatively compare the reaction mixture to full dimers when the $1: 7$ 2-ethoxyethanol and toluene mixture was used as solvent.

Table 4.2 Conditions used for breaking $\mu$-dichloro(pq)Pt(II) dimers.

\begin{tabular}{|c|c|}
\hline Solvent & Temperature \\
\hline acetone & $50^{\circ} \mathrm{C}$ \\
DCM & $50^{\circ} \mathrm{C}$ \\
$\begin{array}{c}1: 7 \text { mixture of 2- } \\
\text { ethoxyethanol and toluene }\end{array}$ & $100^{\circ} \mathrm{C}$ \\
\hline
\end{tabular}

About $0.07 \mathrm{~g}(0.345 \mathrm{mmol})$ of acac-COOH and $0.5 \mathrm{~mL}(3.6 \mathrm{mmol})$ of TEA were added to the filtered solution of broken $\mu$-dichloro(pq)Pt(II) dimers. This reaction mixture was stirred under argon while shielded from light at room temperature and at $40^{\circ} \mathrm{C}$ intermittedly. It seemed that the progression of the reaction could be followed by ${ }^{1} \mathrm{H}$ NMR through comparison of the integrations of the acac-COOH peak $(\delta 5.66 \mathrm{ppm}$ in acetone-d6, $\delta 5.50 \mathrm{ppm}$ in $\mathrm{CDCl}_{3}$ ) and new peaks that appeared near the acac-COOH peak $\left(\delta 5.7 \mathrm{ppm}\right.$ in acetone-d6, $\delta 5.57$ and $5.7 \mathrm{ppm}$ in $\mathrm{CDCl}_{3}$ ). Heat was applied for 24.5 hours in the first reaction attempt, but this ended up being the minimum time that heat was applied for this reaction. With increased NMR monitoring in all subsequent reactions, the reaction mixture was heated for a minimum of 30 hours and up to 5 days total. At least one overnight period where the reaction was left to proceed at room 
temperature was also used in subsequent reactions. A summary of the various conditions used is included in Table 4.8 in section 4.3.3.3.

Purification of $(\mathrm{pq}) \mathrm{Pt}(\mathrm{acac}-\mathrm{COOH})$ from leftover starting materials was first attempted using silica gel chromatography. All solvents used were prepared with $1 \%$ acetic acid by volume included. The conditions that were used to successfully purify (ppy)Pt(acac-COOH) were used as a starting point, therefore the first few silica gel columns used were prepared using toluene. The crude product was dissolved in acetone or DCM and dried onto a small portion of silica. The mobile phase was changed to $30 \%$ EtOAc in DCM or just DCM partway through the columns. The complex was not completely purified using any of these column conditions.

The use of a 1:7 solution of 2-ethoxyethanol and toluene as solvent for the reaction in later cases presented the problem of fully drying the crude product. The 2ethoxyethanol could not be removed under vacuum without the application of heat, but further heating of the reaction mixture was undesirable. Extractions were therefore used in an attempt to remove the product from the 2-ethoxyethanol; these are summarized with the results in Table 4.9.

Following the fourth reaction performed to obtain (pq)Pt(acac-COOH), an excess of diethyl ether added to the 2-ethoxyethanol reaction mixture caused a yellow solid to crash out. The solid was removed by filtration through a fine pore sintered glass funnel and the 2-ethoxyethanol and ether mixture was concentrated back down to 2ethoxyethanol. The precipitate was removed from the funnel by dissolving it in DCM; the DCM was then concentrated under vacuum. An excess of $\mathrm{diH}_{2} \mathrm{O}$ was added to the 2ethoxyethanol, again causing a yellow solid to precipitate. The precipitate was isolated 
through filtration and then recovered from the filter as described above. A silica gel column was performed in an attempt to further purify this second precipitate. This column was prepared using $30 \%$ EtOAc in DCM and the mobile phase was gradually changed to 75 and then $100 \%$ EtOAc in DCM.

The crude reaction solutions in 2-ethoxyethanol from the fifth and sixth reaction attempts were partially purified using ether and $\mathrm{diH}_{2} \mathrm{O}$ as described above.

Instead of performing a column on the product of the fifth reaction, further extractions were performed. The $\mathrm{diH}_{2} \mathrm{O} / 2$-ethoxyethanol mixture was extracted with $20 \times 20 \times 40 \mathrm{~mL}$ of ether. The ether layers were combined and dried with $\mathrm{MgSO}_{4}$ prior to concentration. They did not contain complex. The precipitate from the addition of $\mathrm{diH}_{2} \mathrm{O}$ was found to contain the complex, so it was redissolved in toluene for further purification attempts. Two more sets of ether and $\mathrm{diH}_{2} \mathrm{O}$ addition were performed successively as described above.

In the case of the sixth reaction, silica gel chromatography was performed on the precipitate isolated following the addition of $\mathrm{diH}_{2} \mathrm{O}$ to try to further purify the complex. The column was prepared using $10 \%$ toluene in DCM and the mobile phase was gradually changed to $5 \%$ toluene in DCM, DCM, then $30 \%$ EtOAc in DCM.

4.2.4.3 Synthesis, purification, and characterization of 8,10-dioxoundecanoic acid-(2(2,4-difluorophenyl)pyridyl)platinum(II); (2,4-dfppy)Pt(acac-COOH)

The work described in this section was performed by Boshra Hosseini and was reported in her B.Sc. thesis. ${ }^{189}$ 
$\mu$-Dichlorobis(2-(2,4-Difluorophenyl)pyridylplatinum(II)) $(57.2 \mathrm{mg}, 68 \mathrm{nmol})$ was dissolved in acetone and was heated to $50^{\circ} \mathrm{C}$. Silver trifluoromethanesulfonate $\left(\mathrm{CF}_{3} \mathrm{SO}_{3} \mathrm{Ag}, 0.1 \mathrm{~g}, 0.35 \mathrm{mmol}\right)$ was added and the solution was left to reflux at $50^{\circ} \mathrm{C}$ for 2 hours. The solid $\mathrm{AgCl}$ was removed by gravity filtration as well as filtration through a 45 $\mu \mathrm{m}$ pore microfilter. The modified acac ligand $(37 \mathrm{mg}, 172 \mathrm{mmol})$ and $0.5 \mathrm{~mL}$ of triethylamine were added to the filtered reaction mixture and was left to stir under argon for 45 minutes at room temperature. The reaction was monitored by TLC.

The solvent was removed under vacuum to give both a dark brown solid and a grey solid. The brown solid dissolved in DCM and the grey precipitate was removed by filtration through a $45 \mu \mathrm{m}$ pore microfilter. The dissolved product was then dried onto silica and purified using silica gel column chromatography. The column was prepared with DCM and was washed with DCM until yellow impurities had been removed. The mobile phase was then changed to $\mathrm{MeOH}$ in a slow gradient. The desired product was identified using TLC and isolated through the removal of the solvent under vacuum. The orange crystals obtained were recrystallized using $\mathrm{MeOH}$ and excess DCM. The resultant crystals were filtered from solution and washed with DCM to leave pale beige crystals $(80.8 \mathrm{mg}, 54 \%)$.

${ }^{1} \mathrm{H}$ NMR $\left(\mathrm{CD}_{3} \mathrm{OD}, 300 \mathrm{MHz}\right) \delta 8.99(\mathrm{~m}, 1 \mathrm{H}), 7.98(\mathrm{~s}, 2 \mathrm{H}), 7.29(\mathrm{~m}, 1 \mathrm{H}), 6.97(\mathrm{dd}, 1 \mathrm{H})$, 6.63 (ddd, 1H).

ESI MS, m/z calc'd $599.5\left[(\mathrm{M}+\mathrm{H})^{+}\right], \mathrm{m} / \mathrm{z}$ found $599.3\left[(\mathrm{M}+\mathrm{H})^{+}\right]^{189}$ 


\subsubsection{Absorbance and luminescence studies}

\subsubsection{1 (ppy)Pt(acac-COOH)}

A portion of about $64 \mathrm{mg}$ of purified (ppy) $\mathrm{Pt}(\mathrm{acac}-\mathrm{COOH})$ was used in an initial luminescent study. It was dissolved in $5 \mathrm{~mL}$ of acetone to give a solution of $0.0245 \mathrm{M}$ (Stock 1). This was diluted to give Stock solutions 2 and 3 with concentrations of 0.0049 and $0.00015 \mathrm{M}$, respectively. Solutions were degassed prior to the collection of luminescent data. A $4.5 \mathrm{~mL}$ cuvette was used to hold the samples and they were run on a Fluorolog (Horiba Jobin Yvon). An emission spectrum was obtained with excitation at $370 \mathrm{~nm}$ and an emission peak at $485 \mathrm{~nm}$ was identified.

Table 4.3 Excitation and emission wavelengths for (ppy)Pt(acac-COOH) solutions of different concentrations.

\begin{tabular}{|c|c|c|}
\hline Stock solution & Excitation wavelength $(\mathrm{nm})$ & Emission wavelength $(\mathrm{nm})$ \\
\hline 1 & 360 & 485 \\
2 & 426 & 620 \\
3 & 476 & 626 \\
\hline
\end{tabular}

An excitation spectrum was therefore obtained with emission at $485 \mathrm{~nm}$. This process was repeated with the initial excitation wavelengths for stocks 2 and 3 being 360 and 426 $\mathrm{nm}$, respectively. The excitation wavelengths that were determined to give the best intensities at specific emission wavelengths for each stock solution are summarized in Table 4.3.

A portion of $58 \mathrm{mg}$ of purified (ppy)Pt(acac-COOH) was used to obtain luminescence spectra based on the initial study performed. The complex was dissolved 
in $5 \mathrm{~mL}$ of acetone to give a solution of concentration $0.022 \mathrm{M}$ (Stock 1). Stock 1 was diluted five times to obtain $5 \mathrm{~mL}$ total of Stock $2(0.0044 \mathrm{M})$. Stock 2 was diluted $\sim 29$ times by mixing $109 \mu \mathrm{L}$ of Stock 2 with $3091 \mu \mathrm{L}$ of acetone to obtain Stock $3(0.00015$ M). Each stock solution was degassed by bubbling argon through for 5 minutes prior to obtaining an emission spectrum. Emission spectra with excitation at $426 \mathrm{~nm}$ were obtained for Stocks 1-3.

Absorbance spectra were obtained for Stocks 1 and 3 before they were concentrated to re-isolate the complex. A solution of (ppy)Pt(acac-COOH) in acetonitrile was also prepared at a concentration of $7.5 \times 10^{-5} \mathrm{M}$ for UV-visible analysis. The intensities of the absorbance peaks for this solution were high so it was diluted eight times to one of $9.4 \times 10^{-6} \mathrm{M}$.

\subsubsection{2 (2,4-dfppy)Pt(acac-COOH)}

The luminescence measurements performed on (2,4-dfppy) $\mathrm{Pt}(\mathrm{acac}-\mathrm{COOH})$ were

reported in the B.Sc. thesis of Boshra Hosseini. ${ }^{189}$ Briefly, the measurements were performed on a $0.13 \mathrm{M}$ solution of the complex prepared in methanol that had been degassed through five freeze-pump-thaw cycles. The solution was diluted to $0.01 \mathrm{M}$ following analysis so that the emission properties of a more dilute solution could be obtained. Both solutions were excited at $355 \mathrm{~nm}$. An absorption spectrum was found for a $0.194 \mathrm{mM}$ solution of the complex.

\subsubsection{Conjugation of (ppy)Pt(acac-COOH) to the 15 mer thrombin-binding aptamer}

The 15 mer thrombin-binding aptamer was synthesized with an amino modifier on the 5 '-terminus as shown in the sequence below: 
Thrombin aptamer: ${ }^{8}$ 5'-NH2-( $\left.\mathrm{CH}_{2}\right)_{6}-\mathrm{GGT}$ TGG TGT GGT TGG-3'

The DMS(O)MT protecting group was left on the 5'-amino modifier and was removed manually as described previously. Briefly, the columns containing $500 \AA$ CPG beads were placed on the cleaving chuck and rinsed sequentially with $300 \mu \mathrm{L}$ of anhydrous $\mathrm{MeCN}, 3 \times 300 \mu \mathrm{L}$ of $10 \% \mathrm{DEA}$ in anhydrous $\mathrm{MeCN}$, and $2 \times 200 \mu \mathrm{L}$ of anhydrous MeCN. The DMS(O)MT group was then removed through $2 \times 200 \mu \mathrm{L}$ washes with deblock solution (2\% dichloroacetic acid in DCM) which were followed by 2 more 300 $\mu \mathrm{L}$ rinses with $\mathrm{MeCN}$.

About $5 \mathrm{mg}(8.8 \mu \mathrm{mol})$ of (ppy)Pt(acac-COOH) was measured into a vial along with $\sim 3 \mathrm{mg}(15.6 \mu \mathrm{mol})$ of EDC (1-ethyl-3-(3-dimethylaminopropyl)carbodiimide hydrochloride, MW $191.7 \mathrm{~g} / \mathrm{mol})$ and $\sim 2 \mathrm{mg}(17.4 \mu \mathrm{mol})$ of NHS $(N-$ hydroxysuccinimide, MW $115.10 \mathrm{~g} / \mathrm{mol}$ ). The reactants were dissolved in $1 \mathrm{~mL}$ of anhydrous DMSO and left to react at room temperature for about one hour prior to the addition of the CPG beads from a column of amino-modified DNA prepared as described above. The conjugation reaction was left to proceed at room temperature overnight while shielded from light.

The CPG beads were isolated from the solution through their collection at the bottom of an Eppendorf tube using centrifugation and the removal of the solution via pipette. The DNA was then deprotected overnight at room temperature through the addition of $1 \mathrm{~mL}$ of $\mathrm{NH}_{4} \mathrm{OH}$ to the $\mathrm{CPG}$ beads. The $\mathrm{NH}_{4} \mathrm{OH}$ solution was isolated from the CPG beads using a filter tube and then dried under vacuum.

The crude DNA-Pt conjugate was quantified using the characteristic absorbance of DNA at $260 \mathrm{~nm}$ and $8 \mathrm{nmol}$, was isolated and sent for FIA/MS analysis. The 
remaining product was desalted using a desalting tube prior to reversed-phase HPLC analysis. The sample was run using the conditions outlined in Table 4.4 with absorbance at 260 monitored. Several peaks or peak regions were collected between the 8 and 23 minute points, and the solutions were lyophilized. The DNA in these samples was quantified and then tested for luminescence.

Table 4.4 Conditions used for RP-HPLC analysis of crude Pt(II) complex-TBA conjugate.

\begin{tabular}{|c|c|c|c|c|}
\hline $\begin{array}{c}\text { Time } \\
(\mathrm{min})\end{array}$ & Fraction of $\mathrm{MeCN}(\%)$ & $\begin{array}{c}\text { Fraction of } \mathrm{NH}_{4} \mathrm{OAc} \\
\text { buffer }(\%)\end{array}$ & $\begin{array}{c}\text { Flow rate } \\
(\mathrm{mL} / \mathrm{min})\end{array}$ & $\begin{array}{c}\text { Column } \\
\text { temperature }\left({ }^{\circ} \mathrm{C}\right)\end{array}$ \\
\hline 0 & 5 & 95 & 2 & 25 \\
25 & 25 & 75 & 2 & 25 \\
40 & 100 & 0 & 2 & 25 \\
\hline
\end{tabular}

An initial luminescence study on the DNA collected in the first tube (8 to 9.8 minutes) was performed where tris buffer $(40 \mathrm{mM}, \mathrm{pH} 7.4)$ was used as solvent and a solution with a concentration of $38 \mu \mathrm{M}$ DNA was prepared. An excitation spectrum collected with emission at $485 \mathrm{~nm}$ showed that an excitation wavelength of $300-330 \mathrm{~nm}$ should be used for future studies. The remaining DNA portions were dissolved in a minimum of $600 \mu \mathrm{L}$ of buffer to accommodate the use of a $600 \mu \mathrm{L}$ cuvette or to a concentration of $70 \mu \mathrm{M}$ where possible, as summarized in Table 4.5. Emission spectra with excitation at $320 \mathrm{~nm}$ and at $302 \mathrm{~nm}$ were obtained for each sample. MS results were obtained for the samples in tubes $2,4,5$, and 6 .

The product in tubes 5 and 6 was dissolved in buffer to a final concentration of 70 $\mu \mathrm{M}$ each for a more direct comparison between their luminescence spectra. The solutions 
were degassed by bubbling argon through them for 5 minutes prior to obtaining emission spectra for each with excitation at $302 \mathrm{~nm}$.

Table 4.5 Concentrations for luminescence measurements on DNA isolated using RP-HPLC.

\begin{tabular}{|c|c|c|}
\hline DNA portion & Time collected (minutes) & Concentration $(\mu \mathrm{M})$ \\
\hline 2 & 9.8 to 11 & 37 \\
3 & 11 to 11.9 & 70 \\
4 & 11.9 to 13.2 & 34 \\
5 & 13.2 to 16.2 & 70 \\
6 & 16.2 to 18.4 & 12 \\
\hline
\end{tabular}

The stability of (ppy) Pt(acac-COOH) in $\mathrm{NH}_{4} \mathrm{OH}$ was tested through dissolving 5 mg of the complex in $1 \mathrm{~mL}$ of a $1: 1$ mixture of $\mathrm{MeOH}$ and $\mathrm{NH}_{4} \mathrm{OH}$ and leaving the sample to sit overnight at room temperature. Upon concentration of the mixture under vacuum, an orange oil was isolated. A ${ }^{1} \mathrm{H}$ NMR spectrum of the oil was obtained in acetone-d6.

\subsubsection{Conjugation of (ppy)Pt(acac-COOH) to complementary strands of DNA}

\subsubsection{Conjugation to a 5 '-amino-modified strand}

The random DNA sequence 5'- $\mathrm{NH}_{2}-\left(\mathrm{CH}_{2}\right)_{6}$-CTG ACG TAC TGA CT-3' was synthesized with the DMS(O)MT protecting group left on. The columns were rinsed with $300 \mu \mathrm{L}$ of anhydrous $\mathrm{MeCN}, 3 \times 300 \mu \mathrm{L}$ of $10 \% \mathrm{DEA}$ in anhydrous $\mathrm{MeCN}$ and $2 \times 200 \mu \mathrm{L}$ of anhydrous $\mathrm{MeCN}$ prior to storage or overnight deprotection in $\mathrm{NH}_{4} \mathrm{OH}$ at $37^{\circ} \mathrm{C}$. The solution was then dried under vacuum to isolate the DNA. 
The DNA was deblocked in solution in preparation for the reaction with the $\mathrm{Pt}(\mathrm{II})$ complex. The DMS $(\mathrm{O}) \mathrm{MT}^{+}$group was removed through a 5 minute treatment with $2 \%$ dichloroacetic acid (DCA) in $\mathrm{diH}_{2} \mathrm{O}$. The DNA was extracted from solution using ethanol precipitation as previously described (section 3.2.2). The recovered DNA was dried under vacuum and then re-dissolved in a minimum amount of $\mathrm{diH}_{2} \mathrm{O}$ for CTAB precipitation as described in section 3.2.2 and using the guidelines presented in Table 3.1. The $\mathrm{CTA}^{+}$-DNA pellet was isolated through centrifugation and the removal of most of the solution via pipette. It was fully dried under vacuum.

While the $\mathrm{CTA}^{+}$-DNA pellet dried, the activation of (ppy)Pt(acac-COOH) was performed through the reaction of $\sim 1 \mathrm{mg}(1.8 \mu \mathrm{mol})$ of the complex with $\sim 3.4 \mathrm{mg}(17.7$ $\mu \mathrm{mol})$ of EDC and $\sim 5.1 \mathrm{mg}(44 \mu \mathrm{mol})$ of NHS in $100 \mu \mathrm{L}$ of dimethylformamide (DMF). The reaction was performed at room temperature for about 30 minutes prior to its addition to the $\mathrm{CTA}^{+}$-DNA. The reaction mixture was agitated for about an hour during which time a $3 \%$ solution of lithium perchlorate in acetone was prepared and chilled on dry ice. The reaction mixture and $1 \mathrm{~mL}$ of the lithium perchlorate solution were mixed together and centrifuged at $15,000 \mathrm{~g}$ for 2 minutes to collect precipitated DNA as previously described. ${ }^{175}$ The supernatant was removed with minimal agitation of the pellet, and the pellet was dried under vacuum prior to quantification using UV-visible spectrophotometry. PAGE was used for purification of the crude Pt(II) complex-DNA conjugate on a $19 \%$ polyacrylamide gel prepared as in section 2.2 .3 . The DNA was eluted from the gel, desalted using a desalting tube, and quantified. Luminescence spectra were obtained for a sample where $11 \mathrm{nmol}$ of DNA was isolated. A portion of this sample was also sent for MALDI MS analysis. 
The same 5'-amino-modified DNA sequence that was used as in the solutionphase reaction was synthesized using guanine and adenine bases with ultramild protecting groups. These groups do not require the use of $\mathrm{NH}_{4} \mathrm{OH}$ for deprotection but can be removed using a solution of $0.05 \mathrm{M}$ potassium carbonate in methanol. Following DNA synthesis the CPG beads were rinsed and deblocked as described above. Either EDC and NHS (as described for the solution-phase reaction) or the combination of diisopropylethylamine (DIPEA, MW $129.24 \mathrm{~g} / \mathrm{mol})$ and $O-\left(N\right.$-succinimidyl)- $N, N, N^{\prime}, N^{\prime}$,tetramethyluronium tetrafluoroborate (TSTU, MW $301.05 \mathrm{~g} / \mathrm{mol}$ ) were used for activation of the $\mathrm{Pt}(\mathrm{II})$ complex (as described by Barton et al. ${ }^{194}$ ). For the latter, a portion of $1 \mathrm{mg}(1.8 \mu \mathrm{mol})$ of (ppy)Pt(acac-COOH) was reacted with $1.2 \mu \mathrm{L}(6.9 \mu \mathrm{mol})$ of DIPEA and $1.4 \mathrm{mg}(4.8 \mu \mathrm{mol})$ of TSTU in a 1:1:1 mixture of $\mathrm{MeCN}, \mathrm{DCM}$, and $\mathrm{MeOH}$ for 1 hour. ${ }^{194}$ The EDC/NHS reaction was performed in $1 \mathrm{~mL}$ DMF as in the solution phase reaction. Following activation of (ppy)Pt(acac-COOH), the CPG beads of deblocked DNA were added to the $\mathrm{Pt}(\mathrm{II})$ complex reaction mixture along with another $2.6 \mu \mathrm{L}(15 \mu \mathrm{mol})$ of EDIA when DIPEA/TSTU were used and the mixture was left to react at room temperature overnight. The solution was removed from the $\mathrm{CPG}$ beads via pipette and the beads were then rinsed with $300 \mu \mathrm{L}$ each of $\mathrm{MeCN}, \mathrm{MeOH}$, and $\mathrm{DCM}$ in the DIPEA/TSTU case ${ }^{194}$ or with DMF in the EDC/NHS reaction. For each column's worth of ultramild DNA, $1 \mathrm{~mL}$ of a $0.05 \mathrm{M}$ potassium carbonate in methanol solution was added for overnight deprotection at room temperature. The solution was pipetted from the beads the following day and was neutralized through the addition of $1.5 \mathrm{~mL}$ of 2 $\mathrm{M}$ triethylammonium acetate buffer ${ }^{195}$ or with $6 \mu \mathrm{L}$ of acetic acid prior to being dried under vacuum. 
The DNA thus reacted was then run using PAGE (19\% polyacrylamide gel) or RP-HPLC in an effort to purify Pt(II) complex-conjugated DNA from the crude reaction product. Triethylammonium acetate (TEAA) buffer was prepared containing $5 \% \mathrm{MeCN}$ by volume and the conditions used for RP-HPLC analysis of the samples are summarized in Table 4.6. Absorbance at 260 and $330 \mathrm{~nm}$ was monitored. Samples isolated using PAGE or RP-HPLC were tested for luminescence and promising fractions were sent for MS analysis.

Table 4.6 Conditions for RP-HPLC analysis of crude Pt(II) complex-DNA following solid-phase reactions. Note that the TEAA buffer was prepared with $5 \% \mathrm{MeCN}$, so the fraction of $\mathrm{MeCN}$ could be considered to be $5 \%$ higher than the value recorded here.

\begin{tabular}{|c|c|c|c|c|}
\hline $\begin{array}{c}\text { Time } \\
(\mathrm{min})\end{array}$ & Fraction of MeCN (\%) & $\begin{array}{c}\text { Fraction of TEAA buffer } \\
\text { with 5\% MeCN }(\%)\end{array}$ & $\begin{array}{c}\text { Flow rate } \\
(\mathrm{mL} / \mathrm{min})\end{array}$ & $\begin{array}{c}\text { Column } \\
\text { temperature }\left({ }^{\circ} \mathrm{C}\right)\end{array}$ \\
\hline 0 & 5 & 95 & 2 & 25 \\
40 & 45 & 55 & 2 & 25 \\
\hline
\end{tabular}

The same 5'-amino-modified ultramild DNA sequence was also used in a reaction with acac-COOH alone. About $3 \mathrm{mg}(14 \mu \mathrm{mol})$ of acac-COOH was mixed with $10.3 \mu \mathrm{L}$ $(60 \mu \mathrm{mol})$ of DIPEA and about $13 \mathrm{mg}(43 \mu \mathrm{mol})$ of TSTU and dissolved in a 1:1:1 mixture of $\mathrm{MeCN}, \mathrm{DCM}$, and $\mathrm{MeOH}$. While this was left to react at room temperature for one hour, a column of DNA was manually deblocked as previously described. The CPG beads were then added to the acac- $\mathrm{COOH}$ reaction mixture along with another 22.9 $\mu \mathrm{L}(134 \mu \mathrm{mol})$ of DIPEA and this was left to react overnight. The following isolation and rinsing of the beads and deprotection of the DNA were performed as previously described. 


\subsubsection{Conjugation to a 3 '-amino-modified strand}

3'-Amino-modifier C7 CPG columns (500 $\AA$ ) were obtained from Glen Research and used for the synthesis of a 14mer with sequence 5'-AGT CAG TAC GTC AG$\left(\mathrm{CH}_{2}\right)_{7}-\mathrm{NH}_{2}-3^{\prime}$ under ultramild conditions. Following synthesis, the CPG beads were rinsed with $\mathrm{MeCN}$ and $10 \% \mathrm{DEA}$ in $\mathrm{MeCN}$ as in previous reactions (section 2.2.2) prior

to the removal of the Fmoc amine protecting group as instructed. ${ }^{196}$ Briefly, a $20 \% \mathrm{v} / \mathrm{v}$ solution of piperidine in DMF was prepared and used to rinse the beads in $300 \mu \mathrm{L}$ portions for a total of $7.5 \mathrm{~mL}$. This was followed with a $3 \mathrm{~mL}$ total rinse with $\mathrm{DMF}$ and then a $3 \mathrm{~mL}$ total rinse with $\mathrm{MeCN}$, both in $300 \mu \mathrm{L}$ portions. The beads were then mixed with a solution of (ppy)Pt(acac-COOH) preactivated with TSTU and EDIA as previously described (section 4.2.7.1). Following an overnight reaction period, the DNA was deprotected using ultramild conditions, neutralized, and quantified as previously described (section 4.2.7.1). It was then run on RP-HPLC using the same conditions as in Table 4.6. DNA in the fractions was quantified and the samples were tested for luminescent properties. Promising fractions were sent for MS analysis.

\subsection{Results and discussion}

\subsubsection{Synthesis of $\mathrm{Pt}(\mathrm{II}) \mu$-dichloro-bridged dimers}

The three $\mathrm{C}^{\wedge} \mathrm{N}$ cyclometalating ligands used in this work were selected due to the different $\lambda_{\max }$ values reported for the similar Pt(II) complexes prepared by Thompson et al. in which they were included. It was expected that the Pt(II) complex containing 2phenylpyridine (ppy) and the modified acetylacetone ligand discussed in the following section would emit at a $\lambda_{\max }$ between those of complexes prepared with the other two 
$\mathrm{C}^{\wedge} \mathrm{N}$ ligands. The inclusion of 2-(2,4-difluorophenyl)pyridine was expected to cause the $\lambda_{\max }$ to be blue-shifted from that of the ppy complex while the use of 2-phenylquinoline was expected to lead to emission with a red-shifted $\lambda_{\max }{ }^{186}$

Platinum(II) $\mu$-dichloro-bridged dimers were prepared as described by Thompson et $a .^{186}$ The reaction is shown in Scheme 4.2. Potassium tetrachloroplatinate(II) $\left(\mathrm{K}_{2} \mathrm{PtCl}_{4}\right)$ and 2.5 equivalents of 2-phenylpyridine (ppy) or 2-phenylquinoline were reacted at $80^{\circ} \mathrm{C}$. Two molar equivalents of 2-(2,4-difluorophenyl)pyridine was added to the platinum salt for the preparation of $\mu$-dichlorobis(2- $(2,4-$ difluorophenyl)pyridylplatinum(II)). ${ }^{189}$ In all cases the reaction took place in an inert atmosphere for about 16 hours.

The reaction mixture became a clear yellow solution in the case of 2 phenylpyridine and a brown solution in the case of 2-phenylquinoline within an hour of the application of heat. The hot plate was turned off after the reaction period and the flask was lifted from the oil bath to permit its cooling to room temperature. Once cool, enough deionized water was added to the reaction mixture so that the reaction volume was doubled. The respective products, $\mu$-dichlorobis(2-phenylpyridylplatinum(II)), $\mu$ dichlorobis(2-phenylquinolineplatinum(II)), $\quad$ or $\quad \mu$-dichlorobis(2-(2,4difluorophenyl)pyridylplatinum(II)), were isolated by filtration following their precipitation upon the addition of water to the reaction mixture.. The $\mu$-dichlorobis(2phenylpyridylplatinum(II)) was a yellow solid while $\mu$-dichlorobis(2phenylquinolineplatinum(II)) was a golden yellow solid. $\mu$-Dichlorobis(2-(2,4difluorophenyl)pyridylplatinum(II)) was light green in colour. ${ }^{189}$ 
As 2-ethoxyethanol does not have a high vapor pressure, ${ }^{197}$ the dimers were stored in a desiccator until dry. Typical yields for $\mu$-dichloro(ppy)Pt(II) were in the range of $70-90 \%$. The ${ }^{1} \mathrm{H}$ NMR spectrum obtained on a $300 \mathrm{MHz}$ NMR spectrometer with the sample dissolved in DMSO-d6 is shown in Figure C-1 (Appendix C). No peaks corresponding to unreacted ppy were visible in any of the ${ }^{1} \mathrm{H}$ NMR spectra obtained following the reactions performed to obtain the $\mu$-dichloro(2-phenylpyridineplatinum(II)) dimers and they were used in later reactions without further purification.

A) $\mathrm{K}_{2} \mathrm{PtCl}_{4(\mathrm{~s})}+$<smiles>c1ccncc1</smiles>

$3: 1$ ethoxyethanol/ $/ \mathrm{diH}_{2} \mathrm{O}$<smiles>Cc1ccccc1</smiles>

B) $\mathrm{K}_{2} \mathrm{PtCl}_{4(\mathrm{~s})}+$

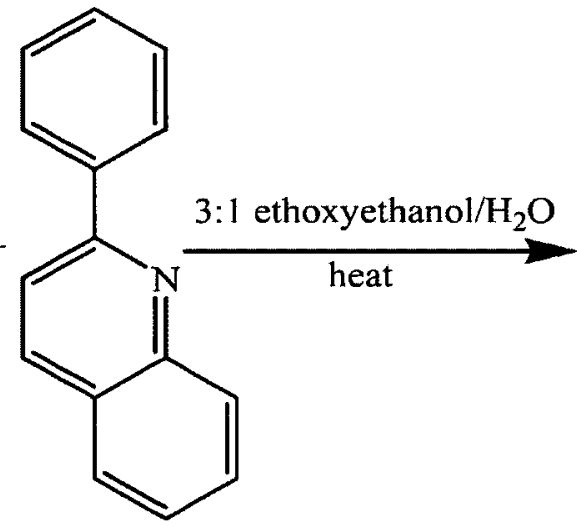<smiles>Cl[PH](Cl)(Cl)[n+]1ccccc1-c1ccccc1</smiles><smiles></smiles><smiles></smiles>

Scheme 4.2 Reaction of potassium tetrachloroplatinate(II) with A) 2-phenylpyridine to form $\mu$ dichloro(2-phenylpyridineplatinum(II)) dimers and B) 2-phenylquinoline to form $\mu$-dichloro(2phenylquinolineplatinum(II)) dimers. ${ }^{198}$ 
Yields could not be calculated for $\mu$-dichloro(pq)Pt(II) which was most likely due to the presence of unreacted 2-phenylquinoline; yields always worked out to greater than $100 \%$ as a result. Suspected pq peaks are labeled in the ${ }^{\prime} \mathrm{H}$ NMR spectrum shown in Figure $\mathrm{C}-2$, while the inset shows the relevant section of a reported ${ }^{1} \mathrm{H}$ NMR spectrum for pq. Though less pure than the $\mu$-dichloro(ppy)Pt(II) dimers isolated, the $\mu$ dichloro(pq)Pt(II) dimers were used in subsequent reactions without further purification as reported by Thompson et al. ${ }^{186}$

\subsubsection{Synthesis of 8,10-dioxoundecanoic acid}

A platinum(II) ion complexed with a 2-phenylpyridine ligand and acetylacetone (acac) was synthesized and characterized by Thompson et al. and was among several complexes described that were intensely emissive at room temperature in solution. ${ }^{186}$ For the conjugation of such a complex to DNA a reactive functional group must be present. The synthesis of a modified acetylacetone ligand in which acac was connected to a hexanoic acid was described by Weizman and Tor for use in creating acetylacetonate complexes with a functionalized linker for nucleotide tagging. ${ }^{188}$ Their modified acac ligand was suitable for use in a Pt(II) complex and so was synthesized from a method modified from that they described.

The synthesis of acac- $\mathrm{COOH}$ was a multistep process and the reactions that occurred in the three stages of the synthesis are shown in Scheme 4.3. The reaction in Scheme $4.3 \mathrm{i}$ represents the reaction of lithium hydride $(\mathrm{LiH})$ and 6-bromohexanoic acid (BrHex). The lithium ion associates with the carboxylate group and hydrogen gas is 
released as a result, as shown in Scheme $4.3 \mathrm{i}$. The lithium-carboxylate interaction is strong, with the affinity of lithium for oxygen resulting in a bond with considerable covalent character. ${ }^{142}$ The reaction mixture of this stage was white and foamy in appearance after about an hour.

i) $\mathrm{LiH}+\mathrm{Br}$<smiles>CC(Br)CBr</smiles>
iia)

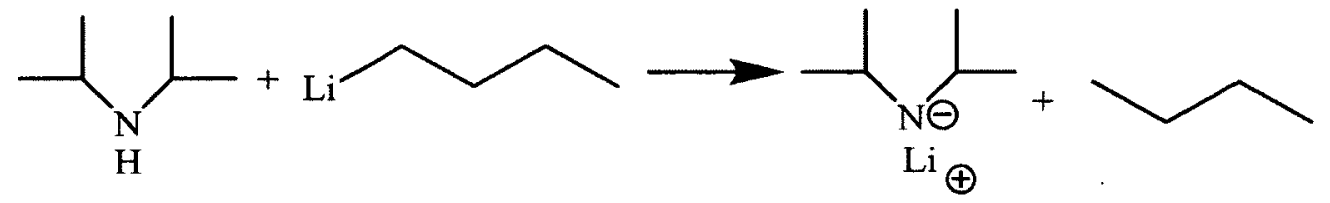

iib)<smiles>CC(=O)CC(C)=O</smiles>
1st equivalent 2nd equivalent

iii)<smiles>CC1=[C+]O[AlH]OC(C)=C1</smiles>

Scheme 4.3 Synthesis of the modified acetylacetone ligand (acac-COOH): 8,10-dioxoundecanoic acid. See text for discussion.

Part 2 of the reaction was performed in two stages. Lithium diisopropylamide (LDA) was prepared in situ, as represented in Scheme 4.3iia. ${ }^{199}$ Scheme 4.3iib illustrates the reaction of LDA with acetylacetone (acac) to form a lithium enolate. The use of such as strong base as LDA for this stage ensures that the acac is quantitatively converted to the enolate form and is therefore reactive in the next part of the reaction (Scheme 
4.3iii). ${ }^{142}$ Dry hexamethylphosphoramide (HMPA) was added along with acac in most of the reactions performed since it helps increase the reactivity of LDA. It does so through the strong association of its basic oxygen atom with the lithium cation, leaving the anionic diisopropylamide unsolvated by HMPA and thus more reactive. ${ }^{142}$ Acetylacetone is therefore deprotonated by the first equivalent of LDA, as in Scheme 4.3iib, and a lithium enolate was formed through reaction with a second equivalent of LDA. This reaction was found to proceed well without the addition of HMPA and it was eliminated from the protocol in later reactions since its absence also gave a cleaner crude product with one fewer compound to remove during purification procedures.

The lithium 6-bromohexanoate suspension was reacted with the reactive lithium enolate intermediateresulting in a modified acetylacetone ligand, as shown in Scheme 4.3iii.

The modified acac ligand consists of pale, nearly colourless, fine crystals when pure. If the acac- $\mathrm{COOH}$ isolated from the column appeared to have few impurities, based on its ${ }^{1} \mathrm{H}$ NMR spectrum, but was orange or red-orange in colour, it was recrystallized to complete its purification. Following successful reactions and purifications, the yield of acac-COOH ranged from $11 \%$ to $19 \%(\sim 240$ to $411 \mathrm{mg})$.

It was found by Weizman and Tor that acac-COOH exists as a tautomeric mixture of the enol and keto forms with $85 \%$ and $15 \%$ of each, respectively. The structures of the two forms are shown in Figure $\mathrm{C}-3$ along with the ${ }^{1} \mathrm{H}$ NMR for the product in $\mathrm{CDCl}_{3}$ and the assignments of peaks. Note that the peak at $5.5 \mathrm{ppm}$ is characteristic of the enol, and therefore the more prevalent form of acac- $\mathrm{COOH}$. When acetone-d6 was used as solvent, the peak shifts to $5.66 \mathrm{ppm}$. Any changes in the chemical shift of this peak in subsequent 
reactions using acac- $\mathrm{COOH}$ were used to monitor the formation of new compounds, as will be discussed below.

\subsubsection{Synthesis of luminescent Pt(II) complexes}

\subsubsection{General synthetic route}

In order to obtain luminescent, square planar $\mathrm{Pt}(\mathrm{II})$ complexes containing the modified acetylacetone ligand and one of the respective cyclometalating $\mathrm{C}^{\wedge} \mathrm{N}$ ligands, the first step was to break the Pt(II) $\mu$-dichloro-bridged dimers through the removal of the chloride ions.

i)

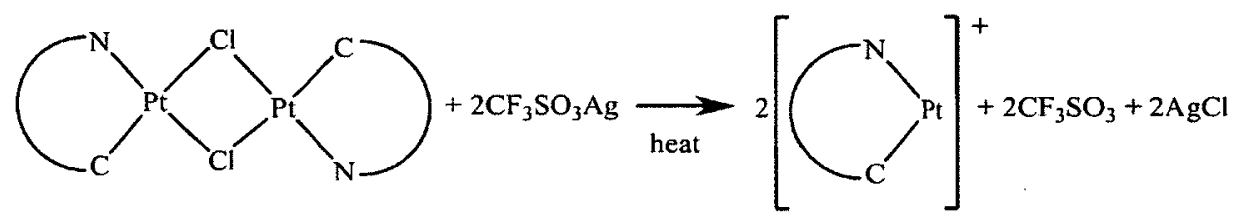

ii)

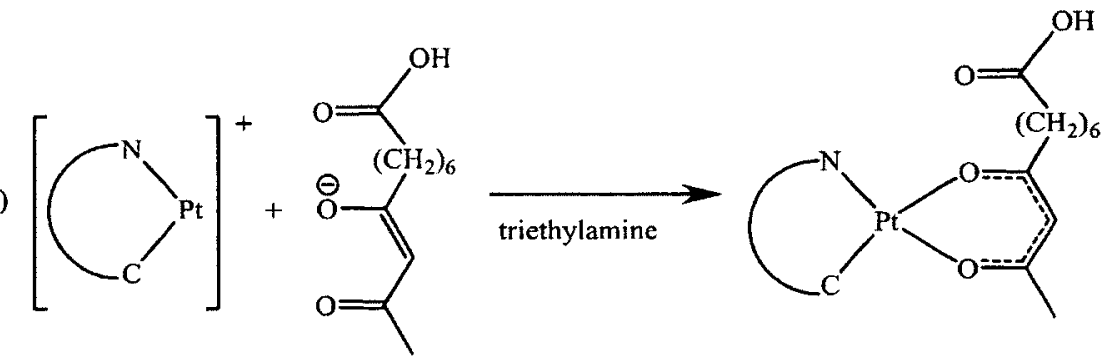

b)<smiles>c1ccc(-c2ccc3ccccc3n2)cc1</smiles>

c)

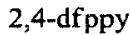

)<smiles>Cc1cc(F)ccc1-c1ccccn1</smiles>

Scheme 4.4 General reaction scheme for the synthesis of square planar Pt(II) complexes containing acac- $\mathrm{COOH}$ and one of the three cyclometalating $\mathrm{C}^{\wedge} \mathrm{N}$ ligands included at the bottom. 
This was achieved by heating a portion of the appropriate dimer in the presence of about 2 equivalents of silver trifluoromethanesulfonate. Upon the formation of solid silver chloride, the dimers were broken in two. This is illustrated in Scheme 4.4i.

Following this stage, the silver chloride was removed from the reaction mixture through gravity filtration and a slight excess of acac- $\mathrm{COOH}$ was added in the presence of triethylamine (Scheme 4.4ii) so as to keep the acac-COOH in the enolate form. ${ }^{142}$ The reaction with acac- $\mathrm{COOH}$ was performed either at room temperature or with the application of heat. The specifics of the reactions for each complex as well as their purification and characterization will be discussed in the following three sections.

\subsubsection{Synthesis, purification, and characterization of 8,10-dioxoundecanoic acid-2-} phenylpyridylplatinum(II); (ppy)Pt(acac-COOH)

The synthesis of 8,10-dioxoundecanoic acid-2-phenylpyridylplatinum(II), or for short (ppy)Pt(acac-COOH), began with breaking the $\mathrm{Pt}-\mathrm{Cl}$ bonds in $\mu$-dichloro(ppy)Pt(II) dimers. As noted in the parameterization summary in Table 4.7, no advantage was discernible when greater than 18 hours was used for this reaction.

To ensure the dimers were predominantly broken at this stage, TLC was used to qualitatively compare the reaction mixture to a sample of $\mu$-dichloro(ppy)Pt(II) dimers. TLC provided a reliable method by which to test for a difference between a sample of unbroken dimers and the reaction mixture. The $\mu$-dichloro(ppy)Pt(II) dimers were monitored through their characteristic green luminescence and were found to run nearly to the solvent front when $30 \%$ EtOAc in DCM was used as the mobile phase. When spotted on a TLC plate, the reaction mixture generally had a faint luminescent green spot 
Table 4.7 Parameterization summary for synthesis and purification of 8,10-dioxoundecanoic acid-2-phenylpyridylplatinum(II); (ppy)Pt(acac-COOH)

\begin{tabular}{|c|c|c|}
\hline Breaking Pt(II) dimers & acac- $\mathrm{COOH}$ addition and reaction period & Purification conditions ${ }^{[a]}$ \\
\hline $\begin{array}{l}\text { - always at } 50^{\circ} \mathrm{C} \\
\text { reacted for a minimum of } \sim 18 \text { hours } \\
\text { (overnight) } \\
\text { reacted for } 20-24 \text { hours several times, } \\
\text { though no discernible advantage was } \\
\text { found } \\
\text { - gravity filtered to remove } \mathrm{AgCl}_{(\mathrm{s})} \\
\text { - determined that } \mathrm{TLC} \text { could be used to } \\
\text { qualitatively demonstrate breaking of } \\
\text { dimers } \\
\text { - TLC: run in } 30 \% \text { EtOAc in DCM, dimers } \\
\text { move nearly to the solvent front and } \\
\text { fluoresce green; broken dimer solution } \\
\text { mostly fluoresced green where spotted and } \\
\text { just above with a faint full dimer spot } \\
\text { present near the solvent front } \\
\text { when TLC monitoring indicated more } \\
\text { unbroken dimer was present than usually } \\
\text { seen, extra } \mathrm{CF}_{3} \mathrm{SO}_{3} \mathrm{Ag} \text { was added and left } \\
\text { for a second night }- \text { no discernible } \\
\text { advantage since purification was } \\
\text { incomplete }\end{array}$ & 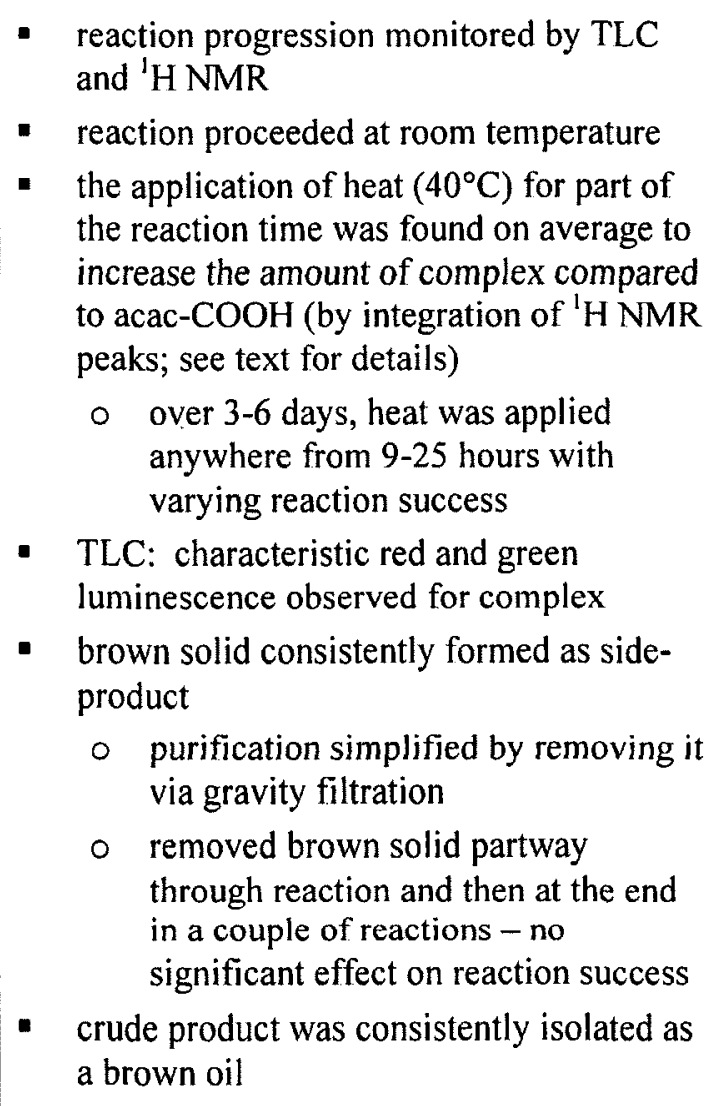 & $\begin{array}{l}\text { crude product oil always dissolved and } \\
\text { dried onto silica for column purification } \\
\text { solvent gradients used in columns where } \\
\text { complex was not completely purified } \\
\text { - } \text { EtOAc to MeOH } \\
\text { - } \text { toluene to } 30 \% \text { EtOAc in DCM } \\
\text { - toluene, } 30 \% \text { EtOAc in DCM, EtOAc } \\
\text { - toluene, } 50 \% \text { DCM in toluene, } 30- \\
75 \% \text { EtOAc in DCM } \\
\text { - toluene+ } 1 \% \text { acid, } 50 \% \text { DCM in } \\
\text { toluene+1\% acid, } 30+\% \text { EtOAc in } \\
\text { DCM+1\% acid } \\
\text { solvent gradients that gave successful } \\
\text { purification } \\
\text { - toluene, slow gradient to DCM then } \\
\text { to } 30(-100) \% \text { EtOAc in DCM } \\
\text { - toluene+ }+1 \% \text { acid, slow gradient to } \\
\text { DCM+1\% acid } \\
\text { - toluene+1\% acid, slow gradient to } \\
\text { DCM+1\% acid, } 30 \% \text { EtOAc in } \\
\text { DCM+1\% acid } \\
\text { - toluene+1\% acid, slow gradient to } \\
30 \% \text { EtOAc in DCM }+1 \% \text { acid }\end{array}$ \\
\hline
\end{tabular}


near the solvent front, likely due to unreacted dimers, but most of the luminescence remained where the sample was spotted or just slightly higher. This is perhaps due to the positively charged (ppy) $\mathrm{Pt}^{+}$halves sticking to the silica (Scheme 4.4i).

For the complexation of acac-COOH with the (ppy) $\mathrm{Pt}^{+}$cations, an excess of acac$\mathrm{COOH}$ was added to the broken dimer solution along with a high excess of triethylamine (TEA) as represented in Scheme 4.4ii. A new peak in the region of the characteristic peak of the enol form of acac-COOH (5.66 ppm in acetone-d6) was found at $5.59 \mathrm{ppm}$ (acetone-d6) in the ${ }^{1} \mathrm{H}$ NMR spectra for the crude product from these first reactions implying that a new chemical species was present. This change in chemical shift demonstrated that the electron density of the enolate portion of acac- $\mathrm{COOH}$ was being shared with Pt(II) and so the proton under consideration is slightly less deshielded in the complex than in free ligand. ${ }^{142}$ The acac-COOH to new species peak integration ratios were $8.3: 1$ and $3.8: 1$, respectively.

At the end of the reaction time, a brown solid could be seen in the reaction mixture. When concentrated following the first reaction for the formation of (ppy)Pt(acac-COOH), the crude product was a dark brown sticky solid. Some solid remained undissolved following the addition of acetone-d6 for an NMR sample and so the sample was filtered prior to obtaining a spectrum. The new peak at $5.59 \mathrm{ppm}$ was observed in this sample, so the undissolved material did not seem to be the desired product. Following the next synthesis, the brown solid was removed from the reaction mixture via gravity filtration and attempts were made to dissolve it in several solvents (30\% EtOAc in DCM, $N, N$-dimethylformamide, $\mathrm{H}_{2} \mathrm{O}$, dilute aqueous acetic acid). It did not dissolve in any of them, which further supported that this was an undesired side- 
product since the luminescent $\mathrm{Pt}(\mathrm{II})$ complexes isolated by Thompson et al. were soluble in organic solvents. ${ }^{186}$ It might have contained silver chloride that formed following the addition of acac-COOH along with other insoluble silver salts. In all subsequent reactions the reaction mixture was gravity filtered to remove the precipitate at the end of the reaction time and it was discarded.

In an attempt to drive the reaction further towards the product, the application of heat $\left(40^{\circ} \mathrm{C}\right)$ intermittently with reaction periods at room temperature was used for remaining syntheses of (ppy)Pt(acac-COOH). The progression of the reaction was monitored using TLC and ${ }^{1} \mathrm{H}$ NMR. Upon successful purification of (ppy)Pt(acac$\mathrm{COOH}$ ), it was confirmed that the peak at $5.59 \mathrm{ppm}$ was characteristic of the complex and could be used to monitor reaction progression when compared to the corresponding acac-COOH peak. The best ratio found in the crude product between acac-COOH and the complex was about $1.2: 1$. While the NMR results were generally used as more concrete proof that the desired complex had been formed, the reaction was also monitored using TLC. The appearance of a luminescent green band that moved with slightly lower mobility than intact $\mu$-dichloro(ppy)Pt(II) dimers in $30 \%$ EtOAc in DCM was used to confirm the presence of (ppy)Pt(acac-COOH) by TLC.

The crude product obtained from the reaction for (ppy) $\mathrm{Pt}(\mathrm{acac}-\mathrm{COOH})$ was always a brown oil and silica gel chromatography was used for purification of the new complex. Interestingly, when run on TLC plates the purified complex sometimes showed red emission in the middle of its band with green luminescence surrounding it. This was promising as it implied that the complex had concentration-dependent luminescence, as the middle of the band would be more concentrated than the outside, and this indicated 
that the complex might form excimers. Yields of 10-24\% pure complex were obtained. A ${ }^{1} \mathrm{H}$ NMR spectrum of purified (ppy)Pt(acac-COOH) is shown in Figure C-4. Mass spectrometry was also used to characterize the product (see section 4.3.3.2).

\subsubsection{Synthesis and purification attempts for 8,10-dioxoundecanoic acid-2-} phenylquinolylplatinum(II); (pq)Pt(acac-COOH)

The synthesis of 8,10-dioxoundecanoic acid-2-phenylquinolylplatinum(II) was performed to expand the number of square-planar, luminescent platinum(II)-based probes available for the study of aptamer-target folding. The synthesis and purification methods initially used to obtain (pq)Pt(acac-COOH) were based on those developed for (ppy)Pt(acac-COOH). The synthesis of (pq)Pt(acac-COOH) therefore began with the reaction of $\mu$-dichloro(pq) $\mathrm{Pt}(\mathrm{II})$ dimers with $\mathrm{CF}_{3} \mathrm{SO}_{3} \mathrm{Ag}$ to break the $\mathrm{Pt}-\mathrm{Cl}$ bonds in the dimers. These dimers proved less soluble in acetone than those containing the 2phenylpyridine ligand, so a greater volume of acetone was required when it was used as the solvent. DCM was used for the third reaction for $(\mathrm{pq}) \mathrm{Pt}(\mathrm{acac}-\mathrm{COOH})$ as the dimers seemed to be slightly more soluble in DCM. The dimers were broken by stirring the reaction mixture at $50^{\circ} \mathrm{C}$ for $20-24$ hours.

TLC was used to check that the dimers were broken in the same way as with the $\mu$-dichloro(ppy)Pt(II) dimers: when 30\% EtOAc in DCM was used as the mobile phase, full dimers had high mobility and nearly reached the solvent front while the reaction mixture had most of the luminescence where it was spotted or just above. For the $\mu$ dichloro(pq)Pt(II) dimers and broken dimers, the luminescence was orange. 
A 1:7 mixture of 2-ethoxyethanol and toluene was also used as solvent for this reaction, permitting a reflux temperature of $100^{\circ} \mathrm{C}$ to be used. The $\mu$-dichloro(pq) $\mathrm{Pt}(\mathrm{II})$ dimers seemed slightly more soluble in this solvent mixture than in acetone or in DCM, and the higher temperature was used in an effort to push the reaction forward. ${ }^{1} \mathrm{H}$ NMR was used to compare the reaction mixture to the $\mu$-dichloro(pq)Pt(II) dimers for all reactions where this solvent mixture was used. The peak patterns were different in the reaction and full dimer spectra, and peaks in the 9-10 ppm region for the dimer spectrum were much diminished in intensity in the reaction spectrum. Table 4.8 contains a summary of the parameters adjusted in both parts of the reaction performed to obtain (pq)Pt(acac-COOH).

Following qualitative TLC or ${ }^{1} \mathrm{H}$ NMR results that supported the progression of the reaction, the brown reaction mixture was gravity filtered into a clean flask so as to remove the precipitated $\mathrm{AgCl}$. To the now orange reaction mixture, acac- $\mathrm{COOH}$ and TEA were added and the reaction mixture changed back to brown very quickly. The first time the reaction was performed, heat $\left(40^{\circ} \mathrm{C}\right)$ was applied to the reaction mixture for a total of 24.5 hours over two days. A 'H NMR spectrum performed on the crude product had a few peaks in the 5.5 to $6.0 \mathrm{ppm}$ region aside from that for leftover acac-COOH, therefore a silica gel column was used to try to purify the (pq)Pt(acac-COOH) complex from leftover starting materials. About a third of the crude product was dried onto silica and added to a column prepared using toluene. The mobile phase was gradually changed to $30 \%$ EtOAc in DCM since this had proven to be a successful solvent system for the purification of (ppy)Pt(acac-COOH). A luminescent orange spot with similar mobility to acac- $\mathrm{COOH}$, but lower mobility than the $\mu$-dichloro(pq)Pt(II) dimers, was observed on 
TLC plates for several fractions. These fractions were concentrated and redissolved in acetone-d6 for ${ }^{3} \mathrm{H}$ NMR. The spectrum obtained is shown in Figure C-5 and a prevalent peak at about 5.7 ppm can be seen. There are many unidentified peaks for this sample in the aliphatic region with high intensities unlike in the case of (ppy)Pt(acac-COOH) (Figure C-4) where peaks could be picked out corresponding to the acac-COOH ligand. Therefore, while the product isolated from this column might have contained a new chemical species that was partially purified, only about $4 \mathrm{mg}$ were isolated, and its identity could not be confirmed at this stage. This result was unfortunately not repeated when columns were performed on the remaining $2 / 3$ of the reaction product or for the next two reaction attempts.

The later reactions performed were regularly monitored using ${ }^{1} \mathrm{H}$ NMR where a peak at $5.7 \mathrm{ppm}$ in acetone-d6 (or $5.57 \mathrm{ppm}$ in $\mathrm{CDCl}_{3}$ ) was thought indicative of (pq) Pt(acac-COOH) complex formation due to this being the chemical shift of the prevalent peak for the partially purified sample described above. The integration of this peak was compared to that of the peak at $5.66 \mathrm{ppm}$ in acetone-d6 (or $5.5 \mathrm{ppm}$ in $\mathrm{CDCl}_{3}$ ) representing acac- $\mathrm{COOH}$, with a ratio of about $1: 10$ typically seen but a ratio of $1: 6$ being the best found over all reactions performed. The 1:6 ratio was observed in one case following about three days of reaction at $40^{\circ} \mathrm{C}$, but the ratio changed to $1: 12$ after one more overnight period at this temperature. Therefore, the reaction was stopped in later reactions when the ratio of complex to acac- $\mathrm{COOH}$ peaks had stabilized.

The switch to a 1:7 mixture of 2-ethoxyethanol and toluene was useful in monitoring the reaction by ${ }^{1} \mathrm{H}$ NMR as neither of these solvents has a peak in the 5-6 ppm region that could interfere with identification of complex or acac-COOH peaks in 
the reaction mixture as was the case for DCM. The 2-ethoxyethanol could not be removed under vacuum, however, without the application of heat, and this was undesirable following the reaction period as additional heat could cause the reaction to reverse as just discussed. Therefore, extractions for the removal of (pq)Pt(acac-COOH) from the 2-ethoxyethanol were attempted for the last three reactions performed to obtain this complex. The extractions and their results are summarized in Table 4.9.

Upon addition of excess diethyl ether to the 2-ethoxyethanol reaction solution, a yellow precipitate formed leaving the reaction mixture golden or brown in colour. ${ }^{1} \mathrm{H}$ NMR analysis was used to determine that this precipitate consisted of $\mu$-dichlorobis(2phenylquinolylplatinum(II)), therefore resulting in partial purification of the reaction mixture. In the first case that this was attempted, the solution was determined to contain about a 1:3 ratio of complex to acac- $\mathrm{COOH}$ based on the peak integrations as discussed above. The 2-ethoxyethanol reaction solution was then mixed with an excess of $\mathrm{diH}_{2} \mathrm{O}$ which again caused a solid to precipitate out of solution. The solid was characterized using NMR and in the first and last cases documented in Table 4.9 it can be seen that the complex to acac- $\mathrm{COOH}$ ratio was found to be about $1: 2$. For both of these reactions, an attempt to further purify the complex from the precipitate using silica gel chromatography was made. The conditions used were described in section 4.2.4.2. In the first of these cases, the complex seemed to be purified with regards to acac-COOH, but some dimer (or broken dimer) was likely mixed with it. This same result was obtained following the column in the second of these cases (reaction 6 in Table 4.9). Therefore, purification of (pq) Pt(acac-COOH) was not achieved in either case. 
Table 4.8 Parameterization summary for synthesis of 8,10-dioxoundecanoic acid-2-phenylquinolylplatinum(II); (pq)Pt(acac-COOH)

\begin{tabular}{|c|c|}
\hline Breaking $\mathrm{Pt}(\mathrm{II})$ dimers & acac- $\mathrm{COOH}$ addition and reaction period \\
\hline $\begin{array}{l}\text { - heated for } 20-24 \text { hours } \\
\text { - } \text { when acetone or DCM was used as solvent it was reacted to } 50^{\circ} \mathrm{C} \\
\text { - when } 1: 7 \text { mixture of } 2 \text {-ethoxyethanol and toluene was used as } \\
\text { solvent it was reacted at } 100^{\circ} \mathrm{C} \\
\text { - progression monitored by TLC and/or }{ }^{1} \mathrm{H} \text { NMR } \\
\text { - TLC } \\
\text { run in DCM or in } 30 \% \text { EtOAc in DCM } \\
\text { full dimers have high mobility and fluoresce orange } \\
\text { reaction mixture of broken dimers showed some remaining full } \\
\text { dimer as well as luminescent orange spots where spotted on the } \\
\text { TLC plate and just above } \\
\text { } \mathrm{HMR} \\
\text { different peak pattern compared to full dimer spectrum }\end{array}$ & $\begin{array}{l}\text { - } \\
\text { - total time heated was } 24.5 \text { hours the first reaction, but more than } 30 \\
\text { hours in all other cases } \\
\text { - }{ }^{\circ} \mathrm{C} \text { and left at room temperature (RT) intermittedly } \\
\text { - }{ }^{1} \mathrm{H} \text { NMR was used predominantly to monitor the progression of the } \\
\text { reaction } \\
\text { o the nearly pure product isolated from the first reaction had a } \\
\text { peak at } 5.7 \text { ppm in acetone-d } 6\left(5.57 \mathrm{ppm} \text { in } \mathrm{CDCl}{ }_{3}\right)-\text { this peak } \\
\text { was predominantly compared to that of acac-COOH for } \\
\text { reaction progression } \\
\text { in } \mathrm{CDCl} \text {, a peak at } 5.7 \text { ppm was often seen as well as that at } \\
5.57 \text { ppm - perhaps an isomer } \\
\text { reaction was ended when the peak ratio of complex to acac- } \\
\mathrm{COOH} \text { stopped changing or reversed }\end{array}$ \\
\hline
\end{tabular}


Table 4.9 Purification strategies for (pq)Pt(acac-COOH). The $5.57 \mathrm{ppm}$ peak in $\mathrm{CDCl}_{3}$ was used as the predominant complex peak for comparison.

\begin{tabular}{|c|c|c|c|c|}
\hline$\overline{\mathrm{Rxn}}$ & Starting solution & Solvent added & Observations & Result \\
\hline \multirow[t]{2}{*}{4} & $\begin{array}{l}\text { crude product in 2-ethoxyethanol, } \\
1: 10 \text { complex to acac-COOH }\end{array}$ & excess ether & $\begin{array}{l}\text { yellow precipitate } \\
\text { golden solution }\end{array}$ & $\begin{array}{l}\text { Ppt: } \mu \text {-dichloro(pq)Pt(II) dimers } \\
\text { Sol'n: } 1: 3 \text { complex to ligand }\end{array}$ \\
\hline & treated 2-ethoxyethanol layer & excess diH $_{2} \mathrm{O}$ & yellow precipitate & $1: 2$ complex to acac- $\mathrm{COOH}$ \\
\hline \multirow{7}{*}{5} & $\begin{array}{l}\text { crude product in 2-ethoxyethanol, } \\
1: 10 \text { complex to acac- } \mathrm{COOH}\end{array}$ & excess ether & $\begin{array}{l}\text { yellow precipitate } \\
\text { orange-yellow solution }\end{array}$ & Ppt: $\mu$-dichloro(pq)Pt(II) dimers \\
\hline & treated 2-ethoxyethanol layer & excess $\mathrm{diH}_{2} \mathrm{O}$ & $\begin{array}{l}\text { yellow precipitate } \\
\text { light yellow solution }\end{array}$ & Ppt: $1: 7$ complex to acac- $\mathrm{COOH}$ \\
\hline & $\mathrm{diH}_{2} \mathrm{O}+2$-ethoxyethanol layers & $\begin{array}{lc}\text { extracted } & \text { with } \\
20 \times 20 \times 40 & \mathrm{~mL} \\
\text { ether } & \end{array}$ & yellow ether layers & $\begin{array}{l}\text { ether contained acac-COOH and } \mu- \\
\text { dichloro(pq)Pt(II) dimers or } \mathrm{pq}\end{array}$ \\
\hline & $\begin{array}{l}\text { precipitate containing complex } \\
\text { dissolved in toluene }\end{array}$ & excess ether & $\begin{array}{l}\text { gold-brown precipitate } \\
\text { ether-toluene dried to brown oil }\end{array}$ & Ppt: $\mu$-dichloro(pq)Pt(II) dimers \\
\hline & $\begin{array}{l}\text { brown oil obtained from ether- } \\
\text { toluene redissolved in toluene }\end{array}$ & excess $\mathrm{diH}_{2} \mathrm{O}$ & $\begin{array}{l}\text { yellow toluene layer dried to yellow- } \\
\text { orange oil } \\
\text { colourless } \mathrm{diH}_{2} \mathrm{O} \text { layer }\end{array}$ & $\begin{array}{l}\text { yellow-orange oil: } 1: 5 \text { complex to } \\
\text { acac-COOH plus } \\
\text { dichloro(pq)Pt(II) dimers }\end{array}$ \\
\hline & $\begin{array}{l}\text { yellow-orange oil dissolved in } 2 \text { - } \\
\text { ethoxyethanol }\end{array}$ & excess ether & no precipitate & $\begin{array}{l}\text { concentrated to yellow 2-ethoxyethanol } \\
\text { solution }\end{array}$ \\
\hline & yellow 2-ethoxyethanol solution & excess $\mathrm{diH}_{2} \mathrm{O}$ & $\begin{array}{l}\text { yellow oil isolated } \\
\text { yellow solution }\end{array}$ & $\begin{array}{l}\text { oil: } 1: 0.5 \text { complex to acac- } \mathrm{COOH} \text {, } \\
\text { maybe contamination in aromatic } \\
\text { region }\end{array}$ \\
\hline \multirow[b]{2}{*}{6} & $\begin{array}{l}\text { crude product in 2-ethoxyethanol, } \\
1: 7.5 \text { complex to acac- } \mathrm{COOH}\end{array}$ & excess ether & $\begin{array}{l}\text { yellow precipitate } \\
\text { red-brown solution }\end{array}$ & moved on without characterization \\
\hline & treated 2-ethoxyethanol layer & excess $\mathrm{diH}_{2} \mathrm{O}$ & $\begin{array}{l}\text { orange precipitate and oil separated } \\
\mathrm{diH}_{2} \mathrm{O} \text { layer: yellow-gold } \\
\text { 2-ethoxyethanol layer: red-orange }\end{array}$ & Ppt/oil: $1: 2$ complex to acac-COOH \\
\hline
\end{tabular}


A higher ratio of $1: 7$ complex to acac- $\mathrm{COOH}$ was found in the precipitate isolated following the addition of $\mathrm{diH}_{2} \mathrm{O}$ to the 2-ethoxyethanol reaction solution following reaction 5 than in the other two cases outlined in Table 4.9. As a result, further extractions were performed. An extraction of the remaining solution with ether merely resulted in isolation of more of the starting materials, so the precipitate itself was the focus of the remaining steps. It was dissolved in toluene and excess ether was added. This caused a gold-brown solid to precipitate out of solution; this was found by NMR to contain $\mu$-dichloro(pq)Pt(II) dimers. The ether-toluene mixture was concentrated to a brown oil which was again dissolved in toluene. The addition of excess $\operatorname{diH}_{2} \mathrm{O}$ did not cause any visual change, but the toluene layer dried to a yellow-orange oil rather than a brown one. The oil was found to have a ratio of 1:5 complex to acac- $\mathrm{COOH}$, as well as likely still containing some $\mu$-dichloro(pq) $\mathrm{Pt}(\mathrm{II})$ dimers. The oil was dissolved in 2ethoxyethanol and the first two steps were repeated. The addition of excess ether did not cause any visible changes and so the mixture was concentrated back to the 2ethoxyethanol solution. The addition of an excess amount of $\mathrm{diH}_{2} \mathrm{O}$ resulted in the separation of a yellow oil which was found to have a complex to acac-COOH peak ratio of 1:0.5 as well as potentially still being contaminated with some dimer or 2phenylquinoline as there were excess peaks in the aromatic region of the ${ }^{1} \mathrm{H}$ NMR. This is illustrated in Figures C-6 and C-7 in Appendix C. The expanded version shown in Figure C-7 includes a number of peaks aside from that thought to correspond to the complex at $5.57 \mathrm{ppm}$. These might represent isomers of the desired complex.

Overall, while it seemed that a new complex was formed, the purification of a single chemical species was not achieved. The purification of the desired complex from 
the reaction mixture might have been complicated by the presence of one or more isomers or related chemical species, as indicated by the presence of several peaks in the 5-6 ppm region of obtained 'H NMR spectra (see Figure C-7). The initial impurity of the $\mu$-dichloro(pq)Pt(II) dimers used in the reaction with excess 2-phenylquinoline might have contributed to the purification difficulties, as unwanted side-products could have been formed or unbound 2-phenylquinoline might be the chemical species that caused the excess aromatic peaks observed in several NMR spectra. It is also of interest to note that in the work reported by Thompson et al. on the synthesis and characterization of phosphorescent cyclometalated platinum complexes, ${ }^{186}$ the 2-phenylquinoline ligand was not used to form a $\mathrm{Pt}(\mathrm{II})$ complex with acetylacetone but only with the other $\beta$-diketonate ligand used in the study, dipivolylmethane (dpm). In the case of 2-phenylpyridine, for example, the authors prepared both the acac and dpm complexes. Though 2phenylquinoline is not the only ligand not used for two $\mathrm{Pt}(\mathrm{II})$ complexes, this might indicate some difficulty in preparing or purifying a (pq) $\mathrm{Pt}(\mathrm{acac})$ complex. The authors do state that changing the $\beta$-diketonate ligand from acac to dpm increased the solubility of the complexes obtained and also greatly simplified their purification using column chromatography. 2-phenylquinoline might therefore not be a suitable ligand for inclusion in the development of luminescent Pt(II) complexes for conjugation to nucleic acids.

4.3.3.4 Synthesis, purification, and characterization of 8,10-dioxoundecanoic acid-(2(2,4-difluorophenyl)pyridyl)platinum(II); (2,4-dfppy)Pt(acac-COOH)

As reported by Boshra Hosseini in her B.Sc. thesis, ${ }^{189}$ the synthesis of 8,10 dioxoundecanoic acid-(2-(2,4-difluorophenyl)pyridyl)platinum(II) was performed over a 
shorter period of time than that required for the synthesis of the complexes prepared using 2-phenylpyridine or 2-phenylquinoline. The desired product in this case was obtained in a higher yield than the complex prepared with 2 -phenylpyridine (54\% versus $24 \%)$ as well. However, (2,4-dfppy)Pt(acac-COOH) seemed to be of low stability when exposed to light and this was not observed for (ppy) Pt(acac-COOH).

Though stored under argon at $4^{\circ} \mathrm{C}$, the purified beige product grew darker in colour over the course of 10 days. A subsequent experiment was performed in which a portion of (2,4-dfppy) Pt(acac-COOH) was irradiated with light for 10 minutes and run on a TLC next to a sample that had not been irradiated (methanol was used as the mobile phase). A second distinct luminescent band was present only in the irradiated sample that seemed to match the migration rate of the 2,4-dfppy ligand. Uncompromised complex also appeared to be present in the irradiated sample. The identity of the second band remained unconfirmed as it could not be isolated for characterization.

The (2,4-dfppy)Pt(acac-COOH) band on TLC exhibited similar luminescent properties as the 2-phenylpyridine complex where the middle of the band was a different colour from the outside. In the case of this complex, the colours were orange and blue, respectively. $^{189}$

\subsubsection{Absorbance and luminescence studies}

\subsubsection{1 (ppy)Pt(acac-COOH)}

The absorbance and luminescence spectra of (ppy)Pt(acac-COOH) were obtained using solutions of the complex dissolved in acetone. An initial luminescence study was performed to determine the wavelength at which this complex emitted. The least 
concentrated solution (Stock 3,0.00015 M) was excited at $370 \mathrm{~nm}$ and an emission spectrum was obtained. It can be seen in Figure 4.3 (solid line) that the complex emitted in the $450-650 \mathrm{~nm}$ range with more than one peak; such structured emission is commonly seen in Pt(II) complexes containing both a monoanionic cyclometalating ligand and a $\beta$ diketonato ligand. ${ }^{186}$ The $\lambda_{\max }$ was at $485 \mathrm{~nm}$, so the emission at this wavelength was monitored while an excitation spectrum was obtained (dashed line in Figure 4.3). This demonstrated that excitation at $360-370 \mathrm{~nm}$ was appropriate for this sample.

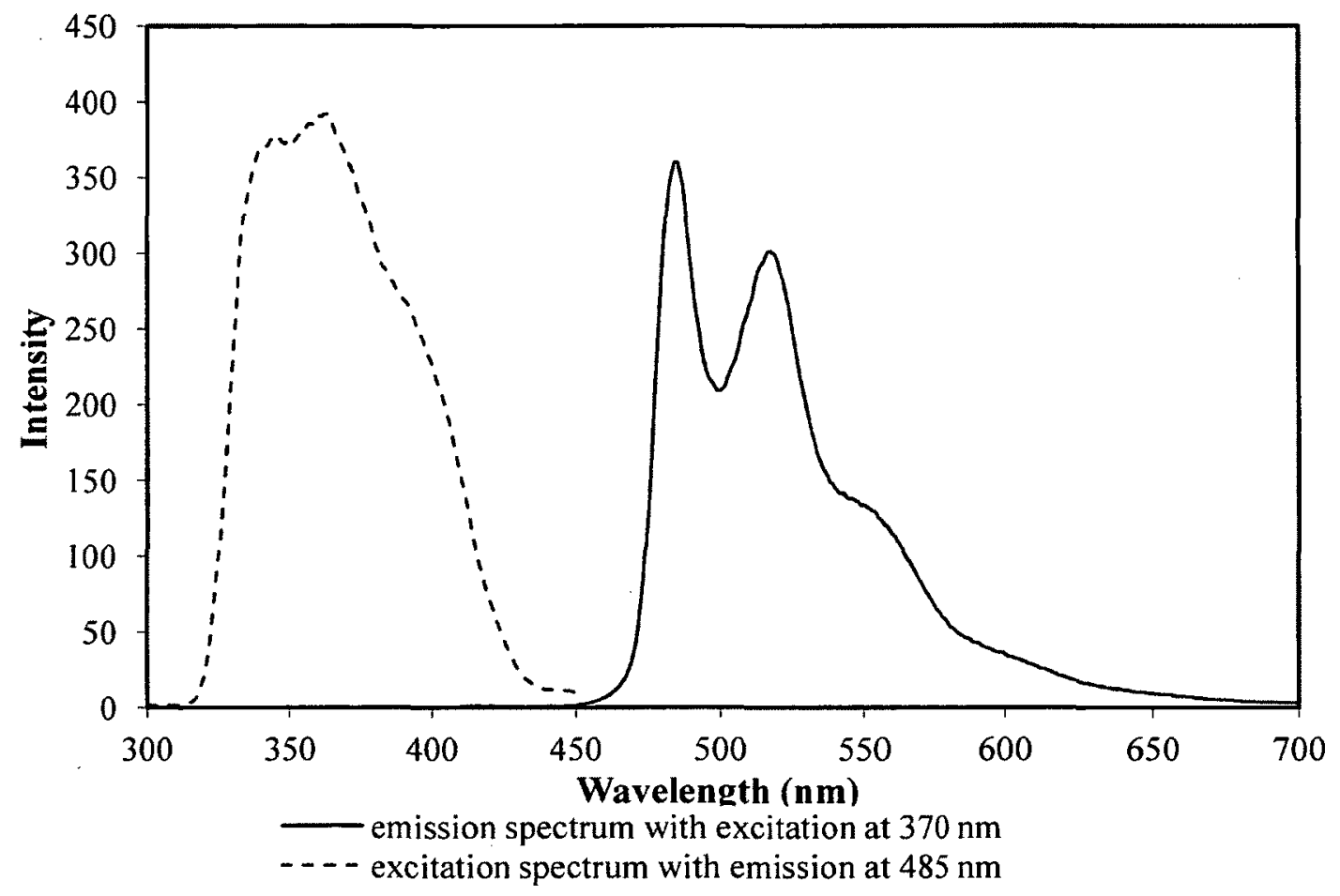

Figure 4.3 Initial emission and excitation spectra obtained for (ppy)Pt(acac-COOH) dissolved in acetone to a concentration of $0.0245 \mathrm{M}$ (Stock 1$)$.

An emission spectrum for stock solution 2, with a concentration of $0.0049 \mathrm{M}$, was therefore obtained using an excitation wavelength of $360 \mathrm{~nm}$. A broad, structureless 
emission peak with a maximum intensity at about $620 \mathrm{~nm}$ was observed in this spectrum alongside the same structured peaks as seen for the more dilute solution. The spectrum was very noisy, so an excitation spectrum with emission at $620 \mathrm{~nm}$ was obtained, and it was found that a more efficient excitation wavelength in this case would be $426 \mathrm{~nm}$. This was used as the first excitation wavelength, therefore, to obtain an emission spectrum of the most concentrated solution (Stock 1, $0.0245 \mathrm{M}$ ). The broad emission peak had a maximum intensity at $626 \mathrm{~nm}$, and the excitation spectrum obtained with emission at this wavelength monitored demonstrated that $476 \mathrm{~nm}$ was the excitation wavelength maximum for this solution. The three spectra obtained for the three (ppy)Pt(acac-COOH) solutions using the optimized excitation wavelengths are illustrated in Figure C-8. The peaks are a little offset from each other in these spectra due to the different excitation wavelengths used. It was therefore determined that the next luminescence study would be on three solutions of similar concentrations to those in this initial study but with all three solutions excited at the optimal wavelength for the middle concentration. The emission spectra obtained for these solutions when excited at a wavelength of $426 \mathrm{~nm}$ are shown in Figure 4.4.

It can be seen in Figure 4.4 that as the concentration of (ppy) Pt(acac-COOH) increased from $0.00015 \mathrm{M}$ to $0.0044 \mathrm{M}$ (green to pink lines), the intensity of the structured peaks in the $450-550 \mathrm{~nm}$ region decreased with the corresponding appearance of a broad, structureless, red-shifted peak for the $0.0044 \mathrm{M}$ solution. This peak increased in intensity with partial loss of the higher energy peaks in the most concentrated solution (blue line). The emergence of this red-shifted band and its increase in intensity as the concentration increased seemed, therefore, to be at the expense of the peaks at shorter 
wavelengths. This is a characteristic associated with excimer formation and emission. ${ }^{182,}, 187$ The concentration-dependent luminescence of (ppy)Pt(acac-COOH) was therefore a promising result when considering its potential use as a luminescent probe for the investigation of aptamer folding. The middle concentration tested here demonstrates what a spectrum might look like if two complex molecules bound to an aptamer strand during conformational analysis overlapped inefficiently to form an excimer; the monomer peaks are still visible but the excimer emission is also present.

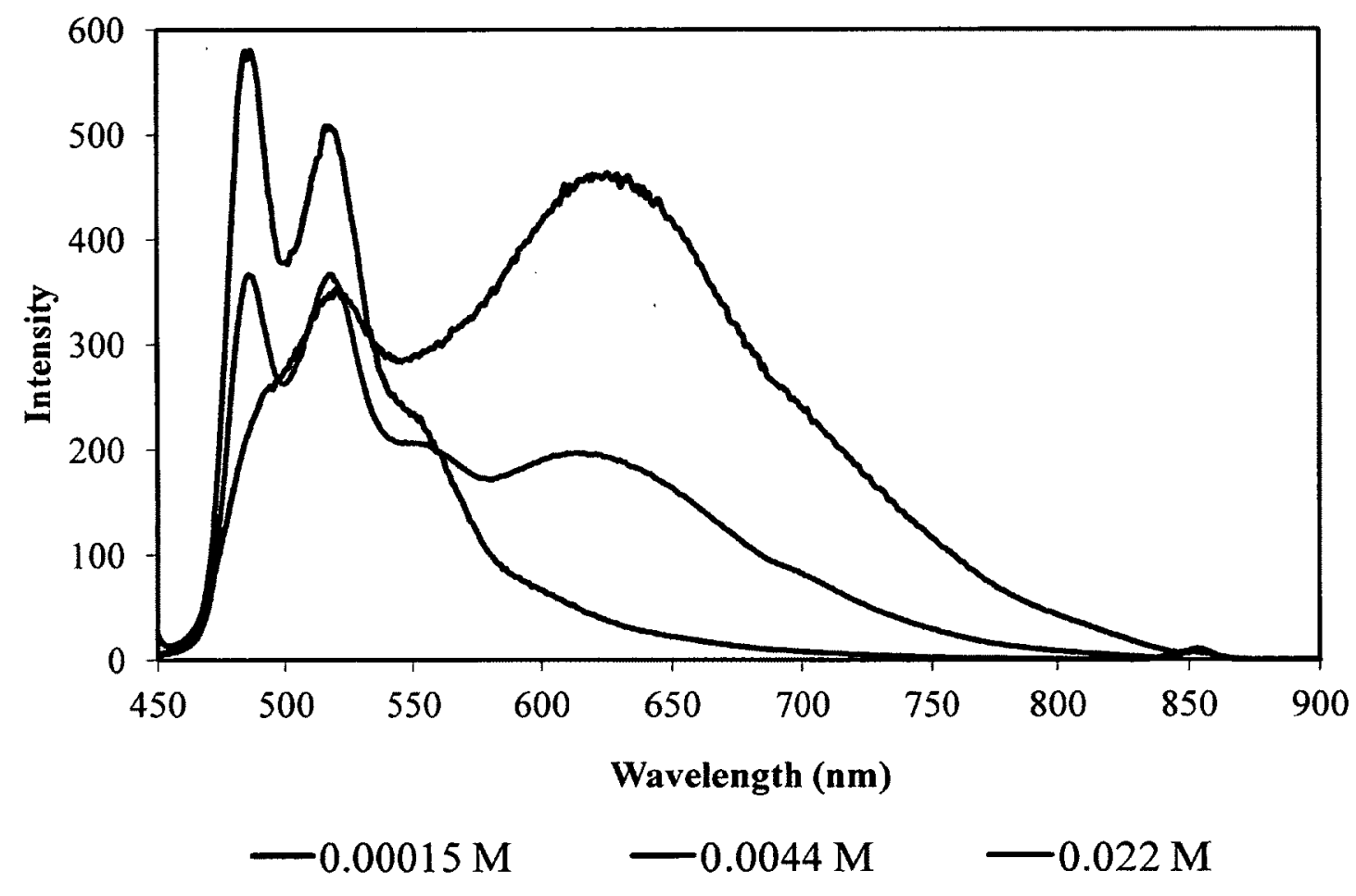

Figure 4.4 Emission spectra for (ppy)Pt(acac-COOH) in acetone with excitation at $426 \mathrm{~nm}$.

The absorbance spectra of the most and least concentrated of the initial solutions studied $(0.024$ and $0.00015 \mathrm{M}$, respectively) were obtained prior to recovery of the 
complex by concentration. The spectra are shown in Figures C-9 and C-10, respectively. The absorbance of acetone interfered at wavelengths below $320 \mathrm{~nm}$, so a portion of the complex was dissolved in acetonitrile and diluted to obtain the spectrum shown in Figure 4.5 .

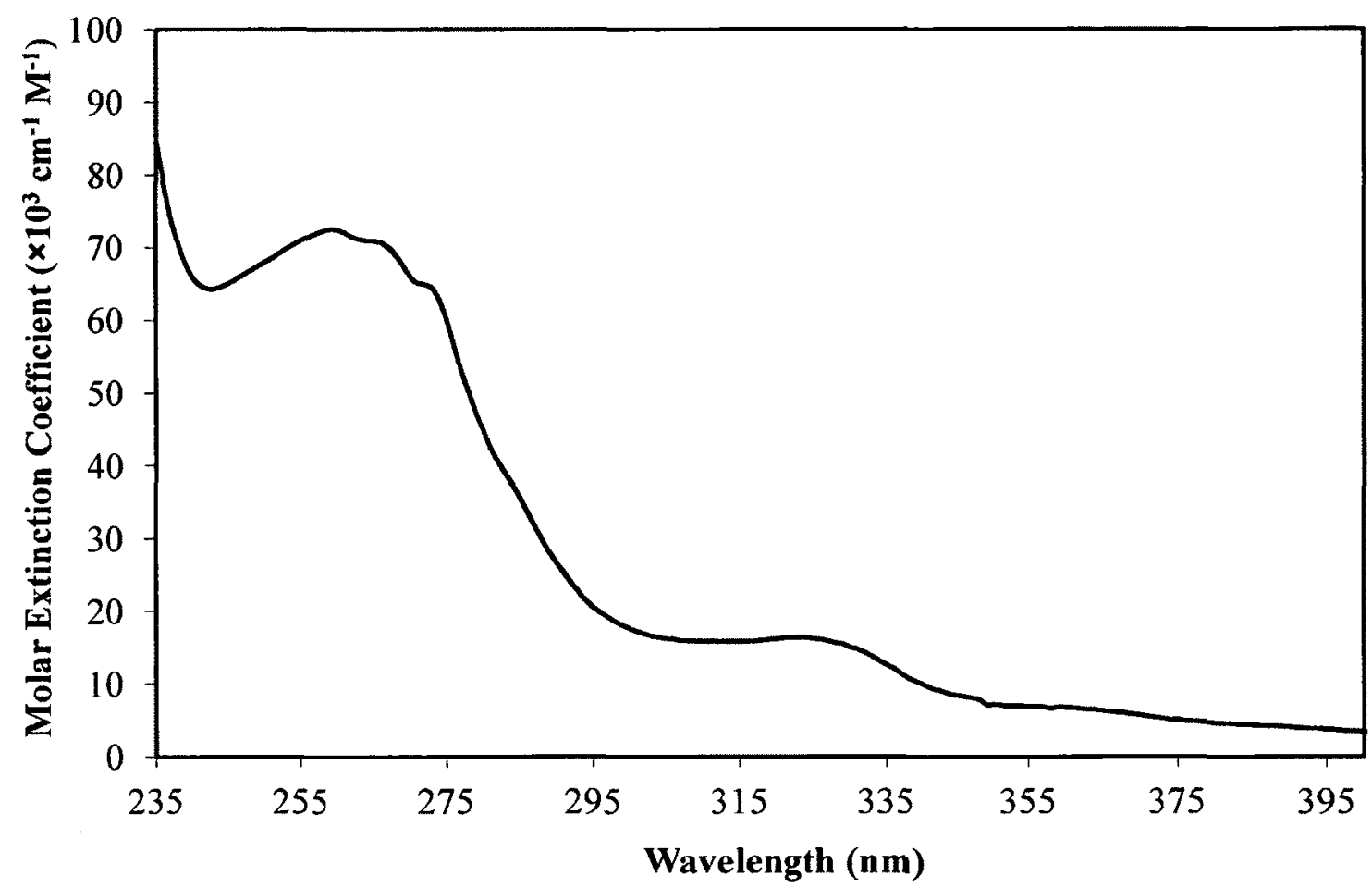

Figure 4.5 Absorbance spectrum for (ppy)Pt(acac-COOH) in acetonitrile at a concentration of $9.4 \times 10^{-6} \mathrm{M}$.

This spectrum demonstrates that a more intensely absorbent band is present at about 260 $\mathrm{nm}$ than the bands observed between 320 and $400 \mathrm{~nm}$ in the spectrum obtained in acetone (Figure C-10). The presence of the $260 \mathrm{~nm}$ absorbance peak is of interest as the presence of excess (ppy)Pt(acac-COOH) in solution with DNA during conjugation reactions might obscure the characteristic absorbance of DNA at this wavelength. The molar absorptivity 
values found for the complex in this case can be seen to be on the range of tens of thousands, however, while short oligodeoxynucleotides have values in the hundreds of thousands. This, coupled with the low solubility of (ppy) $\mathrm{Pt}(\operatorname{acac}-\mathrm{COOH})$ in aqueous solution, should hopefully prevent any difficulty in quantifying DNA where excess complex might be present following conjugation reactions.

\subsubsection{2 (2,4-dfppy)Pt(acac-COOH)}

The absorption and luminescent properties of (2,4-dfppy)Pt(acac-COOH) were explored by Boshra Hosseini as reported. ${ }^{189}$ The absorption spectrum is shown in Figure C-11 and the emission spectra are shown in Figure 4.6. It can be seen that there is an increase in emission intensity at about $600 \mathrm{~nm}$ as the concentration of the complex increases about 10-fold. This is similar to the results seen for the lowest two concentrations used in luminescence studies for (ppy) $\mathrm{Pt}(\mathrm{acac}-\mathrm{COOH})$. The more concentrated solution of (2,4-dfppy)Pt(acac-COOH) represented in Figure 4.6 displays monomer emission between 450 and $550 \mathrm{~nm}$ that is blue-shifted from the monomer emission peaks for (ppy)Pt(acac-COOH) due to the difluoro-substituted 2-phenylpyridine ligand. The peak at about $600 \mathrm{~nm}$ could represent excimer emission, and though it is not as distinct as in the spectra for (ppy) Pt(acac-COOH), it seems that this peak is also blueshifted compared to the excimer peak in Figure 4.4. Therefore, the 2,4-dfppy- and ppycontaining $\mathrm{Pt}(\mathrm{II})$ complexes prepared display concentration-dependent emission properties that are reminiscent of those for excimer-forming compounds. The two $\mathrm{Pt}(\mathrm{II})$ complexes have unique emission properties when compared and would therefore be 
distinguishable from one another if included in the same sensor or on the same DNA strand for conformational analysis.

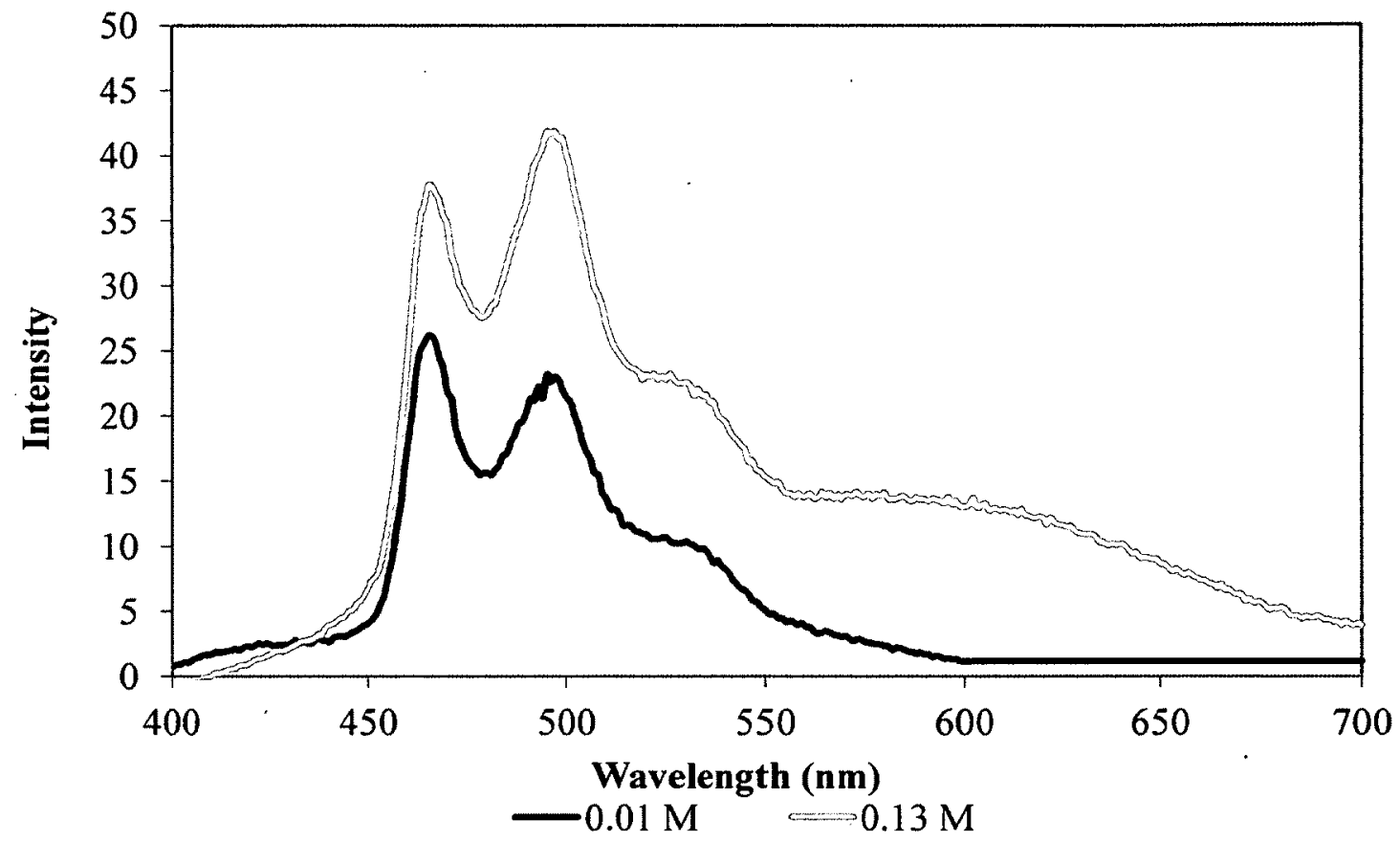

Figure 4.6 Emission spectra of (2,4-dfppy)Pt(acac-COOH) in methanol with excitation at 355 nm. ${ }^{189}$

\subsubsection{Conjugation of (ppy)Pt(acac-COOH) to the 15mer thrombin-binding aptamer}

For use as luminescent probes in the study of aptamer folding, the $\mathrm{Pt}$ (II) complexes discussed herein would need to be conjugated to the aptamer under study. The initial work performed on the conjugation of (ppy)Pt(acac-COOH) to DNA was done using the well-studied 15 mer thrombin-binding aptamer (section 1.2.3). The aptamer was synthesized with an amino-modifier at the 5'-terminus and the DMS(O)MT protecting group was manually removed by washing the $\mathrm{CPG}$ beads as first described in section 2.2.2. 
Prior to the addition of (ppy)Pt(acac-COOH) to the CPG-supported DNA aptamer, the complex was activated through a reaction with EDC (1-ethyl-3-(3dimethylaminopropyl)carbodiimide hydrochloride) and NHS ( $N$-hydroxysuccinimide). As discussed in section 4.1.5, these reagents are used to activate a carboxylic acid group prior to its reaction with an active amine to form a peptide bond. A portion of the $\mathrm{Pt}$ (II) complex was therefore mixed with 10 and 25 molar equivalents of EDC and NHS, respectively, as suggested in the protocol included with the chemicals. ${ }^{200}$ The reagents were dissolved in $1 \mathrm{~mL}$ of DMSO and left to react at room temperature for about an hour. At this point, the CPG beads supporting deblocked DNA were added to the reaction mixture, and the coupling reaction was left to proceed overnight at room temperature.

Following deprotection and quantification, a portion of the crude product was sent for MS analysis. The calculated $\mathrm{m} / \mathrm{z}$ for the thrombin-binding aptamer conjugated to (ppy)Pt(acac-COOH) was 5449.6 while that for the aptamer conjugated to just acac$\mathrm{COOH}$ was 5101.3. The MS data obtained included a peak at $\mathrm{m} / \mathrm{z} 5103.3$ which supported the success of the coupling reaction as it matched the $\mathrm{m} / \mathrm{z}$ of DNA-acac$\mathrm{COOH}$. Some $\mathrm{m} / \mathrm{z}$ values might be the desired target associated with a common ion, such as $\mathrm{Na}^{+}$or $\mathrm{K}^{+}$. Therefore, the presence of a peak in this data with an $\mathrm{m} / \mathrm{z}$ of 5485.6 could be the Pt(II) complex-DNA associated with a potassium cation. The intensity of the DNA-acac-COOH peak was much higher than this latter peak, however, which could indicate that the complex was originally present in full but fragmented in the MS, or it could be a result of decomposition of the complex during deprotection of the DNA in $\mathrm{NH}_{4} \mathrm{OH}$. The previously obtained MS data for the complex alone (section 4.3.3.2) indicated that the complex fragments considerably during MS analysis, so that it doing so 
while conjugated to DNA would not be a great surprise. For example, the MS data obtained for the complex did include a peak that corresponded to the mass of 2 phenylpyridine as well as one that matched the mass of $[\mathrm{Pt}(\mathrm{acac}-\mathrm{COOH})]^{+}$, while the results from the aptamer- $\mathrm{Pt}(\mathrm{II})$ complex reaction included an $\mathrm{m} / \mathrm{z}$ value of 5329.7 which could be the DNA conjugated to the complex missing the 2-phenylpyridine ligand $(\mathrm{m} / \mathrm{z}$ calc'd 5296.1) but associated with a potassium ion. Overall, the results of this reaction were promising but the stability of (ppy) Pt(acac-COOH) in $\mathrm{NH}_{4} \mathrm{OH}$ was called into question.

A portion of (ppy)Pt(acac- $\mathrm{COOH})$ was subsequently dissolved in a 1:1 solution of $\mathrm{MeOH}$ and $\mathrm{NH}_{4} \mathrm{OH}$ to test its stability. Following overnight incubation in this solution at room temperature, the mixture was concentrated under vacuum leaving an orange oil as opposed to the normally yellow (ppy)Pt(acac-COOH) solid. Acetone-d6 was added to the oil; it was not readily soluble, but enough went into solution to give it a yellow colour. The ${ }^{1} \mathrm{H}$ NMR spectrum obtained for this sample is shown in Figure $\mathrm{C}-12$. Though it has a low signal-to-noise ratio, the spectrum indicates that some free acac$\mathrm{COOH}$ was present as its characteristic peak at $5.66 \mathrm{ppm}$ is visible. The characteristic complex peak at $5.59 \mathrm{ppm}$ is also visible but at lower intensity than the acac- $\mathrm{COOH}$ peak. Therefore, these results supported the hypothesis that (ppy) $\mathrm{Pt}(\mathrm{acac}-\mathrm{COOH})$ is not completely stable in $\mathrm{NH}_{4} \mathrm{OH}$ and therefore was likely partially decomposed during DNA deprotection.

Further study was still performed on the crude reaction product to see if any $\mathrm{Pt}(\mathrm{II})$ complex-DNA or DNA-acac-COOH could be isolated. The sample was run using reversed-phase HPLC with the conditions outlined in Table 4.4. As discussed previously, 
DNA has a characteristic absorbance peak at $260 \mathrm{~nm}$. Absorbance at this wavelength was therefore monitored in order to determine when DNA was eluting from the column. The chromatogram in Figure 4.7 illustrates that little separation was achieved with the conditions used, likely because they were non-denaturing and the thrombin-binding aptamer was probably present in a mixture of folded and unfolded strands. Additionally, multiple thrombin aptamers can form intermolecular G-quadruplexes as mentioned in section 1.5.2. ${ }^{84}$ Several portions of DNA were collected from the peaks between 8 and 23 minutes, nonetheless, and analyzed further using UV-visible spectrophotometry. The earliest peaks in RP-HPLC runs on crude DNA samples contain the failed sequences from the synthesis, which is why the peaks that emerged from $0-7$ minutes were not analyzed.

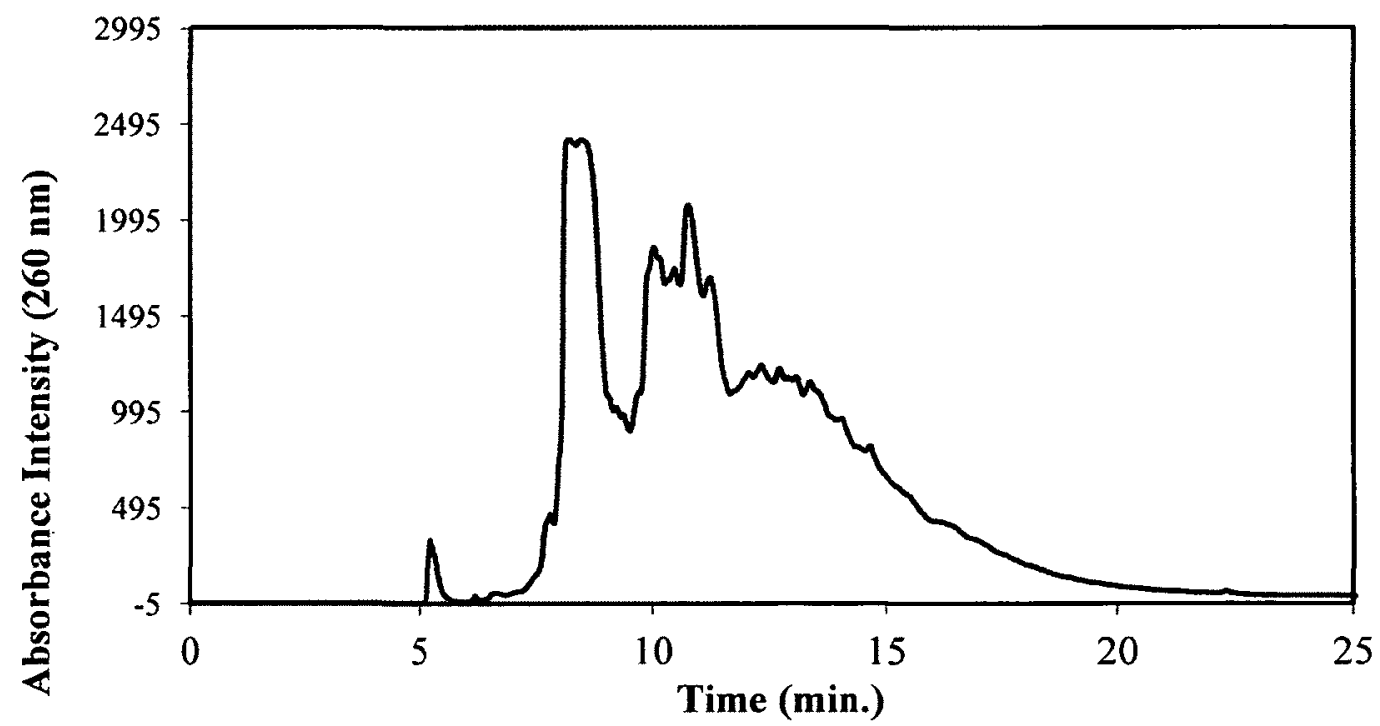

Figure 4.7 RP-HPLC chromatograms from the analysis of crude Pt(II) complex-TBA.

The first of the portions of DNA collected from the RP-HPLC run ( 8 to 9.8 minutes) was dissolved in tris buffer to a concentration of $38 \mu \mathrm{M}$ and was tested for 
luminescence. Emission was recorded at $485 \mathrm{~nm}$ as in the (ppy)Pt(acac-COOH) luminescence studies and it was determined that an excitation wavelength between 300 and $330 \mathrm{~nm}$ should be used for the $\mathrm{Pt}$ (II) complex-DNA samples in this buffer. The remaining DNA portions collected (see Table 4.5) were dissolved to concentrations between 12 and $70 \mu \mathrm{M}$ depending on the amount of DNA present since a minimum of $600 \mu \mathrm{L}$ of solution was required. Emission spectra for all samples were collected with excitation at $320 \mathrm{~nm}$ (see Figure C-13 for representative spectra) and $302 \mathrm{~nm}$. Based on the luminescence intensity and the concentration of DNA in each respective sample, it was determined that MS analysis on the samples in portions 2, 4, 5 and 6 would be performed. Samples 1 and 3 were not sent as the luminescence intensities of these samples were the lowest while their concentrations were among the highest; this seemed to indicate that a lower percentage of $\mathrm{Pt}(\mathrm{II})$ complex-DNA was present in each of these samples.

The MS results are summarized in Table 4.10. A peak representative of the desired (ppy) $\mathrm{Pt}(\mathrm{acac}-\mathrm{COOH})-\mathrm{TBA}$ conjugate associated with a potassium cation was seen in the results for fractions 5 and 6 but not in those samples collected earlier in the RP-HPLC run (2 and 4). These earlier fractions as well as fraction 5 did, however, each have a peak matching the $\mathrm{m} / \mathrm{z}$ of the DNA conjugated to acac- $\mathrm{COOH}$ and the relative intensity of this peak decreased as the fraction number increased. Coupled with the finding that (ppy) Pt(acac-COOH) seemed to decompose in $\mathrm{NH}_{4} \mathrm{OH}$, this indicated that there could have been DNA-acac-COOH in the crude product that was partially separated from the full Pt(II) complex-DNA during RP-HPLC. As previously mentioned, the complex could also be fragmenting during MS analysis and so the peak or a portion of 
the peak for DNA-acac-COOH could originate from the full complex conjugate. It is impossible to determine which process, or if both of these processes, resulted in the DNA-acac-COOH present in these samples with the MS data alone, but since the material in fractions 2 and 4 both demonstrated emission that matched that of the full $\operatorname{Pt}($ II) complex (Figure C-13) it seems likely that at least a small portion of the DNA in each of these fractions was coupled to the complex.

Table 4.10 Summary of MS results for Pt(II) complex-DNA samples.

\begin{tabular}{|c|c|c|c|}
\hline $\mathrm{m} / \mathrm{z}$ calc'd & Target & $\mathrm{m} / \mathrm{z}$ found & Identity \\
\hline \multirow{2}{*}{5449.6} & \multirow{2}{*}{ Pt(II) complex-DNA } & 5485.1 (fraction 5) & target with $\mathrm{K}^{+}$ \\
& & 5484.1 (fraction 6) & \\
\hline \multirow{3}{*}{5101.3} & DNA-acac-COOH & 5101.7 (fraction 2) & target \\
& & $5101.6($ fraction 4) & \\
\hline
\end{tabular}

Considering both the MS and luminescence study results, fractions 5 and 6 were included in one final luminescence study for a direct comparison between the two samples. They were both dissolved in tris buffer to the same final concentration of 70 $\mu \mathrm{M}$ and were degassed prior to obtaining emission spectra with excitation at $302 \mathrm{~nm}$. Figure 4.8 shows the spectra obtained. The lower luminescence intensity of fraction 5 (solid line) implies that a smaller fraction of the DNA in the fraction is conjugated to (ppy)Pt(acac-COOH) than in fraction 6 (dashed line) which has a more intense signal. This agrees with the relative peak intensity values of the Pt(II) complex-DNA with $\mathrm{K}^{+}$in the MS spectra for these samples. The relative peak intensities (relative to the peak with 
the highest intensity in the spectrum) for fractions 5 and 6 were reported as about 45 and $64 \%$, respectively.

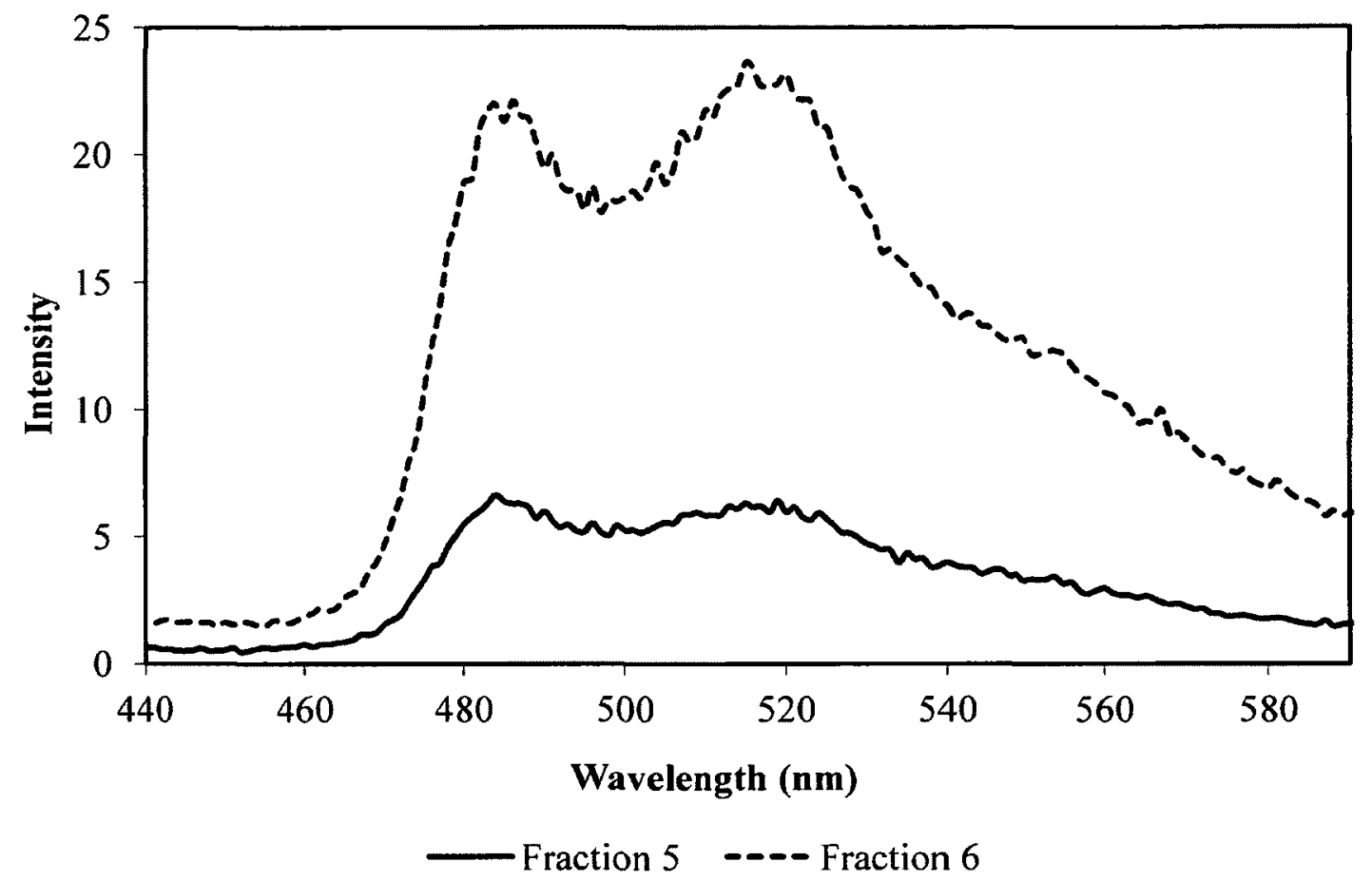

Figure 4.8 Emission spectra for fractions 5 and 6 dissolved in tris buffer to $70 \mu M$. Excitation at $302 \mathrm{~nm}$.

\subsubsection{Conjugation of (ppy)Pt(acac-COOH) to complementary strands of DNA}

The luminescence studies on (ppy)Pt(acac-COOH) confirmed that this complex demonstrated concentration-dependent luminescence and therefore possibly monomer and excimer emission peaks at low and high concentrations, respectively. A proof-ofconcept system was therefore designed using two short DNA strands of random sequence that were complementary to one another. One strand would be conjugated to the $\operatorname{Pt}(\mathrm{II})$ complex at its 5 -terminus while the complementary strand would be conjugated to the complex at its 3 '-terminus, as illustrated in Figure 4.9. While unhybridized, the Pt(II) 
complex molecules conjugated to the two DNA strands would remain farther apart on average and therefore emit at a shorter wavelength than when the strands were hybridized and the two complex molecules would be brought into close proximity. A shift in emission to a longer wavelength, as for the highest concentration solution in Figure 4.4, might be observed in the hybridized case. The conjugation strategies used to label two short, complementary DNA strands with the (ppy)Pt(acac-COOH) complex are detailed in the following sections.
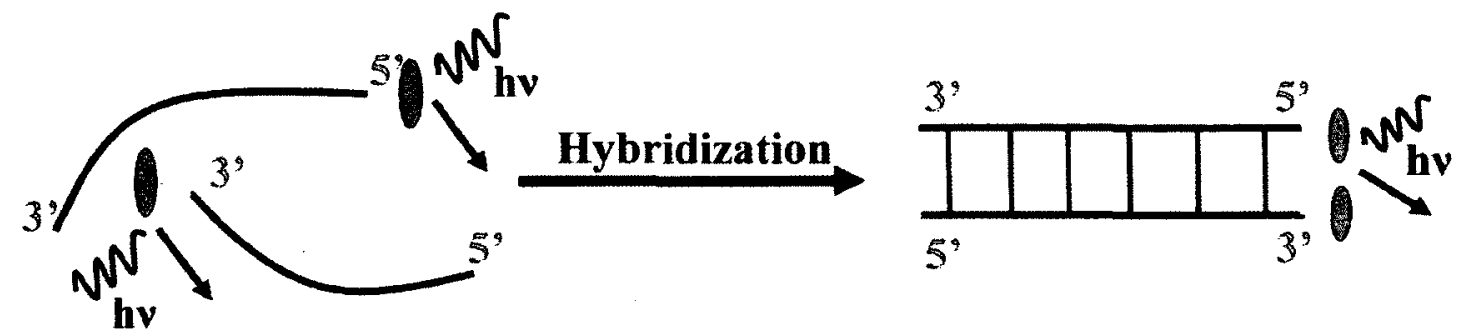

Figure 4.9 Schematic diagram of a proof-of-concept system using complementary DNA strands.

\subsubsection{Conjugation to a 5'-amino-modified strand}

A 14 mer DNA strand with random sequence $5^{\prime}-\mathrm{NH}_{2}-\left(\mathrm{CH}_{2}\right)_{6}-\mathrm{CTG}$ ACG TAC TGA CT-3' was synthesized with the inclusion of the 5'-amino-modifier (as shown) for conjugation to the (ppy)Pt(acac-COOH) complex. Due to the suspected low stability of (ppy)Pt(acac-COOH) in $\mathrm{NH}_{4} \mathrm{OH}$, the initial conjugation attempts with the 5'-modified sequence was performed as a solution-phase reaction. The DNA was therefore cleaved from the CPG beads following synthesis and then the DMS $(O) \mathrm{MT}^{+}$blocking group was removed through a 5 minute treatment with $2 \%$ dichloroacetic acid in $\operatorname{diH}_{2} \mathrm{O}$ (as discussed for the synthesis of DNA-DTPA double-conjugates in Chapter 3). Following ethanol precipitation, the deblocked DNA was precipitated with $\mathrm{CTA}^{+}$to enable its 
solvation in dimethylformamide (DMF). As discussed above, (ppy) $\mathrm{Pt}($ acac-COOH) is not soluble in aqueous solutions which is why organic solvents such as DMSO or DMF must be used in these conjugation reactions.

The $\mathrm{CTA}^{+}$-DNA pellet was reacted with a $1 \mathrm{mg}$ portion of the $\mathrm{Pt}(\mathrm{II})$ complex which had been reacted with a 10 times molar excess of EDC and a 25 times molar excess of NHS in DMF. The DNA and complex conjugation reaction was given about one hour to proceed at room temperature prior to the addition of 10 volume equivalents of a chilled 3\% lithium perchlorate in acetone solution for the precipitation of the DNA. ${ }^{175}$ Following centrifugation for collection of the DNA pellet, the supernatant acetone was removed and the pellet was dried prior to quantification. About $40 \mathrm{nmol}$ of crude Pt(II) complex-DNA was recovered from the reaction of three $1 \mu \mathrm{mol}$ columns of DNA using this method. Similar recoveries were observed at this stage for each of the several times this reaction was attempted. Most of the DNA seemed to be lost at the final stage DNA precipitation through the addition of the lithium perchlorate solution. This was determined through analysis of the leftover DMF/acetone mixture as well as the supernatants from the ethanol and CTAB precipitations using UV-visible spectrophotometry.

The recovered crude product DNA was run on a polyacrylamide gel for purification. The gel images obtained using the absorbance and luminescence channels on the imager are shown in Figure 4.10A. The red outline shows how the less mobile, more luminescent band, was cut out of the gel. About $11 \mathrm{nmol}$ of DNA was recovered from this band. It was dissolved in $60 \mu \mathrm{L}$ of $\mathrm{diH}_{2} \mathrm{O}$ and luminescence spectra were obtained in an effort to determine whether the Pt(II) complex was present. An emission 
spectrum of this sample where excitation was at $302 \mathrm{~nm}$ is shown in Figure 4.10B. The emission peaks are reminiscent of those for the less concentrated complex solutions as shown in Figure 4.4. However, a MALDI (matrix-assisted laser desorption/ionization) MS spectrum obtained for a $2 \mathrm{nmol}$ portion of this sample (shown in Figure C-14) indicated that much of the sample was unreacted amino-modified DNA $(\mathrm{m} / \mathrm{z}$ calc'd $4417.8, \mathrm{~m} / \mathrm{z}$ found 4417 ). It is possible that the sample was incompletely purified by PAGE and so the sample contained both reacted and unreacted DNA or that the conjugate fragmented a great deal during the analysis.

A)

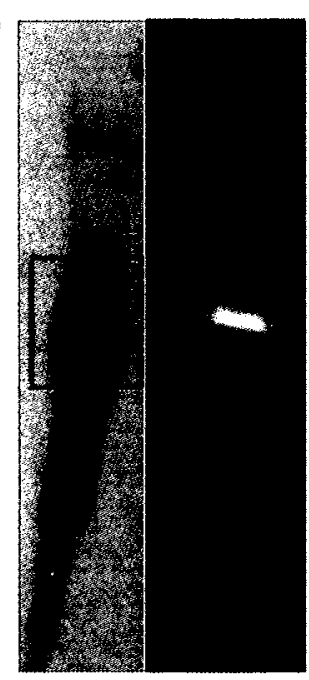

B) 350

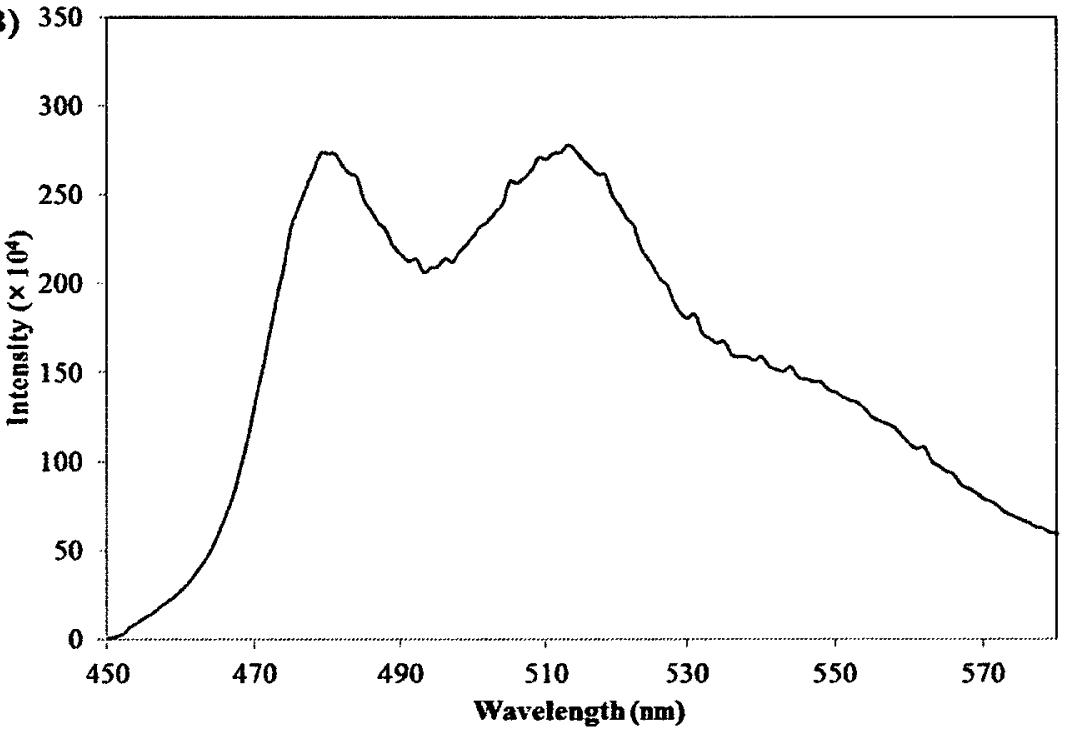

Figure 4.10 Results from solution-phase conjugation of (ppy)Pt(acac-COOH) to the 5'-terminus of a random DNA sequence. A) PAGE images from absorbance (left) and luminescence (right) channels. The red box indicates how the top band was cut out. B) Emission spectrum of the Pt(II) complex-DNA eluted from the band outlined in A with excitation at $302 \mathrm{~nm}$.

The severe loss of DNA during the solution-phase synthesis method seemed likely to be occurring at the stage of precipitation of DNA from DMF, as discussed 
above. The very low amount of DNA recovered following the reaction was inefficient and it made analysis of the crude product as well as purification attempts difficult. A solid-phase reaction where the DNA remained supported on the CPG beads following synthesis worked well for the synthesis of DNA-DTPA conjugates and would be expected to increase the amount of DNA recovered following the reaction of (ppy)Pt(acac-COOH) with amino-modified DNA. Furthermore, the necessity of using organic solvents would be more easily accommodated as the reaction solution containing the Pt(II) complex could simply be removed from the CPG beads prior to deprotection. In order to avoid using $\mathrm{NH}_{4} \mathrm{OH}$ for deprotection, a solid-phase reaction using DNA that incorporated ultramild guanine and adenine bases was therefore developed.

The protecting groups on ultramild guanine and adenine nucleosides can be removed following DNA synthesis through treatment with a $0.05 \mathrm{M}$ potassium carbonate in methanol solution. It was hoped that the Pt(II) complex would be unaffected by these more gentle deprotection conditions. The same random DNA sequence was synthesized with the incorporation of the ultramild bases. Note that thymine does not require a protecting group, and therefore could remain unchanged, and though cytosine is protected during synthesis (see section 1.4) its protecting group can be removed using the ultramild deprotection conditions. Following DNA synthesis, the CPG beads were treated as in previous cases both prior to and during the manual removal of the DMS(O) $\mathrm{MT}^{+}$ protecting group from the amino-modifier.

Two different reactions for the activation of (ppy)Pt(acac-COOH), both of which were introduced in section 4.1.5, were used for the solid-phase synthesis reactions. The first was the EDC/NHS reaction as in the solution-phase case and in previous work with 
the thrombin-binding aptamer (section 4.3.5). The second used another type of peptide coupling agent, TSTU (O-(N-succinimidyl)- $N, N, N^{\prime}, N^{\prime},-$ tetramethyluronium tetrafluoroborate) alongside DIPEA (diisopropylethylamine). Following activation of (ppy)Pt(acac-COOH) using one of these reagent pairs, the CPG beads from one column of newly deblocked DNA was added to the Pt(II) complex solution and the coupling reaction was left to occur overnight at room temperature. The beads were then isolated from solution and the DNA was deprotected overnight in a $0.05 \mathrm{M}$ potassium carbonate in methanol solution. The solution was removed from the CPG beads via pipette and neutralized prior to concentration to avoid damaging the DNA. ${ }^{195}$

The first solid-phase reaction was performed using TSTU/DIPEA and the crude reaction product was run on a polyacrylamide gel. Two bands were isolated and sent for MS analysis. The results indicated that each band was predominantly composed of unreacted amino-modified DNA and all of the $\mathrm{m} / \mathrm{z}$ values found were below that expected for the DNA coupled to acac- $\mathrm{COOH}(\mathrm{m} / \mathrm{z}$ calc'd 4613). Therefore, the MS data did not support that complex conjugation to the DNA strand had occurred (calculated $\mathrm{m} / \mathrm{z}$ 4962 ) as in the previous solid-phase reaction with the thrombin-binding aptamer (section 4.3.5) although the luminescence on the polyacrylamide gel indicated that the complex was present with the DNA.

For a direct comparison between the coupling reagents, the solid-phase reaction was performed using the two peptide coupling reagent pairs concurrently on separate columns of ultramild DNA. The crude products of these reactions were then run side-byside on a polyacrylamide gel. Also, the polyacrylamide gels run thus far on the crude Pt(II) complex-DNA reaction products all showed some luminescence on the gel but MS 
results were inconclusive when it came to demonstrating successful coupling. So a small portion of the isolated $\mathrm{Pt}(\mathrm{II})$ complex solution following a reaction with TSTU and DIPEA was run on the same gel, along with a second portion of the same solution but in the presence of $10 \mathrm{nmol}$ of crude DNA. Finally, the DNA isolated from the two bands on the previously mentioned gel (from the first solid-phase reaction) was run separately on this comparative gel. Images of this gel obtained on the absorbance and luminescence channels are shown in Figure 4.11.

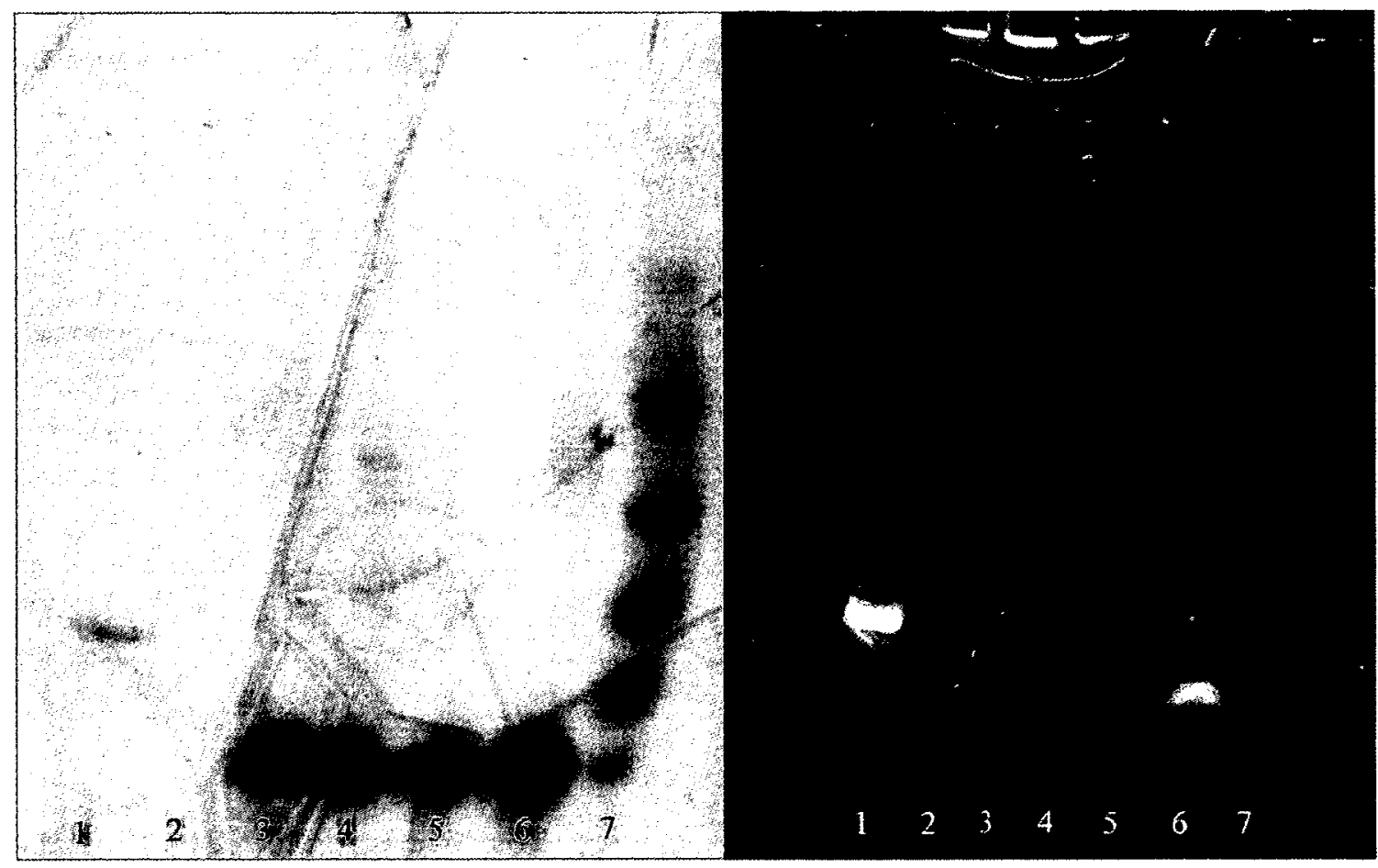

Figure 4.11 Comparative gel for solid-phase Pt(II) complex coupling to ultramild DNA. Lanes: 1 fluorescently-tagged 18mer; 2 - excess Pt(II) complex after reaction with TSTU; 3 - excess Pt(II) complex after reaction with TSTU plus $10 \mathrm{nmol}$ DNA; 4 - $5 \mathrm{nmol}$ DNA from the first reaction with TSTU upper band; 5 - 5 nmol DNA from the first reaction with TSTU lower band; 6 - 10 nmol DNA from the fresh reaction using TSTU; $7-10 \mathrm{nmol}$ DNA from the fresh reaction using EDC/NHS. 
The first lane contained a fluorescently-tagged DNA 18mer to help monitor the progression of DNA on the gel. Its movement on the gel also indicates that the bands located farther down the gel contained smaller species, which would be expected for the random sequence 14 mer used in this work even if conjugated to the $\mathrm{Pt}(\mathrm{II})$ complex. Lane two demonstrates that the complex alone (following reaction with TSTU/DIPEA) has lower mobility in the gel than the heavier DNA strands, which is unexpected based on size alone, but indicated that there was promise for using PAGE for the separation of unconjugated $\mathrm{Pt}(\mathrm{II})$ complex from DNA. When the $\mathrm{Pt}(\mathrm{II})$ complex was mixed with 10 nmol of DNA without a reactive amine group and run in lane 3 , it can be seen that a small amount of the complex seems to stick to the DNA and moves with it to the main DNA band, but most of it has lower mobility and remains separated. This indicated that the highly luminescent bands on this and previous gels were not solely the result of uncoupled $\mathrm{Pt}(\mathrm{II})$ complex sticking to DNA, reacted or unreacted, and moving through the gel with it as a result.

The potential $\mathrm{Pt}(\mathrm{II})$ complex-DNA isolated from two separate bands following the first reaction attempt using TSTU/DIPEA appear to contain the same species when compared in lanes 4 and 5 on this gel. The $5 \mathrm{nmol}$ portions used from each of the previously "purified" product bands look nearly identical on this gel, though the band with higher mobility on the first gel (lane 5) might be slightly less luminescent and could represent a sample with a lower percentage of conjugated DNA than the other band (lane 4).

Finally, $10 \mathrm{nmol}$ of crude product from the newly performed solid-phase reactions using TSTU/DIPEA and EDC/NHS were run in lanes 6 and 7, respectively. The 
EDC/NHS reaction lane appears to contain a number of species with varying mobilities that are all faintly luminescent. This could be an indication of unwanted side-products or poor linearization of the DNA in this sample. By comparison, the reaction using TSTU/DIPEA resulted in a single band of DNA on the gel when considering the image obtained using absorbance (left). However, considering lanes 4 through 6 on both the absorbance and luminescence images, it seems likely that there is incomplete separation of reacted and unreacted DNA as the luminescence is concentrated at the top of each absorbent band.

A gel was run on the remaining crude product from the TSTU/DIPEA reaction run in lane 6 and the portion of the band with the most luminescence was isolated and the DNA eluted. Following quantification, $8 \mathrm{nmol}$ of the sample was sent for MS analysis. The calculated $\mathrm{m} / \mathrm{z}$ value for $\mathrm{Pt}(\mathrm{II})$ complex-DNA did not have a match in the experimentally found $\mathrm{m} / \mathrm{z}$ peaks, nor did the DNA-acac-COOH peak. However, upon consideration of these results and comparison to those obtained from the first solid-phase reaction attempt (using TSTU and ultramild DNA), it was observed that there were several common $\mathrm{m} / \mathrm{z}$ values for the two samples. With most of the $\mathrm{m} / \mathrm{z}$ values below that of DNA-acac-COOH (recall it is 4613), it was hypothesized that the conjugated acac$\mathrm{COOH}$ might be fragmenting during MS analysis. Figure 4.12 represents the modified acac ligand conjugated to amino-modified DNA and includes the $\mathrm{m} / \mathrm{z}$ differences expected to be added to that of the $\mathrm{NH}_{2}$-DNA at various points along the ligand. Table 4.11 contains the $\mathrm{m} / \mathrm{z}$ values found experimentally for the last sample sent for MS analysis in the left-hand column while the differences between these values and that of amino-modified DNA (4417.8) are shown in the right-hand column. Upon comparison of 
the right-hand column and the potential fragment $\mathrm{m} / \mathrm{z}$ values calculated for acac-COOH (Figure 4.12), it can be seen that the values bolded in the table are near matches. These results therefore imply that the modified acac ligand was conjugated to the DNA prior to MS analysis. It is unclear whether the $\mathrm{Pt}(\mathrm{II})$ complex was present, although the peak at $\mathrm{m} / \mathrm{z} 4898.8$ is promising. In the MS data obtained for the complex alone, a prominent peak was present at a value of one hundred less (553) than the expected $\mathrm{m} / \mathrm{z}$ (563). The peak at $\mathrm{m} / \mathrm{z} 4898.8$ could represent this fragmentation of the complex associated with a potassium cation.

Table 4.11 Comparison of $\mathrm{m} / \mathrm{z}$ values found for Pt(II) complex-DNA band cut from a polyacrylamide gel with the calculated $\mathrm{m} / \mathrm{z}$ value differences from that of amino-modified DNA (4417.8).

\begin{tabular}{|c|c|}
\hline $\begin{array}{c}\text { Found } \mathrm{m} / \mathrm{z} \text { values for } \\
\text { narrow band }\end{array}$ & $\begin{array}{c}\text { Difference from calc'd } \mathrm{m} / \mathrm{z} \\
\text { for } \mathrm{NH}_{2} \text {-DNA }\end{array}$ \\
\hline 4418 & 0.2 \\
4448.5 & $\mathbf{3 0 . 7}$ \\
4459.7 & $\mathbf{4 1 . 9}$ \\
4472.3 & $\mathbf{5 4 . 5}$ \\
4514 & $\mathbf{9 6 . 2}$ \\
4532.7 & $\mathbf{1 1 4 . 9}$ \\
4552.4 & 134.6 \\
4593.7 & 175.9 \\
4898.8 & 481 \\
\hline
\end{tabular}

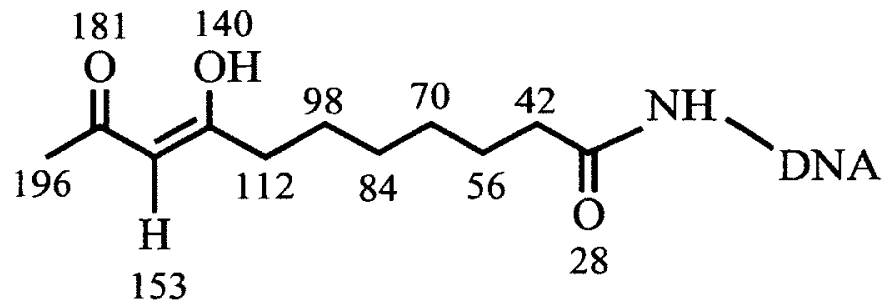

Figure 4.12 Possible fragment mass values of acac-COOH when conjugated to DNA. 
Since the MS results indicated that unreacted amino-modified DNA was still present in the sample, further purification of the remaining $\mathrm{Pt}$ (II) complex-DNA $(\sim 53$ $\mathrm{nmol}$ ) recovered from the gel was performed using RP-HPLC (conditions in Table 4.6). Absorbance at both 260 and $330 \mathrm{~nm}$ were monitored, where $260 \mathrm{~nm}$ was used to identify when DNA was present and it was hoped that $330 \mathrm{~nm}$ might indicate that (ppy)Pt(acac$\mathrm{COOH}$ ) was present. The four peaks evident in the chromatograms in Figure 4.13 were collected, the DNA present was quantified, and the fractions were tested for luminescence. These RP-HPLC results are much clearer with more definitive peaks than in the work with the thrombin-binding aptamer, which is likely due to the use of TEAA buffer as part of the mobile phase and the subsequent denaturation of the DNA.

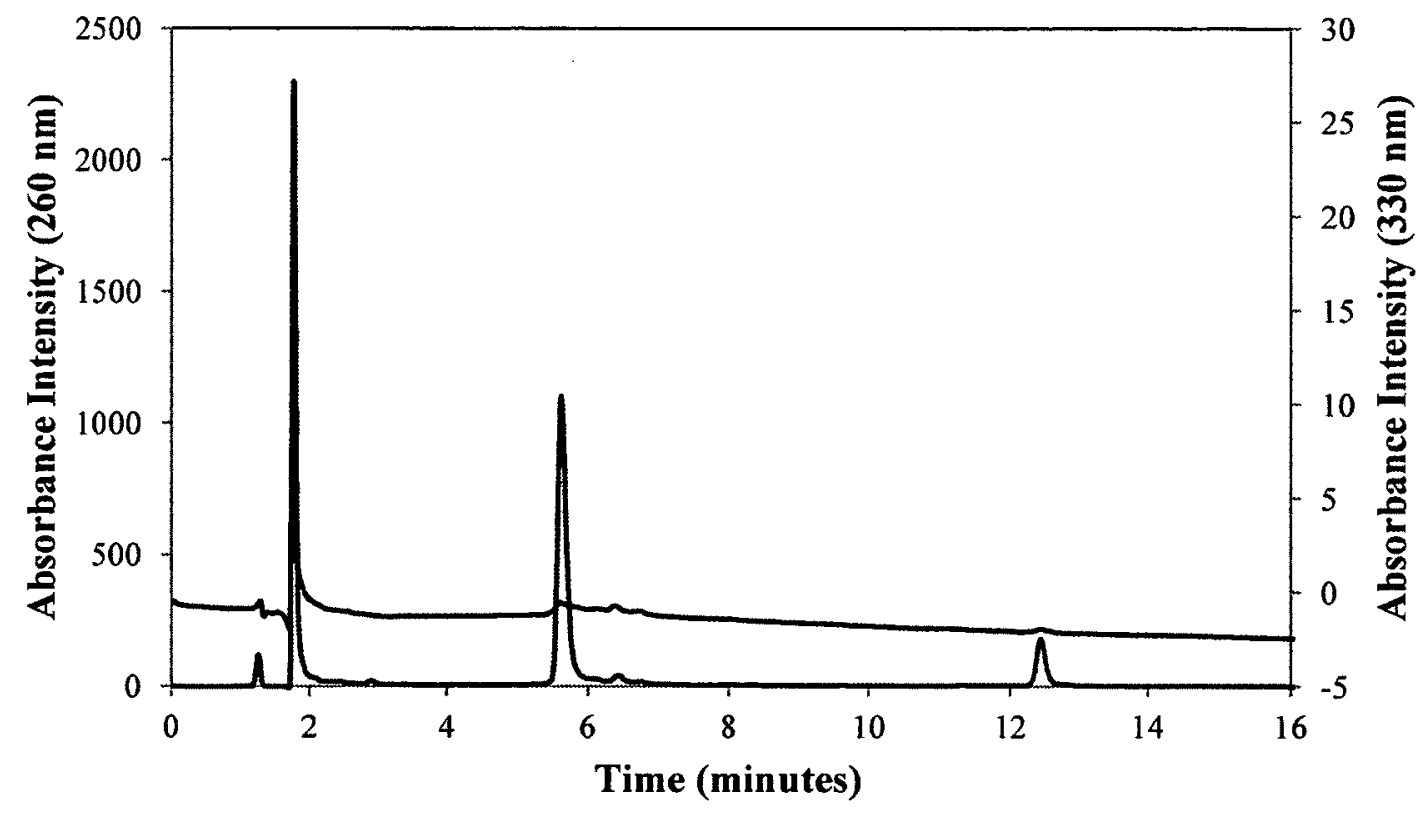

$-260 \mathrm{~nm}-330 \mathrm{~nm}$

Figure 4.13 RP-HPLC chromatograms for the purification of Pt(II) complex-DNA obtained through a solid-phase reaction with 5'-amino-modified DNA. Absorbance at $260 \mathrm{~nm}$ was used to monitor DNA while that at $330 \mathrm{~nm}$ was used to monitor the Pt(II) complex. 
The second peak collected (at about 1.8 to 2 minutes in Figure 4.13) gave promising luminescence results when excited at $320 \mathrm{~nm}$, with the familiar (ppy)Pt(acac$\mathrm{COOH}$ ) evident between 450 and $550 \mathrm{~nm}$ (dotted line in Figure C-15). Peaks 1 and 4 were not as luminescent and did not show the typical $\mathrm{Pt}(\mathrm{II})$ complex peak pattern (Figure C-15). The luminescence of peak 3 was not obtained prior to sending samples of peaks 2 and 3 for MS analysis. MS results indicated that peak 2 was still predominantly aminomodified DNA, though it also contained peaks with $\mathrm{m} / \mathrm{z}$ values similar to the fragmentation values discussed previously for acac-COOH. Peak 3 had only three $\mathrm{m} / \mathrm{z}$ values other than that for unreacted amino-modified DNA and contained comparatively little unreacted DNA. These results will be discussed further below.

In order to determine whether the $\mathrm{m} / \mathrm{z}$ values being found in several of these $\mathrm{Pt}($ II) complex conjugation reactions were in fact representative of fragmented DNA-acac$\mathrm{COOH}$, a coupling reaction using the modified acac ligand instead of the full $\mathrm{Pt}(\mathrm{II})$ complex was performed using TSTU/DIPEA and the same DNA sequence prepared using ultramild bases. Following reaction, deprotection, and quantification of the crude product, RP-HPLC was used for purification. The most intense peak was determined to have the most DNA present using UV-visible spectrophotometry, and a portion of the sample from this fraction was sent for MS analysis.

Upon consideration of the MS results in Table 4.12 , it can be seen that the $\mathrm{m} / \mathrm{z}$ values obtained do not include that of unreacted amino-modified DNA indicating good separation of reacted and unreacted DNA. The peak found at $\mathrm{m} / \mathrm{z} 4609.7$ (bolded) is about what would be expected for the desired product (4613). 
The comparison of the values in the right-hand column to the possible fragmentation values in Figure 4.12 mostly do not overlap. A promising pattern did, however, arise upon comparison of the values in the right-hand column here and in Table 4.11, as well as values obtained for peak 3 from the RP-HPLC purification of Pt(II) complex-DNA (Figure 4.13). All three samples had an $\mathrm{m} / \mathrm{z}$ peak that was about $133-134$ higher than amino-modified DNA, while the peak 3 sample and the DNA-acac-COOH sample both also had a peak about 156 higher. Therefore, though it might be fragmenting in an unpredictable manner, it seems likely that the $5^{\prime}$-amino-modified DNA is successfully coupling with acac-COOH alone or as part of the (ppy)Pt(acac-COOH) complex.

Table 4.12 Comparison of $\mathrm{m} / \mathrm{z}$ values found for DNA-acac-COOH with the calculated $\mathrm{m} / \mathrm{z}$ value differences from that of amino-modified DNA (4417.8).

\begin{tabular}{|c|c|}
\hline Found $\mathrm{m} / \mathrm{z}$ values & $\begin{array}{c}\text { Difference from calc'd } \mathrm{m} / \mathrm{z} \\
\text { for } \mathrm{NH}_{2} \text {-DNA }\end{array}$ \\
\hline 4551.9 & $\mathbf{1 3 4 . 1}$ \\
4566.7 & 148.9 \\
4574 & $\mathbf{1 5 6 . 2}$ \\
4588.1 & 170.3 \\
4592.8 & 175 \\
$\mathbf{4 6 0 9 . 7}$ & $\mathbf{1 9 1 . 9}$ \\
4623.1 & 205.3 \\
4658.4 & 240.6 \\
\hline
\end{tabular}

\subsubsection{Conjugation to a 3'-amino-modified strand}

The sequence 5'-AGT CAG TAC GTC AG- $\left(\mathrm{CH}_{2}\right)_{7}-\mathrm{NH}_{2}-3^{\prime}$ was designed to be complementary to that used for 5 ' labelling in the previous section. It was prepared using ultramild guanine and adenine bases as in the previous section. As synthetically prepared 
DNA is built from the $3^{\prime}$ - to the 5'-terminus, modified CPG beads must be used when a 3'-modifier is desired. Therefore, a CPG column with 3'-amino-modifier present was used for the synthesis of this sequence. As mentioned briefly in section 1.4 , the 3 ' reactive amine is protected with the fluorenylmethoxycarbonyl (Fmoc) group. This group was removed following DNA synthesis through reaction with the base piperidine. The mechanism of this elimination reaction is shown in Scheme 4.5. Piperidine removes the highly acidic proton on the fluorene ring, as illustrated, to give the cyclopentadienyl anion. The cyclopentadienyl anion then releases a carbamate anion and this in turn spontaneously loses $\mathrm{CO}_{2}$ to leave the amine free for reaction with activated (ppy)Pt(acac$\mathrm{COOH}) .{ }^{142}$ Following treatment with piperidine, the CPG beads were therefore added to a solution of (ppy) Pt(acac-COOH) preactivated through reaction with TSTU and DIPEA.

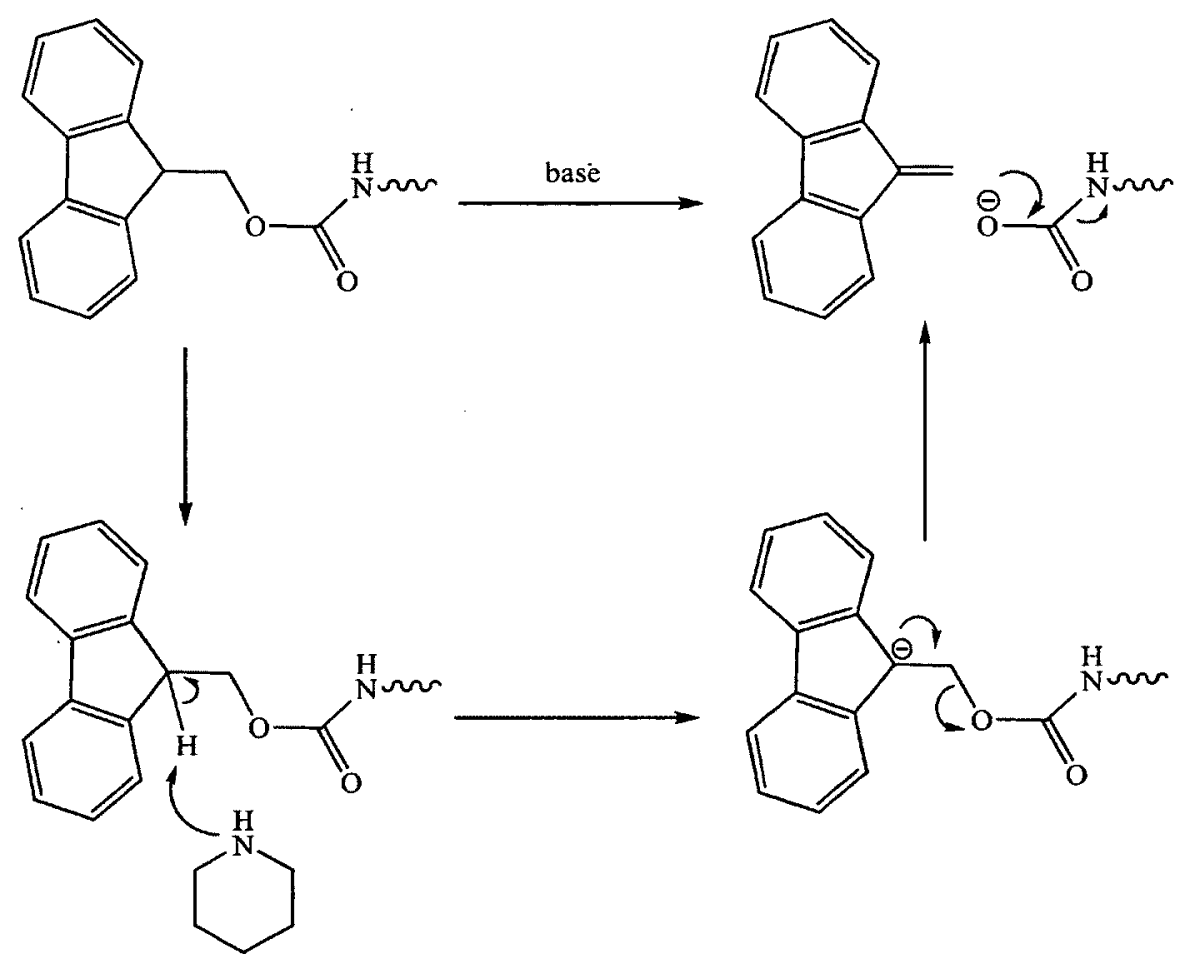

Scheme 4.5 Removal of the Fmoc protecting group using piperidine. ${ }^{142}$ 
The crude reaction product was isolated from the beads using ultramild deprotection conditions as previously discussed and was run using RP-HPLC in an attempt to purify the desired Pt(II) complex-DNA conjugate. A representative chromatogram is shown in Figure C-16. Little DNA was recovered from peaks emerging later than the first group of intense peaks (i.e. after about 5 minutes) which likely contained failed DNA sequences. The peak present at about 8 minutes was tested for luminescence and with excitation at $320 \mathrm{~nm}$ was found to have emission characteristic of (ppy)Pt(acac-COOH) (see Figure C-17). This sample was therefore sent for MS analysis and the results are summarized in Table 4.13. The peak with the highest $\mathrm{m} / \mathrm{z}$ value of 4659.3 could be the DNA conjugated to acac- $\mathrm{COOH}(\mathrm{m} / \mathrm{z}$ calc'd 4662$)$. The other peaks might represent the same fragment of DNA-acac-COOH with an $\mathrm{m} / \mathrm{z}$ of 4607 if it is associated with sodium or potassium ions, as demonstrated in Table 4.13.

Table 4.13 Summary of MS results for possible 3'-Pt(II) complex-DNA following RP-HPLC.

\begin{tabular}{|c|c|c|}
\hline $\begin{array}{c}\text { Found } \mathrm{m} / \mathrm{z} \\
\text { values }\end{array}$ & $\begin{array}{c}\text { Difference from calc'd } \\
\mathrm{m} / \mathrm{z} \text { for } \mathrm{NH}_{2} \text {-DNA }\end{array}$ & Possible identity \\
\hline 4630.3 & 163.4 & fragmented DNA-acac-COOH $(4607)+\mathrm{Na}^{+}$ \\
4646.3 & 179.4 & fragmented DNA-acac-COOH $(4607)+\mathrm{K}^{+}$ \\
$\mathbf{4 6 5 9 . 3}$ & 192.4 & DNA-acac-COOH \\
\hline
\end{tabular}

\subsection{Conclusions and future work}

The synthesis, purification, and characterization of a new square planar, luminescent platinum(II) complex has been performed to add to a group of luminescent probes for potential use in the study of aptamer folding upon target binding. The new complex, prepared using 2-phenylpyridine and a modified acetylacetone ligand, exhibited 
concentration-dependent luminescence compatible with that seen for excimer-forming compounds. The emission peaks of this complex were red-shifted compared to those found by Boshra Hosseini and reported in her B.Sc. thesis ${ }^{189}$ for a complex prepared using a difluoro-substituted phenylpyridine ligand and the same modified acetylacetone ligand as that used in this work. Work towards the synthesis and purification of a third Pt(II) complex for this family of probes was reported but purification of the 2phenylquinoline-containing product was not achieved. It would have been expected to exhibit emission that was red-shifted from that of the complex containing 2phenylpyridine. ${ }^{186}$

The Pt(II) complex that was successfully isolated, 8,10-dioxoundecanoic acid-2phenylpyridylplatinum(II), was subject to several conjugation attempts with aminomodified DNA. MS results for the $15 \mathrm{mer}$ thrombin-binding aptamer following a solidphase conjugation reaction using EDC and NHS as coupling reagents supported the successful coupling of the Pt(II) complex to the 5 -terminus of the aptamer. However, the seeming instability of the complex in ammonium hydroxide during deprotection led to the decision that future reactions would be performed in solution or using ultramild conditions for a solid-phase reaction. It was not conclusively determined whether the solution-phase reaction resulted in successful coupling as little DNA was recovered from the organic solvent in which the reaction was performed. If a better method for DNA recovery from DMF or DMSO could be determined, the solution-phase reaction might still prove useful in conjugating Pt(II) complexes to DNA.

The results following the solid-phase reactions where a potassium carbonate solution was used for deprotection of the ultramild DNA were less promising than those 
from the work with the thrombin-binding aptamer. Luminescence emission matching that of the complex was observed in several samples, but though these samples were sent for MS analysis following different reactions and purification techniques, the full $\mathrm{Pt}(\mathrm{II})$ complex-DNA conjugate was never detected. However, some support for successful conjugation was provided by the MS results as a peak matching the expected $\mathrm{m} / \mathrm{z}$ of the DNA conjugated to the modified acetylacetone ligand was seen in a couple of samples. It also seemed that fragments of this conjugate were present in several sets of MS data. An attempt to confirm fragmentation through the preparation and MS analysis of a DNAacac-COOH conjugate did show some common peaks, thus supporting successful conjugation.

Inefficient purification was a problem following each conjugation reaction with (ppy)Pt(acac-COOH). The RP-HPLC runs using triethylammonium acetate buffer to help denature the DNA seemed to be more successful than earlier attempts using ammonium acetate buffer or the attempts at using PAGE for purification. Therefore, future purification attempts should also be performed using denaturing RP-HPLC.

The completion of the proof-of-concept complementary. DNA sequences study would be useful for both optimizing conjugation of (ppy)Pt(acac-COOH) to aminomodified DNA as well as to prove that a change in emission can be observed for two conjugated Pt(II) complex molecules. Once that has been achieved, work on the conjugation of two (ppy)Pt(acac-COOH) molecules to one DNA strand could be attempted. This need not be an aptamer in the first experiments, but could be a strand that folds into a hairpin structure, for example, as in the pyrene-based thrombin sensor discussed in section 4.1.2.2. An aptamer with a known structure that changes upon target 
binding could then be studied using the luminescent-probes to detect and determine its conformational change prior to moving on to study aptamers that do not have reported structures. 


\section{Appendices}

\section{Appendix A \\ Supporting data for singly-labelled DNA-DTPA conjugates}

Figure A-1 Calibration curve for the initial xylenol orange tests. Each point is the average absorbance from three spectra on the same sample. Xylenol orange was present at $15 \mathrm{nmol} / \mathrm{mL}$ in each sample.

.270

Figure A-2 Attempt to separate SC-Gd(III) from xylenol orange using a stirred cell (filter with $1000 \mathrm{Da}$ cut-off). The numbers refer to the approximately $1 \mathrm{~mL}$ fractions washed through the filter, the last two being repeats of the first and fourth $1 \mathrm{~mL}$ washings, respectively. The inset is an expanded view of the region where the xylenol

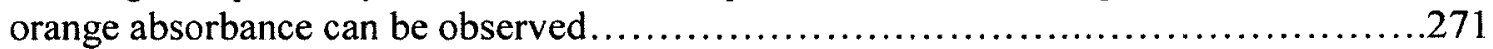

Figure A-3 Calibration curves obtained for the xylenol orange test on the filtrate of a sample of $30 \mathrm{~T} \mathrm{SC-Gd(III).} \mathrm{Xylenol} \mathrm{orange} \mathrm{was} \mathrm{present} \mathrm{at} 15 \mathrm{nmol} / \mathrm{mL}$ in all samples.

Figure A-4 Xylenol orange test on a sample of $15 \mathrm{~T} \mathrm{SC-Gd(III)} \mathrm{stored} \mathrm{for} \mathrm{a} \mathrm{period} \mathrm{of}$ approximately four months at $-20^{\circ} \mathrm{C}$

Figure A-5 Xylenol orange test on SC-Gd(III) heated to $37^{\circ} \mathrm{C}$ in bovine serum for 1 and 24 hours, respectively. Each solution contains $10 \mathrm{nmol}$ of xylenol orange........274

Figure A-6 Xylenol orange test on filtrate from $15 \mathrm{nmol}$ of $\mathrm{Gd}($ III) in bovine serum $(0.075 \mathrm{mM})$. Each solution contained $10 \mathrm{nmol}$ of xylenol orange. 275

Figure A-7 Xylenol orange test for SC-Gd(III) heated at $37^{\circ} \mathrm{C}$ for 3 hours in thrombin aptamer buffer. Each solution contained $15 \mathrm{nmol}$ of xylenol orange.

Figure A-9 Calibration curve for SC-Gd(III) heated at $37^{\circ} \mathrm{C}$ for 3 hours in buffer. Each point is the average absorbance from three spectra on the same sample. Xylenol orange was present at $15 \mathrm{nmol} / \mathrm{mL}$ in each sample.

Figure A-10 Relaxivity plots for Magnevist ${ }^{\circledR}$ and DTPA-Gd(III) at $9.4 \mathrm{~T}, \mathrm{pH} 7.4$ and $25^{\circ} \mathrm{C}$. 278

Figure A-11 Relaxivity plots for SC-Gd(III) alone, with thrombin (target), and with

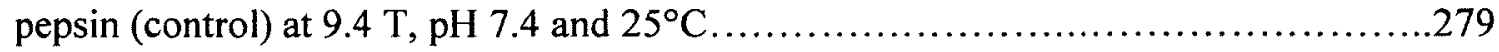

Figure A-12 Relaxivity plots for 15T SC-Gd(III) alone, with thrombin, with pepsin,

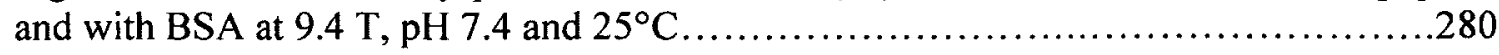

Figure A-13 Relaxivity plots for $15 \mathrm{~T} \mathrm{SC-Gd(III)+polyA} \mathrm{alone,} \mathrm{with} \mathrm{thrombin,} \mathrm{and}$

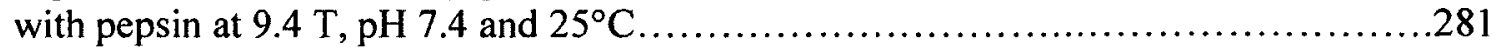


Figure A-14 Relaxivity plots for 30T SC-Gd(III) alone, with thrombin, and with pepsin

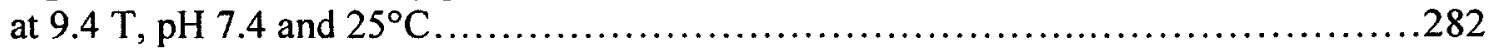

Figure A-15 Relaxivity plots for 30T SC-Gd(III)+polyA alone, with thrombin, and

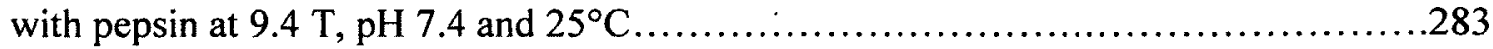

Figure A-16 Relaxivity plots for SC-Gd(III) alone, with thrombin, with pepsin, and

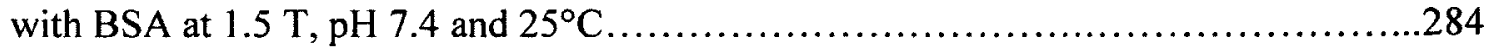




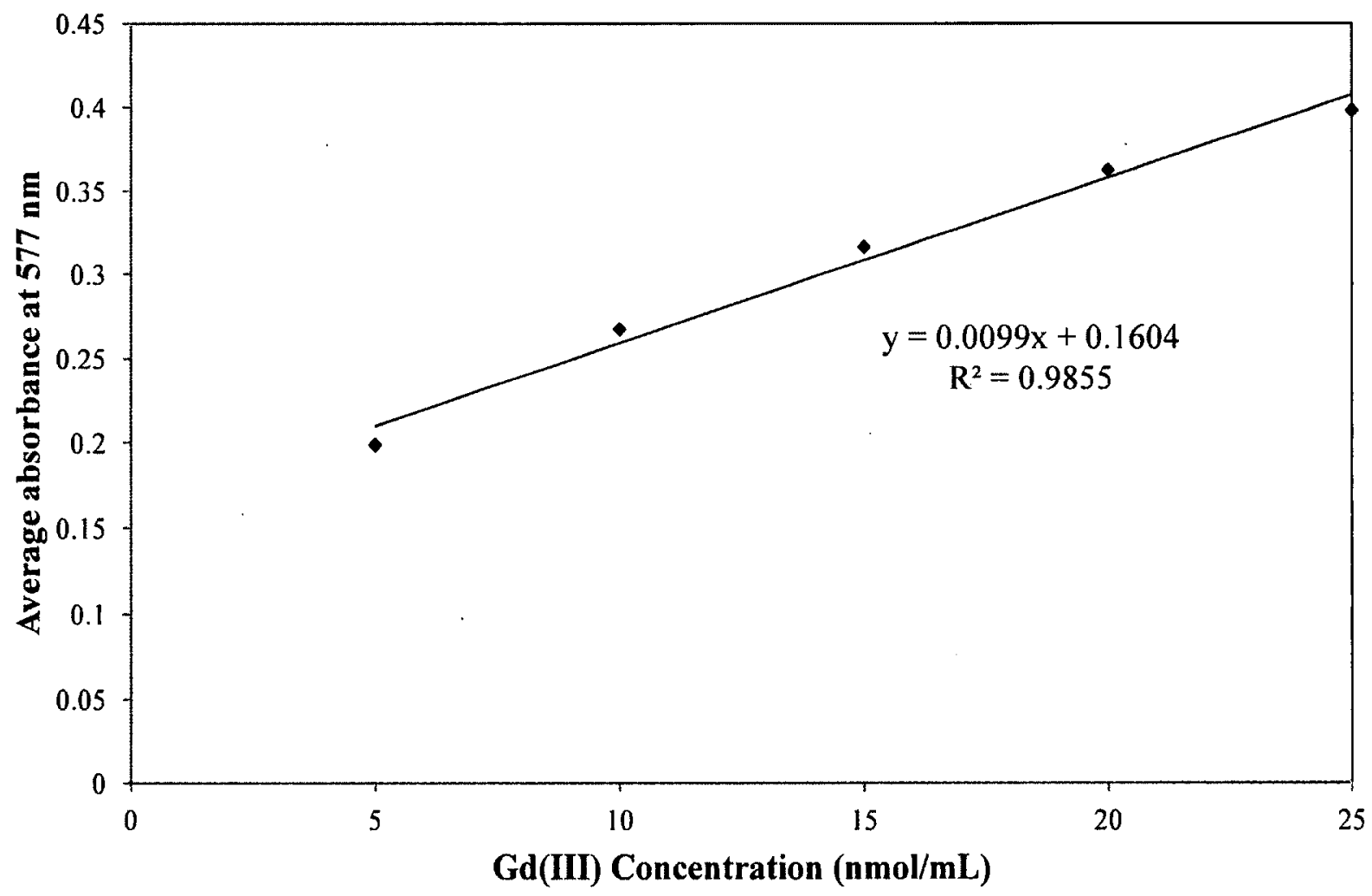

Figure A-1 Calibration curve for the initial xylenol orange tests. Each point is the average absorbance from three spectra on the same sample. Xylenol orange was present at $15 \mathrm{nmol} / \mathrm{mL}$ in each sample. 


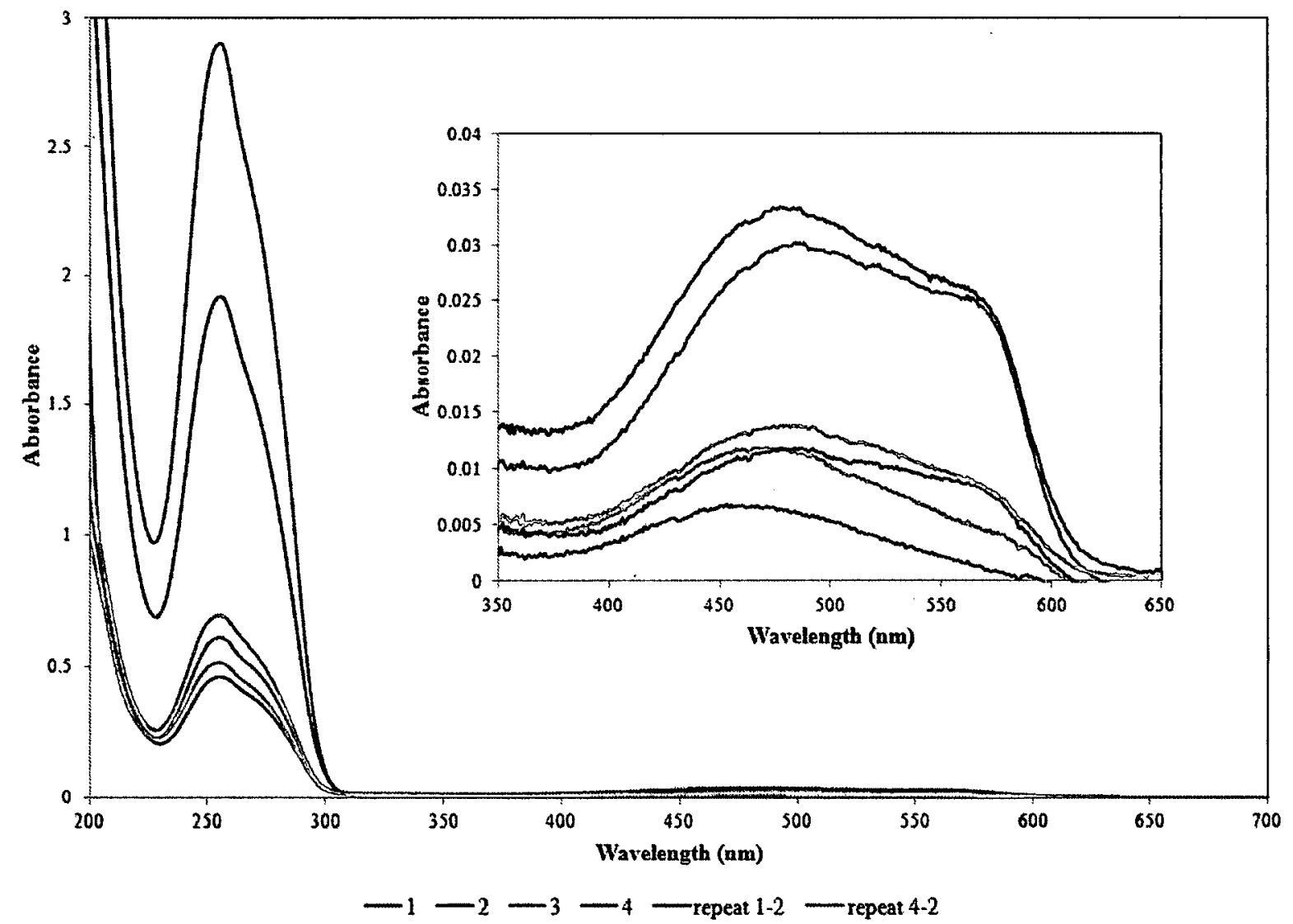

Figure A-2 Attempt to separate SC-Gd(III) from xylenol orange using a stirred cell (filter with 1000 Da cut-off). The numbers refer to the approximately $1 \mathrm{~mL}$ fractions washed through the filter, the last two being repeats of the first and fourth $1 \mathrm{~mL}$ washings, respectively. The inset is an expanded view of the region where the xylenol orange absorbance can be observed. 


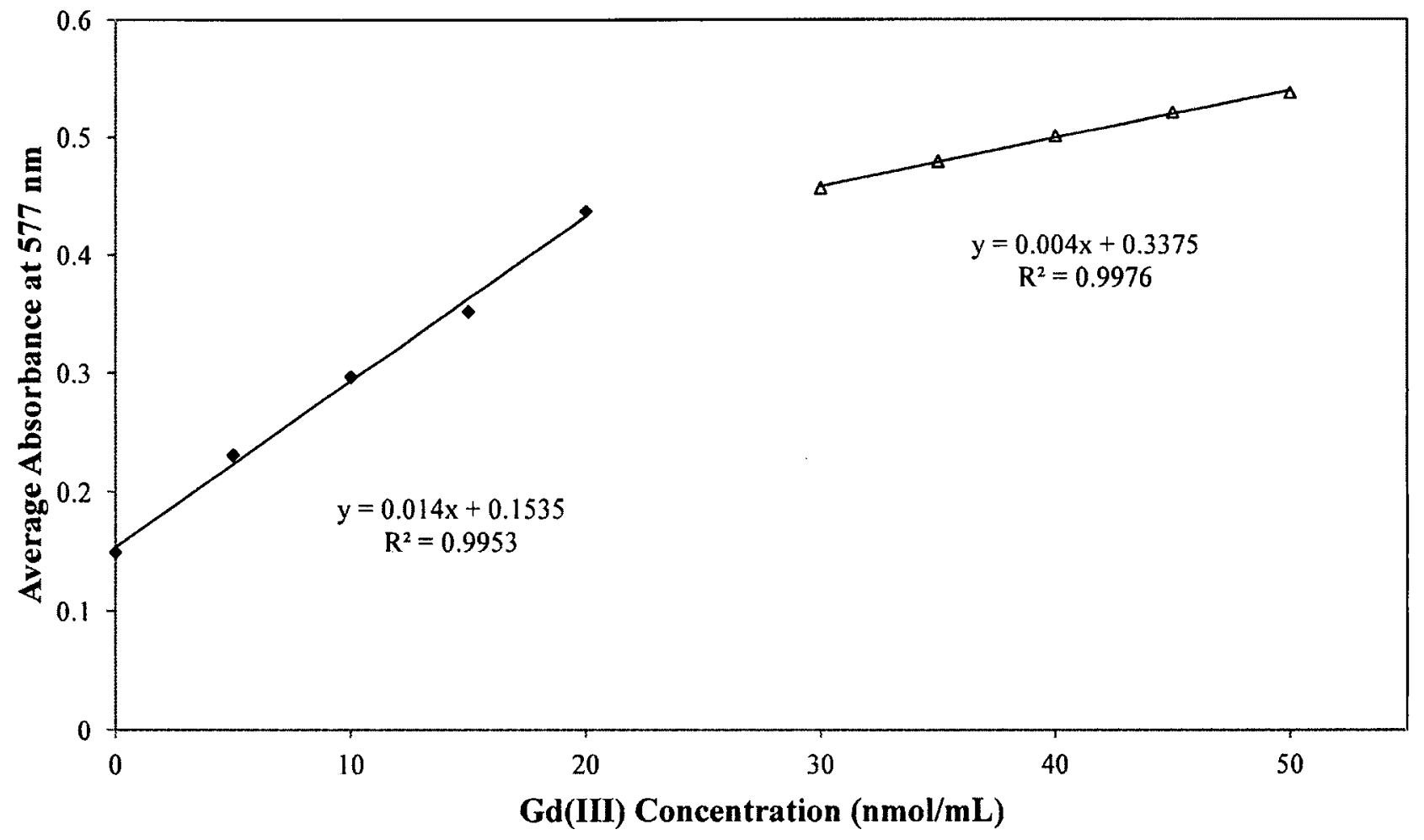

- Low range $\Delta$ High range

Figure A-3 Calibration curves obtained for the xylenol orange test on the filtrate of a sample of 30T SC-Gd(III). Xylenol orange was present at 15 $\mathrm{nmol} / \mathrm{mL}$ in all samples. 


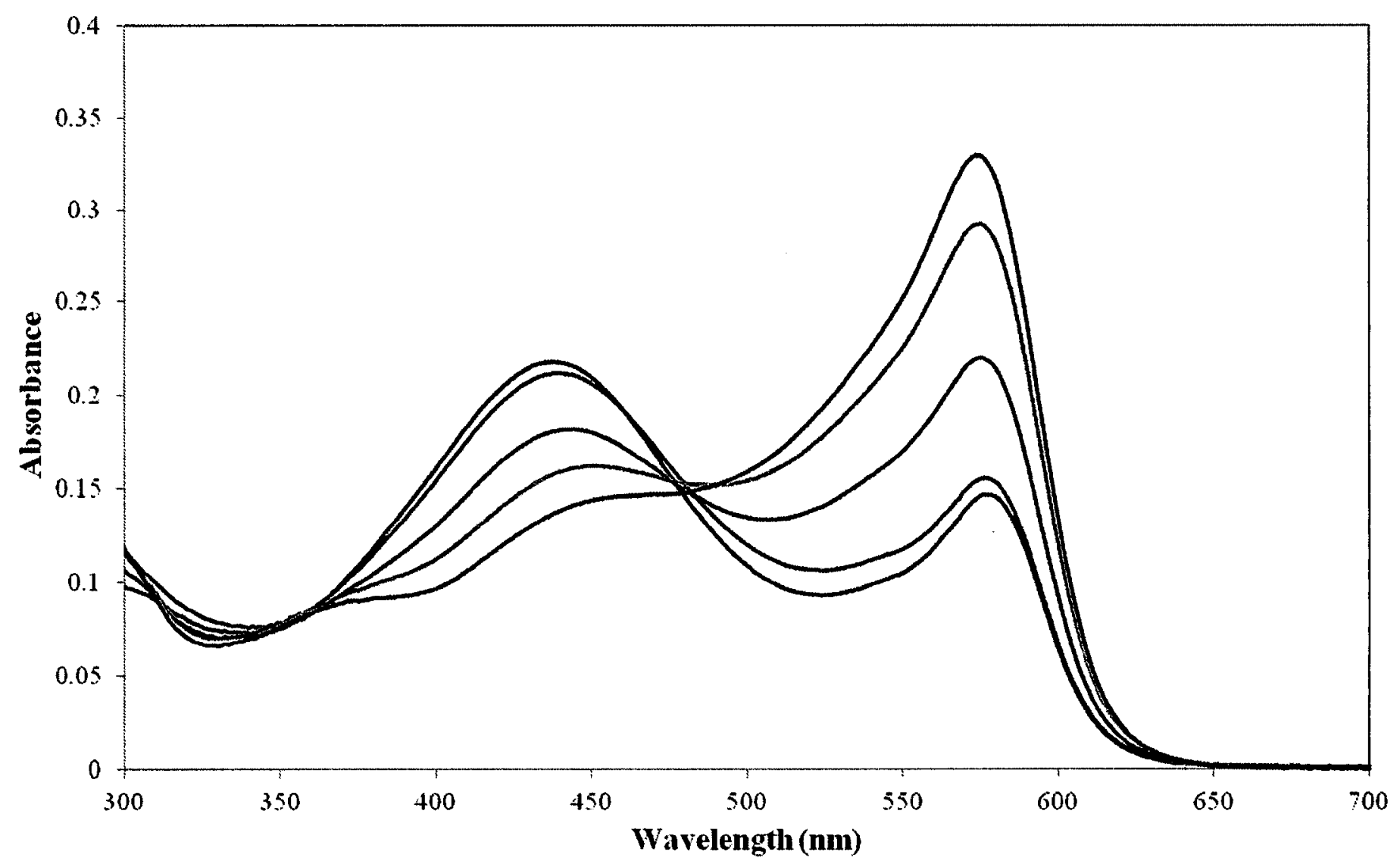

$-15 \mathrm{~T} \mathrm{SC}$ tiltrate $-0 \mathrm{nmol}$ Gd(III) $-5 \mathrm{mmol}$ Gd(III) $-10 \mathrm{mmol}$ Gd(III) $-15 \mathrm{mmol}$ Gd(III)

Figure A-4 Xylenol orange test on a sample of $15 \mathrm{~T} \mathrm{SC-Gd(III)} \mathrm{stored} \mathrm{for} \mathrm{a} \mathrm{period} \mathrm{of} \mathrm{approximately} \mathrm{four} \mathrm{months} \mathrm{at}-20^{\circ} \mathrm{C}$. 


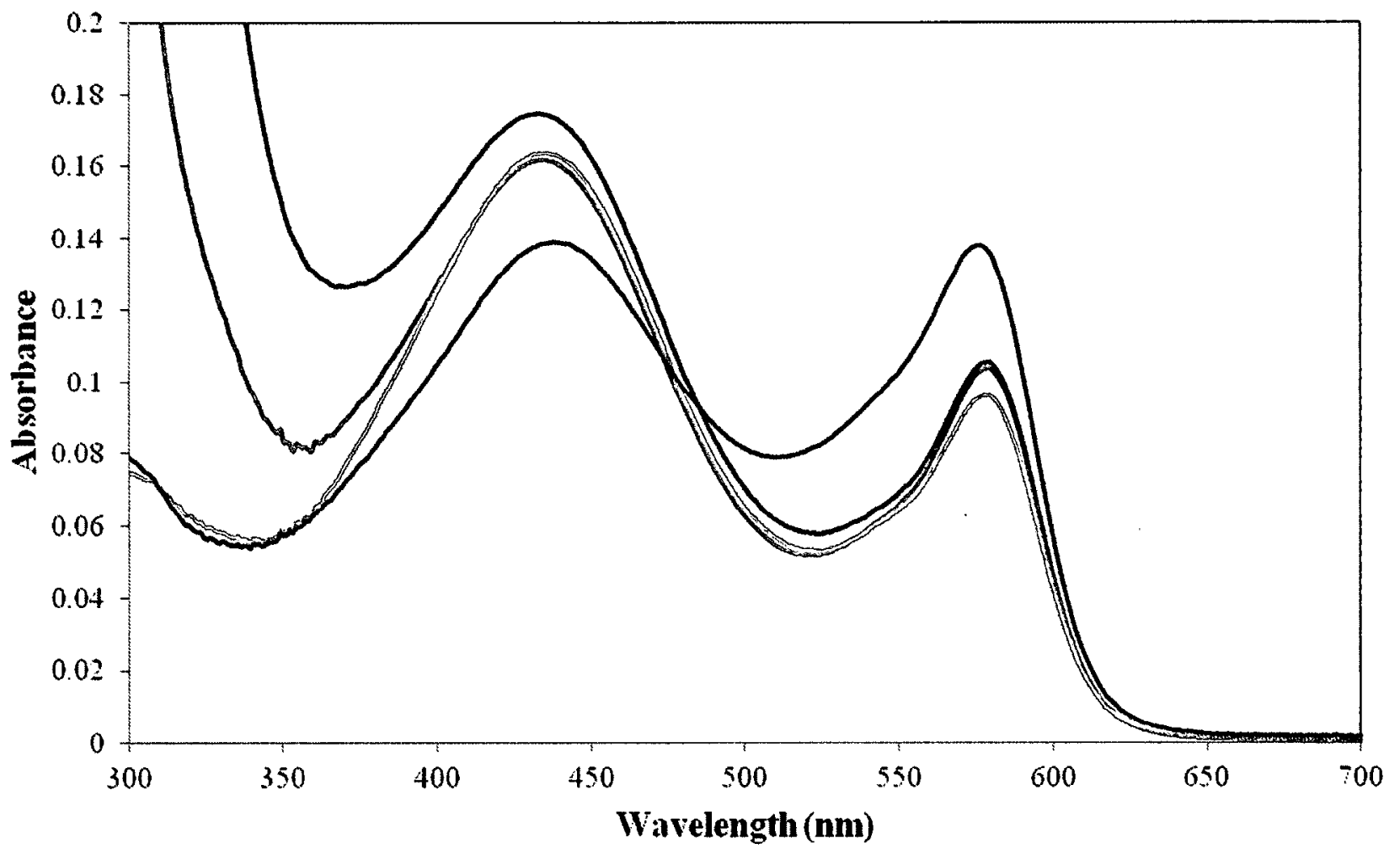

$-\mathrm{SC}-\mathrm{Gd}(\mathrm{III})$ filtrate 1 hour $-\mathrm{SC}-\mathrm{Gd}(\mathrm{III})$ filtrate 24 hours

$\Longrightarrow 01 \mathrm{~mol}$ Gd(III) $\quad-5 \mathrm{mmol}$ Gd(III)

Figure A-5 Xylenol orange test on SC-Gd(III) heated to $37^{\circ} \mathrm{C}$ in bovine serum for 1 and 24 hours, respectively. Each solution contains $10 \mathrm{nmol}$ of xylenol orange. 


$$
M
$$




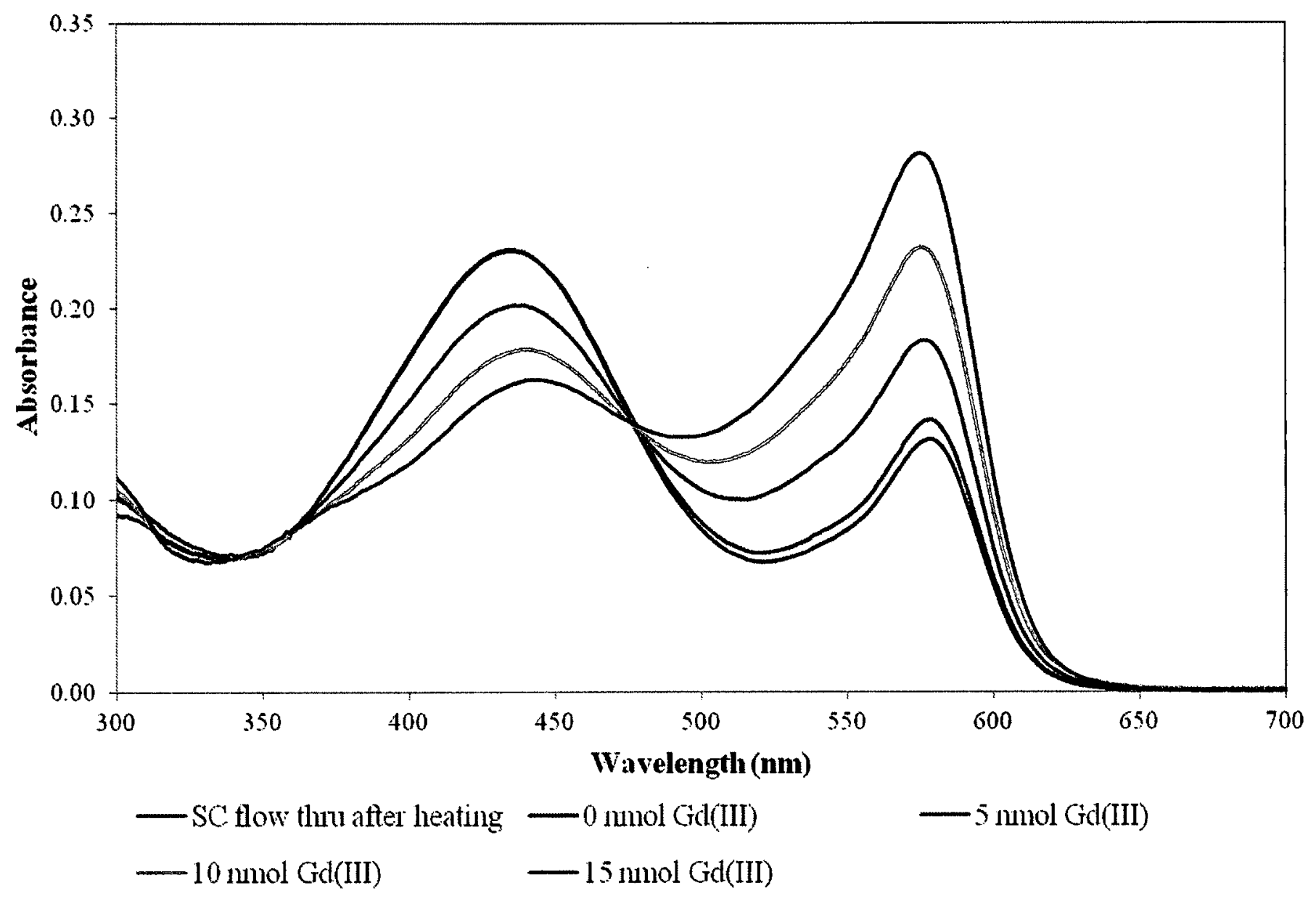

Figure A-7 Xylenol orange test for SC-Gd(III) heated at $37^{\circ} \mathrm{C}$ for 3 hours in thrombin aptamer buffer. Each solution contained $15 \mathrm{nmol}$ of $\mathrm{xylenol}$ orange. 


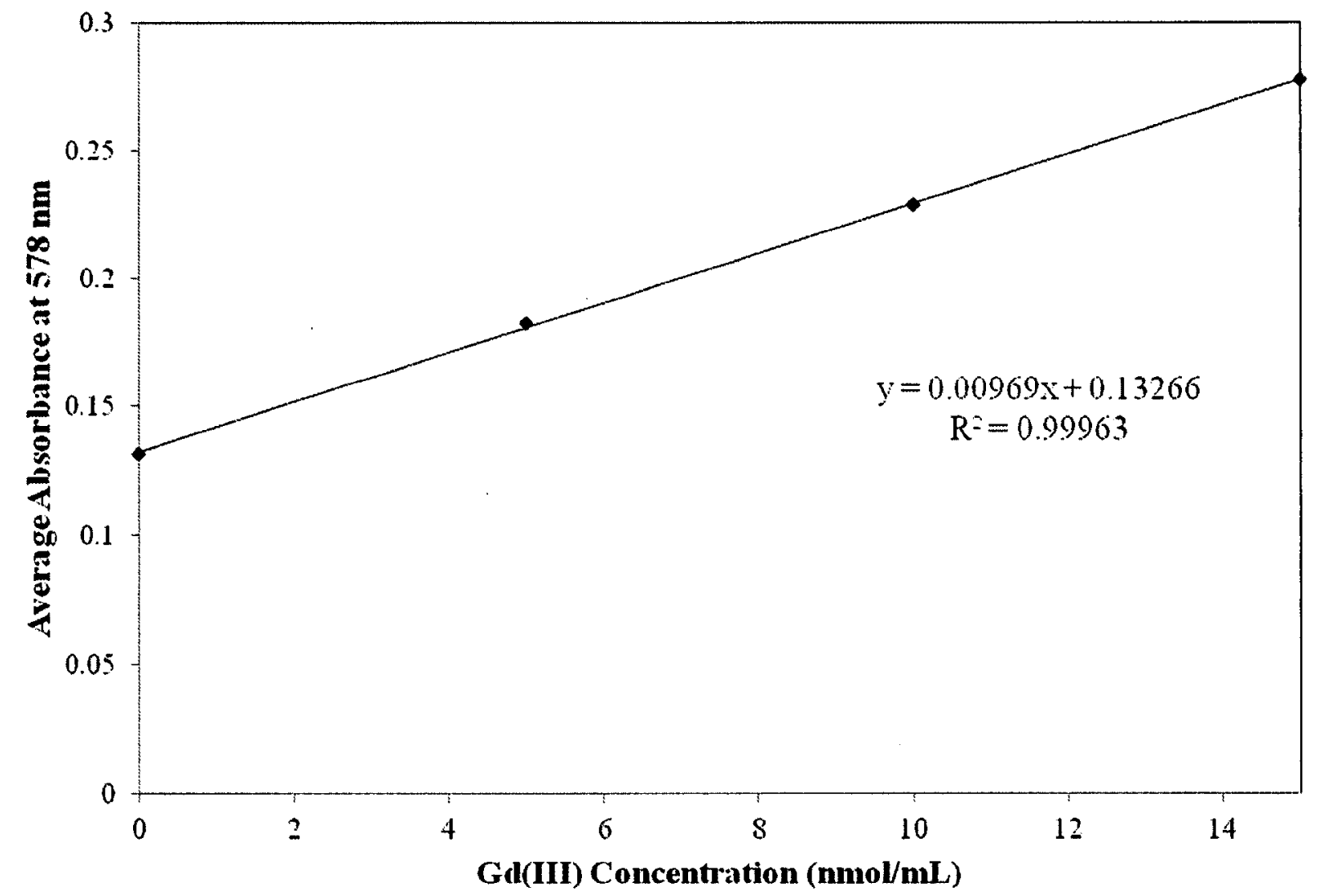

Figure A-9 Calibration curve for SC-Gd(III) heated at $37^{\circ} \mathrm{C}$ for 3 hours in buffer. Each point is the average absorbance from three spectra on the same sample. Xylenol orange was present at $15 \mathrm{nmol} / \mathrm{mL}$ in each sample. 


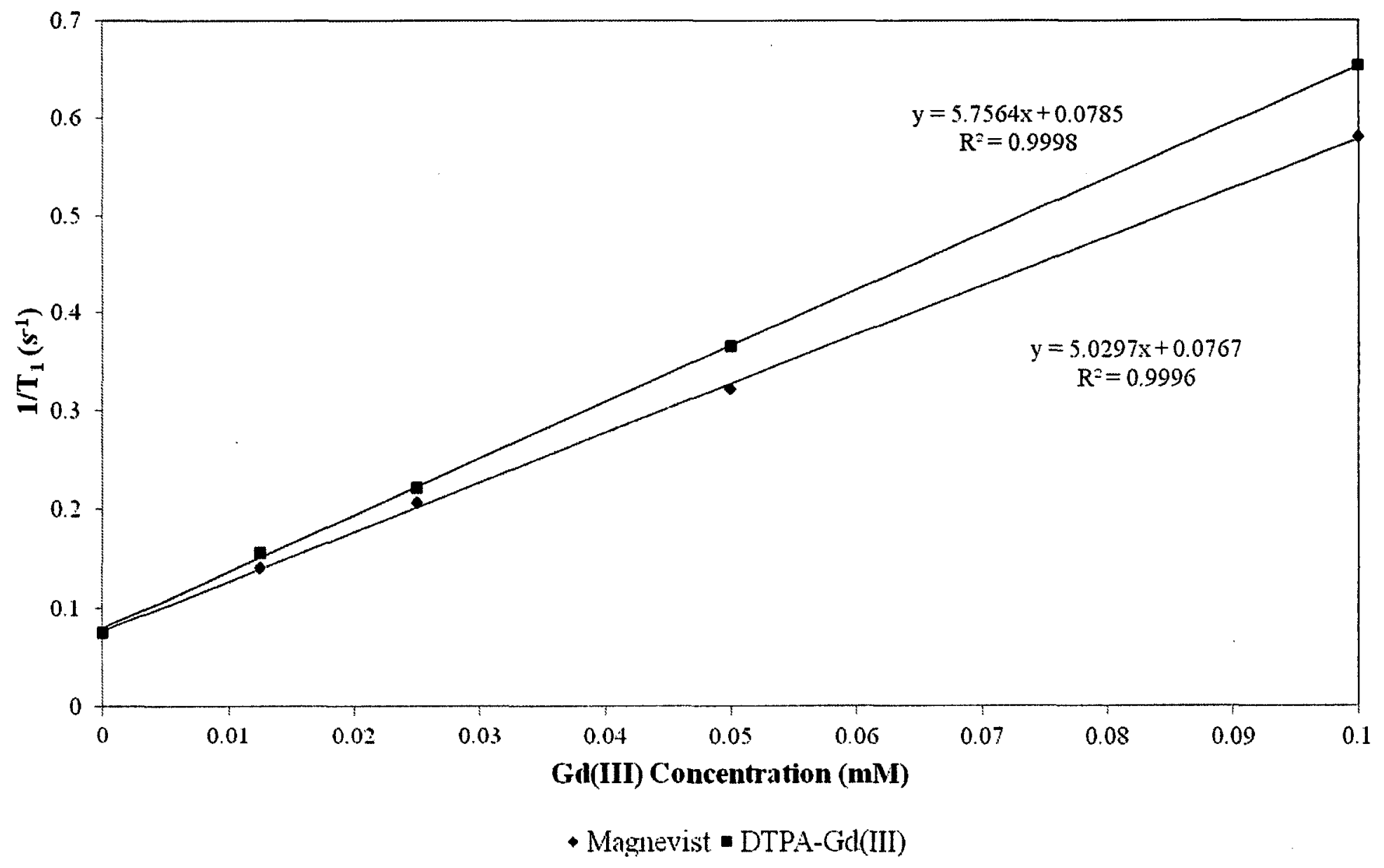

Figure A-10 Relaxivity plots for Magnevist ${ }^{\circ}$ and DTPA-Gd(III) at $9.4 \mathrm{~T}, \mathrm{pH} 7.4$ and $25^{\circ} \mathrm{C}$. 


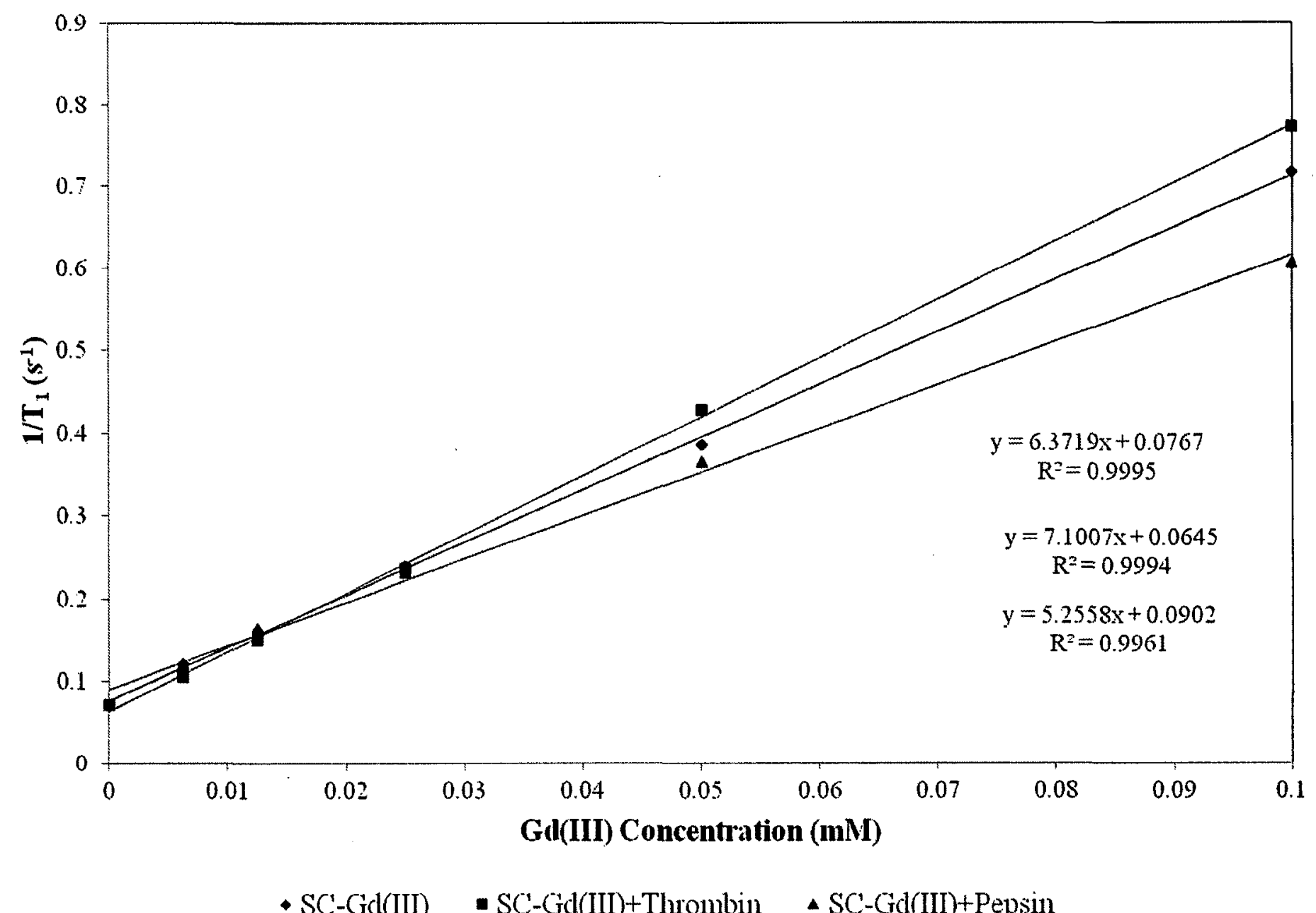

Figure A-11 Relaxivity plots for SC-Gd(III) alone, with thrombin (target), and with pepsin (control) at 9.4 T, pH 7.4 and $25^{\circ} \mathrm{C}$. 


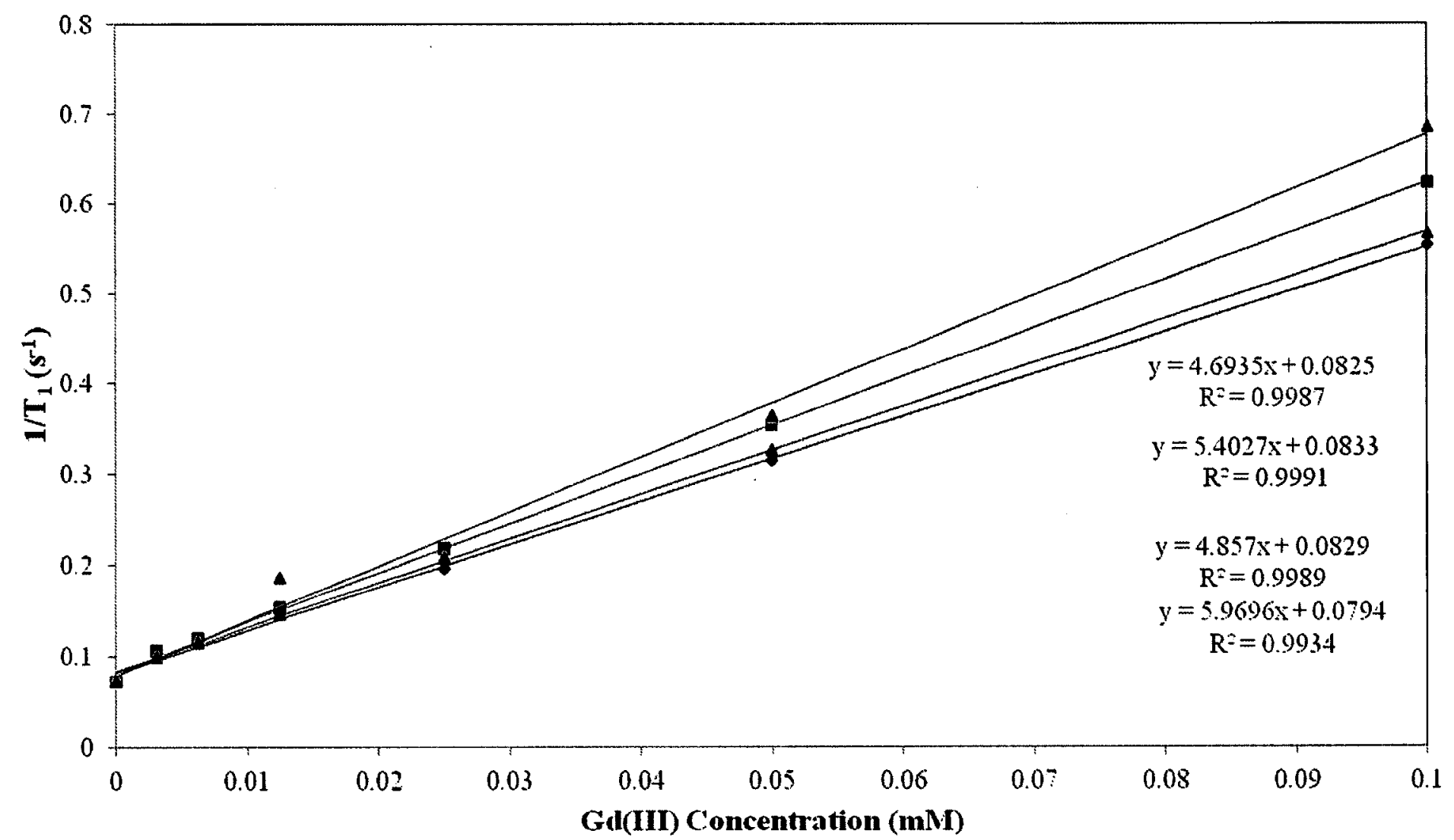

-15T SC-Gd(III) $\quad$ - 15T SC-Gd(III)+Thrombin $\quad 415 \mathrm{~T} \mathrm{SC-Gd(III)+Pepsin} \quad 15 \mathrm{~T} \mathrm{SC-Gd(III)+BSA}$

Figure A-12 Relaxivity plots for 15T SC-Gd(III) alone, with thrombin, with pepsin, and with BSA at 9.4 T, pH 7.4 and $25^{\circ} \mathrm{C}$. 


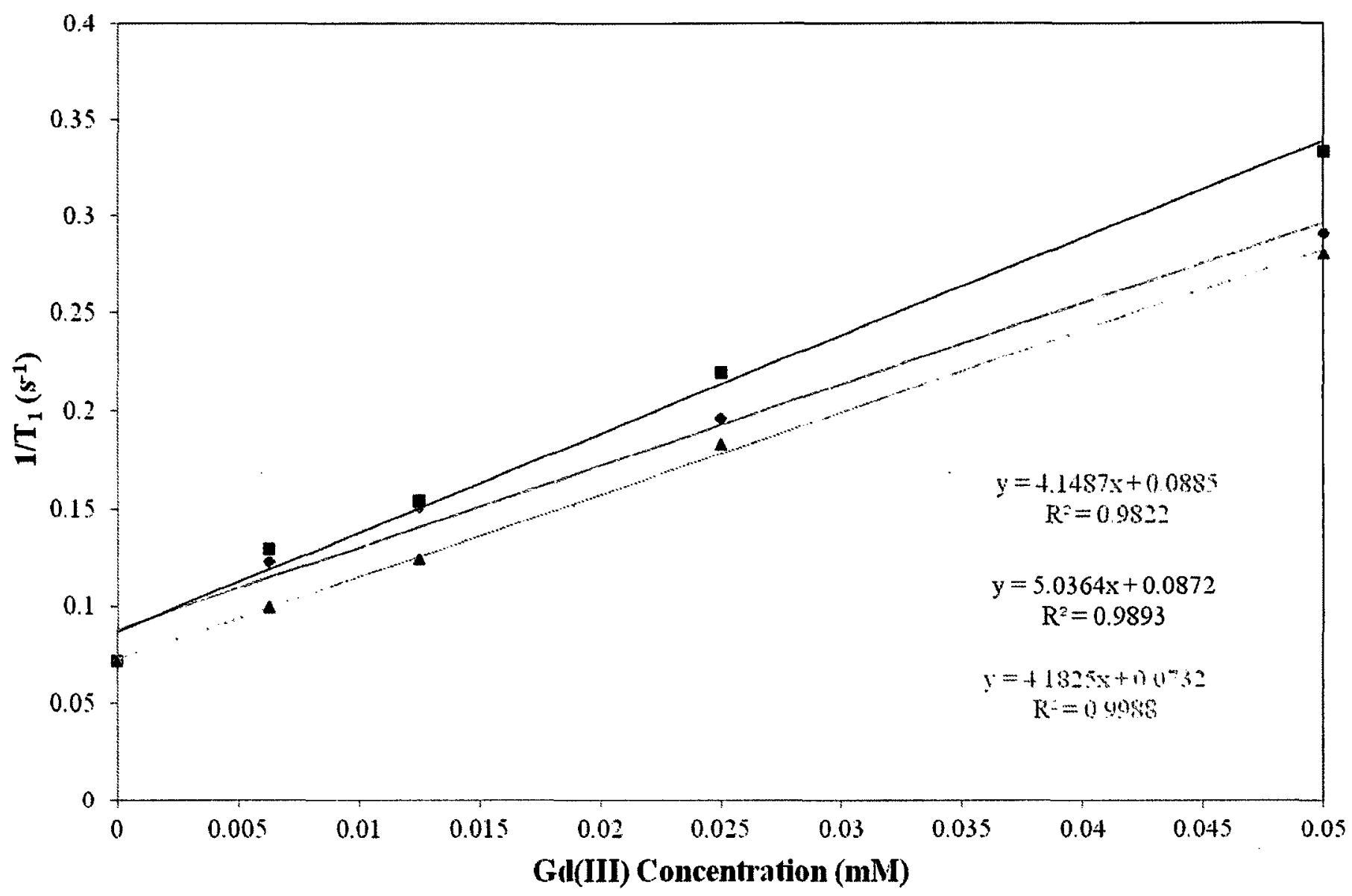

-15T SC-Gd(III)+polyA - $15 \mathrm{~T} \mathrm{SC-Gd(III)+polyA+Thrombin}$-15T SC-Gd(III)+poly A+Pepsin

Figure A-13 Relaxivity plots for 15T SC-Gd(III)+polyA alone, with thrombin, and with pepsin at $9.4 \mathrm{~T}, \mathrm{pH} 7.4$ and $25^{\circ} \mathrm{C}$. 


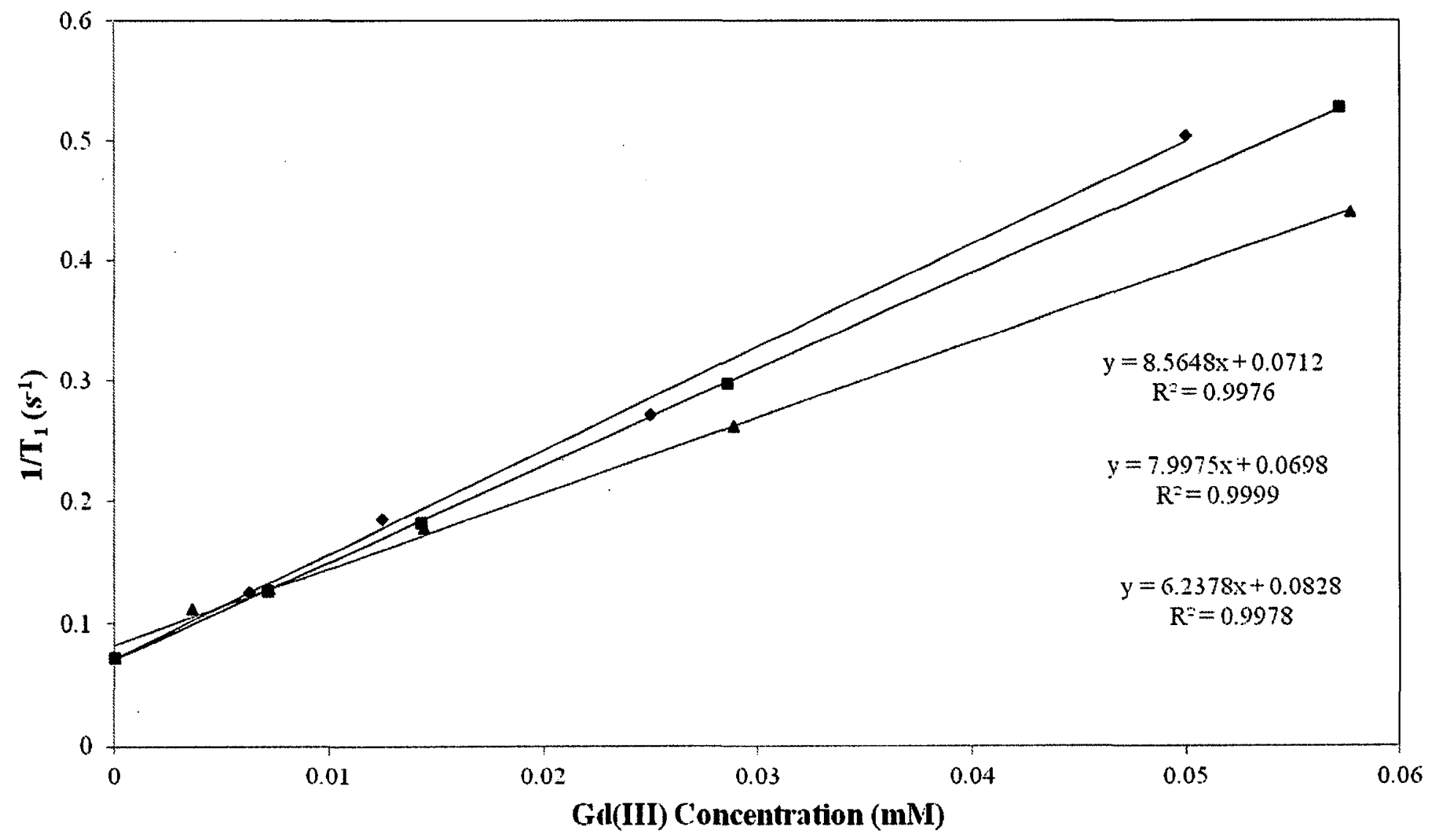

- 30T SC-Gd(III) - 30T SC-Gd(III)+Thrombin $\quad$-30T SC-Gd(III)+Pepsin

Figure A-14 Relaxivity plots for 30T SC-Gd(III) alone, with thrombin, and with pepsin at $9.4 \mathrm{~T}, \mathrm{pH} 7.4$ and $25^{\circ} \mathrm{C}$. 


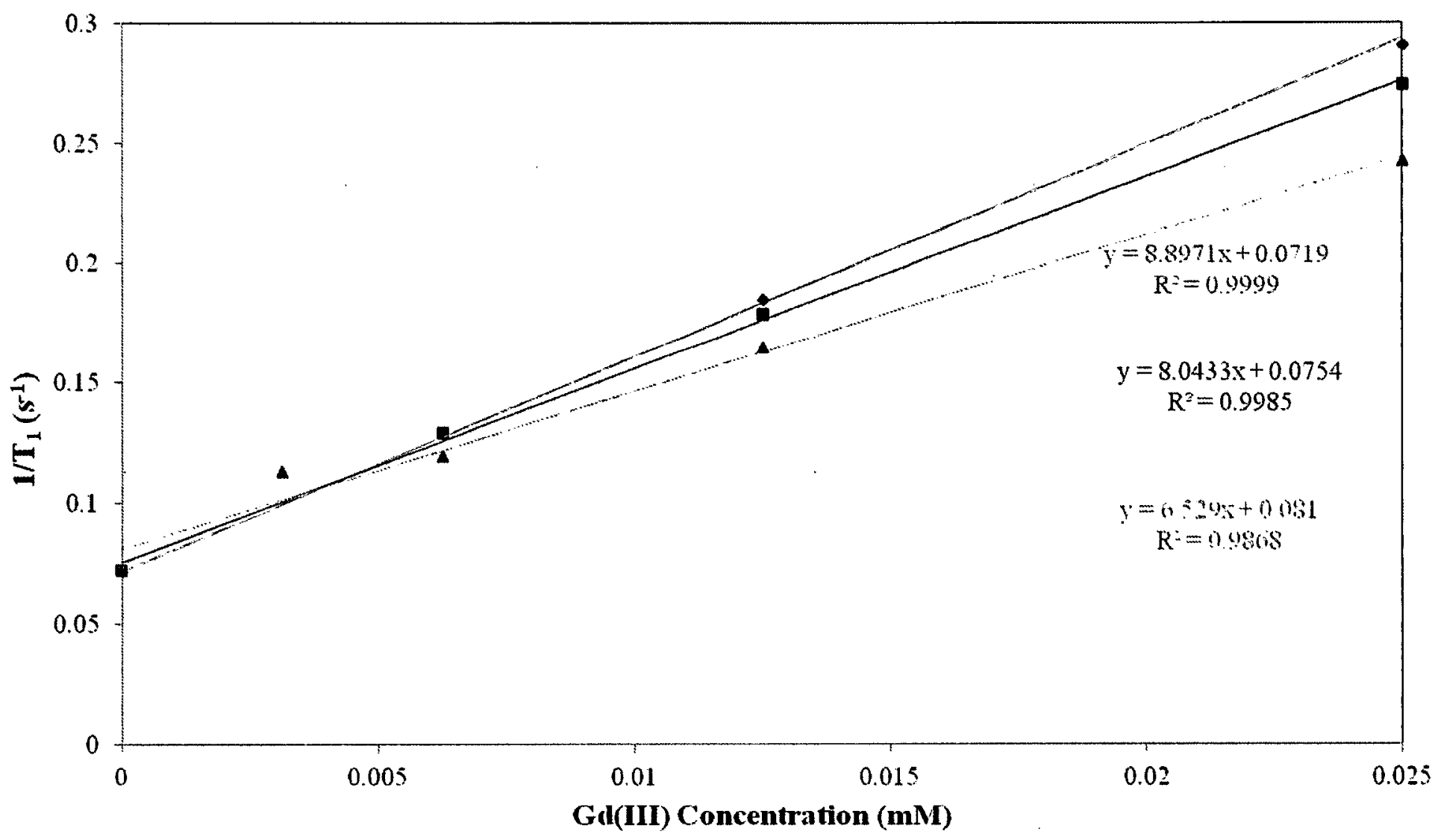

- 30T SC-Gd(III)+polyA - 30T SC-Gd(III)+polyA+Thrombin $\quad$ \30T SC-Gd(III)+polyA+Pepsin

Figure A-15 Relaxivity plots for 30T SC-Gd(III)+polyA alone, with thrombin, and with pepsin at 9.4 T, pH 7.4 and $25^{\circ} \mathrm{C}$. 


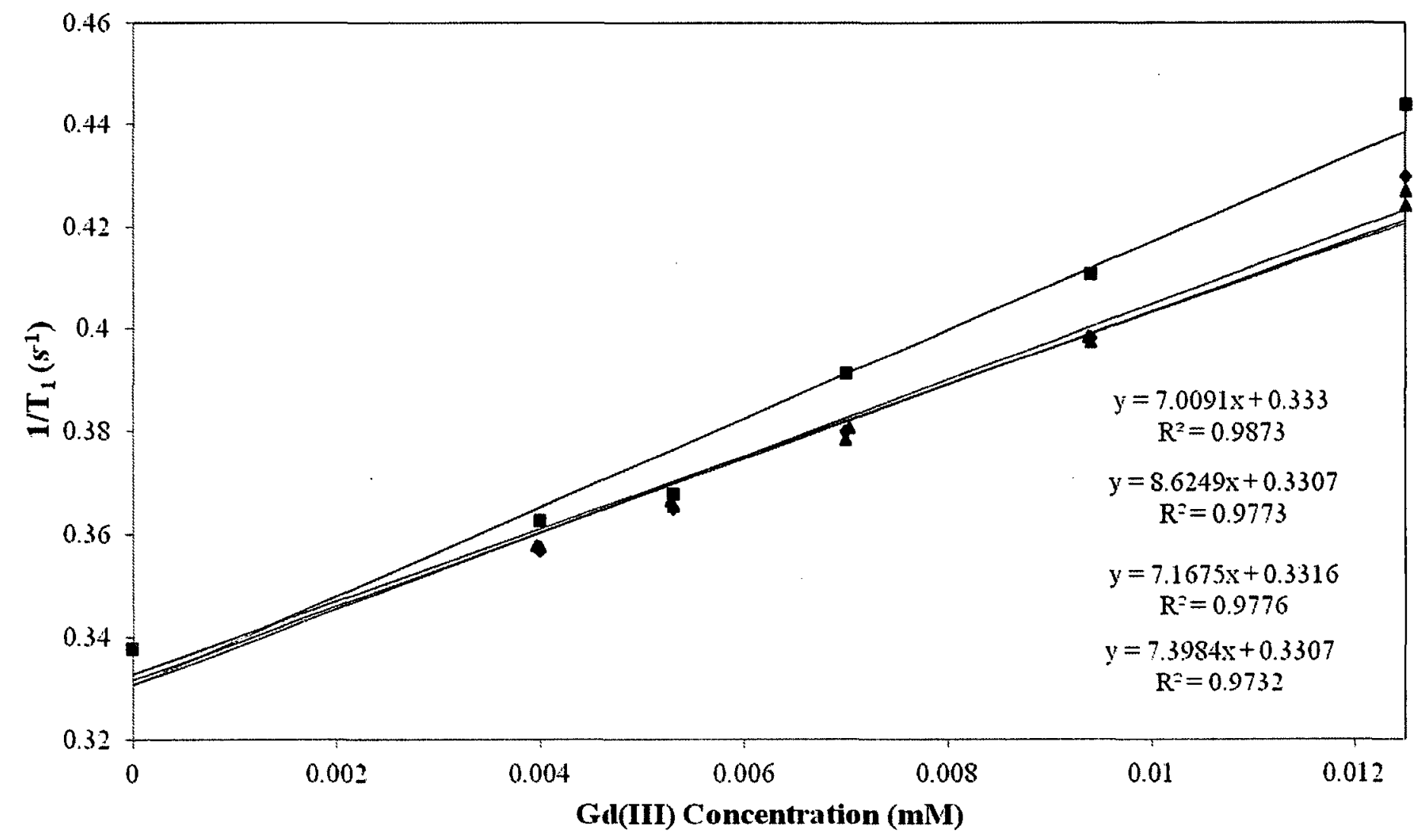

- SC-Gd(III) - SC-Gd(III)+Thrombin $\quad$ A SC-Gd(III)+Pepsin $\quad$ - SC-Gd(III)+BSA

Figure A-16 Relaxivity plots for SC-Gd(III) alone, with thrombin, with pepsin, and with BSA at $1.5 \mathrm{~T}, \mathrm{pH} 7.4$ and $25^{\circ} \mathrm{C}$. 


\section{Appendix B Supporting data for double-labelled DNA-DTPA conjugates}

Figure B-1 Melting curves for 15T DC and 30T DC in a 1:2 ratio with their respective polyA strands.

.286

Figure B-2 Relaxivity plots for DC-Gd(III) alone, with thrombin, and with pepsin at 9.4 T, pH 7.4 and $25^{\circ} \mathrm{C}$. The proteins were tested at both $2: 1$ and $1: 1$ ratios with the conjugate. .287

Figure B-3 Relaxivity plots for $15 \mathrm{~T}$ DC-Gd(III) alone, with thrombin, and with pepsin at $9.4 \mathrm{~T}, \mathrm{pH} 7.4$ and $25^{\circ} \mathrm{C}$. The proteins were tested at both $2: 1$ and $1: 1$ ratios with the conjugate. 288

Figure B-4 Relaxivity plots for $15 \mathrm{~T} \mathrm{DC-Gd(III)} \mathrm{hybridized} \mathrm{with} \mathrm{polyA} \mathrm{alone,} \mathrm{with}$ thrombin, and with pepsin at $9.4 \mathrm{~T}, \mathrm{pH} 7.4$ and $25^{\circ} \mathrm{C}$. The proteins were tested at both $2: 1$ and $1: 1$ ratios with the conjugate.

289

Figure B-5 Relaxivity plots for 30T DC-Gd(III) alone, with thrombin, and with pepsin at $9.4 \mathrm{~T}, \mathrm{pH} 7.4$ and $25^{\circ} \mathrm{C}$. The proteins were tested at both $2: 1$ and $1: 1$ ratios with the conjugate. 290

Figure B-6 Relaxivity plots for $30 \mathrm{~T}$ DC-Gd(III) hybridized with polyA alone, with thrombin, and with pepsin at $9.4 \mathrm{~T}, \mathrm{pH} 7.4$ and $25^{\circ} \mathrm{C}$. The proteins were tested at both $2: 1$ and $1: 1$ ratios with the conjugate

Figure B-7 Relaxivity plots for $15 \mathrm{~T} \mathrm{DC-Gd(III)} \mathrm{alone,} \mathrm{with} \mathrm{thrombin,} \mathrm{and} \mathrm{with} \mathrm{pepsin}$ at $1.5 \mathrm{~T}, \mathrm{pH} 7.4$ and $25^{\circ} \mathrm{C}$. The protein and conjugate were mixed in equimolar amounts, where applicable.

Figure B-8 Relaxivity plots for DC-Gd(III) alone, with thrombin, and with pepsin at $1.5 \mathrm{~T}, \mathrm{pH} 7.4$ and $25^{\circ} \mathrm{C}$. The protein and conjugate were mixed in equimolar amounts, where applicable. 293 


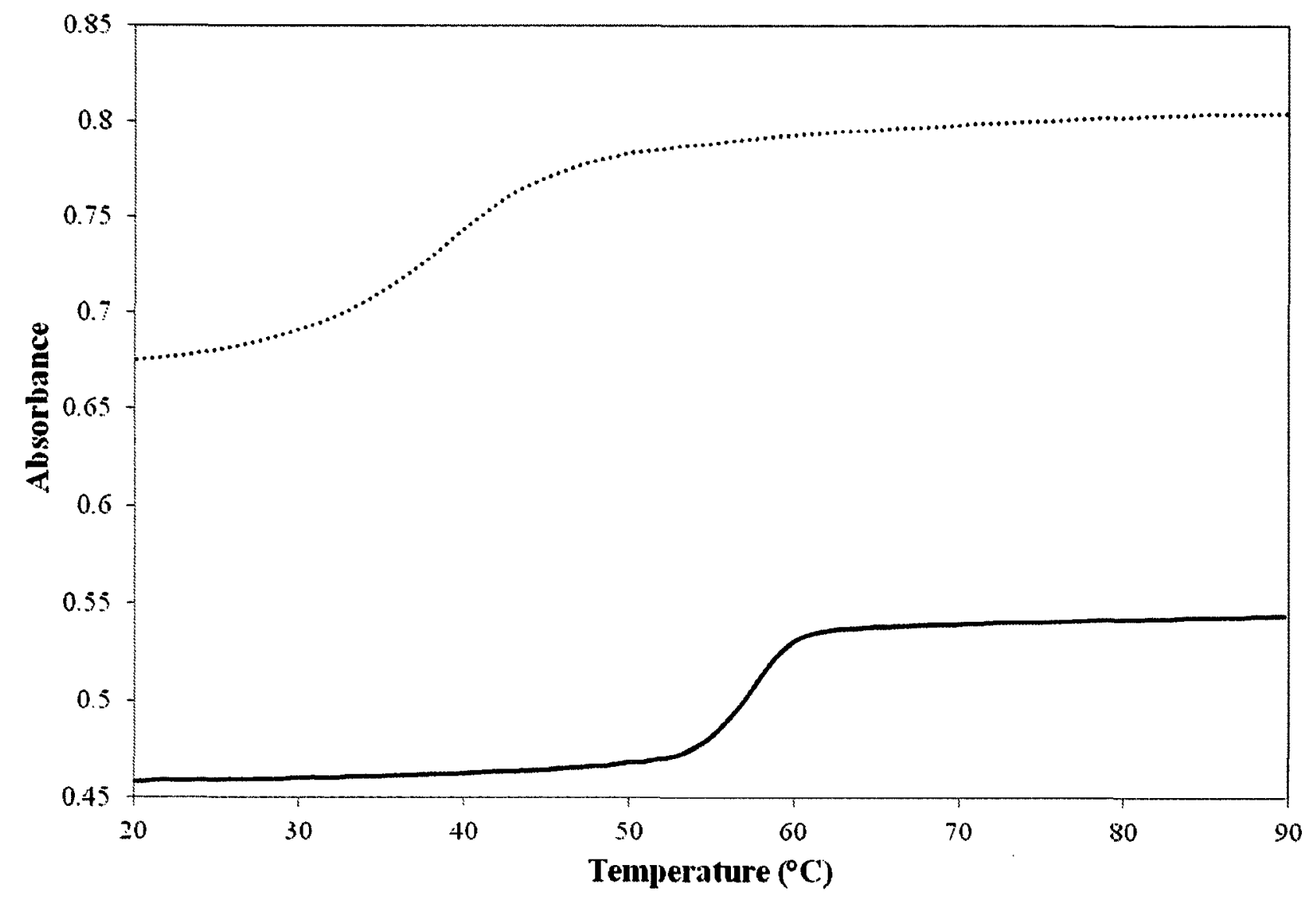

$15 \mathrm{~T} \mathrm{DC}+15$ poly $-30 \mathrm{~T} \mathrm{DC}+30$ polyA

Figure B-1 Melting curves for 15T DC and 30T DC in a 1:2 ratio with their respective polyA strands. 


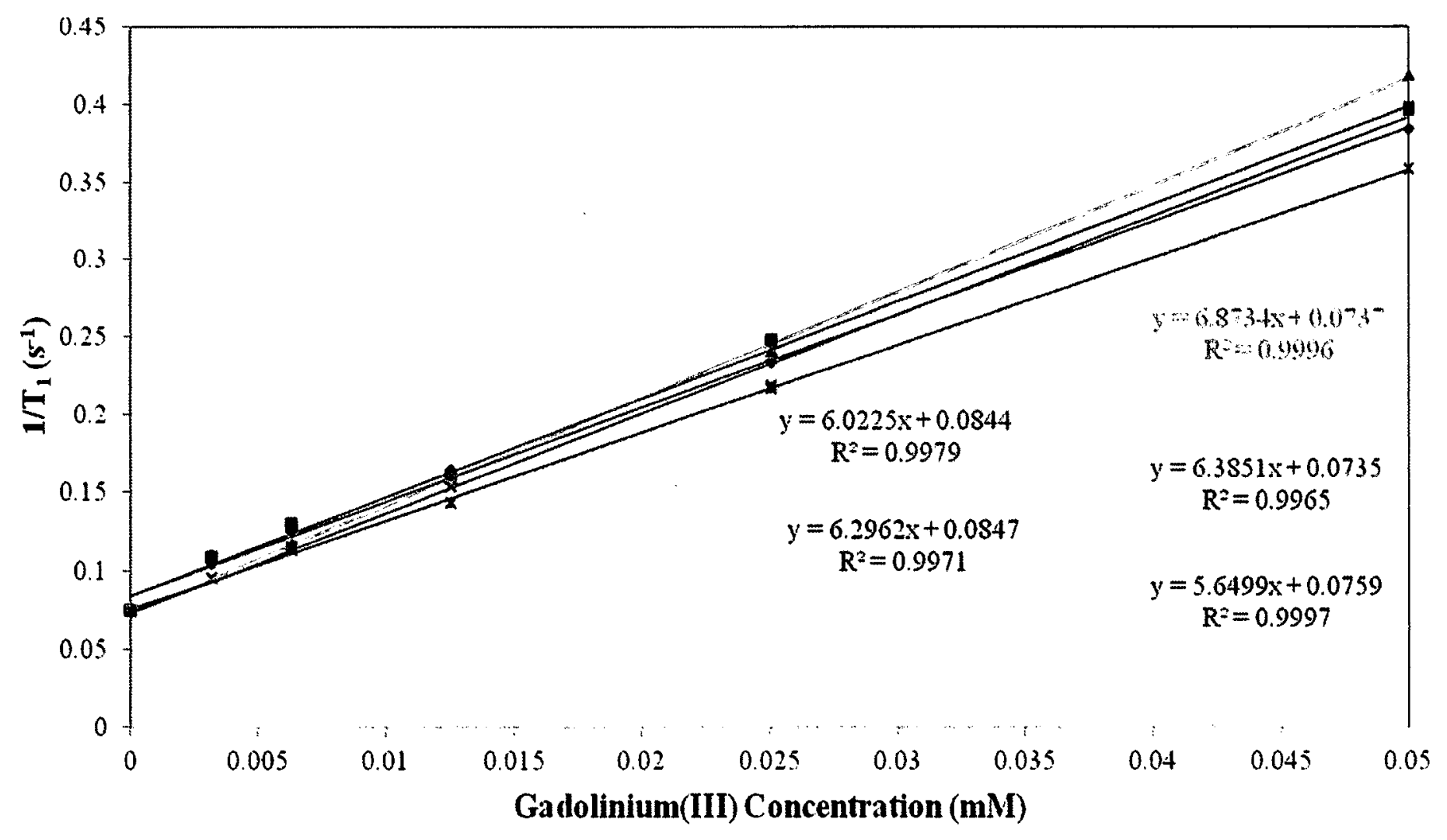

- DC-Gd(III)

- DC-Gd(III)+Thrombin(2:1) $\Delta$ DC-Gd(III)+Pepsin(2:1)

$\times$ DC-Gd(III)+Thrombin(1:1) * DC-Gd(III)+Pepsin(1:1)

Figure B-2 Relaxivity plots for DC-Gd(III) alone, with thrombin, and with pepsin at $9.4 \mathrm{~T}, \mathrm{pH} 7.4$ and $25^{\circ} \mathrm{C}$. The proteins were tested at both $2: 1$ and 1:1 ratios with the conjugate. 


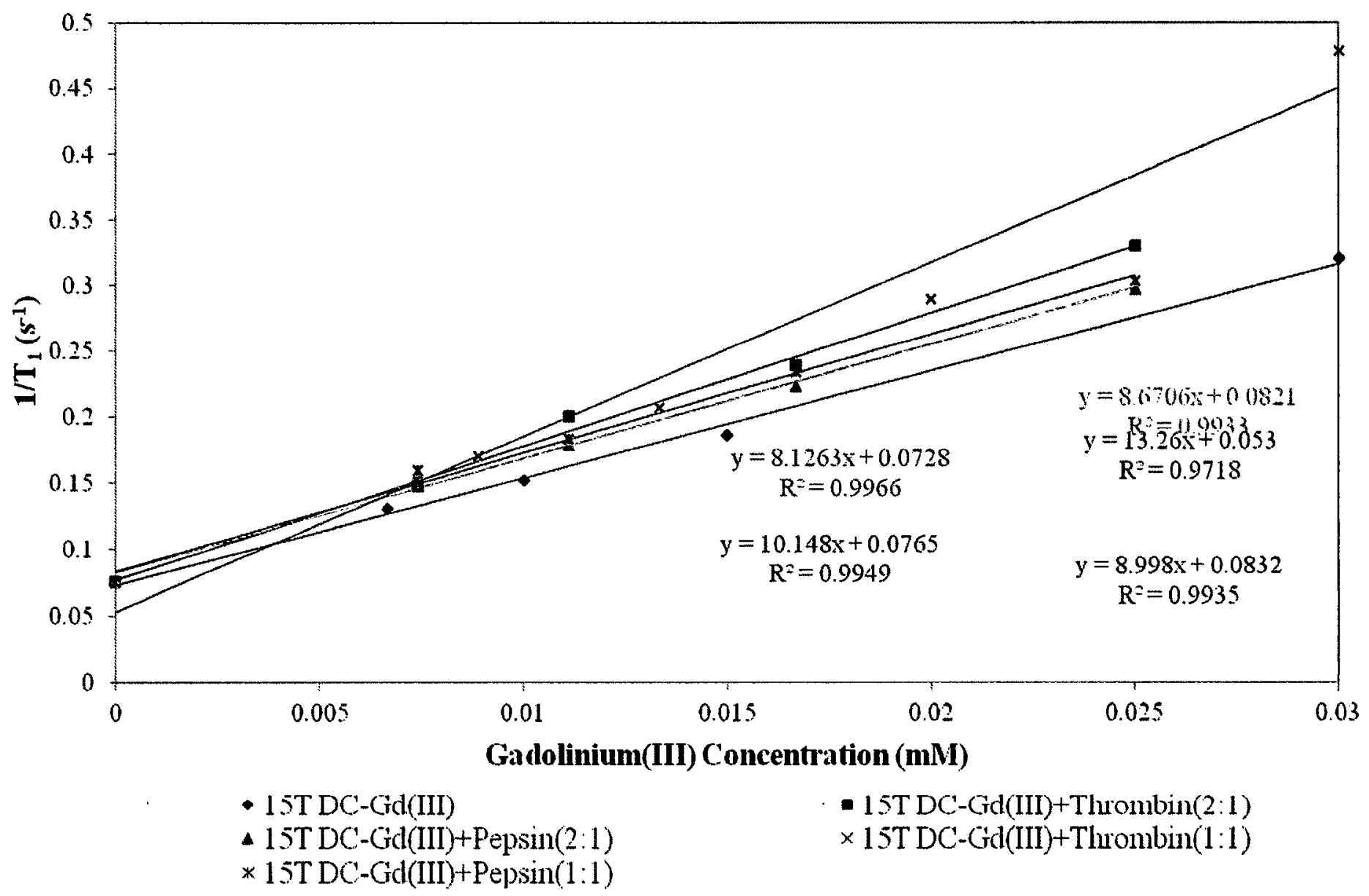

Figure B-3 Relaxivity plots for 15T DC-Gd(III) alone, with thrombin, and with pepsin at $9.4 \mathrm{~T}, \mathrm{pH} 7.4$ and $25^{\circ} \mathrm{C}$. The proteins were tested at both $2: 1$ and 1:1 ratios with the conjugate. 


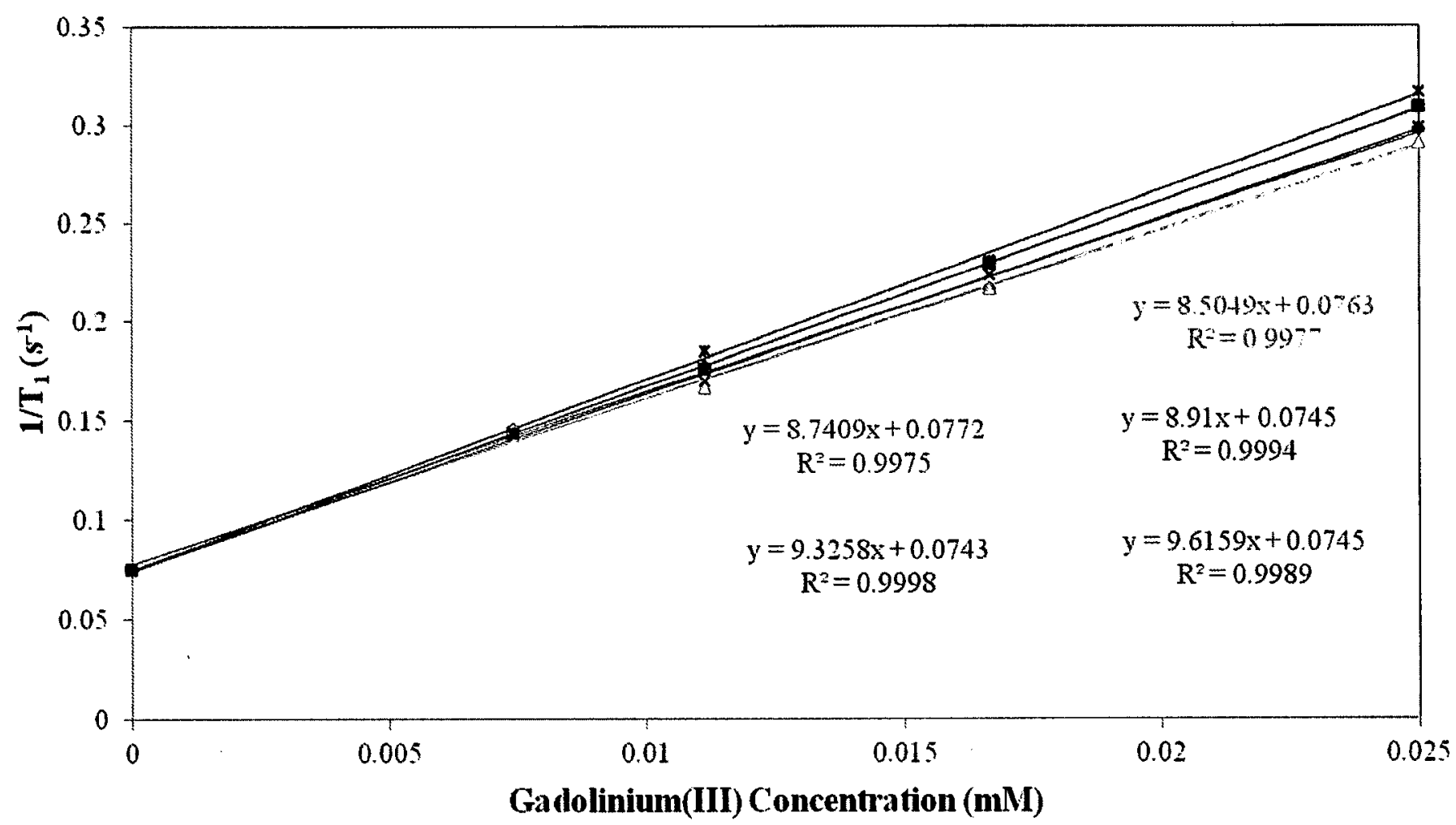

-15T DC-Gd(III)+polyA

$\triangle 1.5 T$ DC-Gd(III)+polyA+Pepsin (2:1)

* 1.5T DC-Gd(III)+polyA+Pepsin(1:1)
- 15 T DC-Gd(III)+polyA+Thrombin(2:1)

$\times 15$ T DC-Gd(III)+polyA+Thrombin(1:1)

Figure B-4 Relaxivity plots for $15 \mathrm{~T} \mathrm{DC-Gd(III)} \mathrm{hybridized} \mathrm{with} \mathrm{polyA} \mathrm{alone,} \mathrm{with} \mathrm{thrombin,} \mathrm{and} \mathrm{with} \mathrm{pepsin} \mathrm{at} 9.4 \mathrm{~T}$, pH 7.4 and $25^{\circ} \mathrm{C}$. The proteins were tested at both $2: 1$ and 1:1 ratios with the conjugate. 


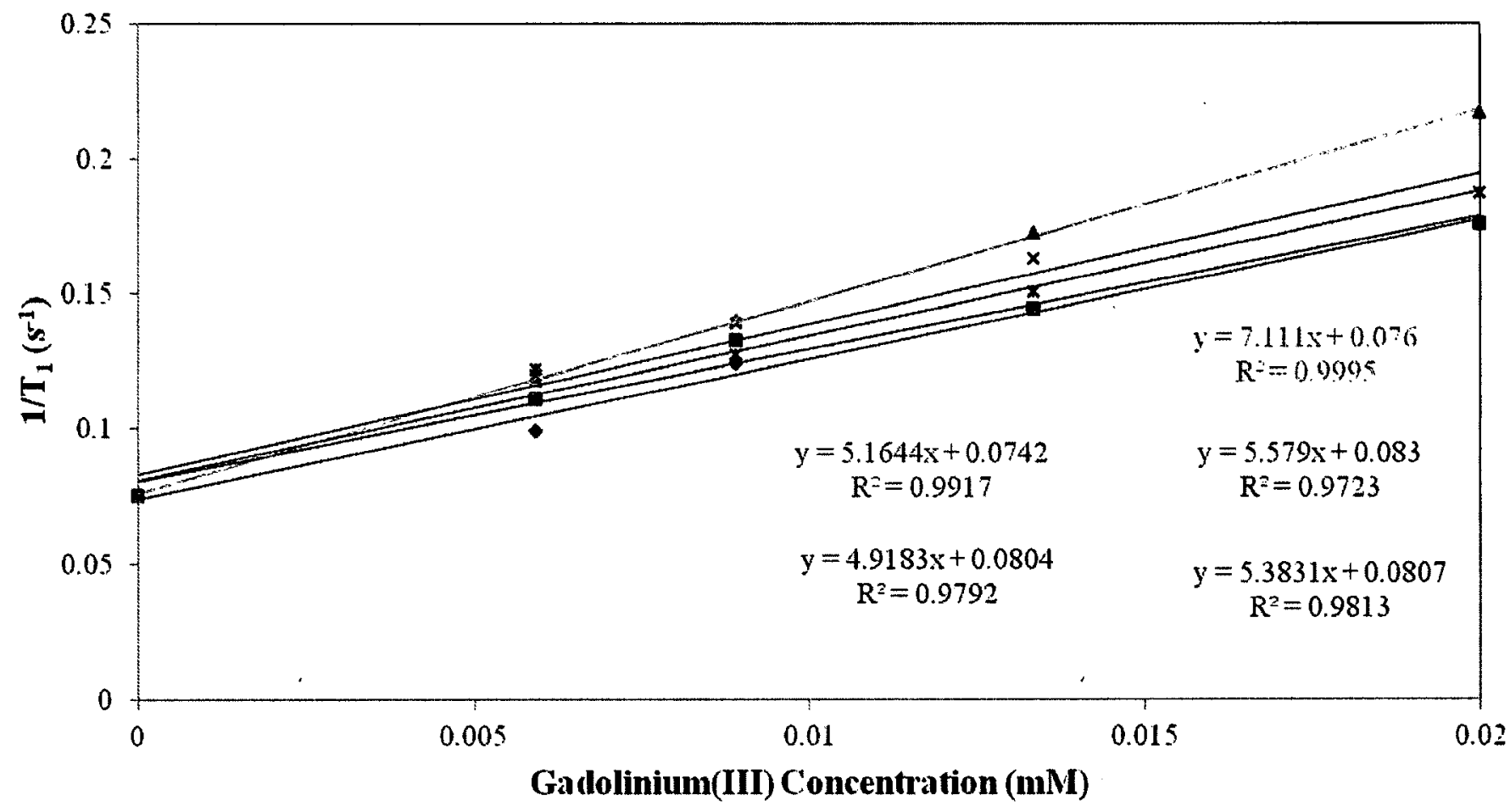

- 30T DC-Gd(III)

- $30 \mathrm{~T} \mathrm{DC-Gd(III)+Thrombin(2:1)}$

- 30T DC-Gd(III)+Pepsin(2:1) × 30T DC-Gd(III)+Thrombin(1:1)

* 30T DC-Gd(III)+Pepsin(1:1)

Figure B-5 Relaxivity plots for 30T DC-Gd(III) alone, with thrombin, and with pepsin at $9.4 \mathrm{~T}, \mathrm{pH} 7.4$ and $25^{\circ} \mathrm{C}$. The proteins were tested at both $2: 1$ and $1: 1$ ratios with the conjugate. 


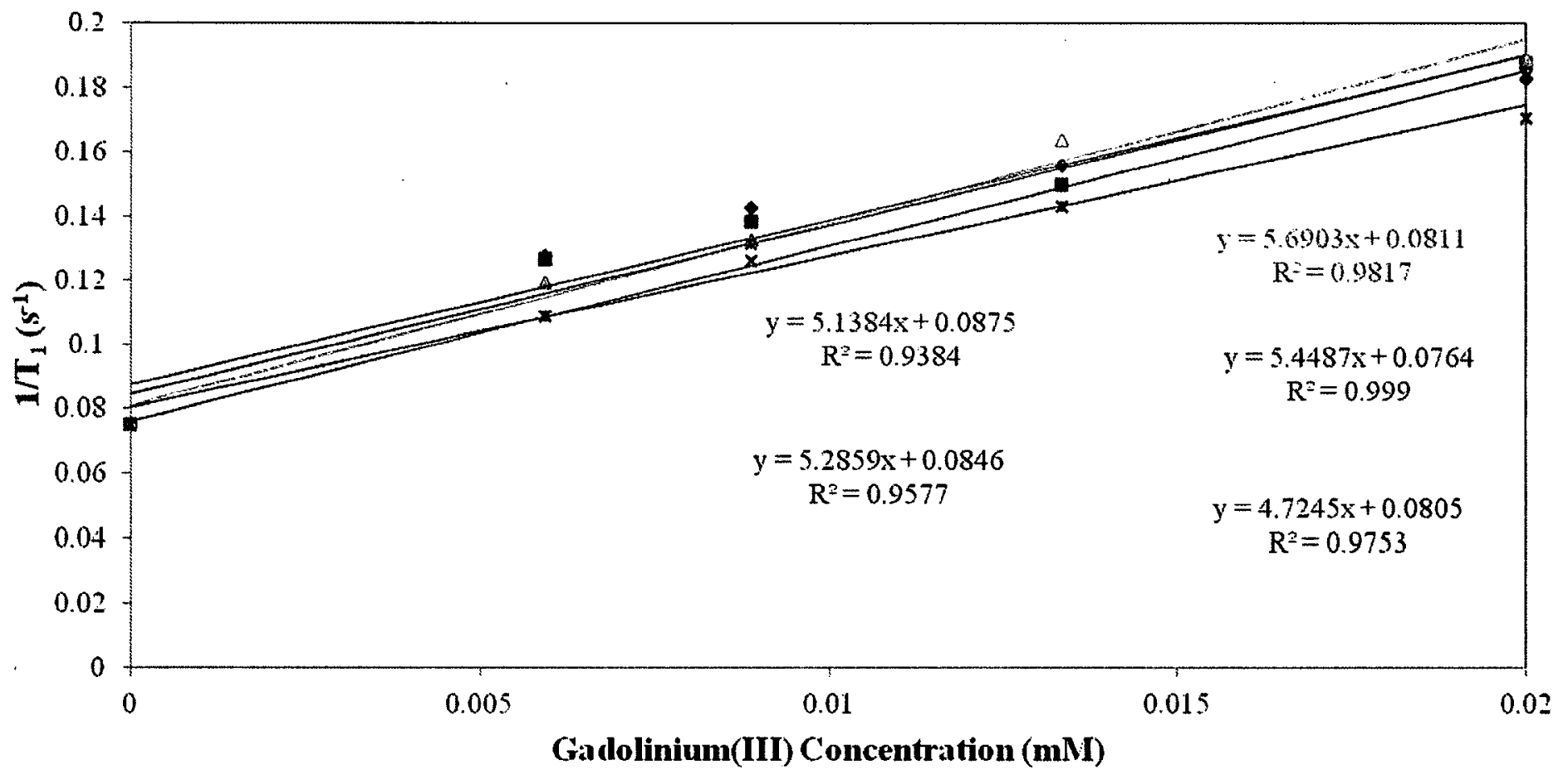

-30T DC-Gd(III)+polyA

$\triangle$ 30T DC-Gd(III)+polyA+Pepsin(2:1)

* 30T DC-Gd(III)+polyA+Pepsin( $1: 1)$
- 30T DC-Gd(III)+polyA+Thrombin(2:1)

$\times 30$ T DC-Gd(III)+polyA+Tluombin $(1: 1)$

Figure B-6 Relaxivity plots for 30T DC-Gd(III) hybridized with polyA alone, with thrombin, and with pepsin at 9.4 T, pH 7.4 and $25^{\circ} \mathrm{C}$. The proteins were tested at both $2: 1$ and $1: 1$ ratios with the conjugate. 


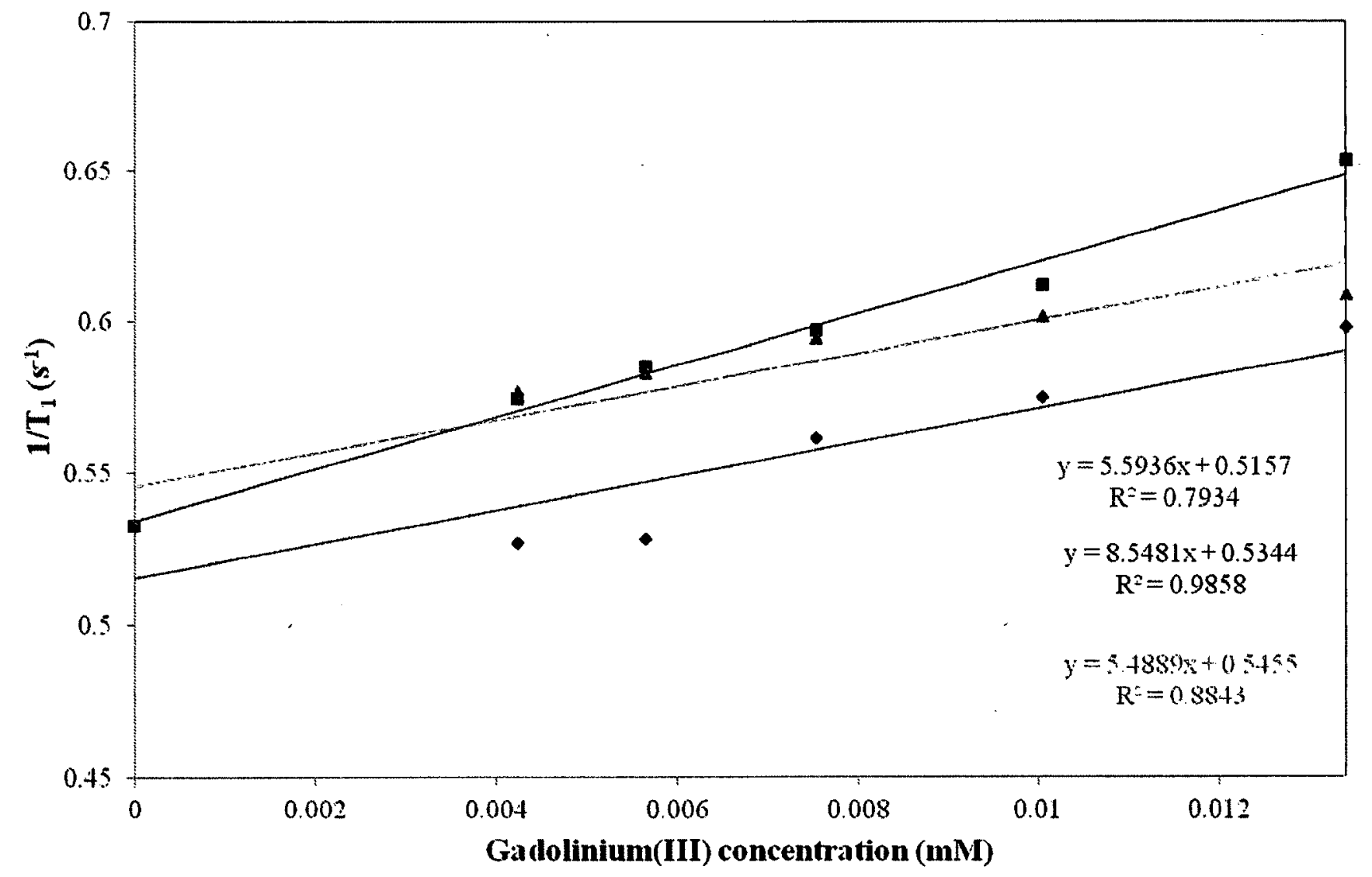

- 15T DC-Gd(III) - $15 \mathrm{~T} \mathrm{DC-Gd(III)+thrombin} \quad$. 15T DC-Gd(III)+pepsin

Figure B-7 Relaxivity plots for $15 \mathrm{~T}$ DC-Gd(III) alone, with thrombin, and with pepsin at $1.5 \mathrm{~T}, \mathrm{pH} 7.4$ and $25^{\circ} \mathrm{C}$. The protein and conjugate were mixed in equimolar amounts, where applicable. 


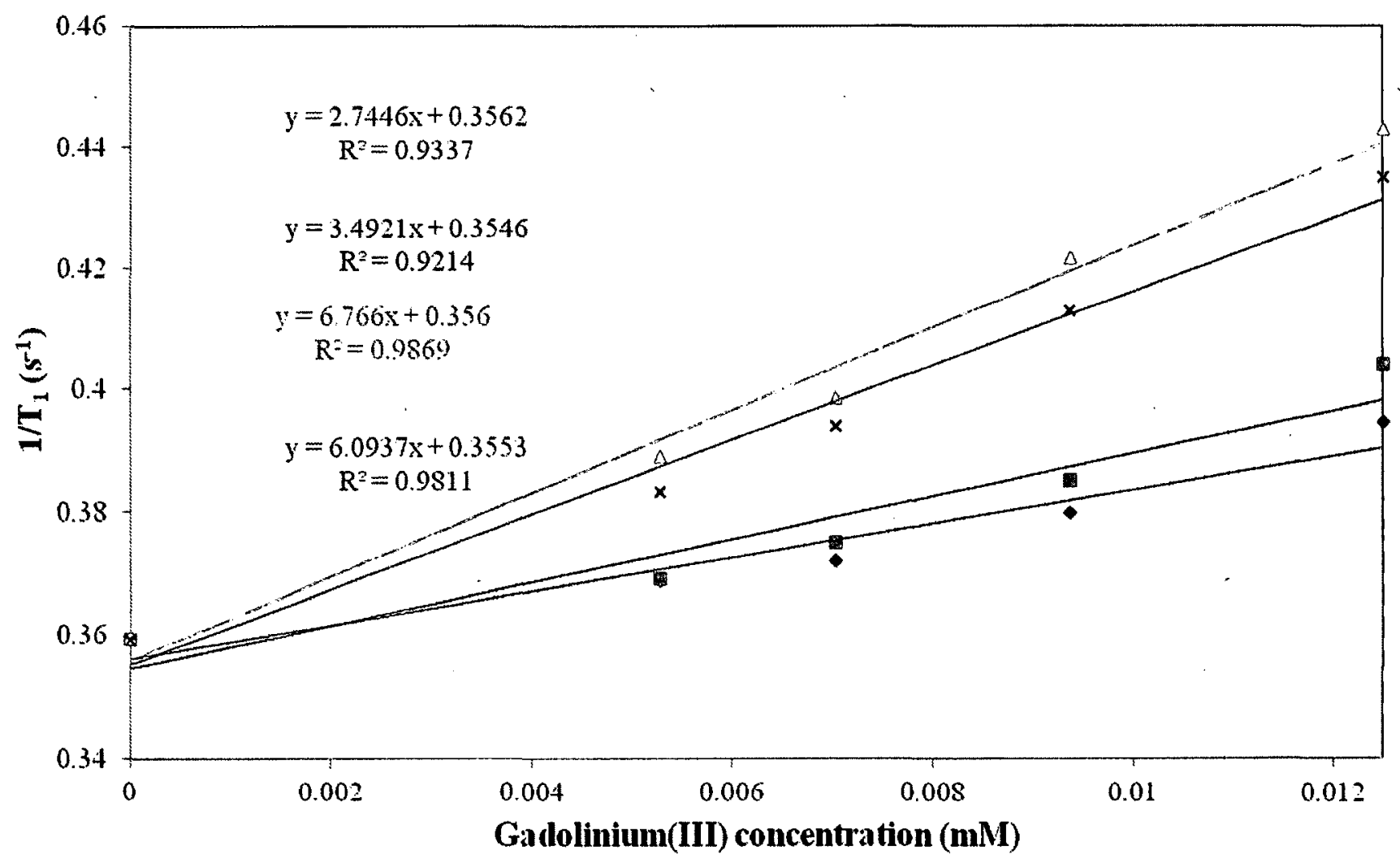

- Magnevist $\quad$ DC-Gd(III) $\quad \Delta \mathrm{DC}-\mathrm{Gd}(\mathrm{III})+$ thrombin $\quad \times \mathrm{DC}-\mathrm{Gd}(\mathrm{III})+$ pepsin

Figure B-8 Relaxivity plots for DC-Gd(III) alone, with thrombin, and with pepsin at $1.5 \mathrm{~T}, \mathrm{pH} 7.4$ and $25^{\circ} \mathrm{C}$. The protein and conjugate were mixed in equimolar amounts, where applicable. 


\section{Appendix C Supporting data for work with Pt(II) complexes}

Figure C-1 ${ }^{1} \mathrm{H}$ NMR (300 MHz) spectrum of $\mu$-dichloro(2-phenylpyridineplatinum(II)) dimers in DMSO-d6.... 296

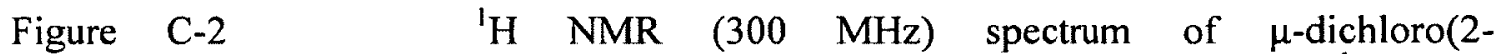
phenylquinolineplatinum(II)) dimers in $\mathrm{CDCl}_{3}$. The inset is a portion of the ${ }^{1} \mathrm{H} \mathrm{NMR}$ spectrum for 2-phenylquinoline in $\mathrm{CDCl}_{3}$ adapted from the Sigma-Aldrich website. $^{201}$ 297

Figure C-3 ' $\mathrm{H}$ NMR (300 MHz) spectrum of 8,10-dioxoundecanoic acid (acac-COOH) in $\mathrm{CDCl}_{3}$ and assigned peaks for the enol $(85 \%)$ and keto $(15 \%)$ forms. ${ }^{188}$ The peak at $\delta$ $5.5 \mathrm{ppm}$ is characteristic of acac-COOH.......................................298

Figure C-4 $\quad{ }^{1} \mathrm{H}$ NMR spectrum of (ppy)Pt(acac-COOH) in acetone-d6..............299

Figure C-5 ${ }^{1} \mathrm{H}$ NMR spectrum of partially purified (pq) $\mathrm{Pt}(\mathrm{acac}-\mathrm{COOH})$ in acetoned6.

Figure C-6 $\quad{ }^{1} \mathrm{H}$ NMR spectrum of partially purified $(\mathrm{pq}) \mathrm{Pt}(\mathrm{acac}-\mathrm{COOH})$ in $\mathrm{CDCl}_{3} \ldots 301$

Figure C-7 Expanded region of ${ }^{1} \mathrm{H}$ NMR spectrum of partially purified (pq)Pt(acac-

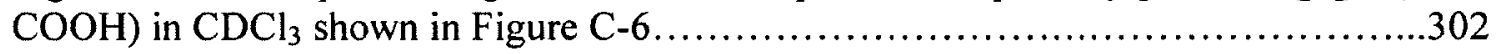

Figure C-8 Emission spectra obtained for (ppy)Pt(acac-COOH) dissolved in acetone with different excitations for each respective solution, as indicated in the legend........303

Figure C-9 Absorbance spectrum for a $0.022 \mathrm{M}$ solution of (ppy)Pt(acac-COOH) in acetone .304

Figure C-10 Absorbance spectrum for a $0.00015 \mathrm{M}$ solution of (ppy)Pt(acac-COOH) in acetone. .305

Figure C-11 Absorbance spectrum for (2,4-dfppy) $\mathrm{Pt}(\mathrm{acac}-\mathrm{COOH})$ in methanol at a

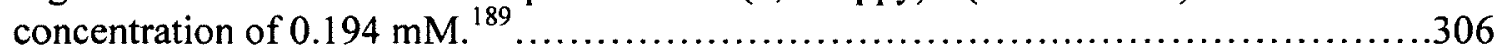

Figure C-12 ${ }^{1} \mathrm{H}$ NMR spectrum of (ppy)Pt(acac-COOH) in acetone-d6 after an

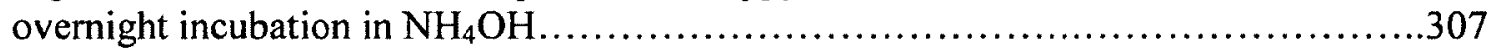

Figure C-13 Emission spectra with excitation at $320 \mathrm{~nm}$ for portions of DNA collected using RP-HPLC following reaction of (ppy)Pt(acac-COOH) with 5'-amino-modified TBA .308

Figure C-14 MALDI MS spectrum of a 2 nmol portion of the DNA isolated following a solution-phase reaction for conjugation of (ppy) $\mathrm{Pt}(\mathrm{acac}-\mathrm{COOH})$ to the 5 '-terminus of a random sequence DNA strand. This is the same sample as that used to obtain the gel and

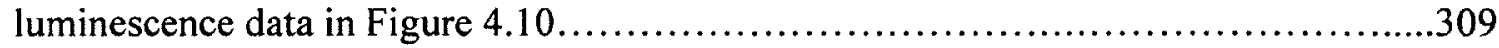


Figure C-15 Emission spectra for peaks from RP-HPLC purification of previously PAGE-purified Pt(II) complex-DNA .............................................. 310

Figure C-16 RP-HPLC chromatogram (absorbance $260 \mathrm{~nm}$ ) for $3^{\prime}-\mathrm{Pt}(\mathrm{II})$ complexDNA ............................................................................ 311

Figure C-17 Emission spectrum for sample isolated from the peak at about 8 minutes

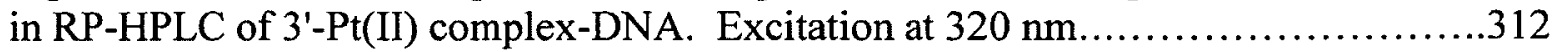




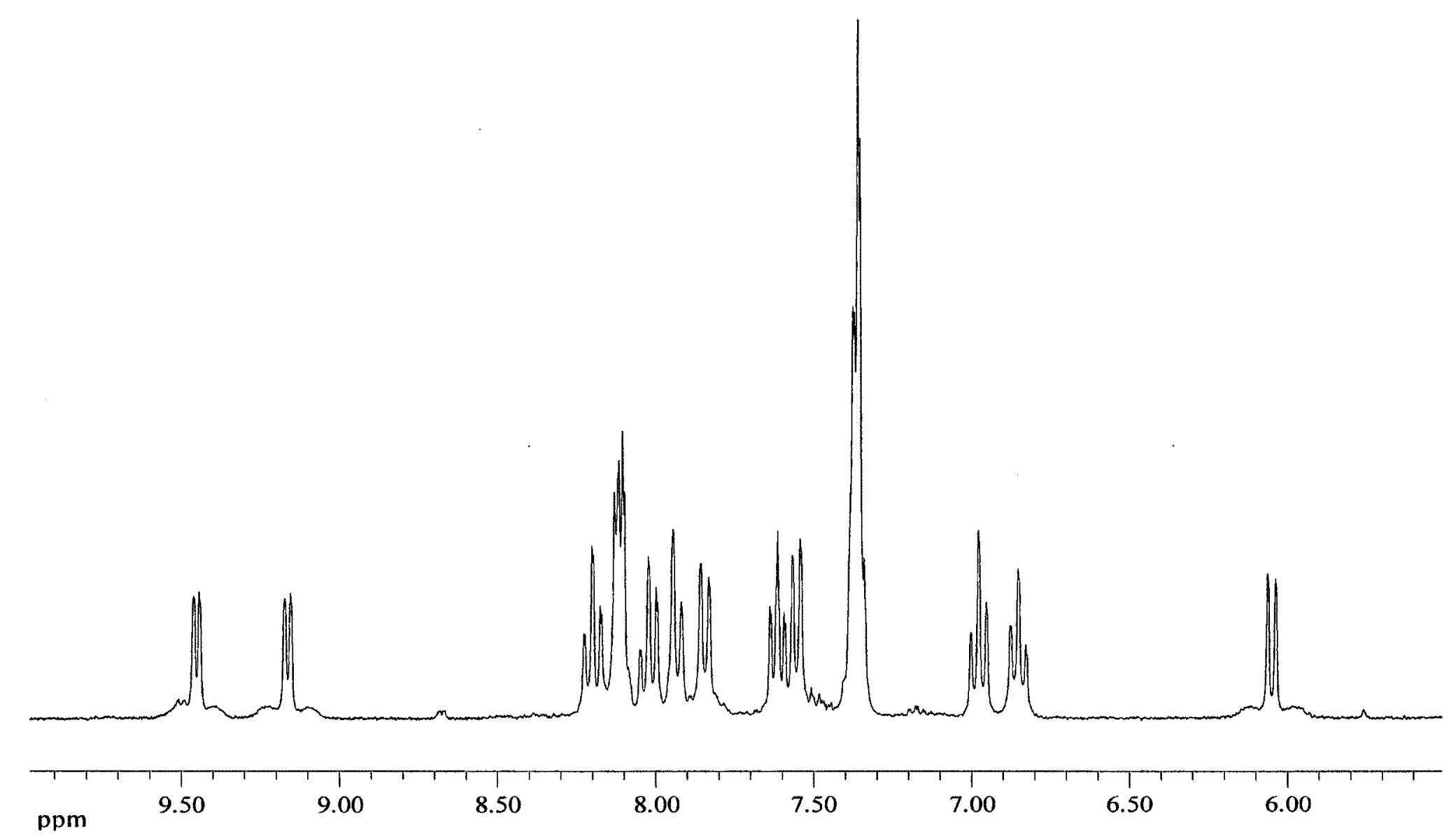

Figure C-1 'H NMR (300 MHz) spectrum of $\mu$-dichloro(2-phenylpyridineplatinum(II)) dimers in DMSO-d6. 

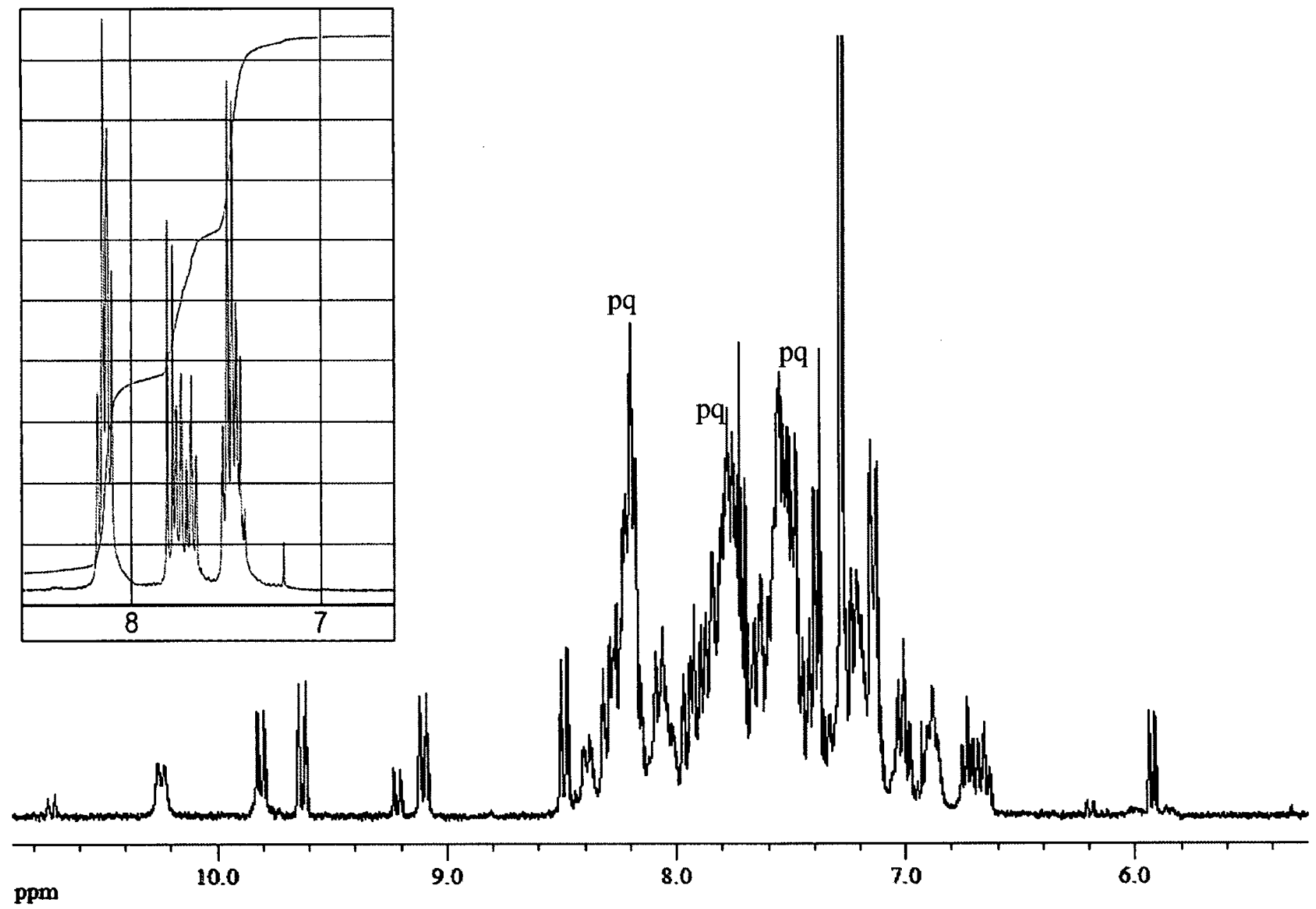

Figure C-2 ${ }^{1} \mathrm{H}$ NMR (300 MHz) spectrum of $\mu$-dichloro(2-phenylquinolineplatinum(II)) dimers in $\mathrm{CDCl}_{3}$. The inset is a portion of the ${ }^{1} \mathrm{H}$ NMR spectrum for 2-phenylquinoline in $\mathrm{CDCl}_{3}$ adapted from the Sigma-Aldrich website. ${ }^{201}$ 

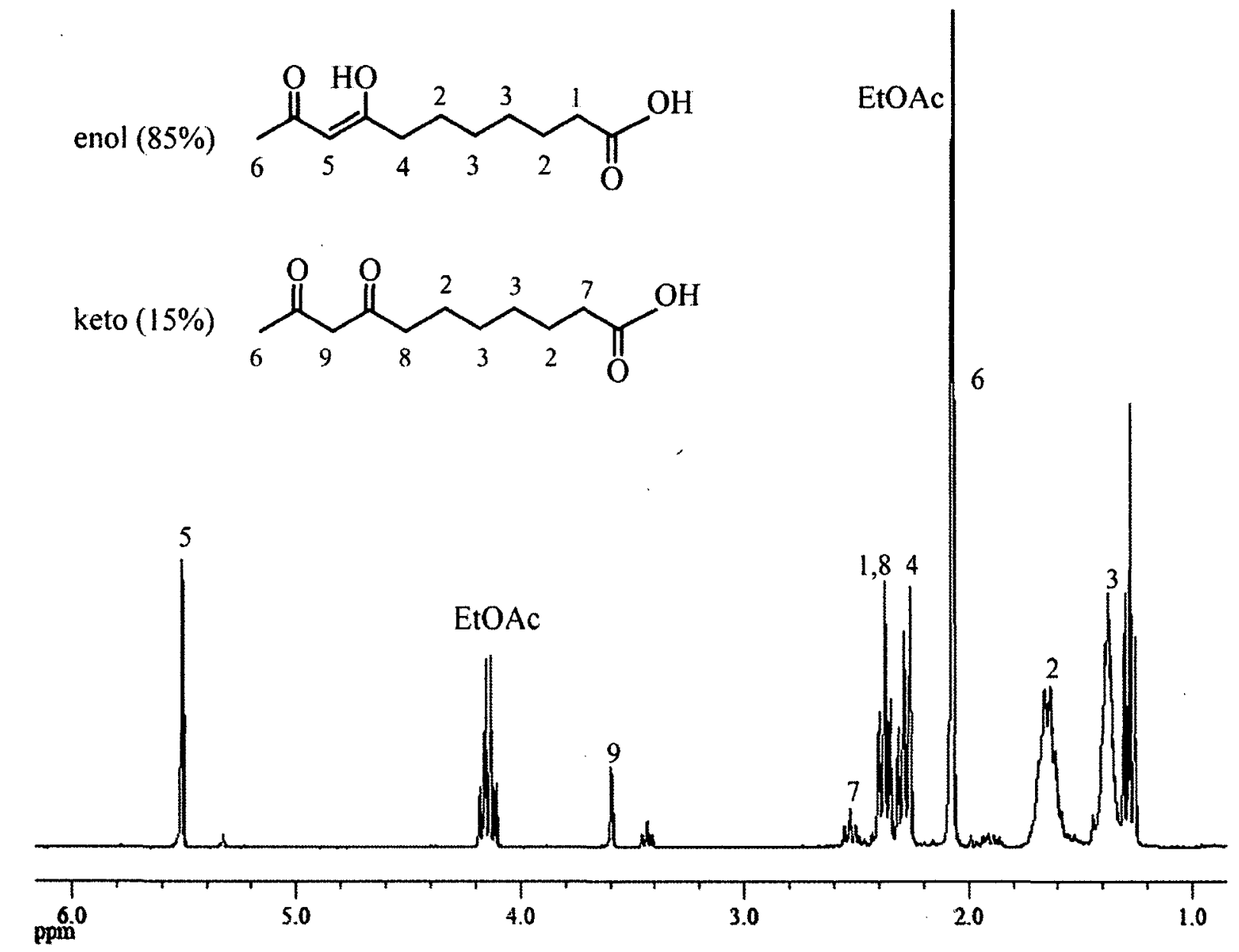

Figure C-3 ${ }^{1} \mathrm{H}$ NMR (300 MHz) spectrum of 8,10-dioxoundecanoic acid (acac-COOH) in $\mathrm{CDCl}_{3}$ and assigned peaks for the enol (85\%) and keto (15\%) forms. ${ }^{188}$ The peak at $\delta 5.5 \mathrm{ppm}$ is characteristic of acac-COOH. 
<smiles>C#CC#CC</smiles> 


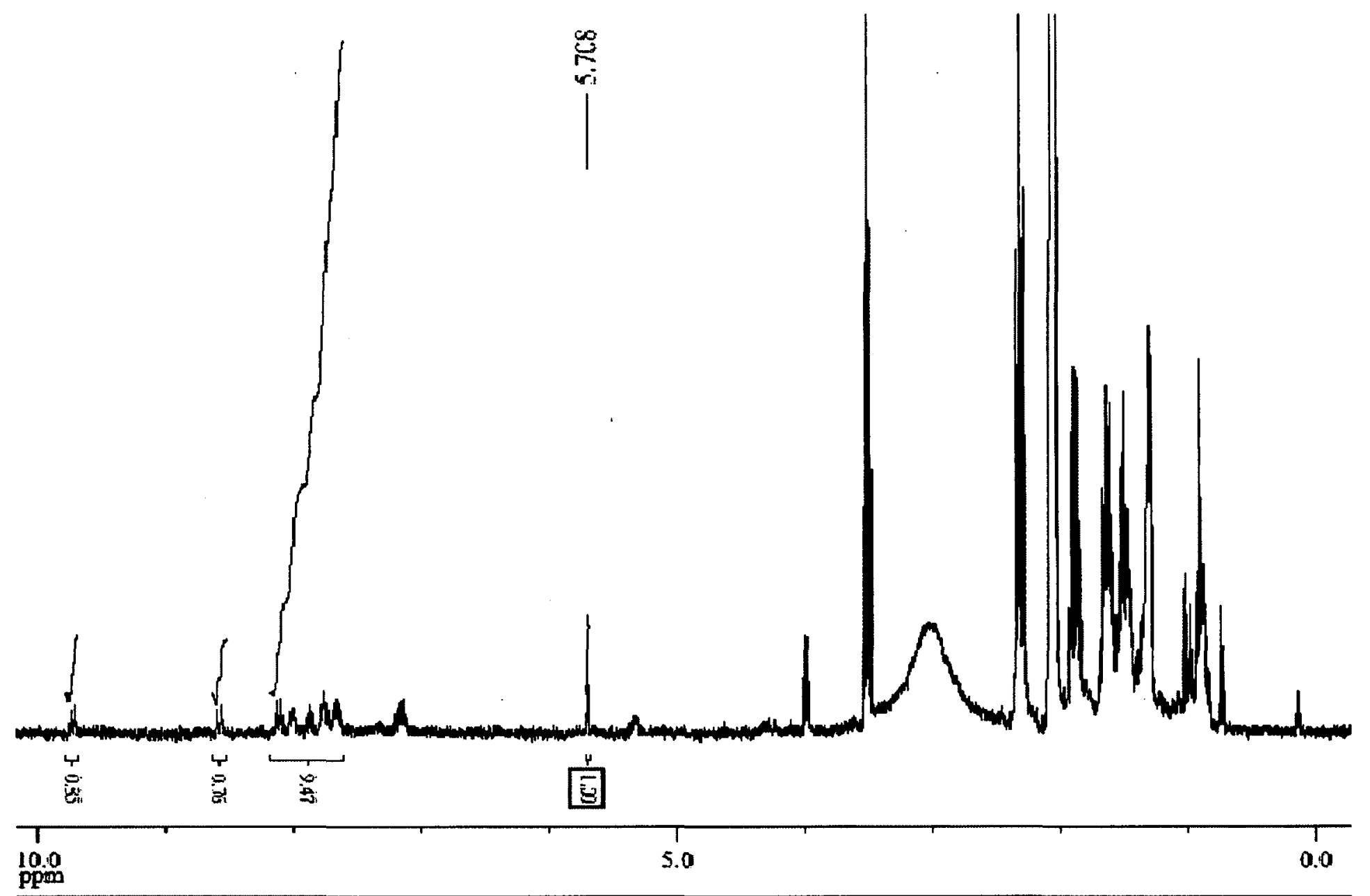

Figure C-5 'H NMR spectrum of partially purified (pq)Pt(acac-COOH) in acetone-d6. 


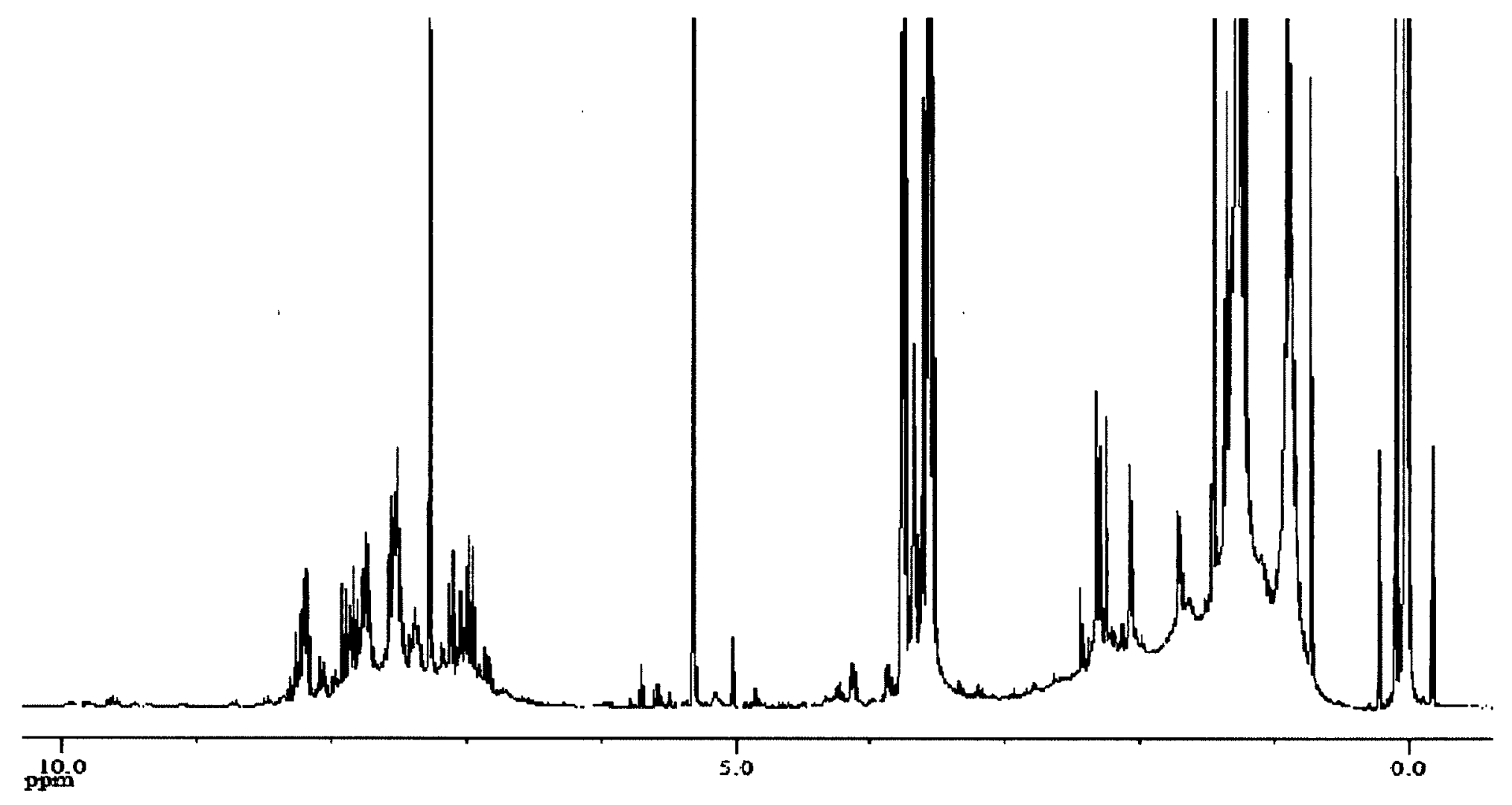

Figure C-6 'H NMR spectrum of partially purified (pq)Pt(acac-COOH) in $\mathrm{CDCl}_{3}$. 


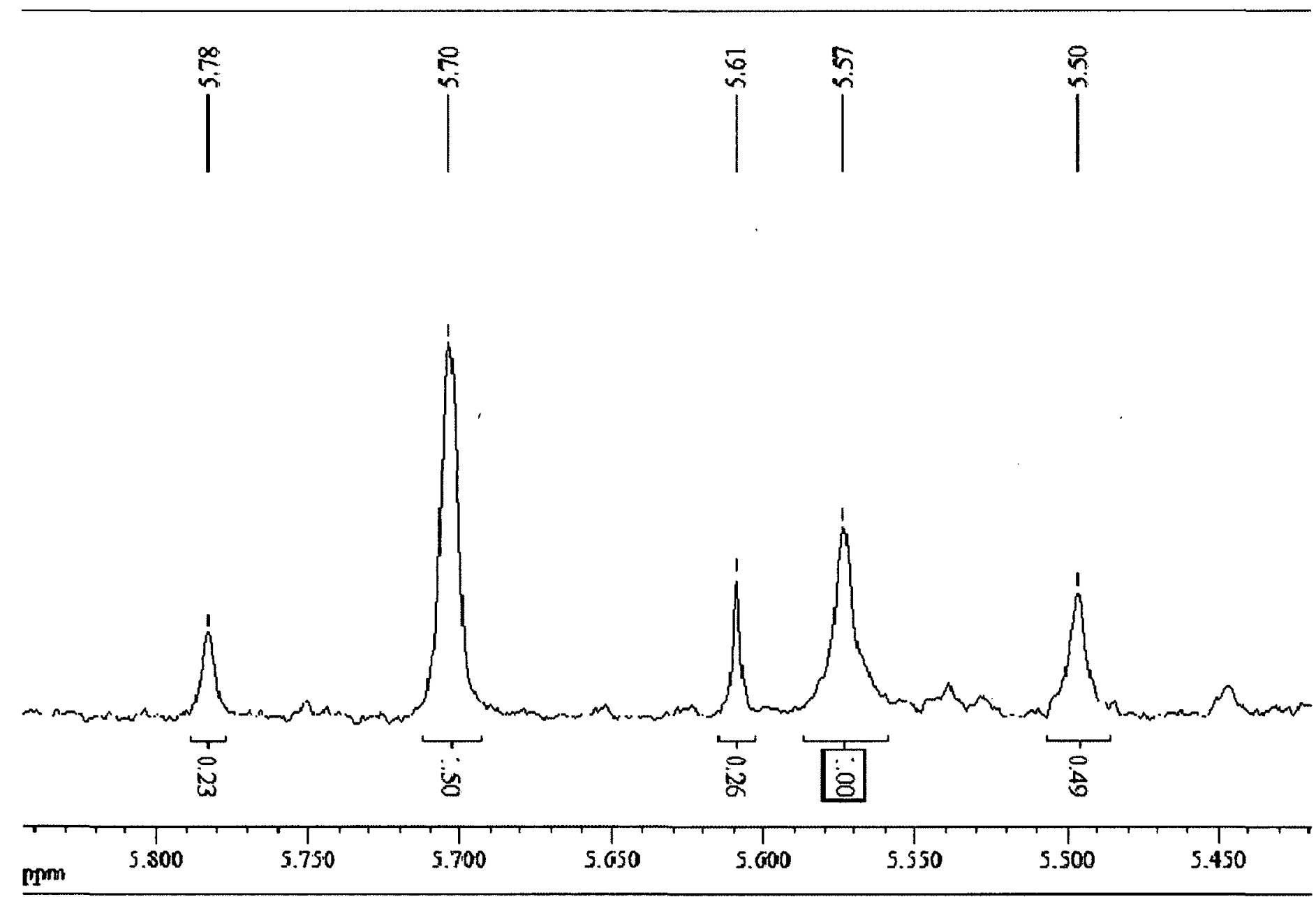

Figure C-7 Expanded region of ${ }^{1} \mathrm{H}$ NMR spectrum of partially purified (pq)Pt(acac-COOH) in $\mathrm{CDCl}_{3}$ shown in Figure C-6. 


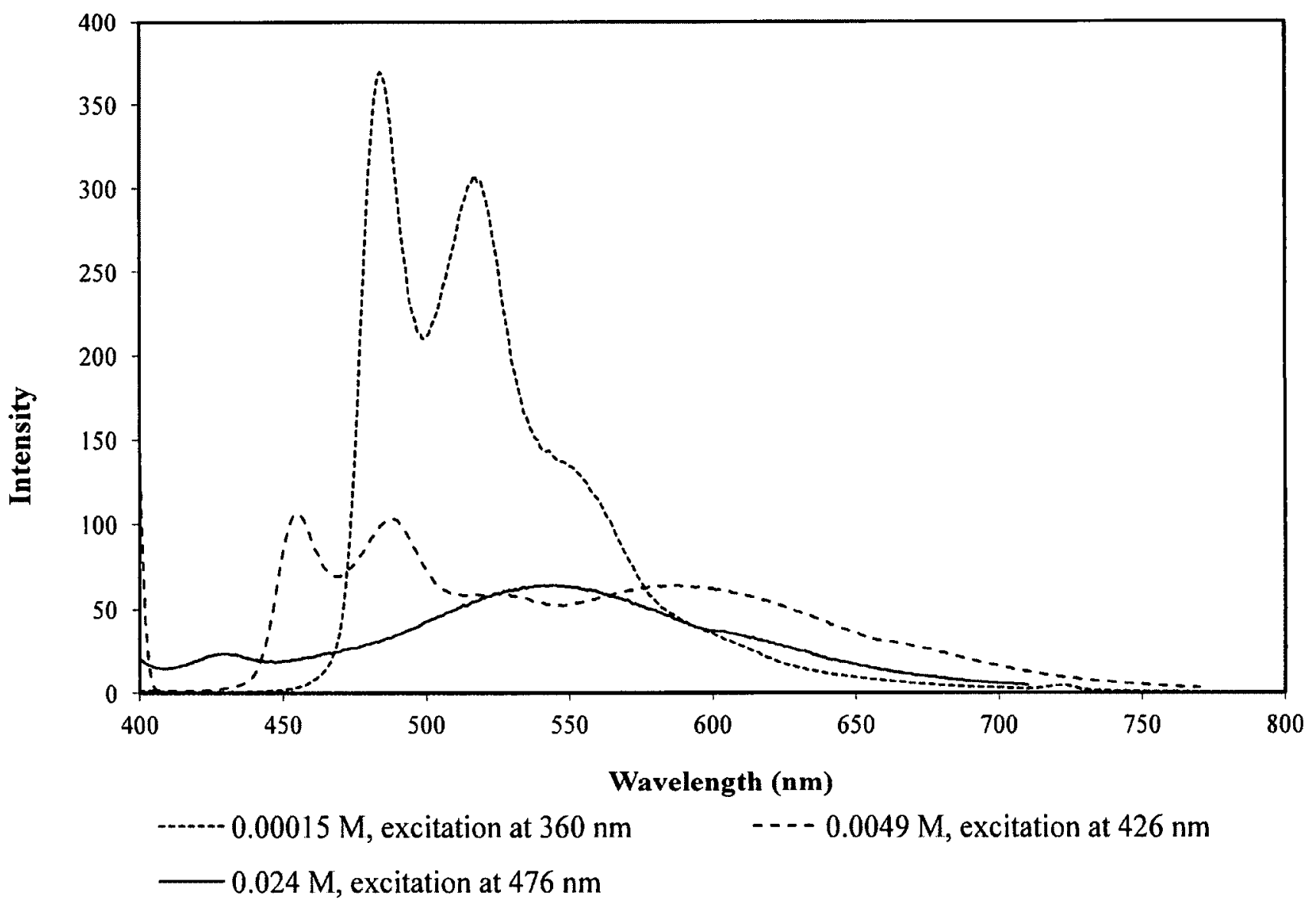

Figure C-8 Emission spectra obtained for (ppy)Pt(acac-COOH) dissolved in acetone with different excitations for each respective solution, as indicated in the legend. 


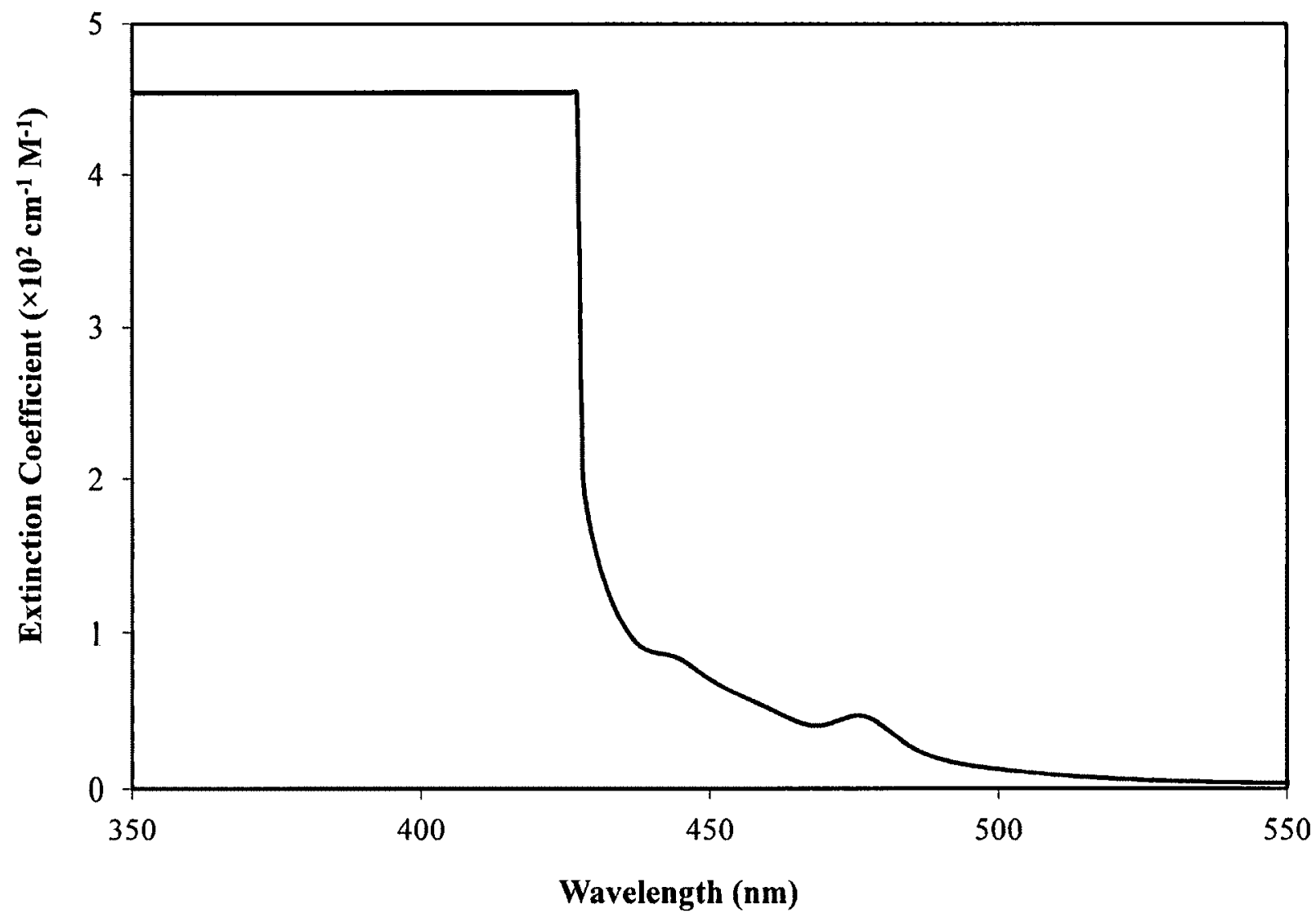

Figure C-9 Absorbance spectrum for a 0.022 M solution of (ppy)Pt(acac-COOH) in acetone. 


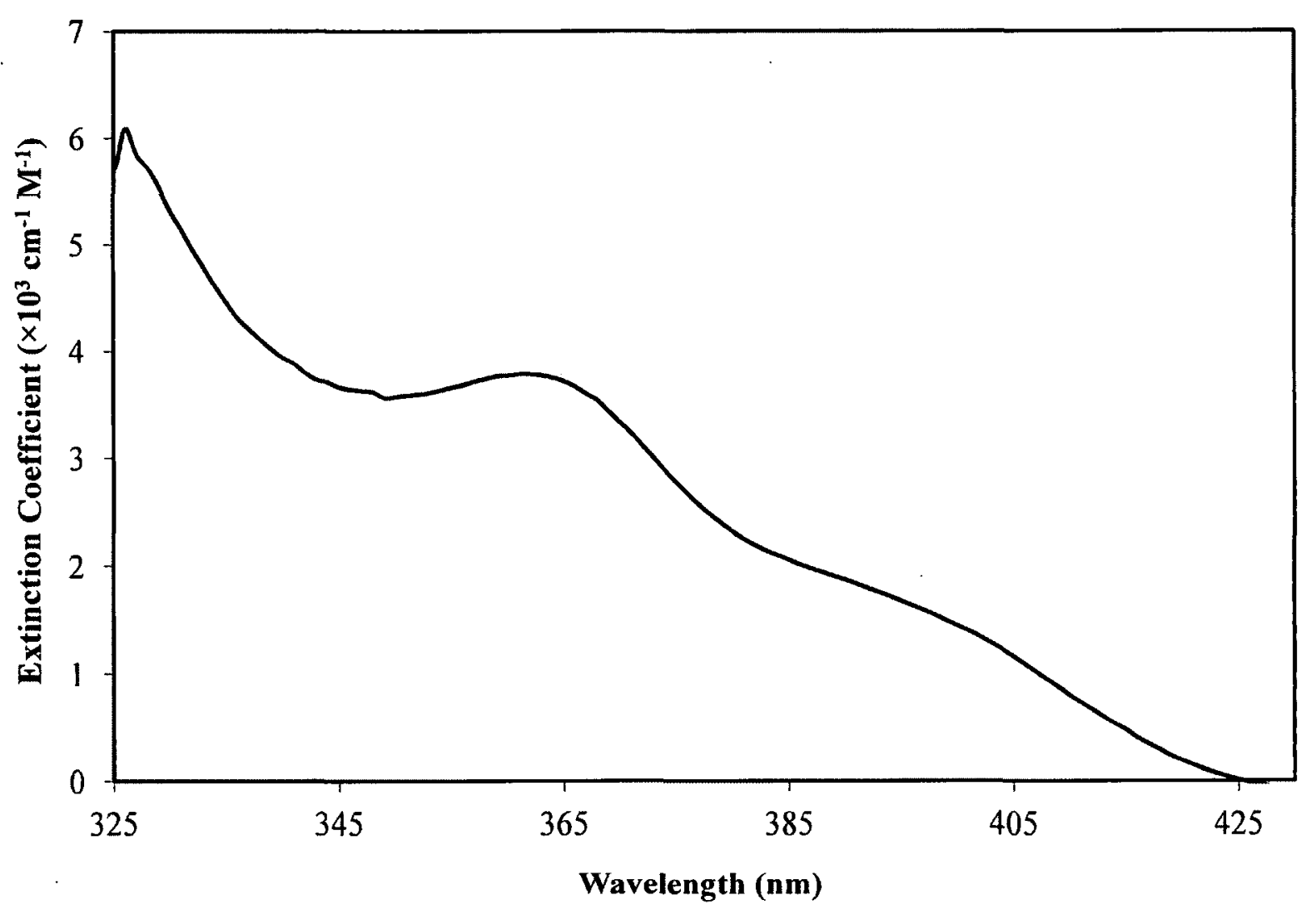

Figure C-10 Absorbance spectrum for a 0.00015 M solution of (ppy)Pt(acac-COOH) in acetone. 


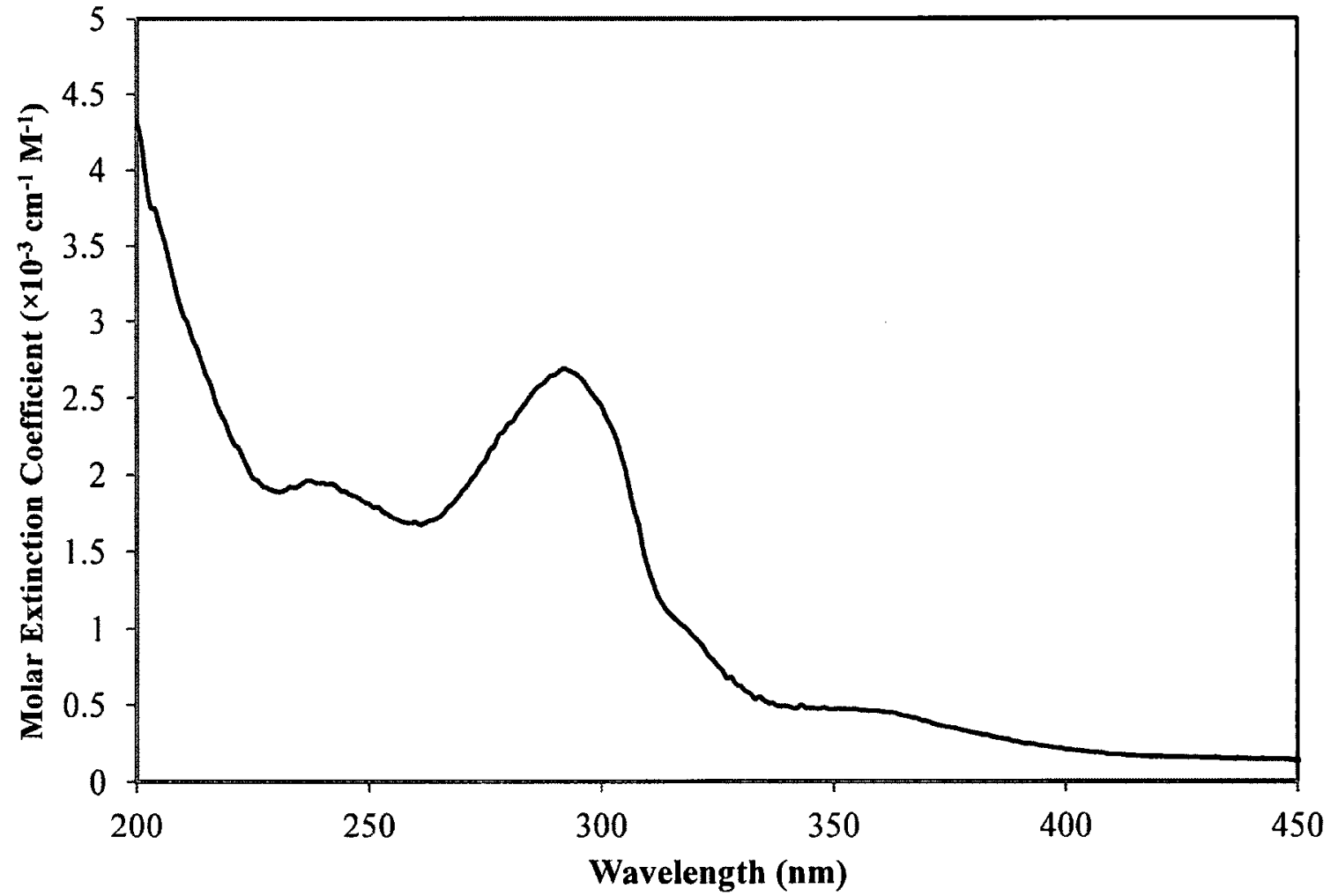

Figure C-11 Absorbance spectrum for (2,4-dfppy)Pt(acac-COOH) in methanol at a concentration of $0.194 \mathrm{mM} .{ }^{189}$ 


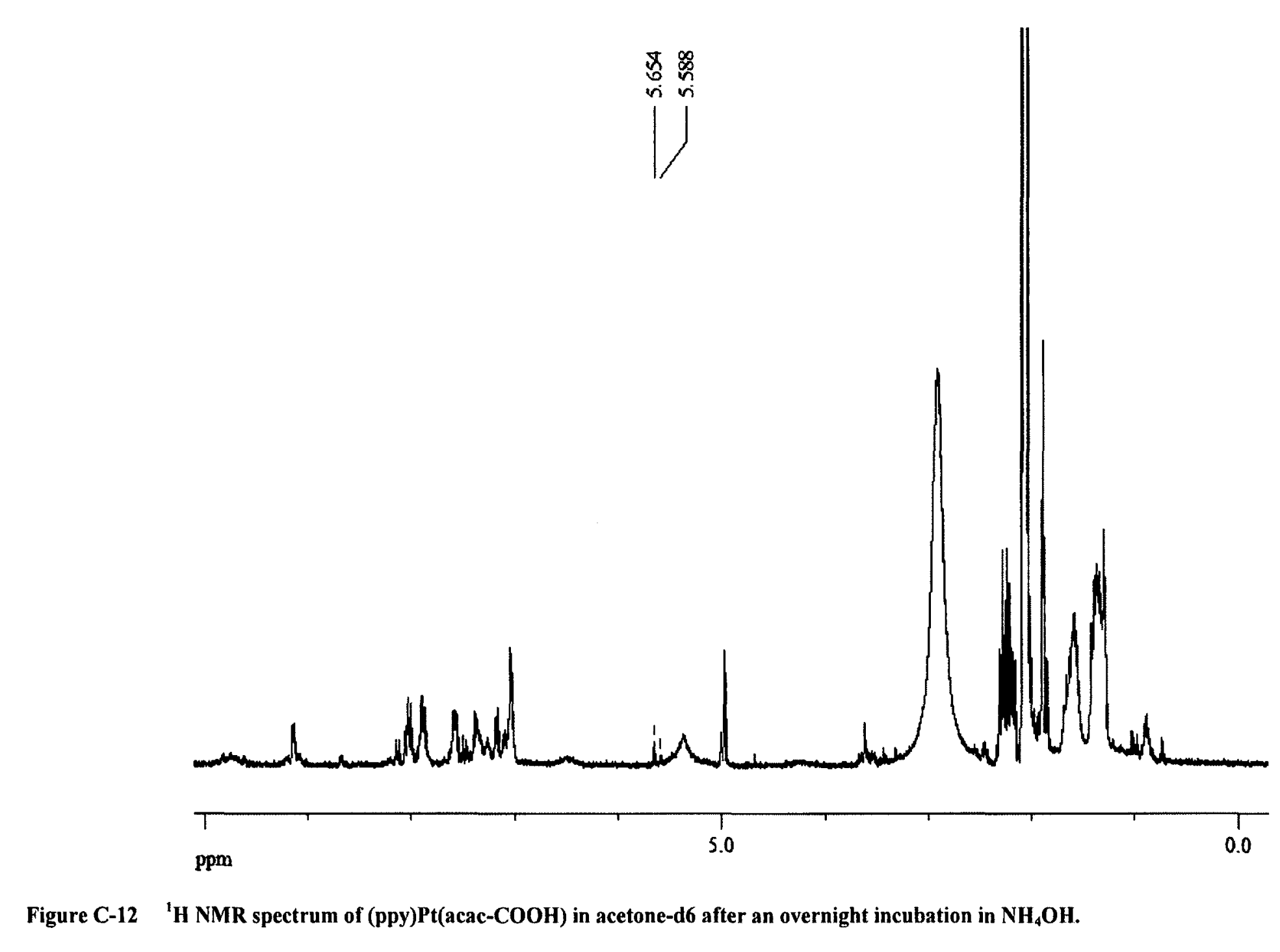




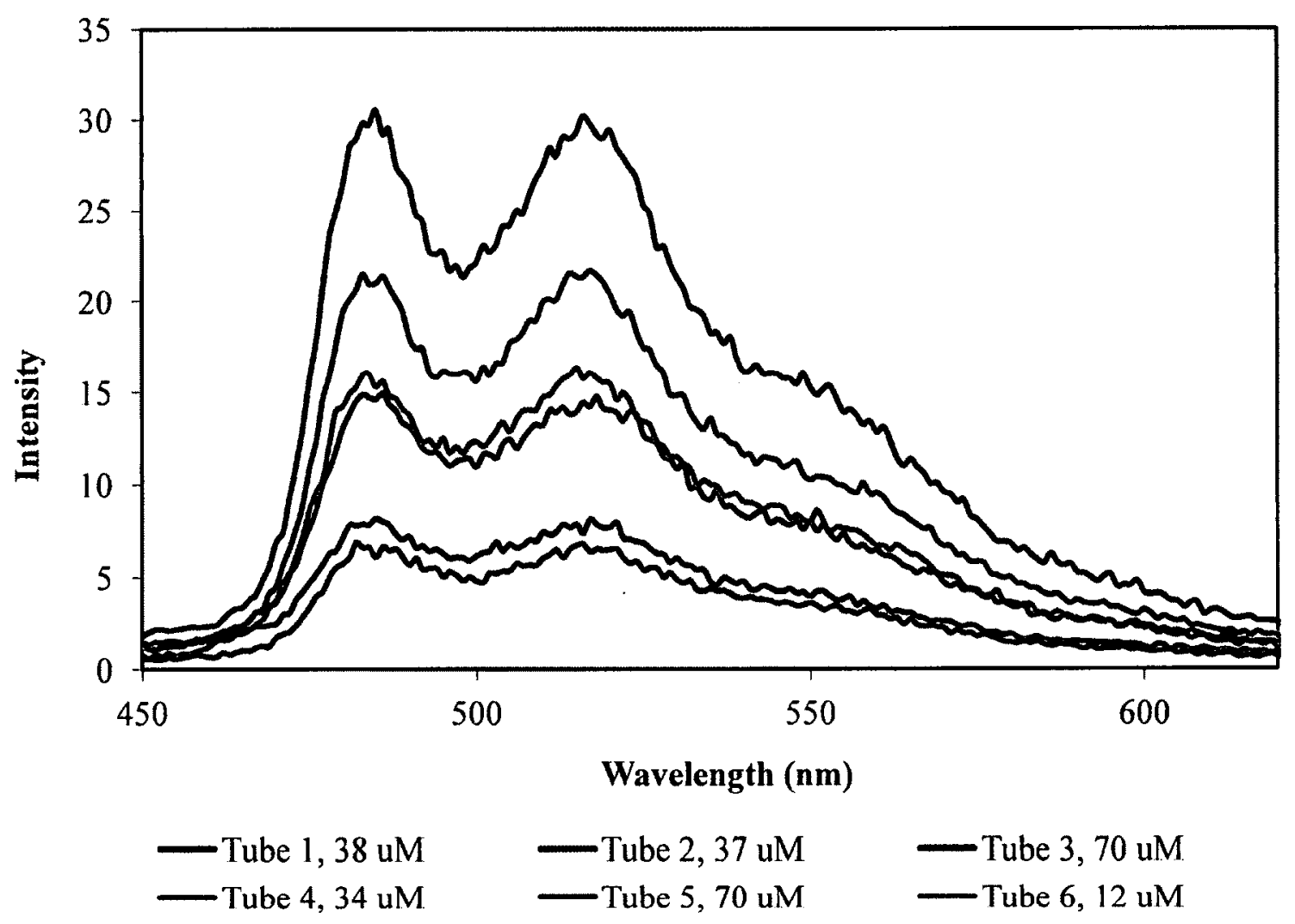

Figure C-13 Emission spectra with excitation at $320 \mathrm{~nm}$ for portions of DNA collected using RP-HPLC following reaction of (ppy)Pt(acac-COOH) with 5'-amino-modified TBA. 


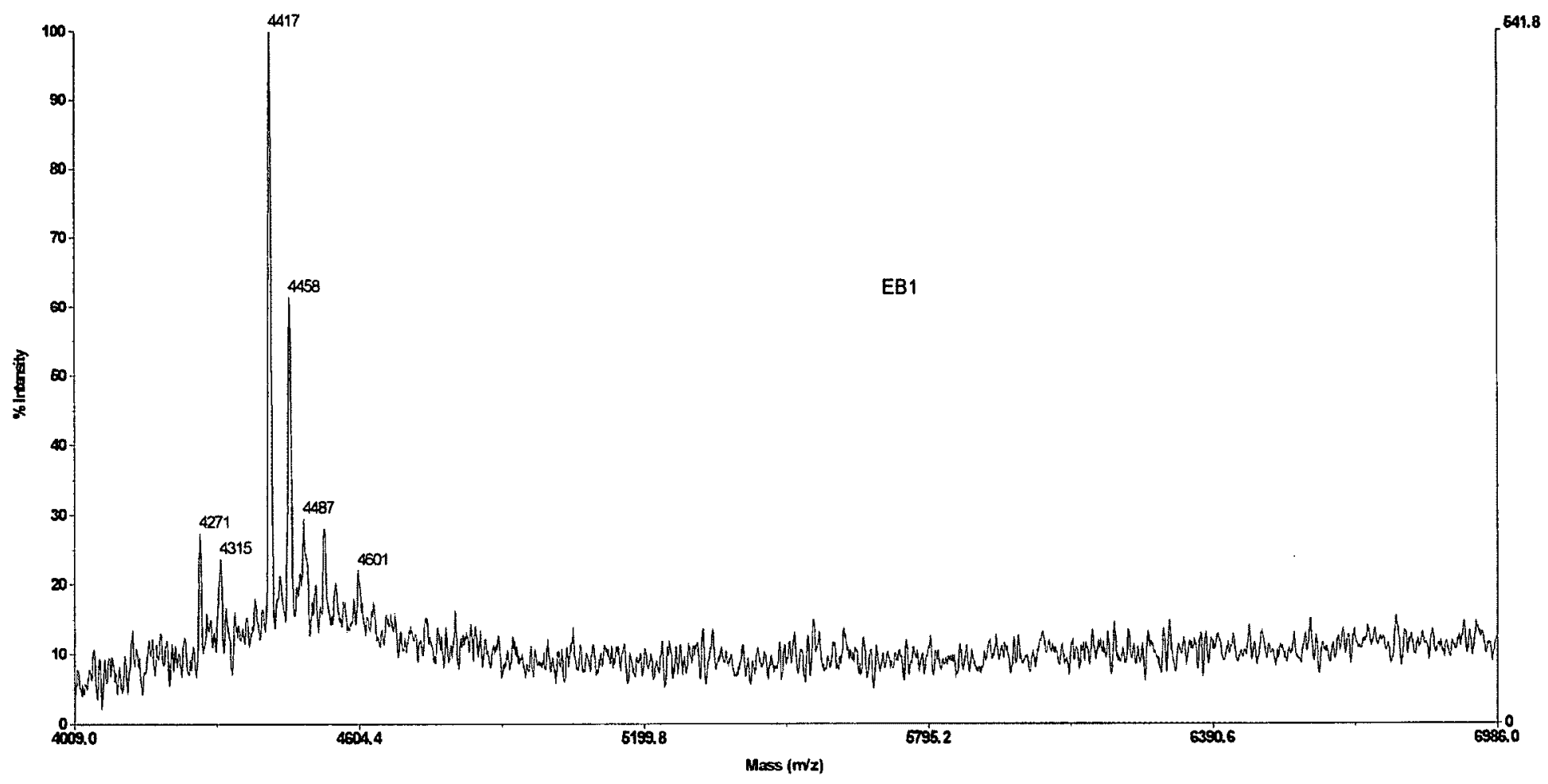

Figure C-14 MALDI MS spectrum of a 2 nmol portion of the DNA isolated following a solution-phase reaction for conjugation of (ppy)Pt(acac-

$\mathrm{COOH}$ ) to the 5'-terminus of a random sequence DNA strand. This is the same sample as that used to obtain the gel and luminescence data in Figure 4.10 . 


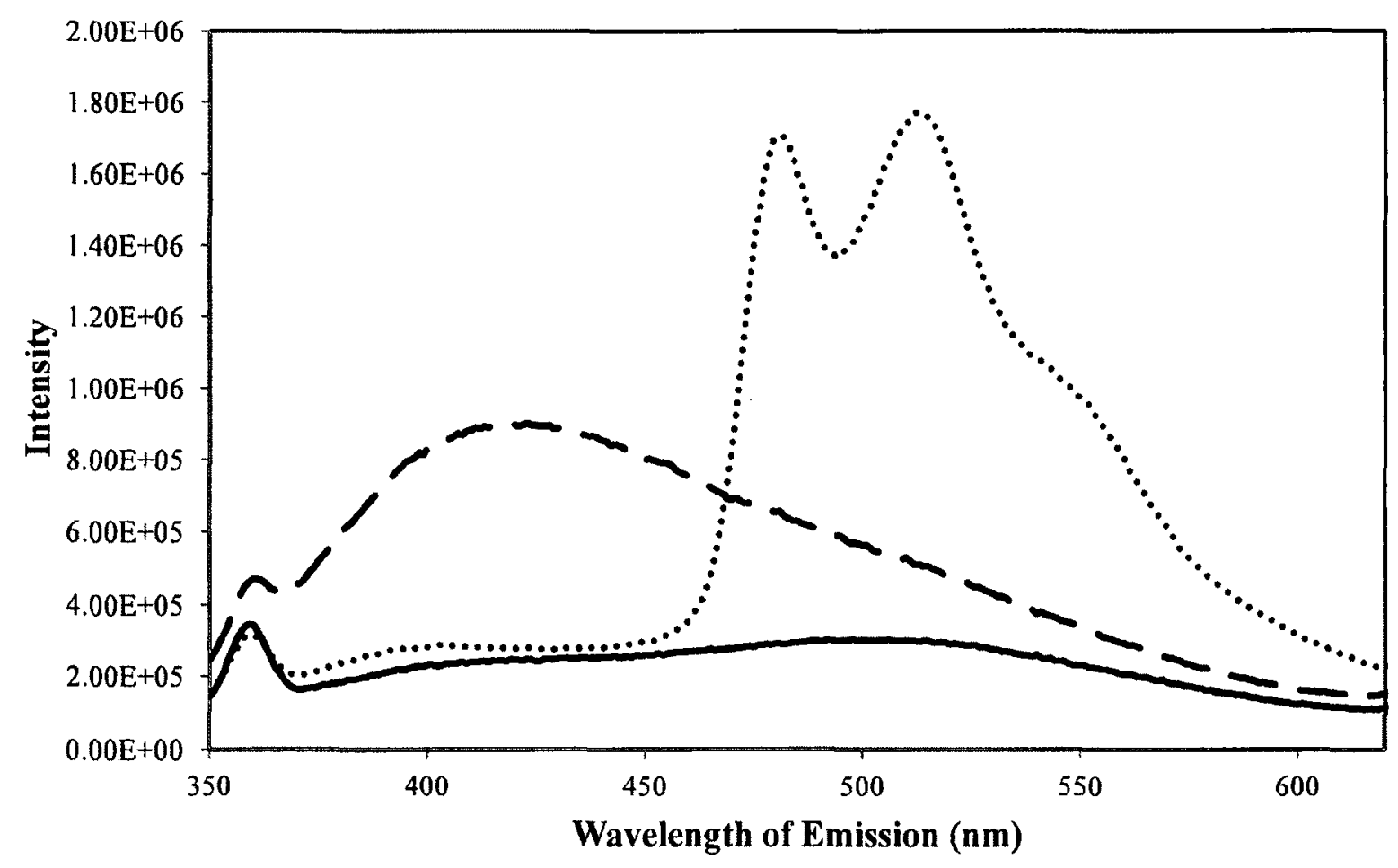

- peak $1 \quad \cdots \cdot$ peak 2 - peak 4

Figure C-15 Emission spectra for peaks from RP-HPLC purification of previously PAGE-purified Pt(II) complex-DNA. 


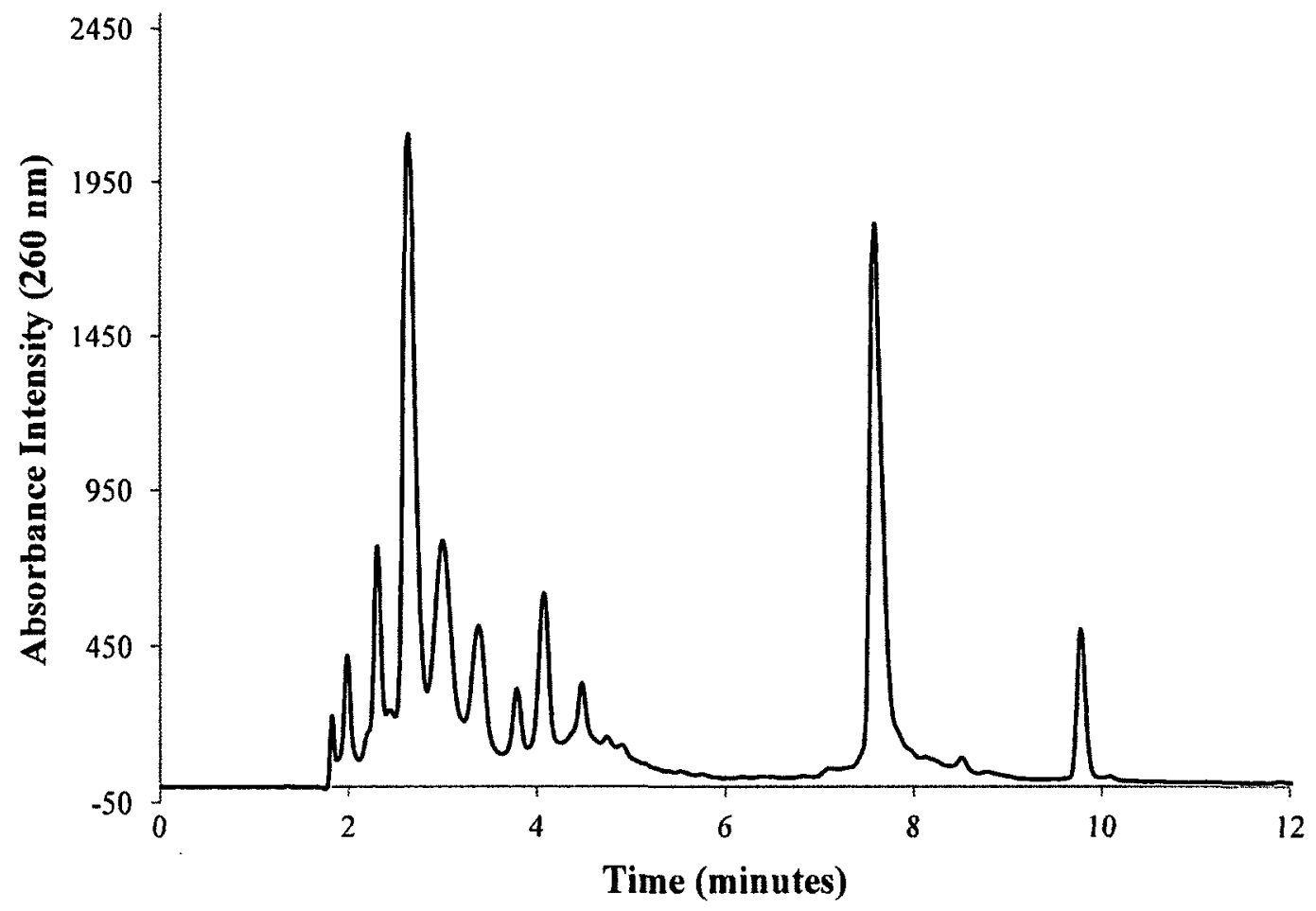

Figure C-16 RP-HPLC chromatogram (absorbance $260 \mathrm{~nm}$ ) for 3'-Pt(II) complex-DNA. 


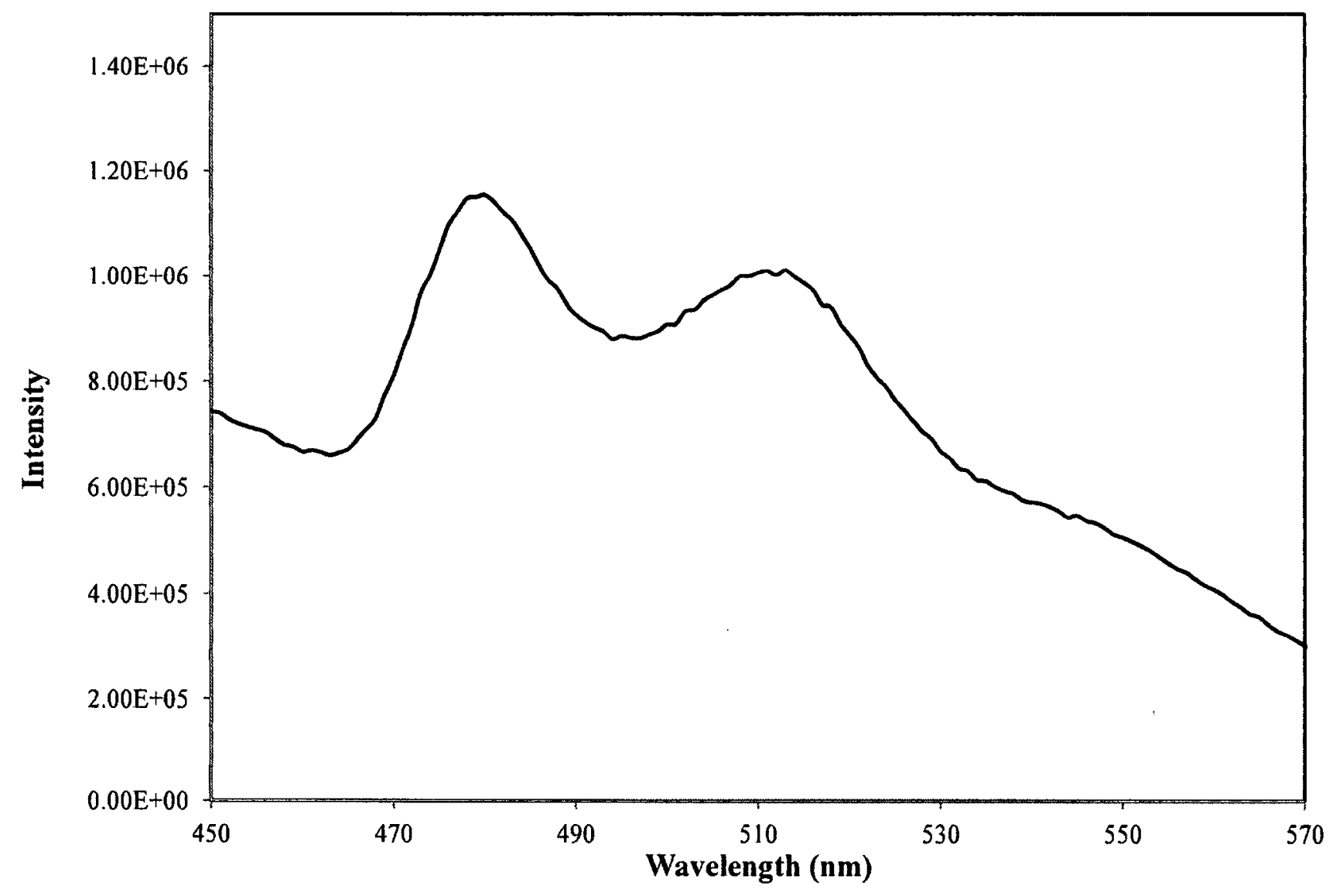

Figure C-17 Emission spectrum for sample isolated from the peak at about 8 minutes in RP-HPLC of 3'-Pt(II) complex-DNA. Excitation at $320 \mathrm{~nm}$. 


\section{References}

(1) Blackburn, G. M.; Gait, M. J.; Loakes, D.; Williams, D. M., Eds.; Nucleic Acids in Chemistry and Biology, 3rd Edition; Royal Society of Chemistry: Cambridge, UK, 2006.

(2) Li, Y.; Lu, Y., Eds.; In Functional Nucleic Acids for Analytical Applications; Springer Science+Business Media, LLC: New York, USA, 2009; .

(3) Kruger, K.; Grabowski, P. J.; Zaug, A. J.; Sands, J.; Gottschling, D. E.; Cech, T. R. Cell 1982, 31, 147-157.

(4) Robertson, D. L.; Joyce, G. F. Nature 1990, 344, 467-468.

(5) Ellington, A. D.; Szostak, J. W. Nature 1990, 346, 818-822.

(6) Tuerk, C.; Gold, L. Science 1990, 249, 505-510.

(7) Breaker, R. R.; Joyce, G. F. Chemistry \& Biology 1994, 1, 223-229.

(8) Bock, L. C.; Griffin, L. C.; Latham, J. A.; Vermaas, E. H.; Toole, J. J. Nature 1992, $355,564-566$.

(9) Ellington, A. D.; Szostak, J. W. Nature 1992, 355, 850-852.

(10) Bunka, D. H. J.; Platonova, O.; Stockley, P. G. Curr. Opin. Pharmacol. 2010, 10, 557-562.

(11) Liu, J.; Cao, Z.; Lu, Y. Chem. Rev. 2009, 109, 1948-1998.

(12) Shevchenko, Y.; Francis, T. J.; Blair, D. A. D.; Walsh, R.; DeRosa, M. C.; Albert, J. Anal. Chem. 2011, 83, 7027-7034.

(13) Ostroff, R. M.; Bigbee, W. L.; Franklin, W.; Gold, L.; Mehan, M.; Miller, Y. E.; Pass, H. I.; Rom, W. N.; Siegfried, J. M.; Stewart, A.; Walker, J. J.; Weissfeld, J. L.; Williams, S.; Zichi, D.; Brody, E. N. PLoS ONE 2011, 5, e15003.

(14) Lee, J. H.; Yigit, M. V.; Mazumdar, D.; Lu, Y. Adv. Drug Delivery Rev. 2010, 62, 592-605.

(15) Willner, I.; Shlyahovsky, B.; Zayats, M.; Willner, B. Chem. Soc. Rev. 2008, 37, 1153-1165.

(16) Xu, W.; Lu, Y. Chem. Commun. 2011, 47, 4998-5000.

(17) Yigit, M. V.; Mazumdar, D.; Kim, H. K.; Lee, J. H.; Odintsov, B.; Lu, Y. ChemBioChem 2007, 8, 1675-1678.

(18) Yigit, M. V.; Mazumdar, D.; Lu, Y. Bioconjugate Chem. 2008, 19, 412-417.

(19) Hermann, T.; Patel, D. J. Science 2000, 287, 820-825. 
(20) Zimmermann, G. R.; Jenison, R. D.; Wick, C. L.; Simorre, J. P.; Pardi, A. Nat. Struct. Biol. . 1997, 4, 644-649.

(21) Tasset, D. M.; Kubik, M. F.; Steiner, W. J. Mol. Biol. 1997, 272, 688-698.

(22) Silverman, S. K. In Artificial Functional Nucleic Acids: Aptamers, Ribozymes, and Deoxyribozymes Identified by In Vitro Selection; Li, Y., Lu, Y., Eds.; Functional Nucleic Acids for Analytical Applications; Springer Science + Business Media, LLC: New York, NY, 2009; pp 47-108.

(23) Bunka, D. H. J.; Stockley, P. G. Nat. Rev. Microbiol. 2006, 4, 588-596.

(24) Aquino-Jarquin, G.; Toscano-Garibay, J. D. Int. J. Mol. Sci. 2011, 12, 9155-9171.

(25) Wiegand, T. W.; Williams, P. B.; Dreskin, S. C.; Jouvin, M. H.; Kinet, J. P.; Tasset, D. The Journal of Immunology 1996, 157, 221-230.

(26) Stojanovic, M. N.; de Prada, P.; Landry, D. W. J. Am. Chem. Soc. 2001, 123, 49284931 .

(27) Mann, D.; Reinemann, C.; Stoltenburg, R.; Strehlitz, B. Biochem. Biophys. Res. Commun. 2005, 338, 1928-1934.

(28) Fang, X.; Tan, W. Acc. Chem. Res. 2010, 43, 48-57.

(29) Xu, Y.; Phillips, J. A.; Yan, J.; Li, Q.; Fan, Z. H.; Tan, W. Anal. Chem. 2009, 81, 7436-7442.

(30) Medley, C. D.; Bamrungsap, S.; Tan, W.; Smith, J. E. Anal. Chem. 2011, 83, 727. 734.

(31) Brody, E. N.; Willis, M. C.; Smith, J. D.; Jayasena, S.; Zichi, D.; Gold, L. Mol. Diagn. 1999, 4, 381-388.

(32) Jenison, R. D.; Gill, S. C.; Pardi, A.; Polisky, B. Science 1994, 263, 1425-1429.

(33) Sefah, K.; Phillips, J. A.; Xiong, X.; Meng, L.; Van Simaeys, D.; Chen, H.; Martin, J.; Tan, W. Analyst 2009, 134, 1765-1775.

(34) Bouchard, P. R.; Hutabarat, R. M.; Thompson, K. M. Annu. Rev. Pharmacol. Toxicol. 2010, 50, 237-257.

(35) Lee, J. F.; Stovall, G. M.; Ellington, A. D. Curr. Opin. Chem. Biol. 2006, 10, 282289.

(36) McKeague, M.; Bradley, C. R.; De Girolamo, A.; Visconti, A.; Miller, J. D.; DeRosa, M. C. Int. J. Mol. Sci. 2010, 11, 4864-4881.

(37) Cella, L. N.; Sanchez, P.; Zhong, W.; Myung, N. V.; Chen, W.; Mulchandani, A. Anal. Chem. 2010, 82, 2042-2047. 
(38) Yan, A. C.; Levy, M. RNA Biol. 2009, 6, 316-320.

(39) Beigelman, L., et al J. Biol. Chem. 1995, 270, 25702-25708.

(40) Keefe, A. D.; Cload, S. T. Curr. Opin. Chem. Biol. 2008, 12, 448-456.

(41) Vira, S.; Mekhedov, E.; Humphrey, G.; Blank, P. S. Anal. Biochem. 2010, 402, $146-$ 150 .

(42) Ohk, S. H.; Koo, O. K.; Sen, T.; Yamamoto, C. M.; Bhunia, A. K. J. Appl. Microbiol. 2010, 109, 808-817.

(43) Mascini, M., Ed.; Aptamers in Bioanalysis; John Wiley \& Sons, Inc.: Hoboken, New Jersey, 2009.

(44) Di Cera, E.; Gruber, A. Chapter 1 Thrombin: Structure, Functions, and Regulation; Maragoudakis, M. E., Tsopanoglou, N. E., Eds.; Thrombin: Physiology and Disease; Springer Science+Business Media, LLC: New York, NY, 2009; pp 1-18.

(45) Paborsky, L. R.; McCurdy, S. N.; Griffin, L. D.; Toole, J. J.; Leung, L. L. K. J. Biol. Chem. 1993, 268, 20808-20811.

(46) Kelly, J. A.; Feigon, J.; Yeates, T. O. J. Mol. Biol. 1996, 256, 417-422.

(47) Shim, J. W.; Tan, Q.; Gu, L. Q. Nucleic Acids Res. 2009, 37, 972-982.

(48) Kankia, B. I.; Marky, L. A. J. Am. Chem. Soc. 2001, 123, 10799-10804.

(49) Zhao, Q.; Li, X. F.; Shao, Y.; Le, X. C. Anal. Chem. 2008, 80, 7586-7593.

(50) Zhao, Q.; Lu, X.; Yuan, C. -.; Li, X. F.; Le, X. C. Anal. Chem. 2009, 81, 7484-7489.

(51) Hasegawa, H.; Taira, K.; Sode, K.; Ikebukuro, K. Sensors 2008, 8, 1090-1098.

(52) Engvall, E.; Perlmann, P. Immunochemistry 1971, 8, 871-874.

(53) Harris, D. C. Quantitative Chemical Analysis; W.H. Freeman and Company: United States of America, 2007; Vol. 7.

(54) Ferreira, C. S. M.; Papamichael, K.; Guilbault, G.; Schwarzacher, T.; Gariepy, J.; Missailidis, S. Anal. Bioanal. Chem. 2008, 390, 1039-1050.

(55) Vivekananda, J.; Kiel, J. L. Lab. Invest. 2006, 86, 610-618.

(56) Lai, R. Y.; Plaxco, K. W.; Heeger, A. J. Anal. Chem. 2007, 79, 229-233.

(57) Green, L. S.; Jellinek, D.; Jenison, R.; Östman, A.; Heldin, C. H.; Janjic, N. Biochemistry 1996, 35, 14413-14424.

(58) Bogomolova, A.; Aldissi, M. Biosens. Bioelectron. 2011, 26, 4099-4103. 
(59) Huizenga, D. E.; Szostak, J. W. Biochemistry 1995, 34, 656-665.

(60) Liu, J.; Mazumdar, D.; Lu, Y. Angew. Chem. Int. Ed. 2006, 45, 7955-7959.

(61) Liz-Marzán, L. M. Langmuir 2006, 22, 32-41.

(62) Mirkin, C. A.; Letsinger, R. L.; Mucic, R. C.; Storhoff, J. J. Nature 1996, 382, 607609.

(63) Becker, R. C.; Povsic, T.; Cohen, M. G.; Rusconi, C. P.; Sullenger, B. Thromb. Haemostasis 2010, 103, 586-595.

(64) Dougan, H.; Lyster, D. M.; Vo, C. V.; Stafford, A.; Weitz, J. I.; Hobbs, J. B. Nuclear Medicine \& Biology 2000, 27, 289-297.

(65) Dougan, H.; Weitz, J. I.; Stafford, A. R.; Gillespie, K. D.; Klement, P.; Hobbs, J. B.; Lyster, D. M. Nucl. Med. Biol. 2003, 30, 61-72.

(66) Kottke-Marchant, K. In The Role of Coagulation in Arterial and Venous Thrombosis; Askari, A. T., Lincoff, A. M., Eds.; Antithrombotic Drug Therapy in Cardiovascular Disease; Humana Press: New York, NY, 2010; pp 19-38.

(67) Keefe, A. D.; Pai, S.; Ellington, A. Nat. Rev. Drug Discovery 2010, 9, 537-550.

(68) Burmeister, P. E.; Wang, C.; Killough, J. R.; Lewis, S. D.; Horwitz, L. R.; Ferguson, A.; Thompson, K. M.; Pendergrast, P. S.; McCauley, T. G.; Kurz, M.; Diener, J.; Cload, S. T.; Wilson, C.; Keefe, A. D. Oligonucleotides 2006, 16, 337-351.

(69) Eckstein, F. Antisense Nucleic Acid Drug Dev. 2000, 10, 117-121.

(70) Kang, J.; Lee, M. S.; Copland III, J. A.; Luxon, B. A.; Gorenstein, D. G. Bioorg. Med. Chem. Lett. 2008, 18, 1835-1839.

(71) King, D. J.; Ventura, D. A.; Brasier, A. R.; Gorenstein, D. G. Biochemistry 1998, 37, 16489-16493.

(72) Takahashi, H.; Hamazaki, H.; Habu, Y.; Hayashi, M.; Abe, T.; Miyano-Kurosaki, N.; Takaku, H. FEBS Lett. 2004, 560, 69-74.

(73) Rusconi, C. P.; Roberts, J. D.; Pitoc, G. A.; Nimjee, S. M.; White, R. R.; Quick Jr., G.; Scardino, E.; Fay, W. P.; Sullenger, B. A. Nat. Biotechnol. 2004, 22, 1423-1428.

(74) Ng, E. W. M.; Shima, D. T.; Calias, P.; Cunningham Jr., E. T.; Guyer, D. R.; Adamis, A. P. Nat. Rev. Drug Discovery 2006, 5, 123-132.

(75) Beaucage, S. L.; Caruthers, M. H. Tetrahedron Lett. 1981, 22, 1859-1862.

(76) Matteucci, M. D.; Caruthers, M. H. J. Am. Chem. Soc. 1981, 103, 3185-3191.

(77) Caruthers, M. H. Acc. Chem. Res. 1991, 24, 278-284. 
(78) McBride, L. J.; Caruthers, M. H. Tetrahedron Lett. 1983, 24, 245-248.

(79) Thornback, J. Microarrays, Nanotechnology and Beyond.

http://www.glenresearch.com/GlenReports/GR19-14.html (accessed 06/20, 2012).

(80) Glen Research. 5'-DMS(O)MT-Amino-Modifier.

http://www.glenresearch.com/Technical/TB_DMSOMT.pdf (accessed 06/20, 2012).

(81) Nelson, P. S.; Kent, M.; Muthini, S. Nucleic Acids Res. 1992, 20, 6253-6259.

(82) Creighton, T. E. In The Physical and Chemical Basis of Molecular Biology; Helvetian Press: 2010.

(83) Ellington, A.; Pollard, J. D. J. In Purification of Oligonucleotides Using Denaturing Polyacrylamide Gel Electrophoresis; Ausubel, F. M., Brent, R., Kingston, R. E., Moore, D. D., Seidman, J. G., Smith, J. A. and Struhl, K., Eds.; Current Protocols in Molecular Biology; John Wiley \& Sons, Inc.: New York, 1998; pp 2.12.1-2.12.7.

(84) Arghavani, M. B.; Romano, L. J. Anal. Biochem. 1995, 231, 201-209.

(85) Ahuja, S., Ed.; In Handbook of Bioseparations; Separation Science and Technology; Academic Press: San Diego, California, 2000; , pp 722.

(86) Leung, K. H.; Yip, S. P. In Denaturing High-Performance Liquid Chromatography (DHPLC) for Nucleic Acid Analysis; Walker, J. M., Rapley, R., Eds.; Molecular Biomethods Handbook; Humana Press: New Jersey, U.S.A., 2008; Vol. 2, pp 89-106.

(87) Cavaluzzi, M. J.; Borer, P. N. Nucleic Acids Res. 2004, 32, el13.

(88) Femino, A. M.; Fogarty, K.; Lifshitz, L. M.; Carrington, W.; Singer, R. H. Methods Enzymol. 2003, 361, 245-304.

(89) Tinoco, I. J. J. Am. Chem. Soc. 1960, 82, 4785-4790.

(90) Bagalkot, V.; Zhang, L.; Levy-Nissenbaum, E.; Jon, S.; Kantoff, P. W.; Langer, R.; Farokhzad, O. C. Nano Lett. 2007, 7, 3065-3070.

(91) Pu, Y.; Zhu, Z.; Liu, H.; Zhang, J.; Liu, J.; Tan, W. Anal. Bioanal. Chem. 2010, 397, $3225-3233$.

(92) Rockey, W. M.; Huang, L.; Kloepping, K. C.; Baumhover, N. J.; Giangrande, P. H.; Schultz, M. K. Bioorg. Med. Chem. 2011, 19, 4080-4090.

(93) Werner, E. J.; Datta, A.; Jocher, C. J.; Raymond, K. N. Angew. Chem. , Int. Ed. Engl. 2008, 47, 8568-8580.

(94) Iggo, J. A. NMR: Spectoscopy in Inorganic Chemistry; Oxford Chemistry Primers; Oxford University Press Inc., New York: United States, 1999. 
(95) Smith, R. C.; Lange, R. C. Understanding Magnetic Resonance Imaging; CRC Press LLC: United States of America, 1998.

(96) Weishaupt, D.; Köchli, V. D.; Marincek, B. How does MRI work? An Introduction to the Physics and Function of Magnetic Resonance Imaging; Springer-Verlag Berlin Heidelberg New York: Germany, 2003.

(97) Nelson, J. H. In Nuclear Magnetic Resonance Spectroscopy; Pearson Education, Inc.: Upper Saddle River, NJ, 2003.

(98) Liney, G. In Chapter I The Basics; MRI in Clinical Practice; Springer-Verlag London Limited 2006: United Kingdom, 2006; pp 3-25.

(99) Keeler, J. . Understanding NMR Spectroscopy, Second Edition; John Wiley \& Sons, Ltd: United Kingdom, 2010.

(100) Tóth, É.; Helm, L.; Merbach, A. Top. Curr. Chem. 2002, 221, 61-101.

(101) Caravan, P.; Ellison, J. J.; McMurry, T. J.; Lauffer, R. B. Chem. Rev. 1999, 99, 2293-2352.

(102) Botta, M.; Tei, L. Eur. J. Inorg. Chem. 2012, 1945-1960.

(103) Cotton, S. Lanthanide and Actinide Chemistry; John Wiley \& Sons Ltd.: Chichester, England, 2006.

(104) Idée, J. M.; Port, M.; Robic, C.; Medina, C.; Sabatou, M.; Corot, C. Journal of Magnetic Resonance Imaging 2009, 30, 1249-1258.

(105) Lin, S. P.; Brown, J. J. Journal of Magnetic Resonance Imaging 2007, 25, 884-899.

(106) Caravan, P. Acc. Chem. Res. 2009, 42, 851-862.

(107) Caravan, P.; Zhang, Z. Eur. J. Inorg. Chem. 2012, , 1916-1923.

(108) Artemov, D. J. Cell. Biochem. 2003, 90, 518-524.

(109) Lauffer, R. B.; Parmelee, D. J.; Dunham, S. U.; Ouellet, H. S.; Dolan, R. P.; Witte, S.; McMurry, T. J.; Walovitch, R. C. Radiology 1998, 207, 529-538.

(110) Schwartz, L. H.; Seltzer, S. E.; Tempany, C. M. C.; Silverman, S. G.; PiwnicaWorms, D. R.; Adams, D. F.; Herman, L.; Herman, L. A.; Hooshmand, R. Journal of Magnetic Resonance Imaging 1995, 5, 566-570.

(111) Kielar, F.; Tei, L.; Terreno, E.; Botta, M. J. Am. Chem. Soc. 2010, 132, 7836-7837.

(112) Kamaly, N.; Kalber, T.; Thanou, M.; Bell, J. D.; Miller, A. D. Bioconjugate Chem. 2009, 20, 648-655. 
(113) Howes, P.; Green, M.; Bowers, A.; Parker, D.; Varma, G.; Kallumadil, M.; Hughes, M.; Warley, A.; Brain, A.; Botnar, R. J. Am. Chem. Soc. 2010, 132, 9833-9842.

(114) Liepold, L. O.; Abedin, M. J.; Buckhouse, E. D.; Frank, J. A.; Young, M. J.; Douglas, T. Nano Lett. 2009, 9, 4520-4526.

(115) Thurecht, K. J.; Blakey, I.; Peng, H.; Squires, O.; Hsu, S.; Alexander, C.; Whittaker, A. K. J. Am. Chem. Soc. 2010, 132, 5336-5337.

(116) Doiron, A. L.; Chu, K.; Ali, A.; Brannon-Peppas, L. Proc. Natl. Acad. Sci. U. S. A. 2008, 105, 17232-17237.

(117) Qiao, R.; Yang, C.; Gao, M. J. Mater. Chem. 2009, 19, 6274-6293.

(118) Bamrungsap, S.; Chen, T.; Shukoor, M. I.; Chen, Z.; Sefah, K.; Chen, Y.; Tan, W. ACS Nano 2012, 6, 3974-3981.

(119) De León-Rodríguez, L. M.; Lubag, A.; Udugamasooriya, D. G.; Proneth, B.; Brekken, R. A.; Sun, X.; Kodadek, T.; Sherry, A. D. J. Am. Chem. Soc. 2010, 132, 12829-12831.

(120) Shapiro, M. G.; Westmeyer, G. G.; Romero, P. A.; Szablowski, J. O.; Küster, B.; Shah, A.; Otey, C. R.; Langer, R.; Arnold, F. H.; Jasanoff, A. Nat. Biotechnol. 2010, 28, 264-272.

(121) Shapiro, M. G.; Szablowski, J. O.; Langer, R.; Jasanoff, A. J. Am. Chem. Soc. 2009, 131, 2484-2486.

(122) Hines, J. V.; Ammar, G. M.; Buss, J.; Schmalbrock, P. Bioconjugate Chem. 1999, $10,155-158$.

(123) Cai, J.; Shapiro, E. M.; Hamilton, A. D. Bioconjugate Chem. 2009, 20, 205-208.

(124) Caravan, P.; Farrar, C. T.; Frullano, L.; Uppal, R. Contrast Media Mol. Imaging 2009, 4, 89-100.

(125) Yang, J. J., et al J. Am. Chem. Soc. 2008, 130, 9260-9267.

(126) Tóth, É.; Burai, L.; Brücher, E.; Merbach, A. Dalton Trans. 1997, 1587-1594.

(127) Powell, D. H.; Dhubhghaill, O. M. N.; Pubanz, D.; Helm, L.; Lebedev, Y. S.; Schlaepfer, W.; Merbach, A. E. J. Am. Chem. Soc. 1996, 118, 9333-9346.

(128) IUPAC In Compendium of Chemical Terminology, 2nd ed. (the "Gold Book"); McNaught, A. D., Wilkinson, A., Eds.; Blackwell Scientific Publications: Oxford, 1997.

(129) Caravan, P.; Parigi, G.; Chasse, J. M.; Cloutier, N. J.; Ellison, J. J.; Lauffer, R. B.; Luchinat, C.; McDermid, S. A.; Spiller, M.; McMurry, T. J. Inorg. Chem. 2007, 46, $6632-6639$. 
(130) Brasch, R. C.; McNamara, M. T.; Ehman, R. L.; Couet, W. R.; Tozer, T. N.; Sosnovsky, G.; Maheswara Rao, N. U.; Prakash, I. Eur. J. Med. Chem. 1989, 24, 335340 .

(131) Hermann, P.; Kotek, J.; Kubíček, V.; Lukeš, I. Dalton Trans. 2008, 23, 3027-3047.

(132) Jing, M.; Bowser, M. T. Anal. Chim. Acta 2011, 686, 9-18.

(133) Grobner, T. Nephrol. Dial. Transplant 2006, 21, 1104-1108.

(134) Broome, D. R. Eur. J. Radiol. 1008, 66, 230-234.

(135) Gupta, A.; Shamseddin, M. K.; Khaira, A. Clinical and Experimental Dermatology 2011, 36, 763-768.

(136) Penfield, J. G.; Reilly, R. F. Seminars in Dialysis 2011, 24, 480-486.

(137) Laurent, S.; Vander Elst, L.; Copoix, F.; Muller, R. N. Invest. Radiol. 2001, 36, 115-122.

(138) Laurent, S.; Vander Elst, L.; Muller, R. N. Contrast Media Mol. Imaging 2006, 1, 128-137.

(139) Munshi, K. N.; Dey, A. K. Microchim. Acta 1968, 56, 1059-1065.

(140) Laurent, S.; Vander Elst, L.; Henoumont, C.; Muller, R. N. Contrast Media Mol. Imaging 2010, 5, 305-308.

(141) Derome, A. E. In Modern NMR Techniques for Chemistry Research; Pergamon: Oxford, U.K., 1987; pp 86-89.

(142) Clayden, J.; Greeves, N.; Warren, S.; Wothers, P. In Organic Chemistry; Oxford University Press: United States, 2001.

(143) Bernard, E. D.; Beking, M. A.; Rajamanickam, K.; Tsai, E. C.; DeRosa, M. C. J. Biol. Inorg. Chem. 2012, DOI 10.1007/s00775-012-0930-z.

(144) Feng, J.; Sun, G.; Pei, F.; Liu, M. Bioorg. Med. Chem. 2003, 11, 3359-3366.

(145) Duarte, M. G.; Gil, M. H.; Peters, J. A.; Colet, J. M.; Vander Elst, L.; Muller, R. N.; Geraldes, C. F. G. C. Bioconjugate Chem. 2001, 12, 170-177.

(146) Marckmann, P.; Skov, L.; Rossen, K.; Dupont, A.; Damholt, M. B.; Heaf, J. G.; Thomsen, H. S. J. Am. Soc. Nephrol. 2006, 17, 2359-2362.

(147) Port, M.; Idée, J. M.; Medina, C.; Robic, C.; Sabatou, M.; Corot, C. BioMetals 2008, 21, 469-490.

(148) Ohyoshi, E.; Kohata, S. J. Inorg. Biochem. 1993, 52, 157-163. 
(149) Luderer, F.; Walschus, U. Top. Curr. Chem. 2005, 260, 37-56.

(150) Duncan, M. R.; Lee, J. M.; Warchol, M. P. Int. J. Pharm. 1995, 120, 179-188.

(151) Sigma-Aldrich. Sigmacote ${ }^{\circledR}$ Product Information.

http://www sigmaaldrich.com/etc/medialib/docs/Sigma/Product_Information_Sheet/1/sl2pis.Par.0001.File. tmp/sl2pis.pdf(accessed 07/14, 2012).

(152) Sun, G.; Feng, J.; Jing, F.; Pei, F.; Liu, M. J. Magn. Magn. Mater. 2003, 265, 123129.

(153) Isaacman, S.; Kumar, R.; del Barco, E.; Kent, A. D.; Canary, J. W.; Jerschow, A. Polyhedron 2005, 24, 2691-2694.

(154) Aime, S.; Botta, M.; Panero, M.; Grandi, M.; Uggeri, F. Magn. Reson. Chem. 1991, $29,923-927$.

(155) Caravan, P. Chem. Soc. Rev. 2006, 35, 512-523.

(156) Millero, F. J.; Dexter, R.; Hoff, E. J. Chem. Eng. Data 1971, 16, 85-37.

(157) "Viscosity of Liquids" in CRC Handbook of Chemistry and Physics, Internet Version 2006, Lide, D.R., Ed.; Taylor and Francis: Boca Raton, FL, 2006.

(158) Rohrer, M.; Bauer, H.; Mintorovitch, J.; Requardt, M.; Weinmann, H. J. Invest. Radiol. 2005, 40, 715-724.

(159) Rudovský, J.; Botta, M.; Hermann, P.; Hardcastle, K. I.; Lukeš, I.; Aime, S. Bioconjugate Chem. 2006, 17, 975-987.

(160) Zhang, Z.; Greenfield, M. T.; Spiller, M.; McMurry, T. J.; Lauffer, R. B.; Caravan, P. Angew. Chem. Int. Ed. 2005, 44, 6766-6769.

(161) Parac-Vogt, T. N.; Kimpe, K.; Laurent, S.; Piérart, C.; Vander Elst, L.; Muller, R. N.; Binnemans, K. Eur. J. Inorg. Chem. 2004, 3538-3543.

(162) Mastarone, D. J.; Harrison, V. S. R.; Eckermann, A. L.; Parigi, G.; Luchinat, C.; Meade, T. J. J. Am. Chem. Soc. 2011, 133, 5329-5337.

(163) Avedano, S.; Tei, L.; Lombardi, A.; Giovenzana, G. B.; Aime, S.; Longo, D.; Botta, M. Chem. Commun. 2007, 4726-4728.

(164) Janas, T.; Janas, T. Cellular \& Molecular Biology Letters 2011, 16, 25-39.

(165) Shangguan, D.; Cao, Z.; Meng, L.; Mallikaratchy, P.; Sefah, K.; Wang, H.; Li, Y.; Tan, W. J. Proteome Res. 2008, 7, 2133-2139.

(166) Berezovski, M. V.; Lechmann, M.; Musheev, M. U.; Mak, T. W.; Krylov, S. N. J. Am. Chem. Soc. 2008, 130, 9137-9143. 
(167) Colman, R. W.; Marder, V. J.; Clowes, A. W.; George, J. N.; Goldhaber, S. Z. In Hemostasis and Thrombosis: Basic Principles and Clinical Practice, Fifth Edition; Lippincott Wiliams \& Wilkins: Philadelphia, PA, 2006; pp 1822.

(168) Oster, Z. H.; Srivastava, S. C.; Som, P.; Meinken, G. E.; Scudder, L. E.; Yamamoto, K.; Atkins, H. L.; Brill, A. B.; Coller, B. S. Proc. Natl. Acad. Sci. U. S. A. 1985, 82, 3465-3468.

(169) Spuentrup, E.; Buecker, A.; Katoh, M.; Wiethoff, A. J.; Parsons Jr, E. C.; Botnar, R. M.; Weisskoff, R. M.; Graham, P. B.; Manning, W. J.; Günther, R. W. Circulation 2005, 111, 1377-1382.

(170) Port, M.; Corot, C.; Raynal, I.; Idée, J. M.; Dencausse, A.; Lancelot, E.; Meyer, D.; Bonnemain, B.; Lautrou, J. Invest. Radiol. 2001, 36, 445-454.

(171) Liu, H.; Zhu, Z.; Kang, H.; Wu, Y.; Sefan, K.; Tan, W. Chem. Eur. J. 2010, 16, 3791-3797.

(172) Kelkar, S. S.; Reineke, T. M. Bioconjugate Chem. 2011, 22, 1879-1903.

(173) Li, S.; Goins, B.; Zhang, L.; Bao, A. Bioconjugate Chem. 2012, 23, 1322-1332.

(174) Kim, D.; Jeong, Y. Y.; Jon, S. ACS Nano 2010, 4, 3689-3696.

(175) Boutorine, A. S.; Sun, J. S. In Postsynthetic Functionalization of Triple HelixForming Oligonucleotides; Derdewijn, P., Ed.; Methods in Molecular Biology, vol. 288: Oligonucleotide Synthesis: Methods and Applications; Humana Press Inc.: Totowa, NJ, 2005; Vol. 288, pp 251-260.

(176) Vargas-Baca, I.; Mitra, D.; Zulyniak, H. J.; Banerjee, J.; Sleiman, H. F. Angew. Chem., Int. Ed. Engl. 2001, 40, 4629-4632.

(177) Lipari, G.; Szabo, A. J. Am. Chem. Soc. 1982, 104, 4546-4559.

(178) Patel, D. J.; Suri, A. K.; Jiang, F.; Jiang, L.; Fan, P.; Kumar, R. A.; Nonin, S. J. Mol. Biol. 1997, 272, 645-664.

(179) de los Santos, C. In 7.03 - Probing DNA Structure by NMR Spectroscopy; Â Â Otto Meth-Cohn,Sir Derek Barton and Koji Nakanishi, Eds.; Comprehensive Natural Products Chemistry; Pergamon: Oxford, 1999; pp 55-80.

(180) Sindbert, S.; Kalinin, S.; Nguyen, H.; Kienzler, A.; Clima, L.; Bannwarth, W.; Appel, B.; Müller, S.; Seidel, C. A. M. J. Am. Chem. Soc. 2011, 133, 2463-2480.

(181) Heyduk, E.; Heyduk, T. Anal. Chem. 2005, 77, 1147-1156.

(182) Birks, J. B. Rep. Prog. Phys. 1975, 38, 903-974.

(183) Østergaard, M. E.; Hrdlicka, P. J. Chem. Soc. Rev. 2011, 40, 5771-5788. 
(184) Shi, C.; Gu, H.; Ma, C. Anal. Biochem. 2010, 400, 99-102.

(185) Roy, R.; Hohng, S.; Ha, T. Nat. Methods 2008, 5, 507-516.

(186) Brooks, J.; Babayan, Y.; Lamansky, S.; Djurovich, P. I.; Tsyba, I.; Bau, R.; Thompson, M. E. Inorg. Chem. 2002, 41, 3055-3066.

(187) Farley, S. J.; Rochester, D. L.; Thompson, A. L.; Howard, J. A. K.; Williams, J. A. G. Inorg. Chem. 2005, 44, 9690-9703.

(188) Weizman, H.; Yitzhak, T. J. Am. Chem. Soc. 2002, 124, 1568-1569.

(189) Hosseini, B. Developing Luminescent Biosensors for the Detection of Structural Changes in DNA Aptamers, Carleton University, Ottawa, 2008.

(190) Han, S. Y.; Kim, Y. A. Tetrahedron 2004, 60, 2447-2467.

(191) El-Faham, A.; Albericio, F. Chem. Rev. 2011, 111, 6557-6602.

(192) Lohse, O.; Thevenin, P.; Waldvogel, E. Synlett 1999, 45-48.

(193) Kinnory, D. S.; Takeda, Y.; Greenberg, D. M. J. Biol. Chem. 1955, 212, 379-383.

(194) Dandliker, P. J.; Núñez, M. E.; Barton, J. K. Biochemistry 1998, 37, 6491-6502.

(195) Glen Research. Procedure for Ultramild Deprotection of Oligodeoxynucleotides. http://www.glenresearch.com/Technical/TB UltraMild Deprotection.pdf (accessed 07/29, 2012).

(196) Glen Research. Pre-synthesis Labelling of Amino modifier C3 or C7 CPG. http://www.glenresearch.com/Technical/TB PreSynthesis Labeling.html (accessed 08/01, 2012).

(197) "Surface Tension of Common Liquids", in CRC Handbook of Chemistry and Physics, Internet Version 2006, Lide, D.R., Ed.; Taylor and Francis: Boca Raton, FL, 2006.

(198) Cockburn, B. N.; Howe, D. V.; Keating, T.; Johnson, B. F. G.; Lewis, J. J. Chem. Soc. , Dalton Trans. 1973, 404-410.

(199) Giubellina, N.; Mangelinckx, S.; Törnroos, K. W.; De Kimpe, N. J. Org. Chem. 2006, 71, 5881-5887.

(200) Pierce Biotechnology. Instructions, NHS and Sulfo-NHS.

(201) Sigma-Aldrich. FTNMR of 2-phenylquinoline. http://www.sigmaaldrich.com/spectra/fnmr/FNMR006032.PDF (accessed 07/28, 2012). 